THE UNIVERSITY OF CHICAGO

DARK MATTER LIMITS FROM A 2L C3F8 FILLED

BUBBLE CHAMBER

A DISSERTATION SUBMITTED TO

THE FACULTY OF THE DIVISION OF THE PHYSICAL SCIENCES

IN CANDIDACY FOR THE DEGREE OF

DOCTOR OF PHILOSOPHY

DEPARTMENT OF PHYSICS

BY

ALAN EDWARD ROBINSON

CHICAGO, ILLINOIS

DECEMBER 2015 
Copyright (c) 2015 by Alan Edward Robinson

All rights reserved 
For Toby, my wife

Love;

there's a hell of a dark universe next door;

let's go.

Based on a quote by E.E. Cummings 


\title{
Dark Matter Limits from a 2L C3F8 Filled \\ Bubble Chamber
}

by

Alan Edward Robinson

\author{
Dissertation Advisor \\ Juan I. Collar, Professor \\ Dissertation Committee \\ Luca Grandi, Assistant Professor \\ Philippe Guyot-Sionnest, Professor \\ Dan Hooper, Associate Professor
}

\begin{abstract}
The PICO-2 $\mathrm{L} \mathrm{C}_{3} \mathrm{~F}_{8}$ bubble chamber search for Weakly Interacting Massive Particle (WIMP) dark matter was operated in the SNOLAB underground laboratory at the same location as the previous $\mathrm{CF}_{3} \mathrm{I}$ filled COUPP-4kg detector. Neutron calibrations using photoneutron sources in $\mathrm{C}_{3} \mathrm{~F}_{8}$ and $\mathrm{CF}_{3} \mathrm{I}$ filled calibration bubble chambers were performed to verify the sensitivity of these target fluids to dark matter scattering. This data was combined with similar measurements using a low-energy neutron beam at the University of Montreal and in situ calibrations of the PICO-2L and COUPP- $4 \mathrm{~kg}$ detectors. $\mathrm{C}_{3} \mathrm{~F}_{8}$ provides much greater sensitivity to WIMP-proton scattering than $\mathrm{CF}_{3} \mathrm{I}$ in bubble chamber detectors.

PICO-2L searched for dark matter recoils with energy thresholds below $10 \mathrm{keV}$. Radiopurity assays of detector materials were performed and the expected neutron recoil background was evaluated to be $1.6_{-0.9}^{+0.3}$ single bubble events during the $211.5 \mathrm{~kg}$-day exposure. Twelve single bubble dark matter candidate events were observed. These events were not uniformly distributed in time, and were likely caused by particulates in the active volume. Despite this background, PICO-2L sets a world-leading upper limit to the WIMP-proton spin dependent scattering cross-section.
\end{abstract}




\section{Acknowledgments}

This thesis and its results rely on an immense amount of collaborative work and history. Many aspects of this thesis rely on research in nuclear physics, physical chemistry, and cosmology. Well written papers from Jack Gibbons, Dick Macklin, and Hal Schmitt at Oak Ridge National Laboratory and Walter John and John Prosser at Lawrence Livermore of difficult precision measurements of neutron producing reactions around 1960 were critical to most of the calibrations presented here. This and other dark matter research would not be possible without precision nuclear physics measurements, and data compilations such as ENDF/B, JENDL, and Mughabghab's Atlas of Neutron Resonances. Similarly, the work and products of the National Institute of Science and Technology were critical in understanding the physics of PICO bubble chambers and radiation within them.

For the use of their equipment and expertise, and for sparking my interest in Nuclear Physics, I thank Ernst Rehm, Claudio Ugalde, and Brad DiGiovine at Argonne National Laboratory. Without their help, we would not have rapidly discovered the poor nucleation efficiency of $\mathrm{CF}_{3} \mathrm{I}$ and PICO-2L would not have set world's best dark matter limits presented here.

As 'the COUPP graduate student', I am indebted to both my student predecessors Drew Fustin and Matthieu Zydagis for their herculean efforts to develop the superheated fluid bubble chamber. These detectors could not have been constructed, run, understood without the 'COUPP Post-doc Committee' of Jeter Hall, Eric Dahl, Hugh Lippincott, Russell Neilson, and Eric Vàsquez-Jauregui. For them and other long-time and recent members of the PICO collaboration, thank you.

Many people have provided me with the opportunities that have brought me to where I am today. I will highlight Glenn Grant, my high-school physics teacher at Mission Secondary School, who introduced me to what physics is. I'd also like to thank the late Erich 
Vogt, my first-year physics professor who has inspired countless students and was titan in the nuclear physics community. I have been financially supported by the National Science and Engineering Research Council of Canada (NSERC) through the Post-Graduate Scholarship program for most of my studies and the University of Chicago, and through several Undergraduate Student Research Awards. The opportunities made possible by these awards have been instrumental in the work presented here and in training me as a physicist.

Juan Collar, my advisor and friend, you live for the advancement of science and deeply understand critical thought, academic freedom, subject literature, and experimental methods. I admire you and have learned a great deal by working with you and with your guidance. Just forgive me if I never give a conference talk like you do.

To my family, you've supported me and my odd passion for physics throughout my life. Though I'm often far away you've always been there for me.

Toby, my love, you keep me together. Thanks for all the edits. 


\section{Table of Contents}

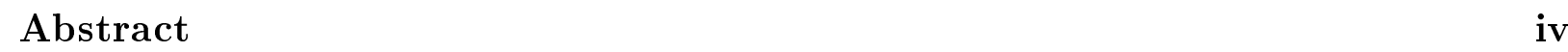

\begin{tabular}{|l|l}
\hline Acknowledgments & v \\
\hline
\end{tabular}

\begin{tabular}{|l|l}
\hline List of Figures & $\mathrm{x}$ \\
\hline
\end{tabular}

List of Tables $\quad$ xiii

\begin{tabular}{llr}
\hline & Bubble Chambers & 1
\end{tabular}

1.1 Bubble Chamber Searches for Dark Matter . . . . . . . . . . . . . . . 1

1.2 Seitz Theory . . . . . . . . . . . . . . . . . . 3

2 Bubble Chamber Technology Development 9

$2.1 \quad \mathrm{CF}_{3} \mathrm{I}$ Chemical Stability . . . . . . . . . . . . . . . . . . . . . . . . . 9

$2.1 .1 \quad \mathrm{CF}_{3} \mathrm{I}$ Test Stands . . . . . . . . . . . . . . . . . . . . . . . . . . . . . . . . . . . . . . . .

2.1 .2 Darkening Measurements . . . . . . . . . . . . . . . . 11

2.1.3 Illumination and Optical Thickness Measurements . . . . . . . . . 15

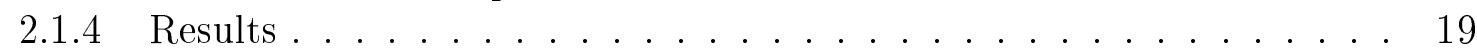

2.2 Buffer $/ \mathrm{C}_{3} \mathrm{~F}_{8}$ Interface Stability . . . . . . . . . . . . . . . . . . . . . . . . . . . . . . . . .

2.2 .1 Surface Forces . . . . . . . . . . . . . . . . . . . 22

2.2 .2 Emulsions . . . . . . . . . . . . . . . . . . . . . . . . . . . . . . . . . 25

2.2 .3 Nucleation at Interfaces . . . . . . . . . . . . . . . . . . . . . . . . . . . . . . 26

2.2 .4 Alternative Buffer Fluids . . . . . . . . . . . . . . . . 29

2.2 .5 Surface Treatments . . . . . . . . . . . . . . . . . . . . . . . . . . . . . . . . . .

2.2 .6 Conclusion . . . . . . . . . . . . . . . . . . . 32

\begin{tabular}{|lll}
\hline 3 & Elements of Nuclear Recoil Calibrations & 34 \\
\hline
\end{tabular}

3.1 Monoenergetic Neutron Sources . . . . . . . . . . . . . . . . . . 34

3.1.1 The ${ }^{51} \mathrm{~V}(p, n)$ and ${ }^{7} \operatorname{Li}(p, n)$ Reactions . . . . . . . . . . . . . . . . 35

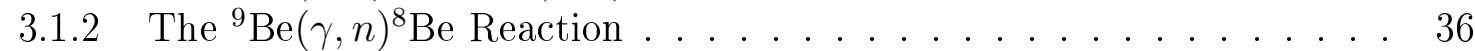

3.2 Low Energy Neutron Simulations . . . . . . . . . . . . . . . . . . 47

$3.3{ }^{241} \mathrm{Am} /$ Be Neutron Spectrum $\ldots \ldots \ldots \ldots . \ldots \ldots$

3.4 Alternative Calibration Methods . . . . . . . . . . . . . . . 56

3.5 Event Rates from Neutron Recoils . . . . . . . . . . . . . . . . . . 57

\begin{tabular}{|lll}
4 & Calibration Bubble Chambers & 64
\end{tabular}

$4.1 \quad(\gamma, n)$ Neutron Sources . . . . . . . . . . . . . . . . . . 64

$4.1 .1 \quad$ STAR $^{88} \mathrm{Y} /$ Be Neutron Source . . . . . . . . . . . . . . . 64

4.1 .2 University of Chicago Sources . . . . . . . . . . . . . . . . . . 66

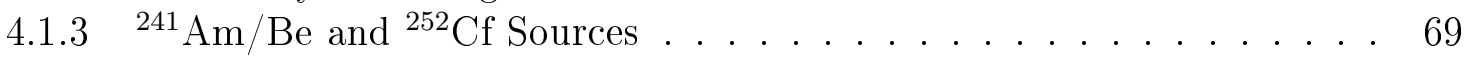


$4.1 .4 \quad \mathrm{He}-3$ measurements $\ldots \ldots \ldots$

4.2 STAR Bubble Chamber $\ldots \ldots \ldots \ldots \ldots \ldots \ldots$

4.2 .1 Contaminants $\ldots \ldots \ldots \ldots \ldots \ldots \ldots \ldots$

$4.2 .2 \quad$ Thermal Stability . . . . . . . . . . . . . . . . . . . . . . . . 79

$4.2 .3 \quad$ Geometry Measurements . . . . . . . . . . . . . . . . . . 83

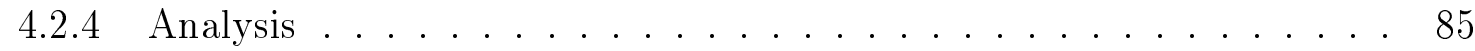

4.3 University of Chicago Bubble Chamber . . . . . . . . . . . . . . 88

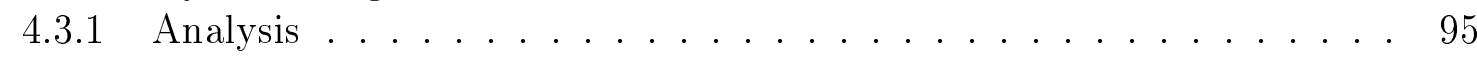

4.4 CYRTE Bubble Chamber $\ldots \ldots \ldots \ldots$

4.4 .1 Temperature Calibrations . . . . . . . . . . . . . . . . . . . 102

$4.4 .2 \quad$ Analysis $\ldots \ldots \ldots \ldots \ldots$. . . . . . . . . . . . . . . 104

$4.4 .3 \quad$ Gamma Sensitivity . . . . . . . . . . . . . . . . . . 107

5 The PICO-2L Bubble Chamber $\quad 111$

5.1 Components . . . . . . . . . . . . . . . . . . . . . . . . . . 114

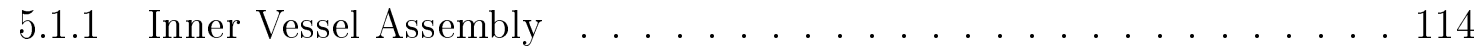

5.1 .2 Pressure Vessel $\ldots \ldots \ldots \ldots$. . . . . . . . . . . . . . . 117

$5.1 .3 \quad$ Hydraulic Controls and DAQ . . . . . . . . . . . . . . 121

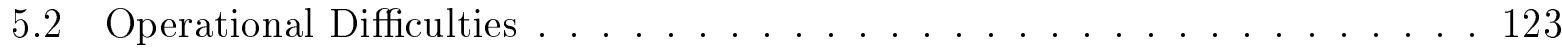

5.3 Analysis Variables and Cuts . . . . . . . . . . . . . . . 126

5.3 .1 Livetime Cuts . . . . . . . . . . . . . . . . . . . . . 127

5.3 .2 Efficiency Cuts . . . . . . . . . . . . . . . . 127

5.3 .3 The Acoustic Parameter . . . . . . . . . . . . . . . . . . . . 129

5.3 .4 Fiducial Volumes . . . . . . . . . . . . . . . . . . . . . . . 132

5.4 Data Sets . . . . . . . . . . . . . . . . . . . . . 138

6 Nuclear Recoil Calibration Results $\quad 140$

$6.1 \quad \mathrm{CF}_{3} \mathrm{I}$ Calibrations . . . . . . . . . . . . . . . . . . . . . 140

6.1 .1 AmBe Measurements . . . . . . . . . . . . . . . . . . . . . . 140

$6.1 .2 \mathrm{Y} /$ Be Measurements . . . . . . . . . . . . . . . . . . . . 143

$6.1 .3 \quad$ Efficiency Limits . . . . . . . . . . . . . . . . . . . . . 147

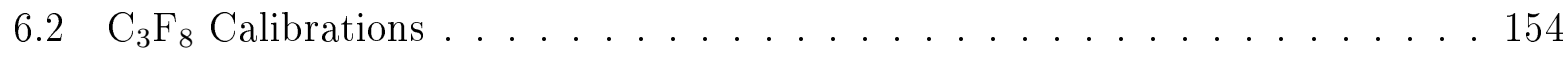

$6.2 .1 \quad$ University of Montreal Neutron Beam. . . . . . . . . . . . . . . . . 154

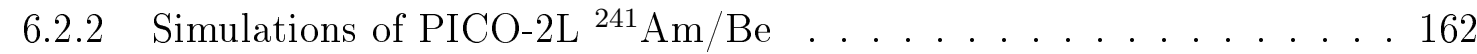

6.2 .3 Photoneutron Source Measurements . . . . . . . . . . . . . . . . . . . 164

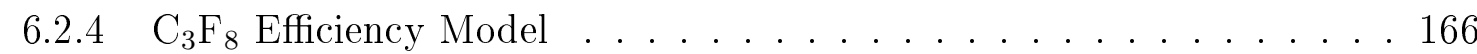

7 Background Studies in Bubble Chambers 173

7.1 Radiopurity Assays . . . . . . . . . . . . . . . . . . . . 173

$7.2 \quad(\alpha, n)$ Reaction Rates . . . . . . . . . . . . . . . . . . . . 180

7.3 COUPP-4kg Background Recalculation . . . . . . . . . . . . . . 186

7.4 Neutron Calibration Backgrounds $\ldots \ldots \ldots \ldots \ldots . \ldots \ldots$ 
$7.5 \quad$ High-energy Backgrounds in Bubble Chambers . . . . . . . . . . . . . . . 195

7.5 .1 Photon Flux Measurements . . . . . . . . . . . . . . . . 195

7.5 .2 Photonuclear Cross-sections in the Active Volume . . . . . . . . . . 199

7.5.3 Photoneutrons from Detector Materials . . . . . . . . . . . . . . . 203

$8 \quad$ Neutron Background Estimates for PICO-2L 206

$8.1 \quad$ Inner Vessel Components . . . . . . . . . . . . . . . . . . . . . 206

8.2 Pressure Vessel Components . . . . . . . . . . . . . . . . . . . . 213

8.3 DAQ Components . . . . . . . . . . . . . . . . . . . . . . . . . . . . 217

8.4 Environmental Neutrons . . . . . . . . . . . . . . . . . . . . . . . . . . . 219

8.5 Other Background Sources . . . . . . . . . . . . . . . . . . . . 221

8.6 PICO-2L Neutron Simulations . . . . . . . . . . . . . . . . . . . . 221

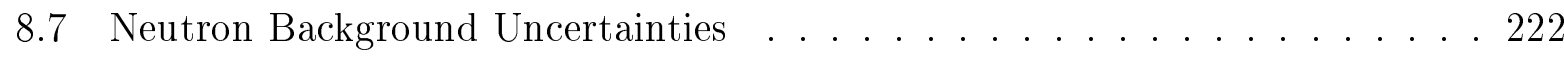

8.8 Total Neutron Background Expectation . . . . . . . . . . . . . . . 225

9 PICO-2L Dark Matter Results $\quad 227$

9.1 Time to Previous Non-timeout Cut . . . . . . . . . . . . . . . 227

9.2 Dark Matter Limits . . . . . . . . . . . . . . . . . . . . . . . . . . . . . 229

\begin{tabular}{|l|l|}
\hline A Results of $\mathrm{CF}_{3} \mathrm{I}$ Darkening Tests & 234
\end{tabular}

B New libraries for simulating neutron scattering in dark matter detector calibrations 242

\begin{tabular}{ll}
\hline C Efficiency Limit Calculator & 249
\end{tabular}

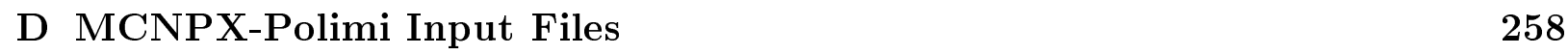

D.1 PICO-2L 4b Geometry . . . . . . . . . . . . . . . . . . . . . . . . . 258

D.2 STAR runs 20111010_1-20111011_2 . . . . . . . . . . . . . 268

D.3 University of Chicago Bubble Chamber, $\mathrm{YBe}_{-} 14 \mathrm{C} \_\mathrm{C} 3 \mathrm{~F} 8 \ldots . . .272$

D.4 CYRTE Geometry, June $2013 \mathrm{C}_{3} \mathrm{~F}_{8}$ fill $\ldots \ldots \ldots \ldots \ldots \ldots$

D.5 Montreal He-3 Calibration . . . . . . . . . . . . . . . . . . . . . . . . 281

\begin{tabular}{ll}
\hline References & 293
\end{tabular} 


\section{List of Figures}

$1.1 \mathrm{C}_{3} \mathrm{~F}_{8}$ Seitz Parameters . . . . . . . . . . . . . . . 7

$1.2 \mathrm{CF}_{3} \mathrm{I}$ Seitz Parameters $\ldots \ldots \ldots \ldots . \ldots . \ldots . \ldots$

$2.1 \quad$ Darkening of COUPP-60 images . . . . . . . . . . . . . . . . . . . . . . 10

$2.2 \quad \mathrm{CF}_{3} \mathrm{I}$ test stand plumbing diagram . . . . . . . . . . . . . . . . 11

2.3 Photograph of a $\mathrm{CF}_{3} \mathrm{I}$ Test Stand . . . . . . . . . . . . . . . . . . 12

2.4 Iodine absorption of red light $\ldots \ldots \ldots \ldots$. . . . . . . . . . . . 14

2.5 Iodine absorption of white light $\ldots \ldots \ldots$. . . . . . . . . . . . . . . . . . . . . . . . . . . . .

2.6 Spectra of LED illumination . . . . . . . . . . . . . . . . . . . . . . . . . . . . . . . . . . . . .

2.7 Effects if venting $\mathrm{CF}_{3} \mathrm{I} \ldots \ldots \ldots \ldots \ldots \ldots$

2.8 Emulsion in COUPP-0.1 . . . . . . . . . . . . . . . . . . . . . . . . . . . . . . . . . . . . . . . . . .

2.9 The triple contact force balance . . . . . . . . . . . . . . . . 23

$2.10 \mathrm{C}_{3} \mathrm{~F}_{8} /$ buffer interface measurements . . . . . . . . . . . . . . . . . . . . . . . . . . . . . . .

2.11 Silane treated pressure vessels $\ldots \ldots \ldots$. . . . . . . . . . . . . . . . . . . . . . . . . . .

$3.1{ }^{51} \mathrm{~V}(p, n)$ cross-section $\ldots \ldots \ldots \ldots \ldots \ldots$

$3.2 \quad$ Existing ${ }^{9} \mathrm{Be}(\gamma, n)^{8} \mathrm{Be}$ Cross-section Measurements . . . . . . . . . . . . . . 39

3.3 Reanalyzed ${ }^{9} \mathrm{Be}(\gamma, n)^{8} \mathrm{Be}$ Cross-section Measurements . . . . . . . . . . . . 45

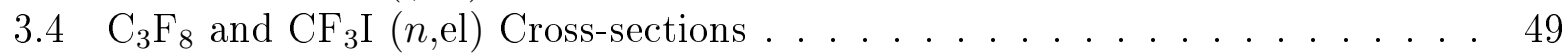

$3.5{ }^{19} \mathrm{~F}(n, \mathrm{el})$ Cross-section $\quad \ldots \ldots \ldots \ldots \ldots \ldots$

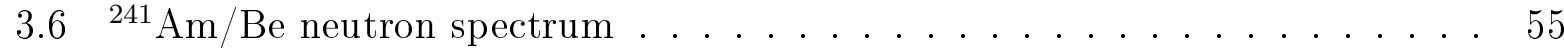

3.7 Range of Nuclear Recoils in $\mathrm{CF}_{3} \mathrm{I}$. . . . . . . . . . . . . . . . . . . . . . . . . . . . . . . 68

3.8 Ratio of ${ }^{88} \mathrm{Y} /$ Be to Am/Be Recoils . . . . . . . . . . . . . . . . . . . . 61

3.9 Setting Efficiency Limits . . . . . . . . . . . . . . . . 62

4.1 STAR Neutron Source . . . . . . . . . . . . . . . . 65

4.2 Chicago Neutron Source Configurations . . . . . . . . . . . . . . . 67

4.3 Chicago Neutron Source $\ldots \ldots \ldots$. . . . . . . . . . . . . . . . . . . . . . . . . .

$4.4{ }^{3}$ He Detector Arrangement . . . . . . . . . . . . . . . . . . . . . . . . . . . . . . . . . . . . . . . . .

$4.5{ }^{3}$ He Detector Measurement . . . . . . . . . . . . . . . . . . . 71

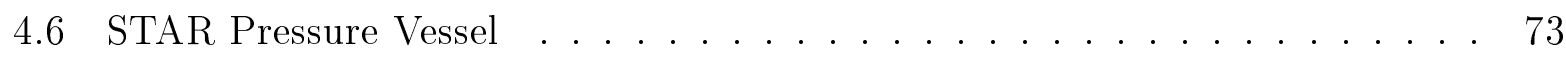

4.7 STAR Containment Vessel . . . . . . . . . . . . . . . . . . . . . . . . . . . . . . . . . . . . . .

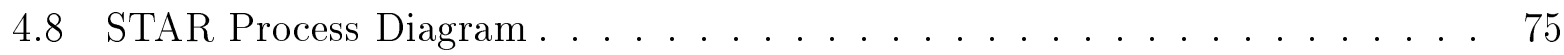

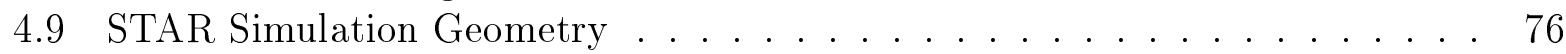

4.10 STAR Contaminants . . . . . . . . . . . . . . . . . . . . . . . . . . . . . . . . . . .

$4.11 \mathrm{CF}_{3} \mathrm{I}$ Vapor Pressure in STAR . . . . . . . . . . . . . . . . . 79

$4.12 T_{1}$ /Event Rate Correlation . . . . . . . . . . . . . . . . . . . . . 82

4.13 STAR Source Position . . . . . . . . . . . . . . . . . . . . 84

4.14 Effect of STAR Geometry Components . . . . . . . . . . . . . . 86 
4.15 STAR Fiducial Volume . . . . . . . . . . . . . . . . . . . . . 88

4.16 University of Chicago Bubble Chamber . . . . . . . . . . . . . . . . 92

4.17 UC Bubble Chamber MCNPX Geometry . . . . . . . . . . . . . . . . . . 93

4.18 UC Bubble Chamber Water Bath . . . . . . . . . . . . . . . . . . . 94

4.19 UC Bubble Chamber Source Position . . . . . . . . . . . . . . . . . . 95

4.20 UC Bubble Chamber Cuts $\ldots \ldots \ldots \ldots$

4.21 CYRTE Bubble Chamber . . . . . . . . . . . . . . . . . . 100

4.22 CYRTE Temperature Calibration . . . . . . . . . . . . . . . . . 103

4.23 CYRTE Temperature vs. Time . . . . . . . . . . . . . . . . . . . . 104

4.24 CYRTE Livetime Cut . . . . . . . . . . . . . . . . . . . 105

4.25 CYRTE $\mathrm{Sb} \& \mathrm{Sb} / \mathrm{Be}$ Rates . . . . . . . . . . . . . . . . . . . . 109

4.26 Measured $\gamma$ Rejection in $\mathrm{C}_{3} \mathrm{~F}_{8}$ and $\mathrm{CF}_{3} \mathrm{I} \ldots \ldots \ldots \ldots$

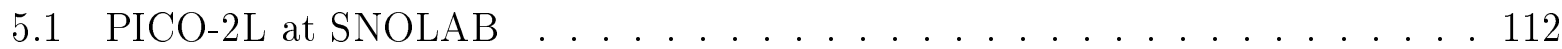

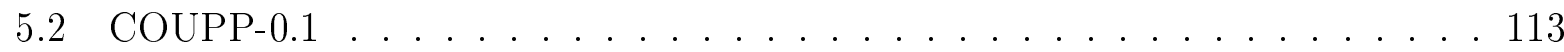

5.3 PICO-2L Inner Vessell. . . . . . . . . . . . . . . . . . . . . . 115

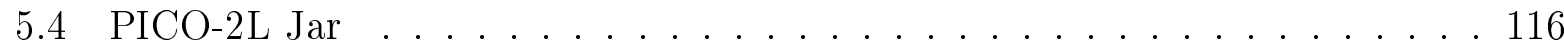

$5.5 \quad$ PICO-2L Pressure Vessel . . . . . . . . . . . . . . . . . . . . . . . . . . 118

5.6 PICO-2L RTD Positions $\ldots \ldots \ldots \ldots \ldots \ldots$

5.7 PICO-2L Hydraulic Cart . . . . . . . . . . . . . . . . . . . . . . . . . . . 121

5.8 PICO-2L Trigger Rates . . . . . . . . . . . . . . . . . . . . . . . . . . . . 124

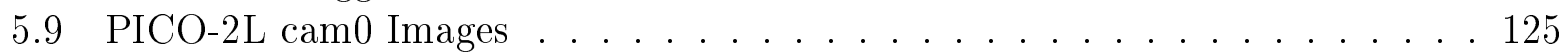

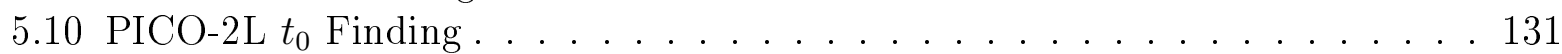

5.11 PICO-2L Piezo 1 Gain Correction . . . . . . . . . . . . . . . . . . . . . . 133

5.12 Acoustic Parameter Distributions . . . . . . . . . . . . . . . . . 134

5.13 Pressure Rise from a Growing Bubble . . . . . . . . . . . . . . . . . . . 135

5.14 PICO-2L Position Reconstruction . . . . . . . . . . . . . . . . . . . 137

$6.1 \quad \mathrm{CF}_{3} \mathrm{I}^{88} \mathrm{Y} /$ Be Event Rates . . . . . . . . . . . . . . . . . . . . 143

6.2 Background Rate in STAR . . . . . . . . . . . . . . . . . . 146

6.3 Background Rate in the UC Bubble Chamber . . . . . . . . . . . . . . 147

6.4 Allowed $\mathrm{CF}_{3} \mathrm{I}$ Bubble Nucleation Efficiencies . . . . . . . . . . . . . 148

6.5 Overlaid $\mathrm{CF}_{3} \mathrm{I}$ Nucleation Efficiency $\ldots \ldots \ldots \ldots$. . . . . . . . . . 151

$6.6 \quad \mathrm{CF}_{3} \mathrm{I}$ Efficiency Functions for Calculating Limits . . . . . . . . . . . . . 152

6.7 Efficiency Function Fit to COUPP-4kg Am/Be Data . . . . . . . . . . . . 153

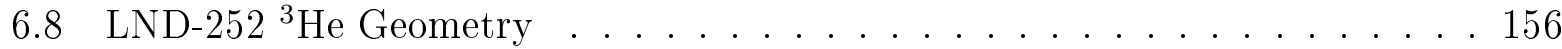

6.9 Neutron Beam Flux Calibration . . . . . . . . . . . . . . . . . 157

6.10 Neutron Beam-end Geometry . . . . . . . . . . . . . . . . . . . . . 158

6.11 Stainless Steel Cross-section . . . . . . . . . . . . . . . . . . . . 159

6.12 MCNPX-Polimi geometry of PICO-0.1. . . . . . . . . . . . . . . 161

6.13 PICO-2L ${ }^{241} \mathrm{Am} /$ Be Source Position . . . . . . . . . . . . . . . . . . . . . 164

6.14 University of Chicago $\mathrm{C}_{3} \mathrm{~F}_{8}{ }^{88} \mathrm{Y} /$ Be Calibration Data $\ldots \ldots \ldots . . . .166$ 
6.15 Allowed $\mathrm{C}_{3} \mathrm{~F}_{8}$ Bubble Nucleation Efficiencies . . . . . . . . . . . . . . 167

$6.16 \mathrm{C}_{3} \mathrm{~F}_{8}$ Efficiency Functions for Calculating Limits . . . . . . . . . . . . . . . . 170

$6.17 \mathrm{C}_{3} \mathrm{~F}_{8}{ }^{88} \mathrm{Y} /$ Be Event Rate . . . . . . . . . . . . . . . . 172

7.1 GES Assay Radon Background Variations . . . . . . . . . . . . . . . . 177

7.2 GES Assay Detector Geometry . . . . . . . . . . . . . . . . . . . . . . . . . . . . . . . . . . . . . .

7.3 GES Assay Count Rate Calibration . . . . . . . . . . . . . . . . . . . 179

$7.4 \quad$ GES Assay Energy and Resolution Calibration Fit Residuals . . . . . . . . . 179

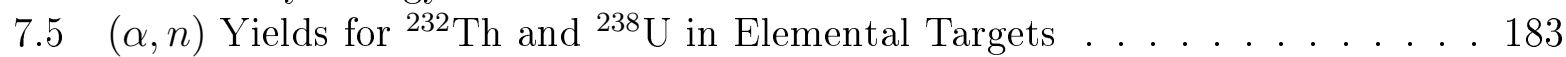

$7.6 \quad(\alpha, n)$ Neutron Energies for ${ }^{232} \mathrm{Th}$ and ${ }^{238} \mathrm{U}$ in Elemental Targets . . . . . . . 184

$7.7{ }^{19} F(n, \alpha)$ Reaction Cross-sections $\ldots \ldots \ldots \ldots \ldots$

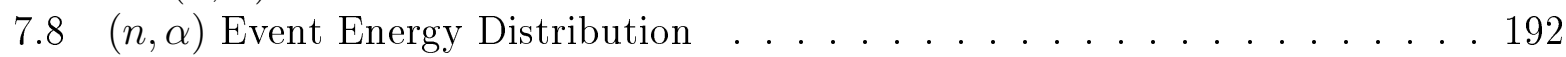

$7.9 \quad 1.78 \mathrm{~kg}$ NaI Internal Background Spectrum . . . . . . . . . . . . . . . . . . . 198

7.10 Comparison of NaI Photon Flux Measurements . . . . . . . . . . . . . . 201

$8.1 \quad$ Retroreflector Neutron Event Multiplicity . . . . . . . . . . . . . . . . . . 207

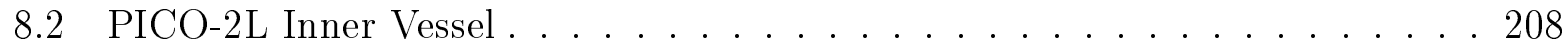

8.3 Assaying the Inner Vessel Top Flange . . . . . . . . . . . . . . . . . . . . 210

8.4 PICO-2L Pressure Vessel Components . . . . . . . . . . . . . . . . . . . . . . . . . . . . . . . . . . . . .

8.5 Versions of the PICO-2L Simulation Geometry . . . . . . . . . . . . . . 223

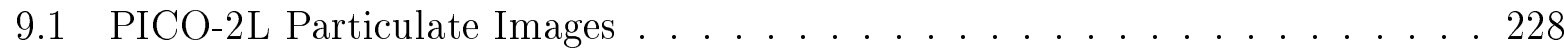

9.2 WIMP-proton Cross-section Limits . . . . . . . . . . . . . . . . . . . . 231

9.3 Comparison to COUPP-4kg $\mathrm{CF}_{3} \mathrm{I}$ Nucleation Efficiency Models . . . . . . 232

A.1 Darkening measurements Oct-Dec . . . . . . . . . . . . . . . 237

A.2 Darkening measurements Dec-Feb . . . . . . . . . . . . . . . . . . . . . . . . . . . . . . . . . . . . . . . . .

A.3 Darkening measurements Mar-May . . . . . . . . . . . . . . . . . . 241

B.1 Dipole anisotropy of neutron scattering . . . . . . . . . . . . . . . . 244

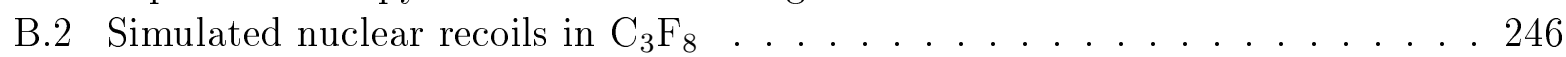

B.3 Simulated nuclear recoil spectrum for XCD . . . . . . . . . . . . . . 247 


\section{List of Tables}

2.1 Illumination used to test $\mathrm{CF}_{3} \mathrm{I}$ darkening $\ldots \ldots \ldots \ldots$

2.2 Nucleation at droplet coalescence . . . . . . . . . . . . . . . . . . . . . . . . . . . . . . . . . . . . . . . . .

$2.3 \quad \mathrm{C}_{3} \mathrm{~F}_{8} /$ buffer interface properties . . . . . . . . . . . . . . . 31

$3.1 \quad$ Isotopes for $(\gamma, \mathrm{n})$ sources $\ldots \ldots \ldots \ldots \ldots$. . . . . . . . . . . . . . . . . . . . . . . . .

3.2 Corrections to ${ }^{124} \mathrm{Sb} /$ Be source yield . . . . . . . . . . . . . . . 41

3.3 Radioisotope sources used in Fujishiro et al. (1982). . . . . . . . . . . . . . . . 44

$3.4 \quad$ Neutron yield of $(\gamma, \mathrm{n})$ sources $\ldots \ldots \ldots$. . . . . . . . . . . . . . 46

4.1 Neutron Yields of University of Chicago Sources . . . . . . . . . . . . . . 66

4.2 List of ${ }^{88} \mathrm{Y}$ and ${ }^{124} \mathrm{Sb}$ Sources $\ldots \ldots \ldots \ldots$. . . . . . . . . . . . . . . . . . . . . . . . . . . . . .

$4.3{ }^{3}$ He Measurements . . . . . . . . . . . . . . . . . . . . . . . . . . . . . . . . . . . .

4.4 STAR Temperature Calibration . . . . . . . . . . . . . . . . . . . . . 81

4.5 STAR Source Positions . . . . . . . . . . . . . . . . . . . . . . . . . . . . . . . . . . . . . . 84

4.6 STAR Run List . . . . . . . . . . . . . . . . . . . . . . 89

4.7 STAR Rate Uncertainties . . . . . . . . . . . . . . . 90

4.8 University of Chicago $\mathrm{CF}_{3} \mathrm{I}$ Run List . . . . . . . . . . . . . . . . . . . . . . . . 97

4.9 UC Bubble Chamber Rate Uncertainties . . . . . . . . . . . . . . . . . 98

4.10 CYRTE 2013 Run List . . . . . . . . . . . . . . . . . . . . . . . . . . . . . . . . . . . . . . . . . . . . . . . . .

4.11 CYRTE 2014 Run List . . . . . . . . . . . . . . . . . 108

5.1 PICO-2L Cut Efficiencies . . . . . . . . . . . . . . . . . . . . . . . . . . . . . . . . 132

5.2 AP Nuclear Recoil Cuts . . . . . . . . . . . . . . . . . 132

5.3 Dytran Cut Efficiencies . . . . . . . . . . . . . . . . 136

5.4 PICO-2L Run Conditions . . . . . . . . . . . . . . . . . 138

5.5 PICO-2L Dark Matter Search Counts . . . . . . . . . . . . . . . . . . 138

5.6 PICO-2L Neutron Calibration Counts . . . . . . . . . . . . . . . . 139

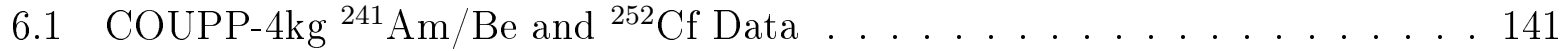

6.2 Montreal Neutron Beam Calibration . . . . . . . . . . . . . . . 160

6.3 PICO-0.1 Rate Uncertainties . . . . . . . . . . . . . . . . . . . . . . . . . . . . . . . . . .

6.4 PICO-0.1 Results . . . . . . . . . . . . . . . . . 162

$6.5 \quad$ PICO-2L ${ }^{241} \mathrm{Am} /$ Be Calibration Uncertainties . . . . . . . . . . . . . . 165

6.6 Measured and Expected $\mathrm{C}_{3} \mathrm{~F}_{8}$ Calibration Counts . . . . . . . . . . . . . 171

7.1 Commonly Fitted Photon Energies for GES Assays . . . . . . . . . . . . . 175

$7.2 \quad(\alpha, n)$ Cross-section References $\ldots \ldots \ldots \ldots \ldots$. . . . . . . . 182

$7.3 \quad(\alpha, n)$ Yield on Detector Components . . . . . . . . . . . . . 185

7.4 Total neutron background predictions . . . . . . . . . . . . . . . . . . 187

$7.5 \quad(n, \alpha)$ Reaction Q-values $\ldots \ldots \ldots$. . . . . . . . . . . . . . . . . . . . . . . . . . . . 
$7.6 \quad(n, \alpha)$ Reaction Rates in $\mathrm{C}_{3} \mathrm{~F}_{8} \quad \ldots \ldots \ldots \ldots \ldots 1$

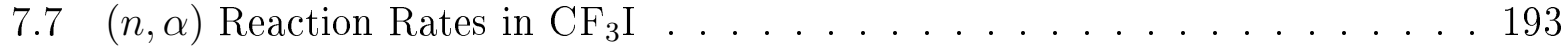

$7.8 \quad \alpha$ Event Rates During COUPP-4kg Neutron Calibrations . . . . . . . . . . . 194

$7.9 \quad$ COUPP-4kg $\gamma$ Flux . . . . . . . . . . . . . . . . . . 197

7.10 SNOLAB High-energy Photon Flux . . . . . . . . . . . . . . . . 200

7.11 Survival of Photons Through COUPP Neutron Shields . . . . . . . . . . . . 200

7.12 Photonuclear Reaction Q-values . . . . . . . . . . . . . . 202

7.13 Photonuclear Event Rates . . . . . . . . . . . . . . . . . . 202

$7.14(\gamma, n)$ Q-values . . . . . . . . . . . . . . . . . . . . . . . . . . . . . . . . . . . . . . . . . . .

$7.15(\gamma, n)$ Rates in COUPP-4kg . . . . . . . . . . . . . . . . . 204

8.1 Inner Vessel Parts List . . . . . . . . . . . . . . . . . . . . . . 209

8.2 Pressure Vessel Parts List . . . . . . . . . . . . . . . . . . . . 214

8.3 Neutron Backgrounds from DAQ Components . . . . . . . . . . . . . 218

8.4 Versions of the PICO-2L Simulation Geometry . . . . . . . . . . . . . . . . . . 224

8.5 Background from PICO-2L Components . . . . . . . . . . . . . . 226

9.1 PICO-2L TNPT Cuts . . . . . . . . . . . . . . . . . . . . 229

A.1 Darkening measurements Oct-Dec . . . . . . . . . . . . . . 236

A.2 Darkening measurements Dec-Feb . . . . . . . . . . . . . . . . . . . . . . . . . . . . . . . . . . . . . . .

A.3 Darkening measurements Mar-May . . . . . . . . . . . . . . . . . 240 


\section{Chapter 1 Bubble Chambers}

\subsection{Bubble Chamber Searches for Dark Matter}

Cold dark matter is necessary to explain the growth of our universe (Planck Collaboration 2014), the formation of galaxies (Blumenthal et al. 1984), and gravitational acceleration within galaxies (Rubin and Ford 1970). Weakly interacting massive particle (WIMP) dark matter is a well-motivated model for dark matter that may solve outstanding problems in both cosmology and particle physics (Jungman, Kamionkowski, and Griest 1996). A WIMP is expected to have a mass comparable to $100 \mathrm{GeV}$ and to interact only via rare processes with cross-sections near or below $0.1 \mathrm{pb}$. In a radiation detector, WIMP dark matter would collide with nuclei and produce recoiling nuclei with energies of $\sim 10 \mathrm{keV}$ (Lewin and Smith 1996). In contrast, most forms of ionizing radiation interact preferentially with electrons.

Bubble chambers are radiation detectors that use superheated liquid as the detector medium. Superheated liquid are held at a pressure below their equilibrium vapor pressure. Particles stopping in the detector cause the liquid to boil. The Seitz theory of bubble nucleation, described in the next section, shows that radiation must surpass both an energy and a stopping power threshold before a bubble will form. This dual threshold allows a bubble chamber to trigger on the high stopping power of a heavily charged nucleus $(\sim$ $1 \mathrm{MeV} / \mu \mathrm{m})$ while ignoring the much lower stopping power of electrons $(<0.01 \mathrm{MeV} / \mu \mathrm{m})$ (Fustin 2012, Section 3.1).

Bubble chambers operate by containing a large volume of liquid at a constant temperature while pistons or bellows cycle the pressure between low pressure expansions, and compressions at high pressure. The liquid is superheated during expansions, and compres- 
sions allow any gas that has formed to condense. In order to use a bubble chamber to search for dark matter, the fraction of time in the expanded state must be maximized. Continuously superheated bubble chambers have been developed by the former COUPP (Behnke et al. 2008) and now PICO collaborations. These bubble chambers' surfaces are constructed of smooth and low-radioactivity materials in order to minimize the rate of bubble formation. For COUPP and PICO bubble chambers to date, the $\mathrm{C}_{3} \mathrm{~F}_{8}$ or $\mathrm{CF}_{3} \mathrm{I}$ active fluid is contained by a high-purity synthetic silica glass jar and high-purity water buffer fluid floating above the active fluid. The active fluid and the buffer fluid are the two components of the inner volume of the detector. The low bubble formation rate is necessary both to maximize the expansion live time, and to minimize the number of background events that can mimic dark matter interactions. Chapter 2 explores some design challenges for selecting the fluids used in the inner volume of a continuously superheated bubble chamber.

The main results of this thesis, in Chapter 9, are world-leading limits on the spindependent nuclear scattering cross-section of WIMP dark matter from the PICO-2L bubble chamber experiment (formally published in Amole et al. 2015). The design and analysis of the experiment is a development of the former COUPP-4kg experiment (Behnke et al. 2012), the subject of Drew Fustin's, my predecessor's, thesis (Fustin 2012). The first three chapters of this thesis provide further discussion of dark matter and the motivation for using a bubble chamber which will not be fully repeated here. For the purposes of this thesis, dark matter can be simply considered as a source of nuclear recoils.

Neutrons, being massibe neutral particles, are the only other naturally present particle that preferentially scatter on nuclei, and can mimic WIMP dark matter. In contrast to dark matter particles that are likely to pass through the entire Earth without scattering, neutrons are likely to scatter every $\sim 10 \mathrm{~cm}$ in most solids or liquids. Neutrons can therefore be used to calibrate a dark matter detector's efficiency. Chapter 3 describes how to model 
the trajectories and propagation of neutrons in matter, how to produce low-energy nuclear recoils using neutrons for use in calibrations, and how to calculate the efficiency for detecting nuclear recoils from neutron calibrations. Three calibration bubble chamber detectors, described in Chapter 4 were operated in the presence of mono-energetic neutron sources to determine their sensitivity to nuclear recoils. In combination with neutron calibration data from another detector at a neutron beam at the University of Montreal and the PICO-2L detector, sensitivity limits are developed in Chapter 6 .

The inadvertent production of neutrons near a dark matter detector creates a background rate of events that limits the ultimate sensitivity of the detector. PICO-2L and other dark matter detectors are surrounded by thick layers of water, plastic, or other hydrogencontaining materials in order to prevent neutrons from natural radioactivity from reaching

the detector. Neutrons passing through the shield and neutrons produced by detector materials inside of the shielding are the primary limitations on the sensitivity of PICO2L. The background of nuclear recoil events produced by neutrons and other radiation will be discussed in Chapter 7 with the background expectations for the PICO-2L experiment presented in Chapter 8 .

\subsection{Seitz Theory}

A superheated liquid, the active component of a bubble chamber, is out of thermal equilibrium. The vapor pressure of the liquid, $P_{v}$, is greater than its pressure, $P_{l}$ so that the equilibrium state of the fluid at this temperature and pressure is a gas. The phase transition to a gas is impeded by the energy required to form the surface of a gas bubble.

Any gas/liquid interface develops a surface tension $\sigma$. This constant tension around a small spherical gas bubble exerts a pressure on the gas that may exceed the pressure excess inside the bubble. Force balance is achieved when the spherical bubble is of a critical radius 
$r_{c}$, and

$$
P_{b}-P_{l}=\frac{2 \sigma}{r_{c}}
$$

The pressure inside the bubble $\left(P_{b}\right)$ is approximately equal to the vapor pressure $\left(P_{b} \approx P_{v}\right)$. If a gas bubble forms with a radius $>r_{c}$, it will grow until either the pressure rises or the temperature falls past the boiling point.

At the edges of a fluid, gas bubbles with large surfaces may form at sharp edges such as scratches or at the merger of droplets. Such cases will be discussed in Section 2.2.3. In the bulk of a fluid, a sufficiently large amount of energy must be concentrated to form a larger than critical bubble. Ionizing particles are able to provide this energy. The Seitz theory of bubble nucleation (Seitz 1958) postulates that most of the energy from the stopping of an ionizing particle instantaneously develops a line source of heat, a 'hot-spike'. If the particle has an energy, $E_{r}$, greater than the critical energy required to form a critical bubble, $E_{c}$, and has a sufficiently large stopping power so as to deliver the required heat within a critical radius, a bubble may form.

The energy required to form a bubble can be divided into the heat required to vaporize the fluid, and the energy required to form the bubble surface (Peyrou 1967; Dahl 2011).

$$
E_{c}=\frac{4 \pi}{3} r_{c}^{3} \rho_{b} \Delta h+4 \pi r_{c}^{2}\left(\sigma+T \frac{d \sigma}{d T}\right)
$$

where $\rho_{b}$ is the density of the gas in the bubble, $\Delta h=h_{b}-h_{l}$ is the specific enthalpy of the fluid, and $T$ is the temperature. $E_{c}$ is called the Seitz threshold energy for forming a bubble. Additional energy loss due to conduction or radiation away from the 'hot-spike' is neglected in the model.

The SuperSeitzModel calculator developed by Dahl $(\overline{2013})$ is used to calculate the threshold. This program uses the NIST REFPROP program (Lemmon, Huber, and McLinden 
2013) to calculate the thermodynamic properties of $\mathrm{C}_{3} \mathrm{~F}_{8}$ and $\mathrm{CF}_{3} \mathrm{I}$ from the equations of state of Lemmon and Span (2006), Lemmon (2006), and Span and Wagner (1996). The calculator accounts for several complications that arise when calculating the values used in Equation 1.2. The pressure of the liquid in equilibrium with a critical bubble is significantly lower than the saturation vapor pressure of the fluid. The vapor pressure, heat of enthalpy, and surface tension of the liquid are all defined in the saturated state. In the presence of a critical bubble, they are (Dahl 2013)

$$
\begin{gathered}
P_{b}=P_{v} \exp \left(-\frac{P_{v}-P_{l}}{P_{v}} \frac{\rho_{v}}{\rho_{l}}\right) \approx P_{v}-\left(P_{v}-P_{l}\right) \frac{\rho_{v}}{\rho_{l}} \\
\Delta H=\Delta H^{\circ}-\int_{P_{v}}^{P_{b}} \frac{T \alpha_{b}(P)}{\rho_{b}(P)} d P+\int_{P_{v}}^{P_{l}} \frac{T \alpha_{l}(P)}{\rho_{l}(P)} d P \approx \Delta H^{\circ}+\frac{P_{v}-P_{l}}{\rho_{l}} T\left(\alpha_{b}-\alpha_{l}\right) \\
\sigma=\sigma_{\circ}-\int_{0}^{P_{b}-P_{l}} \frac{\Gamma}{\rho_{b}-\rho_{l}} d p
\end{gathered}
$$

where $\rho_{v}$ is the density of a saturated vapor, $\alpha$ is the coefficient of thermal expansion, $\Delta H^{\circ}$ is the heat of vaporization in the saturated state, and $\Gamma$ is the surface density of the liquid. The dimension $\delta=\frac{\Gamma}{\rho_{b}-\rho_{l}}$ is known as the Tolman length (Tolman 1949). As it has not been measured in $\mathrm{C}_{3} \mathrm{~F}_{8}$ or $\mathrm{CF}_{3} \mathrm{I}$, a constant Tolman length of $0.4 \pm 0.4 \mathrm{~nm}$, approximately the intermolecular spacing, is assumed.

The SuperSeitzModel calculator was slightly modified in order to determine the Seitz threshold of mixtures of $\mathrm{CF}_{3} \mathrm{I}$ and $\mathrm{CO}_{2}$. REFPROP is able to calculate the thermodynamic properties of mixed fluids given a mixing model for the fluids. The provided mixing model parameters for $\mathrm{CF}_{3} \mathrm{H}$ and $\mathrm{CO}_{2}$ were used to model the $\mathrm{CF}_{3} \mathrm{I} / \mathrm{CO}_{2}$ mixture.

The calculated thresholds and critical radii for $\mathrm{C}_{3} \mathrm{~F}_{8}$ and $\mathrm{CF}_{3} \mathrm{I}$ are shown in Figures 1.1 and 1.2. Theoretical uncertainties on these values are of approximately $5 \%$ propagated mainly from the uncertainties in the surface tension and Tolman lengths of the fluids. These 
theshold values will be used throughout the rest of this thesis to predict the sensitivity and behavior of $\mathrm{C}_{3} \mathrm{~F}_{8}$ and $\mathrm{CF}_{3} \mathrm{I}$ filled bubble chambers. 


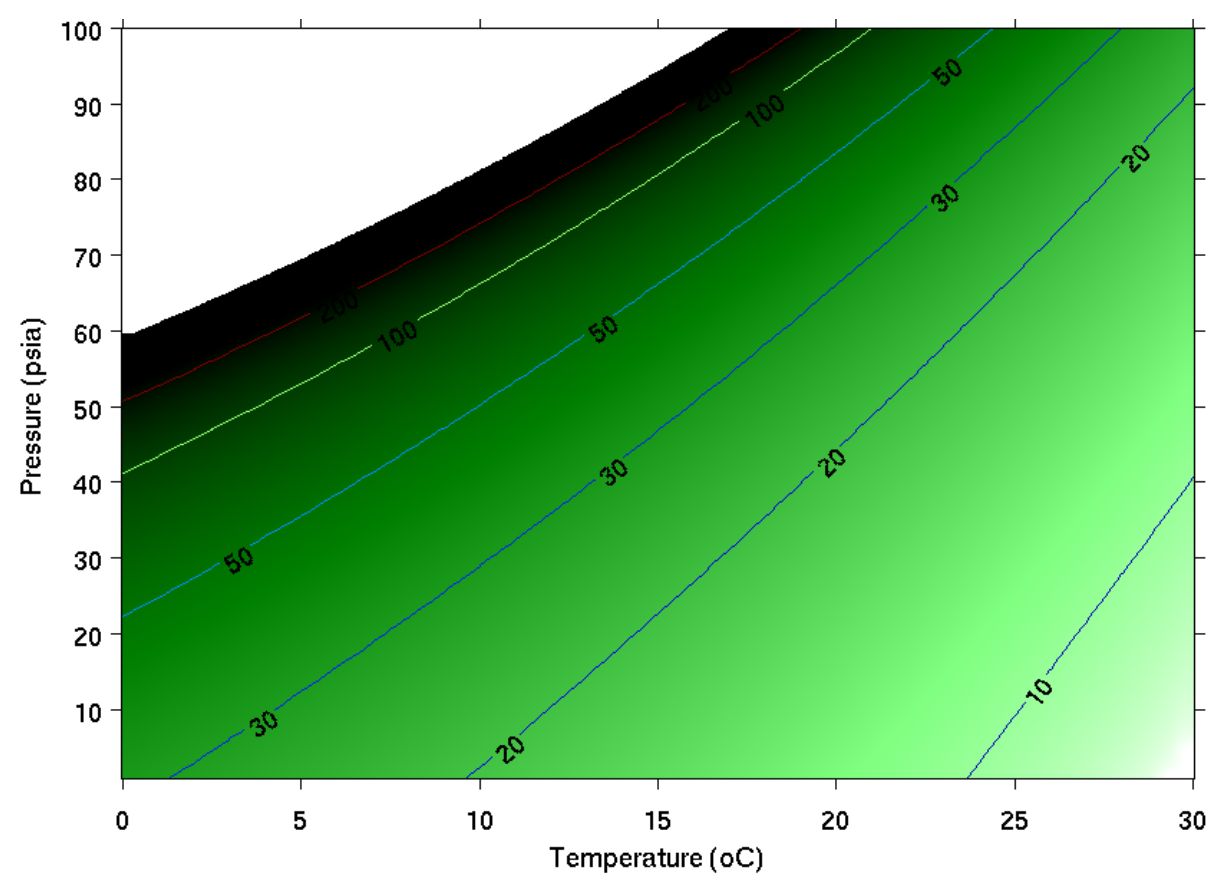

(a) Critical radius in $\mathrm{nm}$.

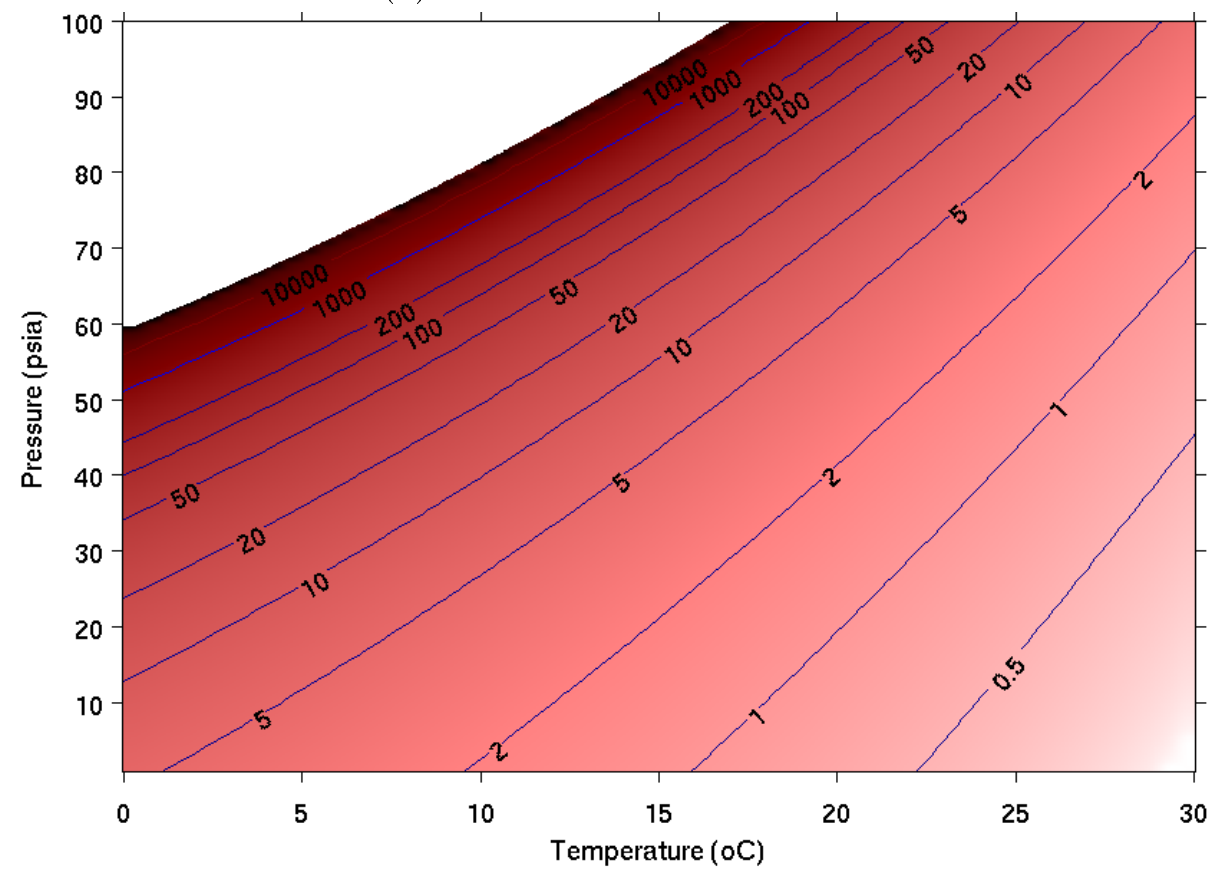

(b) Threshold energy in keV.

Figure 1.1: Calculated Seitz model parameters for $\mathrm{C}_{3} \mathrm{~F}_{8}$. 


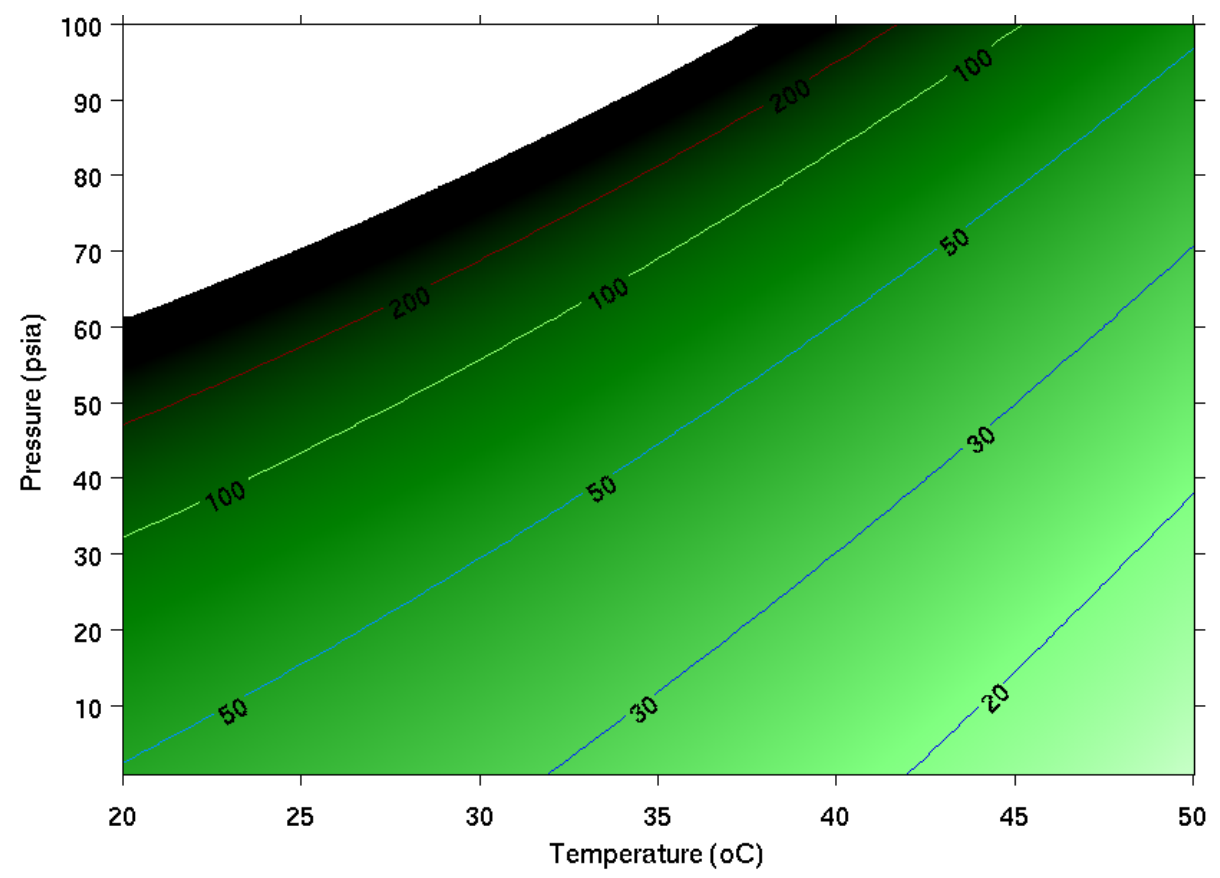

(a) Critical radius in $\mathrm{nm}$.

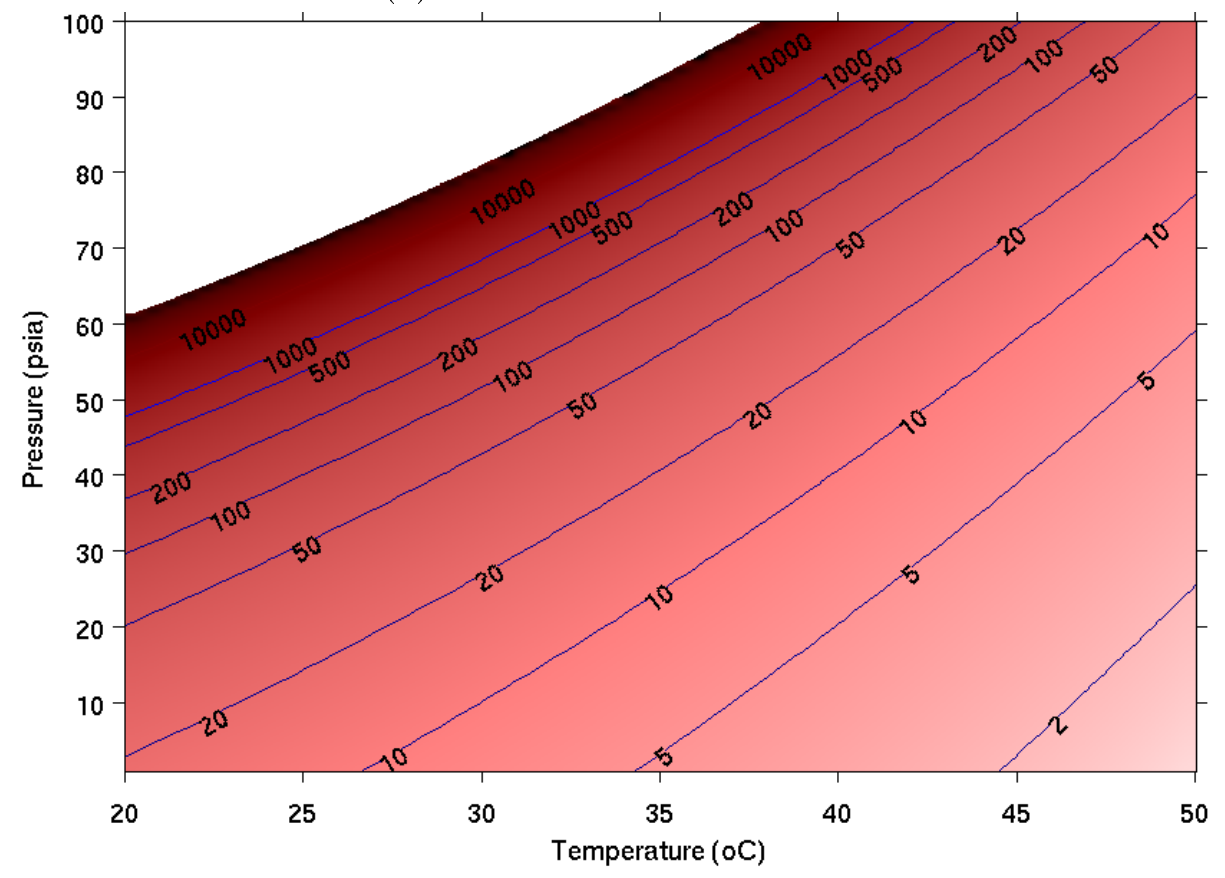

(b) Threshold energy in keV.

Figure 1.2: Calculated Seitz model parameters for $\mathrm{CF}_{3} \mathrm{I}$. 


\section{Chapter 2 \\ Bubble Chamber Technology Development}

In order to search for dark matter, bubble chambers first need to operate stabily. The selection of materials can affect how a bubble chamber operates by either introducing a rate bubble nucleation events on surfaces or by reducing the effectiveness of the data acquisition system. Two considerations for bubble chamber material selection are explored in this chapter.

\section{1 $\quad \mathrm{CF}_{3} \mathrm{I}$ Chemical Stability}

The 2010 run of COUPP-60 $105 \mathrm{~m}$ underground in the NuMI cavern at Fermilab 1 ended due to operational difficulties, most severe of which was darkening of the target fluid. As the primary trigger in PICO and COUPP bubble chambers is from video image analysis, darkening of the target fluid and the loss of image contrast, as shown in Figure 2.1, prevented trigger operation. This darkening was caused by diatomic iodine liberated from the $\mathrm{CF}_{3} \mathrm{I}$ target fluid producing a deep violet color. From the amount of light attenuation in COUPP60 , the ultimate concentration of diatomic iodine was estimated to be $20 \mathrm{ppm}$ (Lippincott 2010). In the COUPP-4kg chamber at NuMI and at SNOLAB, no darkening of the target fluid was observed under similar operating conditions.

The iodine-carbon bond in $\mathrm{CF}_{3} \mathrm{I}$ is very weak. It has a dissociation energy of $53.4 \mathrm{kcal} /-$ mol (Hwang and El-Sayed 1992), the same energy as a $535 \mathrm{~nm}$ green photon. In order to prevent dissociation, $630 \mathrm{~nm}$ red LEDs are used to illuminate COUPP bubble chambers and all paths for outside light to enter are blocked. While filling COUPP-60 in 2010, some white light was permitted to reach the $\mathrm{CF}_{3} \mathrm{I}$ indirectly through a long tube. Nearly all of

1. Fermilab is located in Batavia, IL, USA 60510 

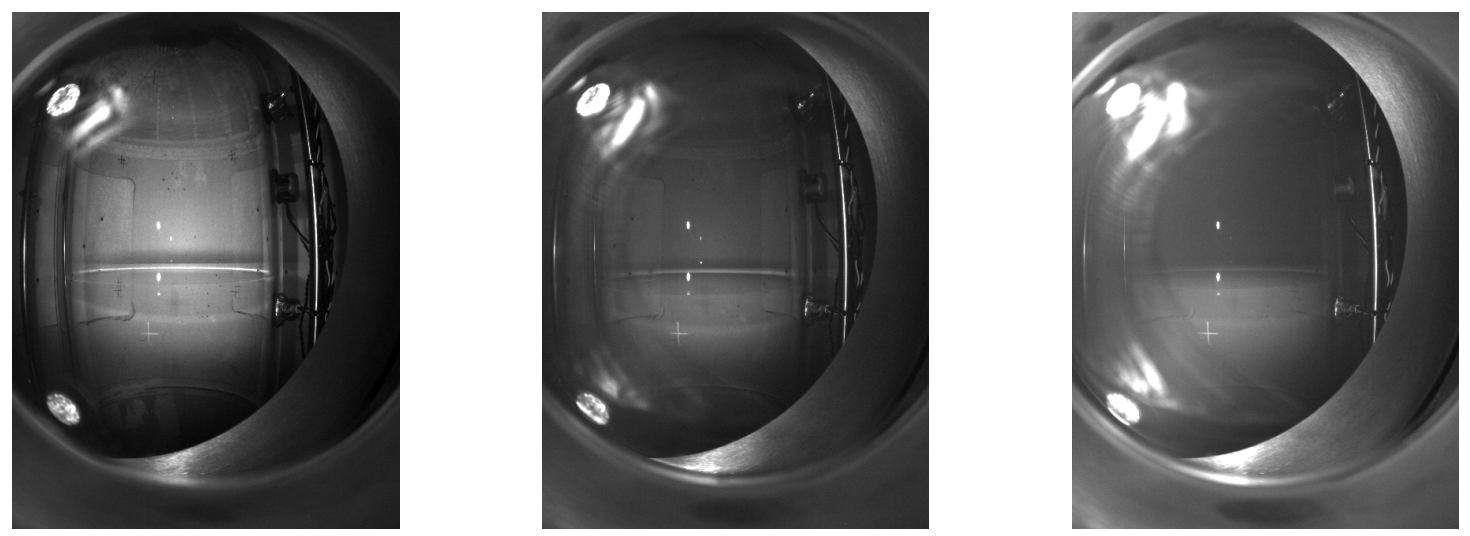

Figure 2.1: Camera_0 images from the failed COUPP-60 2010 NuMI run. Images are from the start of the run (left), 14 days into the run (center), and at the end of the 34 day run with LED illumination increased to compensate for the darkened active fluid (right).

the $\mathrm{CF}_{3} \mathrm{I}$ dissociation occurred after this source of light was blocked off.

In order to investigate whether stray light or some other mechanism caused iodine dissociation in COUPP-60, liquid $\mathrm{CF}_{3} \mathrm{I}$ in a transparent glass pressure container was exposed to several sets of controlled conditions. The rate of darkening due to diatomic iodine formation was measured.

\subsection{1 $\quad \mathrm{CF}_{3} \mathrm{I}$ Test Stands}

A set of three $\mathrm{CF}_{3} \mathrm{I}$ test stand apparatuses were constructed and used in a dark room. Each stand consisted of an aluminum frame, a 1/8" thick polycarbonate shield, a Chemglass brand glass pressure vessel, and plumbing as shown in Figure 2.2 .

As chemical impurities may accelerate or inhibit iodine dissociation, the provenance of the water and $\mathrm{CF}_{3} \mathrm{I}$ used was tracked. Water was stored and transported in polypropylene containers and sourced from either leftover SNOLAB ultrapure water from the filling of the COUPP-60 or deionized water produced in the A0 building at Fermilab. The $\mathrm{CF}_{3} \mathrm{I}$ was taken from the COUPP-60 fill cart and either distilled directly or via a transport cylinder into a test stand. The plumbing of the first test apparatus was cleaned using Radiacwash 


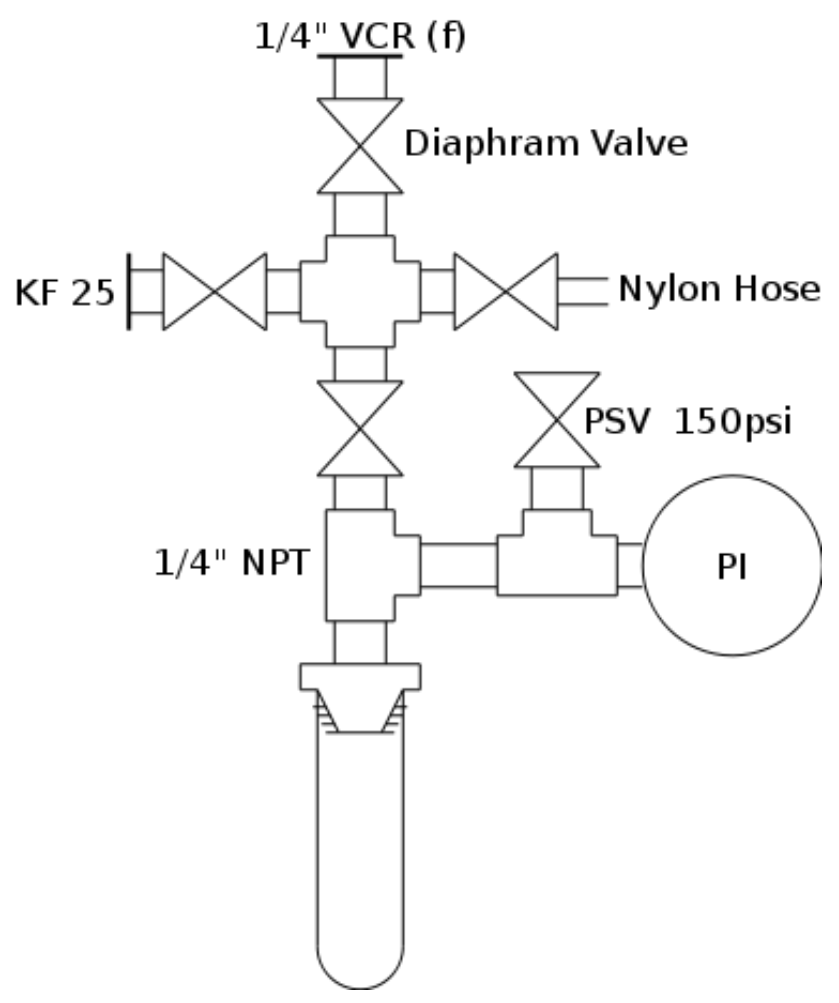

Figure 2.2: Plumbing diagram of the $\mathrm{CF}_{3} \mathrm{I}$ test stands.

cleaning solution (Biodex 2015) and assembled in the A0 clean room at Fermilab. All later stands were simply rinsed in deionized water prior to filling or refilling. Wetted components of the apparatus were made of PTFE, 316 grade stainless steel, and borosilicate glass.

The test stands used either a 38 mL CG-1880-03 Chemglass pressure vessel with an exterior diameter of $25.4 \mathrm{~mm}$ (1") or a $350 \mathrm{~mL}$ CG-1880-12 pressure vessel with an exterior diameter of $82.5 \mathrm{~mm}\left(3.25^{\prime \prime}\right)$. The wall thickness of the vessels is estimated to be $4.5 \mathrm{~mm}$ based on calculations of their size vs. nominal volume and measurements of the wall of a shattered CG-1880-12. This gives an inner diameter of $16.4 \mathrm{~mm}$ and $73.5 \mathrm{~mm}$ respectively.

\subsubsection{Darkening Measurements}

The production of diatomic iodine was measured in the presence of the light sources given in Table 2.1. Changes in the rate of dissociation were measured in the presence or absence 


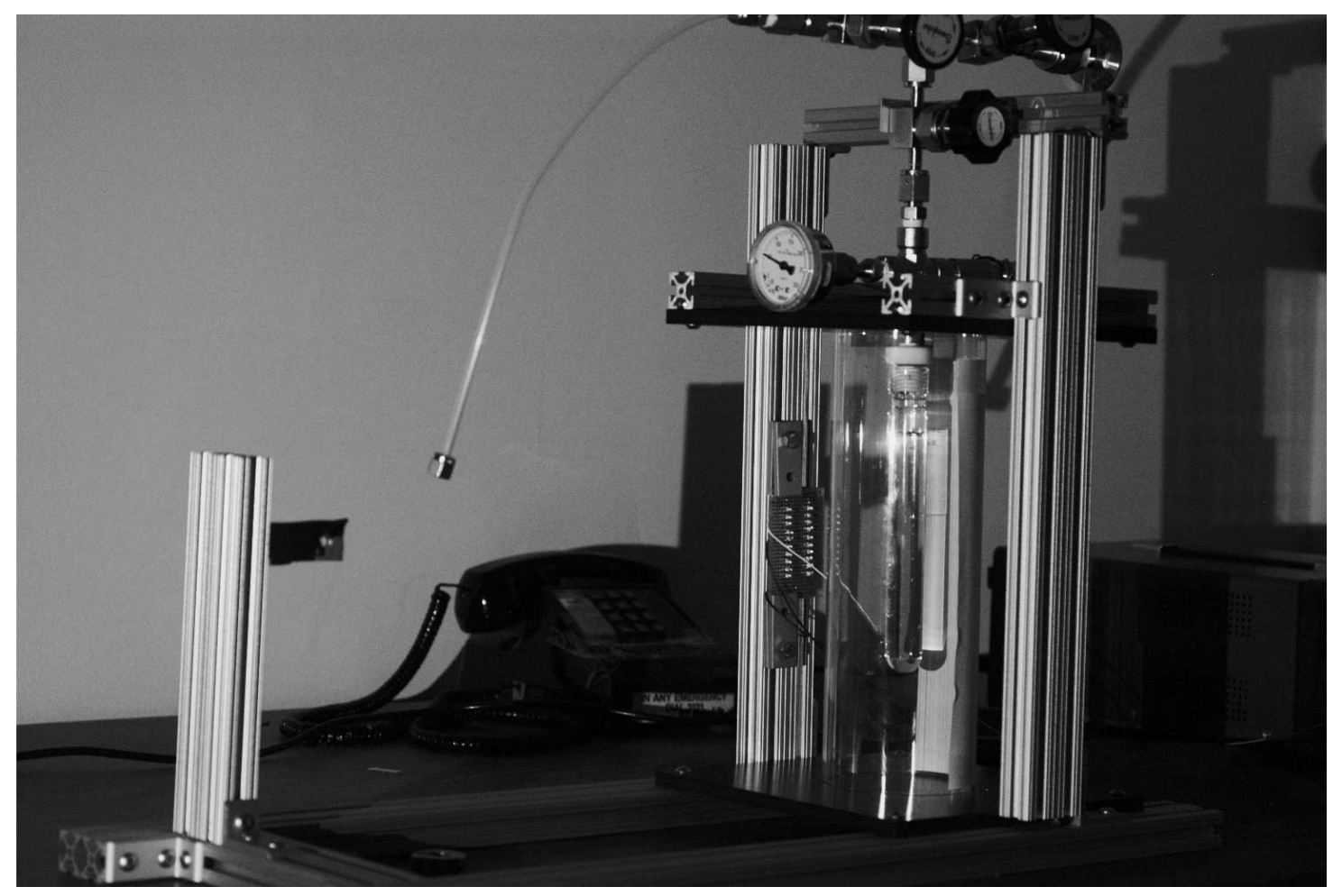

Figure 2.3: A photograph of a $\mathrm{CF}_{3} \mathrm{I}$ Test Stand apparatus. The digital camera mounts to the aluminum post on the left.

of stainless steel, gold, air, water, ethanol, $\mathrm{Na}_{2} \mathrm{SO}_{3}$, or with the use of previously darkened $\mathrm{CF}_{3} \mathrm{I}$. Tests were performed between $15^{\circ} \mathrm{C}$ and $25^{\circ} \mathrm{C}$. To measure darkening, a Nikon D100 digital camera was mounted to the aluminum frame of the test stands. The camera measured the absorption of either diffused white incandescent light, red LED light, or green LED light through the test stand as iodine concentration increased. These three methods were crosscalibrated against each other, and normalized to the absorption of the near-monochromatic red LED light.

The digital camera images used identical exposures and aperture settings, and were saved in the .raw image file format in order to prevent automatic white balance or contrast stretching of the images. Once downloaded from the camera, the images taken with a white halogen light as illumination were white balance corrected for a 2618 Kelvin blackbody 
spectrum using the open source ufraw program (Fuchs and Jensen 2014). The images were reduced in resolution by a factor of four and converted into . fits formated files for further processing using Python scripts.

To measure darkening, the step discontinuity in the brightness of the image across the $\mathrm{CF}_{3} \mathrm{I} /$ water interface was measured. For the thin pressure vessels, the images were averaged across approximately the central third of the vessel before fitting for the step discontinuity in the vertical direction. On either side of the discontinuity, the brightness was fit with either a quadratic or cubic function (see Figure 2.4). The range of the fit region of interest and cut regions were manually selected image-by-image in order to avoid dark spots and areas of poor illumination. For the thick pressure vessels, an automatic script masked obscured pixels and the light intensity in the horizontal direction was fitted to the following model of the optical thickness of the $\mathrm{CF}_{3} \mathrm{I}$ :

$$
D=e \sqrt{r_{y}^{2}-\left(y-y_{o}\right)^{2}}-\left(I-I_{o}\right)
$$

where $e$ is the eccentricity of the ellipse in $y$ - $I$ space, $r_{y}$ is the $y$-axis radius, $y$ is the horizontal pixel position, $I$ is the pixel intensity, and $\left(y_{o}, I_{o}\right)$ is the vessel center and zero-optical depth pixel intensity. This function was fit to the intensity in the region of interest averaged in the vertical direction in order to fix $r_{y}$ and $y_{o}$. The intensity across each one pixel high row of the region of interest was then refit to obtain the intensity, $I$, as a function across the meniscus. The step discontinuity in this function was then fitted with the same method as with the thin pressure vessels.

By taking images in white light, and comparing the color of $\mathrm{CF}_{3} \mathrm{I}$ versus the color of the water, the quadratic or cubic shape of the background illumination can be divided out and the step discontinuity determined with less uncertainty. Measurements of the light received 

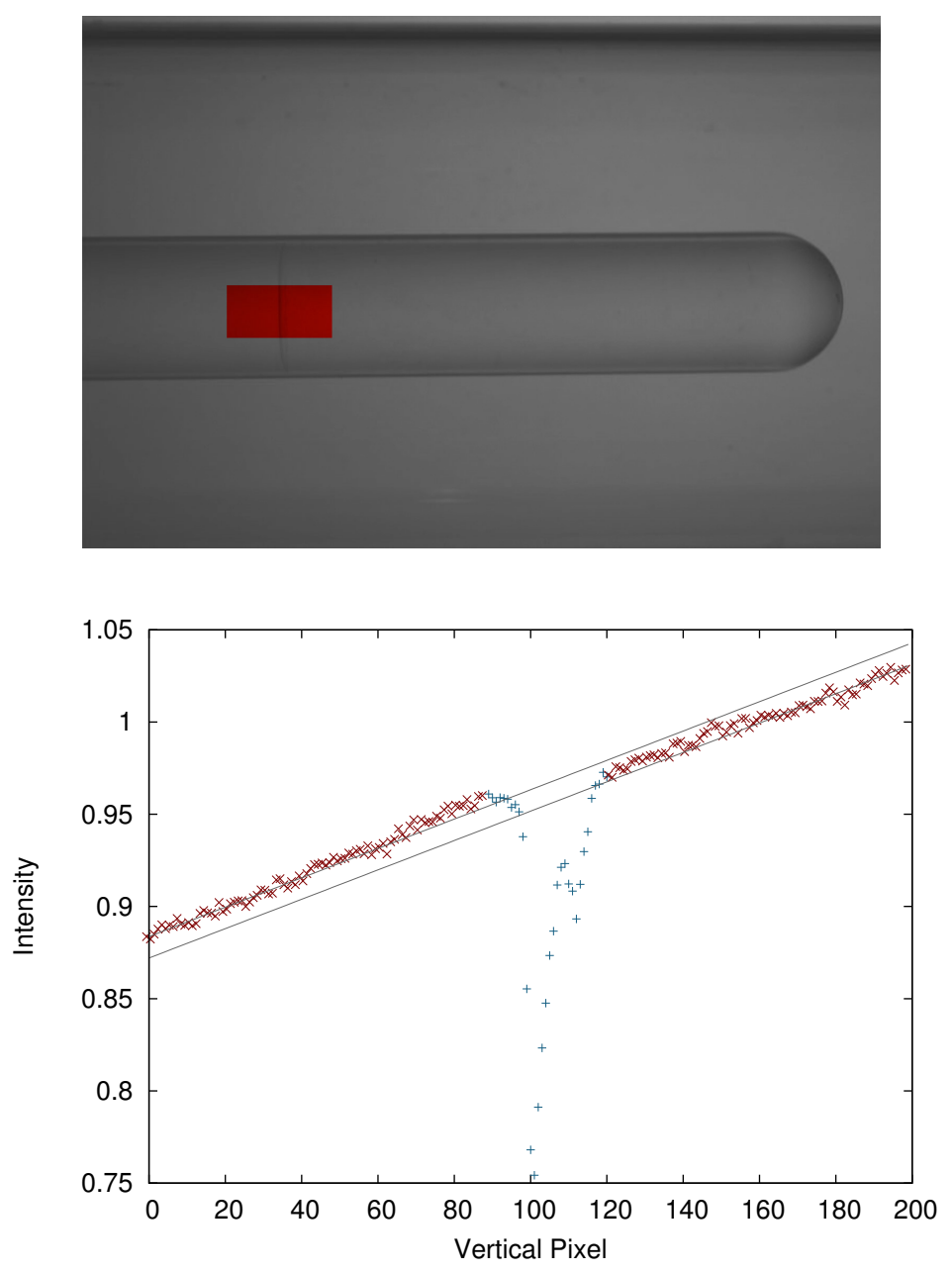

Figure 2.4: Example of a darkening measurement from a red backlit image of the thin test stand filled with $\mathrm{CF}_{3} \mathrm{I}$. The image is rotated to point along the plot's $\mathrm{x}$-axis and the fit region of interest is highlighted. Up and the $-x$-direction is to the left. Within a manually selected region of interest spanning the $\mathrm{CF}_{3} \mathrm{I}$ /water interface, the light intensity through the vessel is plotted as a function of vertical pixel position. A linear model for background illumination is modeled with a step in the function, and the size of the step is taken to be the difference in absorption across the water $/ \mathrm{CF}_{3} \mathrm{I}$ interface. The large maroon data points have been manually selected to be included in the fit. $0.61 \% \pm 0.22 \%$ absorption is measured. 
by the red and green pixels of the camera produce a darkening figure given by

$$
\begin{aligned}
\text { Darkening } & =1-\frac{R_{\mathrm{H}_{2} \mathrm{O}} G_{C F_{3} I}}{G_{\mathrm{H}_{2} \mathrm{O}} R_{C F_{3} I}} \\
& =\frac{(G / R)_{\mathrm{H}_{2} \mathrm{O}}-(G / R)_{C F_{3} I}}{(G / R)_{\mathrm{H}_{2} \mathrm{O}}}
\end{aligned}
$$

where $G$ and $R$ are the green and red values of camera image averaged over two manually selected 25 pixel tall range on either side of the meniscus, as shown in Figure 2.5 .

The systematic uncertainty in darkening measurements was determined for each method from the variance of taking a series of at least five images of the same chamber, resetting the illumination and repositioning the optics each time. This systematic uncertainty was combined with the statistical uncertainty of each fitted image.

Several darkening measurements early during testing used the ambient illumination provided by white fluorescent lights rather than a backlight. These fluorescent light images were analyzed similarly to the white backlit images, but with large unknown uncertainties.

\subsubsection{Illumination and Optical Thickness Measurements}

Several potential sources of illumination may have caused darkening in COUPP-60. An initial light leak from fluorescent lamps, low-power green and yellow status LED's on the COUPP-60 cameras, and bright red LED light supplied by a fiber-optic bundle were all present. To produce darkening of the stands, illumination sources were used as shown in Table 2.1. The SugarCUBE LED fiber illuminator was the same one used in COUPP-60 and the Luxeon III Star LEDs are identical to those used on COUPP-4kg. The yellow and green LED's are similar to the status LED's used on COUPP-60's cameras. The infrared LEDs were tested as a alternative to red illumination. A $35 \mathrm{~W}$ halogen work lamp at approximately a one meter distance was used to illuminate the camera images. Its low 


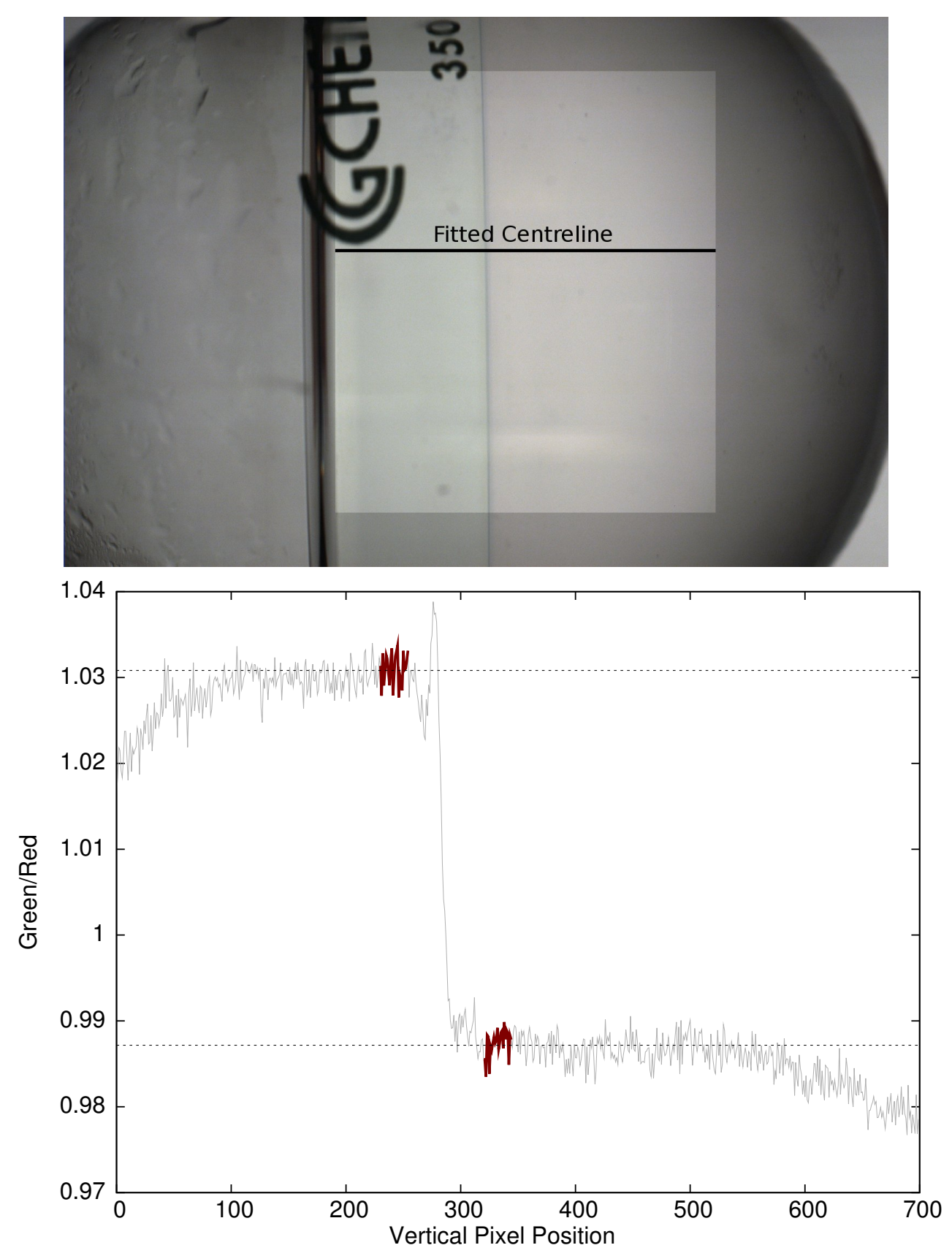

Figure 2.5: Example of a darkening measurement from a white halogen lamp backlit image of a thick test stand. Within the highlighted region of interest in the top image, a centerline at the maximum brightness of the image is found, and the ratio of red to green pixel values averaged perpendicular to the centerline is plotted along it. A discontinuity in the image color appears at the meniscus. Within this plot, regions on either side and distinct from the meniscus are selected, shown in maroon. The difference of the average of these regions, shown by dashed lines, is used to measure the darkening. 


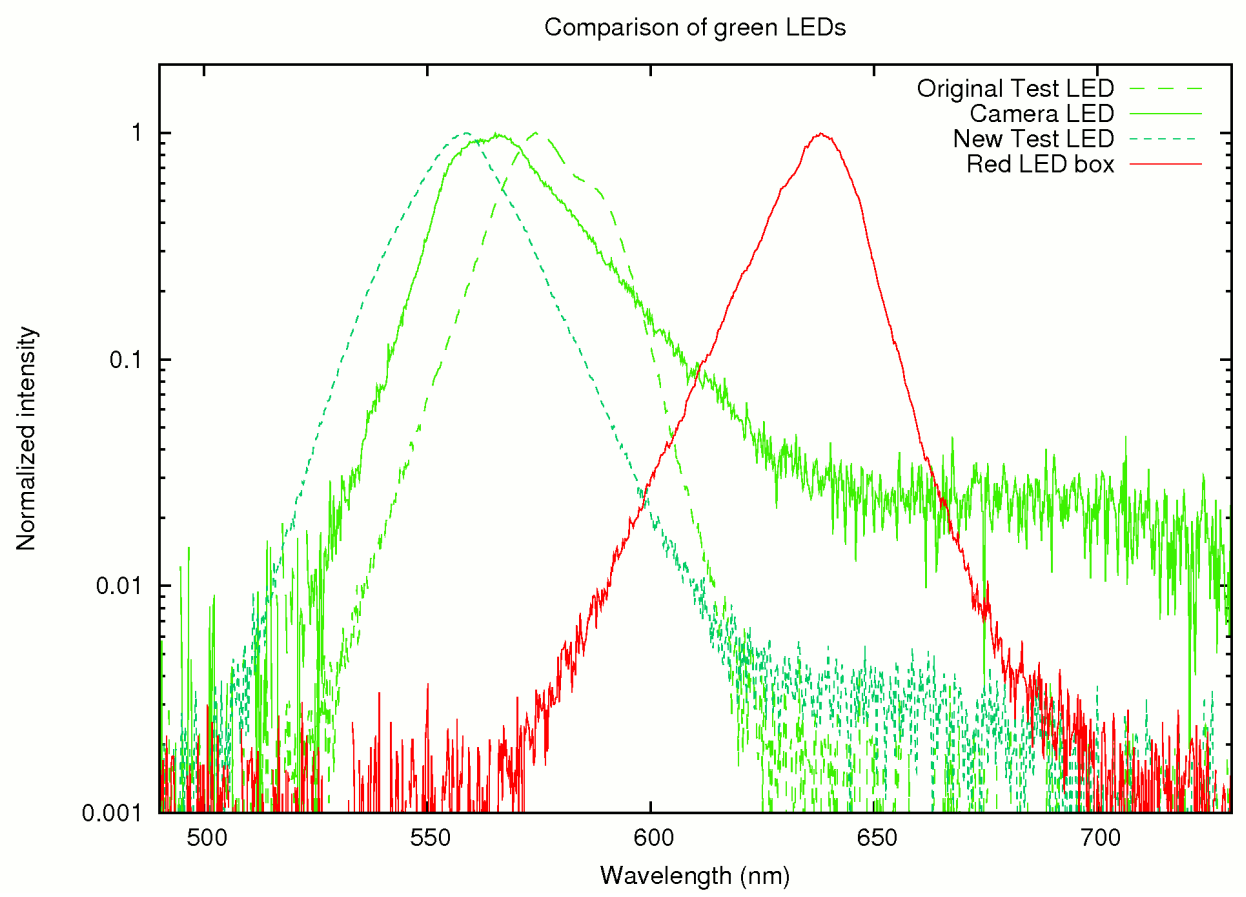

Figure 2.6: Spectra of the test apparatus LEDs. The two types of green LEDs used bracket the wavelength of the green LEDs of the COUPP-60 cameras. The camera LED was measured with an integration time of ten seconds resulting in increased noise as compared to the ten millisecond integration of the other spectra.

intensity and brief usage are not expected to contribute significantly to iodine dissociation. A double lined dark box and a dark room were used to transport and store the apparatuses when not undergoing a controlled exposure.

To verify the performance of the illumination sources, the spectra of most of the LED illumination sources used for the test stands were measured using an Ocean Optics USB4000 UV-VIS spectrometer. The spectra are shown in Figure 2.6.

The rate of photodegradation of the $\mathrm{CF}_{3} \mathrm{I}$ depends on the amount of light reaching the test stand. For the monochromatic lights, this was determined from the intensity and angular distribution specifications of the illumination sources and geometry of the stand. The angular distribution of light from the red LED box was measured as a specification was not available. The power of these light sources has been derated to account for LED 


\begin{tabular}{lcl}
\hline Color & $\begin{array}{c}\text { Typ. Intensity } \\
\mathrm{mW} / \mathrm{cm}^{2}\end{array}$ & LED/Lamp Model \\
\hline Red $630 \mathrm{~nm}$ & 140 each & SugarCUBE Red \\
Yellow $590 \mathrm{~nm}$ & 0.1 total & Kingbright WP7104SYC \\
Green $570 \mathrm{~nm}$ & 0.05 total & Kingbright WP7104CGCK \\
Green $550 \mathrm{~nm}$ & 0.006 total & Lumex SSL-LX5093XPGC \\
White & .35 total & F32T9/841 Philips Alto lamps \\
IR 940 nm & 32 each & OSRAM SDF4751 \\
IR $850 \mathrm{~nm}$ & 32 each & OSRAM SDF4750 \\
Red 630 nm & 3.3 each & Luxeon III Star Red \\
\hline
\end{tabular}

Table 2.1: The illumination sources used to test the dissociation of iodine from $\mathrm{CF}_{3} \mathrm{I}$.

temperature, but not for absorption through windows or environmental variables. As the sources of illumination were placed very close to the test stands, the average intensity of the light was numerically integrated across the volume of the thick test stand, and along the center axis of the thin test stand. The illumination intensity varied up to $10 \%$ between runs depending on the $\mathrm{CF}_{3} \mathrm{I}$ fill level.

To measure the intensity of the white fluorescent light, a commercial light meter was used. They illuminated the test stands with between 320 lux and 380 lux depending on the stand's location. Assuming a luminous efficacy of $100 \mathrm{~lm} / \mathrm{W}$, the test stands were exposed to approximately $35 \mathrm{~mW} / \mathrm{cm}^{2}$ of white light. As the $\mathrm{CF}_{3} \mathrm{I}$ would have been partially shaded by the test stand frame and the luminous efficacy conversion is unknown, the actual level of illumination is uncertain to $\pm 50 \%$.

Iodine photoabsorption is much greater for green light than it is for red light. In air, $\mathrm{I}_{2}$ has an absorption cross-section of approximately $2.7 \times 20^{-19} \mathrm{~cm}^{2}$ per molecule at $630 \mathrm{~nm}$ and $1.3 \times 10^{-18} \mathrm{~cm}^{2}$ per molecule at $570 \mathrm{~nm}$ (Saiz-Lopez et al. 2004). The same absorption cross-section is assumed in $\mathrm{CF}_{3} \mathrm{I}$ to obtain the iodine concentration. This rapidly changing absorption as a function of wavelength makes darkening measurements in green light more sensitive than those in red light. In order to cross-calibrate the different darkening 

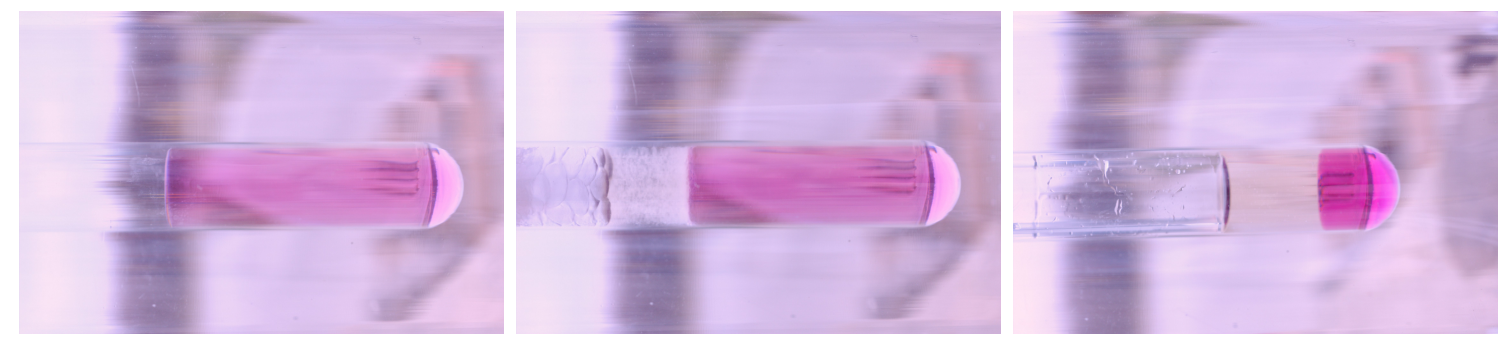

Figure 2.7: The emptying of darkened $\mathrm{CF}_{3} \mathrm{I}$ from a test stand. From left to right, the initial darkened $\mathrm{CF}_{3} \mathrm{I}$, the formation of klatrates as the $\mathrm{CF}_{3} \mathrm{I}$ boils off, and the further concentration of iodine in the remaining $\mathrm{CF}_{3} \mathrm{I}$ and darkening of the water are visible.

measurements, five illumination methods were used to measure the darkening of an iodine saturated thin test stand: $630 \mathrm{~nm}$ backlit, $590 \mathrm{~nm}$ backlit, $550 \mathrm{~nm}$ backlit, halogen backlit, and ambient fluorescent lighting. For comparison, all darkening values given in this chapter are normalized to the halogen backlit result in a thin test stand with an optical thickness of $16 \mathrm{~mm}$. For the thick test stand, the exponential extinction of the light is accounted for when converting to the optical thickness of the thin test stands.

\subsubsection{Results}

The full results and list of variables tested from the darkening test stands are presented in Appendix A. All sources of illumination tested, except the $950 \mathrm{~nm}$ IR LED's produced observable darkening of $\mathrm{CF}_{3} \mathrm{I}$ through a single photon absorbtion process, despite the $630 \mathrm{~nm}$ and $850 \mathrm{~nm}$ photons having insufficient energy to directly break the carbon-iodine bond. The darkening could be reversed in the presence of either steel or $\mathrm{Na}_{2} \mathrm{SO}_{3}$. Darkening did not occur immediately in clean recently assembled test stands, but would occur in test stands that had been refilled without an intermediate cleaning. The rate of darkening decreased as the iodine concentration increased until an equilibrium darkening of $60 \%$ was reached and this equilibrium was maintained when the $\mathrm{CF}_{3} \mathrm{I}$ was stored without illumination present.

From measuring the absorption of $550 \mathrm{~nm}$ light, a conversion factor of approximately $0.37 \mathrm{ppm}$ per percent darkening (as defined in Section 2.1.3) is applied. The equilibrium 
iodine concentration under $630 \mathrm{~nm}$ illumination is found to be $22 \mathrm{ppm}$ in $\mathrm{CF}_{3} \mathrm{I}$, the same concentration observed at the end of 2010 run of COUPP-60. This concentration is lower than the solubility of iodine in $\mathrm{CF}_{3} \mathrm{I}$, as the concentration of iodine could be increased by boiling off the $\mathrm{CF}_{3} \mathrm{I}$ solvent, as shown in Figure 2.7.

Darkening had not been observed in COUPP-4kg either in two runs at SNOLAB or in the NuMI hall at Fermilab. There are several differences between the COUPP-4kg bubble chamber and COUPP-60 bubble chamber during the 2010 run:

- COUPP-60 uses a gold seal between the inner vessel and the belows where previous chambers used a PTFE coated seal.

- The 2010 COUPP-60 run use constant illumination whereas some of the COUPP-4kg runs only illuminated the chamber when needed during a camera exposure.

- COUPP-60 experienced an air leak while filling with water. The chamber was pumped out before $\mathrm{CF}_{3} \mathrm{I}$ was introduced, but some oxygen may have remained dissolved in the water.

- The camera's used on COUPP-60 had green and red indicator LEDs on their rear whereas the COUPP-4kg cameras did not.

- COUPP-60 had more illumination intensity than previous chambers, although it had less integrated light exposure than the 2009 COUPP-4kg run.

- The bellows steel is much further from the active volume in COUPP-60 than in COUPP-4kg.

- The COUPP-60 inner vessel has not been rebuilt and thoroughly cleaned since it was assembled in early 2010 whereas COUPP-4kg had been rebuilt before every run. The COUPP-60 inner vessel had been filled and emptied before installation at NuMI.

All of these differences except the last two have been ruled out by the test stands as 
potential causes of the different rate of $\mathrm{CF}_{3} \mathrm{I}$ degradation between COUPP-4kg and COUPP60. Given that high levels of metal oxide particulate and steel corrosion have been found in the COUPP-60 chamber as of the 2014 run, a catalysis of the $\mathrm{CF}_{3} \mathrm{I}$ by these particulates seems to be the likely cause of the darkening.

To counteract the effects of darkening in the 2014 run of COUPP-60 at SNOLAB, a dilute concentration of $\mathrm{Na}_{2} \mathrm{SO}_{3}$ was added to the buffer water. The $\mathrm{Na}_{2} \mathrm{SO}_{3}$ oxidizes molecular iodine into iodide ions that are highly soluble and transparent in the water buffer. The iodide ions also combine with molecular iodine to form transparent water soluble triiodide ions, further removing molecular iodine. Darkening was not observed during the 2014 COUPP-60 run.

\subsection{Buffer $/ \mathrm{C}_{3} \mathrm{~F}_{8}$ Interface Stability}

The surface tension of the active fluid in a bubble chamber provides the metastability that allow the detector to operated. The surface properties of the active fluid and its container change the amount of energy required to form a bubble and provide new mechanisms for its formation. While boiling events at these interfaces can be identified and cut from a dark matter search, they can very easily reduce the livetime of the detector or ensure the detector never reaches its intended threshold.

When changing target fluids from $\mathrm{CF}_{3} \mathrm{I}$ to $\mathrm{C}_{3} \mathrm{~F}_{8}$, emulsions of $\mathrm{C}_{3} \mathrm{~F}_{8}$ in water were observed to form and remain stable around the $\mathrm{CF}_{3} \mathrm{I} /$ water/fused silica triple contact point (see Figure 2.8). Additional events at the buffer and glass interfaces were also observed. These excess rates have been mitigated through either the use of a linear alkyl benzene buffer fluid, or the application of a hydrophobic coating to the glass.

This section explores how surface chemistry affects bubble chambers, and how materials need to be selected to ensure stable bubble chamber operation. 


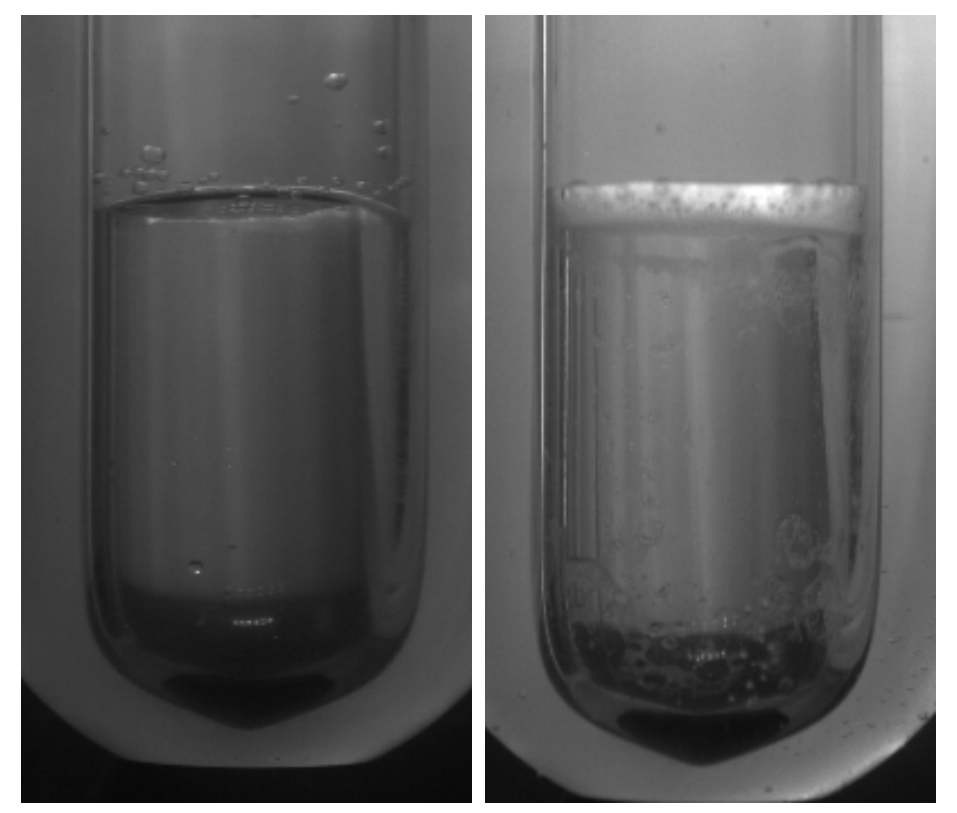

Figure 2.8: COUPP-0.1 (left) at the start of its 2013 run and (right) three weeks later. The $\mathrm{C}_{3} \mathrm{~F}_{8}$ has formed an emulsion in the water buffer at the interface. Droplets are also adhered to the glass.

\subsubsection{Surface Forces}

The surface properties of the liquids in bubble chambers determine our Seitz threshold energy, how solid particulates are transported, and possibly the rate of bubble formation at interfaces and the detector livetime lost due to this rate. The surface tension, the energy required to create additional liquid surface, is measured between the liquid compound and its vapor at equilibrium. Interfacial energy is similarly defined for liquid-liquid and liquidsolid interfaces. As some adhesion always occurs at the liquid-liquid interface, the interfacial energy is always less than the sum of the two surface tensions. The amount of adhesion depends on the similarity of the two liquids; their molecular size, their polar or non-polar nature, and presence of hydrogen bonding. The Good-Girifalco equation (Girifalco and Good 1957) can be used to estimate the interfacial energy, $\sigma_{A B}$ between two fluids when their surface tensions, $\sigma_{A}$ and $\sigma_{B}$, and an interaction parameter, $\Phi$, are known. 

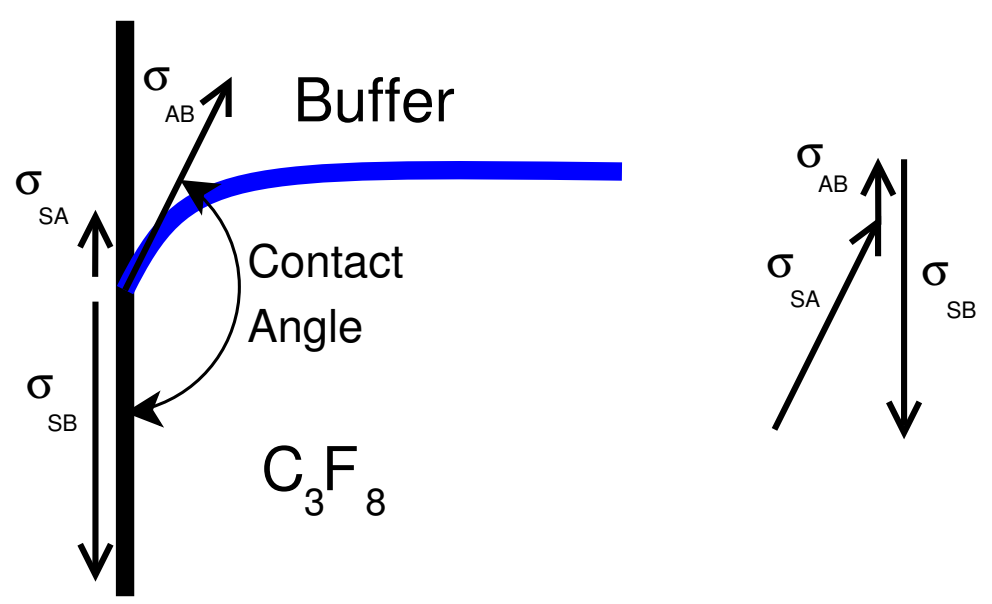

Figure 2.9: Force balance at the triple contact point between a solid wall, active fluid and buffer fluid. The solid-active interfacial energy $\sigma_{\mathrm{SA}}$, solid-buffer interfacial energy $\sigma_{\mathrm{SB}}$, and active-buffer interfacial tension $\sigma_{A B}$, must balance in the plane of the wall.

$$
\sigma_{A B}=\sigma_{A}+\sigma_{B}-2 \Phi\left(\sigma_{A} \sigma_{B}\right)^{1 / 2} \quad \text { Good-Girifalco Equation }
$$

The strong carbon-fluorine bonds in $\mathrm{C}_{3} \mathrm{~F}_{8}$ limit the polarizability of the molecule, keeping intermolecular dispersion forces and surface tension very low. In contrast, water molecules have relatively strong intermolecular forces. At a typical bubble chamber operating temperature of $14^{\circ} \mathrm{C}$, the surface tension of water is $73.8 \mathrm{mN} / \mathrm{m}$ compared to $4.9 \mathrm{mN} / \mathrm{m}$ for $\mathrm{C}_{3} \mathrm{~F}_{8}$ (Lemmon and Span 2006). The interfacial energy of perfluorocarbon liquids, perfluorohexane through perfluorononane, and water has been measured (Halper, Timmons, and Zisman 1972$)$ to be $56.1 \mathrm{mN} / \mathrm{m}$ at $20^{\circ} \mathrm{C}$. This value is adopted for the interfacial energy of $\mathrm{C}_{3} \mathrm{~F}_{8}$ and water. In $\mathrm{CF}_{3} \mathrm{I}$ at $37^{\circ} \mathrm{C}$ and using $\Phi=0.66$ from iodobenzene (Girifalco and Good 1957) and Equation 2.4, the interfacial energy of $\mathrm{CF}_{3} \mathrm{I}$ with pure water is $62.5 \mathrm{mN} / \mathrm{m}$.

At the triple contact point between the active fluid, the buffer, and the inner vessel wall, the interfacial energy of the water $/ \mathrm{C}_{3} \mathrm{~F}_{8}$ interface must be vertically balanced against the adhesive forces to the quartz in order that the position of the contact point remains stationary, as shown in Figure 2.9. This sets the equilibrium contact angle $\theta$ by Young's 
equation (Becher 2001).

$$
\cos \theta_{A}=\frac{\sigma_{S A}-\sigma_{S B}}{\sigma_{A B}}
$$

The same equation with different subscripts can be used at the liquid, vapor, and solid triple contact point.

$$
\cos \theta=\frac{\sigma_{S A}-\sigma_{S V}}{\sigma}
$$

where $\sigma_{S V}$ is the surface energy of the solid surface in the presence of vapor.

Contact angle hysteresis commonly occurs where the angle formed by an advancing interface is greater than that of a receding interface. This hysteresis creates a net retarding force on a droplet, adhering it to the wall and preventing it from falling back into its bulk phase (Dussan V. and Chow 1983). Small contact angle hysteresis is normally unavoidable while large hysteresis is often caused by a rough surface or surface impurities (Neumann 1974). The adhesion of water to fused silica is time-dependent as the silica surface bonds with mono-layers of water (Bernett and Zisman 1969; Janczuk and Zdziennicka 1994). The presence and absence of this water monolayer leads to a large contact angle hysteresis for water glass interfaces.

In Equation 2.5, when the adhesion of the active liquid to the solid equals the liquid interfacial tension, $\sigma_{S C}-\sigma_{S A}=\sigma_{A B}$, the surface is fully wetted, and $\theta_{A}=0$. By measuring the contact angle of a family of chemically similar fluids, $\sigma_{S A}-\sigma_{S V}$ can be held constant and $\sigma$ varied. A critical surface tension, $\sigma_{c}$, can be defined at the $\theta_{A}=0$ intercept. Fluids with a surface tension less than the critical surface tension will fully wet the surface. For polyfluorotetraethylene, PTFE, the critical surface tension lies between $17 \mathrm{mN} / \mathrm{m}$ and $22 \mathrm{mN} / \mathrm{m}$, depending on the chemistry of the fluid (Fox and Zisman 1950). While the critical surface tension of some hydrophobic coatings can be as low at $12 \mathrm{mN} / \mathrm{m}$ (Arkles 2006), nearly all other solids have higher values for the critical surface tension. 


\subsubsection{Emulsions}

Emulsions are the suspension of droplets, the internal phase, in another immiscible liquid, the external phase (Becher 2001). In bubble chambers, emulsion droplets can be formed either when a bubble is recondensed in the buffer fluid or when a bubble turbulently breaches the buffer fluid/target fluid interface. As droplets increase interface area, emulsions are energetically unstable. The refrigerant droplets will fall through the buffer and eventually coalesce with other droplets or the bulk phase.

Droplet coalescence proceeds in at least two steps. As the droplet approaches the bulk interface, the external phase must be displaced from under the droplet. As both the droplet and buffer/target interface deform as the droplet approaches, a dimple will form trapping a thicker volume of the external phase at the center of the droplet than at the edges. The dimple, and thus the volume of fluid that is trapped, is smaller when the interfaces have greater rigidity due to large interfacial energy. Small droplet size, high interfacial energy, low external phase viscosity, and low buoyancy all increase the rate at which the external phase film thins. As the dimple volume decreases, the droplet slowly lowers onto the bulk phase, and creates a thin film around edge of the droplet.

As the droplet approaches a sloped interface, it will slide down the slope. If the contact angle $\theta_{A}<\pi / 2$, the meniscus will curve down at the wall and droplets will collect there. Here, the of the gravitational force driving coalescence is reduced by a factor of $\sin \theta_{A}$. Also, droplets may attach to the wall and be held against gravity above and away from the interface due to contact angle hysteresis. With $\theta_{A}>\pi / 2$, the droplets will fall towards the center of the meniscus, and coalesce more quickly.

The second step of coalescence, the rupture of the thin film of the external phase, has poorly understood dynamics. However, the addition of surface active agents or concentrated fine particulates can create an additional energy barrier that hinders coalescence. 
Particulate stabilized emulsions have been studies extensively due to their role in oil and bitumen extraction. When the contact angle between the particulate solid and the two liquids is far from either $0^{\circ}$ or $180^{\circ}$, the particulates are strongly held to the interface (Levine, Bowen, and Partridge 1989a, 1989b). For a spherical droplet with radius $r$, the work required to extract a particle from the surface is

$$
W=\pi r^{2} \sigma_{A B}(1-\cos \theta)^{2}
$$

For a $100 \mathrm{~nm}$ particulate at the $\mathrm{C}_{3} \mathrm{~F}_{8} /$ water interface, $W=4 \cdot 10^{5} k_{B} T$. The energy available from gravity is negligible for particulates smaller than $100 \mu \mathrm{m}$.

If the particulates cover a large fraction of the interface, they can impede coalescence. To cover a $200 \mathrm{~cm}^{2}$ interface, similar to that in PICO-2L, with close packed $0.1 \mu \mathrm{m}$-size particulates, $0.0024 \mathrm{~cm}^{3}$ of particulates are required. If there are insufficient particulates to fully cover the interface and they have a high density, they will fall towards the lowest point of the interface meniscus, where most of the droplets reside.

\subsubsection{Nucleation at Interfaces}

In a review paper, Cole (1974) explored the necessary conditions for both homogeneous and heterogeneous spontaneous bubble nucleation. For many different interface shape, he finds

or cites the ratio, $f$, of the activation energy for nucleation in the presence and absence the interface.

Unlike for the spontaneous nucleation Cole studied, no thermal reservoir is available to help form a bubble during the short pulse of heat generated by radiation induced boiling. Whereas Cole studied activation energy,

$$
E_{x}=\frac{4 \pi}{3} r_{e}^{3} \rho^{*} \Delta G+4 \pi r_{e}^{2} \sigma
$$


in bubble chambers the threshold energy for radiation induced boiling, Equation 1.2 , is more important. For nucleation at interfaces, Equation 1.2 becomes

$$
E_{c}=V \rho^{\prime} \Delta H+A_{A V}\left(\sigma+T \frac{d \sigma}{d T}\right)+A_{S V}\left(\sigma_{S V}+T \frac{d \sigma_{S V}}{d T}-\sigma_{S A}-T \frac{d \sigma_{S A}}{d T}\right)
$$

where AV, SV, and SA refer to the active fluid/vapor, solid/vapor, and solid/active fluid interfaces respectively. From Equation 2.5.

$$
\sigma_{S A}+T \frac{d \sigma_{S A}}{d T}-\sigma_{S A}-T \frac{d \sigma_{S A}}{d T}=\cos \theta\left(\sigma+T \frac{d \sigma}{d T}\right)+T \sigma \frac{d \cos \theta}{d T}
$$

In most cases far from the critical temperature, contact angles do not vary significantly with temperature (Phillips and C. 1965, Petke and Ray 1969) and the last term can be neglected. Equation 2.9 can then be factored as,

$$
E_{c} \approx V \rho^{\prime} \Delta H+\left(A_{A V}+A_{S V} \cos \theta\right)\left(\sigma+T \frac{d \sigma}{d T}\right)
$$

When $f$ is a function of only $\theta$, the interfacial tensions, and the interface geometry, the derivations proceed as in Cole Cole $(1974)$. Since Cole uses the relationship $\Delta G=\frac{2 \sigma}{\rho^{*} r_{e}}$, the analogy no longer applies if $f=f\left(r_{e}\right)$.

Due to the low surface tensions of both $\mathrm{CF}_{3} \mathrm{I}$ and $\mathrm{C}_{3} \mathrm{~F}_{8}$, they fully wet all surfaces and particulates observed in COUPP and PICO bubble chambers, therefore more energy is required to nucleate bubbles at $\mathrm{C}_{3} \mathrm{~F}_{8}$ and $\mathrm{CF}_{3} \mathrm{I}$ interfaces than in the bulk. When considering other target fluids, the threshold energy at planar surfaces can be reduced by

$$
f_{\text {plane }}=\frac{1}{4}\left(2+3 \cos \theta-\cos ^{3} \theta\right)
$$


while at liquid-liquid interfaces,

$$
f_{\mathrm{LL}}=\left[\frac{1}{2}\left(\sigma^{3}+\sigma_{B}^{3}\right)+\frac{1}{16} \sigma_{A B}^{3}+\frac{3}{8} \frac{\sigma^{2} \sigma_{B}^{2}}{\sigma_{A B}}-\frac{3}{8} \sigma_{A B}\left(\sigma^{2}+\sigma_{B}^{2}\right)-\frac{3}{16} \frac{\sigma^{4}+\sigma_{B}^{4}}{\sigma_{A B}}\right]^{\frac{1}{3}}
$$

which is defined when $\left|\sigma-\sigma_{A B}\right| \leq \sigma_{B}$.

Trapped gas or vapor trapped in poorly wetted cavities can also nucleate bubbles. Again, due to the excellent wetting properties and high gas solubility of $\mathrm{C}_{3} \mathrm{~F}_{8}$ and $\mathrm{CF}_{3} \mathrm{I}$ (Lemal 2004), entrapped gas pockets are not expected to survive the bubble chamber's compression cycle.

When a refrigerant droplet coalesces with the bulk fluid, energy is released by the rapid reduction in the interface surface area. This energy can be sufficient to form the gas-liquid surface of a bubble nucleus. Little of the release of heat from the interface would be donated to the active fluid; only mechanically transfered energy from the rupture of the buffer film is available to form a bubble. Therefore, the free energy released must be greater than the total energy required to form a bubble surface, or

$$
\sigma_{A B}>\sigma-T \frac{d \sigma}{d T}
$$

For $\mathrm{C}_{3} \mathrm{~F}_{8}$ and water at $14^{\circ} \mathrm{C}$ and $\mathrm{CF}_{3} \mathrm{I}$ and water at $37^{\circ} \mathrm{C}$, this relation is satisfied. For either fluid with a linear alkyl benzene buffer, the relation is not satisfied.

Nucleation can also be initiated at the triple contact point between the active fluid, the buffer fluid, and the vessel wall. Here, surface imperfections that create contact angle hysteresis can trap a segment of the triple contact line. When the segment releases, a kink forms in the active fluid/buffer fluid interface. The change in angle at the kink, $\Delta \theta$ is at most the difference between the triple contact angle at release and the equilibrium contact 


\begin{tabular}{lcccc}
\hline Interface & $\begin{array}{c}\text { Temperature } \\
{ }^{\circ} \mathrm{C}\end{array}$ & $\begin{array}{c}\sigma_{A B} \\
(\mathrm{mN} / \mathrm{m})\end{array}$ & $\begin{array}{c}\sigma-T \frac{d \sigma}{d T} \\
(\mathrm{mN} / \mathrm{m})\end{array}$ & $\Delta \theta_{n}$ \\
\hline $\mathrm{C}_{3} \mathrm{~F}_{8} /$ water & 14 & 56.1 & 34.4 & $103^{\circ}$ \\
$\mathrm{CF}_{3} \mathrm{I} /$ water & 37 & 62.5 & 45.1 & $116^{\circ}$ \\
$\mathrm{C}_{3} \mathrm{~F}_{8} /$ hexyl alkyl benzene & 14 & 18 & 34.4 & \\
$\mathrm{CO}_{2} /$ mercury & 5 & 400 & 53.3 & $43^{\circ}$ \\
\hline
\end{tabular}

Table 2.2: The interfacial tension of several active fluid/buffer fluid pairs compared to the surface energy required to form a bubble nucleus (Lemmon and Span 2006; Lemmon 2006, Fox and Zisman 1950, Span and Wagner 1996, Girifalco and Good 1957). If Equation 2.14 holds and $\Delta \theta_{n}$ exists, a droplet of the active fluid merging with the bulk can nucleate a bubble.

angle. Equation 2.14 is extended to account for the lower free energy available:

$$
\sigma_{A B}\left(1-\cos \Delta \theta_{n}\right)=2\left(\sigma-T \frac{d \sigma}{d T}\right) \quad \Delta \theta>\Delta \theta_{n}
$$

The critical change in angle required to form a bubble, $\Delta \theta_{n}$, for various fluids is given in

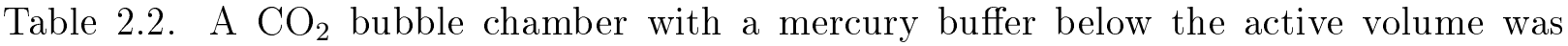
constructed at Argonne National Laboratory. The low value of $\Delta \theta_{n}$ allowed the formation of a nucleation site at the triple contact point on nearly every expansion (E. Rehm, priv. comm.).

\subsubsection{Alternative Buffer Fluids}

The interfaces of several potential buffer fluids and $\mathrm{C}_{3} \mathrm{~F}_{8}$ have been observed in a borosilicate glass vessel at room temperature. A Chemglass $15 \mathrm{~mL}$ pressure vessel with a $3.18 \mathrm{~mm}\left(1 / 8^{\prime \prime}\right)$ outer diameter and $0.71 \mathrm{~mm}(0.028 ")$ thick wall stainless steel fill tube contained the $\mathrm{C}_{3} \mathrm{~F}_{8}$ and buffer under pressure. The interfacial tension, the advancing and receding contact angles, and emulsion coalescence were roughly measured.

For each buffer fluid, the pressure vessel was rinsed clean with distilled water. With 


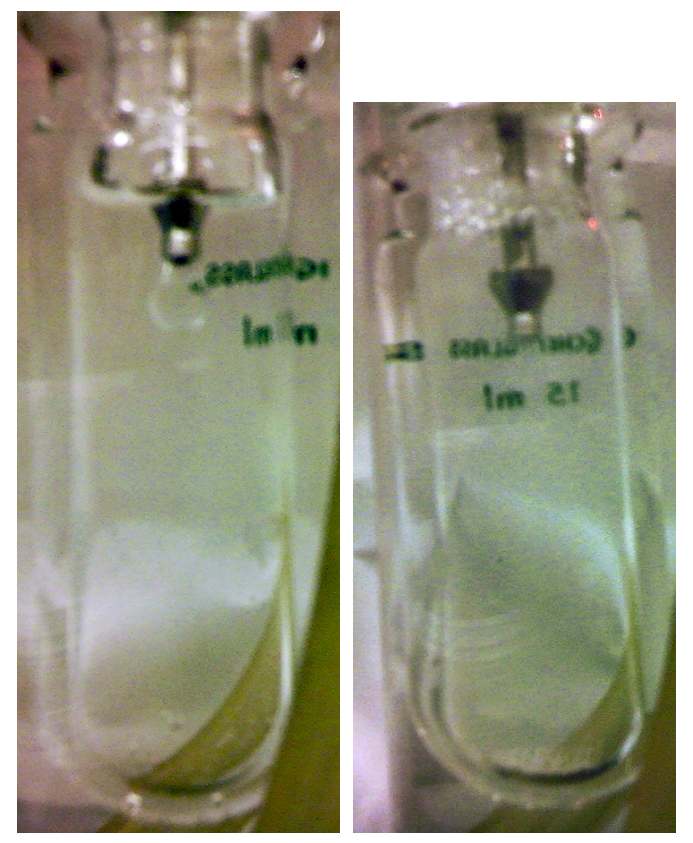

Figure 2.10: Images of pendant drops of $\mathrm{C}_{3} \mathrm{~F}_{8}$ in water (left) and $0.3 \%$ Triton $\mathrm{X}-100$ solution (right). Neither gaseous nor liquid $\mathrm{C}_{3} \mathrm{~F}_{8}$ droplets were observed to coalesce in the Triton $\mathrm{X}-100$ solution.

the pressure vessel cap removed, the vessel was filled with the buffer fluid. The buffer fluid level was set to submerge the tip of the fill tube with the cap in place. The cap was sealed without pumping down, thus leaving approximately four milliliters of air in the system. $\mathrm{C}_{3} \mathrm{~F}_{8}$ was slowly added through the fill tube, and the interfacial tension was measured using the pendant drop method (Fordham 1948). By this method, the surface tension is found by measuring the maximum force it can exert to hold a droplet against gravity. The mass of the droplet and the narrowest diameter of the pendant drop must be measured.

Images of several forming and falling drops were captured for each measurement, and the width and length of the drops recorded. The mass of the drops was estimated to be that of ellipsoidal drops with the same width and length. The minimum diameter of the pendant drop was constrained to be between the inner diameter and outer diameter of the fill tube. The coverage of the fill tube end was roughly measured. Due to the inexact design 


\begin{tabular}{lcccc}
\hline Buffer Fluid & $\sigma_{A B}(\mathrm{mN} / \mathrm{m})$ & $\theta_{A} \max$ adv. & $\theta_{A}$ min rec. & Emulsion lifetime \\
\hline water & $47 \pm 5$ & $140^{\circ} \pm 10^{\circ}$ & $85^{\circ} \pm 10^{\circ}$ & 5 sec \\
$20 \%$ methanol $/ \mathrm{H}_{2} \mathrm{O}$ & $33 \pm 12$ & $140^{\circ} \pm 10^{\circ}$ & $85^{\circ} \pm 10^{\circ}$ & 5 sec \\
$50 \%$ glycerol $/ \mathrm{H}_{2} \mathrm{O}$ & $53 \pm 16$ & \multicolumn{2}{c}{$\mathrm{n} / \mathrm{a}$} & $\gg 10$ minutes \\
$0.3 \%$ Triton $\mathrm{X}-100 / \mathrm{H}_{2} \mathrm{O}$ & $8 \pm 3$ & \multicolumn{2}{c}{$\mathrm{n} / \mathrm{a}$} & $\gg 24$ hours \\
Propylene glycol & $9 \pm 3$ & $135^{\circ} \pm 10^{\circ}$ & $110^{\circ} \pm 10^{\circ}$ & see caption \\
Ethylene glycol & $18 \pm 5$ & $140^{\circ} \pm 10^{\circ}$ & $135^{\circ} \pm 10^{\circ}$ & see caption \\
\hline
\end{tabular}

Table 2.3: Interfacial tension, contact angle, and emulsion lifetime measurements of $\mathrm{C}_{3} \mathrm{~F}_{8}$ and various buffer fluids. The emulsion lifetime in propylene glycol and ethylene glycol was limited by the low terminal velocity of the falling $\mathrm{C}_{3} \mathrm{~F}_{8}$ droplets. Droplet coalescence was rapid. Contact angle measurements could not be performed in the Triton X-100 solution or the glycerol solution as a persistent emulsion was created on filling.

of the apparatus, the interfacial tension could only be measured with large uncertainties. Results are shown in Table 2.3.

Once two to three milliliters of $\mathrm{C}_{3} \mathrm{~F}_{8}$ had been added, the pressure vessel was tilted to observe the advancing and receding contact angles. The angles were measured by comparing the interface shape against a protractor held outside the pressure vessel, and results shown in Table 2.3.

Finally, the pressure vessel was vigorously shaken for up to a minute, and an emulsion formed. The rate of degradation of the emulsion was observed to establish whether unwanted emulsions would persist in a bubble chamber. These rates are also shown in Figure 2.3.

\subsubsection{Surface Treatments}

In order to modify the contact angle at the water $/ \mathrm{C}_{3} \mathrm{~F}_{8} /$ quartz interface, the adhesion of the two liquids to the glass surface can be modified by attaching chemical functional groups to the surface of the quartz surface (Arkles 2006). These groups are bonded to the glass by a siloxane bond. A hydrophobic treatment, with the trade name Dynasylan 8261, was used to modify the surface energy of a Chemglass pressure vessel and a glass pressure vessel 


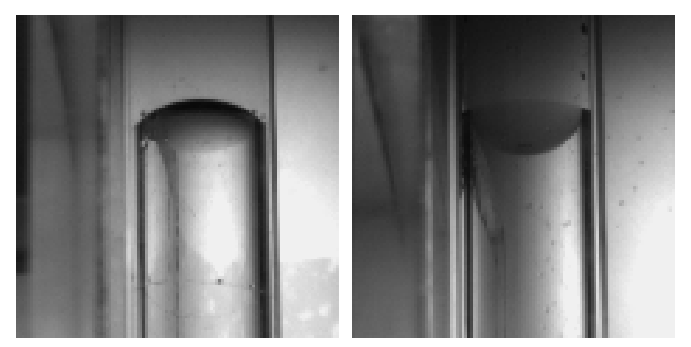

Figure 2.11: The CYRTE bubble chamber $\mathrm{C}_{3} \mathrm{~F}_{8}$ /water interface before (left) and after (right) silane treatment. The equilibrium contact angle $\theta_{A}$ decreased from $130^{\circ}$ to $35^{\circ}$ with the treatment.

for the CYRTE and University of Chicago bubble chambers described in Chapter 4. The treatment reversed the shape of the interface meniscus, as shown in Figure 2.11. Contact angle hysteresis was also reduce so that water droplets no longer adhered to the vessel wall. The treatment dramatically reduced the rate of nucleations at the wall of the bubble chambers.

\subsubsection{Conclusion}

Continuously superheated bubble chambers can only operate when the interfaces with the active volume are free of nucleation sites. First, this requires the active fluid to completely wet the vessel walls. It requires the active fluid/buffer fluid interfacial tension to be sufficiently low so that it does not have the strength to mechanically rip the active fluid. Emulsions need to be suppressed by having a sufficiently high active/buffer interfacial tension at either a flat or concave meniscus, $\theta_{A}<90^{\circ}$, or a low contact angle hysteresis. Finally, the buffer fluid needs to be transparent and of high purity. For use with $\mathrm{C}_{3} \mathrm{~F}_{8}$, ethylene glycol and linear alkyl benzene in a silica vessel meets these requirements. While water meets most of these requirements when the silica is treated with a hydrophobic coating, the interfacial tension is sufficiently high for a coalescencing $\mathrm{C}_{3} \mathrm{~F}_{8}$ droplet to nucleate a bubble.

The PICO-2L experiment described in Chapter 5 and used to set dark matter limits in Chapter 9 uses a water buffer fluid with a $\mathrm{CF}_{3} \mathrm{I}$ active fluid and an uncoated synthetic silica 
jar. The bubble chamber was operable, but did experience periods of high bubble nucleation rate at interfaces, and a background rate due to the presence of particulate matter released from the water $/ \mathrm{C}_{3} \mathrm{~F}_{8}$ interface. The use of alternative buffer fluids or a hydrophobic coating may reduce these problems in future bubble chambers. 


\section{Chapter 3 Elements of Nuclear Recoil Calibrations}

Neutron radiation can produce nuclear recoils that mimic a dark matter signal. While this makes them an good calibration source, such sources are not ideal as neutrons are difficult to measure, collimate, shield, or produce at energies below $1 \mathrm{MeV}$. The low data-taking rate of bubble chambers precludes the use of low efficiency coincidence detectors for detecting scattered neutrons as used to calibrate other dark matter detector technologies such as time-projection chambers (Alexander et al. 2013) or inorganic scintillators (Collar 2013b).

Without coincidence detection, the event-by-event nuclear recoil energy from neutron scattering cannot be measured. Either the dark matter detector response needs to be deconvolved from the response to various broad nuclear recoil spectra, or alternative methods of nuclear recoil production need to be used. When using the former strategy with a threshold detector, sharp features in the nuclear recoil spectrum at relevant energies $(\approx 10 \mathrm{keV})$ eliminate degeneracies in the deconvolution. Monoenergetic neutron sources provide a sharp cutoff in the recoil spectrum at the maximum elastic recoil energy,

$$
E_{r}^{\max }=\frac{4 m_{n} m_{X}}{\left(m_{n}+m_{X}\right)^{2}} E_{n}
$$

where $m_{n}$ and $m_{X}$ are the masses of the neutron and nucleus and $E_{n}$ is the neutron energy in the lab-frame.

\subsection{Monoenergetic Neutron Sources}

Applying Equation 3.1 using the mass of a fluorine nucleus, a $10 \mathrm{keV}$ maximum recoil is provided by a $52.2 \mathrm{keV}$ neutron. Neutrons with such a low energy can be produced by $(p, n)$ 
or $(\gamma, n)$ reactions. The former requires the use of an accelerator while the latter is best used with $\gamma$-rays from radioactive decay. The $(\gamma, n)$ reaction proffers several advantages including being portable, inexpensive, and easy to use (Collar 2013a). While it produces approximately $10^{5}$ more $\gamma$ particles than neutrons, the large electron recoil rejection of bubble chambers should prevent these $\gamma$-rays from being measured. The key advantage of the $(p, n)$ reaction is the ability to tune the neutron energy to values that are unobtainable from radioactive sources.

\subsubsection{The ${ }^{51} \mathbf{V}(p, n)$ and ${ }^{7} \mathbf{L i}(p, n)$ Reactions}

Two reactions are commonly used to produce low-energy monoenergetic neutrons at accel-

erator facilities of which the ${ }^{7} \operatorname{Li}(p, n){ }^{7} \mathrm{Be}$ reaction is the most common. ${ }^{7} \mathrm{Li}(p, n){ }^{7} \mathrm{Be}$ has a wide resonance near threshold for which both the cross-section and angular distributions have been measured (Herrera, Moreno, and Kreiner 2014). The calibrations of competing dark matter detector technologies at neutron beam facilities use the ${ }^{7} \operatorname{Li}(p, n)$ reaction (Alexander et al. 2013).

The monoenergetic neutron beam used for PICO calibrations at the University of Montreal uses the ${ }^{51} \mathrm{~V}(p, n)^{51} \mathrm{Cr}$ reaction. At forward neutron energies below $120 \mathrm{keV}$, this reaction provides a comparable or better yield than the ${ }^{7} \operatorname{Li}(p, n)^{7} \mathrm{Be}$ reaction (Gibbons, Macklin, and Schmitt 1958). A large number of narrow resonances in the reaction crosssection provides an internal calibration of the neutron energy to less than $1 \mathrm{keV}$, as shown in Figure 3.1. Unfortunately, the angular distribution of neutrons from these resonances has only been roughly measured, leading to uncertainties in the measurement of the neutron flux used to normalize the calibration (see Section 6.2.1). 


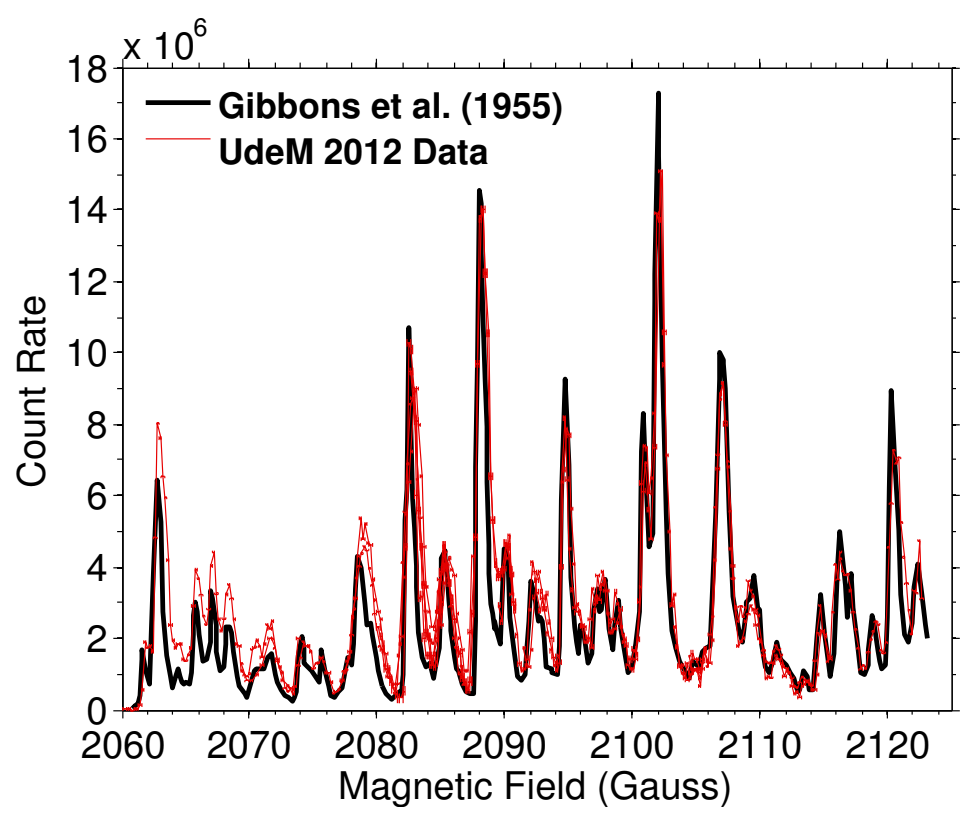

Figure 3.1: The relative cross-section of the ${ }^{51} \mathrm{~V}$ cross-section from Gibbons, Macklin, and Schmitt (1958, Fig. 1) compared to the neutron monitor count rate at the University of Montreal neutron beam. The positions of the measured resonances is used to calibrate the energy of the neutron beam. Plot by Matthieu Lafrenière.

\subsubsection{The ${ }^{9} \operatorname{Be}(\gamma, n)^{8} \operatorname{Be}$ Reaction}

Two stable target isotopes have threshold energies for $(\gamma, n)$ reaction that are sufficiently small to be surpassed by isotopic radioactive sources: ${ }^{9} \mathrm{Be}(Q=-1664.54 \mathrm{keV})$ and ${ }^{2} \mathrm{H}$ $(Q=-2224.57 \mathrm{keV})$. The pure natural abundance of beryllium, lower moderating power, and lower threshold energy make it the preferred target material. Several commercially available radioisotopes are available for use in a ${ }^{9} B e(\gamma, n)$ source, as given in Table 3.1.

For photon energies below $4.7 \mathrm{MeV}$, the ${ }^{9} \mathrm{Be}(\gamma, n)^{8} \mathrm{Be}$ reaction proceeds to the ground state of ${ }^{8}$ Be via one of three broad resonances: $J^{\pi}=1 / 2^{+}$near threshold, $J^{\pi}=1 / 2^{-}$at 2.88 $\mathrm{MeV}$, and $J^{\pi}=5 / 2^{-}$at $3.0 \mathrm{MeV}$. A narrow resonance at $2.4 \mathrm{MeV}$ is not excitable by our candidate radioisotopes and the three-body breakup reaction $\left({ }^{9} \operatorname{Be}(\gamma, n+2 \alpha)\right)$ cross-section is extremely small (Alburger et al. 2004). The low energy neutrons required for calibrations are mostly produced by by the lowest energy $1 / 2^{-}$resonance. 


\begin{tabular}{|c|c|c|c|}
\hline Isotope & $t_{1 / 2}$ & $\begin{array}{l}E_{\gamma} \text { (Intensity) } \\
(\mathrm{keV})\end{array}$ & $\begin{array}{c}E_{\gamma}+Q \\
(\mathrm{keV})\end{array}$ \\
\hline${ }^{56} \mathrm{Co}$ & $77.24 \mathrm{~d}$ & $\begin{array}{l}847.77(99.94 \%) \\
1037.84(14.05 \%) \\
1238.29(66.46 \%) \\
1771.36(15.41 \%) \\
2015.22(3.016 \%) \\
2034.79(7.77 \%) \\
2598.5(16.97 \%) \\
3202.03(3.21 \%) \\
3253.50(7.92 \%) \\
\text { others > } 1664 \mathrm{keV}\end{array}$ & $\begin{array}{c}106.82 \\
350.68 \\
370.25 \\
933.96 \\
1537.49 \\
1588.96 \\
(6.42 \%)\end{array}$ \\
\hline${ }^{88} \mathrm{Y}$ & $106.63 \mathrm{~d}$ & $\begin{array}{l}898.04(93.7 \%) \\
1836.06(99.2 \%) \\
2734.0(0.71 \%) \\
3219.7(0.0070 \%)\end{array}$ & $\begin{array}{c}171.52 \\
1069.46 \\
1555.16\end{array}$ \\
\hline${ }^{124} \mathrm{Sb}$ & $60.20 \mathrm{~d}$ & $\begin{array}{l}602.73(97.8 \%) \\
722.78(10.76 \%) \\
1690.97(47.57 \%) \\
2090.93(5.49 \%) \\
\text { others > } 1664 \mathrm{keV}\end{array}$ & $\begin{array}{c}26.43 \\
426.39 \\
(0.52 \%)\end{array}$ \\
\hline${ }^{207} \mathrm{Bi}$ & $31.55 \mathrm{y}$ & $\begin{array}{l}569.70(97.75 \%) \\
1063.66(74.5 \%) \\
1770.23(6.87 \%)\end{array}$ & 105.69 \\
\hline${ }^{226} \mathrm{Ra}$ & $1600 \mathrm{y}$ & $\begin{array}{l}609.32(45.49 \%) \\
1764.49(15.30 \%) \\
2204.06(4.924 \%) \\
\text { others }>1664 \mathrm{keV}\end{array}$ & $\begin{array}{c}99.95 \\
539.52 \\
(9.69 \%)\end{array}$ \\
\hline${ }^{228} \mathrm{Th}$ & $1.912 \mathrm{y}$ & $\begin{array}{l}238.63(43.6 \%) \\
583.19(30.55 \%) \\
2614.51(35.85 \%) \\
\text { others > } 1664 \mathrm{keV}\end{array}$ & $\begin{array}{r}949.97 \\
(0.09 \%)\end{array}$ \\
\hline
\end{tabular}

Table 3.1: Commercially available $\gamma$ emitting isotopes with large high-energy branching ratios for use in a ${ }^{9} \operatorname{Be}(\gamma, n)$ neutron source. Intensities for all neutron producing $\gamma$ emissions from the listed and daughter isotopes and the most important lower energy emissions are given (Browne and Tuli 2013, McCutchan and Sonzogni 2014 Katakura and Wu 2008; Kondev and Lalkovski 2011; Singh, Jain, and Tuli 2011; Wu 2009; Artna-Cohen 1997; Browne 2005: Martin 2007). 
The ${ }^{9} \mathrm{Be}(\gamma, n){ }^{8} \mathrm{Be}$ reaction has been measured many times, but with little agreement between measurements, especially at the $1 / 2^{-}$resonance. While using photons from radioisotopes is the simplest method to measure the cross-section (Chadwick and Goldhaber 1935: Halban 1938; Russell et al. 1948; Snell, Barker, and Sternberg 1950 Hamermesh and Kimball 1953; Gibbons et al. 1959, John and Prosser 1962, Fujishiro et al. 1982), it cannot provide a complete measurement as a function of energy. Photon beams generated by the bremsstrahlung of a stopping electron beam have been used to measure the crosssection (Jakobson 1961; Berman, Hemert, and Bowman 1967), but the continuous spectrum of photon energies is difficult to deconvolve. Measurements at recently built inverse Compton photon beams (Utsunomiya et al. 2000; Arnold et al. 2012), such as the High-Intensity $\gamma$ Source (HI $\gamma$ S), use tunable photon energies with much better resolution. All of these techniques require accurate knowledge of the absolute photon source strengths, the neutron detection efficiencies, and the photon energy spectra. The cross-section can also be measured using the inelastic scattering of charged particles (Tucker SN 1970; Spencer, Phillips, and Young 1960; Ngoc, Hors, and Jorba 1963; Clerc, Wetzel, and Spamer 1968; Kuechler, Richter, and Witsch 1987; Dixit et al. 1991; Burda et al. 2010), but these measurements suffer from background, low production cross-sections near threshold resonance, and the need to adjust the cross-section to account for the higher momentum transfer. A selection of measured cross-sections are shown in Figure 3.2 .

Measurements of the ${ }^{9} \mathrm{Be}(\gamma, n)^{8} \mathrm{Be}$ cross-section using radioisotopes nominally provide infinitesimal energy resolution and simple absolute calibrations. However, there are significant discrepancies even amongst the radioisotope measurements as there are with the more complicated experiments at accelerator facilities. Most of the radioisotope measurements were performed in the 1940's, 50's, and 60's, before high precision neutron standards, crosssections, and simulation programs were available. Fortunately, several of these experiments 


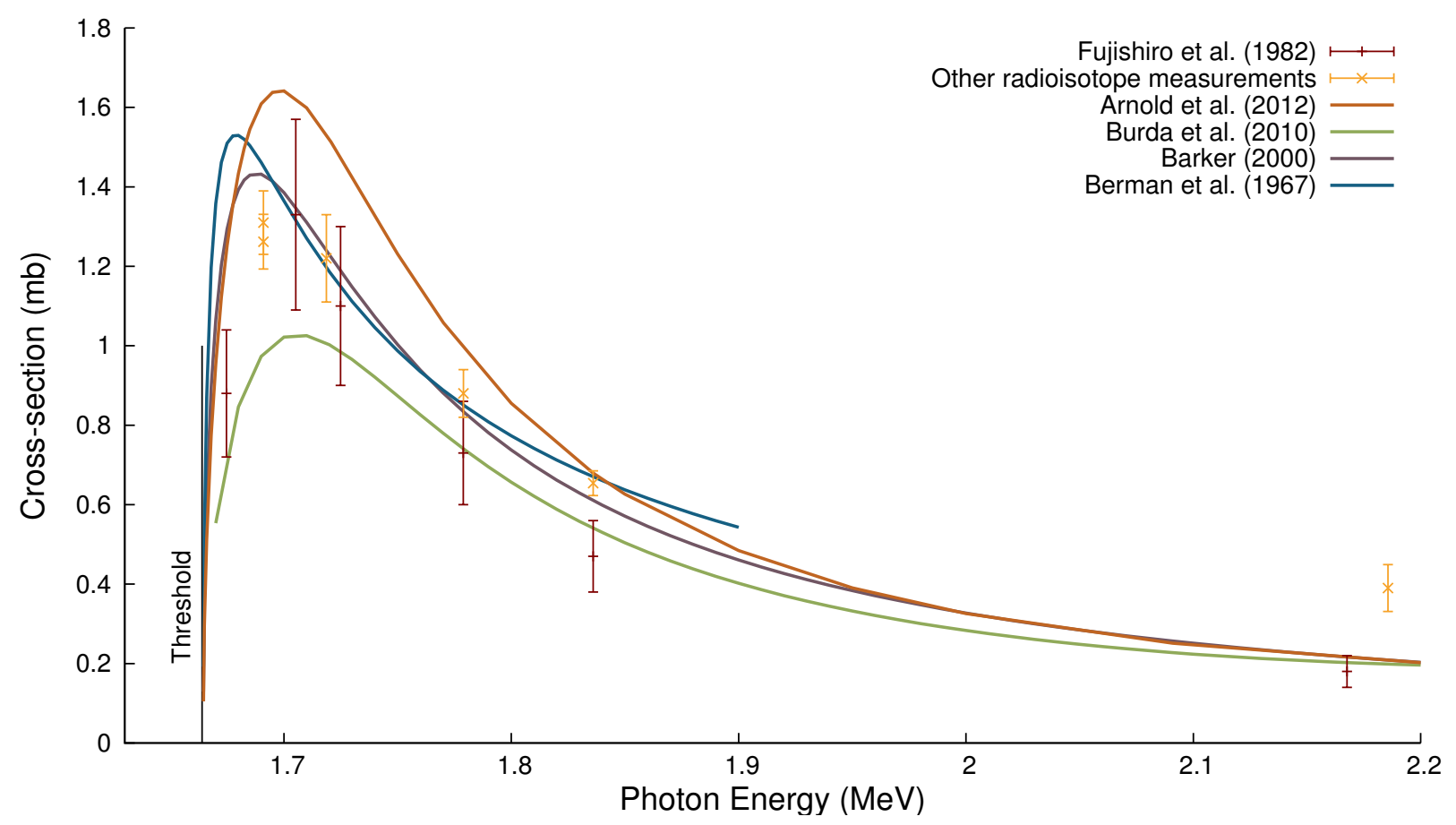

Figure 3.2: Existing measured cross-sections for the ${ }^{9} \mathrm{Be}(\gamma, n){ }^{8} \mathrm{Be}$ reaction below $5 \mathrm{MeV}$. Radioisotope measurements are from Snell, Barker, and Sternberg (1950), Hamermesh and Kimball (1953), Gibbons et al. (1959), John and Prosser (1962), and Fujishiro et al. (1982). Lines show the cross-section from fitted resonance parameters. The parameters from Barker (2000) were a fit to data from Kuechler, Richter, and Witsch (1987).

have been well documented with results that are traceable to modern calibrations. I will apply corrections to the originally measured cross-section values to construct a trusted set of measured cross-sections.

The most precisely calibrated neutron source in the world, NBS-1, owned by the National Institute of Standards and Technology (formerly the National Bureau of Standards), produces neutrons via the ${ }^{9} \mathrm{Be}(\gamma, n)^{8} \mathrm{Be}$ reaction ${ }^{1}$. This source has been calibrated using the $\mathrm{MnSO}_{4}$ bath method to within $0.85 \%$ (McGarry and Boswell 1988). Unfortunately, the source's $\gamma$-activity has not been measured.

John and Prosser (1962) used the same $\mathrm{MnSO}_{4}$ bath technique to measure the yield of a

1. Around $0.1 \%$ of the neutrons may also be produced via ${ }^{79,81} \operatorname{Br}(\alpha, n)$, the beta-delayed neutron decay of ${ }^{210} \mathrm{Tl}$, or from bremsstrahlung photons. These processes will be neglected. 
${ }^{124} \mathrm{Sb}$ source and traced their measured neutron yields with NBS calibrated sources. A list of corrections to the measurement was provided in Table I of their paper, and is reproduced with corrections in Table 3.2. The largest correction, and the largest uncertainty in the paper, comes from the measurement of the peak to Compton ratio of ${ }^{124} \mathrm{Sb}$ photons in a $2^{\prime \prime}$ thick $1.75 "$ diameter NaI crystal. While John and Prosser quote a measured ratio of 0.216 , an MCNP simulation of the detector with a $1.41 \mathrm{MeV}$ threshold gives a ratio of 0.2244 . A $3.5 \%$ uncertainty $(5 \% / \sqrt{2})$ in the gamma-ray source strength is retained to account for sub-dominant uncertainties that were not specified in the paper. Several percent and subpercent level photon lines have been found in ${ }^{124} \mathrm{Sb}$ since 1962 that can contribute to the 1.69 $\mathrm{MeV}$ peak. Assuming a $\mathrm{NaI}(\mathrm{Tl})$ detector with 5.4\% energy resolution at the peak, I applied an additional $0.42 \%$ background subtraction. The NBS-1 neutron source strength has also been revised slightly upward since the 1955 calibration (De Juren and Chin 1955).

John and Prosser calculated the cross-section at the strongest neutron producing line in ${ }^{124} \mathrm{Sb}(1.691 \mathrm{MeV})$ by subtracting background from neutrons generated by the $2.09 \mathrm{MeV}$ line. and obtaining a cross-section of $1.31 \pm 0.08 \mathrm{mb}$. Using the same technique and assuming the cross-sectional shape from Arnold et al. (2012), this is corrected to $1.40 \pm 0.07 \mathrm{mb}$. More generally, the sum of the photon branching ratios $\alpha_{i}$ and cross-sections $\sigma_{i}$ is $\sum_{i} \alpha_{i} \sigma_{i}\left({ }^{124} \mathrm{Sb}\right)=$ $0.678 \pm 0.032$.

John and Prosser's ${ }^{124} \mathrm{Sb} /$ Be source was also compared to sources using ${ }^{24} \mathrm{Al}$ and ${ }^{206} \mathrm{Bi}$. As the neutron emission rate of these two sources was too low to measure using the $\mathrm{MnSO}_{4}$ bath method, a 'Long Counter' was used in the comparison. The cross-section ratios given by John and Prosser at the principal photon energies were converted to ratios of $\sum_{i} \alpha_{i} \sigma_{i}$ by undoing their correction calculation for other $\gamma$-ray branches. MCNPX-Polimi simulations of the Harwell IV Long Counter (Allen 1955) found that the counter had equal sensitivity (within 1\%) to neutrons from each of the sources. I reduced John and Prosser's 3\% correction 


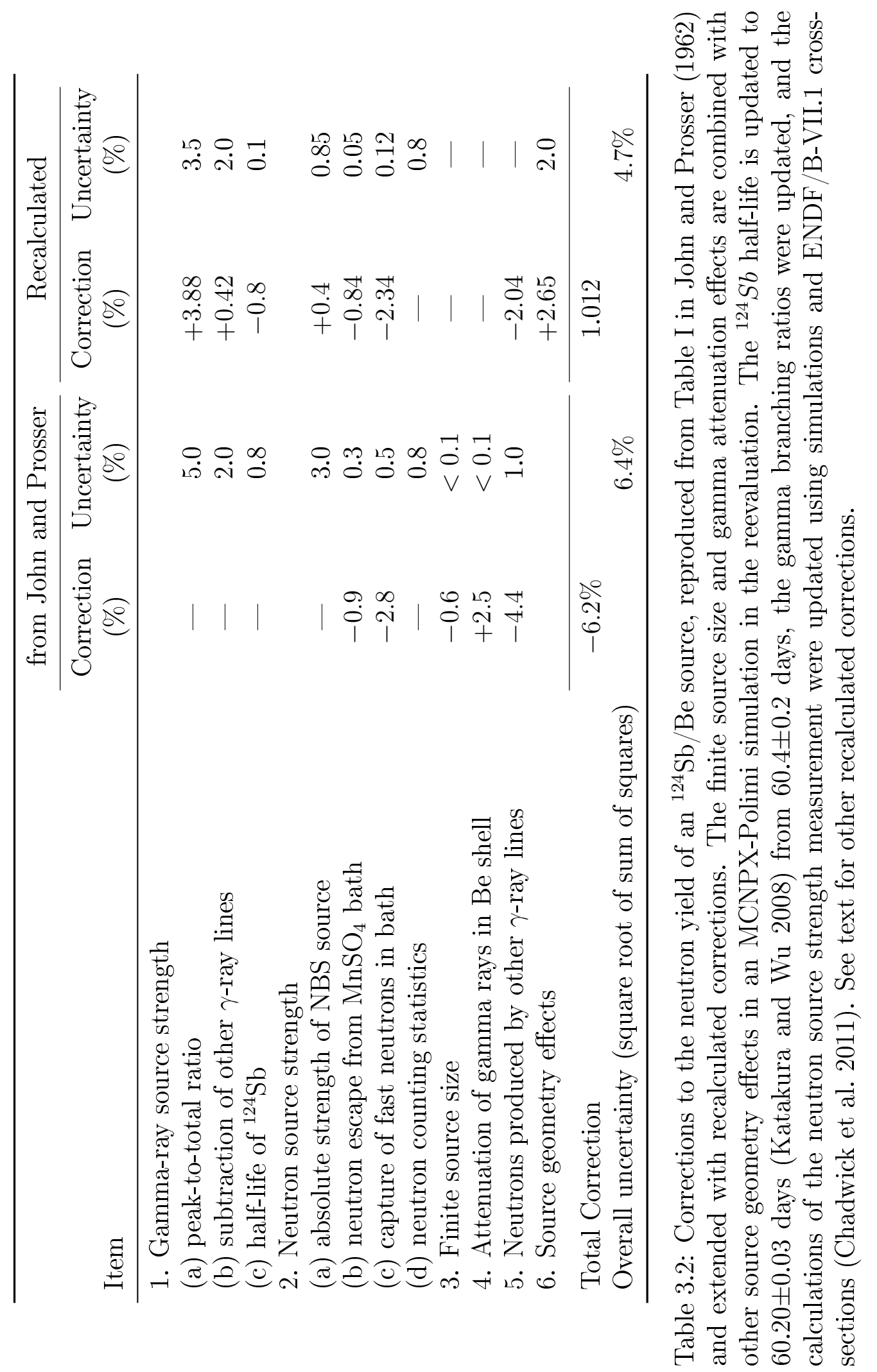


to the sensitivity of neutrons from ${ }^{28} \mathrm{Al} / \mathrm{Be}$ to $0.7 \%$. No other corrections to the cross-section beyond those in the original paper were added.

Gibbons et al. (1959) measured both ${ }^{124} \mathrm{Sb} / \mathrm{Be}$ and ${ }^{88} \mathrm{Y} / \mathrm{Be}$ neutron sources to high precision using a different technique than John and Prosser (1962). The activity of the sources was determined using a $4 \pi$ ionization counter and a scintillation counter while the neutron source strength was measured using a 5-foot diameter graphite moderating sphere and $\mathrm{BF}_{3}$ thermal neutron detectors. The neutron count rate was calibrated against a source traceable to NBS-1. After correcting for the source $\gamma$ branching ratios and for the calibration of NBS-I, Gibbons et al. found a cross-section of $\sum_{i} \alpha_{i} \sigma_{i}\left({ }^{124} S b\right)=0.669 \pm 0.029$ and $\left.\sum_{i} \alpha_{i} \sigma_{i}{ }^{88} Y\right)=0.660 \pm 0.029$

Snell, Barker, and Sternberg (1950) measured the neutron yields from both beryllium and deuterium targets using ${ }^{72} \mathrm{Ga}$ and ${ }^{24} \mathrm{Na}$ radioisotopes. Both isotopes produce $\gamma$-rays above the 2.2 MeV deuterium dissociation threshold. The neutrons were measured by sampling epithermal neutrons in large volume of paraffin moderator using indium foil sandwiched between two cadmium foils. The activated indium was counted using a thin-walled Geiger counter. Neutron losses from the moderator, energy dependence of the sensitivity, and the capture on other elements is minimized in this design. The absolute neutron flux was calibrated against a ${ }^{226} \mathrm{Ra} / \mathrm{Be}$ source that was traceable to several neutron standards available at the time. This calibration is traceable to NBS-1 via the LANL \#37 and LANL \#40 sources (Walker 1946; De Juren, Padgett, and Cutis 1955). As the pressed Ra-Be $(\alpha, n)$ LANL \#40 source would have increased in rate as the concentration of ${ }^{210}$ Po increases, I included a correction for this growth between 1944 and 1953 of 0.9715 in addition to the factor of 0.9809 from the intercalibrations. Where Snell et al. use a Ra/Be source rate of $4.17 \times 10^{6}$, a rate of $3.974 \times 10^{6}$ will be adopted here. The source activities were absolutely counted by evaporating aliquots onto a mica windowed Geiger counter calibrated against 
the specific activity of Th-234. The specific activity cited by Snell, Barker, and Sternberg was corrected to 740.8 disintegrations per minute per milligram of natural abundance uranium Browne and Tuli (2007), reducing the measured cross-section by a factor of 0.9773 .

The neutron energy dependence of the neutron rate measurements of Snell, Barker, and Sternberg (1950) has been recalculated using MCNPX-Polimi (Padovani et al. 2012). The simulated corrections to the neutron detection efficiency are very close to those analytically calculated by Snell, Barker, and Sternberg. One correction they neglected, the thermalization of neutrons reentering the deuterium or beryllium from the surrounding moderator, leads to an small additional loss of efficiency. The ratio of the detection efficiency for each source compared to the ${ }^{226} \mathrm{Ra} / \mathrm{Be}$ source was recalculated. For ${ }^{24} \mathrm{Na} / \mathrm{Be} \sum_{i} \alpha_{i} \sigma_{i}=0.611 \mathrm{mb}$ while for ${ }^{72} \mathrm{Ga} / \mathrm{Be} \sum_{i} \alpha_{i} \sigma_{i}=0.194 \mathrm{mb}$. The original uncertainty in the absolute source activity is retained, and it dominates the total uncertainty of $5 \%$. To reduce these uncertainties, the ratio of the measured beryllium and deuterium cross-sections are compared to the known modern deuterium cross-section (Chadwick et al. 2011). Using the ratio, for ${ }^{24} \mathrm{Na} / \mathrm{Be} \sum_{i} \alpha_{i} \sigma_{i}=0.620 \mathrm{mb}$ while for ${ }^{72} \mathrm{Ga} / \mathrm{Be} \sum_{i} \alpha_{i} \sigma_{i}=0.171 \mathrm{mb}$ with $3 \%$ uncertainties.

Finally, the measurements of Fujishiro et al. (1982) are the most recent radioisotope measurements of the ${ }^{9} \mathrm{Be}(\gamma, n)^{8} \mathrm{Be}$ and have heavily influenced modern evaluations of the cross-section (Barker 1983; Burda et al. 2010; Angulo et al. 1999). Fujishiro et al. selected a large variety of short-lived radioisotopes that produce $\gamma$-rays with energies in the $1 / 2^{-}$ resonance region, shown in Table 3.3. Paraffin embedded BF-3 detectors were used to measure the neutron flux. Except for the neutrons at $8 \mathrm{keV}$, an MCNPX-Polimi simulation approximating these detectors agrees with that relative efficiency vs. neutron energy relationship calculated in the paper using a one dimensional Monte Carlo simulation. A 5\% lower detection efficiency was found at $8 \mathrm{keV}$. I have corrected the photon branching ratios from the paper, with the largest correction factor of 1.125 applied to ${ }^{38} \mathrm{Cl}$. Fujishiro et 


\begin{tabular}{lcccl}
\hline Isotope & $t_{1 / 2}$ & $E_{\gamma}(\mathrm{keV})$ & Intensity $(\%)$ & Reference \\
\hline${ }^{58} \mathrm{Co}$ & $70.86(6)$ days & $1674.725(7)$ & $0.517(10)$ & Nesaraja, Geraedts, and \\
& & & & Singh 2010 \\
${ }^{105} \mathrm{Ru}$ & $4.44(2)$ hours & $1698.1(2)$ & $0.076(14)$ & Frenne and Jacobs 2005 \\
& & $1721.36(15)$ & $0.033(9)$ & \\
& & others $>1664 \mathrm{keV}$ & $0.0017(12)$ & \\
${ }^{65} \mathrm{Ni}$ & $2.51719(26)$ hours & $1724.92(6)$ & $0.399(12)$ & Browne and Tuli 2010 \\
${ }^{28} \mathrm{Al}$ & $2.245(2)$ minutes & $1778.987(15)$ & 100 & Basunia 2013 \\
${ }^{88} \mathrm{Y}$ & & See Table 3.1 & \\
${ }^{38} \mathrm{Cl}$ & $37.230(14)$ minutes & $2167.54(7)$ & 44.4 & Cameron and Singh \\
& & others $>1664 \mathrm{keV}$ & $0.041(32)$ & 2008 \\
\hline
\end{tabular}

Table 3.3: Radioisotope sources used in Fujishiro et al. (1982).

al. did not consider the uncertainty in the branching ratios. The $\pm 21 \%$ uncertainty in the high-energy photon branching ratio dominates all other uncertainties for ${ }^{105} \mathrm{Ru}$.

Fujishiro et al. reported large uncertainties in the absolute efficiency of their measurements, and their measured values underestimate the results from other radioisotope data by a factor of 0.8 . The discrepancy is increased when applying corrections for finite source size, finite target thickness, and Compton scattering that were not considered in the original paper. They provided neither the mass nor precise dimensions of the beryllium and the photon sources that could be used to reextract these corrections. Due to these untraceable corrections to the absolute count rate, only the ratios of cross-sections measured by Fujishiro were considered. The common $6 \%$ systematic uncertainty from the uncertainty of the neutron detection efficiency is subtracted when converting from the absolute to relative cross-section values.

A least-squares fit of the $1 / 2^{-}$resonance to the reanalyzed radioisotope data using the parameterization and fixed parameters for other resonances from Arnold et al. (2012) was performed. The $\gamma$-ray energies, branching ratios, and measured $\sum_{i} \alpha_{i} \sigma_{i}$ for each radioisotope measurement was input to the fit. Relative cross-section values were used for the data from Fujishiro et al. and the ${ }^{206} \mathrm{Bi}$ and ${ }^{28} \mathrm{Al}$ measurements of John and Prosser (1962) 


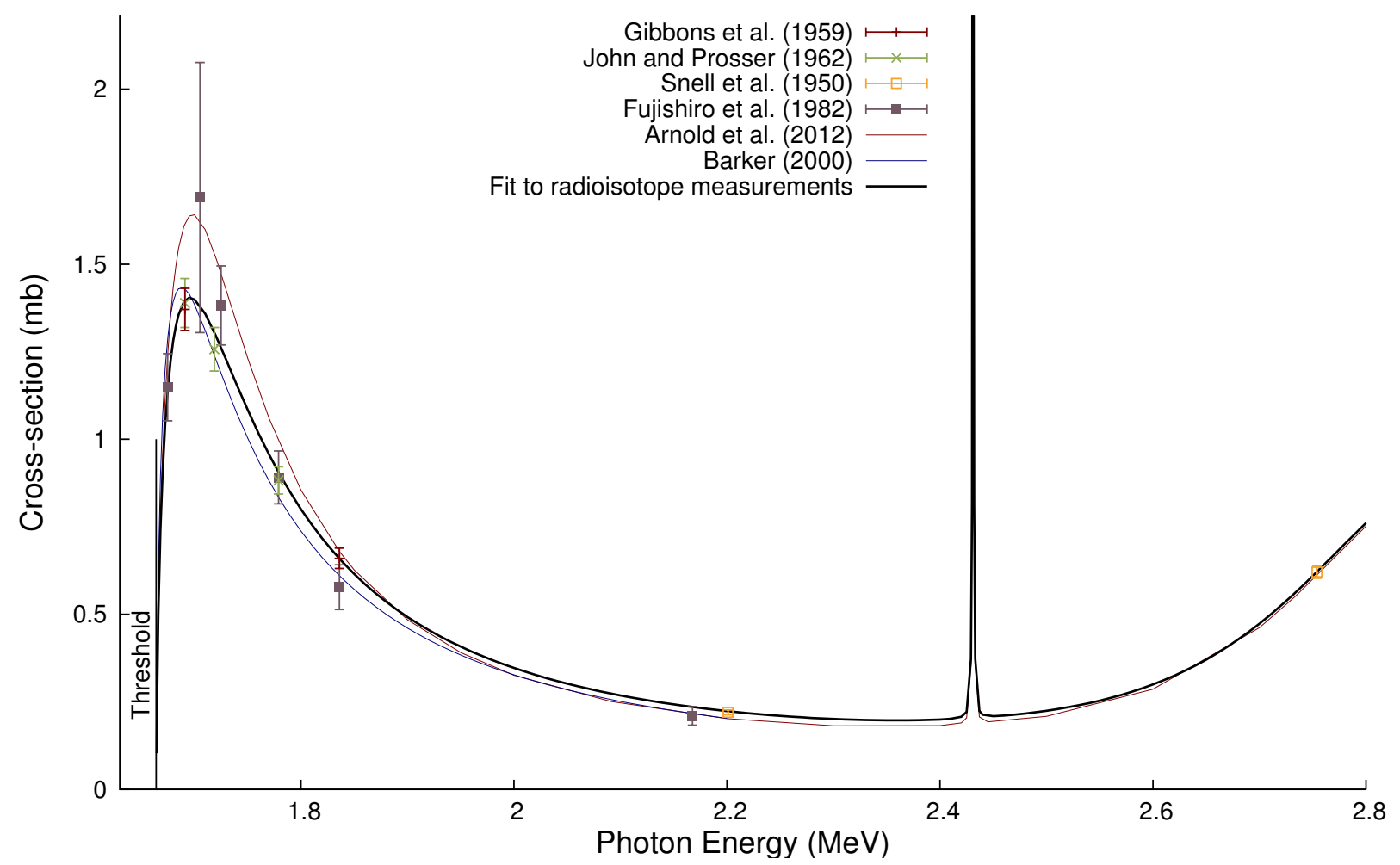

Figure 3.3: Reanalyzed cross-sections from radioisotope measurements of the ${ }^{9} \mathrm{Be}(\gamma, n)^{8} \mathrm{Be}$ cross-section below $3 \mathrm{MeV}$. Only the cross-sections for the highest intensity photon energy of each radioisotope are shown, assuming that the cross-sections at other photon energies follows in proportion to the fit. The absolute yield of the measurements of Fujishiro et al. (1982) are floated. Fit parameters are $E_{n}=1738.8 \pm 2.1 \mathrm{keV}, \Gamma_{\gamma}=0.7715 \pm 0.032 \mathrm{eV}$, and $\Gamma_{n}=268.7 \pm 16 \mathrm{keV}$.

while absolute values were used for all other data. The ${ }^{24} \mathrm{Na}$ data from Snell, Barker, and Sternberg (1950) was excluded as it is well above the $1 / 2^{-}$resonance, although its agreement with Arnold et al. validates the use of their parameters for the higher energy resonances. Results from the fit are shown in Figure 3.3 .

Fit parameters are $E_{n}=1738.8 \pm 2.1 \mathrm{keV}, \Gamma_{\gamma}=0.7715 \pm 0.032 \mathrm{eV}$, and $\Gamma_{n}=268.7 \pm$ $16 \mathrm{keV}$ with a goodness of fit of $\chi^{2}=2.2 / 7$ dof $(p=0.948)$. Fit uncertainties were determined by applying the Monte Carlo method. The normally distributed measured data was randomly varied and refit 1000 times, from which the standard deviation of the fit 


\begin{tabular}{|c|c|c|c|c|c|}
\hline Isotope & $\begin{array}{c}E_{\gamma} \\
(\mathrm{keV})\end{array}$ & $\begin{array}{l}E_{n}^{c . m .} \\
(\mathrm{keV})\end{array}$ & $\alpha_{i}$ & $\begin{array}{c}\sigma_{i} \\
(\mathrm{mb})\end{array}$ & $\sum_{(\mathrm{mb})} \alpha_{i} \sigma_{i}$ \\
\hline \multirow[t]{8}{*}{${ }^{56} \mathrm{Co}$} & 1771.36 & 94.9 & 0.1541 & & \\
\hline & 2015.22 & 351 & 0.03016 & & \\
\hline & 2034.79 & 370 & 0.0777 & & \\
\hline & 2598.5 & 994 & 0.1697 & & \\
\hline & 3202.03 & 1537 & 0.0321 & & \\
\hline & 3253.50 & 1589 & 0.0792 & & \\
\hline & others & & 0.0642 & 0.034 & \\
\hline & total & & 0.6078 & 0.311 & \\
\hline \multirow[t]{4}{*}{${ }^{88} \mathrm{Y}$} & 1836.06 & 152 & 0.992 & 0.659 & \\
\hline & 2734.0 & 950 & 0.0071 & 0.567 & \\
\hline & 3219.7 & 1381 & 0.000070 & 0.431 & \\
\hline & total & & 0.999 & $0.656 \pm 0.024$ & \\
\hline \multirow[t]{4}{*}{${ }^{124} \mathrm{Sb}$} & 1690.97 & 23.5 & 0.4757 & 1.397 & \\
\hline & 2090.93 & 379 & 0.0549 & 0.274 & \\
\hline & others & & 0.0052 & & 0.003 \\
\hline & total & & 0.5358 & & $0.682 \pm 0.020$ \\
\hline${ }^{207} \mathrm{Bi}$ & 1770.23 & 93.9 & 0.0687 & 0.955 & $0.0656 \pm 0.0027$ \\
\hline \multirow[t]{4}{*}{${ }^{226} \mathrm{Ra}$} & 1764.49 & 88.8 & 0.1530 & 0.990 & \\
\hline & 2204.06 & 479 & 0.04924 & 0.223 & \\
\hline & others & & 0.0969 & & 0.066 \\
\hline & total & & 0.2991 & & $0.228 \pm 0.007$ \\
\hline \multirow[t]{3}{*}{${ }^{228} \mathrm{Th}$} & 2614.51 & 844 & 0.3585 & 0.320 & \\
\hline & others & & 0.0009 & & 0.0007 \\
\hline & total & & 0.3595 & & 0.1154 \\
\hline
\end{tabular}

Table 3.4: Neutron yields for commercially available $\gamma$ emitting isotopes using the ${ }^{9} \operatorname{Be}(\gamma, n)$ reaction. Uncertainties are given where more than $90 \%$ of the neutron production is from the $1 / 2^{-}$resonance. 
parameters over the trials was calculated. The fit parameters are strongly correlated, with correlation parameters of 0.65 between $E_{n}$ and $\Gamma_{\gamma}, 0.83$ between $E_{n}$ and $\Gamma_{n}$, and 0.95 between $\Gamma_{\gamma}$ and $\Gamma_{n}$.

Both ${ }^{88} \mathrm{Y} / \mathrm{Be}$ and ${ }^{124} \mathrm{Sb} / \mathrm{Be}$ neutron sources are used in the calibrations presented in Chapters 4 and 6 . To calculate the ${ }^{88} \mathrm{Y} / \mathrm{Be}$ neutron production yield, the measurement of Gibbons et al. (1959) is combined with constraints provided by the fits to other radioisotope measurements. Fits excluding the Gibbons et al. were performed assuming a range of fixed ${ }^{88} \mathrm{Y} / \mathrm{Be}$ neutron source yields. A likelihood function was constructed from the probabilities

of obtaining the fitted $\chi^{2}$ values, with 6 degrees of freedom, assuming a flat prior probability on the ${ }^{88} \mathrm{Y} /$ Be source yield. From the maximum likelihood and the $68 \%$ confidence interval of the likelihood ratio test, an extrapolated measurement of the ${ }^{88} \mathrm{Y} / \mathrm{Be}$ source yield is made giving $\sum_{i} \alpha_{i} \sigma_{i}=0.648 \pm 0.043 \mathrm{mb}$. Combined with the result of Gibbons et al., a yield of $\sum_{i} \alpha_{i} \sigma_{i}=0.656 \pm 0.024 \mathrm{mb}$ is adopted. For ${ }^{124} \mathrm{Sb} / \mathrm{Be}$, the combined fit gives $\sum_{i} \alpha_{i} \sigma_{i}=0.682 \pm 0.020 \mathrm{mb}$ which is adopted. Neutron yield for other $(\gamma, n)$ sources are given in Table 3.4 .

\subsection{Low Energy Neutron Simulations}

For 50 years, the International Atomic Energy Agency (IAEA) and various national nuclear data groups (Jawerth 2015) have compiled a vast library of measured and evaluated neutron interaction cross-sections below $20 \mathrm{MeV}$. This data permits accurate Monte Carlo calculations of neutron propagation through matter. The Monte Carlo for Neutral Particles (MCNP) program and Geant4 (Agostinelli et al. 2003) implement these libraries and the their low-energy nuclear physics processes. The MCNPX-Polimi program (Padovani et al. 2012 ) is used for most simulations described in this thesis while Geant4 version 9.6 or later has been used to cross-check certain simulations. As a low-rate threshold detector, COUPP 
and PICO rely more heavily on the accuracy of these simulations for calibrations and evaluations of background processes than other dark matter detector technologies. These extensive calculations require accurate cross-section data, geometries, source distributions, and interpretation, and an evaluation of their uncertainties.

Most neutron cross-section data used in PICO simulations are from the ENDF/B-VII neutron libraries (Chadwick et al. 2011), and reflect the best current understanding of lowenergy neutron cross-sections. For most isotopes of interest, the ENDF/B-VII evaluations include data on elastic, inelastic, and reaction cross-sections, energy and angle distributions of products, and cross-section uncertainties including covariances across energy.

The uncertainty in the neutron elastic scattering cross-section on carbon and fluorine affects every simulation involving our detector target fluids. Figures 3.4 and 3.5 show the ENDF/B-VII.1 cross-section, uncertainties, and cross-section covariances for these nuclei. The fluorine cross-section dominates the elastic scattering rate, contributing between $67 \%$ and $82 \%$ of recoils above $5 \mathrm{keV}$ for the various neutron calibrations of PICO and COUPP. For mono-energetic neutron sources, the relative ${ }^{19} \mathrm{~F}$ neutron elastic cross-section uncertainty at the calibration neutron energy is applied to the measured bubble rate. For ${ }^{241} \mathrm{Am}$ and ${ }^{252} \mathrm{Cf}$ calibration, two simulation of PICO-2L where the density of the $\mathrm{C}_{3} \mathrm{~F}_{8}$ differed by $5 \%$ and the random number seed was identical. Each history that differed between the simulations was weighted by the cross-section uncertainty at the recoil where the two simulations branced. An uncertainty of $\pm 3 \%$ per bubble nucleation was found for calibrations in both PICO-2L and COUPP-4kg, where the using the assumption that the cross-section uncertainty is fully correlated across all relevant neutron energies.

The role of the three large cross-section resonances in the ${ }^{19} \mathrm{~F}$ neutron elastic crosssection at $27.0 \mathrm{keV}, 48.9 \mathrm{keV}$, and $97.9 \mathrm{keV}$, were examined. These resonances enhance the scattering cross-section for neutrons approaching the nucleus with non-zero angular 


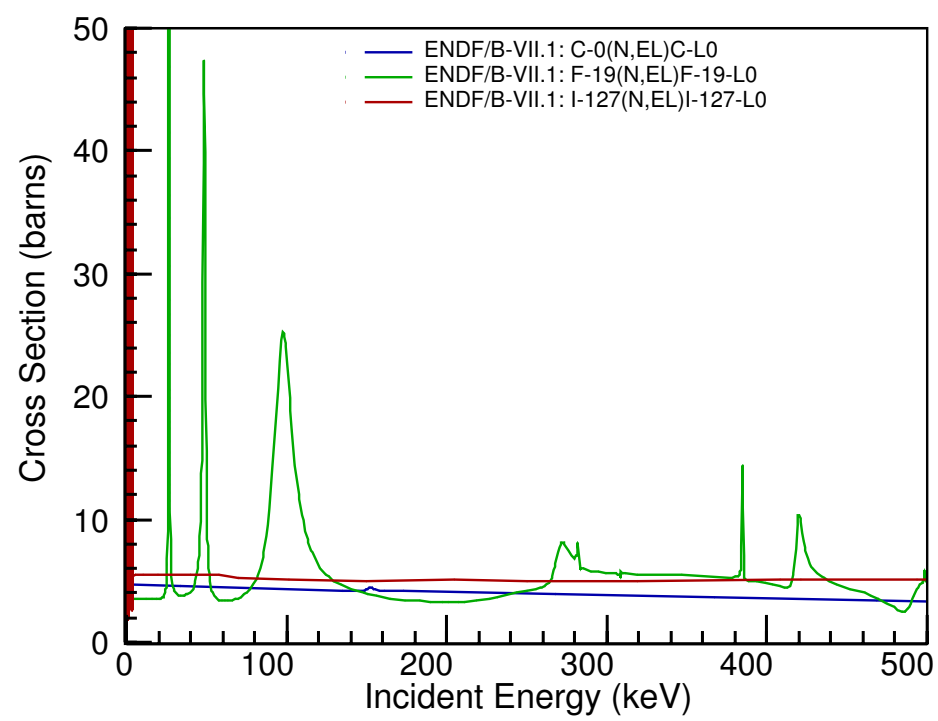

Figure 3.4: Neutron elastic cross-section for the elements of $\mathrm{C}_{3} \mathrm{~F}_{8}$ and $\mathrm{CF}_{3} \mathrm{I}$ from ENDFB/VII.1 (Chadwick et al. 2011). As fluorine has a large cross-section and is more prevalent than iodine or carbon in these compounds, its recoils dominate during neutron calibrations.

momentum. Therefore the scattering angle distribution at these and nearby energies is nonisotropic. In A. E. Robinson (2014, Appendix B), I showed that due to a missing feature in the program that translates nuclear data libraries into the library files for both Geant 4 and MCNP, isotropic angular distributions were being used when simulating neutron recoils from fluorine and many other isotopes. All simulations of calibrations in PICO use the corrected libraries from A. E. Robinson (2014).

The resonances in fluorine can be exploited in order to separately measure the nucleation efficiency of carbon and fluorine. By measuring the bubble nucleation rate on resonance, for example at $50 \mathrm{keV}$, and off-resonance at $61 \mathrm{keV}$, a measured enhancement in the scattering rate at $50 \mathrm{keV}$ can be attributed to fluorine while the scattering rate from carbon remains nearly constant. Such a measurement will require using the bubble chamber data to constrain the existing poorly measured strength of the $48.9 \mathrm{keV}$ resonance.

Uncertainties in the geometry of materials through which neutrons must pass before 


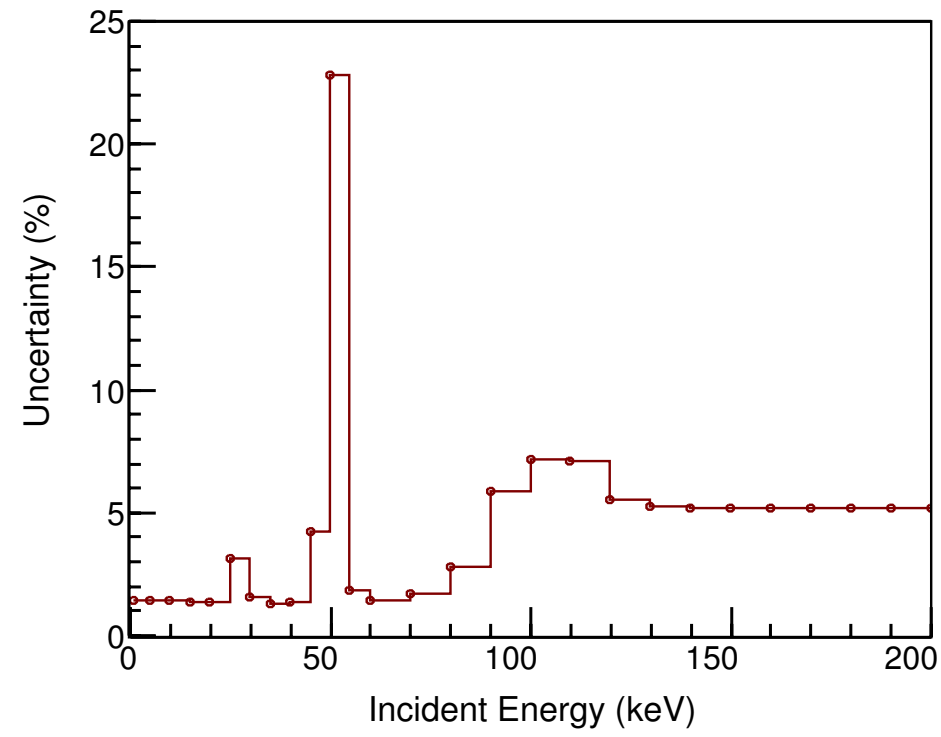

(a) ${ }^{19} \mathrm{~F}(n, \mathrm{el})$ cross-section relative uncertainty

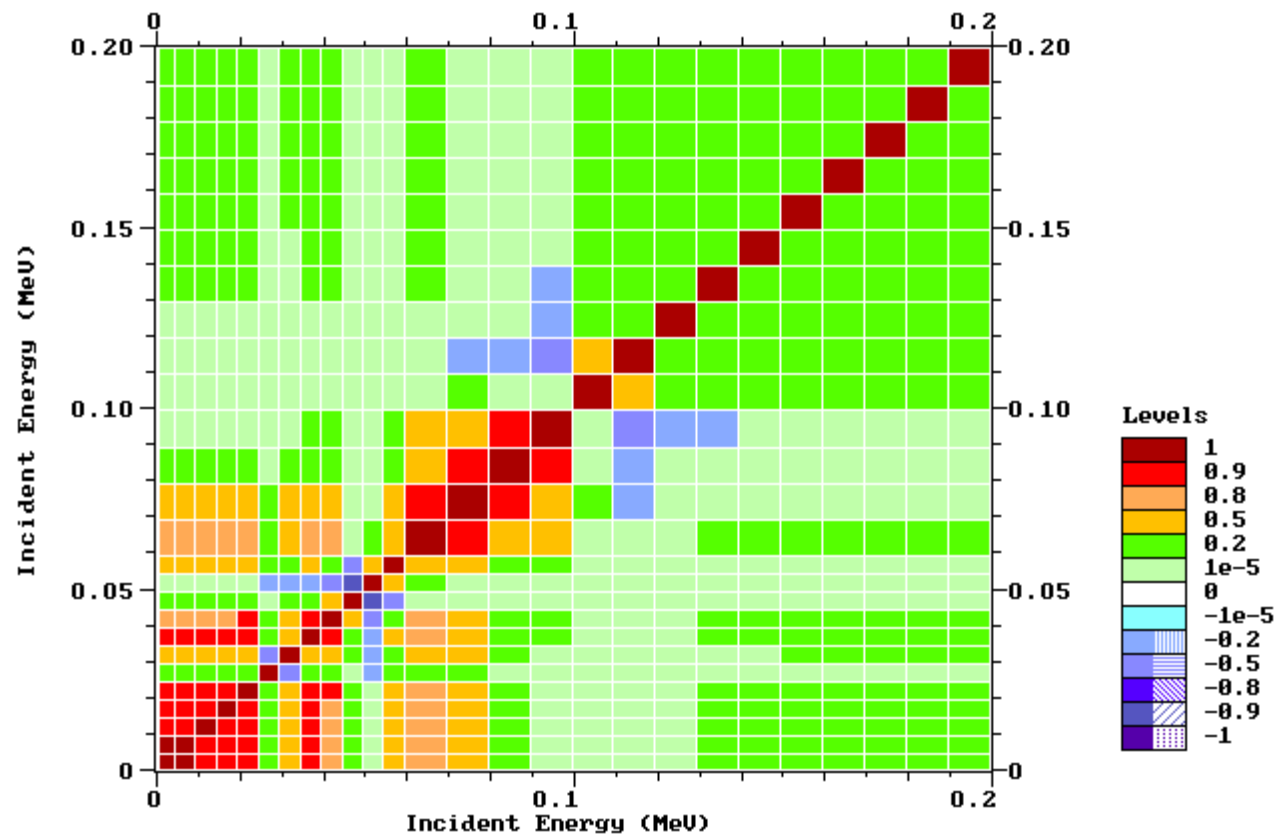

(b) ${ }^{19} \mathrm{~F}(n, \mathrm{el})$ cross-section uncertainty correlations

Figure 3.5: The ${ }^{19} \mathrm{~F}$ neutron elastic cross-section uncertainty and uncertainty correlation from ENDF-B/VII.1 (Chadwick et al.2011). This cross-section and its uncertainty dominate generation of nuclear recoils from neutrons in both $\mathrm{C}_{3} \mathrm{~F}_{8}$ and $\mathrm{CF}_{3} \mathrm{I}$. While the uncertainties for scattering cross-sections at different neutron energies are only partly correlated, a scale uncertainty (full correlation) is conservatively assumed for PICO simulations. 
entering the active volume include uncertainties in cross-sections, material compositions, and material densities. Several important detector materials, such as grades 304 and 316 stainless steel, aluminum alloy 6061, and silicon, have neutron scattering cross-section uncertainties of approximately $10 \%$ at relevant neutron energies. While cross-section uncertainties limit the ultimate accuracy of the simulated passage of neutrons through detector components, the dimensions and masses of components can be easily overlooked and miscalculated. Where possible, and especially for critical components, the mass of materials was measured in order to validate the simulation geometry.

Unknown isotopic contaminants and concentrations in materials with resonances at the calibration neutron energies that reflect neutrons. In the case of the PICO-2L mineral oil hydraulic fluid, the detector sensitivity to background and calibration neutrons depended strongly on the hydrogen content of the oil, see Section 6.2.2. Uncertainties will be explored on a case-by-case basis for each simulation geometry in later chapters.

The energies, angles, and positions of source particles in a simulation need to be set correctly. In the case of radioisotope sources, each of these variables is normally independent, although correlations between different source emissions, such as the multiple neutrons generated by spontaneous fission, can sometimes be important when measuring bubble multiplicity and other correlated variables. In the case of $(\gamma, n)$ sources, strong correlations exist between the energy, angle, and position of an emitted neutron. While the distribution of ejected neutrons from the reaction is isotropic and monoenergetic in the rest frame, conversion to the lab-frame boosts the energies and angles for each generated neutron depending on the original photon direction. Using MCNPX-Polimi, these correlations are produced by simulating the radiation propagation of photons from the radioisotope source and their conversion into neutrons. A library was written for MCNPX that encodes the ${ }^{9} \operatorname{Be}(\gamma, n)$ cross-section found in the previous section. 
In order to efficiently simulate the ${ }^{9} \operatorname{Be}(\gamma, n)$ reaction, biasing was used. Every $\gamma$-ray entering the geometric cell volume containing BeO was forced to collide, and a photoneutron was created for each collision. Simulated photoneutron had weights of $\mathcal{O}\left(10^{-} 5\right)$ and these weights were tracked through post-processing of simulation outputs. As the uncollided weight of photons could not exit and reenter a beryllium containing cell, no more than one neutron per generated photon was simulated. Had more than one neutron per history been generated, the correlations between neutron recoils and bubble multiplicity would have been corrupted.

For simulating the ${ }^{51} \mathrm{~V}(p, n)$ reaction at the University of Montreal neutron beam, the energy-angle relationship was analytically calculated from the center-of-mass to lab frame boost and the center-of-mass dipole anisotropies given in Gibbons, Macklin, and Schmitt (1958, Table I), and the neutrons were directly generated in the simulation. In contrast to the $(\gamma, n)$ reaction, the position of the generated neutrons was point-like and the angle of the incoming protons was uniform for the $(p, n)$ reaction. The energy-angle-position distribution could be simply calculated without simulating the incident protons.

MCNPX-Polimi produces an output file with one line for every nuclear and/or electron recoil generated in the requested cells of the simulation geometry. The event history, incident particle type, interaction type, target nucleus, interaction position, deposited energy, particle energy before collision, and particle weight are recorded. For most calibration simulations, the elastic and inelastic neutron recoils in the target volume are extracted from this file, with the deposited energy, target nucleus, and particle history number retained in order to predict event rates and bubble multiplicities. In addition to elastic and inelastic recoils, incoming neutrons may have sufficient energy to undergo a nuclear reaction. For elastic and inelastic scattering, MCNPX-Polimi records a reaction type or -99 or -1 respectively. For neutron induced nuclear reactions, the reaction type is the number of ejected neutrons 
from the reaction, either zero or positive. MCNPX can create a particle tracking, or ptrac, output file in addition to the Polimi output file, with two lines per step of the particle's simulation. Due to the large file size and non-optimal data format generated by the ptrac output, the Polimi output format is generally preferred for PICO simulations.

It was found that MCNPX-Polimi version 2.0.0 did not correctly calculate the deposited energy from nuclear reactions when both neutrons and charged particles were in the final state. The MCNPX-Polimi code calculated the deposited energy from the differences in the initial and final state momenta and energies of the neutrons and the reaction Q-value during a collision. Any excess energy above the energy of the neutrons was assumed to be transported away from the interaction site by photons - the energy deposited by charged particles was not counted. This bug was reported and fixed in MCNPX-Polimi version 2.0.9 (Padovani 2014). For PICO simulations with multi-MeV neutrons, such as those described in Section 6.2.2, both a ptrac and an MCNP-Polimi output were generated, where the ptrac output identified the nuclear reaction process and kinematic variables required to reconstruct the true deposited energy.

\section{$3.3 \quad{ }^{241}$ Am/Be Neutron Spectrum}

The particle energies for all radiation sources used to calibrate PICO and COUPP are well known except for the neutrons from ${ }^{241} \mathrm{Am} /$ Be sources. ${ }^{241} \mathrm{Am} / \mathrm{Be}$ sources generate neutrons via the ${ }^{9} \mathrm{Be}(\alpha, n)$ reaction by pressing a mixture of fine $\mathrm{AmO}_{2}$ and metallic beryllium powders into a capsule. As highly ionizing $\alpha$ particles traverse the powder granules, they rapidly lose energy. The energy of the $\alpha$ determines the final-state branching ratio of the $(\alpha, n)$ reaction. These branching ratios dominate the uncertainties in the neutron energy spectrum. A variety of ${ }^{241} \mathrm{Am} / \mathrm{Be}$ neutron spectrum evaluations have been produced, mostly based on semi-empirical calculations (ISO 2001; Geiger and Zwan 1975). A ${ }^{241} \mathrm{Am} /$ Be source may be 
specified in MCNPX-Polimi with a pre-coded energy spectrum based on Geiger and Zwan (1975). This spectrum is used for PICO simulations.

In order to determine the simulation uncertainties, SOURCES-4C was used to calculate the effect of $\mathrm{AmO}_{2}$ grain sizes on the neutron energy spectrum (Wilson et al. 2002). There are four final states accessible to $5.5 \mathrm{MeV} \alpha$-rays from ${ }^{241} \mathrm{Am}$ (Geiger and Zwan 1975): the ground state and first two excited states of ${ }^{9} \mathrm{Be}(\alpha, n){ }^{12} \mathrm{C}$, and the three-body breakup reaction ${ }^{9} \operatorname{Be}(\alpha, n+\alpha){ }^{8} \mathrm{Be}$. As described in Section 7.2, SOURCES-4C was modified with the ${ }^{9} \mathrm{Be}(\alpha, n)$ cross-section and final state branching ratios from the JENDL-AN/05 evaluation (Shibata et al. 2011). As SOURCES is designed to only model two-body final states of the $(\alpha, n)$ reaction, the three-body neutron energy spectrum was approximated using an array of two-body final state with incrementing Q-values. A similar approximation procedure was used in Shores, Mueller, and Schlapper (2003).

Figure 3.6 shows the spectrum calculated by SOURCES-4C and the contributions from the different final states. The spectrum shown in the plot assumes that $\alpha$ particles are generated in the beryllium metal. A second calculation was performed assuming that the energy of the $\alpha$ 's entering the beryllium was degraded after exiting a thick layer $\mathrm{AmO}_{2}$. The branching ratios to the ground, first excited, and second exited states and the 3-body final state changed by factors of $1.35,1.01,0.26$, and 0.81 respectively between these calculations.

Simulations of the PICO-2L ${ }^{241} \mathrm{Am} / \mathrm{Be}$ calibration using the 4 a geometry (see Section 6.2.2 were performed using the SOURCES-4C calculated spectra for each final state with both full-energy and degraded $\alpha$ 's. The MCNPX-Polimi ${ }^{241} \mathrm{Am} / \mathrm{Be}$ neutron spectrum binned above $6 \mathrm{MeV}$, between 2 and $6 \mathrm{MeV}$, and below $2 \mathrm{MeV}$ was used to normalize the SOURCES-4C calculation. These bins approximately select neutrons from each of the $(\alpha, n)$ reaction final states, with the $\left(\alpha, n_{2}\right)$ and $(\alpha, \alpha+n)$ spectra combined in the lowest bin. The recoil energy spectra were similar for all the simulations, but the recoil rates differed. 


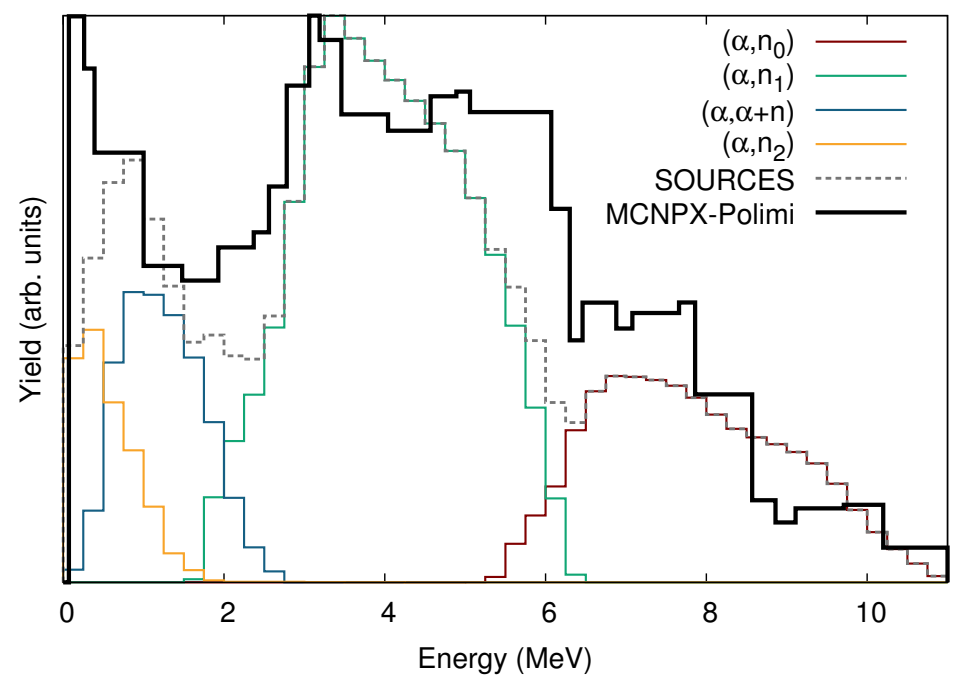

Figure 3.6: ${ }^{241} \mathrm{Am} / \mathrm{Be}$ neutron energy spectra from MCNPX-Polimi as used in simulations, and calculated by a modified version of SOURCES-4C (see Section 7.2). The SOURCES-4C spectrum is divided into contributions from the 4 final states of the reaction.

The simulated nuclear recoil rate above $3 \mathrm{keV}$ in the active volume for undegraded $\alpha$ 's was a factor of 1.26 larger than the rate for those with fully degraded energies. A $1 \sigma$ rate uncertainty of $26 / \sqrt{6}=10.6 \%$ is applied to the total rate of recoils in ${ }^{241} \mathrm{Am} / \mathrm{Be}$ calibration simulations of both PICO-2L and COUPP-4kg.

As shown in Figure 3.6 and Chartier (2009), SOURCES-4C does not reproduce the ${ }^{241} \mathrm{Am} / \mathrm{Be}$ neutron spectrum well, even when the source code and libraries are modified. Recent calculations of the spectrum using code developed in Japan (Tsujimura, Yoshida, and Momose 2007) and the JENDL-AN/05 $(\alpha, n)$ cross-sections are able to closely match the empirically determined neutron spectra. If the internal geometry of a ${ }^{241} \mathrm{Am} / \mathrm{Be}$ source is well known, the neutron spectrum may now be calculable from first principles with smaller uncertainties than those presented here. 


\subsection{Alternative Calibration Methods}

As mentioned in this chapter's introduction, neutron scattering cannot provide an eventby-event recoil energy measurement when calibrating bubble chambers. Charged particle scattering can provide a recoil energy measurement and sources of monoenergetic nuclear recoils are available. The former method was used with the COUPP Iodine Recoil Threshold Experiment (CIRTE), and is well described in Behnke et al. (2013). The CIRTE experiment measured the nucleation efficiency for iodine recoils in $\mathrm{CF}_{3} \mathrm{I}$ and found that the efficiency was consistent with $100 \%$ efficiency above the Seitz threshold.

Monoenergetic recoils at low energy can be provided by photoabsorbtion or thermal neutron capture reactions. Two thermal neutron capture reactions are of potential interest: $\left.{ }^{35} \mathrm{Cl}(n, p)\right)^{35} \mathrm{~S}(Q=615.0 \mathrm{keV})$ and ${ }^{14} \mathrm{~N}(n, p){ }^{14} \mathrm{C}(Q=625.9 \mathrm{keV})$. The former produces a sulfur recoil of $17.6 \mathrm{keV}$ while the latter produces a carbon recoil of $44.7 \mathrm{keV}$ with the remaining energy going to the proton. Both reactions are near the energies and atomic masses of interest in a $\mathrm{CF}_{3} \mathrm{I}$ bubble chamber, assuming a suitable chemical can be used to dope the chamber with these elements. The sensitivity to thermal neutrons via the ${ }^{35} \mathrm{Cl}(n, p){ }^{35} \mathrm{~S}$ reaction complicates the use of refrigerants containing chlorine in a dark matter search bubble chamber.

High energy photoabsorbtion reactions may be initiated directly on the target fluids without introducing a dopant. Such a calibration would be similar to the recent cross-

section measurement of the ${ }^{19} \mathrm{~F}(\gamma, \alpha){ }^{15} \mathrm{C}$ reaction at the High Intensity $\gamma$ Source (HI $\gamma$ ) using a bubble chamber (DiGiovine et al. 2015). A combination of poor energy resolution, beam-related neutron background, backgrounds from competing processes, and low reaction cross-sections near threshold preclude the use of photoabsorbtion reactions for studying nuclear recoils in either $\mathrm{C}_{3} \mathrm{~F}_{8}$ or $\mathrm{CF}_{3} \mathrm{I}$. 


\subsection{Event Rates from Neutron Recoils}

In Chapter 6, models for the bubble nucleation efficiency are developed using count rates from neutron calibrations of $\mathrm{C}_{3} \mathrm{~F}_{8}$ and $\mathrm{CF}_{3} \mathrm{I}$ bubble chambers. Given a model of the efficiency for detecting a nuclear recoil as a function of the recoil energy $E_{r}$, temperature, pressure, the recoiling nucleus $z$, and the target fluid, the expected count rates $R$ for these calibrations can be calculated from the simulated recoil rate $\rho$.

$$
R\left(E_{c}\right)=\sum_{z} \int_{E_{c}}^{\infty} \eta\left(E_{c}, E_{r}, z\right) \rho\left(E_{r}\right) d E_{r}
$$

The inverse problem is not trivially solved. To approach a valid solution, several simplifications of the efficiency function are made. First, a physically relevant bubble nucleation function must monotonically increase as the recoil energy, temperature, or stopping power of the recoiling nucleus increases or the pressure decreases. Ordered by stopping power, iodine recoils should be more efficient than fluorine recoils which should be more efficient than carbon recoils. To further constrain the problem, the pressure and temperature are combined into a Seitz threshold variable $E_{c}$. Finally, assumptions that minimize the sensitivity to recoils from low mass dark matter candidates will be used: the carbon and fluorine recoil efficiencies are assumed to be equal and the efficiency for iodine recoils is assumed to be $100 \%$ above the Seitz threshold energy. These assumptions are all found to be consistent with the available data and theoretical expectations (see Figure 3.7).

In order to recover the efficiency function, the allowed parameter space for the efficiency function is mapped. An upper limit to the efficiency function in $E_{r}$ at a given Seitz threshold can be set when the expected recoil rate from low-energy neutron recoils is higher than the observed rate. A lower limit is set by observing more high-energy neutron or multiple bubble events than predicted. The model that maximizes the allowed efficiency at a given 


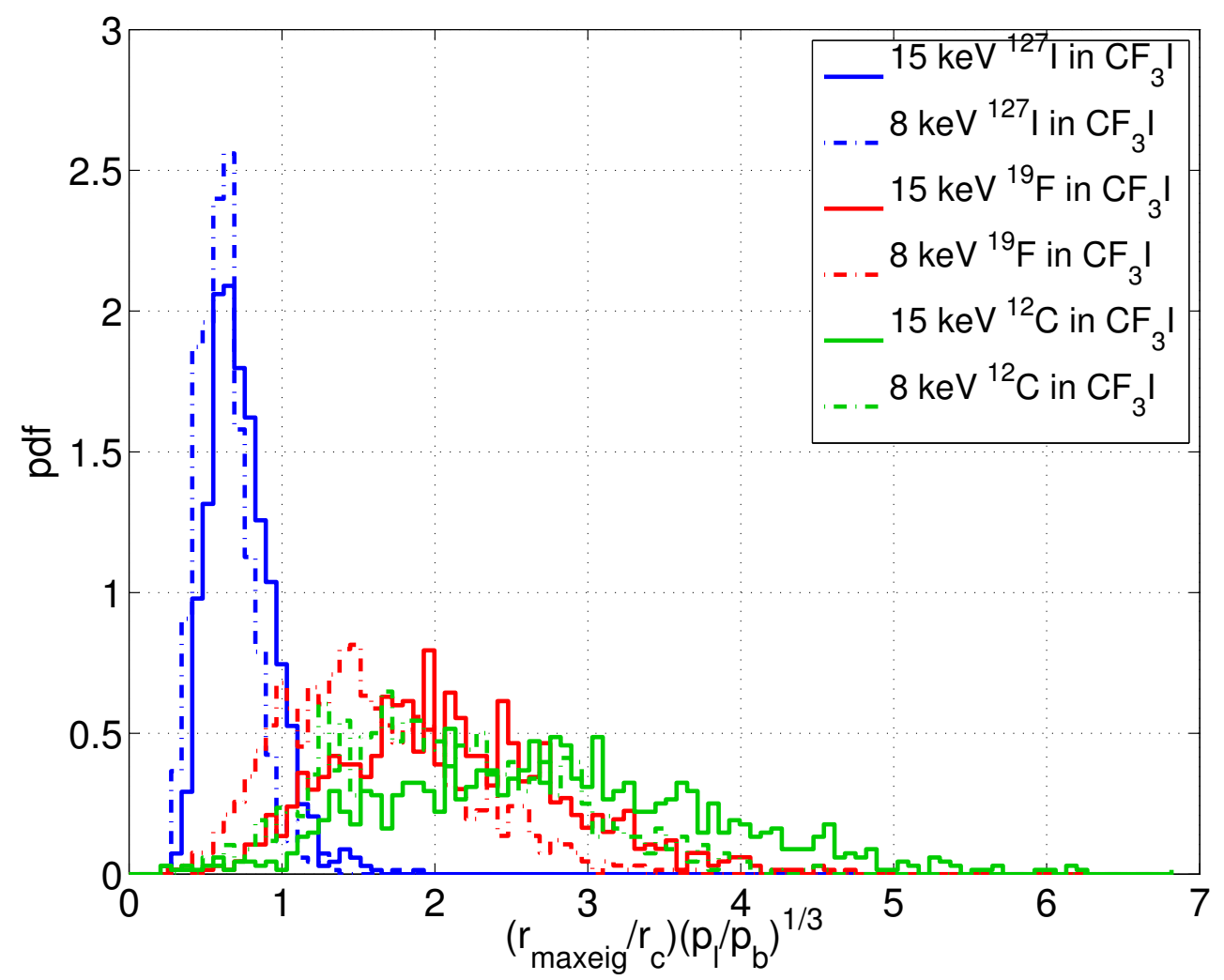

Figure 3.7: The simulated probability distribution function of nuclear recoils ranges in $\mathrm{CF}_{3} \mathrm{I}$. The plot and calculation is by Dahl (2011). Simulations of nuclei stopping in $\mathrm{CF}_{3} \mathrm{I}$ were performed using the SRIM program (Ziegler, Biersack, and Ziegler 2008). The length shown is the rms position of the simulated displacement sites projected along the longest dimension of the energy distribution divided by the critical bubble radius at the Seitz threshold corresponding to the recoil's energy. Carbon and fluorine recoils in $\mathrm{CF}_{3} \mathrm{I}$ may deposit most of their energy outside of one critical radius, and thus have a lower bubble nucleation efficiency than iodine recoils, which are almost entirely contained within one critical radius. As carbon and fluorine recoils have a broad distribution of stopping powers, any inefficiency due to the stopping power threshold is expected to vary slowly with recoil energy. 
$E_{r}$ and $E_{c}$ while remaining consistent with the count rate is a step function in $E_{r}$, as per the following theorem.

Theorem 1. Consider a threshold counting experiment with a threshold energy of $E_{c}$ measuring an event rate $R_{L}$ given by Equation 3.2 from a recoil energy distribution $\rho_{L}$. Furthermore, $0 \geq \eta \geq 1$ and $\eta\left(E_{r}, z\right)$ is monotonically increasing in $E_{r}$ for every nuclear recoil species $z$. The predicted rate for a second experiment using the same $\eta$ but a different distribution of recoil energies, $\rho_{U}\left(E_{r}\right)$ is $R_{U}$. If for every $z, \rho_{U} / \rho_{L}$ is monotonically decreasing, then, the predicted rate $R_{U}$ is minimized when

$$
\eta\left(E_{r}, z\right)=\Theta\left(E_{r}-E_{s}\right)= \begin{cases}1 & E_{r}>E_{s} \\ 0 & E_{r}<E_{s}\end{cases}
$$

Proof. Let $\eta=\Theta\left(E_{r}-E_{s}\right)+\delta$ where $\delta \geq 0$ for $E_{r}>E_{c}$, and $\delta \leq 0$ for $E_{r} \leq E_{c}$. By construction, this set of functions contains all possible efficiency models. By construction, $E_{s}$ is set such that

$$
\begin{aligned}
R_{L} & \equiv \int_{E_{c}}^{\infty}\left(\delta\left(E_{r}\right)+\Theta\left(E_{r}-E_{s}\right)\right) \rho_{L}\left(E_{r}\right) d E_{r} \\
& =\int_{E_{c}}^{\infty} \Theta\left(E_{r}-E_{s}\right) \rho_{L}\left(E_{r}\right) d E_{r}
\end{aligned}
$$

therefore,

$$
\int_{E_{c}}^{E_{s}} \delta\left(E_{r}\right) \rho_{L}\left(E_{r}\right) d E_{r}=-\int_{E_{s}}^{\infty} \delta\left(E_{r}\right) \rho_{L}\left(E_{r}\right) d E_{r}>0
$$

As $\rho_{U} / \rho_{L}$ is monotonically decreasing

$$
\int_{E_{c}}^{E_{s}} \delta\left(E_{r}\right) \rho_{U}\left(E_{r}\right) \frac{\rho_{L}\left(E_{s}\right)}{\rho_{U}\left(E_{s}\right)} d E_{r} \geq-\int_{E_{s}}^{\infty} \delta\left(E_{r}\right) \rho_{L}\left(E_{r}\right) d E_{r}
$$


and

$$
\int_{E_{c}}^{E_{s}} \delta\left(E_{r}\right) \rho_{L}\left(E_{r}\right) d E_{r} \geq-\int_{E_{s}}^{\infty} \delta\left(E_{r}\right) \rho_{U}\left(E_{r}\right) \frac{\rho_{L}\left(E_{s}\right)}{\rho_{U}\left(E_{s}\right)} d E_{r}
$$

therefore

$$
\begin{aligned}
& \int_{E_{c}}^{E_{s}} \delta\left(E_{r}\right) \rho_{U}\left(E_{r}\right) d E_{r} \geq-\int_{E_{s}}^{\infty} \delta\left(E_{r}\right) \rho_{U}\left(E_{r}\right) d E_{r} \\
& \int_{E_{s}}^{\infty} \delta\left(E_{r}\right) \rho_{U}\left(E_{r}\right) d E_{r} \geq 0 \\
& R_{U} \equiv \int_{E_{c}}^{\infty}\left(\delta\left(E_{r}\right)+\Theta\left(E_{r}-E_{s}\right)\right) \rho_{U}\left(E_{r}\right) d E_{r} \\
& \geq \int_{E_{c}}^{\infty} \Theta\left(E_{r}-E_{s}\right) \rho_{U}\left(E_{r}\right) d E_{r}
\end{aligned}
$$

$R_{U}$ is minimized when $\eta=\Theta\left(E_{r}-E_{s}\right)$.

The assumptions used in this theorem are only approximated for PICO calibrations, as shown in Figure 3.8, but step functions will be used regardless.

An upper limit efficiency function consistent with the data can be mapped out for all recoil energies as shown in Figure 3.9a. Similarly, a lower limit efficiency model can be mapped by finding the maximum rate consistent with a model through $\left(E_{r}, \eta\right)$ as shown in Figure 3.9b. By construction, any efficiency model consistent with the data through $\left(E_{r}, \eta\right)$ must be within the envelope

$$
\eta\left(E_{r}^{\prime}\right)= \begin{cases}\leq \eta_{\max }\left(E_{r}^{\prime}\right) & \\ \leq \eta, & E_{r}^{\prime} \leq E_{r} \\ \geq \eta_{\min }\left(E_{r}^{\prime}\right) & \end{cases}
$$




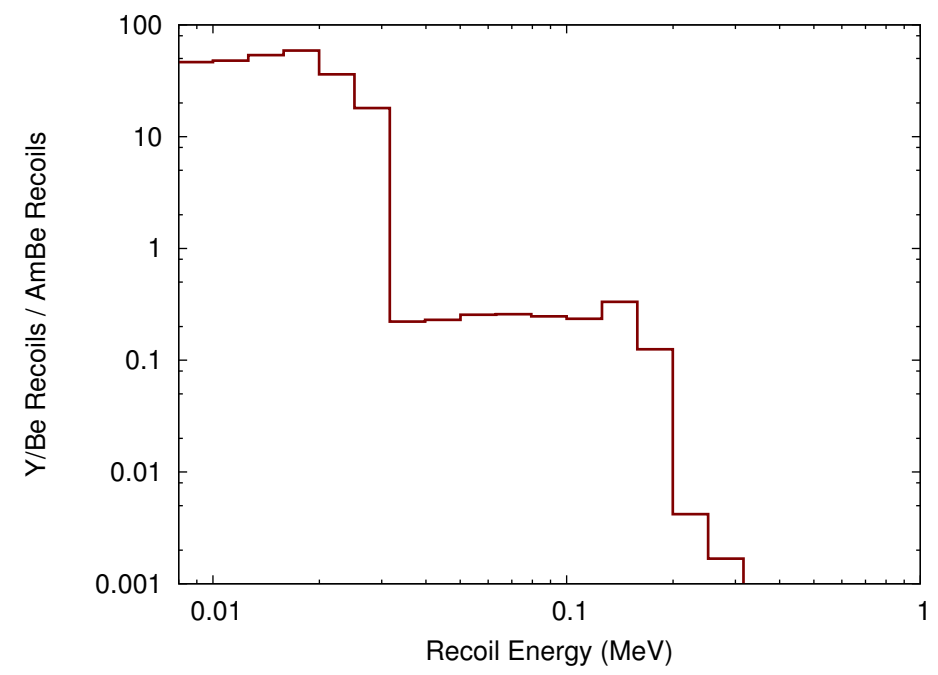

Figure 3.8: The ratio of simulated fluorine recoils in the STAR bubble chamber in the presence of ${ }^{88} \mathrm{Y} / \mathrm{Be}$ neutrons versus the COUPP-4kg bubble chamber in the presence of $\mathrm{Am} / \mathrm{Be}$ neutrons. While the function is not strictly decreasing, the limit finding procedure based on Theorem 1 will still find the approximate limits of allowed bubble nucleation efficiency functions.

where $\eta_{\max }$ and $\eta_{\min }$ are previously found limits to the efficiency function. By Theorem 1 , the maximum expected count rate consistent with the data for a model through $\left(E_{r}, \eta\right)$ is provided by a step function between the minimum of this window and the maximum of this window. This double step efficiency model, shown in Figure 3.9b, is used to further constrain the limits on the efficiency function. The search for upper and lower limit efficiency functions is iterated three times in order to calculate the worst-case efficiency functions that are consistent with both limits.

Theorem 1 can also be used to find the efficiency function consistent with the calibration that produces the lowest possible WIMP detection efficiency. Where the dark matter recoil energy distribution is softer or harder than the neutron recoil spectrum used to set the lower limit of the efficiency function, i.e. $d R / d E_{r} \div \rho_{L}$ is monotonic, an efficiency functions with a single step between the higher and lower limit curves bound the expected dark matter sensitivity. The position of the step is set to maximize consistency with the data. As these 


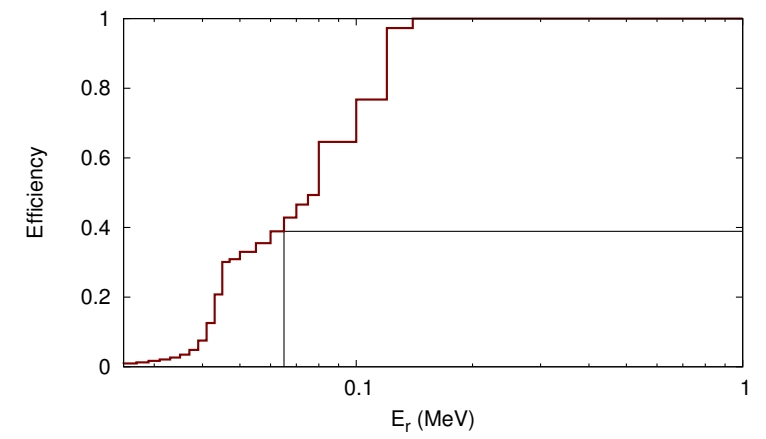

(a) Setting the upper limit efficiency. For each bin, a step function (black) is calculated that maximizes the size of the step while not overpredicting any measured count rates. The upper limit of all allowed step functions is shown in maroon.

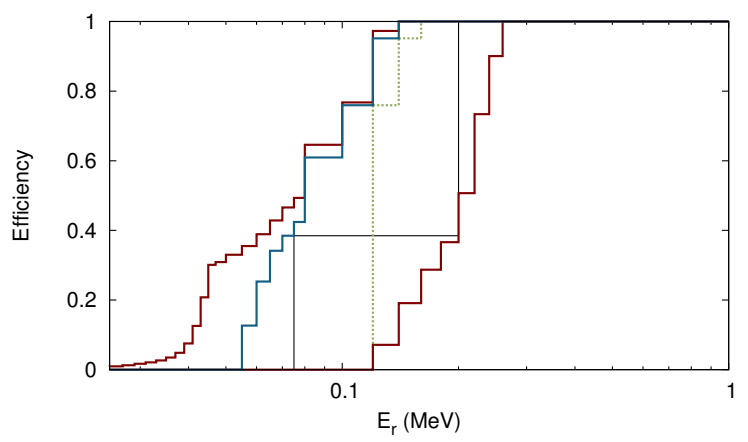

(c) The upper limit efficiency is recalculated with a double step function so as to be consistent with the lower limit efficiency. Where the ends of the double step cross the upper or lower limit curves, the efficiency model follows the curves, as shown by the upper limit model at $0.05 \mathrm{MeV}$ shown in dashed green.

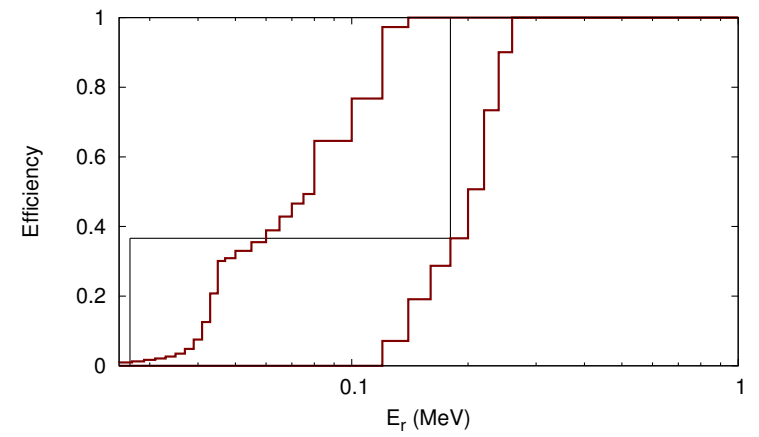

(b) Similarly, the lower limit is set using a double step function that does not underpredict any measured count rate.

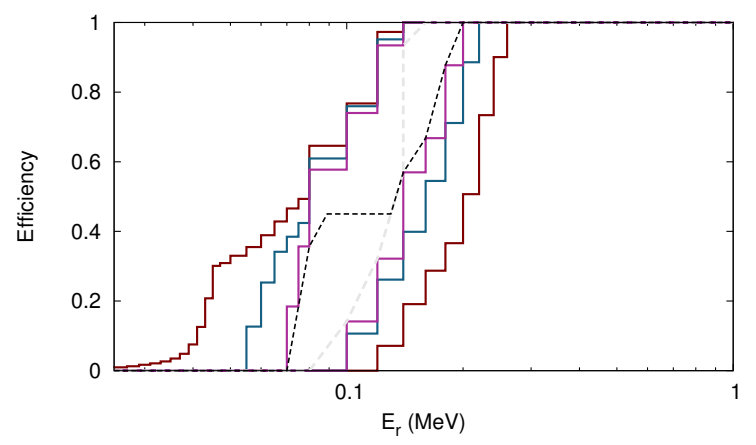

(d) The limit finding algorithm is iterated 3 times for each requested confidence level. The dashed lines shows the efficiency model that bound the allow dark matter sensitivity at the requested confidence level (see text).

Figure 3.9 
step functions, shown in Figure $3.9 \mathrm{~d}$, touch the limit curves at more than one point, they are less consistent with the data than the double step functions used to set the efficiency function limits. For dark matter particle masses for which $d R / d E_{r} \div \rho_{L}$ is not monotonic, the step functions are still used to estimate the bounds on the dark matter sensitivity. At these masses, the ${ }^{241} \mathrm{Am} / \mathrm{Be}$ neutron recoil spectrum well appoximates the expected dark matter recoil spectrum, and the dark matter sensitivity does not depend strongly on the shape of the efficiency function.

Using the theoretical tools presented in this chapter, the bubble nucleation efficiency and dark matter sensitivity of bubble chambers described in the next two chapters can be analysed and measured. 


\section{Chapter 4 \\ Calibration Bubble Chambers}

\section{1 $(\gamma, n)$ Neutron Sources}

Two $(\gamma, n)$ neutron source designs were used to calibrate the response of $\mathrm{CF}_{3} \mathrm{I}$ and $\mathrm{C}_{3} \mathrm{~F}_{8}$ to nuclear recoils. The first source, containing up to $5 \mathrm{mCi}$ of ${ }^{88} Y$ was used with the STAR bubble chamber while the second design, with an activity of $100 \mathrm{mCi}$, was used with the other bubble chambers. Both source designs use commercially available radioactive material from Eckert \& Ziegler, and use beryllium in the form of sintered beryllium oxide with a nominal density of $2.85 \mathrm{~g} / \mathrm{cm}^{2}$ in order to avoid the possibility of toxic exposure to elemental beryllium. The beryllium oxide was provided by American Beryllia Inc.1 $\left.\right|^{1}$ at reduced cost through their R\&D Assistance Program.

\subsubsection{STAR ${ }^{88} \mathrm{Y} / \mathrm{Be}$ Neutron Source}

The STAR ${ }^{88} \mathrm{Y} /$ Be neutron source was designed to have a high neutron yield, producing up to 6,100 neutrons per second in 39 grams of $\mathrm{BeO}$ when containing five millicuries of ${ }^{88} \mathrm{Y}$. The ${ }^{88} \mathrm{Y}$ was in solution as $\mathrm{YCl}$. The solution was evaporated into the bottom of a one milliliter crimp-top high-recovery V-vial. The vial was filled with epoxy resin, sealed, and placed in a well surrounded by beryllium oxide one centimeter thick. The entire source was contained in a stainless steel container sealed with a 2-1/8" Conflat (CF) flange. The source was packed with several millimeters of padding above it to prevent rattling in order to maintain the source geometry and avoid damage. Figure 4.1 shows the source geometry.

Due to the large radiation dose rate near the source, up to $33 \mathrm{mR} / \mathrm{hr}$ at $30 \mathrm{~cm}$, the source was handled using a 4" diameter PVC pipe as a long handle with the source wadded

\footnotetext{
1. http://www. americanberyllia.com
} 

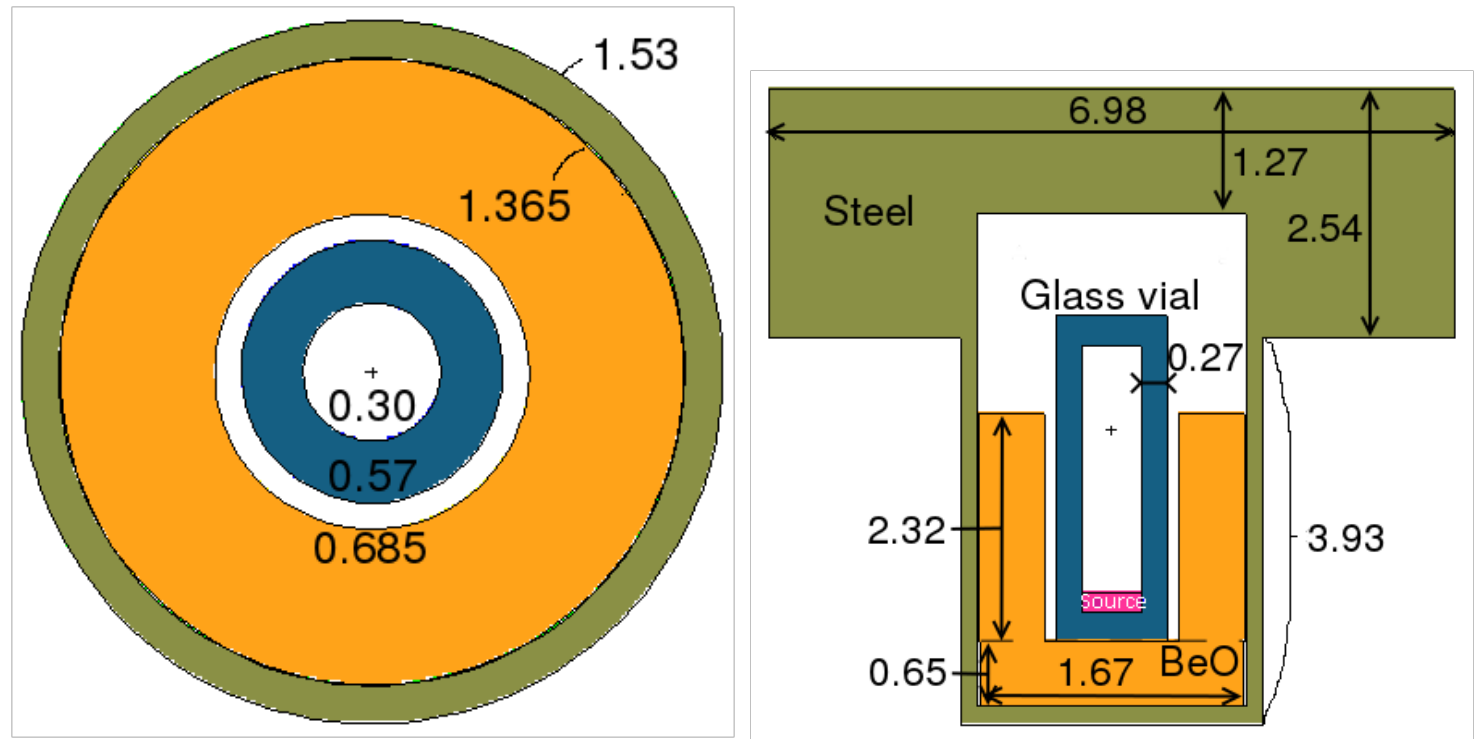

Figure 4.1: Design of the ${ }^{88} \mathrm{Y} / \mathrm{Be}$ monoenergetic neutron source used with the STAR bubble chamber at Argonne National Laboratory. All dimensions are in centimeters and on the horizontal cross-section, dimensions are given for the radius.

into one end. Each time the source was placed next to the bubble chamber, the positions of the source were recorded with millimeter accuracy. Neutron recoil rate simulations were rerun for each position of the source.

This source could be operated in two configurations: as a neutron source with the beryllium oxide in place, or as a pure $\gamma$ source with the BeO replaced by aluminum. Changing out the beryllium oxide and the aluminum required opening and resealing the CF flange and handling the fragile glass encapsulated source inside. When changing the source configuration from pure gamma back to a neutron source in November 2011, the glass vial had cracked and the inner surface was exposed during handling. The failure of the source ended data taking with the STAR bubble chamber.

The gamma activity of the source was not precisely measured. The neutron activity was measured using a ${ }^{3}$ He detector as described in Section 4.1.4. 


\begin{tabular}{lcc}
\hline Configuration & $\begin{array}{c}{ }^{88} \mathrm{Y} \text { Yield } \\
\text { (neutrons } / \mathrm{s} / \mu \mathrm{Ci})\end{array}$ & $\begin{array}{c}{ }^{124} \mathrm{Sb} \text { Yield } \\
(\text { neutrons } / \mathrm{s} / \mu \mathrm{Ci} \text { ) }\end{array}$ \\
\hline 1 & $0.462 \pm 0.041$ & $0.472 \pm 0.040$ \\
2 & $0.474 \pm 0.042$ & $0.484 \pm 0.041$ \\
3 & $2.083 \pm 0.089$ & $2.077 \pm 0.076$ \\
\hline
\end{tabular}

Table 4.1: Neutron yields of the source configurations shown in Figure 4.2. Uncertainties from both the cross-section from Table 3.4 and geometric uncertainties are included.

\subsubsection{University of Chicago Sources}

Later $(\gamma, n)$ sources at the University of Chicago used a simpler source design based on commercially available encapsulations. These sources used an Eckert \& Ziegler (2007) Type D calibrated $\gamma$ source with one of three configurations of beryllium oxide shown in Figure 4.2 .

The neutron yield in Table 4.1 of the three geometries shown in Figure 4.2 is very sensitive to the distance between the source and the BeO. Depending how the activity is distributed within the the $3.18 \mathrm{~mm}$ depth of the active element, in the extreme case, the source yield may vary by up to $60 \%$. Relative measurements of the source strength with sources $1714-43$ and 1742-25 in their normal orientation and flipped so that their decals faced the BeO were used to determine the average depth of the activity within the source. The source activity in both positions was consistent with the activity being equally distributed within the epoxy resin plug. This source was simulated as a cylindrical active element extending from $0.277 \mathrm{~mm}$ to $0.5945 \mathrm{~mm}$ from the source face with a $0.25 \mathrm{~cm}$ radius. Uncertainties in the position of the activity result in an $\pm 8.0 \%$ uncertainty in the source rate for configurations 1 and 2 . For configuration 3 , the uncertainty is $\pm 2.2 \%$.

Due to the short half-lives of ${ }^{88} \mathrm{Y}$ and ${ }^{124} \mathrm{Sb}$, several Type-D $\gamma$ sources had been procured for bubble chamber calibrations. They are listed in Table 4.2 . 
Configuration 1

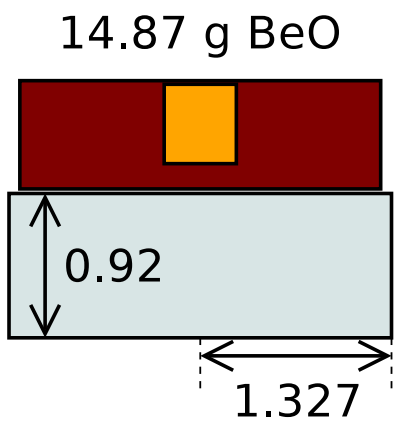

\section{Configuration 3}

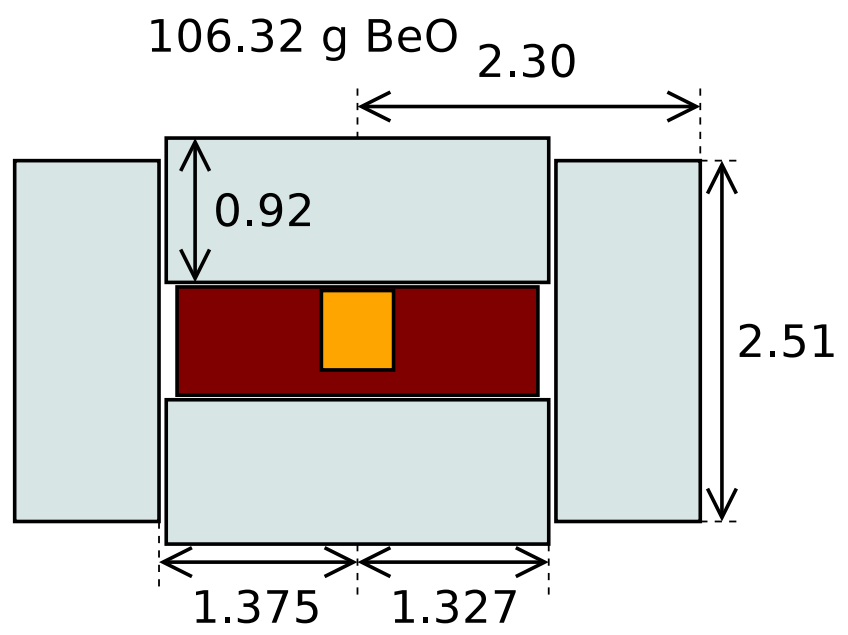

Configuration 2 $16.29 \mathrm{~g} \mathrm{BeO}$

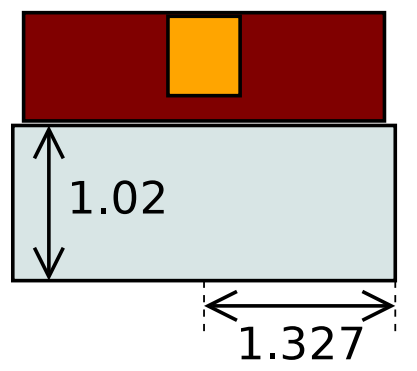

\section{Type-D source}

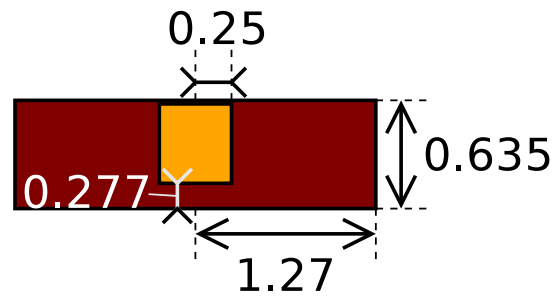

Figure 4.2: Design, and measured dimensions and masses of $(\gamma, n)$ source configurations used at the University of Chicago. Each configuration is cylindrically symmetric. The BeO cylinders used in Configurations 1 and 2 were constructed of multiple thin disk held together with tape around their circumference. A $0.025 \pm 0.025 \mathrm{~cm}$ gap above and below the Type D source is used to model the effect of slack in the alignment of the source components. The active material is evaporated into a well and mixed with epoxy resin in the acrylic disks. The activity is assumed to be evenly distributed within the epoxy. 


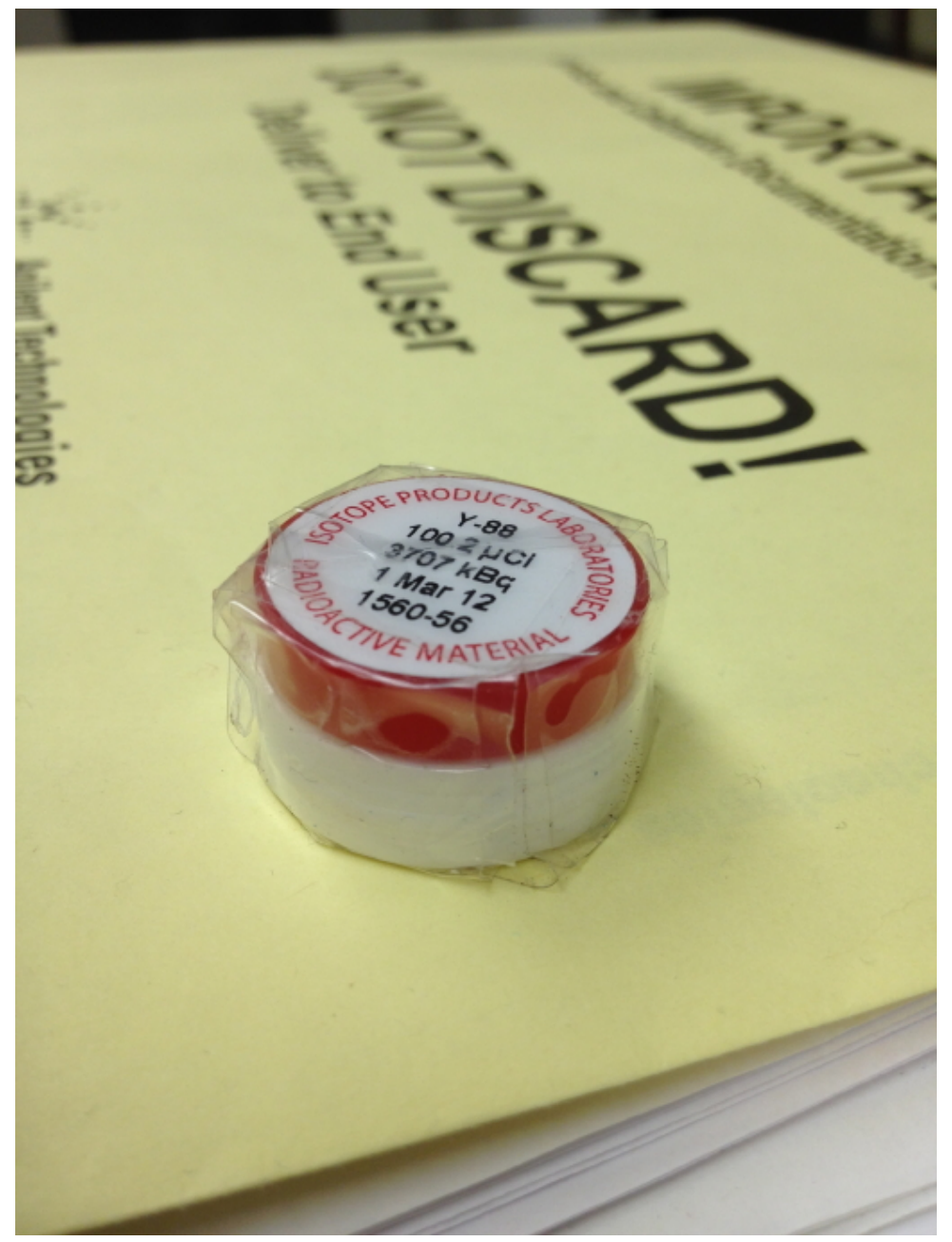

Figure 4.3: $\mathrm{An}{ }^{88} \mathrm{Y} /$ Be source in configuration 2 used with the University of Chicago Bubble Chamber.

\begin{tabular}{cccc}
\hline Isotope & Source ID & Date & Activity $(\mu \mathrm{Ci})$ \\
\hline${ }^{88} \mathrm{Y}$ & $1560-56$ & 1-Mar-12 & 100.2 \\
${ }^{88} \mathrm{Y}$ & $1637-69$ & 15-Dec-12 & 102.1 \\
${ }^{88} \mathrm{Y}$ & $1671-7$ & 1-Apr-13 & 103.5 \\
${ }^{88} \mathrm{Y}$ & $1714-43$ & 15-Jan-14 & 91.8 \\
${ }^{124} \mathrm{Sb}$ & $1742-25$ & 1-Jun-14 & 104.1 \\
\hline
\end{tabular}

Table 4.2: Type-D ${ }^{88} \mathrm{Y}$ and ${ }^{124} \mathrm{Sb}$ sources used to produce $(\gamma, n)$ neutrons at the University of Chicago. 


\subsection{3 $\quad{ }^{241} \mathrm{Am} / \mathrm{Be}$ and ${ }^{252} \mathrm{Cf}$ Sources}

A variety of weak ${ }^{241} \mathrm{Am} / \mathrm{Be}$ and ${ }^{252} \mathrm{Cf}$ sources have been used to test the response of COUPP and PICO bubble chambers. Data using these sources at Argonne and the University of Chicago has not been analyzed as high multiplicity data is available from neutron calibrations with COUPP-4kg and PICO-2L at SNOLAB. At SNOLAB, an ${ }^{241} \mathrm{Am} / \mathrm{Be}$ pressed powder source and a ${ }^{252} \mathrm{Cf}$ were used. The sources are described in Section 6.2 of Drew Fustin's thesis (2012).

\subsubsection{He-3 measurements}

To verify the neutron production rates given in Table 4.1 from the source configurations of Figure 4.2 , the sources were measured using an LND-252 ${ }^{3}$ He thermal neutron detector (LND Inc 2015). The detector configuration is shown in Figure 4.4. MCNPX-Polimi simulations of both the sources and the detector were used to determine the absolute efficiency of the detector. Measurements and count rates from these measurements are shown in Table 4.3 .

These measured rates are all near the expected rates given in Table 4.1, although the rates for configuration 3 are slightly low. The nominal rates given in Table 4.1 will be used in the analysis.

Geometric uncertainties that cause the source rate to be under-measured in the ${ }^{3} \mathrm{He}$ detector could lead to an overly optimistic calibration of dark matter sensitivity. To verify that no ${ }^{3} \mathrm{He}$ had leaked out of the tubes, three nearly identical ${ }^{3}$ He tubes (two LND-252 and a steel bodied LND-25192) were inter-calibrated using an Am/Be sources. All three were found to have the same sensitivity to within $1.3 \%$. Uncertainties in material compositions, cross-sections, or densities may have also resulted in neutrons being reflected away from the detector. All materials used in the measurement were of high purity, and have low neutron cross-section uncertainties. 


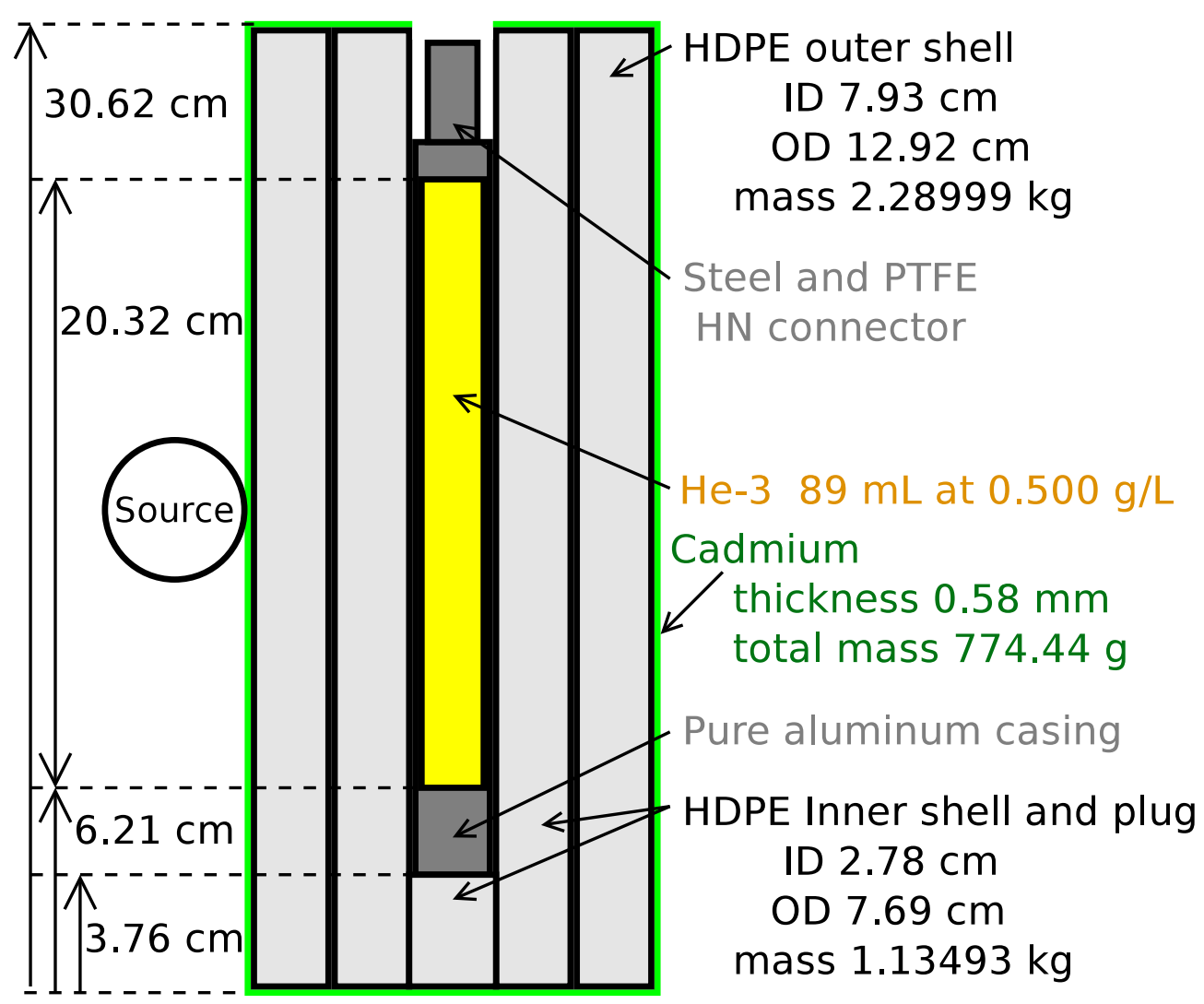

Figure 4.4: Arrangement of an LND-252 (LND Inc 2015) ${ }^{3} \mathrm{He}$ detector for measuring the neutron production rate of $(\gamma, n)$ sources. The cadmium layer surrounding the polyethylene moderator prevents ambient thermal neutrons from contributing a background. The ceramic insulators holding the cathode wire of the detector are approximated in this geometry by using pure aluminum.

The measured rate of the ${ }^{88} \mathrm{Y}$ STAR source was inconsistent between the two measurements in July and October 2011. With 99 days between the two measurements, a rate of 2073 neutrons/sec was expected for the measurement Oct 26. The actual measurement was $6 \%$ below this value. The half-life adjusted average of the two measurements will be adopted, a neutron rate of $3830 \pm 150$ neutrons/sec on 19 July, 2011. 


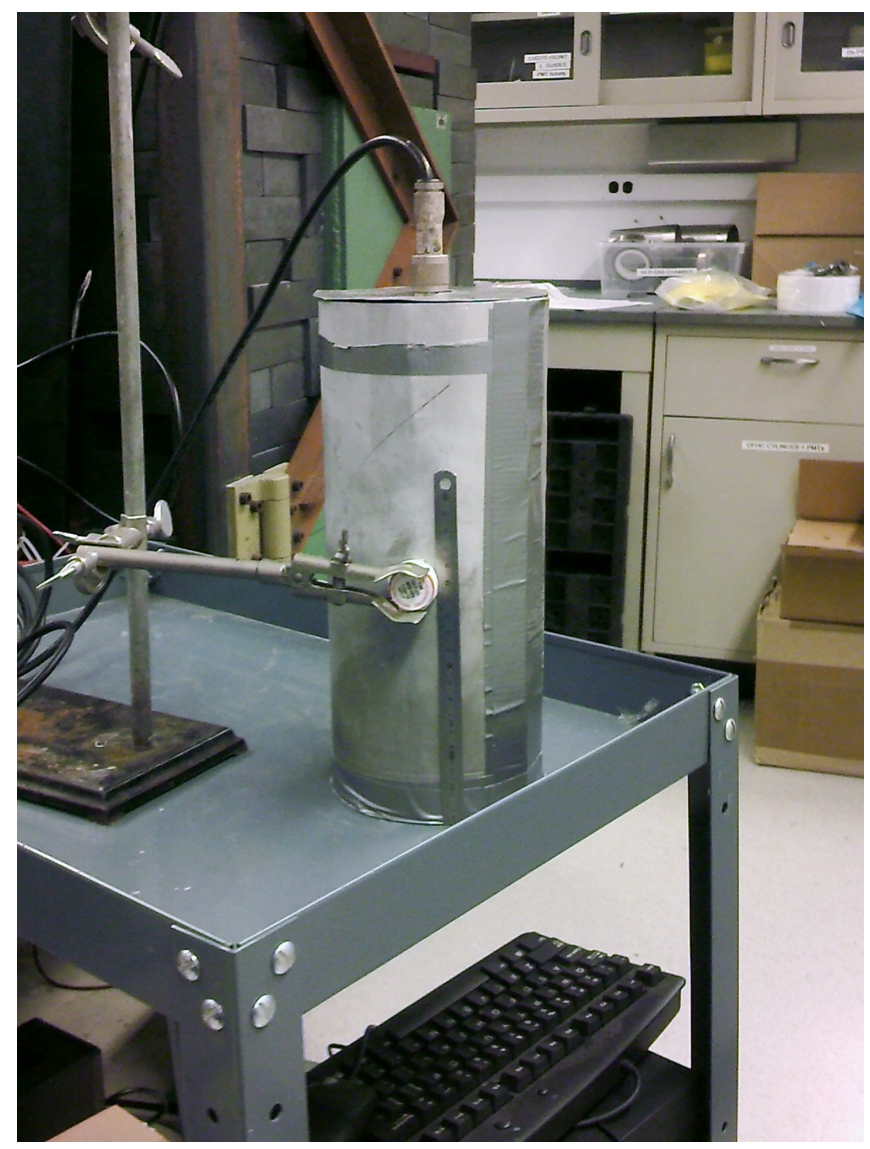

Figure 4.5: Measurement of ${ }^{88} \mathrm{Y}$ source 1671-7 in Configuration 212 July, 2013.

\subsection{STAR Bubble Chamber}

The STAR bubble chamber, unlike all other COUPP and PICO chambers, did not use a bellows. Rather, pressure was controlled using a pneumatic piston pressing directly against the water inner volume buffer fluid. The pressure was set using a regulator on a reservoir connected to the pneumatic side of the piston. The pressure set by this regulator was transmitted through the piston. Sticking of the piston precluding fine control of the $\mathrm{CF}_{3} \mathrm{I}$ pressure. A 3-way valve switched between the regulated and high pressure air reservoirs during expansions and compressions.

The active volume of the bubble chamber was contained within a thick $(4.03 \mathrm{~mm})$ glass 


\begin{tabular}{llccc}
\hline Source & Date & $\begin{array}{c}\text { Count Rate } \\
(\text { counts/sec })\end{array}$ & $\begin{array}{c}\text { Source Rate } \\
(\text { neutrons/sec })\end{array}$ & $\begin{array}{c}\text { Source Yield } \\
(\text { neutrons } / \mathrm{s} / \mu \mathrm{Ci})\end{array}$ \\
\hline${ }^{88}$ Y STAR & 19-Jul-11 & $72.44 \pm 0.35$ & $3950 \pm 150$ & - \\
${ }^{88}$ Y STAR & 26-Oct-11 & $35.81 \pm 0.39$ & $1951 \pm 74$ & - \\
${ }^{88}$ Y 1637-69 Conf. 2 & 10-Jan-13 & $0.820 \pm 0.030$ & $39.1 \pm 2.0$ & $0.454 \pm 0.023$ \\
${ }^{88}$ Y 1671-7 Conf. 2 & 12-Jul-13 & $0.518 \pm 0.007$ & $24.7 \pm 1.0$ & $0.463 \pm 0.018$ \\
${ }^{252}$ Cf & 17-Jul-13 & $0.442 \pm 0.009$ & $28.3 \pm 1.2$ & - \\
${ }^{88}$ Y 1671-7 Conf. 3 & 14-Aug-13 & $1.535 \pm 0.013$ & $84.6 \pm 3.2$ & $1.999 \pm 0.077$ \\
${ }^{88}$ Y 1671-7 Conf. 3 & 19-Nov-13 & $0.787 \pm 0.021$ & $43.4 \pm 1.8$ & $1.893 \pm 0.079$ \\
${ }^{88}$ Y 1671-7 Conf. 1 & 20-Nov-13 & $0.2078 \pm 0.0043$ & $11.42 \pm 0.48$ & $0.502 \pm 0.021$ \\
${ }^{88}$ Y 1714-43 Conf. 3 & 14-Jan-14 & $3.304 \pm 0.028$ & $182.3 \pm 7.0$ & $2.004 \pm 0.077$ \\
${ }^{124}$ Sb 1742-25 Conf. 1 & 12-Jun-14 & $0.962 \pm 0.007$ & $48.50 \pm 0.36$ & $0.5349 \pm 0.0040$ \\
\hline
\end{tabular}

Table 4.3: Measurements of the neutron source rates using the ${ }^{3}$ He detector shown in Figure 4.4. Statistical uncertainties and a $3.7 \%$ systematic uncertainty due to play in the geometry of the He-3 moderator are applied. The source yield values can be compared to those calculated in Table 4.1. An LND-25192 used to verify the sensitivity of the LND-252 detectors uses the same geometry with the pure aluminum casing replaced by stainless steel.

CG-1880-4 48 mL Chemglass pressure vessel. Air surrounded the pressure vessel - there was no hydraulic fluid bath that would act as a moderator and thermal bath. The pressure control system and pressure vessel was surrounded by a steel containment vessel. The containment vessel formed a nearly closed volume with small openings to allow readout wires and the source holder through.

Two Optek OPA730RD red LED arrays on the inside of the containment vessel backilluminated the chamber through glass diffusers. The LEDs consumed $10 \mathrm{~W}$ of power and were mounted on a water cooled heat sink. For all the analyzed runs, no water was supplied to the heat sink. Two perpendicular camera ports held Basler 602f cameras to image the $\mathrm{CF}_{3} \mathrm{I}$. These cameras were placed at $60 \pm 1 \mathrm{~cm}$ from the center of the chamber for cam 0 and $58 \pm 1 \mathrm{~cm}$ from the center of the chamber for cam1.

Two two-wire resistive thermometers (RTDs) bonded to the exterior of the pressure vessel measured the temperature of the system. These are described in Section 4.2.2. 


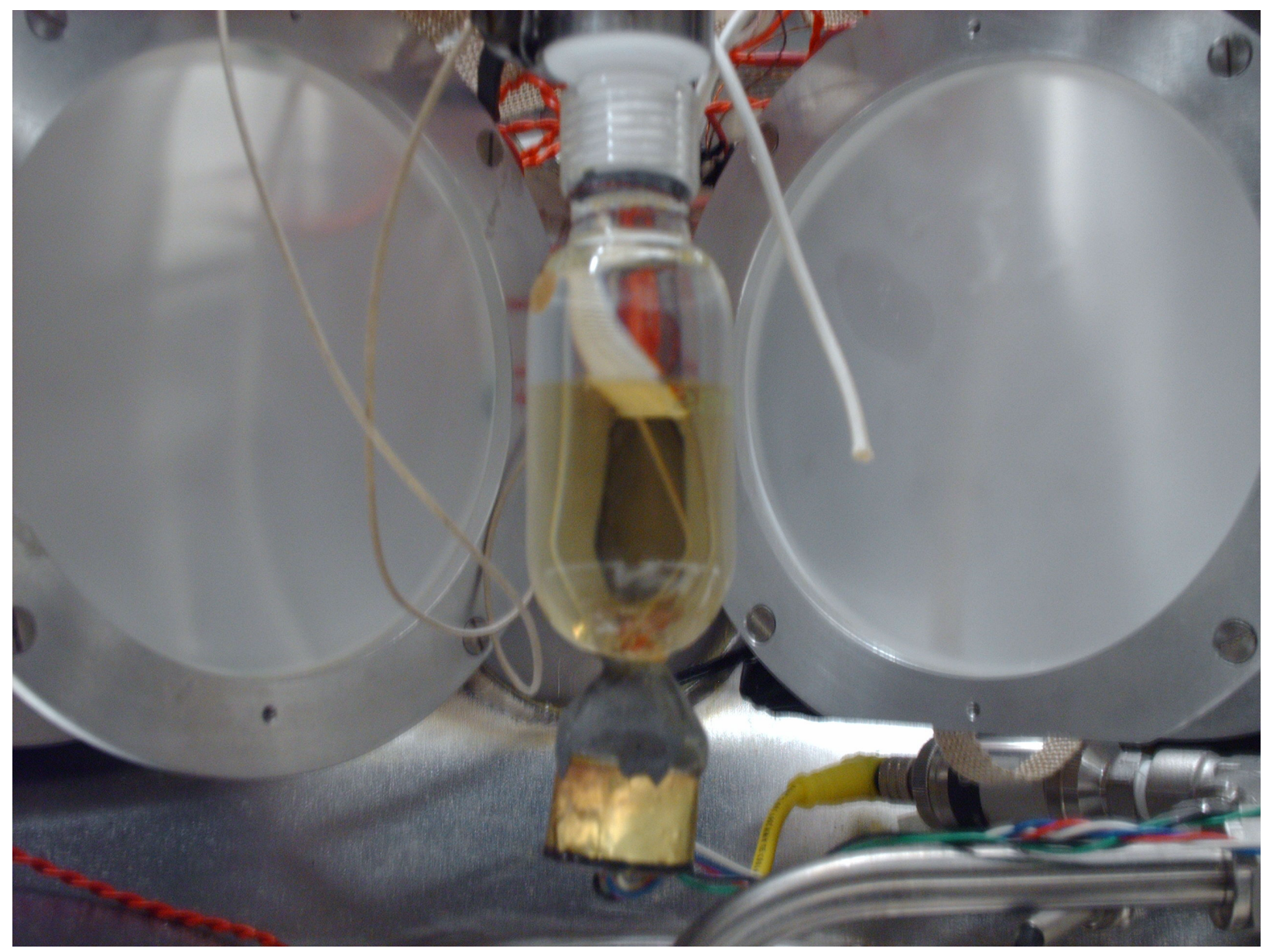

Figure 4.6: The STAR bubble chamber pressure vessel with yellowed $\mathrm{CF}_{3} \mathrm{I}$ and insulation removed looking through the source mounting port. The LED diffusers are visible behind and to either side of the pressure vessel. 


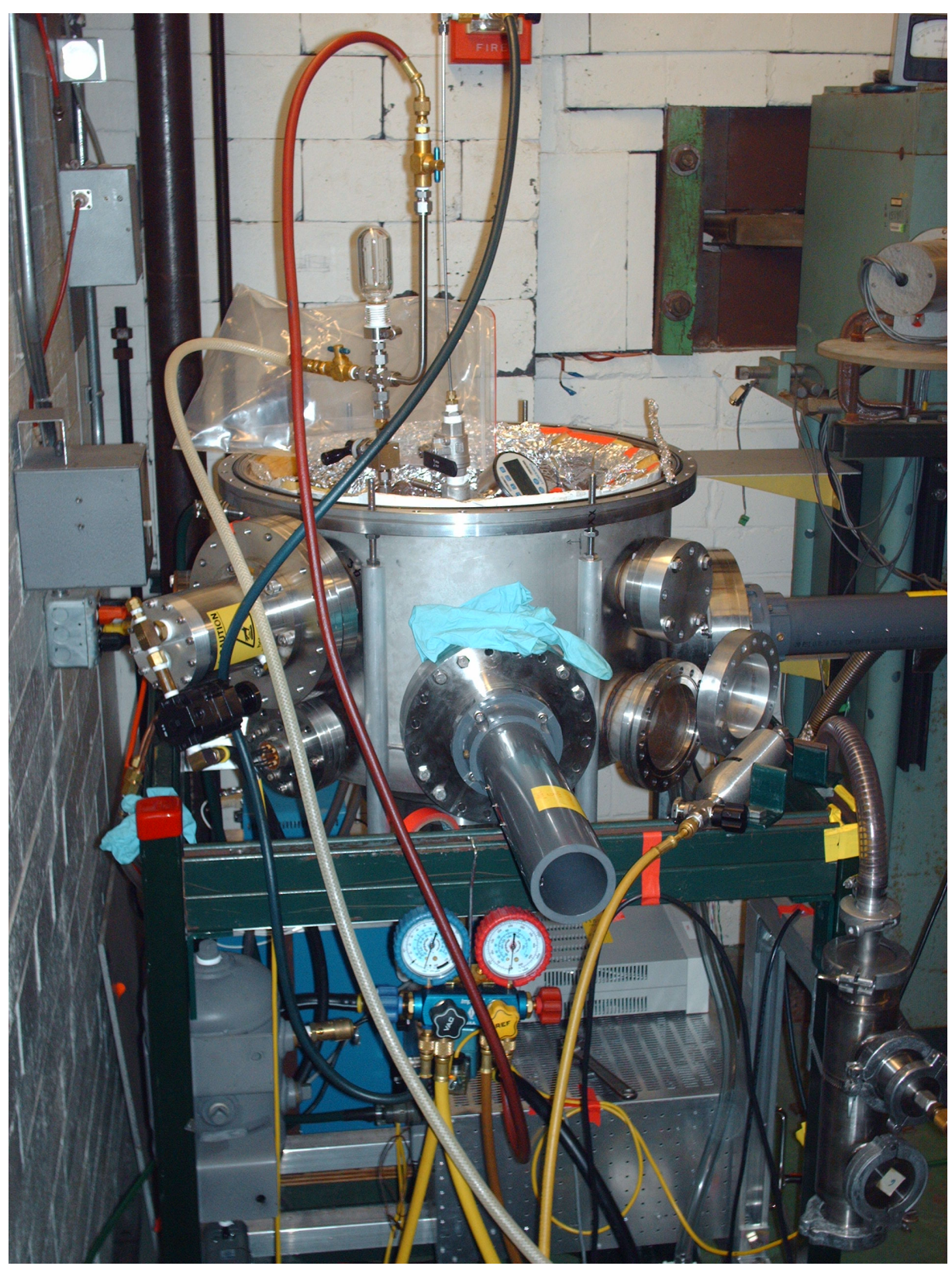

Figure 4.7: The STAR containment vessel during filling. 


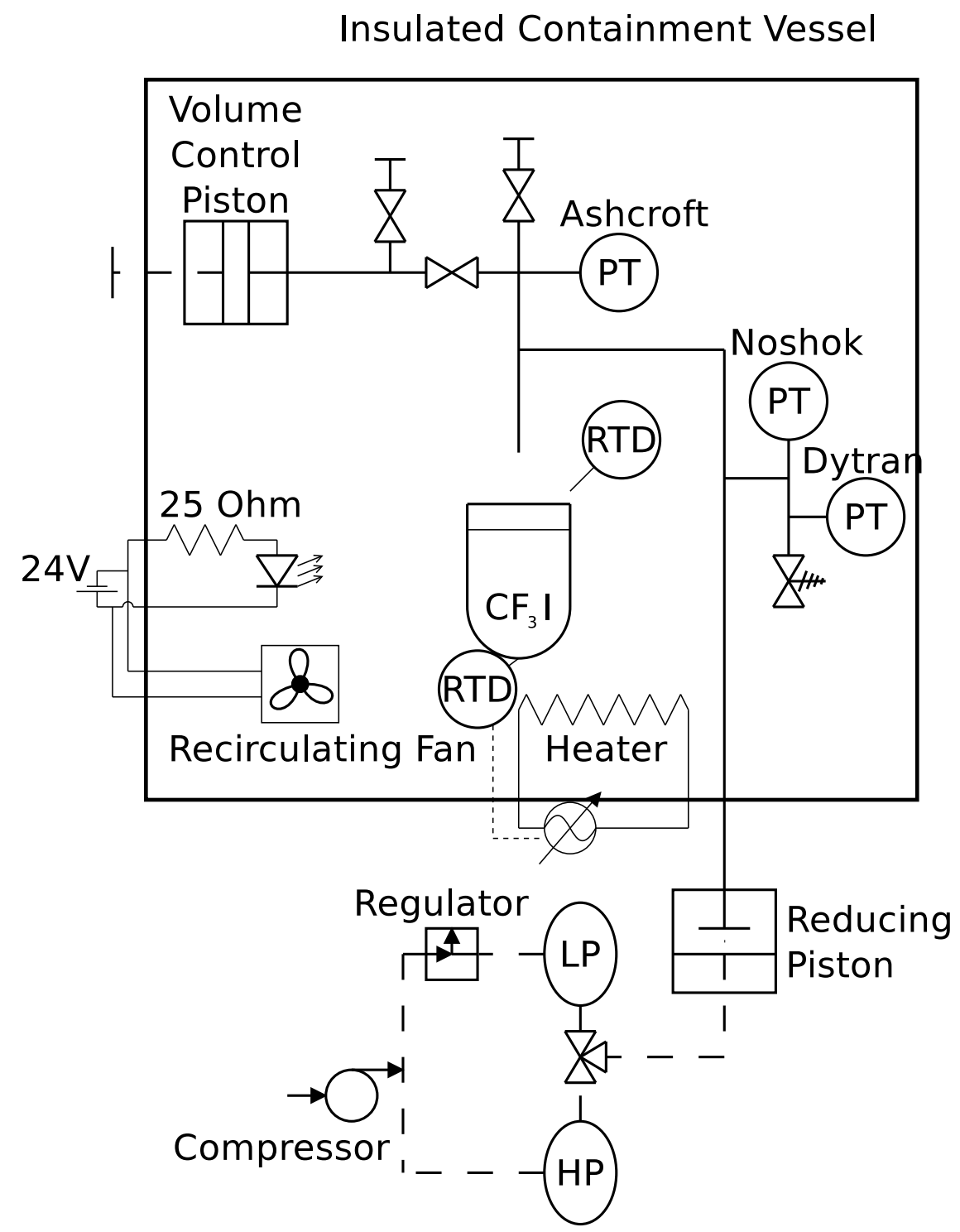

Figure 4.8: Process flow diagram of the STAR bubble chamber including thermally relevant electrical components. The following acronyms are used: pressure transducer (PT), resistive thermometer (RTD), low-pressure reservoir (LP), and high-pressure resevoir (HP). 


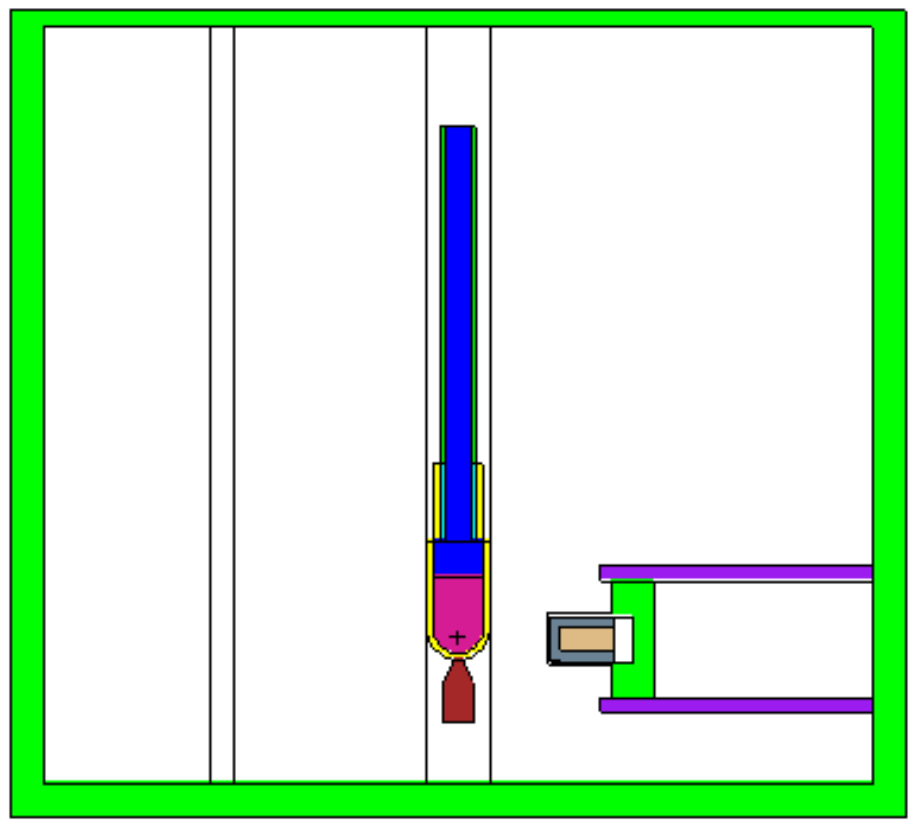

Figure 4.9: Cross-sections of the MCNPX-Polimi geometry of the STAR bubble chamber in the plane of the ${ }^{88} \mathrm{Y} / \mathrm{Be}$ source. The source, the source holder, the pressure vessel, the active and buffer fluids, steel tubing, the piezo electric transducer, and the containment vessel are visible. The LED mounts, diffusers, and inner volume pipes to the piston are out of the plane shown.

The hydraulic volume was instrumented with a Noshok brand (slow), and a Dytran brand (fast) pressure transducers that were read by the data aquisition system (DAQ). The $\mathrm{CF}_{3} \mathrm{I}$ was elevated approximately $5 \mathrm{~cm}$ in comparison to the transducers, contributing a negligible offset of $0.5 \mathrm{kPa}$ to pressure measurements. A calibrated Ashcroft pressure transducer was used during filling operations and to cross-calibrate against the Noshok transducer. The two transducers agreed within the $0.7 \mathrm{psi}(5 \mathrm{kPa})$ tolerance of the more sensitive Ashcroft gauge. While the system pressure could not be precisely controlled, it was precisely measured.

A piezoelectric transducer was mounted to the bottom of the STAR pressure vessel, but its data has not been used in this analysis. The piezo was attached with a cone of J-B Weld brand epoxy resin. 


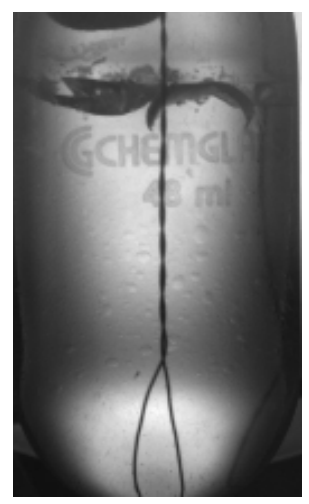

Figure 4.10: Dislodged PTFE tape in STAR, run 20111115_0 event 2155. The DAQ RTD is just visible at the top left of the image while the wires to the RTD controlling the heater are visible behind the chamber.

As the STAR bubble chamber contained a reasonably large volume of target fluid at a surface location, its background event rate was higher than most other COUPP and PICO bubble chambers, with an event approximately every 90 seconds. In order to maximize the counting statistics obtained from the bubble chamber, a very short dead time between events was set, with a compression time of 8 seconds for most events and a compression time of 30 seconds every $10^{\text {th }}$ event. The compression time was extended to 10 seconds beginning with run $20111017 \_3$, and to 30 seconds with a 60 second expansion every $10^{\text {th }}$ event for run 20111110_3.

\subsubsection{Contaminants}

While the STAR bubble chamber was cleaned before assembly, it did contain wetted materials that were not present in other COUPP and PICO bubble chambers that could degrade. Wetted materials included 316 grade stainless steel, borosilicate glass, solid PTFE, PTFE tape, Viton fluroelastomer, epoxy resin, and Apiezon Type N vacuum grease. During $\mathrm{CF}_{3} \mathrm{I}$ filling, the gaseous $\mathrm{CF}_{3} \mathrm{I}$ was also exposed to brass, nylon, and other elastomers through a 4 port refrigerant handling manifold designed for use with R-134a refrigerant.

Several wetted materials degraded in the presence of $\mathrm{CF}_{3} \mathrm{I}$. The original reducing piston 
in STAR was made of aluminum. The piston corroded, formed $\mathrm{Al}(\mathrm{OH})_{3}$ in solution, created a foam at the $\mathrm{CF}_{3} \mathrm{I}$ interface, and finally seized in May and June 2011. It was replaced with a stainless steel piston. PTFE tape used to seal the inner volume was observed to discolor and degrade. Sections of the tape detached and migrated to the $\mathrm{CF}_{3} \mathrm{I}-$-buffer interface as shown in Figure 4.10.

Towards the end of October 2011, the eroded PTFE tape and other particulates allowed for the formation of an emulsion at the top of the bubble chamber. The event rate from the emulsion dominated the event rate from the rest of the chamber. The emulsion would alternate between periods of activity and benignity. During active periods, full expansion could not be reached.

The $\mathrm{CF}_{3} \mathrm{I}$ used to fill STAR was from the same batch used to fill the COUPP-60 run at NuMI in 2010, as described in Section 2.1. This $\mathrm{CF}_{3} \mathrm{I}$ contained between $0.6 \%$ and $3.0 \%$ $\mathrm{mol} / \mathrm{mol}$ of dissolved $\mathrm{CO}_{2}$. The vapor pressure of the $\mathrm{CF}_{3} \mathrm{I} \mathrm{CO}_{2}$ mixture was significantly higher than that of pure $\mathrm{CF}_{3} \mathrm{I}$, as shown in Figure 4.11. $\mathrm{A} \mathrm{CO}_{2}$ concentration of $1.25 \pm 0.63 \%$ is adopted in the calculation of the Seitz threshold. A precise measurement of the vapor pressure in situ was not possible due to the intermediate layer of water between the liquid and gaseous $\mathrm{CF}_{3} \mathrm{I}$ during measurements.

The $\mathrm{CF}_{3} \mathrm{I}$ used in STAR was exposed to both intense LED illumination and some white light which darkened the $\mathrm{CF}_{3} \mathrm{I}$ at a rapid rate. A saturated concentration of iodine was present for some preliminary runs taken before October 2011. Unlike in COUPP-60, the STAR bubble chamber was sufficiently thin so that this darkening did not obscure images and prevent bubble chamber operation. During the runs that were analyzed, $5 \mathrm{mM} \mathrm{Na} \mathrm{Na}_{3}$ solution was used as the buffer fluid. 


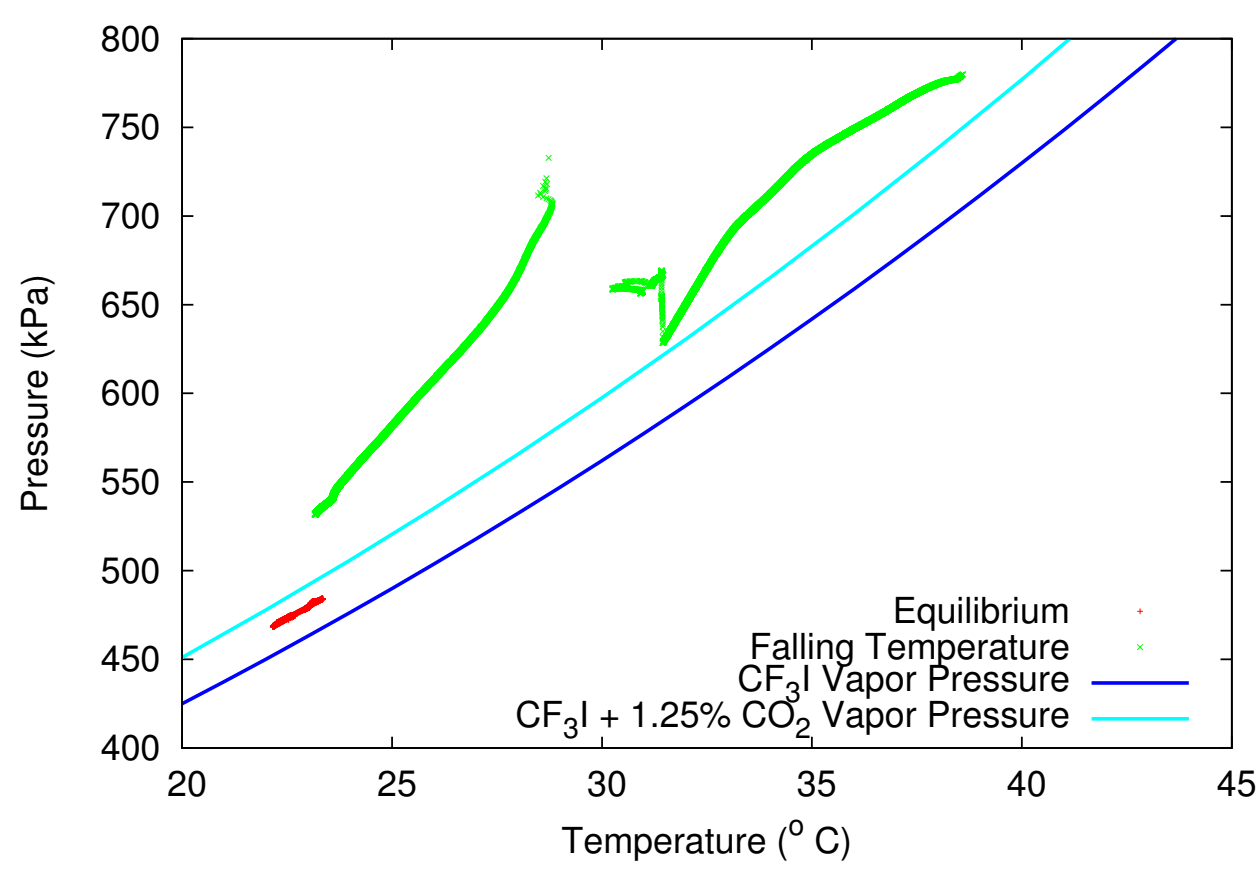

Figure 4.11: Vapor pressure vs. temperature of pure $\mathrm{CF}_{3} \mathrm{I}$, and that of $\mathrm{CO}_{2}$ contaminated $\mathrm{CF}_{3} \mathrm{I}$ in STAR. In thermal equilibrium (in red), the observed vapor pressure is lower than the adopted pressure. As $\mathrm{CO}_{2}$ has a higher solubility than $\mathrm{CF}_{3} \mathrm{I}$ in water, the buffer fluid may reduce the equilibrium concentration of $\mathrm{CO}_{2}$ in the vapor space. As the temperature was ramping (in green), the vapor space may have been several degrees Celsius warmer the measured $\mathrm{CF}_{3} \mathrm{I}$ temperature. Given these considerations, the extremes of the red and green curves provide upper and lower limits on the active fluid vapor pressure.

\subsubsection{Thermal Stability}

Two 2-wire RTDs bonded to the outside of the pressure vessel monitored the system. The RTD mounted to the bottom of the pressure vessel controlled a heater on the floor of the containment vessel below the pressure vessel. The temperature measured by the RTD mounted to the top of the vessel was recorded by the DAQ. Additional heat was produced by the $25 \Omega$ current limiting resistors for the LED illumination, providing a constant $3.2 \mathrm{~W}$

of power. With the LEDs on and the heater disabled, these resistor raised the temperature at the $\mathrm{CF}_{3} \mathrm{I}$ to between $29^{\circ} \mathrm{C}$ and $36^{\circ} \mathrm{C}$ depending on the ambient temperature outside the containment vessel. The inside of the containment vessel was lined with 1" of dense 
fiberglass insulation except at the camera ports and the source mounting port.

The current limiting resistors released their heat at an elevation slightly above the pressure vessel. This location did not force air convection inside the chamber and allowed a temperature gradient within the containment vessel. A reference thermometer was used to measure the gradient both inside and outside the active volume. With the recirculating fan off, no cooling of the LEDs, and with the heater supplying minimal power at a setpoint temperature of $38.5^{\circ} \mathrm{C}$, the top of the containment vessel was $7^{\circ} \mathrm{C}$ hotter than the bottom of the vessel. For the brief period when the recirculating fan was in operation that includes runs 20111110_4 to 20111114_2, the difference was reduced to $0.5^{\circ} \mathrm{C}$.

The paired wires connecting the RTDs to the DAQ and to the heater controller were long and of unequal length. The additional resistance in the wire leads to the RTDs created a miscalibration in the temperature readout of the DAQ. After the ${ }^{88} \mathrm{Y} / \mathrm{Be}$ measurements, an additional pair of voltage sensing wires was connected $5 \mathrm{~cm}$ away from the DAQ RTD. The 4-wire temperature measurement was significantly more accurate than the 2-wire measurement. The RTDs were calibrated against a precision $\left( \pm 0.1^{\circ} \mathrm{C}\right)$ reference thermometer placed inside the water filled pressure vessel in October and November 2011. The corrections used for various operating conditions are given in Table 4.4. The temperature was allowed to equilibrate for 30 minutes prior to each measurement. Two measurements outside the pressure vessel were made after leaving the chamber to equilibrate overnight, with the reference thermometer placed next to the pressure vessel.

When calibrated against the equilibrium temperature outside the pressure vessel, the calibrations of $T_{\text {set }}$ and $T_{1}$ in Table 4.4 reveal a temperature gradient of between $0.6^{\circ} \mathrm{C}$ and $1.3^{\circ} \mathrm{C}$ between the bottom and top of the pressure vessel. During operation, the gradient was found to vary and could reach a value as large at $2.3^{\circ} \mathrm{C}$. The gradient was correlated with the setpoint temperature and with the event rate inside the chamber. The largest gradients 


\begin{tabular}{lll}
\hline Condition & $\begin{array}{l}\text { DAQ Offset } \\
T_{\text {ref }}-T_{1} \\
\left({ }^{\circ} \mathrm{C}\right)\end{array}$ & $\begin{array}{l}\text { Heater setpoint offset } \\
T_{\text {ref }}-T_{\text {set }} \\
\left({ }^{\circ} \mathrm{C}\right)\end{array}$ \\
\hline \multicolumn{2}{c}{ 2-wire DAQ RTD measurements } \\
\hline Outside the pressure vessel & $-4.74 \pm 0.20$ & $-1.57 \pm 0.20$ \\
Chiller at $8{ }^{\circ} \mathrm{C}, 31.5^{\circ} \mathrm{C}$ heater setpoint & $-5.64 \pm 0.4$ & $-1.4 \pm 0.3$ \\
Chiller at $8{ }^{\circ} \mathrm{C}, 35.0^{\circ} \mathrm{C}$ heater setpoint & $-5.17 \pm 0.4$ & $-1.4 \pm 0.3$ \\
Chiller off, $38.5^{\circ} \mathrm{C}$ heater setpoint & $-5.21 \pm 0.3$ & $-1.2 \pm 0.2$ \\
Chiller off, $41.5^{\circ} \mathrm{C}$ heater setpoint & $-5.39 \pm 0.3$ & $-1.2 \pm 0.2$ \\
Chiller off, $44.5^{\circ} \mathrm{C}$ heater setpoint & $-5.54 \pm 0.3$ & $-1.1 \pm 0.2$ \\
\hline & 4 -wire DAQ RTD measurement \\
\hline
\end{tabular}

Table 4.4: Measured temperature offsets between the heater setpoint temperature $T_{\text {set }}$, the DAQ readout temperature $T_{1}$, and the temperature on the inside of the pressure vessel $T_{\text {ref }}$. $T_{1}$ is shown without corrections applied by the DAQ software. Uncertainties are based on the offset difference when ramping up vs. ramping down the temperature at $41.5^{\circ} \mathrm{C}$.

occurred when $T_{\text {set }}=38.5^{\circ} \mathrm{C}$ with no cooling applied. Figure 4.12 shows how $T_{1}$, and the gradient, increased during periods of high rate in these conditions. With the recirculating fan on during runs 20111110_4 - 20111114_2, the gradient was dramatically reduced, to $<0.5^{\circ} \mathrm{C}$. The mean of the calibrated $T_{\text {set }}$ and $T_{1}$ temperatures is used to determine the temperature of a given calibration run. As the $\mathrm{CF}_{3} \mathrm{I}$ is better coupled to $T_{\text {set }}$, this will result in a slight upward bias to the temperature measurement.

In order to verify that the RTDs were well coupled to the target fluid, the $\mathrm{CF}_{3} \mathrm{I}$ was allowed to boil into a measured fixed gas volume. The drop in temperature caused by the boiling was recorded by the RTDs. $T_{1}$ measured a temperature drop of $0.06 \pm 0.01^{\circ} \mathrm{C}$ within 16 seconds after the $\mathrm{CF}_{3} \mathrm{I}$ boiled. A temperature drop of up to $0.10^{\circ} \mathrm{C}$ was expected, assuming no heat gain from the environment outside the pressure vessel. While the measured change in temperature was small, the RTDs were shown to be reasonably well coupled to the active volume. 


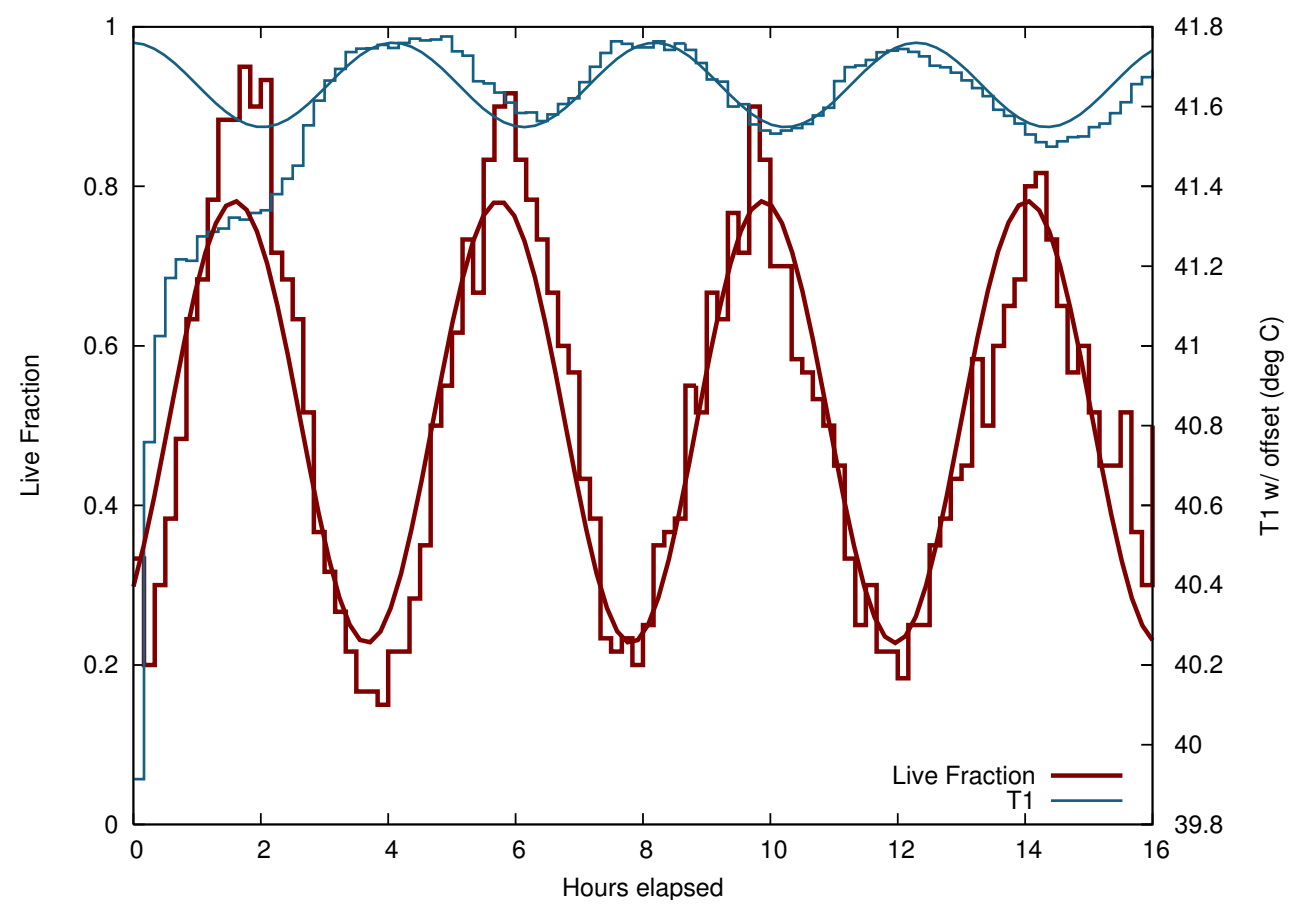

Figure 4.12: Correlation between $T_{1}$ and event rate in run 20111010_1. High rates occurred as when the expansion pressure, and thus the bubble nucleation energy threshold, was lower. The expansion pressure setpoint was cycled for all STAR runs. 
During an expansion, the $\mathrm{CF}_{3} \mathrm{I}$ decompresses and undergoes adiabatic cooling. From a compression pressure of $1.5 \mathrm{MPa}$ (200 psig) to an expansion at $200 \mathrm{kPa}$ (15 psig), the temperature will drop $0.84^{\circ} \mathrm{C}$ (Dahl 2011) with the active volume changing by $0.25 \mathrm{~mL}$. The temperature of the $\mathrm{CF}_{3} \mathrm{I}$ in the presence of pressure cycling depends on the expansion and compression history of the chamber, and the thermal coupling of the $\mathrm{CF}_{3} \mathrm{I}$ to the environment. Given the short compression and expansion times of the bubble chamber, the $\mathrm{CF}_{3} \mathrm{I}$ temperature would have rarely reached equilibrium in STAR. A temperature offset of $-0.42 \pm 0.25^{\circ} \mathrm{C}$ is applied to the data to approximate the effect of adiabatic cooling. As this temperature offset correlates with the event rate, it introduces both an absolute threshold uncertainty and a relative uncertainty between different operating conditions of the STAR bubble chamber.

Uncertainties in the run temperature, shown in Table 4.6 are based on the measured temperature gradient, calibration uncertainties, and temperature variations during the run.

\subsubsection{Geometry Measurements}

Precise measurements were made of key dimensions of the STAR bubble chamber geometry. The position of the source was measured every time it was inserted using the DAQ's cameras. By extending the fields of view of the stereo cameras outside of the active volume, the position of the source relative to the bubble chamber was measured in three dimensions to high accuracy. As the source position could not be reproduced to this accuracy, the

measurement, and simulations of the experiment, were repeated for each replacements of the source. The measurements are shown in Table 4.5.

The circumferences of another pressure vessel of the same model as the one used in STAR were measured using a caliper. The outer circumference was found to have a slight ellipticity, with the radius varying between $1.880 \mathrm{~cm}$ and $1.905 \mathrm{~cm}$. The internal circumference was 


\begin{tabular}{llll}
\hline Runs & $r$ & $x$ & $z$ \\
& $(\mathrm{~cm})$ & $(\mathrm{cm})$ & $(\mathrm{cm})$ \\
\hline 20111010_1-20111011_2 & $4.788 \pm 0.044$ & $0.032 \pm 0.044$ & $2.099 \pm 0.041$ \\
20111013_1-20111017_0 & $4.543 \pm 0.044$ & $0.311 \pm 0.044$ & $1.821 \pm 0.060$ \\
20111110_3-20111115_2 & $4.476 \pm 0.028$ & $0.009 \pm 0.028$ & $1.916 \pm 0.060$ \\
\hline
\end{tabular}

Table 4.5: Position of the ${ }^{88} \mathrm{Y} /$ Be source for analyzed STAR bubble chamber runs. $r$ is measured between the center of the chamber and the face of the source, $x$ is the distance of closest approach between the axis of the source and the axis of the chamber, and $z$ is the vertical distance from the axis of the chamber to the bottom of the pressure vessel. A separate neutron propagation simulation was performed for each row in this table.

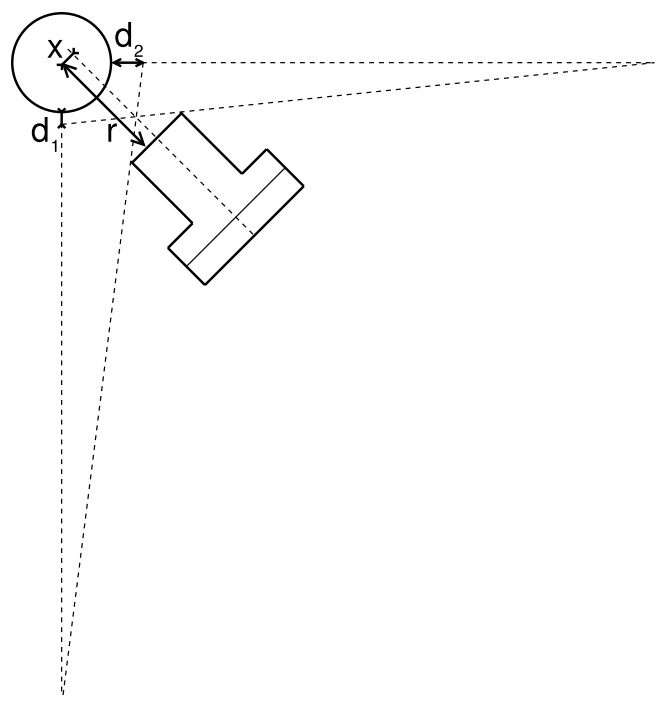

Figure 4.13: Geometry of the STAR ${ }^{88} \mathrm{Y} / \mathrm{Be}$ source and the rays from the stereo cameras used to measure the position. The normal vector to the face of the source is assumed to lie at a $45^{\circ}$ angle bisecting the angle between the two cameras. 
circular with a radius of $1.495 \pm 0.005 \mathrm{~cm}$. The $\mathrm{CF}_{3} \mathrm{I}$ fill level was measured in September 2011 by filling the empty STAR pressure vessel with a water to the same level as the $\mathrm{CF}_{3} \mathrm{I}$. A volume of $29.0 \pm 0.4 \mathrm{~mL}$ was found. The vessel was refilled, and the volume of this fill was measured from camera images by comparing the $\mathrm{CF}_{3} \mathrm{I}$ fill level to the September fill. The difference in height, $0.58 \pm 0.02 \mathrm{~cm}$, was multiplied by the inner cross-section, $\pi(1.495 \mathrm{~cm})^{2}=7.022 \mathrm{~cm}^{2}$, and added to the September fill level to give $33.1 \pm 0.4 \mathrm{~mL}$ of $\mathrm{CF}_{3} \mathrm{I}$. The fill level was monitored throughout the analyzed runs, and it remained constant.

The bottom of the pressure vessel was assumed to be hemispherical and the wall thickness constant in the simulation geometry of the vessel. While a slight asymmetry in the pressure vessel was measured, it was approximated as being rotationally symmetric for all simulations.

Other components inside of and including the containment vessel wall were measured and closely approximated in simulations. The effect of these components on the neutrons reaching the $\mathrm{CF}_{3} \mathrm{I}$ was tracked in the simulations. Figure 4.14 shows the integrated spectrum of the number of simulated recoils vs. energy that have collided in various detector components before reaching the $\mathrm{CF}_{3} \mathrm{I}$. Most neutrons have been moderated within the source or through the pressure vessel, in the direct line of sight to the $\mathrm{CF}_{3} \mathrm{I}$. Approximately $10 \%$ of the recoils are affected by materials out of the line of sight. Components outside the containment vessel can be neglected.

\subsubsection{Analysis}

The STAR bubble chamber was operated in a pressure ramping mode, where the pressure setpoint changed up and down slowly over the course of a run. Each run provided an

efficiency measurement at a continuum of Seitz thresholds. In the analysis, these operating conditions are binned in pressure with $1.7 \mathrm{kPa}(0.25 \mathrm{psi})$ bin widths. The number of events 


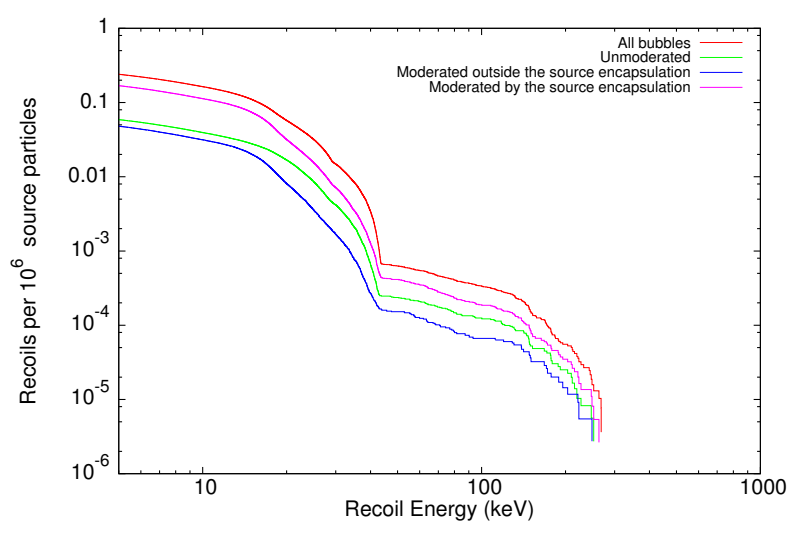

(a) All neutrons.

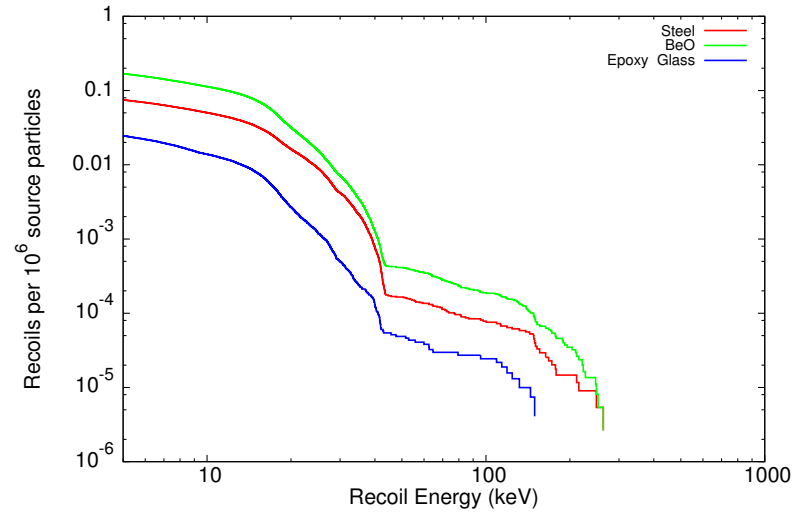

(b) Moderated neutrons in the ${ }^{88} \mathrm{Y} /$ Be source.

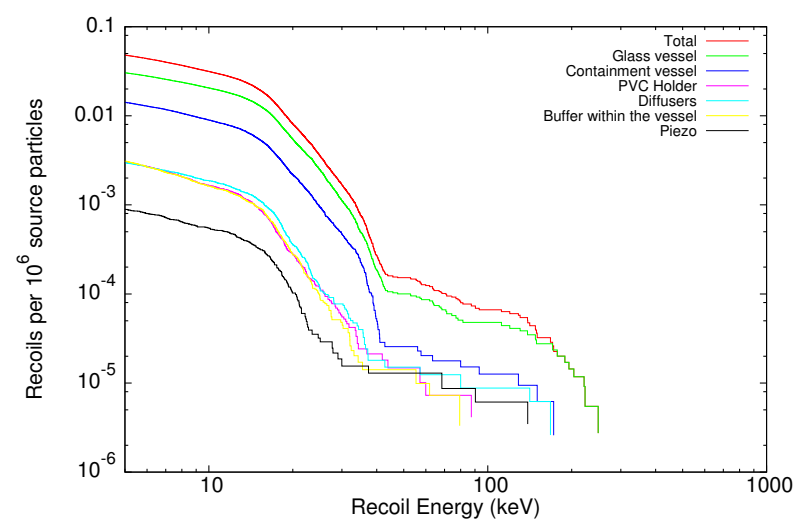

(c) Moderated neutrons in detector components.

Figure 4.14: Integrated recoil energy spectra from ${ }^{88} \mathrm{Y} / \mathrm{Be}$ neutrons in STAR with the source in position for run 20111013_1, as defined in Table 4.5. Recoils generated from neutrons that have been moderated by various detector components are shown. For a given recoil energy, the relative contribution of various moderating materials to the total recoil rate can be obtained from these plots. 
and the livetime for each bin was summed from the raw data after passing a series of cuts.

The thermodynamic properties of the bubble chamber were measured and recorded asynchronously approximately 100 times per second for the first 10 seconds of each expansion, and once every second thereafter. The pressure and temperature at any given time was linearly extrapolated from the recorded pressure history and the livetime per pressure bin was summed. Livetime within the first $0.5 \mathrm{~s}$ of an expansion or when the pressure was changing by more than $500 \mathrm{~Pa}(0.07 \mathrm{psi})$ between measurements was cut. An allowed range of $T_{1}$ temperatures was set for each run, and excursions outside of these ranges were cut. The span of the allowed temperature ranges varied from $0.35^{\circ} \mathrm{C}$ to $0.6^{\circ} \mathrm{C}$. Events were cut if the livetime immediately before the trigger was received was cut.

All non-fiducial cuts are applied equally to the events and livetime so that the cut efficiencies are assumed to be equal and cancel. Unlike the other non-fiducial cuts where the cut variable changes slowly over time, the pressure differential cut removes short periods of livetime within an expansion. Any delayed correlation between sudden pressure changes and bubble formation could result in a different cut efficiency for cuts of the livetime versus the number of triggers. A $10 \%$ uncertainty in the differential pressure cut efficiency is taken, resulting in a $5 \%$ uncertainty in the event rate.

All the data analyzed from STAR was handscanned in order to identify fiducial events. Events were cut if there was no video trigger or if no bubble was formed within the fiducial region of cam0 defined by Figure 4.15. The fiducial volume of $22.7 \pm 0.4 \mathrm{~mL}$ was measured using the cam0 images and the known inner and outer diameters of the pressure vessel.

To estimate the uncertainty due to geometric error, the MCNPX-Polimi geometry was resimulated with the density of materials in the line of sight between the source and active volume increased by the $1 \sigma$ uncertainties on their cross-sections and thicknesses. The uncertainty was set by the change in the integrated reoil rate between the simulations at 

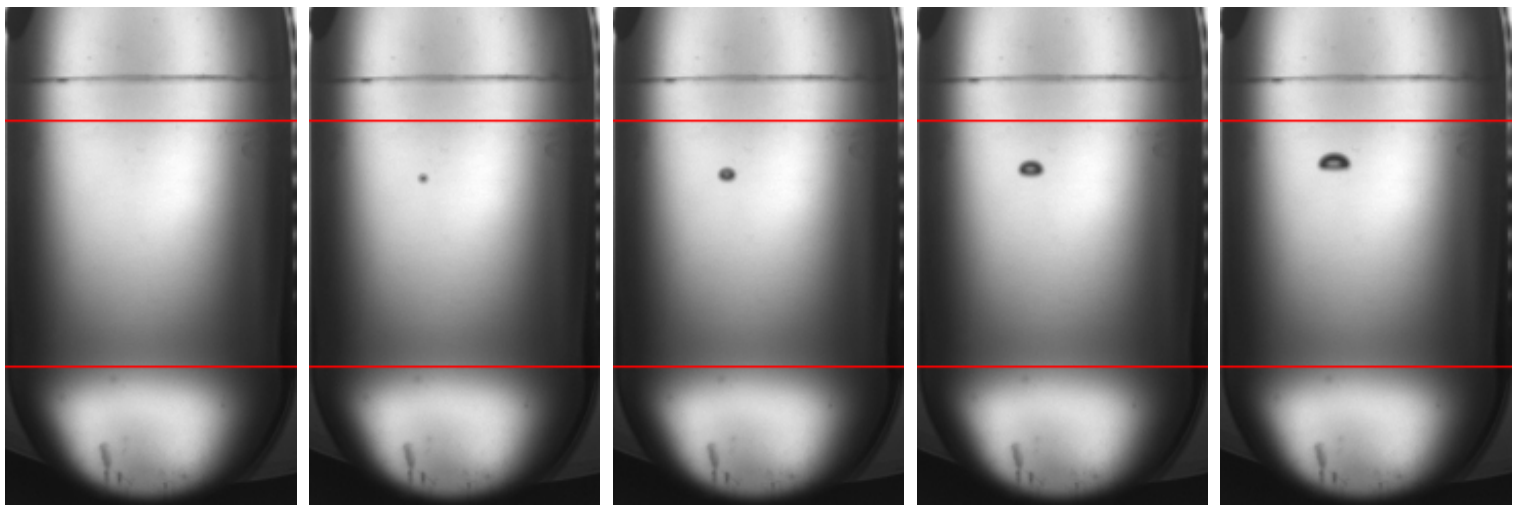

Figure 4.15: STAR events were handscanned to ensure they avoided the bottom hemisphere and the buffer interface. No wall fiducial cut was applied. Bubbles were accepted if they originated btween the red horizontal lines.

energies between $15 \mathrm{keV}$ and $45 \mathrm{keV}$. A further $1 \%$ uncertainty was added for the $10 \%$ of neutrons entering the active volume that scatter in materials out of the line of sight. A total uncertainty of $2.5 \%$ is obtained. This and other rate uncertainties are shown in Table 4.7 .

A small number of multiple bubble events, $\approx 1 \%$ of the total rate, were observed in STAR. An event was accepted as a multiple bubble event if multiple coincident bubbles were observed by the handscan with at least one bubble inside the fiducial volume. The total number of events in each runs is shown in Table 4.6.

\subsection{University of Chicago Bubble Chamber}

A simplified bubble chamber was constructed and operated by Juan Collar at the University of Chicago in 2012 in order to validate the results from the STAR bubble chamber. The temperature control and inner volume purity were much improved over that of STAR.

This chamber again used a $48 \mathrm{~mL}$ Chemglass pressure vessel and pneumatic controls to set the pressure. However, the chamber construction and data acquisition (DAQ) system are very different. The inner volume consisted of the pressure vessel, a baseplate, a steel bellows above the plate, a small plugged fill port, and a slow pressure transducer. Most of these 


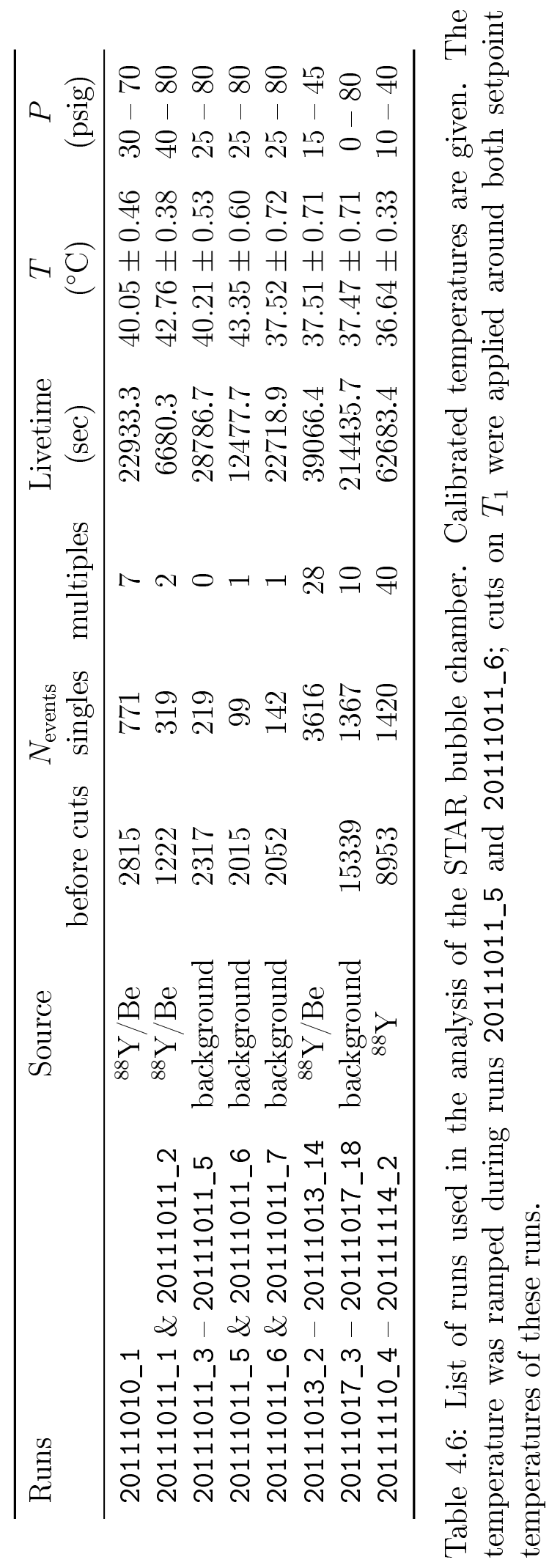




\begin{tabular}{lc}
\hline Cause & $\begin{array}{c}\text { Rate Uncertainty } \\
(\%)\end{array}$ \\
\hline Source strength & 3.9 \\
Simulation geometry & 2.5 \\
Fiducial volume & 1.8 \\
Cut efficiency & 5.0 \\
${ }^{19} \mathrm{~F}(n, \mathrm{el})$ cross-section at $152 \mathrm{keV}$ & 5.2 \\
\hline Total & 8.7 \\
\hline
\end{tabular}

Table 4.7: Global systematic uncertainties in the event rate of the STAR bubble chamber. components are visible in Figure 4.16. Only borosilicate glass, solid PTFE, and stainless steel were wetted. The bellows was connected to a pneumatic piston via a push rod. The chamber was compressed by opening an inlet valve between a high pressure reservoir and the piston. On decompression, the inlet valve was closed and an outlet valve to a small-bore leak valve was opened. The chamber pressure very slowly decreased over several minutes through the expansion. There was no software controlled expansion pressure setpoint, although the DAQ would force a compression after $210 \mathrm{~s}$ if no bubble formed. A 3 minute compression followed every expansion.

Volumetric graduations were marked on the pressure vessel every $5 \mathrm{~mL}$ before assembly. The vessel was filled with $15.5 \pm 0.5 \mathrm{~mL}$ of $\mathrm{CF}_{3} \mathrm{I}$ in April 2012, with $16.0 \pm 0.5 \mathrm{~mL}$ of $\mathrm{CF}_{3} \mathrm{I}$ in January 2013, and with $14.8 \pm 0.5 \mathrm{~mL}$ of $\mathrm{C}_{3} \mathrm{~F}_{8}$ in July 2013.

The pressure vessel was immersed in a small aluminum walled water bath with inner dimensions of approximately $5.4 \mathrm{~cm} \times 4.4 \mathrm{~cm} \times 12.4 \mathrm{~cm}$. A resistive thermometer (RTD) and two thermocouples below and above the pressure vessel monitored the temperature. The RTD was used to control a Huber brand recirculating heater/chiller that flowed water in a coil around the outside of the bath. Adhesive backed foam, and aluminized bubble pack insulation was wrapped around the water bath. The temperature measured by the three thermometers rarely differed by more than $0.2^{\circ} \mathrm{C}$ from the water bath setpoint temperature 
at any point during a run. The setpoint temperature, either $37.0^{\circ} \mathrm{C}, 39.0^{\circ} \mathrm{C}$, or $14.0^{\circ} \mathrm{C}$ is used as the $\mathrm{CF}_{3} \mathrm{I}$ temperature in the analysis with a $\pm 0.3^{\circ} \mathrm{C}$ uncertainty. The slow pressure cycling of the bubble chamber and the presence of a thermally coupled water bath reduced temperature uncertainties due to adiabatic cooling of the $\mathrm{CF}_{3} \mathrm{I}$. No adiabatic cooling correction is applied.

After an initial unsuccessful fill using the same $\mathrm{CO}_{2}$ contaminated $\mathrm{CF}_{3} \mathrm{I}$ used in STAR, the chamber was filled with $\mathrm{CF}_{3}$ I that had been purified through a molecular sieve by Hugh Lippincott at Fermilab to remove carbon dioxide and other contaminants. The background event rate was much lower than that of the STAR bubble chamber and no emulsions or discoloration were observed during running. Black cloth and sheeting was wrapped around the water bath and cameras to prevent any white light leaking into the bubble chamber and causing discoloration. The reduction in light leakage allowed high purity water to be used as the buffer fluid. $5 \mathrm{mM} \mathrm{Na} \mathrm{SO}_{3}$ solution was used as a buffer fluid for runs in January 2013 in order to test its effects on nucleation efficiency. No difference was observed in the nucleation efficiency.

Runs filled with $\mathrm{C}_{3} \mathrm{~F}_{8}$ in July 2013 were prematurely stopped due to excessive wall boiling. The pressure vessel did not have a hydrophobic coating, and thus the contact angle hysteresis described in Section 2.2 caused sporatic boiling. Far less boiling occured at the walls in early runs as the ${ }^{88} \mathrm{Y} /$ Be data was being taken. Planned background runs, in addition to the runs that were obtained, were curtailed due to excessive spontaneous boiling.

Two cameras were used to detect bubbles and trigger the DAQ. Images were captured every $\approx 30 \mathrm{~ms}$. A sum of the image pixel values across one dimension was performed and the sums compared to the previous frame. Any differences above the trigger threshold initiated a compression. The $\mathrm{CF}_{3} \mathrm{I}$ was illuminated with $850 \mathrm{~nm}$ infrared LEDs shining from the 


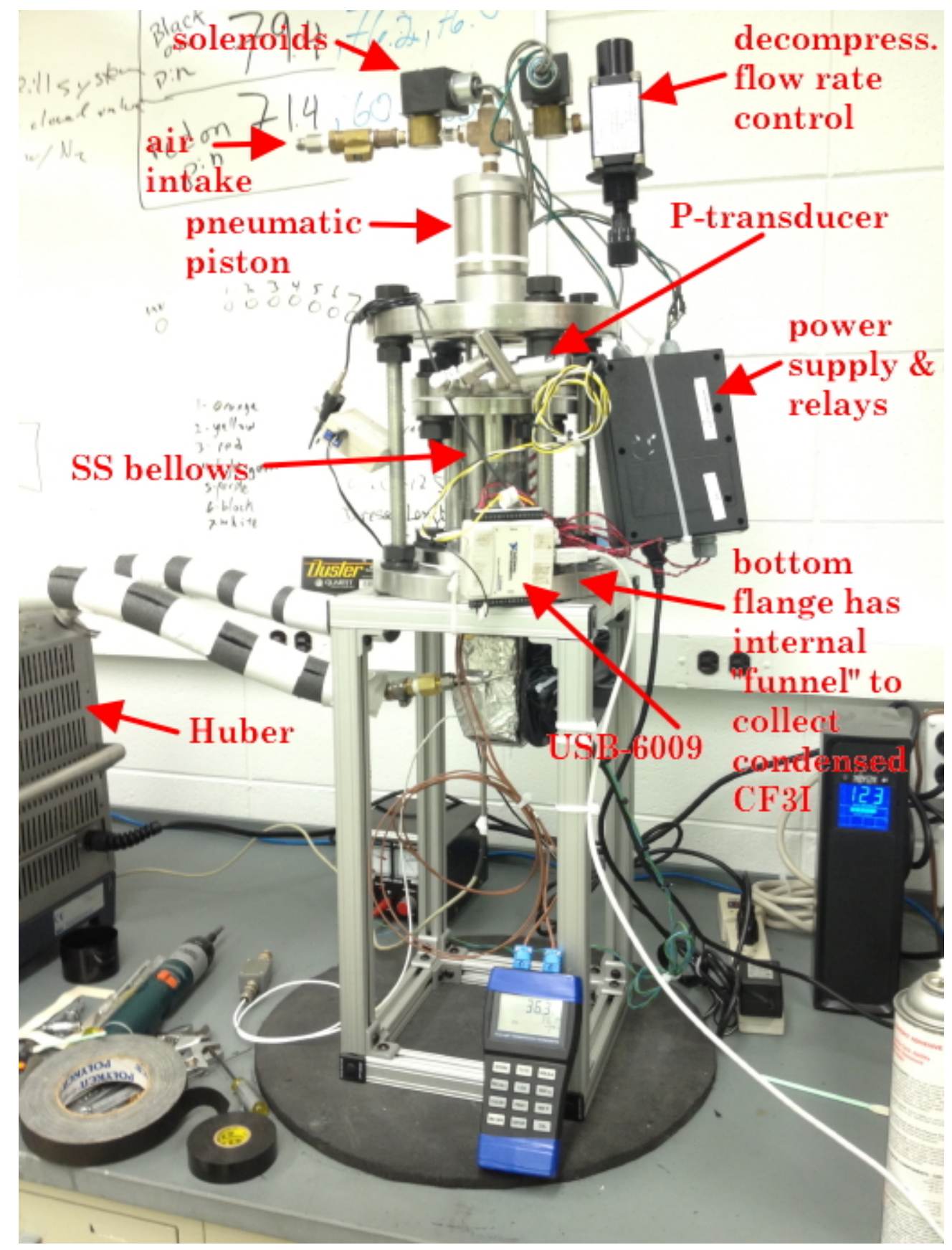

Figure 4.16: The University of Chicago bubble chamber with components labeled. Green components are stainless steel and yellow components are silica. 


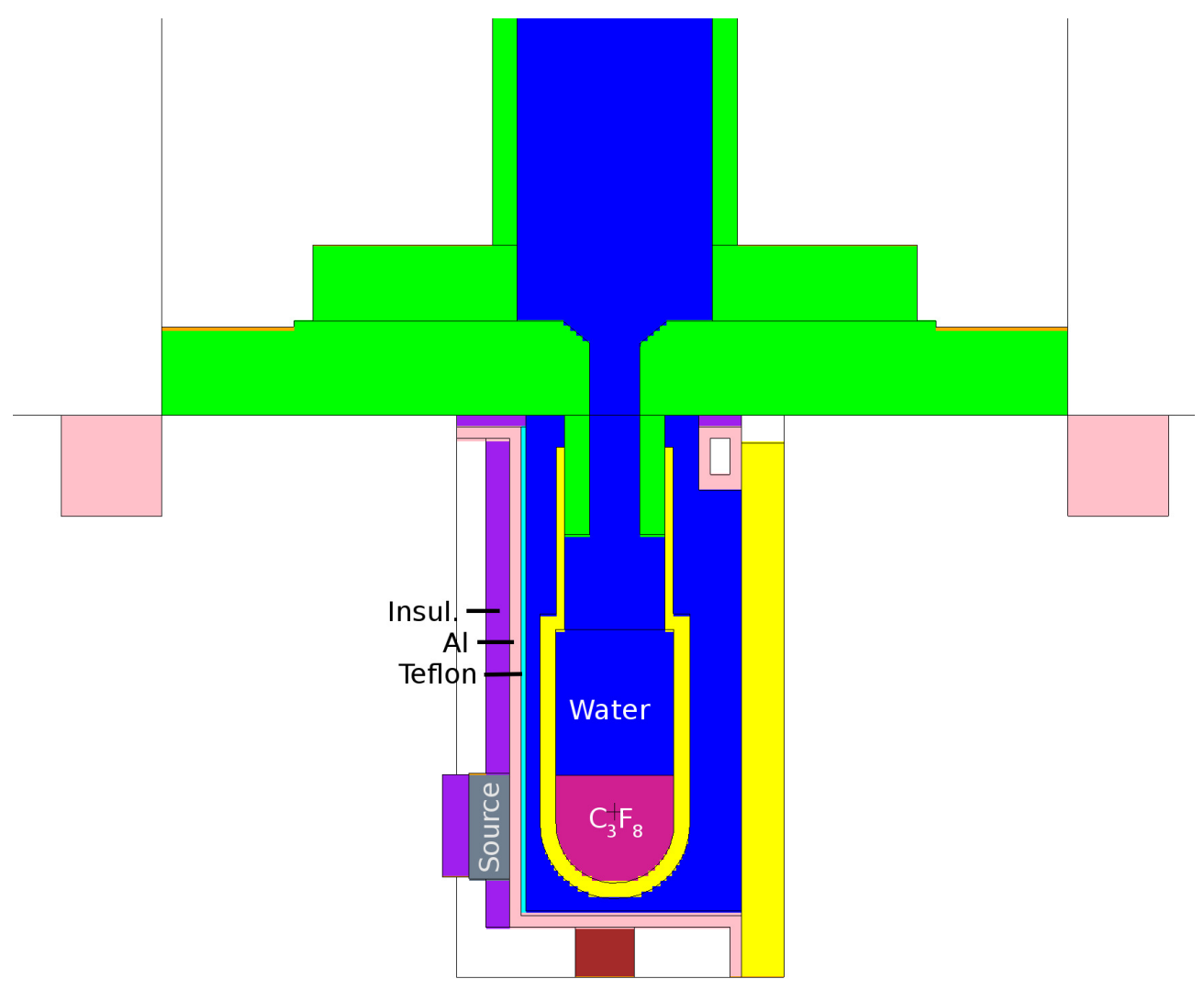

Figure 4.17: A cross-section of the MCNPX-Polimi geometry used to simulate the University of Chicago bubble chamber. 


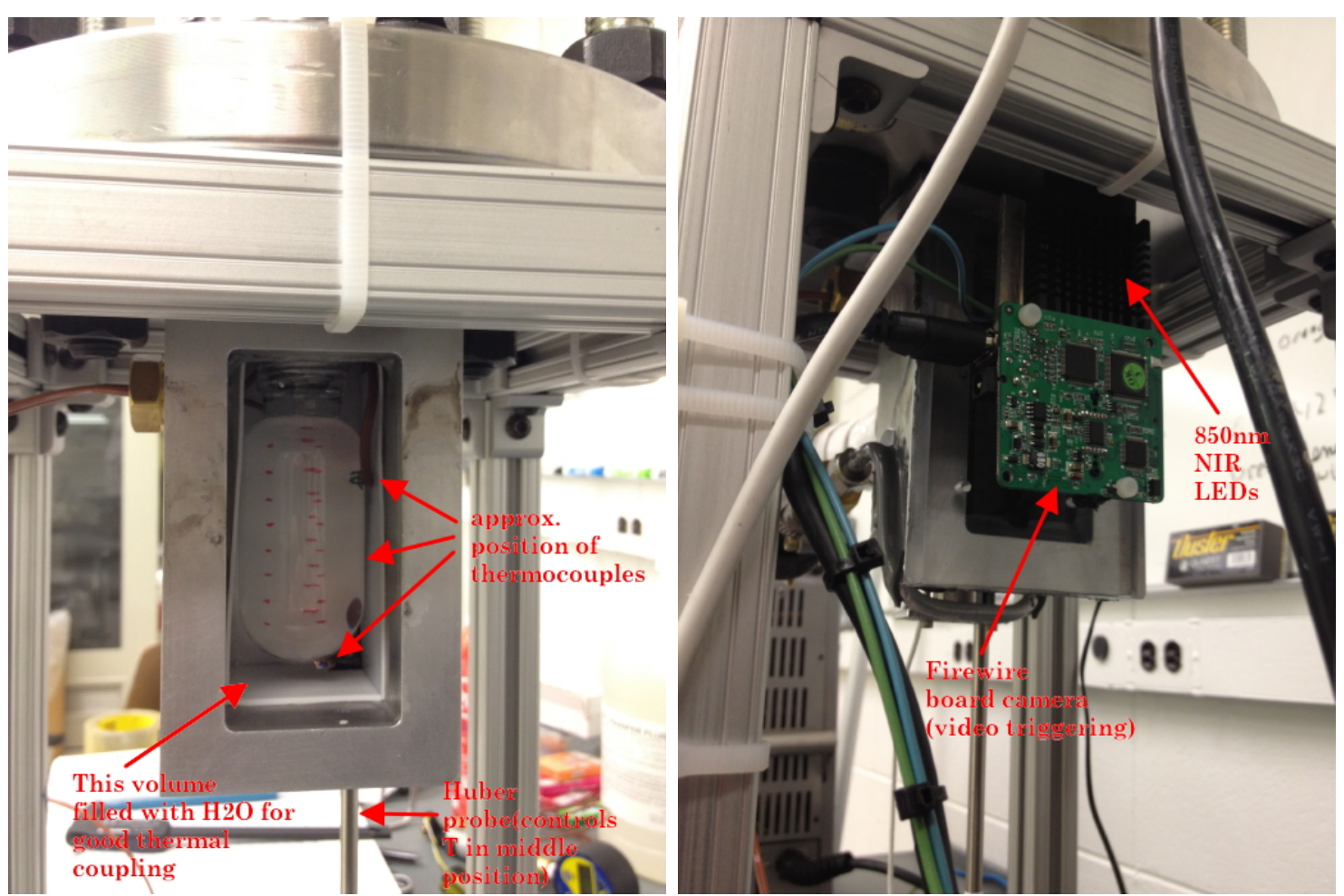

Figure 4.18: The pressure vessel and water bath of the University of Chicago Bubble chamber with acrylic window and cameras removed (left) and in place (right).

front. PTFE reflectors were placed behind the pressure vessel along the walls of the water bath.

${ }^{88} \mathrm{Y} /$ Be sources 1560-56 and 1637-69 in configuration 2, and source 1671-7 in configuration 1 (see Figure 4.2 and Table 4.2 were placed flush against the outside wall of the water bath opposite the cameras as shown in Figure 4.19. The position was reproducible to within $2 \mathrm{~mm}$ in the plane of the face of the source. $1.39 \mathrm{~cm}$ of material (aluminum, PTFE, water, and borosilicate glass) separated the source from the $\mathrm{CF}_{3} \mathrm{I}$.

The DAQ did not record images. The DAQ did generate a 1 sample per second history of the inner pressure and a record of the time, expansion time, and pressure at each trigger. A separate log of the temperature was made. Fiducial cuts could not be made to the data and event classes that were not constant in time, such as 'collar' and 'wall' events, could 


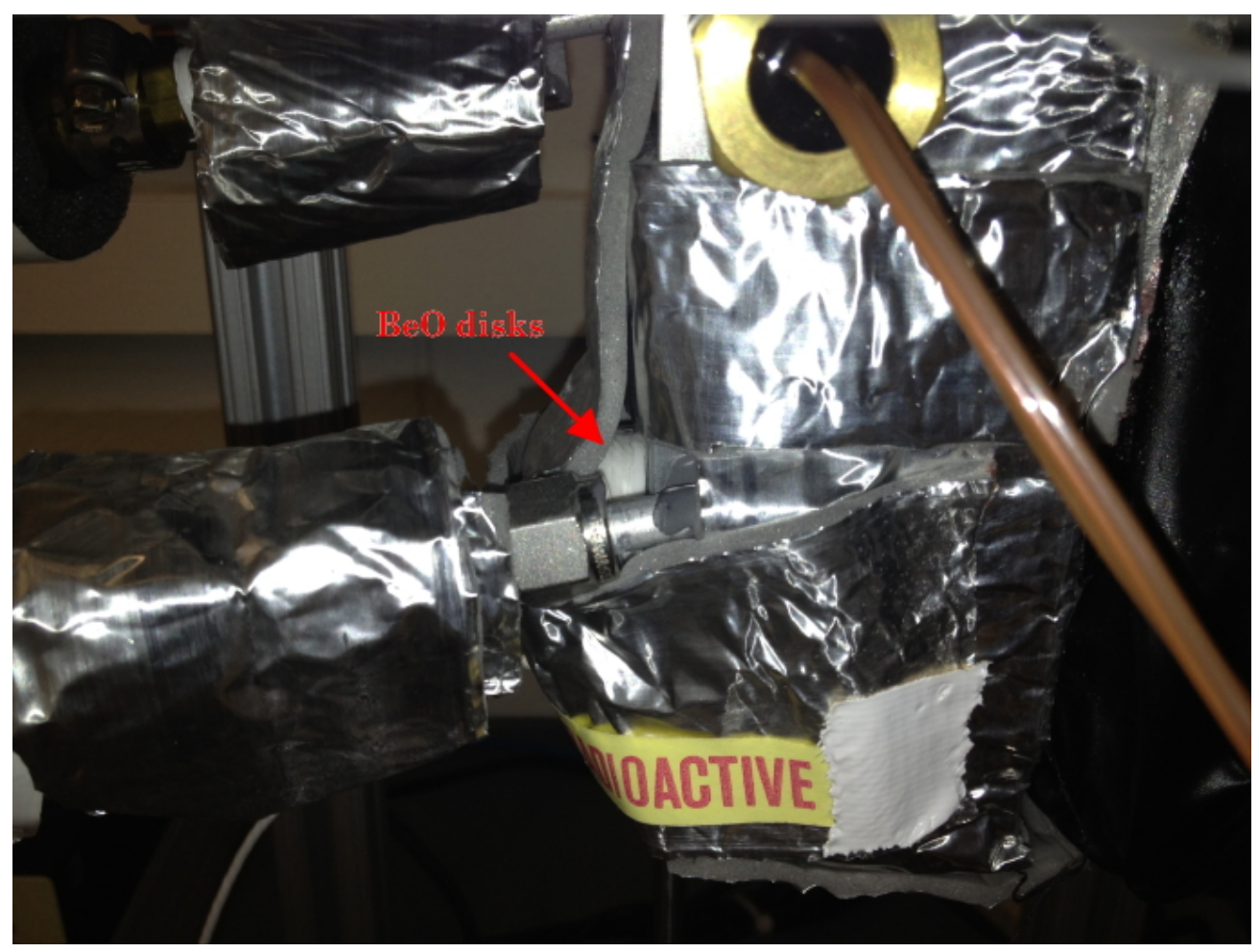

Figure 4.19: The University of Chicago bubble chamber with the ${ }^{88} \mathrm{Y} /$ Be source and insulation in place. The thermocouples enter the water bath at the port on the top right of the picture.

not be cut or background subtracted.

Both background runs, with no source in place, and ${ }^{88} \mathrm{Y}$-only runs, with the BeO replaced by PTFE, were taken. Only a slight sensitivity to the ${ }^{88} \mathrm{Y}$ photons was observed at the lowest measured threshold. Runs using an ${ }^{241} \mathrm{Am} /$ Be neutron source were also performed but not analyzed here.

\subsubsection{Analysis}

Due to the simplicity of the University of Chicago Bubble chamber DAQ, few cuts were applied to its data. Events and livetime were cut during periods of high bubble nucleation rate as shown in Figure 4.20 or if the expansion time exceeded the timeout time of 210 seconds. High rate periods were caused by the existence of nucleation sites, often at the $\mathrm{CF}_{3} \mathrm{I}$-water- 


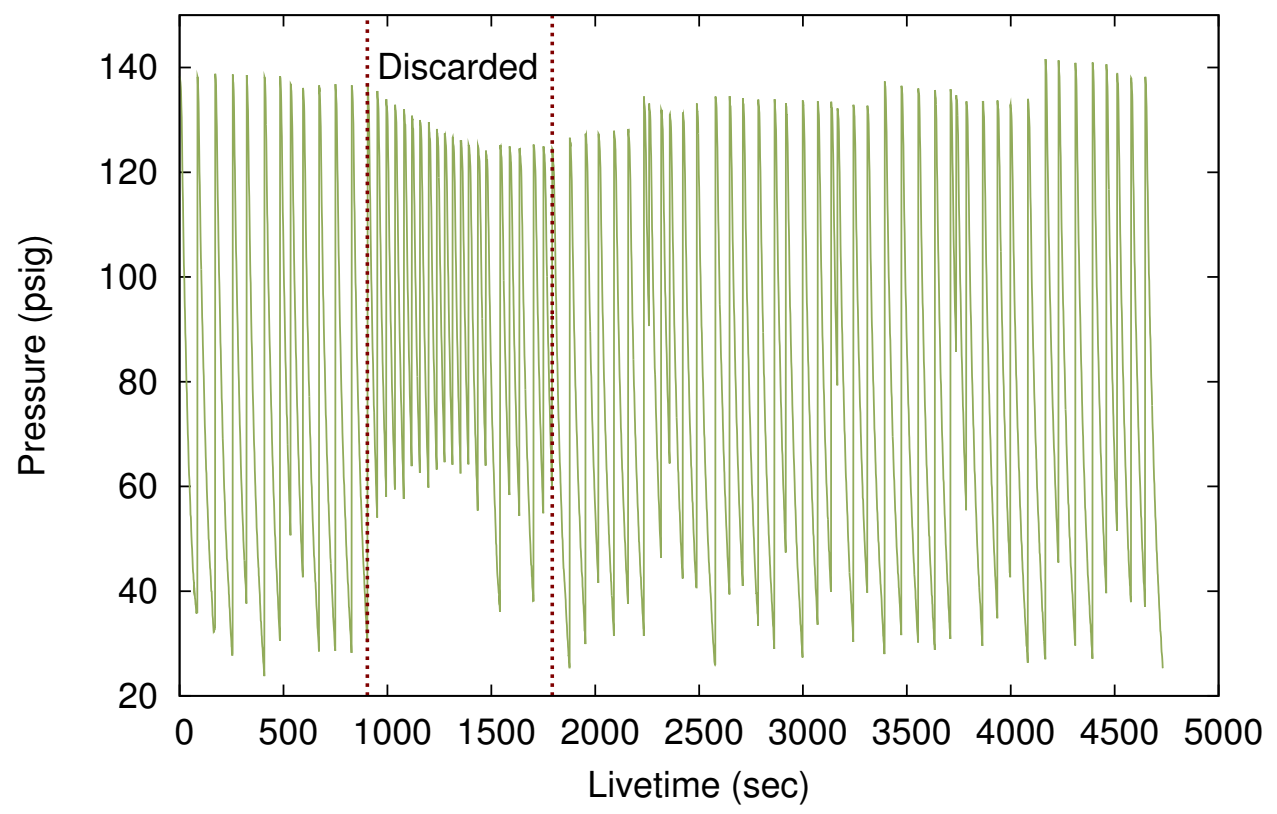

Figure 4.20: Pressure and event history of run YBe_37C_2 of the University of Chicago bubble chamber showing the discarded high-rate periods. Every local minimum in the pressure trace is an event.

wall triple interface, that nucleated at a high pressure. From live observations of the start and end of each run by Juan Collar, the rate of wall and surface events was found to be negligible outside of these high-rate periods.

As with the STAR bubble chamber data, the pressure recorded by the history file was used to determine the livetime binned in $1.7 \mathrm{kPa}(0.25 \mathrm{psi})$ wide steps. The history file was also used to extrapolate the pressure at the trigger, as the pressure often rose before being recorded in the trigger file. A $1 \%$ livetime uncertainty is added due to these extrapolations. The number of counts and livetime were summed for each bin and run. The total number of events and livetime below $580 \mathrm{kPa}(80 \mathrm{psig})$ are shown in Table 4.8 .

Most event rate uncertainties are given in Table 4.9. An additional systematic uncertainty of $10 \%$ of the background rate is also applied as the rate of spontaneous nucleations may have varied outside of the high-rate periods. When calculating the Seitz threshold, a 


\begin{tabular}{lccc}
\hline Runs & Start Date & $N_{\text {events }}$ & $\begin{array}{c}\text { Livetime } \\
\text { (sec) }\end{array}$ \\
\hline YBe_37C_1 & 6-Apr-2012 & 477 & 75626.9 \\
Y_37C_1 & 9-Apr-2012 & 165 & 40491.2 \\
Y_37C_2 & 13-Apr-2012 & 309 & 132675.2 \\
YBe_37C_2 & 17-Apr-2012 & 819 & 96050.2 \\
YBe_39C_1 & 20-Apr-2012 & 827 & 76738.8 \\
Y_39C_1 & 23-Apr-2012 & 595 & 169114.6 \\
blank_37C_1 & 10-May-2012 & 147 & 83195.6 \\
blank_39C_1 & 12-May-2012 & 117 & 58883.5 \\
ambient_sulfite_39C_1 & 9-Jan-2013 & 60 & 15848.9 \\
ambient_sulfite_39C_2 & 9-Jan-2013 & 30 & 17170.8 \\
YBe_sulfite_39C_1 & 10-Jan-2013 & 133 & 13509.2 \\
YBe_sulfite_39C_2 & 10-Jan-2013 & 389 & 38262.6 \\
Y_sulfite_39C_1 & 11-Jan-2013 & 119 & 43821.3 \\
blank_14C_C3F8 & 11-Jul-2013 & 367 & 13481.6 \\
YBe_14C_C3F8 & 12-Jul-2013 & 577 & 12642.2 \\
Y_14C_C3F8 & 14-Jul-2013 & 297 & 8392.4 \\
Y_14C_C3F8_2 & 15-Jul-2013 & 383 & 9166.7 \\
Y_14C_C3F8_3 & 16-Jul-2013 & 57 & 593.7 \\
\hline
\end{tabular}

Table 4.8: List of runs used in the analysis of the University of Chicago bubble chamber. Cuts are applied to all event numbers and livetimes. All runs operated at pressures above 40 psia $(275 \mathrm{kPa})$. 


\begin{tabular}{lccc}
\hline Cause & \multicolumn{3}{c}{$\begin{array}{c}\text { Rate Uncertainty } \\
(\%)\end{array}$} \\
\hline Source strength & \multicolumn{3}{c}{8.7} \\
Target Mass & \multicolumn{3}{c}{3.2} \\
${ }^{19} \mathrm{~F}(n, \mathrm{el})$ cross-section at $152 \mathrm{keV}$ & \multicolumn{3}{c}{5.2} \\
Livetime & \multicolumn{3}{c}{1.0} \\
\hline Moderator & Geom. & $\sigma_{\mathrm{n}, \mathrm{el}}$ & \# of ${ }_{1}^{0} n$ affected \\
\cline { 2 - 5 } BeO & 0.5 & 2 & 38 \\
Water & 7 & 0.5 & 27 \\
Aluminum & 7 & 10 & 13.5 \\
Glass & 7 & 1.3 & 10.2 \\
Teflon & 5 & 2.65 & 4.8 \\
Other & 30 & 30 & 4.0 \\
Subtotal & \multicolumn{4}{c}{3.2} \\
\hline Total & \multicolumn{4}{c}{11.1} \\
\hline
\end{tabular}

Table 4.9: Global systematic uncertainties in the event rate of the University of Chicago bubble chamber. The neutron propagation uncertainties are given per material in the MCNPXPolimi geometry. The relative geometric and cross-section uncertainties for each material are added in quadrature, multiplied by the number of neutrons affected, then totaled in quadrature over all materials. This method overestimates the simulation uncertainty slightly. It adds an uncertainty for neutrons scattered away from the active volume but does not reduce the uncertainty from neutrons that are rescattered back into the active volume.

$\pm 0.2^{\circ} \mathrm{C}$ uncertainty in temperature is applied, as discussed, and a $2.7 \mathrm{kPa}(0.3 \mathrm{psi})$ uncertainty in the expansion pressure is applied. The uncertainties in the purity of the $\mathrm{CF}_{3} \mathrm{I}$ and $\mathrm{C}_{3} \mathrm{~F}_{8}$ are considered negligible.

\subsection{CYRTE Bubble Chamber}

The bubble chamber used for the COUPP Iodine Recoil Threshold Experiment (CIRTE) (Behnke et al. 2013) was moved from the Fermilab to the University of Chicago in February 2012 and renamed the COUPP Y/Be Recoil Threshold Experiment (CYRTE). The DAQ and pressure control systems for CYRTE are a scaled version of those used for PICO-2L. 
The chamber, shown in Figure 4.21, uses a $1.0 \mathrm{~cm}$ inner diameter $1 \mathrm{~mm}$ wall thickness synthetic silica pressure vessel designed to hold the full compression pressure of the chamber. The vessel is immersed in a polymethylmethacrylate (PMMA) walled recirculating water bath that replaced the bath used for CIRTE. The bath walls are positioned to minimize the amount of moderator between the active volume and a source placed on the front of the bath. A compound bellows contained in a steel cylinder above the pressure vessel separates the inner volume from the hydraulic volume. One or two cameras imaged the chamber from the side using illumination from a red LED array opposite the cameras. The light passed through two tissue paper diffusers separated by $2.5 \mathrm{~cm}$ of PMMA. The entire system was suspended from an aluminum frame.

Three RTDs at the outlet, center, and inlet of the bath, $T_{3}, T_{4}$, and $T_{5}$ respectively, and a temperature sensor in the hydraulic volume above the pressure vessel, $\mathrm{T}_{1}$, monitor the system. A heater in the upper hydraulic volume was controlled using $\mathrm{T}_{1}$ and set so as to minimize the thermal gradient vertically in the inner volume. The heater was used only during runs using $\mathrm{CF}_{3} \mathrm{I}$ where the operating temperature was above room temperature. Three pressure sensors were installed on the hydraulic cart, another measured the pressure outside the bellows, and one measured the inner volume pressure. All the sensors were calibrated before their installation for the previous CIRTE experiment to within $700 \mathrm{~Pa}$ $( \pm 0.1 \mathrm{psi})$. No drift in their inter-calibration was observed. The rating for the pressure transducers guarantees that pressure measurements are reproducable to within \pm 0.7 psi. As with other unbiased uncertainties defined by a bounded range of values in this thesis, $\pm 1 / \sqrt{12}$ of the full range was used as the $68 \%$ confidence interval, or \pm 0.4 psi. This is the standard deviation of a flat probability distribution over the bounded range. In the case of the gradient correction, where a potential bias exists, $\pm 1 / \sqrt{6}$ of the range was used.

An ultrasonic acoustic sensor was installed for later CYRTE runs that could have been 


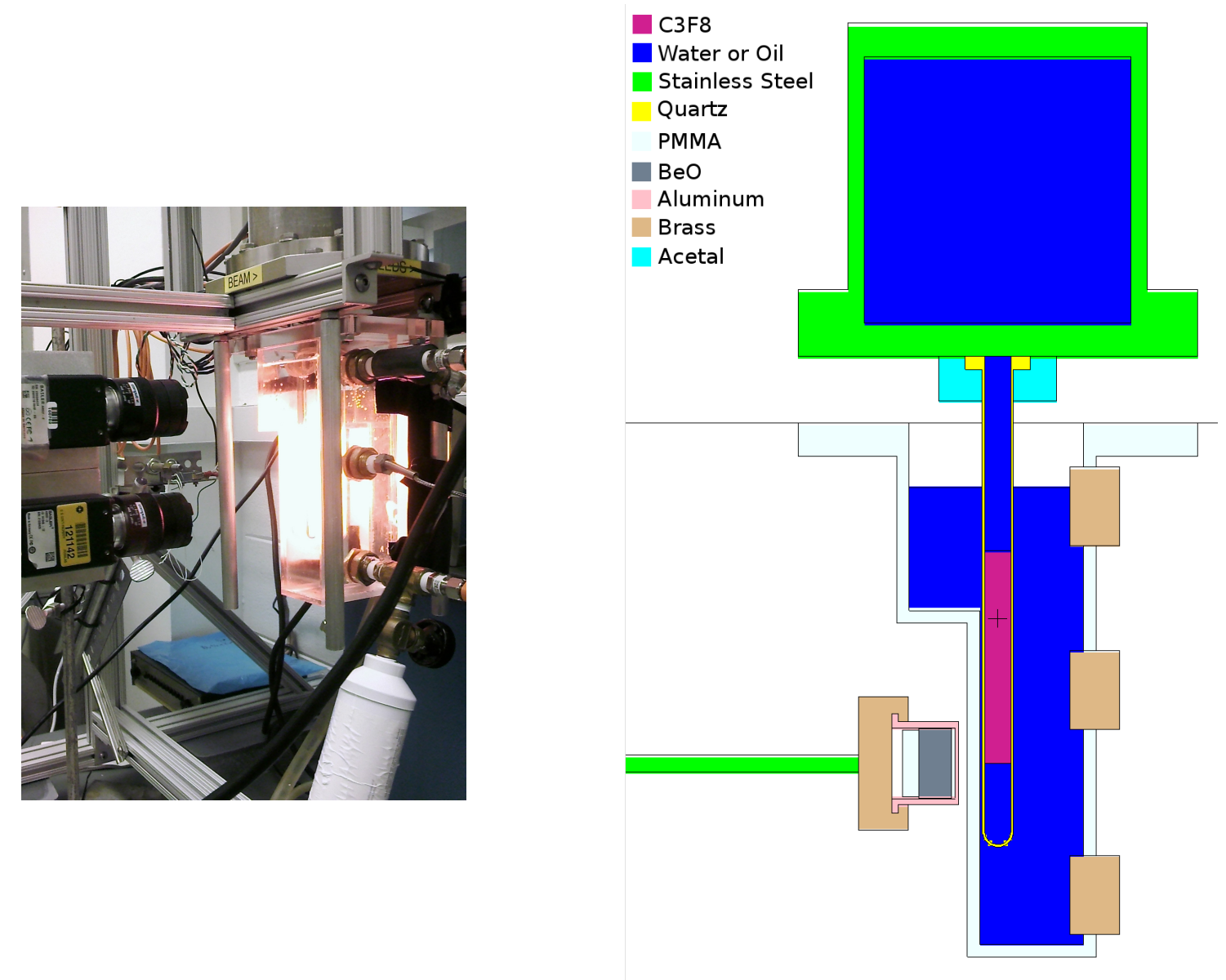

Figure 4.21: Picture and MCNP geometry cross-section of the CYRTE bubble chamber. The MCNP geometry shows the ${ }^{88} \mathrm{Y} /$ Be source fully extended with the June $2013 \mathrm{C}_{3} \mathrm{~F}_{8}$ fill.

used to measure the sound created by growing bubbles and to identify neutron and $\alpha$ initiated bubbles. The same acoustic sensor used for CIRTE was used for CYRTE. Significant ultrasonic noise was generated by cavitation in the outlet hose of the water bath and by the NESLAB recirculating heater/chiller located beside and behind the chamber. Rubber feet for the chiller and a metering clamp valve on the bath outlet were installed to minimize these sources of noise. A Dytran brand fast pressure transducer was also installed to monitor the inner volume. Neither sensor has been used in this analysis.

The analysis uses ${ }^{88} \mathrm{Y} /$ Be source data with $\mathrm{CF}_{3} \mathrm{I}$ from a fill in February $2013,{ }^{88} \mathrm{Y} / \mathrm{Be}$ 
source data with $\mathrm{C}_{3} \mathrm{~F}_{8}$ from a fill in June 2013, and ${ }^{124} \mathrm{Sb} /$ Be source data with $\mathrm{C}_{3} \mathrm{~F}_{8}$ from a fill in May 2014. The fill level for each run was determined by measuring the height of the active fluid in the pressure vessel and calculating the volume based on the known $1.00 \mathrm{~mm}$ inner diameter of the vessel. The February 2013, June 2013, and May 2014 fills contained $6.7 \pm 0.1 \mathrm{~mL}, 6.4 \pm 0.2 \mathrm{~mL}$, and $3.3 \pm 0.1 \mathrm{~mL}$ of target fluid respectively. Due to the restricted diameter of the pressure vessel with only one narrow flow path to the inner volume, large droplets of $\mathrm{C}_{3} \mathrm{~F}_{8}$ and water could not pass each other. During the June 2013 fill, $2.6 \mathrm{~mL}$ of water remained trapped below the active volume during the run, as seen in Figure 4.21 . CYRTE required a filling procedure reversed from that used with other COUPP and PICO bubble chambers. The active fluid was distilled into the chamber first, then water or, in the case of the $\mathrm{CF}_{3} \mathrm{I}$ fill, $5 \mathrm{mM} \mathrm{Na} \mathrm{SO}_{3}$ solution was forced into the vessel under pressure.

The $(\gamma, n)$ sources used with CYRTE were installed at the end of a pneumatic piston. The piston extended after a settling time of 15 to 25 seconds during the 2013 runs or 90 seconds during the May 2014 runs carrying the source toward the active volume. The source mover allowed the active volume to reach thermal equilibrium after expansion before bringing the source near and increasing the event rate. The $\mathrm{Y} /$ Be source was contained in an aluminum and brass source holder with a $1.3 \mathrm{~mm}$ aluminum window between the source and the active volume. The source holder slid and was supported on thin aluminum angle stock on its path towards and away from the active volume. A normally open spring switch was connected to the source holder with a string. When the source was in position, the spring switch closed. The switch position was recorded by the DAQ. This source position indicator failed itermittently during running by failing to indicate that the source was in place. 


\subsubsection{Temperature Calibrations}

During the first run of CYRTE in January 2013, the inner volume plumbing was modified to accommodate a $36 \mathrm{~cm}$ long Omega brand PR-21 RTD tipped stainless steel probe immersed in the active volume labeled $\mathrm{T}_{2}$. The probe was used to measure the $\mathrm{CF}_{3} \mathrm{I}$ temperature directly and calibrate the other sensors. The chamber was operable with the tip of the probe in the active volume, but it was a strong source of bubble nucleation, limiting the livetime. While the probe was in place, a series of temperature calibrations were performed.

To calibrate the temperature of the data taken for CIRTE in the $\pi^{-}$beam at Fermilab (Behnke et al. 2013), the bubble chamber was operated with the inner volume RTD and the same duty cycle as was used with CIRTE: a 35 second maximum expansion time and 25 second compression. The calibration was performed at both the CIRTE run temperature of $34.2^{\circ} \mathrm{C}$ and at $23^{\circ} \mathrm{C}$. The inner volume temperature, $T_{2}$, was calibrated against the average of $T_{3}, T_{4}$, and $T_{5}$ and an offset of $-0.2^{\circ} \mathrm{C}$ was applied. The calibrated temperature and the variation in the four temperature sensors over all CYRTE runs is shown in Figure 4.22 and an uncertainty of $0.2^{\circ} \mathrm{C}$ was adopted in the calibration. This calibration is only valid for the exact temperature, 25 second compression time, and 30-35 second expansion time used with CYRTE.

During each expansion, the inner volume temperature is reduced by up to $1^{\circ} \mathrm{C}$ by the adiabatic expansion of $\mathrm{C}_{3} \mathrm{~F}_{8}$ or $\mathrm{CF}_{3} \mathrm{I}$. This temperature swing was observed using the inner volume temperature probe, and used to determine the time required to equilibrate the inner volume temperature after expansion. As seen in Figure 4.23, the thin glass walls of the inner vessel allow the inner volume to equilibrate rapidly with the water bath, with the temperature difference decaying with a decay constant of $54 \mathrm{~s}$. Thermal mass from the pressure vessel walls, the inner vessel RTD, and the buffer fluid reduce the temperature jump from the $0.92^{\circ} \mathrm{C}$ calculated from the fluid properties of $\mathrm{CF}_{3} \mathrm{I}$. After accounting for the 


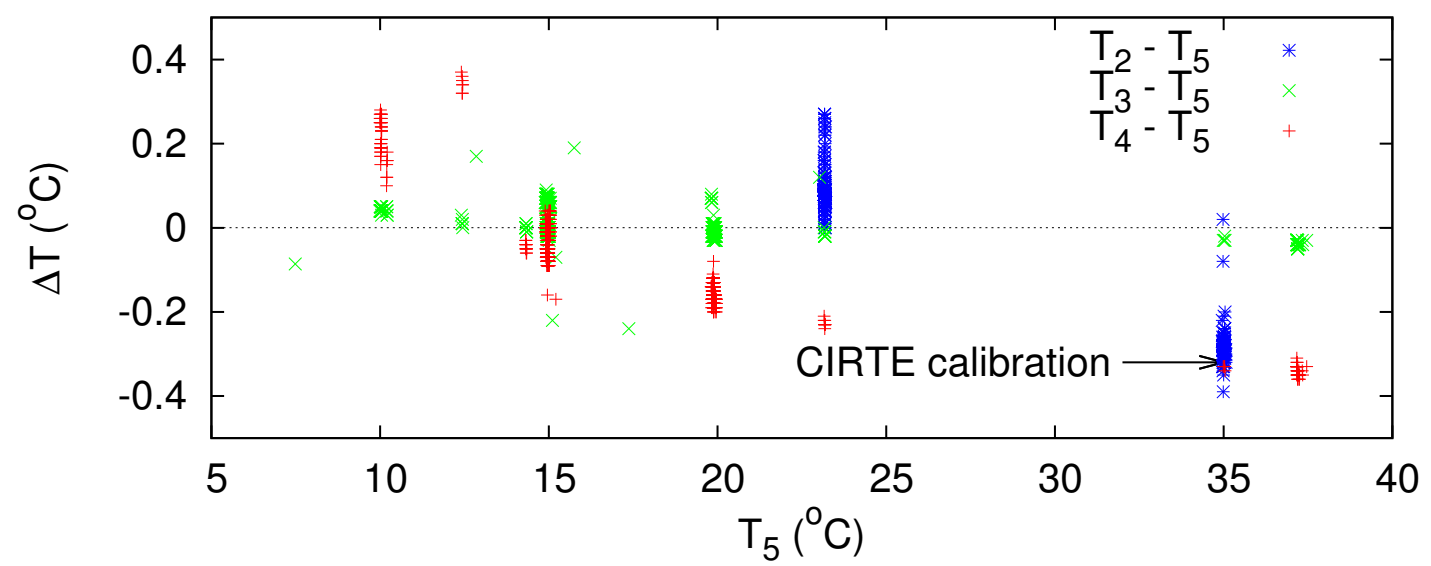

Figure 4.22: Difference between the three measured water bath temperatures and the inner volume temperature for all runs of CYRTE. $T_{2}$ is the inner volume temperature, $T_{3}$ is the bath outlet temperature, $T_{4}$ is measured at the center of the bath, and $T_{5}$ is the bath inlet temperature. The $T_{2}$ values shown are the measured at the time of a trigger. The CYRTE calibration point at $34^{\circ} \mathrm{C}$ is labeled.

response time of the inner vessel RTD, the temperature jump on expansion from $275 \mathrm{kPa}$ (40 psia) to $1540 \mathrm{kPa}(223 \mathrm{psia})$ is $0.55^{\circ} \mathrm{C}$. The bellows temperature, $T_{1}$ also varied during each bubble chamber cycle. The $T_{1}$ sensor is located near the hose connection between the bellows assembly and the hydraulic cart. On each expansion, warm hydraulic fluid was pushed passed the sensor while on compression, cold fluid was pushed into the bellow assembly.

As the runs conditions used for CYRTE $\mathrm{CF}_{3} \mathrm{I}$ data were similar to those used for CIRTE, the same temperature calibration is used with the temperature uncertainty increased to $0.3^{\circ} \mathrm{C}$ to account for variations in the pressure duty cycle. For $\mathrm{C}_{3} \mathrm{~F}_{8}$ operation, the deadtime at the start of an expansion was either $25 \mathrm{~s}$ or $90 \mathrm{~s}$. For a typical expansion from 210 psia $(1450 \mathrm{kPa})$ to $40 \mathrm{psia}(276 \mathrm{kPa})$ at $15^{\circ} \mathrm{C}$, the $\mathrm{C}_{3} \mathrm{~F}_{8}$ is cooled by $0.84^{\circ} \mathrm{C}$. The $90 \mathrm{~s}$ deadtime after expansion allowed the active volume to reach thermal equilibrium with the water bath to within a temperature difference of $0.16^{\circ} \mathrm{C}$. With a $25 \mathrm{~s}$ deadtime, $0.53^{\circ} \mathrm{C}$ of the $0.92^{\circ} \mathrm{C}$ of expansion cooling remains. As the water bath temperature sensor, $T_{4}$, was recessed 


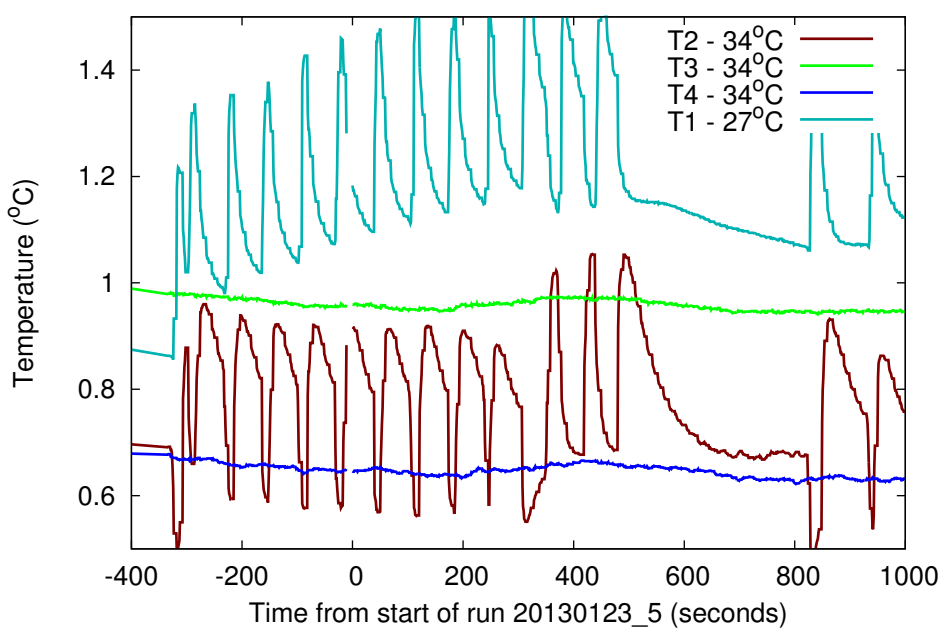

Figure 4.23: CYRTE temperatures vs. time. For each expansion and compression, $T_{2}$ decreases and increases by approximately $0.3^{\circ} \mathrm{C}$. The inner volume RTD equilibrates with the $\mathrm{CF}_{3} \mathrm{I}$ with a time constant of 3 seconds. During both the long compression at 600 seconds and long expansion at 300 seconds, the $\mathrm{CF}_{3} \mathrm{I}$ temperature is observed to approach equilibrium with a time constant of 54 seconds. $T_{1}$ is the temperature measured in the hydraulic fluid of the bellow assembly.

from the bulk of the water bath, its coupling to the bath temperature is imperfect and it does not track the other two bath temperatures. For CYRTE $\mathrm{C}_{3} \mathrm{~F}_{8}$ data with the long expansion deadtime, the average of the water bath inlet and outlet temperatures, $T_{3}$ and $T_{5}$, is adopted as the equilibrium inner volume temperature, with an uncertainty of $0.16^{\circ} \mathrm{C}$. With a short expansion deadtime, $0.2^{\circ} \mathrm{C}$ is subtracted from the measured temperature and the temperature uncertainty is increased to $0.3^{\circ} \mathrm{C}$

\subsubsection{Analysis}

Due to poor illumination at the bottom of the inner vessel, automatic bubble finding was not reliable. Events were manually examined to validate the position and number of bubbles for each event. A significant event rate at the active fluid/buffer fluid interface was cut by requiring bubbles to be formed more than $2 \mathrm{~mm}$ away from the interface. Every analyzed 


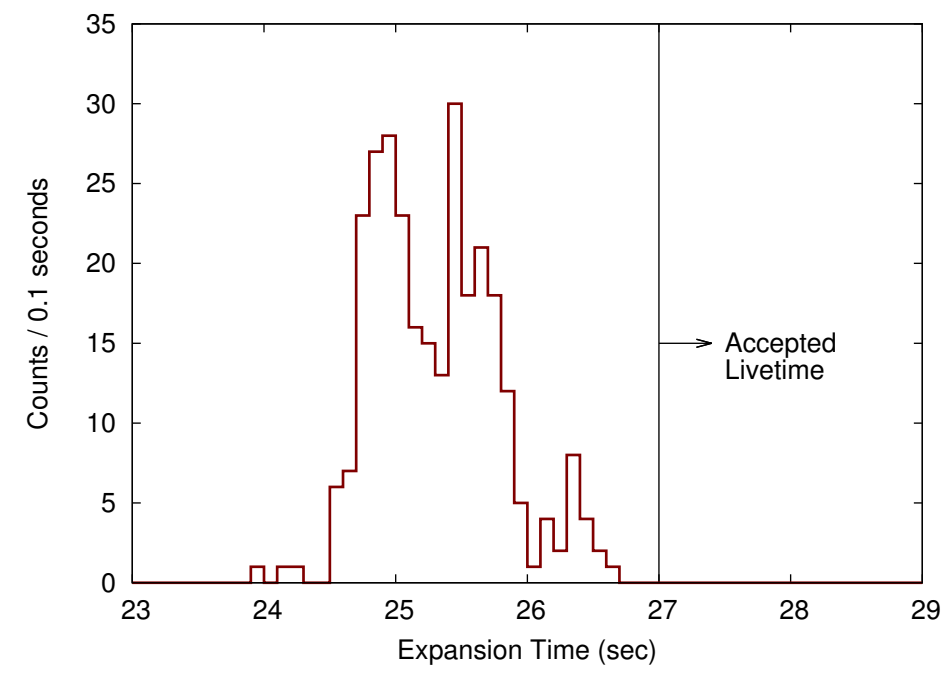

Figure 4.24: Distribution of source extension times and the selected livetime cut for CYRTE $\mathrm{CF}_{3} \mathrm{I}$ data.

event was handscanned to determine the number of bubbles in the event and whether the bubbles formed in the fiducial region.

Livetime for CYRTE was counted only when both the expansion deadtime had expired and the source (if applicable) was in place. As the source mover operated reliably but the source position indicator in the DAQ did not, an expansion time cut, set from the start of the expansion, was used to determine when the source was in place and livetime could be counted. The livetime cut was set by finding the maximum time required for the source mover to extend, as measured by the source position indicator when it was operable. Figure 4.24 shows a distribution of source extension times and the adopted livetime cut of 27 seconds for the CYRTE $\mathrm{CF}_{3} \mathrm{I}$ data.

CYRTE was operated without ramping temperature or pressure. Rather, an expansion pressure was randomly selected from a list of possible pressures before each expansion. Pressures were selected so as to explore Seitz thresholds between $2 \mathrm{keV}$ and $40 \mathrm{keV}$.

The livetimes and number of counts for all analyzed runs of CYRTE are shown in Tables 4.10 and 4.11 . 


\begin{tabular}{|c|c|c|c|c|c|c|c|}
\hline \multicolumn{2}{|l|}{$\mathrm{CF}_{3} \mathrm{I}$ Background } & \multicolumn{4}{|c|}{$\begin{array}{l}\text { Runs 20130218_2 } \\
\& \text { 20130227_3-20130312_0 }\end{array}$} & \multicolumn{2}{|c|}{$36.9 \pm 0.2^{\circ} \mathrm{C}$} \\
\hline Pressure (psia) & 26.3 & 32.0 & 36.4 & 40.0 & 42.7 & 45.1 & 47.1 \\
\hline Threshold (keV) & $8.52(29)$ & $10.5(4)$ & $12.6(5)$ & $14.8(6)$ & $16.7(8)$ & $18.8(9)$ & $20.8(10)$ \\
\hline Livetime (sec) & 482 & 823 & 2072 & 3285 & 4413 & 3888 & 4583 \\
\hline Singles & 8 & 15 & 13 & 10 & 14 & 9 & 2 \\
\hline Multiples & 0 & 0 & 0 & 0 & 0 & 0 & 1 \\
\hline \multicolumn{2}{|c|}{$\mathrm{CF}_{3} \mathrm{I} 99.93 \mathrm{mCi}^{88} \mathrm{Y} / \mathrm{Be}$} & \multicolumn{4}{|c|}{ Runs 20130318_2 - 20130321_1 } & \multicolumn{2}{|c|}{$36.9 \pm 0.2^{\circ} \mathrm{C}$} \\
\hline Pressure (psia) & 26.3 & 32.0 & 36.4 & 40.0 & 42.7 & 45.1 & 47.1 \\
\hline Threshold (keV) & $8.52(29)$ & $10.5(4)$ & $12.6(5)$ & $14.8(6)$ & $16.7(8)$ & $18.8(9)$ & $20.8(10)$ \\
\hline Livetime (sec) & 788 & 880 & 2067 & 5788 & 3091 & 5732 & 6266 \\
\hline Singles & 1 & 3 & 2 & 9 & 11 & 7 & 11 \\
\hline \multicolumn{2}{|l|}{$\mathrm{C}_{3} \mathrm{~F}_{8}$ Background } & \multicolumn{4}{|c|}{$\begin{array}{l}\text { Runs 20130621_2 } \\
\& \text { 20130715_0 - 20130717_0 }\end{array}$} & \multirow{5}{*}{\multicolumn{2}{|c|}{$14.8 \pm 0.4^{\circ} \mathrm{C}$}} \\
\hline Pressure (psia) & 38.9 & 47.0 & 55.5 & 61.1 & 66.9 & & \\
\hline Threshold (keV) & $4.14(29)$ & $6.2(5)$ & $10.4(10)$ & 15.8(17) & $26.5(35)$ & & \\
\hline Livetime (sec) & 12549 & 12738 & 13024 & 26773 & 42660 & & \\
\hline Singles & 14 & 17 & 11 & 18 & 16 & & \\
\hline \multicolumn{2}{|c|}{$\mathrm{C}_{3} \mathrm{~F}_{8} 49.34 \mathrm{mCi}^{88} \mathrm{Y}$} & \multicolumn{4}{|c|}{ Runs 20130702_1 - 20130709_0 } & \multicolumn{2}{|c|}{$14.8 \pm 0.4^{\circ} \mathrm{C}$} \\
\hline Pressure (psia) & 32.2 & 38.9 & 47.0 & 55.5 & 61.1 & 66.9 & \\
\hline Threshold (keV) & $3.09(20)$ & 4.14(29) & $6.42(5)$ & 10.4(10) & $15.8(17)$ & $26.5(35)$ & \\
\hline Livetime (sec) & 3.84 & 8125 & 9850 & 9438 & 19467 & 27898 & \\
\hline Singles & 2 & 76 & 18 & 7 & 8 & 8 & \\
\hline Multiples & 0 & 1 & 0 & 0 & 0 & 0 & \\
\hline \multicolumn{2}{|c|}{$\mathrm{C}_{3} \mathrm{~F}_{8} 54.32 \mathrm{mCi}^{88} \mathrm{Y} / \mathrm{Be}$} & \multicolumn{4}{|c|}{ Runs 20130620_6 - 20130621_1 } & \multirow{5}{*}{\multicolumn{2}{|c|}{$14.8 \pm 0.4^{\circ} \mathrm{C}$}} \\
\hline Pressure (psia) & 38.9 & 47.0 & 55.5 & 61.1 & 66.9 & & \\
\hline Threshold (keV) & $4.14(29)$ & $6.2(5)$ & $10.4(10)$ & $15.8(17)$ & $26.5(35)$ & & \\
\hline Livetime (sec) & 153.7 & 189.7 & 205.1 & 472.9 & 781.0 & & \\
\hline Singles & 34 & 30 & 29 & 25 & 4 & & \\
\hline \multicolumn{2}{|c|}{$\mathrm{C}_{3} \mathrm{~F}_{8} 48.07 \mathrm{mCi}^{88} \mathrm{Y}$} & \multicolumn{4}{|c|}{ Runs 20130709_1 - 20130709_2 } & \multirow{5}{*}{\multicolumn{2}{|c|}{$19.7 \pm 0.3^{\circ} \mathrm{C}$}} \\
\hline Pressure (psia) & 41.4 & 47.2 & 51.5 & 57.6 & 65.1 & & \\
\hline Threshold (keV) & $2.09(11)$ & $2.63(15)$ & $3.17(19)$ & $4.24(28)$ & $6.4(5)$ & & \\
\hline Livetime (sec) & 23.5 & 72.7 & 251.2 & 778.5 & 427.8 & & \\
\hline Singles & 18 & 13 & 28 & 14 & 1 & & \\
\hline
\end{tabular}

Table 4.10: List of runs from 2013 used in the analysis of the CYRTE bubble chamber. The threshold uncertainties only include the uncertainties propagated from the temperature and pressure uncertainties. Continued on next page. 


\begin{tabular}{|c|c|c|c|c|c|}
\hline \multicolumn{2}{|l|}{$\mathrm{C}_{3} \mathrm{~F}_{8}$ Background } & \multicolumn{3}{|c|}{ Runs 20130709_1 - 20130709_2 } & $19.7 \pm 0.3^{\circ} \mathrm{C}$ \\
\hline Pressure (psia) & 47.2 & 51.5 & 57.6 & 65.1 & \\
\hline Threshold (keV) & $2.63(15)$ & $3.17(19)$ & $4.24(28)$ & $6.4(5)$ & \\
\hline Livetime (sec) & 28.2 & 194 & 905 & 416 & \\
\hline Singles & 0 & 1 & 2 & 0 & \\
\hline
\end{tabular}

Table 4.10: Continued.

\subsubsection{Gamma Sensitivity}

When filled with $\mathrm{C}_{3} \mathrm{~F}_{8}$, CYRTE was very sensitive to electron recoils. Figure 4.25 shows the measured count rates in the presence of both photon and neutron radiation from ${ }^{124} \mathrm{Sb}$ and $\mathrm{Sb} /$ Be sources. CYRTE's electron recoil sensitivity is 100 (at $2 \mathrm{keV}$ ) to 1,000,000 (at $4 \mathrm{keV}$ ) times greater than the sensitivity measured by other bubble chambers shown in Figure 4.26 . Given that the sensitivity is only observed with CYRTE, a contaminant must caused this sensitivity.

Daniel Baxter and C. Eric Dahl 2015 have thoroughly investigated the properties and potential causes of this sensitivity. In addition to measurements with ${ }^{88} \mathrm{Y}$ and ${ }^{124} \mathrm{Sb}$ sources, the anomalous sensitivity has been confirmed in CYRTE using both ${ }^{137} \mathrm{Cs},{ }^{241} \mathrm{Am}$, and ${ }^{207} \mathrm{Bi}$ $\gamma$ sources with a range of photon energies and in the presence of various attenuators. CYRTE is most sensitive to interactions with low-energy $\gamma$-rays, below $100 \mathrm{keV}$. The sensitivity is also observed to vary over time and between fills of the bubble chamber.

In order to generate a sufficiently large stopping power to nucleate a bubble, photon interactions may produce an Auger cascade. A Compton scattering or photoabsorbtion process may remove an electron from the inner shells of an atom. Remaining electrons in the atom may decay into the open inner shell. Every time an electron transitions to a lower energy state, its previous energy state become available. Electrons cascade until the ion's electron configuration is in its ground state. The energy released by each transition may be reemitted as a photon, or donated to an ejected valence electron. While no single 


\begin{tabular}{|c|c|c|c|c|c|c|c|}
\hline \multicolumn{2}{|c|}{$113.06 \mathrm{mCi}{ }^{124} \mathrm{Sb} / \mathrm{Be}$} & \multicolumn{4}{|c|}{$\begin{array}{l}\text { Runs 20140514_2 - 20140519_7 } \\
\& \text { 20140603_17 - 20140605_3 }\end{array}$} & \multicolumn{2}{|c|}{$15.0 \pm 0.2^{\circ} \mathrm{C}$} \\
\hline Pressure (psia) & 27.5 & 32.2 & 36 & 38.9 & 43.6 & 52 & 61.1 \\
\hline Threshold (keV) & $2.50(9)$ & $3.00(12)$ & $3.52(15)$ & $4.00(18)$ & $5.01(21)$ & $8.0(5)$ & $15.0(11)$ \\
\hline Livetime (sec) & 4985 & 5699 & 8945 & 18389 & 23465 & 20981 & 35337 \\
\hline Singles & 126 & 186 & 205 & 238 & 271 & 134 & 138 \\
\hline \multicolumn{2}{|c|}{ Background w/ BeO } & \multicolumn{4}{|c|}{ Runs 20140513_3 \& 20140514_0 } & \multirow{5}{*}{\multicolumn{2}{|c|}{$15.0 \pm 0.2^{\circ} \mathrm{C}$}} \\
\hline Pressure (psia) & 27.5 & 32.2 & 36 & 38.9 & 43.6 & & \\
\hline Threshold (keV) & $2.50(9)$ & $3.00(12)$ & $3.52(15)$ & $4.00(18)$ & $5.01(21)$ & & \\
\hline Livetime (sec) & 8588 & 16002 & 22629 & 12912 & 19616 & & \\
\hline Singles & 26 & 26 & 29 & 30 & 28 & & \\
\hline \multicolumn{2}{|l|}{$113.90 \mathrm{mCi}{ }^{124} \mathrm{Sb}$} & \multicolumn{4}{|c|}{ Runs 20140523_1 - 20140526_2 } & \multirow{5}{*}{\multicolumn{2}{|c|}{$15.0 \pm 0.2^{\circ} \mathrm{C}$}} \\
\hline Pressure (psia) & 27.5 & 32.2 & 36 & 38.9 & & & \\
\hline Threshold (keV) & $2.50(9)$ & $3.00(12)$ & $3.52(15)$ & $4.00(18)$ & & & \\
\hline Livetime (sec) & 238.6 & 3099 & 13737 & 37320 & & & \\
\hline Singles & 28 & 135 & 204 & 228 & & & \\
\hline \multicolumn{2}{|c|}{$113.57 \mathrm{mCi}{ }^{124} \mathrm{Sb} / \mathrm{Be}$} & \multicolumn{4}{|c|}{$\begin{array}{l}\text { Runs 20140519_8 - 20140522_9 } \\
\& \text { 20140530_1 - 20140602_9 }\end{array}$} & \multicolumn{2}{|c|}{$19.9 \pm 0.2^{\circ} \mathrm{C}$} \\
\hline Pressure (psia) & 41.4 & 43 & 45 & 47.2 & 51.5 & 57.6 & \\
\hline Threshold (keV) & $2.03(8)$ & $2.16(9)$ & $2.33(10)$ & $2.54(11)$ & $3.06(14)$ & $4.07(21)$ & \\
\hline Livetime (sec) & 29.1 & 8.00 & 36.9 & 416.6 & 1967 & 12778 & \\
\hline Singles & 12 & 5 & 15 & 109 & 238 & 348 & \\
\hline \multicolumn{2}{|c|}{ Background w/ BeO } & \multicolumn{4}{|c|}{$\begin{array}{l}\text { Runs 20140508_6 } \\
\& \text { 20140512_0-20140513_2 }\end{array}$} & \multicolumn{2}{|c|}{$19.9 \pm 0.2^{\circ} \mathrm{C}$} \\
\hline Pressure (psia) & 33.1 & 41.4 & 43 & 45 & 47.2 & 51.5 & 57.6 \\
\hline Threshold (keV) & $1.52(5)$ & $2.03(8)$ & $2.16(9)$ & $2.33(10)$ & $2.54(11)$ & $3.06(14)$ & $4.07(21)$ \\
\hline Livetime (sec) & 5776 & 20182 & 21317 & 10733 & 33223 & 45665 & \\
\hline Singles & 20 & 47 & 11 & 14 & 56 & 52 & 48 \\
\hline \multicolumn{2}{|l|}{$113.82 \mathrm{mCi}^{124} \mathrm{Sb}$} & \multicolumn{4}{|c|}{ Runs 20140526_4 - 20140527_25 } & \multicolumn{2}{|c|}{$19.9 \pm 0.2^{\circ} \mathrm{C}$} \\
\hline Pressure (psia) & 41.4 & 43 & 45 & 47.2 & 51.5 & 57.6 & \\
\hline Threshold (keV) & $2.03(8)$ & $2.16(9)$ & $2.33(10)$ & $2.54(11)$ & $3.06(14)$ & $4.07(21)$ & \\
\hline Livetime (sec) & 6.22 & 6.74 & 24.1 & 81.5 & 682.8 & 4315 & \\
\hline Singles & 3 & 5 & 10 & 15 & 58 & 64 & \\
\hline
\end{tabular}

Table 4.11: List of runs from 2014 used in the analysis of the CYRTE bubble chamber filled with $\mathrm{C}_{3} \mathrm{~F}_{8}$. 


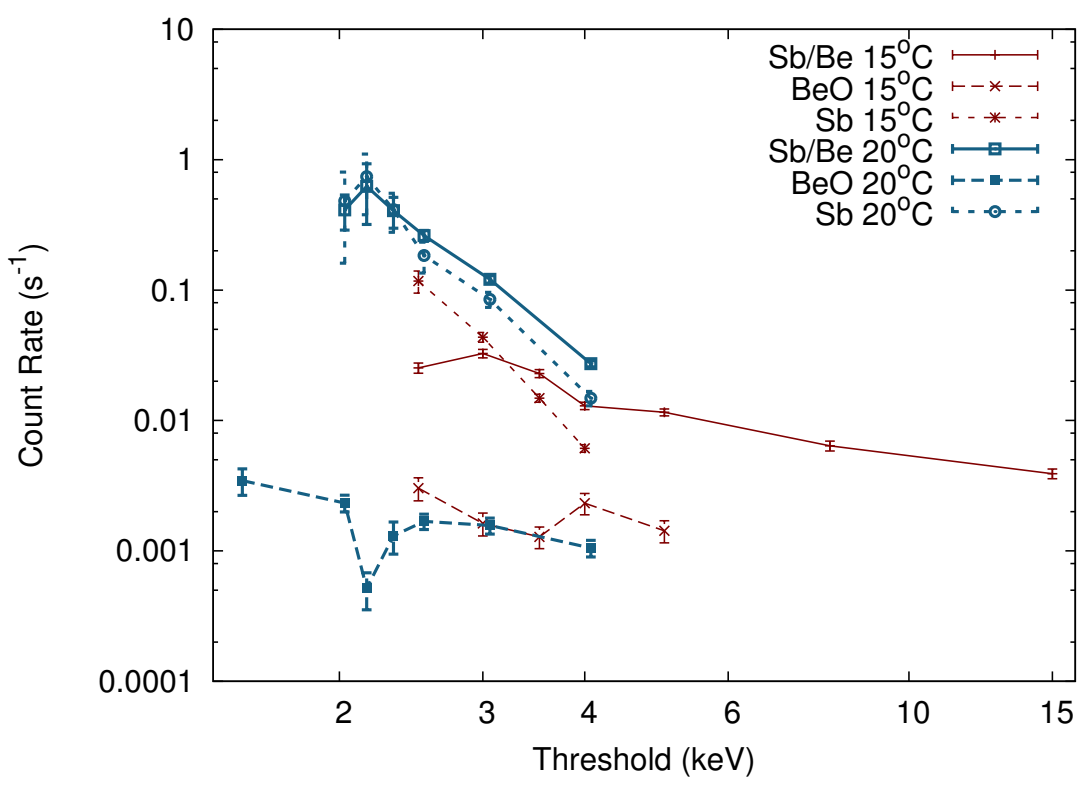

Figure 4.25: Measured bubble nucleation rate in the presence of and ${ }^{124} \mathrm{Sb} / \mathrm{Be}$ source and in background configurations with either the $\mathrm{BeO}$ or the ${ }^{124} \mathrm{Sb}$ source removed. CYRTE demonstrates a high sensitivity to photons. See discussion in text.

valence electron has sufficient stopping power to nucleate a bubble, a large number of ejected valence electrons in combination with the original Compton electron may nucleate a bubble. Riepe and Hahn (1961) found that a $\mathrm{CBrF}_{3}$ filled bubble chamber was partially sensitive to the Auger electrons from the decay of ${ }^{37} \mathrm{Ar}$ at a threshold that is insensitive to individual electrons.

As the total cascade energy and number of electrons available to eject by an Auger cascade increases with the nuclear charge, bubble chambers filled with heavy elements are more susceptible to $\gamma$-induced bubble nucleation. Either iodine, tungsten, or another heavy element may have been present in CYRTE. The cleaning of the bubble chamber after the CIRTE experiment was insufficient to remove all the iodine that may have dissociated during the $\mathrm{CF}_{3} \mathrm{I}$ fill. However, the only other bubble chamber to have been filled with $\mathrm{C}_{3} \mathrm{~F}_{8}$ after a $\mathrm{CF}_{3} \mathrm{I}$ run, the University of Chicago bubble chamber, observed an electron recoil sensitivity consistent with measurements in PICO-2L and COUPP-0.1, much lower than that observed 


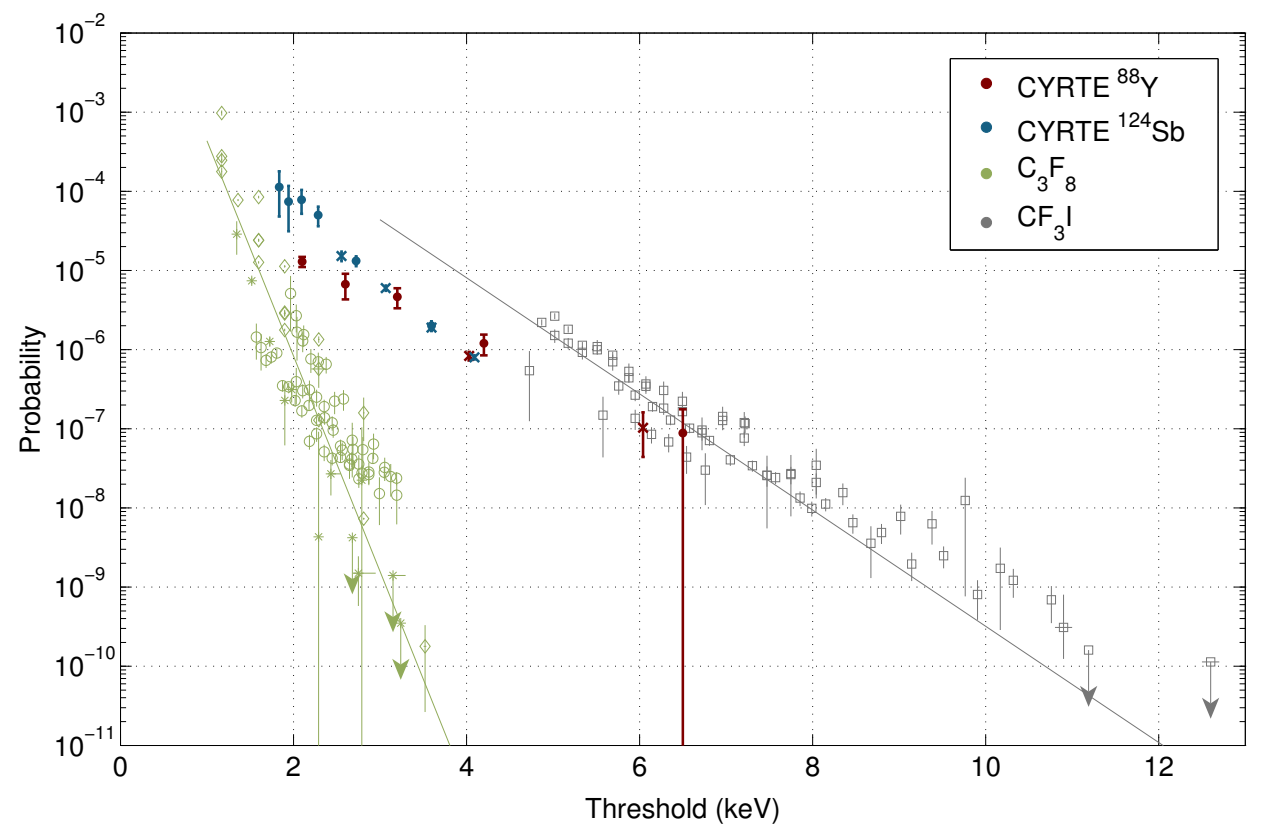

Figure 4.26: Gamma rejection measurements of $\mathrm{C}_{3} \mathrm{~F}_{8}$ (light green) and $\mathrm{CF}_{3} \mathrm{I}$ (grey) using both dark matter detectors and calibration bubble chambers. Only CYRTE demonstrates an anomalous rejection. All rejection factors for both $\mathrm{C}_{3} \mathrm{~F}_{8}$ and $\mathrm{CF}_{3} \mathrm{I}$ fit exponential functions in Seitz threshold energy.

with CYRTE.

The data and experiments presented in this chapter are used in Chapter 6 to set bubble nucleation efficiency functions for dark matter search detectors such as PICO-2L, described in the next chapter. 


\section{Chapter 5 The PICO-2L Bubble Chamber}

PICO-2L (Amole et al. 2015) is a successor to the COUPP-4kg experiment at SNOLAB (Behnke et al. 2012, Fustin 2012). PICO-2L was designed to overcome the low sensitivity of COUPP-4kg to spin-dependent WIMP-nucleon couplings and COUPP-4kg's neutron background rate. From ${ }^{241} \mathrm{Am} / \mathrm{Be}$ and preliminary ${ }^{88} \mathrm{Y} / \mathrm{Be}$ calibrations with $\mathrm{CF}_{3} \mathrm{I}$ in 2012 , we had learned that low carbon and fluorine recoil efficiencies in $\mathrm{CF}_{3} \mathrm{I}$ severely limited the sensitivity of $\mathrm{CF}_{3} \mathrm{I}$ to dark matter candidates with masses below $10 \mathrm{GeV}$ or with spin-dependent couplings to the nucleon. Neutron calibrations with PICASSO (Archambault et al. 2011) had demonstrated that the perfluorocarbon $\mathrm{C}_{4} \mathrm{~F}_{10}$ could be operated at a lower Seitz threshold and had improved bubble nucleation efficiency compared to $\mathrm{CF}_{3} \mathrm{I}$. Both a dark matter search bubble chamber filled with perfluorocarbon fluid and calibrations of the two fluids, see Chapters 4 and 6, were pursued.

The COUPP-0.1 calibration bubble chamber was constructed in 2012 above ground by Mike Crisler and others at Fermilab to test the feasibility of a perfluorocarbon filled bubble chamber. COUPP-0.1 was a near copy of the CYRTE bubble chamber with a larger pressure vessel, a much larger water bath, and modified fill ports. $\mathrm{C}_{3} \mathrm{~F}_{8}$ was selected as the target fluid as its operating temperature was the closest to room temperature of all the perfluorocarbon fluids. For $\mathrm{C}_{3} \mathrm{~F}_{8}$, a $3-10 \mathrm{keV}$ Seitz threshold at 30 psia $(200 \mathrm{kPa})$ is set at temperature between $6.6^{\circ} \mathrm{C}$ and $14.5^{\circ} \mathrm{C}$ compared to $45.0^{\circ} \mathrm{C}$ to $53.5^{\circ} \mathrm{C}$ for $\mathrm{C}_{4} \mathrm{~F}_{10}$. COUPP-0.1 demonstrated that such a bubble chamber was operable but did suffer from the formation of bubbles at the triple interface of the water, $\mathrm{C}_{3} \mathrm{~F}_{8}$ and quartz, especially in the suspected presence of particulate contamination as discussed in Section 2.2 and Figure 2.8. COUPP-0.1 was subsequently shipped to the University of Montreal in 2013 and used with 


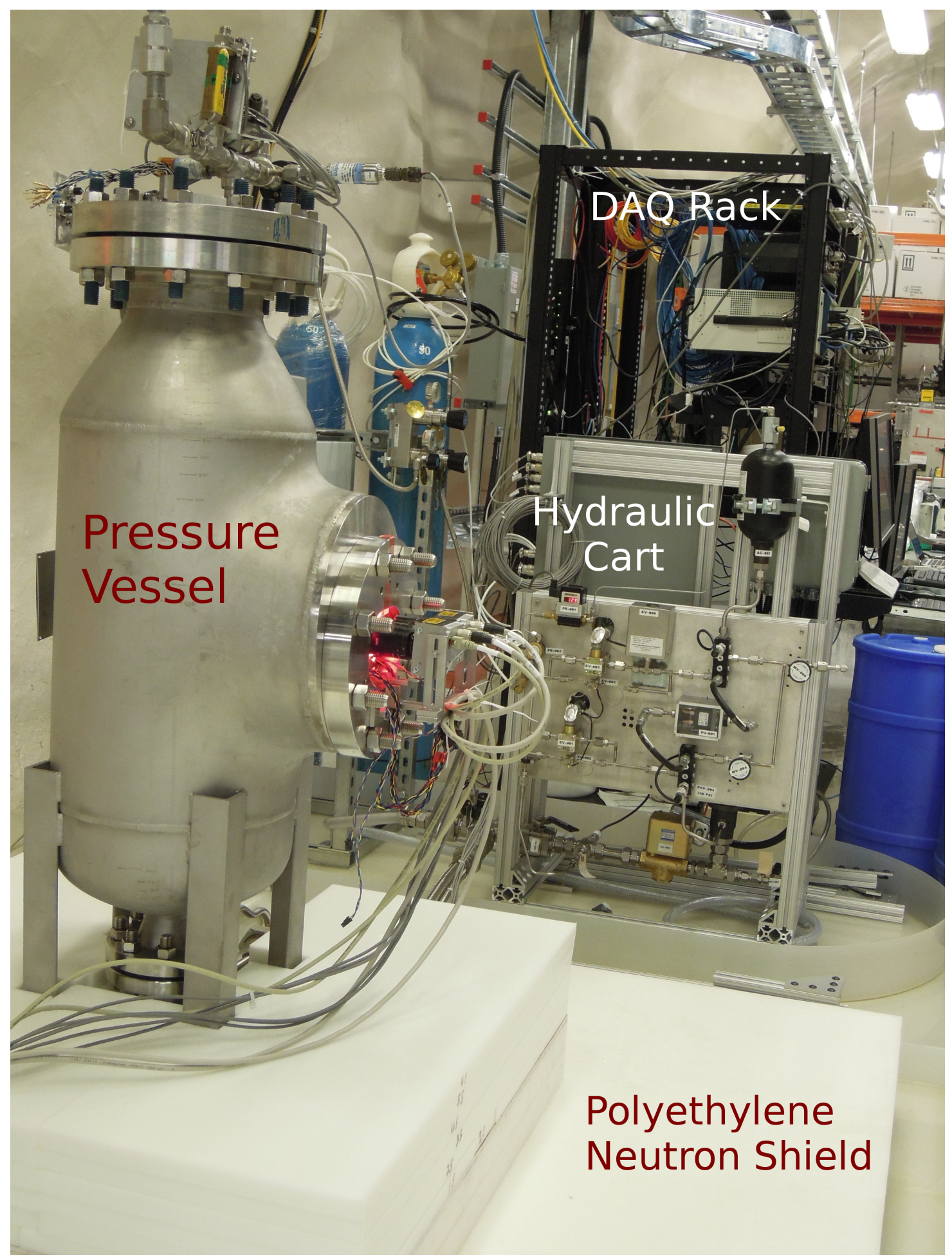

Figure 5.1: PICO-2L at SNOLAB with major components labeled. 


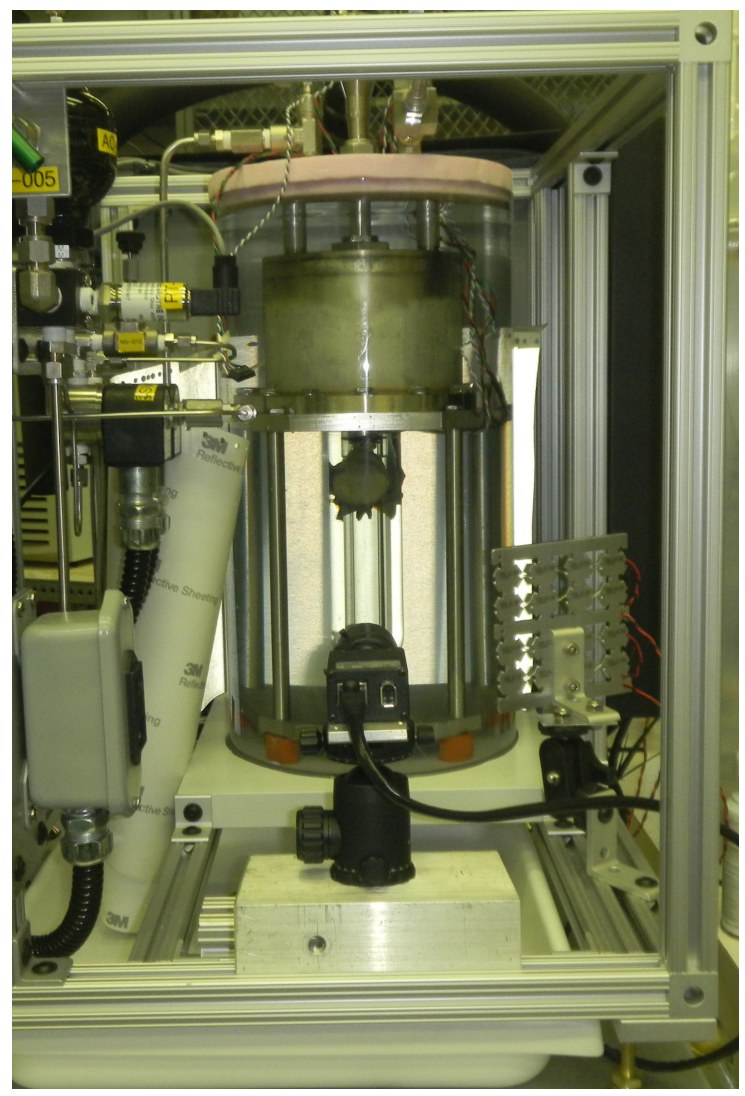

Figure 5.2: The COUPP-0.1 bubble chamber used to test the operation of a $\mathrm{C}_{3} \mathrm{~F}_{8}$ bubble chamber. The $100 \mathrm{~mL}$ pressure vessel is centered in a cylindrical water bath. The hemispherical bottom of the pressure vessel is obsured by one of two cameras taking 100 fps digital images of the $\mathrm{C}_{3} \mathrm{~F}_{8}$ active volume. Hydraulic components are above and to the left of the pressure vessel.

the monoenergetic neutron beam there to calibrate the bubble nucleation efficiency of $\mathrm{C}_{3} \mathrm{~F}_{8}$. The bubble chamber was renamed PICO-0.1 after the move.

PICO-2L was located in SNOLAB under 6000 meters water equivalent of overburden at the Vale Creighton Mine \#9 in Greater Sudbury, Ontario, Canada (Duncan, Noble, and Sinclair 2010). The large rock overburden reduces the cosmic muon flux by over seven orders of magnitude in comparison to at the earth's surface. Neutron spallation and other nuclear processes induced by muons can produce backgrounds in bubble chambers. At SNOLAB, these backgrounds were completely negligible for PICO-2L. PICO-2L operated at SNOLAB 
from October 2013 to May 2014. A second run of PICO-2L was started in March 2015 and is currently operating.

\subsection{Components}

The PICO-2L bubble chamber uses the same layout and many of the same components as the previous COUPP-4kg. PICO-2L is located in the same position that COUPP-4kg was, and reused the polypropylene neutron shield, recirculating water heater/chiller, cameras, and DAQ computers as COUPP-4kg. However, the pressure vessel, inner vessel, the hydraulic control system, and the slow DAQ digitizers were completely redesigned in order to overcome the neutron background and some operational limitations of COUPP-4kg. Details of the

reused COUPP-4kg components can be found in Fustin (2012, chap. 4). Details regarding neutron production in materials and the neutron backgrounds of COUPP-4kg components can be found in Chapter 7 .

\subsubsection{Inner Vessel Assembly}

The inner vessel assembly contains $2.104 \mathrm{~L}$ of $\mathrm{C}_{3} \mathrm{~F}_{8}$ and the water buffer fluid in a radioclean environment. Only synthetic and natural fused silica, PTFE, and various grades of stainless steel are wetted by the inner volume. All components were ultrasonically cleaned, assembled in a Class 10 (ISO 4) cleanroom area, and spray rinsed for several hours with ultrapure water before shipment to SNOLAB.

The bellows arrangement of the PICO-2L inner vessel assembly (see Figure 5.3) is improved over COUPP-4kg. Instead of a single bellows, two bellows operating differentially allow the jar position to remain fixed, simplifying operation and bubble position reconstruction. This design change does reduce the operational range of the bellows to slightly greater than the amount required to safely accommodate the volume growth of the active fluid 


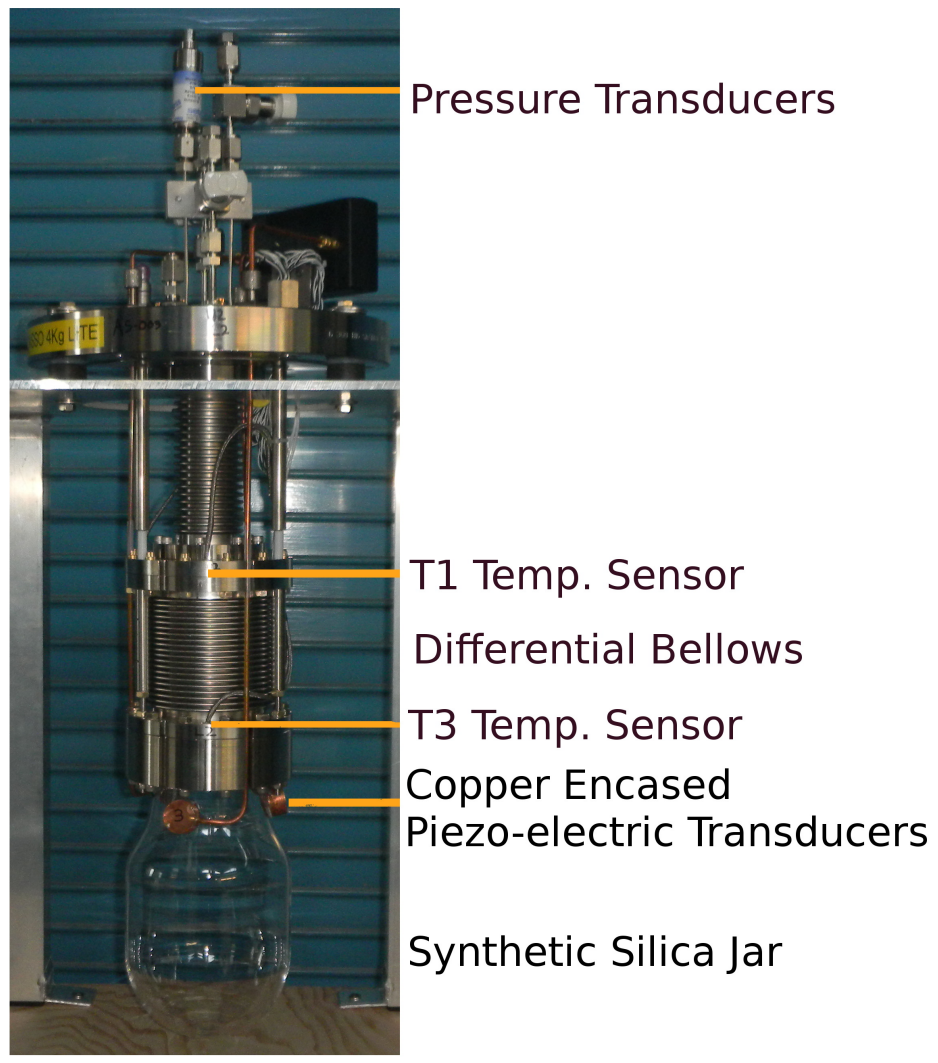

Figure 5.3: PICO-2L assembled inner vessel. The distance from the top of the large top flange to the bottom of the synthetic silica jar is $64.2 \mathrm{~cm}$. 


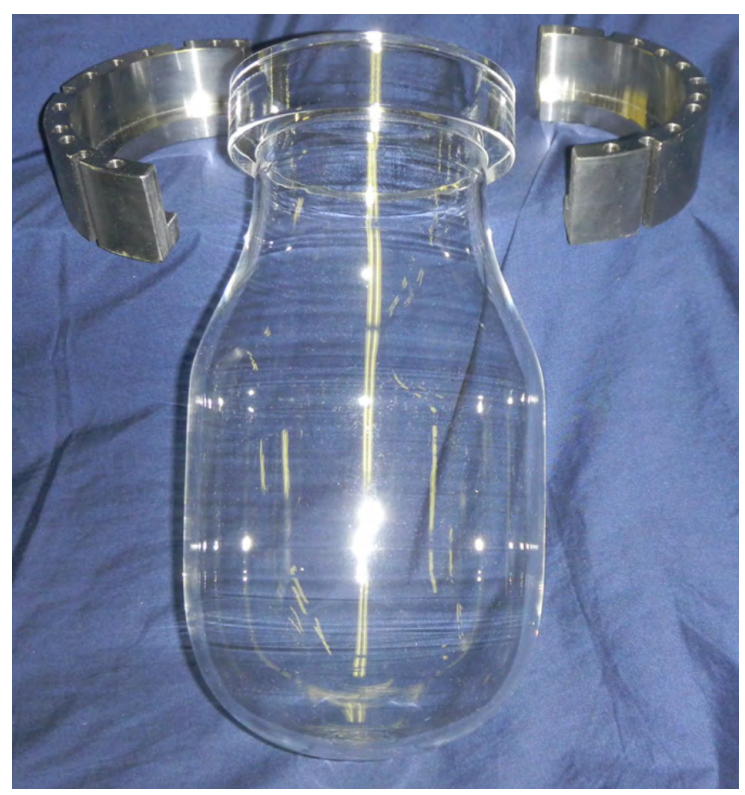

Figure 5.4: The synthetic silica jar and retaining ring of the PICO-2L bubble chamber used contain the active fluid.

during expansion and bubble growth. Large changes in the operating temperature could not be accommodated due to thermal expansion or contraction of the $\mathrm{C}_{3} \mathrm{~F}_{8}$.

Each bellows end is sealed to the adjacent component using a PTFE coated nickel alloy c-ring seal. In order to cushion the natural silica flange of the synthetic silica jar against the seal, a $3 \mathrm{~mm}\left(1 / 8^{\prime \prime}\right)$ thick ring of Garlock 9900 graphite loaded nitrile gasket material was placed between the jar flange and the backing flange, opposite the sealing surface. The jar and its backing flange are shown in Figure 5.4 .

Three custom built low-radioactivity piezo electric transducers, in Alloy 10100 copper housings, were bonded to the exterior of the jar. Four resistive thermometers (RTDs) were embedded in flanges of the bellows assembly: T1 and T2 were located between the small and large bellows while T3 and T4 were located in the bottom flange adjacent to the jar. Two Setra GCT-225 pressure transducers measure the inner vessel (T4) and hydraulic fluid pressure (PT5) at the vessel vessel. An AC-coupled Dytran 2005V pressure transducer was connected to the inner volume to measure the bubble growth before compression. The 
signals from the Dytran sensor and the piezoelectric transducers were digitized at $2.5 \times 10^{6}$ samples per second while the other sensors were sampled 200 times per second. These traces are captured from $160 \mathrm{~ms}$ before the trigger to between $120 \mathrm{~ms}$ and $280 \mathrm{~ms}$ after the trigger.

As the inner vessel pressure transducer was located $65 \mathrm{~cm}$ above $\mathrm{C}_{3} \mathrm{~F}_{8}$ /water interface, a correction for the static head between the sensor and the $\mathrm{C}_{3} \mathrm{~F}_{8}$ of $1.09 \mathrm{psi}(7.52 \mathrm{kPa})$ was applied. This accounts for the static head of the water and the average pressure through the height of the active volume. The accuracy of the pressure transducer dominates the systematic uncertainty of $\pm 0.4 \mathrm{psi}( \pm 3 \mathrm{kPa})$.

A fluid handling cart was constructed to measure, filter, and distill both water and $\mathrm{C}_{3} \mathrm{~F}_{8}$ into the pressure vessel. The cart held either the stainless steel water receptacle or $\mathrm{C}_{3} \mathrm{~F}_{8}$ source bottle in a heater blanket on a refrigerant scale. During the water distillation, the vapor passed through a flow meter and a Gaskleen V series $3 \mathrm{~nm}$ particulate filter located directly before the inner vessel. During the $\mathrm{C}_{3} \mathrm{~F}_{8}$ distillation, the vapor passed through a Gaskleen II reactive gas purifier near the source bottle before passing through the particulate filter. Except for the Dytran pressure transducer, all plumbing in the fluid handling cart and inner vessel used either welded or Swagelok VCR metal gasket fittings with uncoated stainless steel gaskets.

\subsubsection{Pressure Vessel}

A new simplified pressure vessel was built for PICO-2L. This pressure vessel has fewer and less massive components compared to the COUPP-4kg pressure vessel which may have contained radioactive uranium and thorium and contributed to the COUPP-4kg neutron background. The central component of the PICO-2L pressure vessel is a Grade 316L stain-

less steel $12^{\prime \prime}$ pipe tee with a bottom cap, a reducer to a $6 "$ top flange into which the inner vessel lowers, and a synthetic silica viewport shared by two stereo vision cameras. 


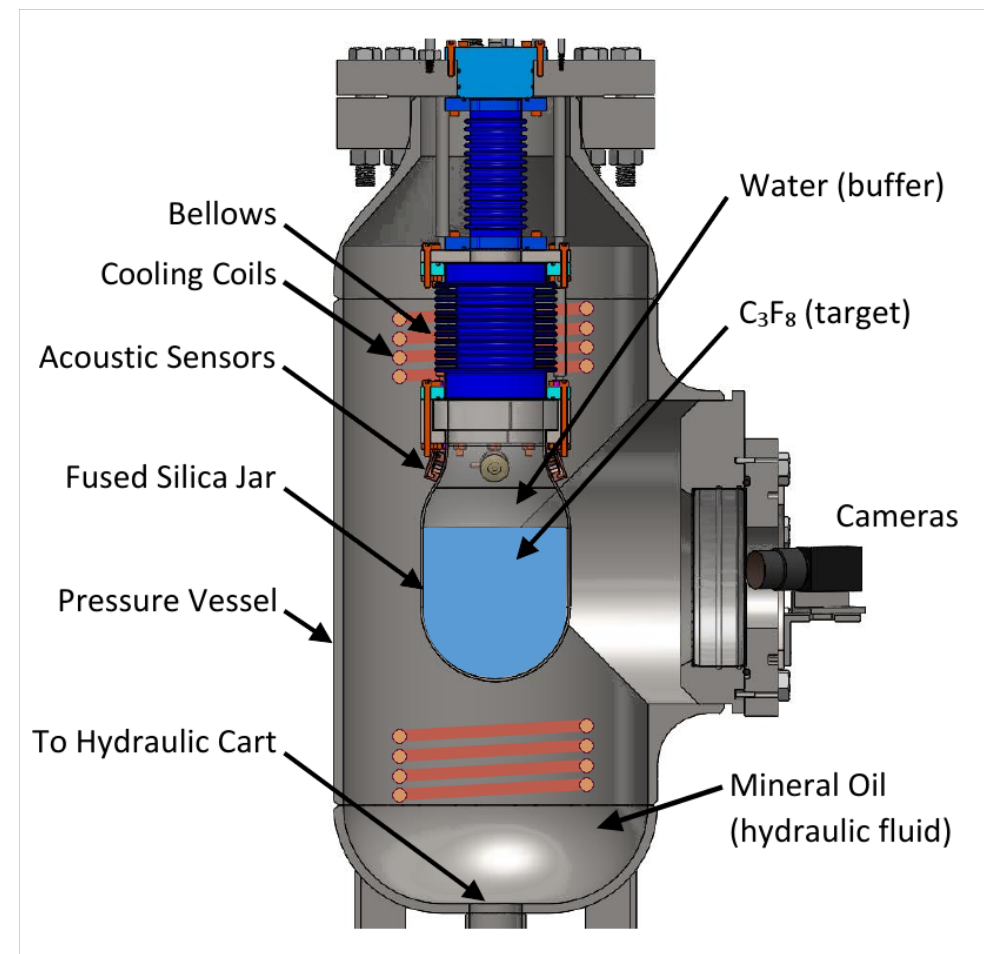

Figure 5.5: A Solidworks CAD generated diagram of PICO-2L showing the pressure vessel and all major components within it. 
As gasket materials can cause neutron backgrounds, an elastomer o-ring was used to seal the viewport. The as-built pressure vessel dimensions (Figure 5.6) were checked against the planned dimensions (Figure 5.5). The geometry differed significantly at the $12^{\prime \prime}$ to 6 " reducer. The as-built dimensions are used for neutron calibration simulations while the planned dimensions were retained for most background simulations.

The pressure vessel was filled with mineral oil as a hydraulic fluid. COUPP-4kg used propylene glycol as a hydraulic fluid. Propylene glycol is a less suitable hydraulic fluid for future larger bubble chambers as it is more compressible than mineral oil and yellows as it decomposes slowly over time. Mineral oil's primary disadvantage is the difficulty in cleaning spills and residue. Both buffer fluids have high hydrogen density and are of high chemical purity, thus providing a neutron shield while not contributing significantly to the rate of background events.

A recirculating flow of water was used to control the temperature of the hydraulic and active fluids. Inside the pressure vessel shell, a continuous length of 1/4" Alloy 10100 copper tube was formed into a set of loops above and below the active volume, in the approximate positions shown in Figure 5.5. Cooling water from a NESLAB RTE-740 recirculating heater/chiller flowed through the tube. As T1 and T2 were located above the cooling coils while T3 and T4 were located below, a temperature gradient was observed between them. In order to determine the temperature of the active volume based on these remote temperature sensors, a temperature calibration was performed at the end of the run (Neilson 2014). With the $\mathrm{C}_{3} \mathrm{~F}_{8}$ drained and the system full of depressurized hydraulic and buffer fluid, a string of temperature sensors was lowered into the pressure vessel to the positions shown in Figure 5.6. The average of T13 and T14 was compared to T4 and a temperature dependent offset was found. When cooling was applied to set $\mathrm{T} 4$ at $15.0^{\circ} \mathrm{C}$, the hydraulic fluid around the inner vessel was $0.1^{\circ} \mathrm{C}$ cooler. As with the calibration bubble chambers, adiabatic cool- 


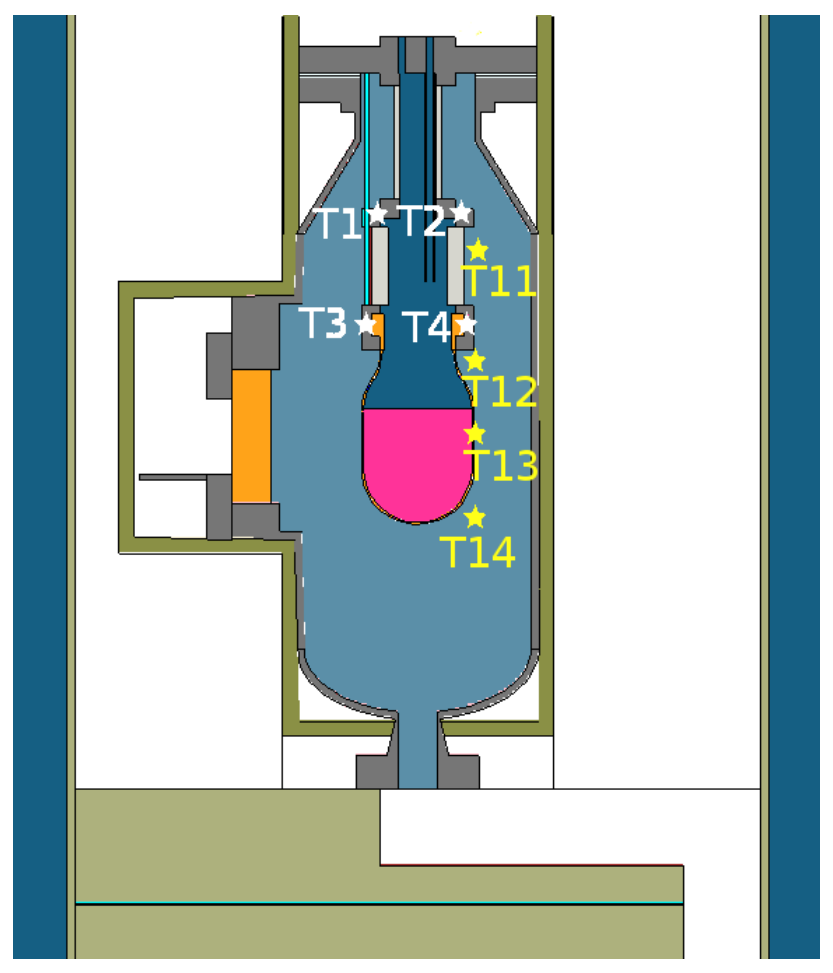

Figure 5.6: RTD positions in PICO-2L during temperature calibrations, May 2015, overlaid on a cross-section of the PICO-2L 2b MCNP geometry. Sections 8.6 and 6.2 .2 describe the details of the PICO-2L MCNP geometry. 


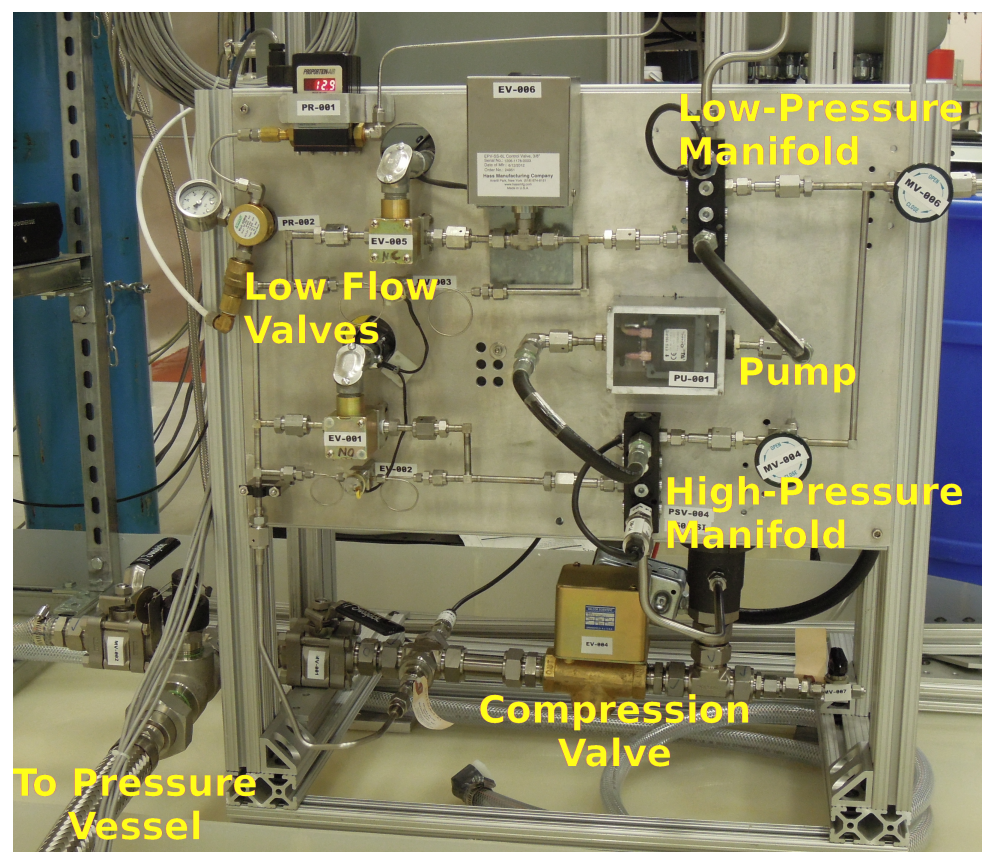

Figure 5.7: The PICO-2L Hydraulic Cart with most major components labeled. Two rubber bladder accumulator tanks, the high-pressure and low-pressure reservoirs, and the cart's electronic components are mounted on the opposite side of the main panel.

ing during expansions lowers the active fluid temperature. An additional $0.1^{\circ} \mathrm{C}$ offset was applied to the data. The temperature used in the analysis is given by

$$
T_{C_{3} F_{8}}=\mathrm{T} 4+0.016\left(21^{\circ} \mathrm{C}-\mathrm{T} 4\right)-0.1^{\circ} \mathrm{C}
$$

with a $\pm 0.3^{\circ} \mathrm{C}$ systematic uncertainty. The total uncertainties shown in Table 5.4 near the end of this chapter, include the standard deviation of the temperature measured during each run condition.

\subsubsection{Hydraulic Controls and DAQ}

A new hydraulic cart design was developed following the COUPP-4kg experiment. The new design was first tested with the CIRTE bubble chamber before being scaled up to PICO-2L. The PICO-2L hydraulic cart is shown in Figure 5.7. To control the fluid pressure, two 
pressure reservoirs are connected to the pressure vessel via computer controlled valves. The high-pressure reservoir was held at the compression pressure of 195 psia (1.34 MPa) while the low-pressure reservoir was slightly above atmospheric pressure. A National Instruments (NI) CompactRIO real-time Field Programmable Gate Array (FPGA) controller on the hydraulic cart processed all of the bubble chamber's pressure and temperature data and controlled all of the automated valves. During an expansion, a path from the low pressure reservoir through a $\frac{3}{8} "$ metering valve and a solenoid valve is opened, and the pressure lowers towards the expansion setpoint as the volume of the system expands. The FPGA closes the valve once the target pressure is reached. To finely adjust the pressure, two small orifice $(1.52 \mathrm{~mm})$ normally closed solenoid valves connected to the pressure reservoirs are pulsed open. When a trigger is received, a large solenoid valve is rapidly opened to the high pressure reservoir. The active volume reaches full compression pressure within $20 \mathrm{~ms}$. A pump between the two reservoirs recharges them during compressions.

Most of COUPP-4kg's DAQ components were reused for PICO-2L. Only the SC-2345 digitizer box was replaced by the CompactRIO controller and the main DAQ chassis was upgraded to an NI PXIe-1062Q. The DAQ software was redesigned so that backup triggers and control of the bubble chamber's operational state were handled by the FPGA controller. In COUPP-4kg, a software failure of the main DAQ computer could prevent the processing of trigger signals.

During normal operation, the formation of a bubble causes rapid changes between 100 frames per second camera images. These differences were detected by the main DAQ computer and a trigger signal was sent to the hydraulic cart. The main DAQ then wrote the fast digitizer data and data for each event to file, started a new event file structure, and waited 30 seconds until commanding a new expansion. The main DAQ also provided expansion timeout triggers. In $\mathrm{COUPP}-4 \mathrm{~kg}$, the solubility of $\mathrm{CF}_{3} \mathrm{I}$ in water allowed $\mathrm{CF}_{3} \mathrm{I}$ to 
be transported through the buffer fluid and form vapor on the rough surfaces of the steel components of the inner vessel. Expansions in both COUPP-4kg and early PICO-2L runs were triggered after 500 seconds in order to prevent mass transport out of the active volume, overextension of the bellows, and spurious triggers from the boiling of droplets away from the active volume. This expansion timeout was increased to 1000 seconds for late PICO-2L runs, and may not have been necessary at all given the extremely low solubility of $\mathrm{C}_{3} \mathrm{~F}_{8}$ in water at a molar fraction of $1.5 \times 10^{-6} / \mathrm{atm}$ at $10^{\circ} \mathrm{C}$ (Wen and Muccitelli 1979 ).

The hydraulic cart monitored the pressures inside PICO-2L and triggered the chamber when a pressure excursion was observed. Such excursions included a rapid rise of the inner vessel pressure that would occur during boiling, a large pressure differential between the inner vessel and the pressure vessel due to overextension of the bellows, or low pressure in the high-pressure reservoir indicating a loss of hydraulic volume or pump failure. The hydraulic cart also generated email alarms in the case of mechanical or operational failures.

\subsection{Operational Difficulties}

PICO-2L was operated similarly to COUPP-4kg. However, some additional operational difficulties arose with the $\mathrm{C}_{3} \mathrm{~F}_{8}$ filled bubble chamber.

During filling, because the water distillation slowed to a stop when nearly full, vacuum was to applied to the inner vessel to remove any remaining air in the system. The vacuum pump was accidentally connected backwards and pushed air at approximately $300 \mathrm{kPa}$ directly into the inner vessel. Within 5 minutes, this air was pumped back out and pumping continued for the full day. When PICO-2L was sampled for particulates after the end of the run, several milligrams of particulates, mostly metal oxide and silica particulates, were observed. A small minority of particulates may have been mine dust or other external particulates. The seal material of the vacuum scroll pump used with PICO-2L was also 


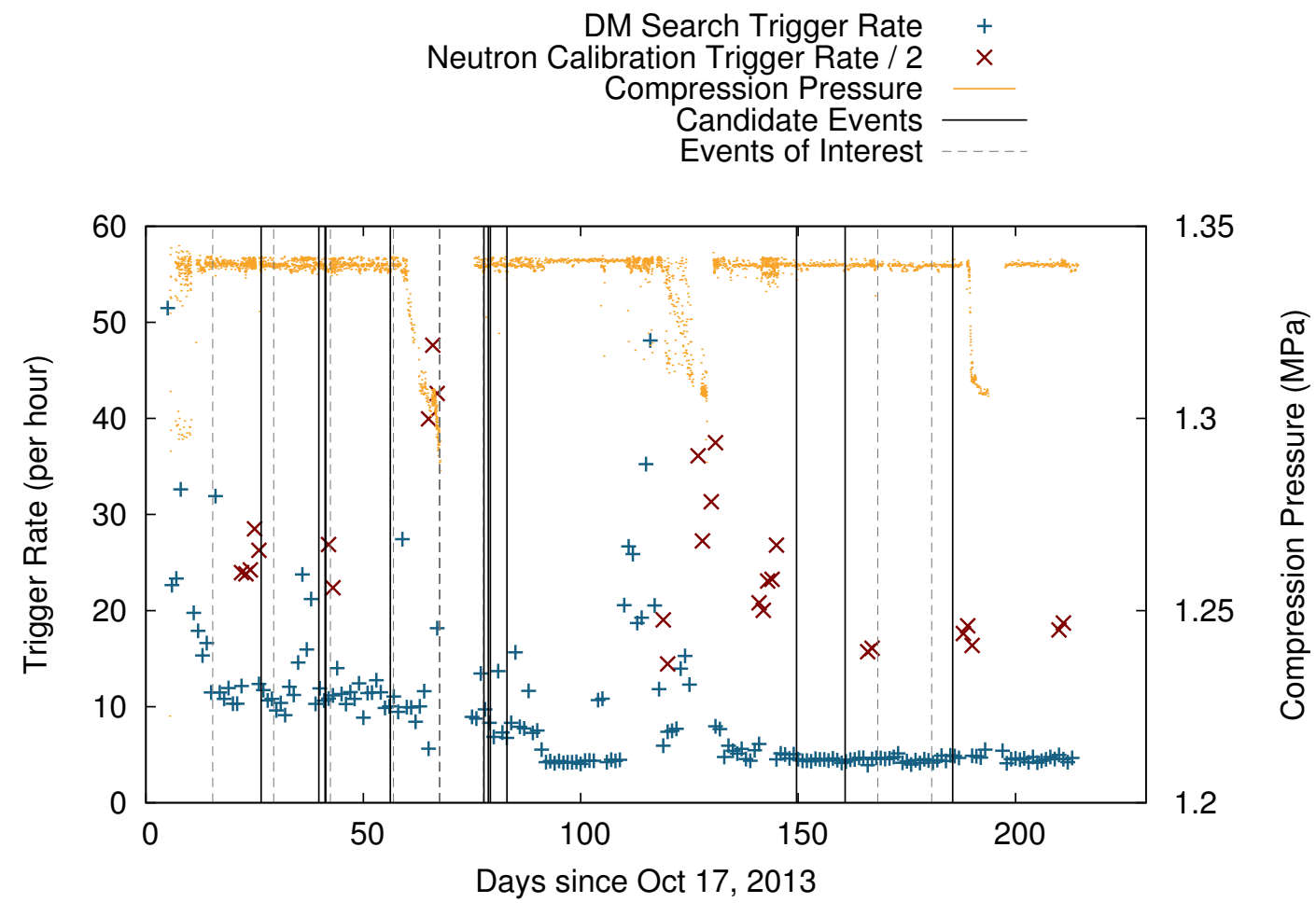

Figure 5.8: Trigger rates, compression pressure, and candidate events over the course of PICO-2L running. As mineral oil slowly leaked from the hydraulic cart, the amount of reserve hydraulic fluid in the low pressure reservoir decreased. Low compression pressures occurred when the reserve was depleted. Events of interest that were cut during periods of low compression pressure or other data quality cuts.

examined, and no particulates matching that material were found.

As described in Section 2.2, spontaneous bubble nucleation at interfaces is much more likely in $\mathrm{C}_{3} \mathrm{~F}_{8}$ that in $\mathrm{CF}_{3} \mathrm{I}$. In PICO-2L, and to a greater extent in the CYRTE bubble chamber before siloxane treatment, these nucleations would occur in time clustered clumps. This event rate could be suppressed by compressing the chamber for long periods. The rate could increase when temperatures changed or bubbles formed near the $\mathrm{C}_{3} \mathrm{~F}_{8} /$ water interface. Figure 5.8 shows the background event rate that spiked throughout the dark matter search dataset.

As with COUPP-0.1 (see Figure 2.8) and CYRTE, interfacial tensions also trapped water droplets against the wall of the inner vessel below the interface as shown in Figure 5.9. These 

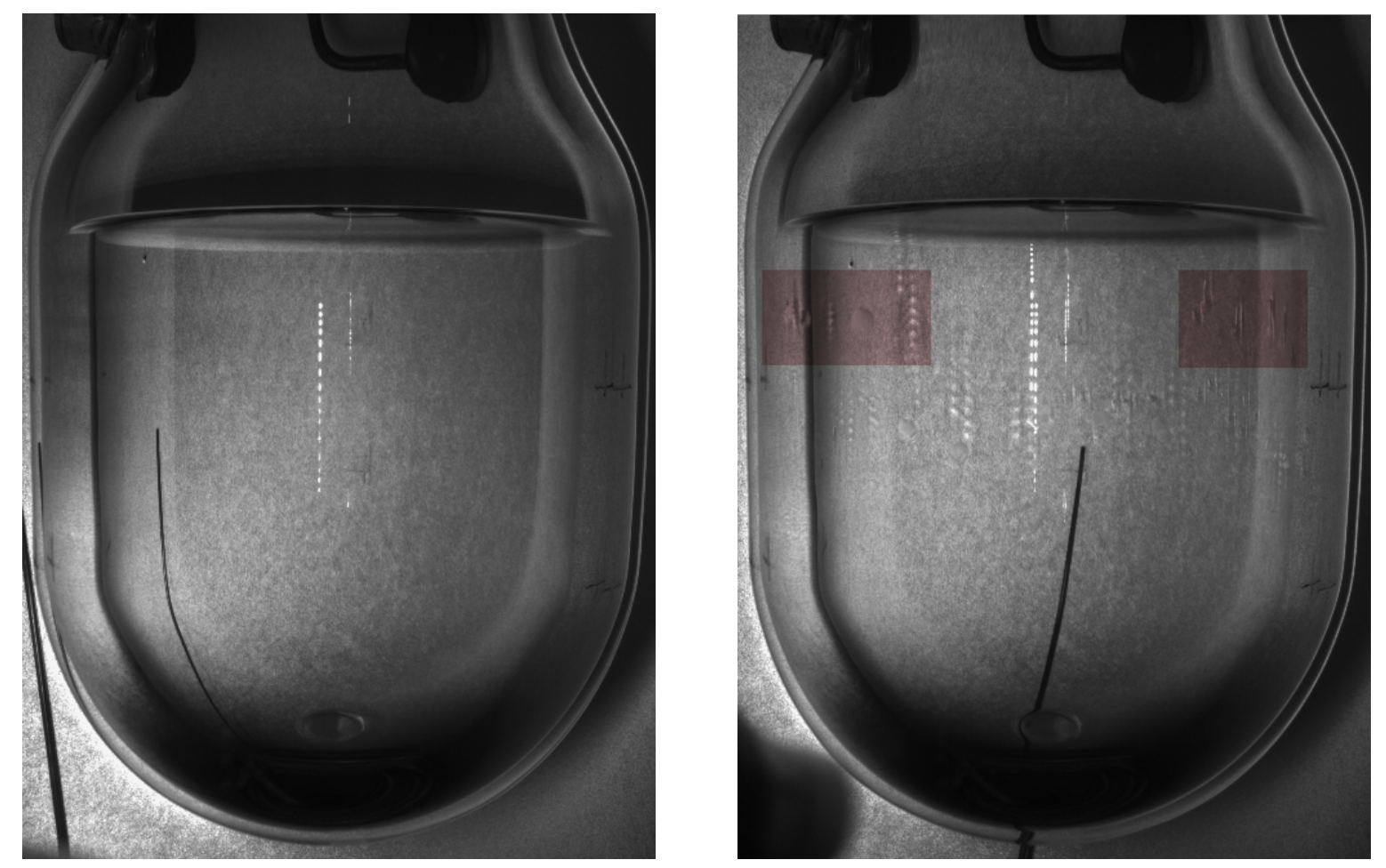

Figure 5.9: PICO-2L cam0 images at the start of running in October 2013 (left) and near the end of running in May 2014 (right). During running, water droplets formed below the $\mathrm{C}_{3} \mathrm{~F}_{8}$ / water interface as shown with red tint. The stainless steel rod visible behind the inner vessel was used to measure the final position of components in the pressure vessel. It dropped into the pressure vessel and out of reach before PICO-2L was filled with $\mathrm{C}_{3} \mathrm{~F}_{8}$.

droplets refracted the images of bubbles and slightly decreased the accuracy of the 3D bubble position reconstruction. They were also a source of bubble nucleation.

Two of the three piezoelectric transducers installed on PICO-2L failed during the dark matter search, and the third developed instability in its gain. Similar failures occurred to some piezos during both runs of COUPP-4kg and COUPP-60. The other failures occurred in bubble chambers using epoxy resin encased transducers submerged in conductive propylene glycol, and failure of the piezo casing had been suspected as the cause of the signal failure. In PICO-2L, the non-conductivity of mineral oil and the use of more robust casings precluded this failure mechanism. Instead, the failures are suspected of being caused by overvoltage on the internal preamplifiers of the transducers (Levine 2014). The Analog Devices AD8065 
low-noise amplifiers used in the housings are designed to measure AC coupled acoustic signals of up to $1 \mathrm{mV}$ from the sensor elements, and can safely tolerate voltages up to $24 \mathrm{~V}$ (Devices 2013). The rapid pressure change during a compression can generate voltages of $\mathcal{O}(100 \mathrm{~V})$ and likely damaged the piezo electric sensors. For PICO-2L run 2 and later bubble chambers, voltage clamping diodes have been installed at the preamplifier input and the preamplifier power supply is intentionally interrupted when compression occurs in order to prevent damage from overvoltage and overheating.

\subsection{Analysis Variables and Cuts}

Analysis cuts in PICO-2L are used to both investigate the potential dark matter signal, and to classify events that may be of operational significance. Notably, an acoustic paramter cut allows nuclear recoil events that are a signal of dark matter to be discriminated from events caused by $\alpha$-decay. Analysis cuts may cut both events and livetime, or just events. Livetime is cut during periods where the thermodynamic state of the bubble chamber is uncertain or changing or the expected rate of events is above background. Where events are cut and livetimes are kept, the cut efficiencies need to be accurately determined in order to calculate the detector sensitivity.

In order to validate the cuts and determine cut efficiencies, 7839 of 10540 non-timeout events from the neutron calibration data from PICO-2L were examined by at two independent experimenters to validate the automated reconstruction.

All the analysis cuts for PICO-2L are motivated by or were implemented in the operation of previous bubble chambers. Except for the time to previous non-timeout cut (TNTP) described in Section 9.1, all cuts were either pre-set or tuned to calibration data.

This section is based on the PICO-2L 2013-2014 Analysis Tech Note (Harris, Lippincott, and Neilson 2014). 


\subsubsection{Livetime Cuts}

Livetime is cut for the first $25 \mathrm{~s}$ after each expansion of the bubble chamber. Adiabatic cooling and flows generated within the $\mathrm{C}_{3} \mathrm{~F}_{8}$ during expansion are allowed to subside during this period. The $25 \mathrm{~s}$ cut period starts when the inner vessel pressure is within $1 \mathrm{psi}(6.9 \mathrm{kPa})$ of the expansion setpoint pressure. In COUPP-4kg, a $30 \mathrm{~s}$ cut was applied after the start of the expansion. As the setpoint pressure is reached after approximately $5 \mathrm{~s}$, the cuts are similar.

The low compression pressure periods shown in Figure 5.8 were cut from the background data. Nucleation sites, especially those on particulates in the chamber, may not have been passivated during these runs. Any run with at least 10 events with a compression pressure below 190 psia (1.31 MPa) was cut in its entirety. An large excess of 5 potential nuclear recoil-like events in run 20131223_3 (day 67) was cut due to low expansion pressure.

Runs were cut when the expected neutron background rate was higher than normal. This could be due to either the possible presence of an Am/Be neutron source outside the shield near the experiment or to a missing polypropylene plug in the calibration source port of the neutron shielding. The Am/Be neutron source could have contributed up to one background event every 3.2 days when near the experiment. The source was present immediately before and after neutron calibration runs in PICO-2L and during neutron calibrations of the neighboring DAMIC experiment March 12, 2014 (day 146).

Runs 20131108_0, and 4 runs on December 17 and 18, 2014 were cut due to corrupted data or ongoing changes to the DAQ.

\subsubsection{Efficiency Cuts}

The main video trigger of PICO-2L relied on 100 frames per second video images downloaded and compared in real-time. Depending on other processes using the main DAQ computer, 


\begin{tabular}{cc}
\hline Cut & Efficiency \\
\hline Frame skip & $97.7 \pm 0.1$ \\
Video trigger & $100.0 \pm 0.0$ \\
Bubble finder & $98.9 \pm 0.3$ \\
Piezo noise & $99.3 \pm 0.2$ \\
Pressure & $100.0 \pm 0.0$ \\
\hline Total (no fiducial, no acoustic) & $96.1 \pm 0.3$ \\
Acoustic & $90.8 \pm 0.9$ \\
Dytran fiducial & $92 \pm 2$ \\
XYZ fiducial & $82 \pm 1$ \\
\hline Total (Dytran fiducial) & $80.2 \pm 2.2$ \\
Total (XYZ fiducial) & $71.5 \pm 1.4$
\end{tabular}

Table 5.1: Cut efficiencies for PICO-2L. Two different fiducial cuts were applied this process skipped at least one camera exposure $2.3 \%$ of the time whenever insufficient computational power was available. If a bubble had formed during these frame skips, the trigger time was offset from the bubble formation time and the acoustic and Dytran pressure signals were not fully captured. A frame skip cut was used to ensure data quality. Similarly, events would have been cut if a video trigger was not recorded. Hand scans of PICO-2L and COUPP-4kg have found no bubbles unassociated with a video trigger or frame skip.

The PICO bubble finder software, Getbub, looked for clumps of pixels changing between camera image frames. Getbub can reliably detect the formation and position of single bubbles. Events for which Getbub did not detect a single bubble in both camera images are cut. Of the hand scanned fiducial single bubbles events passing the above cuts, $98.9 \pm 0.3 \%$ were correctly identified by Getbub.

In order to verify that the Acoustic Parameter (AP) is correctly calculated (see the next section) the root mean square noise of the operational piezos before the trigger must be low. Of all the event passing the previous cuts, $99.3 \pm 0.2 \%$ pass this cut.

Finally, events would have been cut if the pressure during a trigger was further than 
$0.5 \mathrm{psi}(3.4 \mathrm{kPa})$ from the setpoint pressure.

\subsubsection{The Acoustic Parameter}

The calculation of the acoustic parameter (AP) used to identify background $\alpha$-decay events follows the procedure used with COUPP-4kg. Almost identically to Fustin (2012, Eq. 5.3), AP is defined as

$$
\mathrm{AP}=A(T, P) \sum_{j} G_{j} \sum_{n} C_{n}(\vec{x}) \sum_{f_{\min }^{n}}^{f_{\max }^{n}} f \times \mathrm{PSD}_{f}^{j},
$$

where $A(T, P)$ is a temperature and pressure-dependent scale factor, $G_{j}$ is the gain of piezo $j, C_{n}(\vec{x})$ is the correction factor for the bubble position dependence (position $\vec{x}$ ) in frequency bin $n, f$ is the frequency, $f_{\min }^{n}$ and $f_{\max }^{n}$ are the boundaries of frequency bin $n$, and $\operatorname{PSD}_{f}^{j}$ is the power spectral density for the bin with center frequency $f$ for piezo $j$. AP is normalized to 1 for nuclear recoil events.

The frequency bins giving the best sensitivity in PICO-2L were different than those used in COUPP-4kg. Bins between $20-35 \mathrm{kHz}, 42-50 \mathrm{kHz}, 100-150 \mathrm{kHz}$, and 150-200 kHz provided the best combination of gain and stability for measurements of $\alpha$ discrimination. The position correction for each piezo and frequency bin was a separable function of the cylindrical coordinates of the inner vessel: $r^{2}, \cos \left(\theta-\theta_{o}\right)$, and $z$. Polynomial functions of these parameters were tuned to single bubble events of neutron calibrations.

A correction for the pressure in the bubble chamber pressure at trigger was added. The bubble chamber was operated at pressures between 25 psia and 50 psia (170-345 kPa) depending on the intended Seitz threshold. Neutron calibrations at the run pressures found that the power spectral density varies exponentially with pressure, with an exponential scale

of -4.005 psi. A much smaller temperature correction was found valid between $11^{\circ} \mathrm{C}$ and 
$15^{\circ} \mathrm{C}$. Combined, these corrections are

$$
A(T, P)=\left(e^{-0.2497 \mathrm{P} 4} * 0.1473\left(\mathrm{~T} 4-T_{o}\right)\right)^{-1}
$$

where $\mathrm{P} 4$ is in psi and $\mathrm{T} 4$ is in ${ }^{\circ} \mathrm{C}$.

The power spectral density is calculated over a $10 \mathrm{~ms}$ time window around the start of bubble growth, $t_{0}$, with the background noise power subtracted. As the main camera trigger of the DAQ has a temporal resolution worse than $10 \mathrm{~ms}, t_{0}$ must be found by processing the acoustic signal. The algorithm to find $t_{0}$ used for COUPP-4kg was unreliable for PICO$2 \mathrm{~L}$ due to additional sources of noise. To find $t_{0}$, a discrete Daubechies 12-tap wavelet transform is taken over the zero padded acoustic trace with $2^{20}$ samples. This generates 20 wavelet functions each containing half the number of points of the previous one and is sensitive to half the frequency. Given the Nyquist frequency $f_{N}$ of $1.25 \mathrm{MHz}$, these wavelets are sensitive to frequencies of approximately $f_{N} / 2^{8}$ to $f_{N} / 2^{5}$, or $5 \mathrm{kHz}$ to $39 \mathrm{kHz}$. The $6^{\text {th }}$, $7^{\text {th }}$, and $8^{\text {th }}$ wavelets of the transform were rescaled to real-time and multiplied by each other. As phase information is preserved by wavelets, the amplitude of this product detects the coherent broad spectrum pulse from the start of bubble growth. The tallest peak of this function before compression is identified as coming from the bubble, and $t_{0}$ is set at the first peak reasonably associated with the tallest peak. Figure 5.10 demonstrates the $t_{0}$ finder.

A correction for the gain instability that developed in Piezo 1 was needed to analyze the data after the failure of the other two acoustic sensors. The power spectral density between $420 \mathrm{kHz}$ and $470 \mathrm{kHz}$ was used to normalize the gain of the piezo event by event. At these frequencies, the power measured by the piezo is dominated by Johnson noise of the preamplifier's gain resistors and preamplifier shot noise. Above $470 \mathrm{kHz}$, the power spectral function did not provide a linear measurement of the gain while at lower frequencies, the acoustic power contributes to the measured signal. Figure 5.11 shows the result of this 


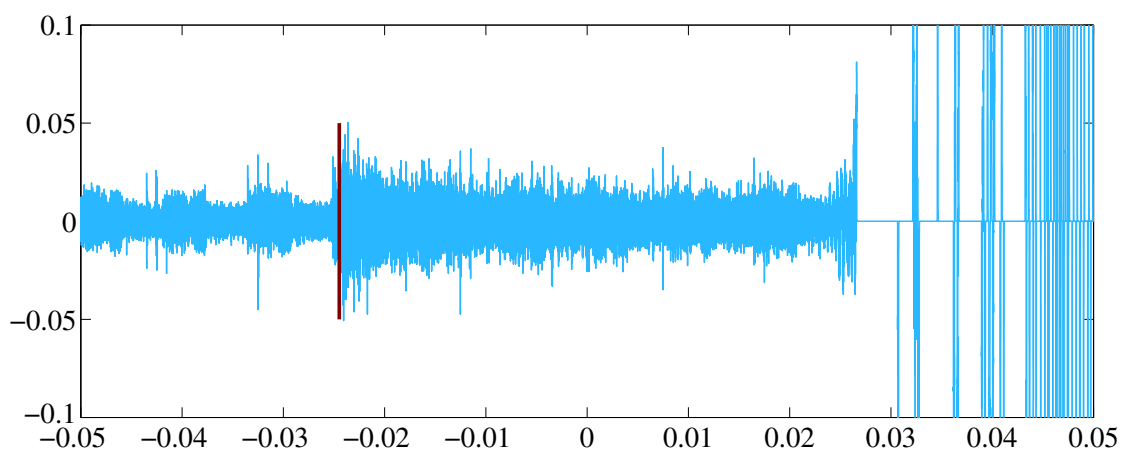

(a) $t_{0}$ in maroon over an unprocessed acoustic trace.

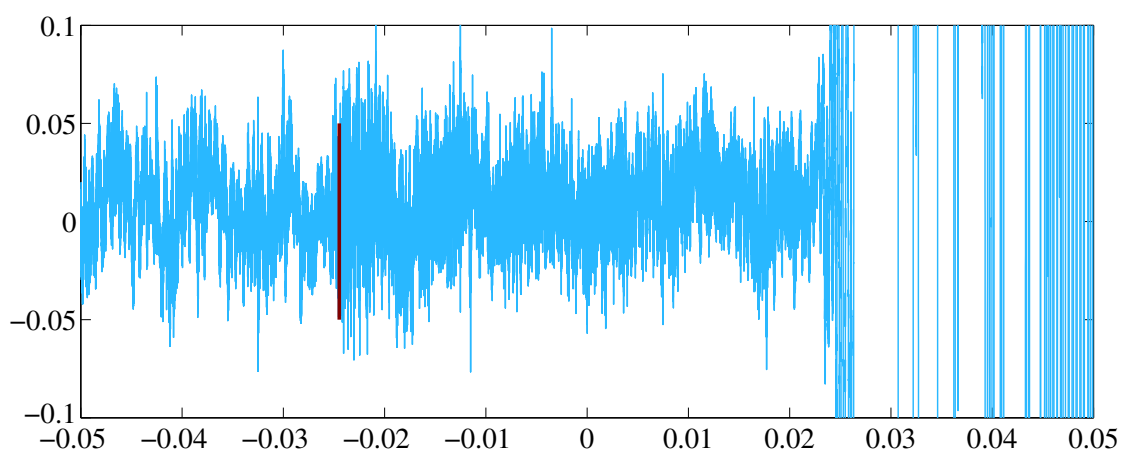

(b) The trace filtered with a $500 \mathrm{~Hz}$ high-bass filter as used in COUPP-4kg

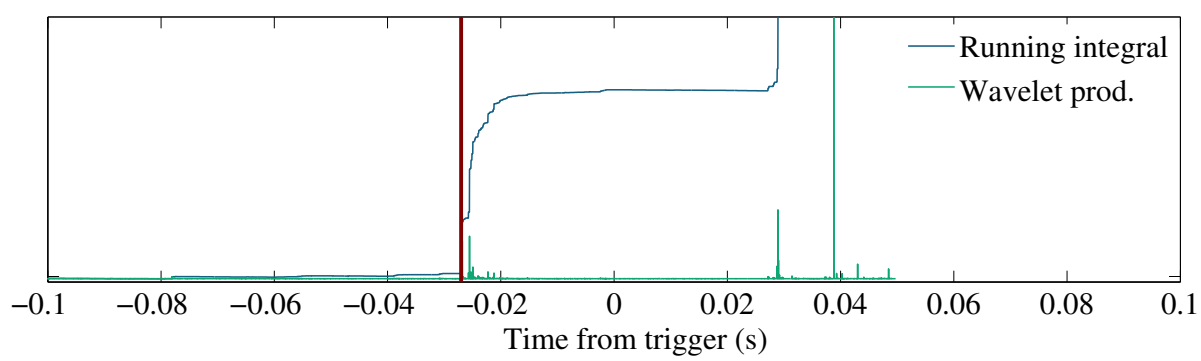

(c) The squared product of wavelets and their integral

Figure 5.10: $t_{0}$ finding using wavelet transformations in PICO-2L for event 20140503_0/28. The wavelet decomposition effectively filters and extracts the $t_{0}$ information. 


\begin{tabular}{ccc}
\hline Condition & Nuclear Recoil & $\alpha$ \\
\hline $4 \mathrm{keV}$ & $0.7<\mathrm{AP}<1.3$ & $\mathrm{AP}>2.5$ \\
$3 \mathrm{keV}$ & $0.7<\mathrm{AP}<1.3$ & $\mathrm{AP}>3.0$ \\
$5.5 \mathrm{keV}$ & $0.55<\mathrm{AP}<1.45$ & $\mathrm{AP}>6.0$ \\
$7 \mathrm{keV}$ & $\mathrm{AP}<2.0$ & $\mathrm{AP}>6.0$ \\
\hline
\end{tabular}

Table 5.2: Cut values on AP for selecting nuclear recoil events and alpha events.

normalization.

The acoustic parameter distributions at each dark matter search threshold are shown in Figure 5.12. At $3 \mathrm{keV}$ and $4 \mathrm{keV}$ with an expansion pressure of 30 psia, the same nuclear recoil acceptance cut as was used for COUPP-4kg is reused for PICO-2L. As the resolution of AP rapidly degrades with increasing expansion pressure, the acceptance region was expanded for data at higher thresholds. Cut parameters are shown in Table 5.2 .

The acceptance for the AP cut was determined using neutron calibration data by assuming that all events with an insufficiently large AP to be considered an $\alpha$ were nuclear recoils. The cut value for considering an event to be caused by an $\alpha$ is shown in Table 5.2 . As not all neutron induced events are nuclear recoils, this underestimates the cut acceptance. Section 7.4 explores the role of the processes that contribute the high-side tail to the main nuclear recoil peak in AP.

\subsubsection{Fiducial Volumes}

In COUPP-4kg, fiducial bubble events were determined by measuring the pressure rise due to early bubble growth. The growth of a bubble in the bulk of the fluid is quadratic in time with a predictable growth rate. Surfaces and interfaces near the bubble alter the growth rate. Figure 5.13 shows typical pressure rise profiles for various locations of bubbles. Quadratic fits to the bubble growth were performed with cuts on the growth rate and the goodness of fit tuned to maximize the separation between event classes. Table 5.3 presents 

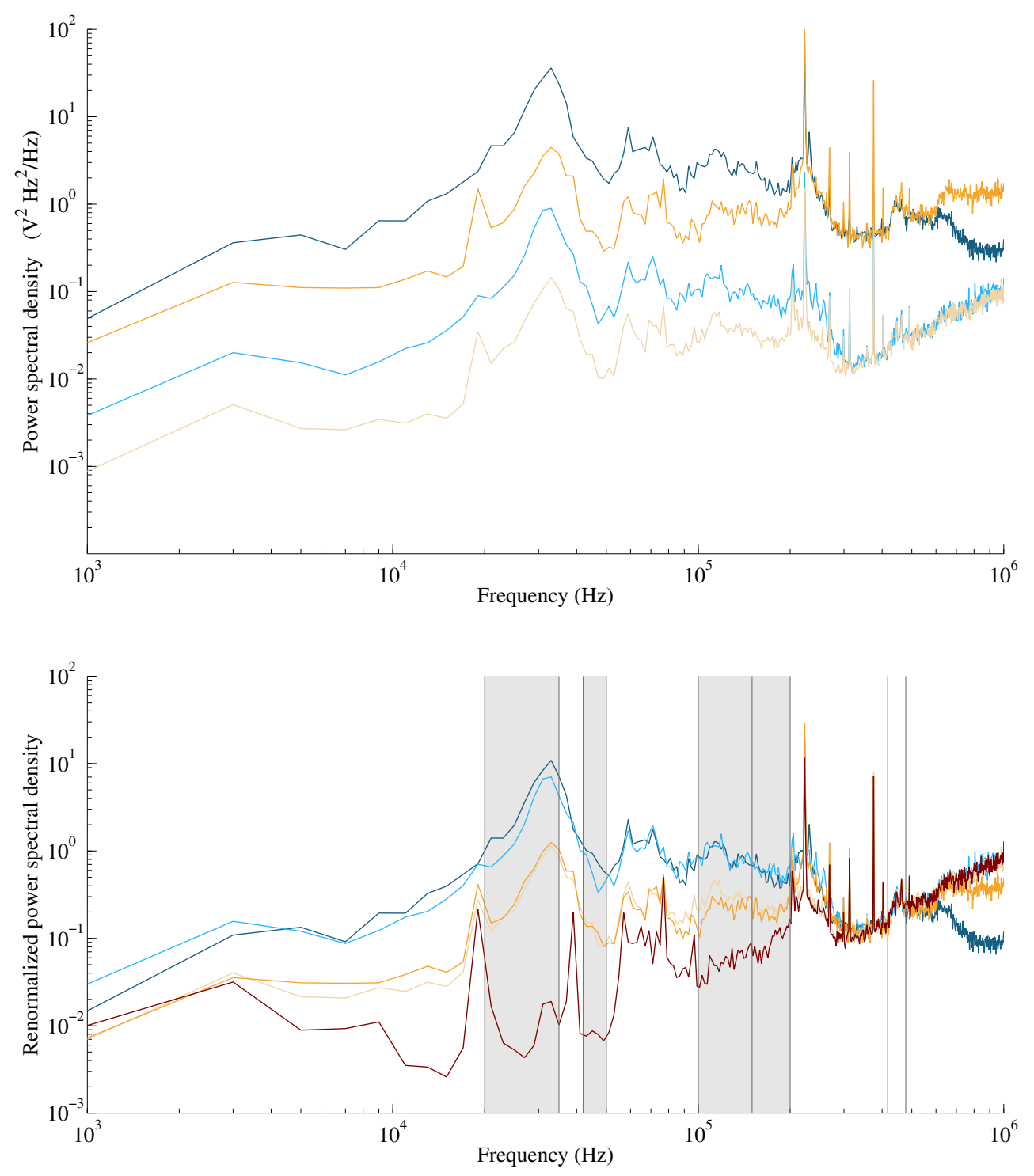

Figure 5.11: Uncorrected (above) and gain corrected (below) power spectral densities measured by Piezo 1. Plots are by Hugh Lippincott. The blue traces are from single bubble neutron calibration events near the wall at $12^{\circ} \mathrm{C}$ and $35 \mathrm{psia}(240 \mathrm{kPa})$ and the yellow traces are from events at $14^{\circ} \mathrm{C}$ and $30 \mathrm{psia}(205 \mathrm{kPa})$. In the bottom figure, the maroon trace shows the background noise spectrum. The piezo gain changed between when the light shaded traces were recorded and the dark shaded traces were recorded. By normalizing to the noise in the unshaded bin near $450 \mathrm{kHz}$, the power spectral density from similar events become aligned at the frequencies relevant to the acoustic analysis. The bottom figure shades the bins used in the calculation of AP (see Equation 5.2). 


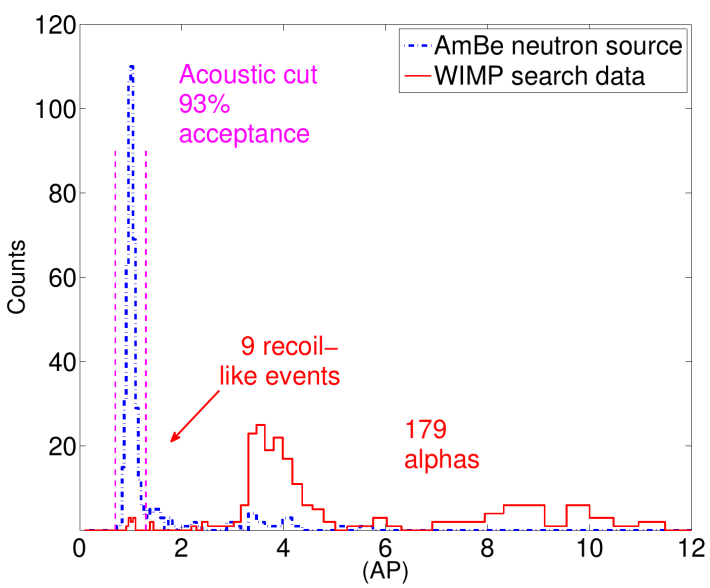

(a) $3 \mathrm{keV}$

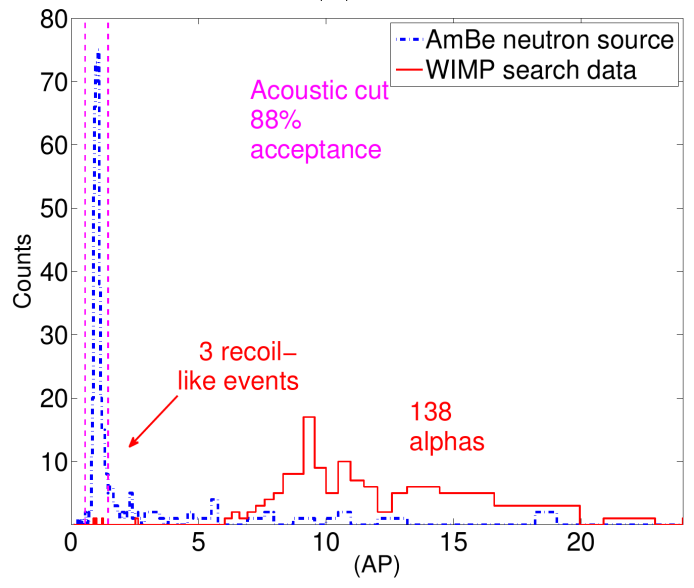

(c) $5.5 \mathrm{keV}$

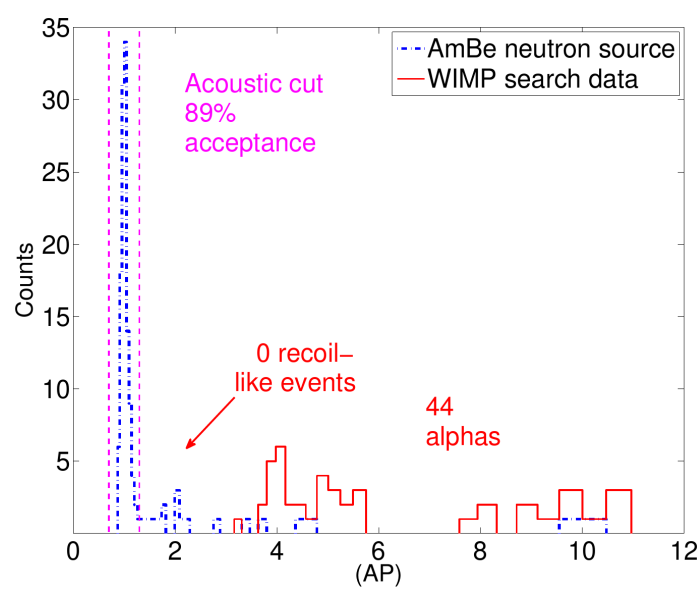

(b) $4 \mathrm{keV}$

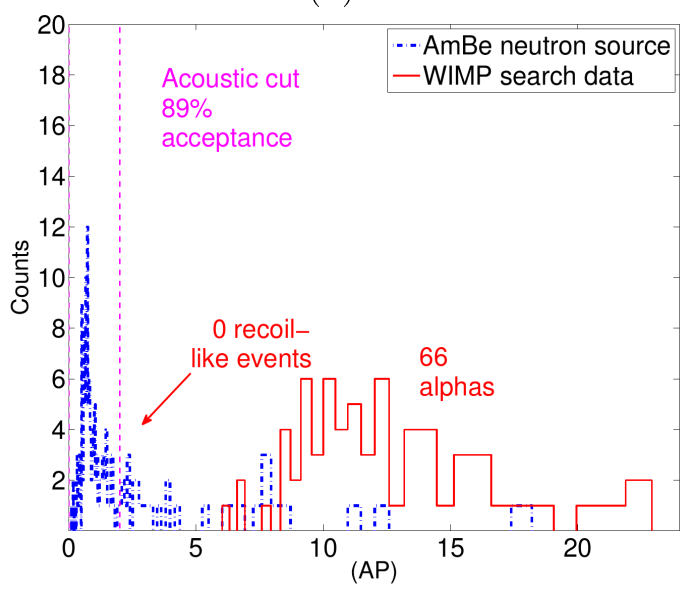

(d) $7 \mathrm{keV}$

Figure 5.12: Distributions of the acoustic parameter, AP, for dark matter search and neutron calibration data, from Harris, Lippincott, and Neilson (2014). The cuts for accepting nuclear recoil events are rescaled at each threshold to the width of the main peak of the neutron calibration distribution. The cut acceptances at each individual threshold are statistically consistent with the combined cut acceptance of $90.8 \pm 0.9 \%$. The combined acceptance is used for the analysis. 


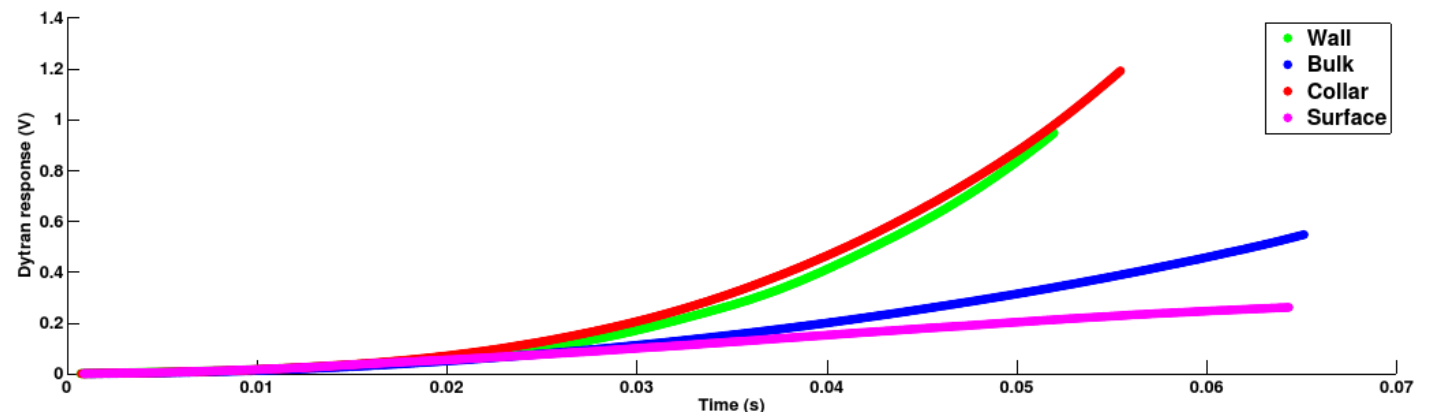

Figure 5.13: Typical pressure measurements of bubble growth using the Dytran fast pressure transducer. Bubble growth is slowed by the presence of the $\mathrm{C}_{3} \mathrm{~F}_{8}$ /water interface and is accelerated by the presence of the $\mathrm{C}_{3} \mathrm{~F}_{8}$ /glass interface. Collar events occur near the triple interface. The plot is by Chanpreet Amole and copied from Amole (2014).

the estimates of the efficiency and efficacy of this cut.

The Dytran fiducial cut rejects events that touch the boundaries of the active volume during early bubble growth. Bubbles that started growing within $1.5 \mathrm{~mm}$ of the boundaries of the inner volume are cut. As the geometry and bubble growth rates are nearly the same as for COUPP-4kg, the same $92 \pm 2 \%$ fiducial cut efficiency is applied with increased uncertainty. The COUPP-4kg fiducial volume measurement relied on measuring the proportion of neutron calibration events that passed the fiducial cut. As the background wall event rate in PICO-2L increased as the bulk event rate increased, the same calibration could not be repeated. However, studies of the expected bubble size at compression in PICO-2L find a fiducial efficiency consistent with the COUPP-4kg measurement.

Improvements to the hydraulic cart and DAQ in PICO-2L reduced the amount of time between bubble formation and compression to $50 \pm 10 \mathrm{~ms}$. Bubbles did not grow to sufficient size for the Dytran fiducial cut variable to be accurately measured. For PICO-2L runs after run 20131101_2, a delay of 10-40 ms was implemented before the start of the compression that increased the bubble growth sufficiently to allow the Dytran fiducial cut to be used at $3 \mathrm{keV}$ and $4 \mathrm{keV}$ Seitz thresholds. At the higher pressures required to reach higher thresholds, bubble growth was too slow to measure reliably, even with a reasonable trigger 


\begin{tabular}{lccc}
\hline Run type & $3 \mathrm{keV}, 40 \mathrm{~ms}$ & $3 \mathrm{keV}, 10 \mathrm{~ms}$ & $4 \mathrm{kev}, 10 \mathrm{~ms}$ \\
\hline Total events & 363 & 758 & 312 \\
Single bulk (hand scan) & 144 & 351 & 150 \\
Passed cuts & 147 & 357 & 153 \\
False negatives & 0 & 2 & 2 \\
False positives & 3 & 8 & 5 \\
Efficiency & $100 \%$ & $99.4 \%$ & $98.7 \%$ \\
Contamination & $1.4 \%$ & $2.0 \%$ & $3.1 \%$ \\
\hline
\end{tabular}

Table 5.3: Dytran cut efficiency and contamination at the given Seitz thresholds and compression delays (see text). Table from Amole (2014, Table 14). False negatives are events from the neutron calibration data that pass the XYZ fiducial cut and are identified as bulk bubbles in the hand scan, but fail the Dytran cut. False positives are bubbles that are identified as wall bubbles by the hand scan and pass the Dytran cut. The inefficiencies shown here are only due to misidentification. They are negligible compared to the inefficiency due to the reduction of the fiducial volume (see text).

delay.

When the Dytran fiducial cut was not available, a fiducial cut based on 3-D reconstruction of the bubble position in the stereo camera images was used, shown in Figure 5.14 . Three dimensional ray reconstruction mapped bubble positions in the inner volume to pixel positions on the cameras. The bubble chamber geometry used for the ray tracing was tuned to resolve bubbles from the inner vessel wall. As the bubble chamber had only one camera viewport, the stereo angle of the cameras was only $6^{\circ}$, far less than would be ideal. The bubble position along the axes of the cameras was poor. As the stereo angle became smaller towards the rear of active volume and water droplets on the quartz lenses the images of bubble towards the rear, the accuracy of the position reconstruction varied between $\pm 2 \mathrm{~mm}$ at the front face of the quartz jar to $\pm 5 \mathrm{~mm}$ at the rear face. The 3 -D reconstruction (XYZ) fiducial cut rejected events within $4 \mathrm{~mm}$ of the front of the cylindrical portion of the jar, $7 \mathrm{~mm}$ from the rear, $5 \mathrm{~mm}$ from the wall of the semi-spherical part of the jar, and $10 \mathrm{~mm}$ from the triple interface region. This cut retains $82 \pm 1 \%$ of the active volume while contributing a $1 \%$ contamination from mis-identified boundary events. 

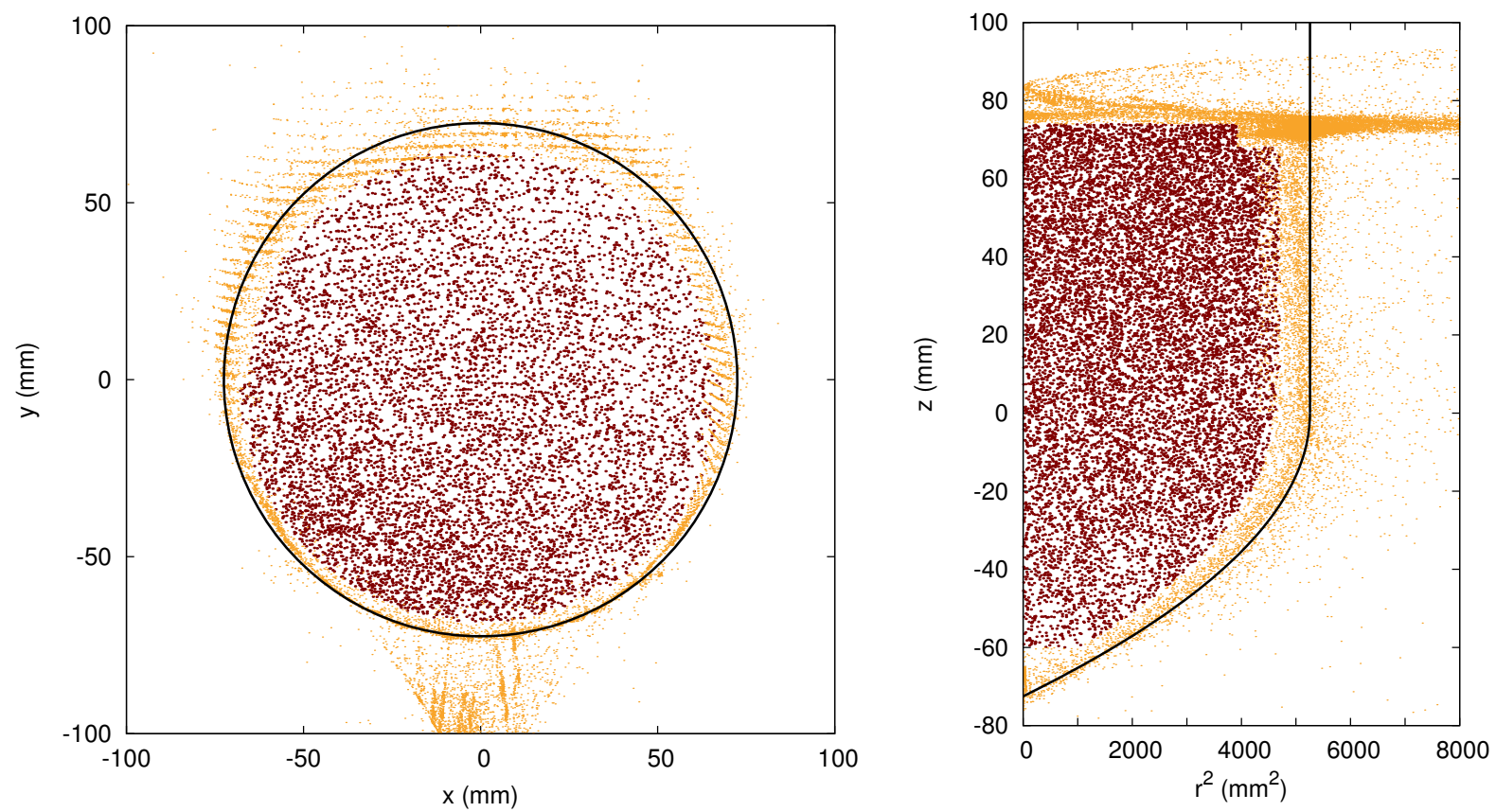

Figure 5.14: 3-D position reconstruction of all PICO-2L bubbles, both during dark matter search and neutron calibrations runs. Events passing the XYZ fiducial cut are shown in maroon while events failing the cut are shown in yellow. Black lines show the position of the vessel walls. The plot on the left shows the bubble reconstructed positions for $0<z<74 \mathrm{~mm}$ projected into the horizontal plane while the plot on the right shows the position of all bubbles projected into cylindrical coordinates. The DAQ cameras are located in $-y$, the bottom of the left plot.

While dark matter is expected to produce recoil events with equal probability over the entire target, ${ }^{241} \mathrm{Am} / \mathrm{Be}$ neutrons do not. Attenuation of $\mathrm{Am} / \mathrm{Be}$ neutrons through the active volume increased the probability of bubbles forming in regions subject to the fiducial cut. Using a neutron simulation of PICO-2L, the expected distribution of single bubble events is calculated assuming a step threshold for both carbon and fluorine recoils in $\mathrm{C}_{3} \mathrm{~F}_{8}$ at $4 \mathrm{keV}$. The cut efficiencies were recalculated for Am/Be data using the same fiducial geometry. An efficiency of $70 \% \pm 2 \%$ is found for the $3-\mathrm{D}$ position reconstruction cut while an efficiency of $87 \pm 3 \%$ is found for the Dytran fiducial cut. 


\begin{tabular}{cccccc}
\hline Run Condition & Start Date & End Date & $\begin{array}{c}\text { Threshold } \\
(\mathrm{keV})\end{array}$ & $\begin{array}{c}\text { Pressure } \\
(\mathrm{psia})\end{array}$ & $\begin{array}{c}\text { Temperature } \\
\left({ }^{\circ} \mathrm{C}\right)\end{array}$ \\
\hline $4 \mathrm{keV}(\mathrm{XYZ})$ & 28-Oct-2013 & 2-Nov-2013 & $4.35 \pm 0.25 \pm 0.26$ & $31.09 \pm 0.02$ & $12.22 \pm 0.20$ \\
$4 \mathrm{keV}$ & 2-Nov-2013 & 9-Nov-2013 & $4.35 \pm 0.25 \pm 0.26$ & $31.09 \pm 0.02$ & $12.22 \pm 0.20$ \\
$3 \mathrm{keV}$ & 10-Nov-2013 & 8-Jan-2014 & $3.24 \pm 0.15 \pm 0.22$ & $31.10 \pm 0.02$ & $14.18 \pm 0.03$ \\
$5.5 \mathrm{keV}$ & 24-Feb-2014 & 2-May-2014 & $6.14 \pm 0.33 \pm 0.33$ & $36.08 \pm 0.02$ & $11.58 \pm 0.04$ \\
$6.5 \mathrm{keV}$ & 24-Mar-2014 & 28-Mar-2014 & $6.75 \pm 0.42 \pm 0.36$ & $34.79 \pm 0.02$ & $10.61 \pm 0.08$ \\
$7 \mathrm{keV}$ & 21-Feb-2014 & 19-May-2014 & $8.08 \pm 0.48 \pm 0.40$ & $41.08 \pm 0.02$ & $11.58 \pm 0.04$ \\
\hline
\end{tabular}

Table 5.4: Thermodynamic parameters for each PICO-2L run condition. Runs are labeled by their nominal Seitz threshold energy. The $4 \mathrm{keV}$ data is separated into runs using the 3 -D reconstruction fiducialization (XYZ) and the Dytran pressure growth fiducialization. Two uncertainties are given for each threshold. The first is the propagated uncertainty in temperature and pressure. In addition to the statistical errors shown, a $\pm 0.3^{\circ} \mathrm{C}$ systematic temperature uncertainty and $\pm 0.7 \mathrm{psi}( \pm 5 \mathrm{kPa})$ pressure uncertainty are applied to the first term of the threshold uncertainty. The second threshold uncertainty is due to uncertainties in the thermodynamic properties of $\mathrm{C}_{3} \mathrm{~F}_{8}$.

\begin{tabular}{cccc}
\hline Condition & $\begin{array}{c}\text { Exposure } \\
\text { (kg-days) }\end{array}$ & Candidate Events & Alpha Events \\
\hline $4 \mathrm{keV}$ & 16.8 & 0 & 44 \\
$3 \mathrm{keV}$ & 74.8 & 9 & 179 \\
$5.5 \mathrm{keV}$ & 82.2 & 1 & 138 \\
$7 \mathrm{keV}$ & 37.8 & 0 & 66 \\
\hline \hline
\end{tabular}

Table 5.5: PICO-2L dark matter search exposure and counts after all cuts and efficiencies are applied.

\subsection{Data Sets}

The PICO-2L bubble chamber was operated at the thresholds shown in Table 5.4. Both dark matter search data (Table 5.5) and neutron calibrations (Table 5.6) were performed at each threshold, although all of the dark matter data at $6.5 \mathrm{keV}$ was cut due to low compression pressure. The exposures in these tables include all relevant cut efficiencies and their uncertainties. Dark matter cross-sections limits will be set in Chapter 9 using this data and the bubble nucleation efficiency functions calculated in the next chapter. 


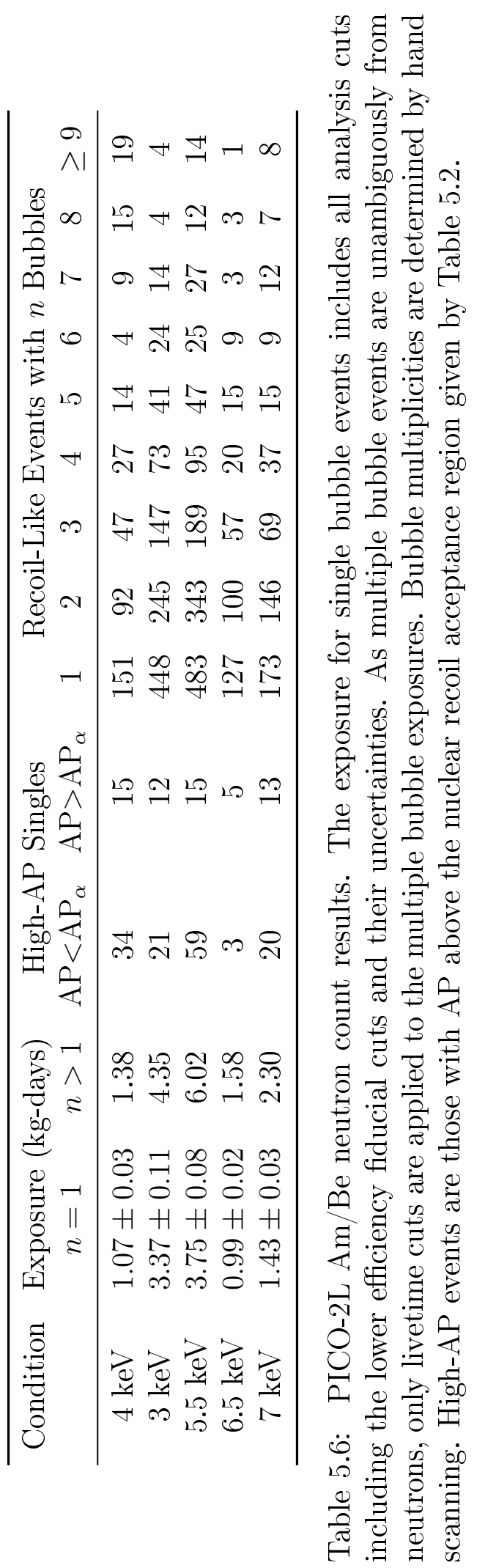




\section{Chapter 6 Nuclear Recoil Calibration Results}

\section{1 $\mathrm{CF}_{3} \mathrm{I}$ Calibrations}

This analysis will search for upper and lower limits to the bubble nucleation efficiency at a set of target threshold energies, as per the procedure given in Section 3.5. From these limits, a family of efficiency models consistent with the calibration data will be generated for calculating dark matter limits. Five target energies below $30 \mathrm{keV}$ corresponding to the highest precision measurements of the event rates from ${ }^{241} \mathrm{Am} / \mathrm{Be}$ neutrons in COUPP$4 \mathrm{~kg}$ were selected for the analysis. Data at these energies provide the best constraints on possible efficiencies models. To compare these ${ }^{241} \mathrm{Am} / \mathrm{Be}$ and other measurements at different Seitz thresholds, the energies of simulated recoils were scaled to the threshold energy of an ${ }^{241} \mathrm{Am} / \mathrm{Be}$ measurement. Measurements with Seitz threshold energies within $8 \%$ of a given ${ }^{241} \mathrm{Am} / \mathrm{Be}$ Seitz threshold energy were compared. The data was compared following the procedure from Section 3.5 with the details of the modeled distribution of count rates for each experiment described below.

\subsubsection{AmBe Measurements}

All high-energy neutron data used to measure the nucleation efficiency of $\mathrm{CF}_{3} \mathrm{I}$ in this analysis come from measurements using COUPP-4kg at SNOLAB as described in Drew Fustin's thesis (Fustin 2012). The count rates in the presence of the ${ }^{241} \mathrm{Am} / \mathrm{Be}$ and ${ }^{252} \mathrm{Cf}$ sources, when placed at 36" and 54" respectively, was obtained from Table 5.4 of Drew Fustin's thesis, and shown here in Table 6.1. The simulation of these measurements uses Drew Fustin's simulation geometry with the updated neutron scattering libraries and simulation 


\begin{tabular}{|c|c|c|c|c|c|c|c|}
\hline \multirow[t]{3}{*}{ Data Set } & \multirow{3}{*}{$\begin{array}{c}\text { Threshold } \\
\text { (keV) }\end{array}$} & \multirow{3}{*}{$\begin{array}{l}\text { Exposure } \\
\text { (kg-days) }\end{array}$} & \multicolumn{5}{|c|}{ Events with $n$ bubbles } \\
\hline & & & \multicolumn{2}{|l|}{1} & 2 & 3 & $\geq 4$ \\
\hline & & & high-AP & recoil & & & \\
\hline $\mathrm{DM}-34^{\circ} \mathrm{C}$ & $15.83_{-1.31}^{+1.46}$ & 393.64 & 1734 & 8 & 1 & 0 & 0 \\
\hline $\mathrm{DM}-37^{\circ} \mathrm{C}$ & $11.21_{-0.87}^{+0.97}$ & 88.41 & 424 & 6 & 0 & 2 & 0 \\
\hline $\mathrm{DM}-40^{\circ} \mathrm{C}$ & $8.00_{-0.61}^{+0.67}$ & 70.98 & 328 & 6 & 0 & 0 & 0 \\
\hline AmBe- $36^{\prime \prime}-34^{\circ} \mathrm{C}-30$ psia & $15.92_{-1.31}^{+1.46}$ & 6.09 & 41 & 556 & 162 & 30 & 7 \\
\hline AmBe- $36^{\prime \prime}-34^{\circ} \mathrm{C}-40$ psia & $26.67_{-2.68}^{+3.06}$ & 2.00 & \multicolumn{2}{|c|}{165} & 29 & 8 & 1 \\
\hline AmBe- $36^{\prime \prime}-34^{\circ} \mathrm{C}-50$ psia & $51.92_{-6.32}^{+7.48}$ & 3.00 & \multicolumn{2}{|c|}{170} & 12 & 0 & 0 \\
\hline AmBe- $36^{\prime \prime}-34^{\circ} \mathrm{C}-60$ psia & $128.13_{-20.98}^{+26.63}$ & 3.33 & \multicolumn{2}{|c|}{70} & 2 & 1 & 0 \\
\hline AmBe- $36^{\prime \prime}-37^{\circ} \mathrm{C}-30$ psia & $11.27_{-0.92}^{+1.02}$ & 4.23 & 29 & 384 & 105 & 31 & 11 \\
\hline AmBe- $36^{\prime \prime}-37^{\circ} \mathrm{C}-35$ psia & $14.10_{-1.19}^{+1.33}$ & 1.69 & \multicolumn{2}{|c|}{150} & 29 & 11 & 2 \\
\hline AmBe- $36^{\prime \prime}-40^{\circ} \mathrm{C}-30$ psia & $8.01_{-0.60}^{+0.66}$ & 2.07 & 13 & 179 & 74 & 19 & 7 \\
\hline AmBe- $36 "-41^{\circ} \mathrm{C}-30$ psia & $7.21_{-0.53}^{+0.58}$ & 0.17 & 1 & 20 & 8 & 2 & 2 \\
\hline Cf252-54"-34 ${ }^{\circ} \mathrm{C}-30$ psia & $15.99_{-1.32}^{+1.46}$ & 29.58 & 131 & 479 & 130 & 32 & 10 \\
\hline Cf252-54"-34 ${ }^{\circ} \mathrm{C}-40$ psia & $26.78_{-2.62}^{+2.98}$ & 10.81 & \multicolumn{2}{|c|}{194} & 26 & 8 & 1 \\
\hline
\end{tabular}

Table 6.1: COUPP-4kg ${ }^{241} \mathrm{Am} / \mathrm{Be}$ and ${ }^{252} \mathrm{Cf}$ run counts and exposures retrieved from Table 5.4 of Drew Fustin's thesis (Fustin 2012).

procedures described in Section 3.2. For the ${ }^{241} \mathrm{Am} / \mathrm{Be}$ simulation, $10^{8}$ neutrons and $5 \times 10^{4}$ recoils were generated. The ${ }^{252} \mathrm{Cf}$ simulation generated $7.53 \times 10^{7}$ neutrons and $4 \times 10^{5}$ recoils.

Events from ${ }^{19} \mathrm{~F}(n, \alpha)$ reactions during a neutron calibration may be acoustically cut (see Section 7.4). With COUPP-4kg, the number of bulk single bubble events cut using the acoustic measurement was measured both in the presence and absence of neutron sources. The rate with the sources in place, 84 events in 12.56 kg-days, was slightly larger than, but consistent with the sum of the background rate, $4.50 \pm 0.16$ events per kg-day, plus the expected number of charged particle reactions, totaling $6.51 \pm 0.62$ events per kg-day. As speculated in Section 7.4 an excess of high Acoustic Parameter (AP) events in PICO2L and COUPP-4kg may be due to energetic elastic recoils. An AP cut was applied to 
simulated recoil events with energies greater than $560 \mathrm{keV}$. In combination with the cut on charged particle events, these two cuts were selected to have a total efficiency of $95.9 \%$, equal to the measured recoil-like AP cut found in Drew Fustin's thesis. An addition 85.07\% cut efficiency is applied to all simulated single bubble events. For event rates without an acoustic cut applied, the $\alpha$ event rate is added to the expected rate.

Using the results of Section 6.2.2 for the PICO-2L ${ }^{241} \mathrm{Am} / \mathrm{Be}$ simulations, a $30 \%$ Gaussian uncertainty to the ${ }^{241} \mathrm{Am} / \mathrm{Be}$ recoil rate is applied. The uncertainty is reduced to $20 \%$ for the ${ }^{252} \mathrm{Cf}$ simulations as both the uncertainties in the energy spectrum and the amount of moderating material are reduced. As large uncertainties are applied to the total event rate, the ratio of multiple bubble events to single bubble events is used in the fit instead of the multiple bubble event rate.

Poisson statistics are combined with other Gaussian uncertainties in the models of the count rate distribution. For the ratio of multiple bubble events to single bubble events from high-energy neutron recoils, the correlated Gaussian uncertainty of $3 \%$ for double bubble events, $6 \%$ for triple bubble events, and $9 \%$ for higher multiplicity events due to the uncertainty in the ${ }^{19} \mathrm{~F}(n, \mathrm{el})$ cross-section (see Section 3.2 is combined with the $\sqrt{N+1}$ uncertainty from the single bubble counting statistics. Where both ${ }^{241} \mathrm{Am} / \mathrm{Be}$ and ${ }^{252} \mathrm{Cf}$ data are available at the same threshold energy, the multi-bubble count rates and expected rates from the two datasets are summed. The Gaussian uncertainties of the expected event rates are assumed to be fully correlated. The joint cumulative distribution function over all multiplicities of a Poisson distribution with the randomly distributed expectation value is numerically calculated by approximating the Gaussian distribution using 100 expectation values spaced in their percent point function. See Appendix $\mathrm{C}$ for the code used in this calculation. 


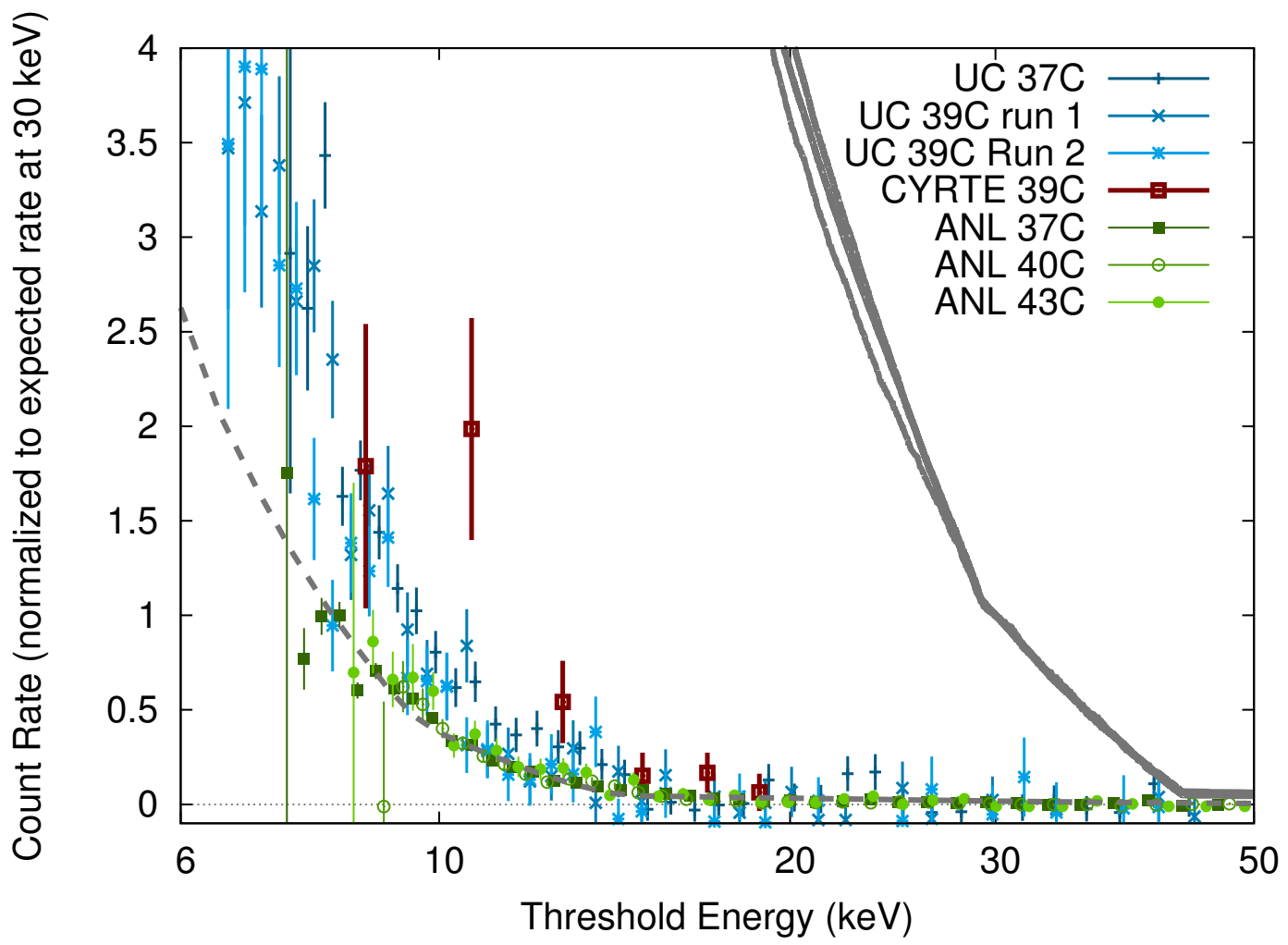

Figure 6.1: Comparison of the background subtracted event rates from different ${ }^{88} \mathrm{Y} / \mathrm{Be}$ calibration experiments normalized to the simulated recoil rate at $30 \mathrm{keV}$. The solid gray lines are the simulated recoil rate for each bubble chamber while the dashed line is the event rate predicted by the best fit efficiency model calculated in Section 6.1.3.

\subsection{2 $\mathrm{Y} / \mathrm{Be}$ Measurements}

Simulations for all three $\mathrm{CF}_{3} \mathrm{I}^{88} \mathrm{Y} / \mathrm{Be}$ calibrations were made with at least $10^{7}$ source particles and between $2 \times 10^{4}$ and $1.2 \times 10^{5}$ recoils above $1 \mathrm{keV}$ generated in each simulation. Separate simulations were performed for each fill of each bubble chamber. For each data set, the simulation was normalized to the total ${ }^{88} \mathrm{Y} /$ Be source exposure. Figure 6.1 compares the event rate observed in each experiment normalized to the simulations. While both single and double bubble event rates were measured for the STAR bubble chamber, only the total event rates are used for measurements using the three bubble chambers.

Over the $6^{\circ} \mathrm{C}$ temperature spread shown in Figure 6.1, the observed count rate does not 
appear to depend strongly on temperature independently of the Seitz threshold energy. In $\mathrm{CF}_{3} \mathrm{I}$ near $40^{\circ} \mathrm{C}$, the critical radius at fixed Seitz threshold changes by $0.5 \% /{ }^{\circ} \mathrm{C}$, so that a several percent effect may be expected. As no change in event rate versus temperature is observed and the expected effect is smaller than the uncertainties in the measurements, it is ignored.

The recoil energy distribution of simulated events in Figure 6.1 shows two kinks. At high recoil energies, the simulated rate is caused by recoils of $950 \mathrm{keV}$ neutrons from the $0.71 \%$ branching ratio $2734 \mathrm{keV}$ line from ${ }^{88} \mathrm{Y}$ decays. In the presence of $152 \mathrm{keV}$ neutrons, carbon and fluorine recoils have maximum recoil energies of $43 \mathrm{keV}$ and $29 \mathrm{keV}$ respectively. The two kinks are at these maximum recoil energies.

While far more source neutrons recoiled in the calibration bubble chambers than background environmental neutrons, the observed event rate from background neutrons is significant at thresholds where the efficiency for detecting source neutrons is low. As the background neutrons are at $\mathrm{MeV}$ scale energies, the rate of background events is both expected and observed to only vary slowly with energy. Background rate measurements across many different thresholds in a given experiment were combined in order to improve the statistics of the measurements.

For the STAR bubble chamber, an exponentially falling background rate,

$$
R_{b g}=a \cdot \exp \left(-E_{t h r} / b\right)
$$

was modeled as shown in Figure 6.2. A constant term to the model was added only for single bubble events. The threshold energy scale of the exponential, $b$, was floated for the single bubble event rate and fixed for the double bubble rate. The single bubble event rate fit found an energy scale of $100 \mathrm{keV}$, approximately one third of the energy scale that would be expected from $(\alpha, n)$ or spontaneous fission neutrons in surrounding materials. This 
$1 / 3$ factor is consistent with event rates versus simulation rates shown in Figure 6.1. For such a background neutron source, the energy scale for double bubble events is expected to be approximately a factor of 2.2 smaller $(b=100 \mathrm{keV} / 2.2=45 \mathrm{keV})$. A $30 \%$ uncertainty on the background rate from single bubble events and a $100 \%$ uncertainty on the rate of double bubble events was applied to account for statistics and possible time variation in the background. After this background subtraction, the single and double bubble event rates are summed in the analysis. A large neutron background was observed in November due to the operation of ${ }^{14} \mathrm{C}$ ion beams at the ATLAS accelerator in the adjacent building. Neutron generation from the accelerator during the analyzed runs was much lower.

The background from the University of Chicago bubble chamber was measured with high statistics. The background rate was rebinned in order to improve the statistical uncertainty in any given bin as shown in Figure 6.3. Only Gaussian statistical uncertainties are considered in the background rate.

Due to the low statistics of the CYRTE $\mathrm{CF}_{3} \mathrm{I}$ data, a constant background rate of $0.0018 \pm 0.0006$ counts per second was used for all thresholds. The uncertainty is purely statistical.

As with the ${ }^{241} \mathrm{Am} / \mathrm{Be}$ multiple bubble events, Poisson and Gaussian statistics were combined. The expected and measured rates from all measurements were independently summed. A set of 100 values for this total expected rate was generated spanning possible values over their Gaussian distribution. The Poissonian probability of measuring an equal or, greater or lesser number of counts, depending on whether the upper or lower limit is being sought, was calculated for each possible expected rate. The probabilities were then averaged over the uncertainty of the expected rates. For each measurement, the uncertainties from background rates, simulations, and source strengths are assumed to be independent. Between measurements in the same bubble chamber, the total uncertainties are assumed to 

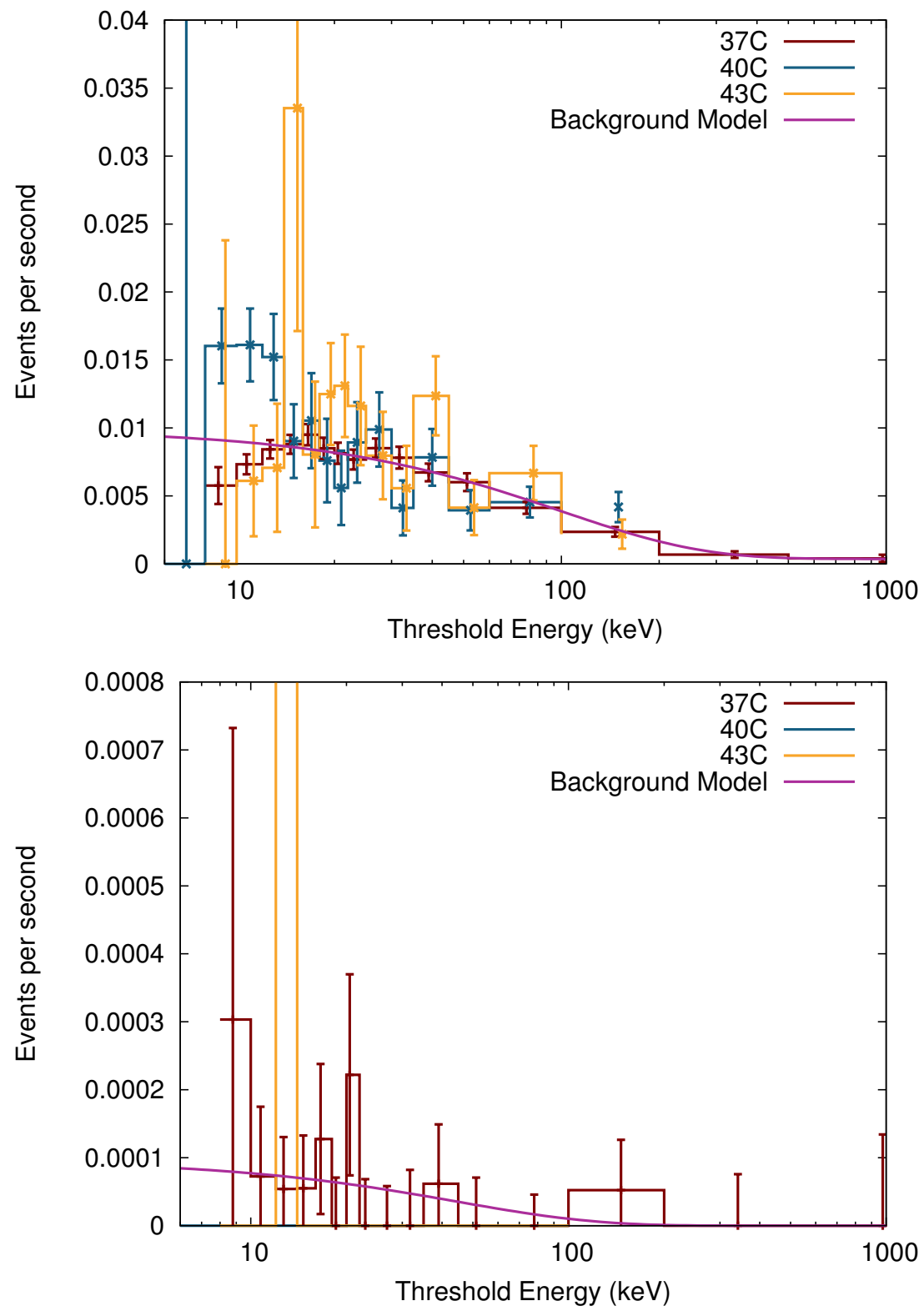

Figure 6.2: The measured and modeled single bubble (above) and double bubble (below) event rate in the STAR bubble chamber. An exponentially falling efficiency model was fitted to the measured data. 


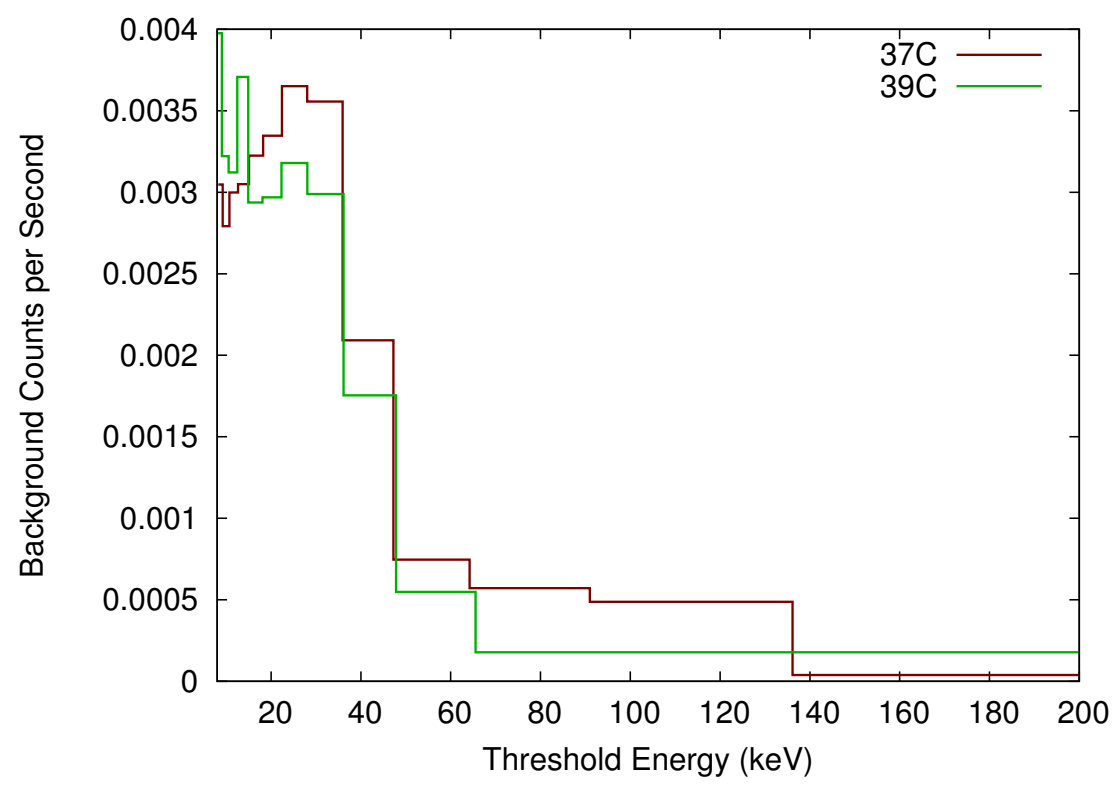

Figure 6.3: The measured background event rates in the University of Chicago bubble chamber. Wider energy bins are used as compared to the ${ }^{88} \mathrm{Y} /$ Be source data.

be fully correlated.

To account for significant Seitz threshold uncertainties, the total probability were calculated at the nominal Seitz threshold and at $\pm 1 \sigma$ values of the Seitz threshold. A weighed sum of these probabilities is used to calculate the likelihood. The weights correspond to the area of the Gaussian distribution below, above, and between $-0.5 \sigma$ and $0.5 \sigma$.

\subsubsection{Efficiency Limits}

To find a bubble nucleation efficiency function given the calibration data, the method described in Section 3.5 is used. The details of some practical issues, including binning, were omitted in that section.

To calculate the event rate for a given efficiency model, a loop over the events from each simulation is needed. This must be repeated for every test efficiency model over all the data. The calculation was performed at the edges of the bins shown in Figure 3.9, Figure 6.4, and 


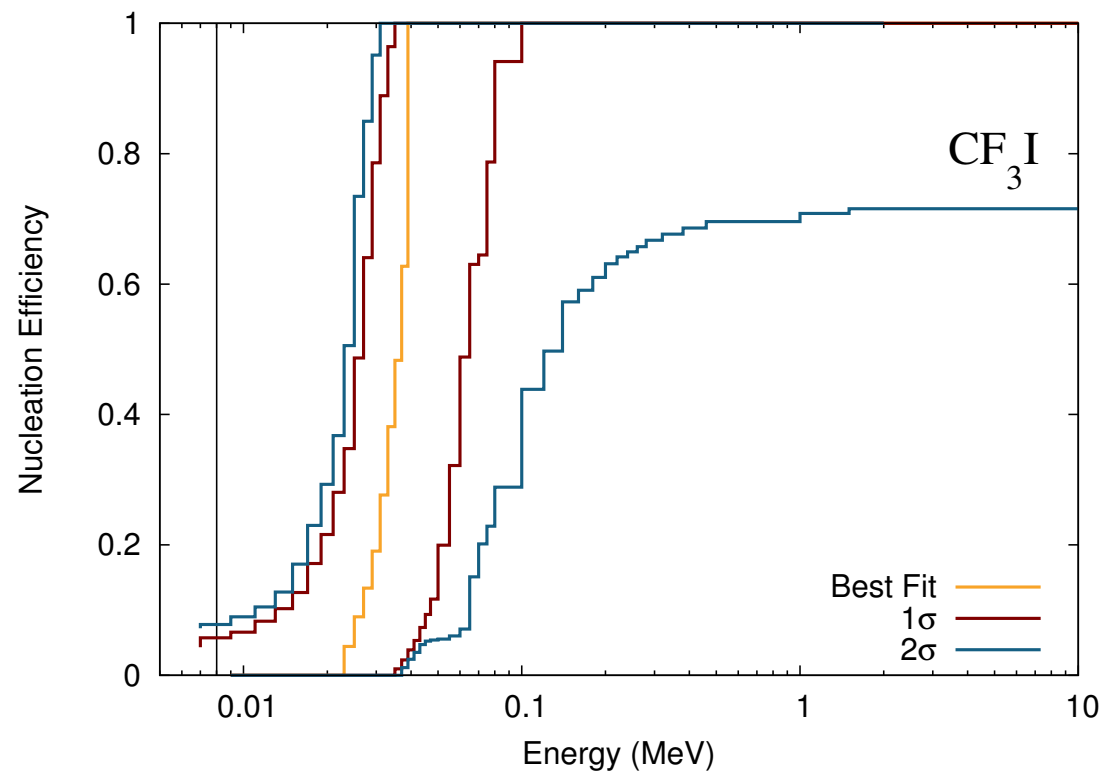

(a) $8.01 \mathrm{keV}$

Figure 6.4: Allowed values of the bubble nucleation efficiency of carbon and fluorine recoils in $\mathrm{CF}_{3} \mathrm{I}$ at the specified Seitz threshold energies. The conservative fluorine efficiency functions used to set dark matter limits in Amole et al. (2015) are also plotted. Figure continues on the next two pages.

other efficiency function plots in this thesis. Where upper limits are shown, the calculation is at the highest energy of the bin while for lower limits, the calculation is at the lowest energy of the bin. The efficiency models used to calculate dark matter limits are shown as lines between these calculated points.

While the total bubble rate could be calculated by binning the simulated recoils in energy and multiplying by the efficiency function, the combinatoric calculation of the event rate from multiple scattering events requires a loop over all simulated recoils. A hybrid calculation was performed where simulated single scatter events and the efficiency function were binned and multiplied and a loop was performed over multiple scattering events.

To set limits, the consistency of an efficiency function with the calibration data was measured by using the likelihood function of obtaining count rates more extreme than the 


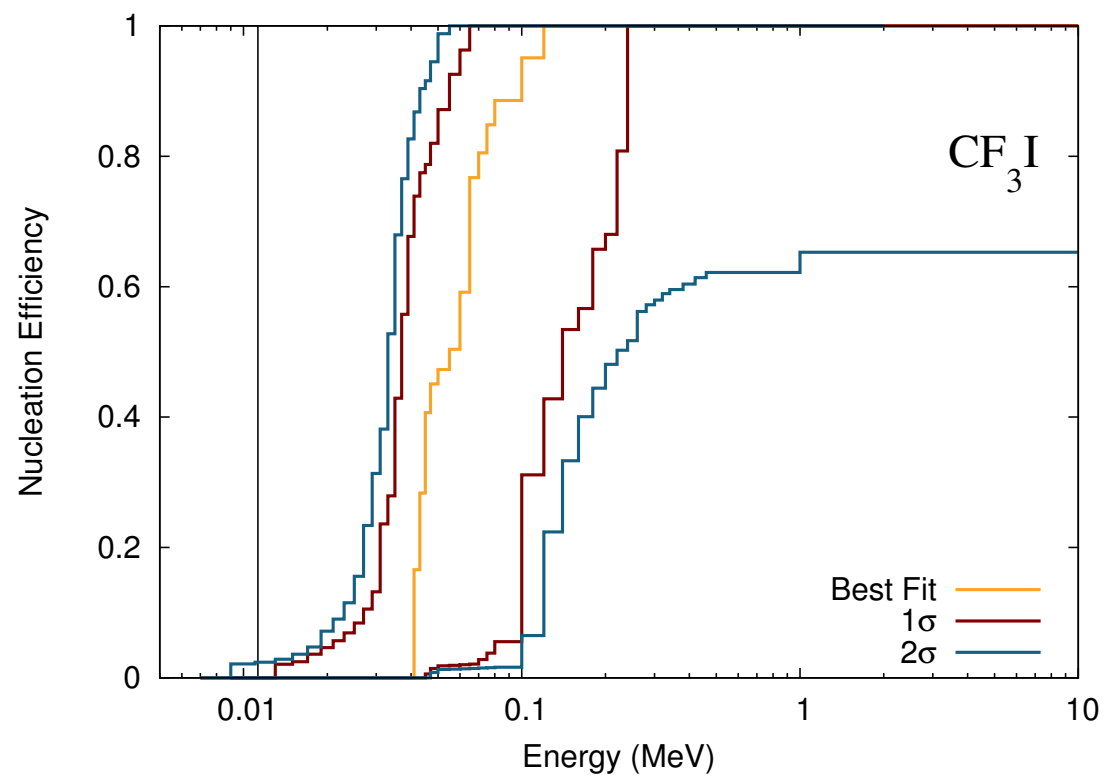

(b) Figure 6.4 continued. $11.27 \mathrm{keV}$

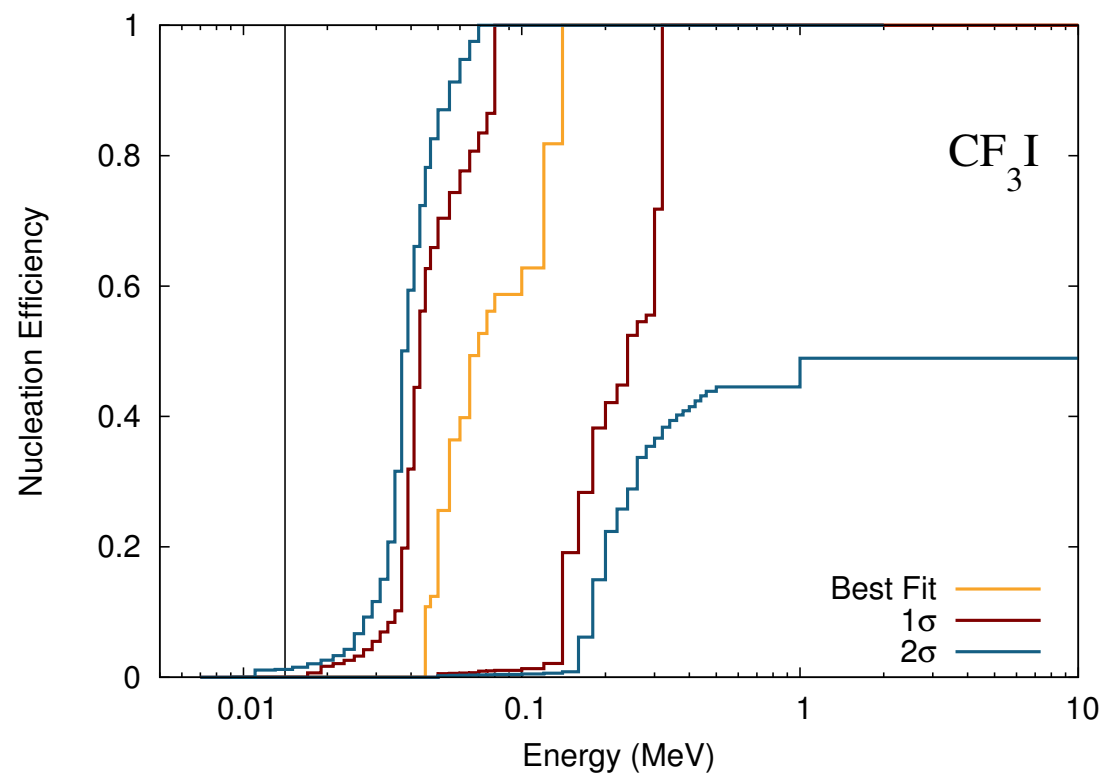

(c) Figure 6.4 continued. $14.10 \mathrm{keV}$ 


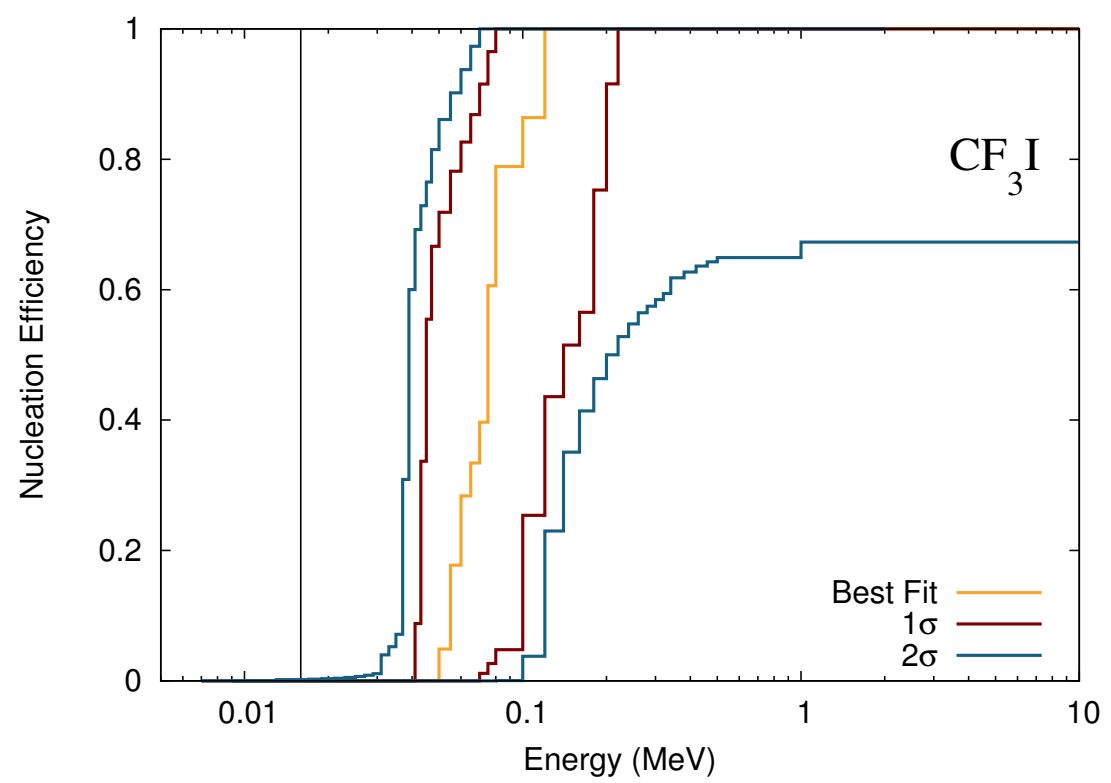

(d) Figure 6.4 continued. $15.92 \mathrm{keV}$

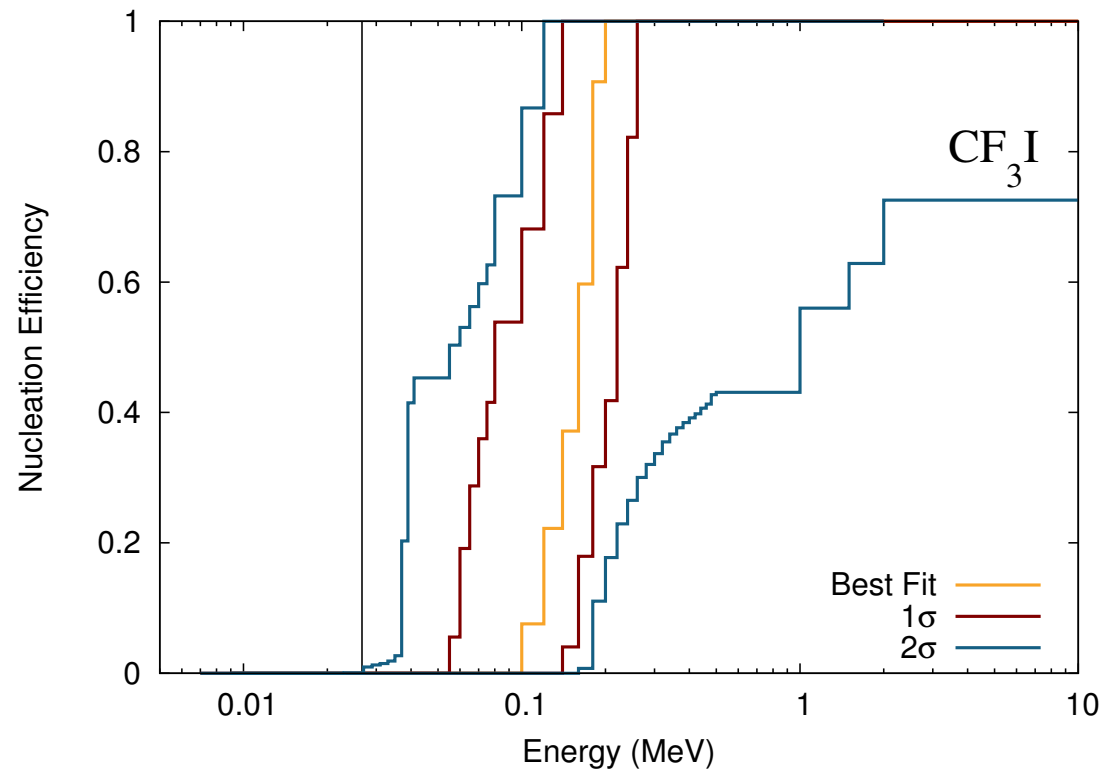

(e) Figure 6.4 continued. $26.67 \mathrm{keV}$ 


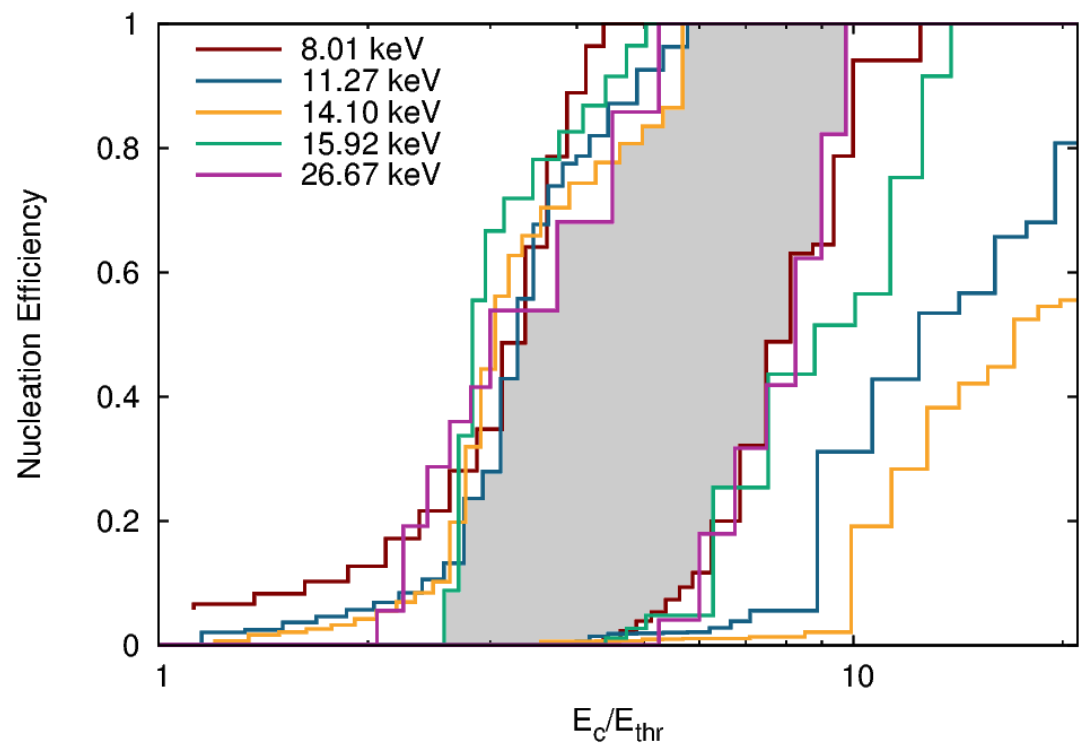

Figure 6.5: Overlaid $1 \sigma$ allowed efficiency function regions from Figure 6.4. The shaded region is allowed at all 5 threshold energies.

ones measured. To set the lower limit, the likelihood is the cumulative distribution function at the observed number of counts, while for the upper limit it is one minus the cumulative distribution function. The likelihood at which the upper limit curve equals the lower limit curve, $\mathcal{L}_{\max }$, was found, and the upper and lower limits at $\mathcal{L}_{\max } / e^{0.5}$ and $\mathcal{L}_{\max } / e^{2}$ likelihood ratios was calculated. These correspond approximately to $1 \sigma$ and $2 \sigma$ confidence intervals of the likelihood ratio test with the efficiency at $E_{s}$ as the only degree of freedom.

The calculated upper and lower limits for $\mathrm{CF}_{3} \mathrm{I}$ are shown in Figure 6.4. Figure 6.5 shows that when the the allowed efficiency function regions are scaled to their Seitz threshold energies, the allowed regions overlap. An efficiency model of the form $\eta\left(E_{r} / E_{c}\right)$ will be consistent with the calibration data. A search for allowed efficiency functions combining the available data at thresholds between $7 \mathrm{keV}$ and $20 \mathrm{keV}$ was performed. Viable dark matter limit functions compatible with the calculated efficiency bounds are shown in Figure 6.6. These functions jump between the lower and limit efficiency functions to approximate effi- 


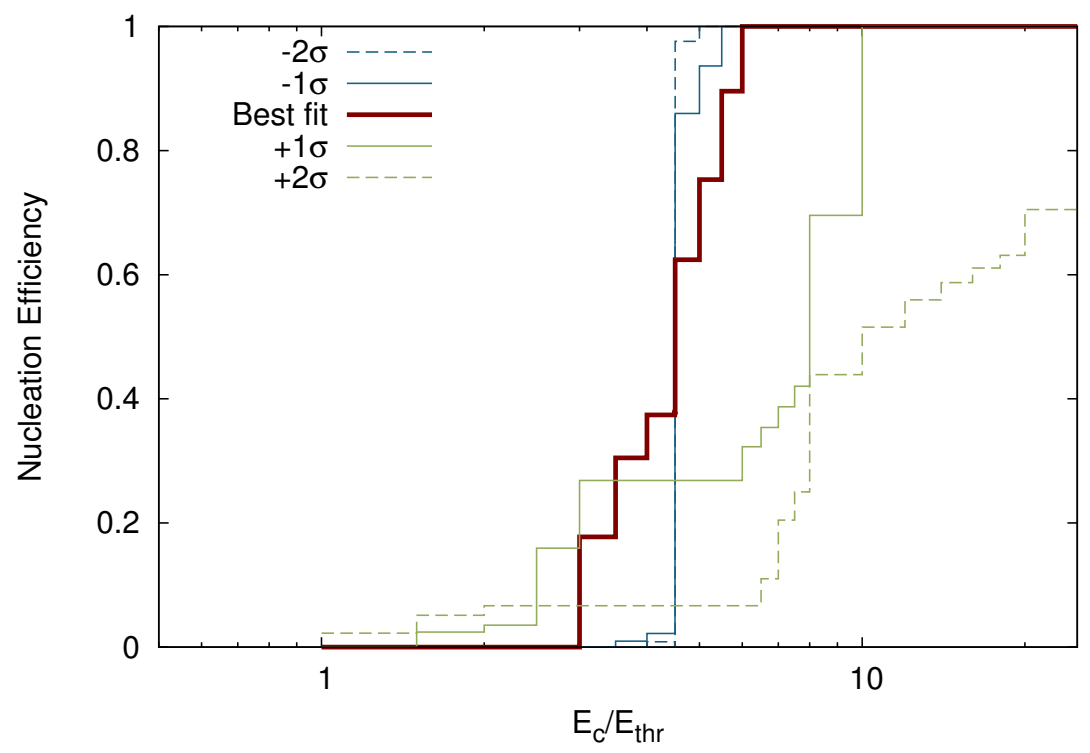

Figure 6.6: $\mathrm{CF}_{3} \mathrm{I}$ bubble nucleation efficiency functions compatible at $>1 \sigma$ and $>2 \sigma$ with the calibration data between $7 \mathrm{keV}$ and $20 \mathrm{keV}$. Functions that predict a low dark matter sensitivity $(-1 \sigma$ and $-2 \sigma)$ follow the lower limit bubble nucleation efficiency at low recoil energies and jump to the upper limit efficiency at high recoil energies. Functions that predict a high dark matter efficiency jump from the upper limit to the lower limit. The position of the jumps is set to maximize the consistency with calibration data. Effectively, these functions show how the shape of the efficiency function can varied while maintaining consistency with measured count rates during calibrations. These functions may be underreporting the bubble nucleation efficiency given comparisons (see text). 


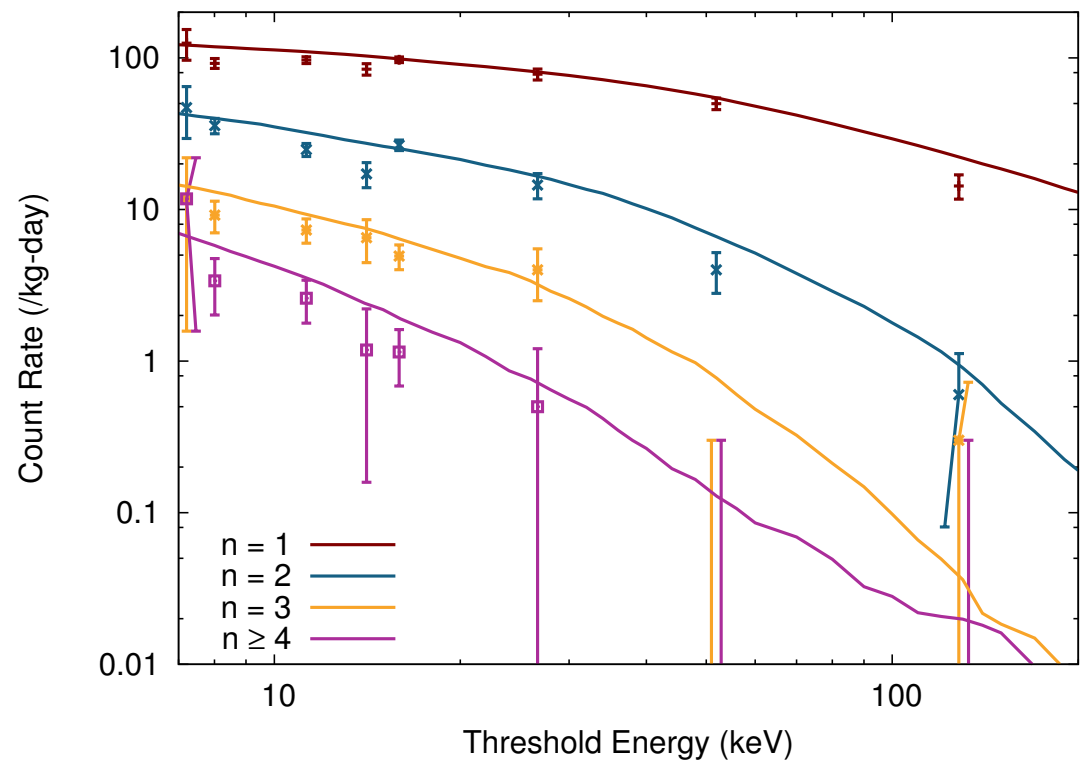

Figure 6.7: The observed and predicted event rates from ${ }^{241} \mathrm{Am} / \mathrm{Be}$ calibrations at 36 " in COUPP-4kg. This plot may be compared to the Figure 6.9 in Fustin (2012). A 85.07\% analysis efficiency is applied to the predicted single bubble event rate.

ciency functions with the highest and lowest sensitivity to low-mass dark matter particles while remaining consistent with the calibration data.

The best fit efficiency function significantly underpredicts the observed rate of recoils from CYRTE and the University of Chicago bubble chamber shown in Figure 6.1 while overpredicting the observed rate in COUPP-4 $\mathrm{kg}{ }^{241} \mathrm{Am} / \mathrm{Be}$ calibrations shown in Figure 6.7. While these count rates appear discrepant, little inconsistency is reported by the fit. The most extreme inconsistency reported, between the predicted and measured University of Chicago bubble chamber count rates in Figure 6.1 gives a p-value of 0.09 for obtaining the observed count rates given the prediction after all statistical and systematic uncertainties are considered. This seems low, and a preliminary independent fit of the $\mathrm{CF}_{3} \mathrm{I}$ calibration data by Eric Dahl is reporting greater $\mathrm{CF}_{3} \mathrm{I}$ efficiency than that shown here, consistent with the University of Chicago data. 


\section{2 $\quad \mathrm{C}_{3} \mathrm{~F}_{8}$ Calibrations}

The treatment of probabilities for the $\mathrm{C}_{3} \mathrm{~F}_{8}$ calibration is similar to that for the $\mathrm{CF}_{3} \mathrm{I}$ calibration. Namely, the systematic uncertainties between different data sets from the same calibration experiment are assumed to be $100 \%$ correlated, while the uncertainties between experiments are uncorrelated. For this purpose, the CYRTE ${ }^{88} \mathrm{Y} / \mathrm{Be}$ and ${ }^{124} \mathrm{Sb} / \mathrm{Be}$ data are considered to be separate experiments. The PICO-2L ${ }^{241} \mathrm{Am} / \mathrm{Be}$ calibration measures the multiplicity ratio before calculating probabilities while the absolute rate of events with different bubble multiplicities is used for the monoenergetic neutron calibrations. Other experimental details are described below.

\subsubsection{University of Montreal Neutron Beam}

Calibrations using the monochromatic ${ }^{51} \mathrm{~V}(p, n)$ neutron beam at the University of Montreal were performed as described in Dahl and Jin (2014) with $40 \mathrm{keV}, 61 \mathrm{keV}$, and $97 \mathrm{keV}$ neutrons. For each neutron energy, an MCNPX-Polimi simulation of the beam line and the PICO- 0.1 bubble chamber was made to determine the neutron recoil spectrum in the $\mathrm{C}_{3} \mathrm{~F}_{8}$. A simplified PICO-0.1 geometry was simulated using both MCNPX-Polimi and by Miaotianzi Jin using Geant4. The results between the simulations was discrepant by $10 \%$. Further comparisons with neutrons emitted within a sphere of $\mathrm{C}_{3} \mathrm{~F}_{8}$ were also discrepant, with the MCNPX-Polimi simulation producing a recoil rate consistent with analytic calculations. MCNPX-Polimi simulations are used exclusively for this analysis.

At the University of Montreal, an LND-25291 ${ }^{3} \mathrm{He}$ counter in an unknown moderator positioned at $90^{\circ}$ to the beam axis was used to monitor the neutron flux during calibrations of the PICO- 0.1 bubble chamber filled with $\mathrm{C}_{3} \mathrm{~F}_{8}$. As the neutron flux at $90^{\circ}$ may be different than the flux at $0^{\circ}$, a second LND-252 ${ }^{3} \mathrm{He}$ detector was used to measure the neutron flux in the forward direction and to calibrate the off-axis neutron flux monitor. The detector 
had been cross-calibrated with the detectors at the University of Chicago (see Section 4.1.4) and is used as an absolute standard. This calibration was performed for neutrons at $97 \mathrm{keV}$, $61 \mathrm{keV}, 50 \mathrm{keV}, 40 \mathrm{keV}, 34 \mathrm{keV}$, and $4.8 \mathrm{keV}$ corresponding to resonances in the ${ }^{51} \mathrm{~V}(p, n)$ cross-section (see Section 3.1.1 and Gibbons, Macklin, and Schmitt 1958). The vanadium target was sufficiently thin to achieve sub-keV neutron energy resolution. The LND-252 was encased in $2.311 \mathrm{~kg}$ of paraffin contained in a 4" Schedule 40 expanded ABS pipe 13.5" $(34.5 \mathrm{~cm})$ long, including end caps. This moderator dimension was selected to minimize the effect of geometric errors on the sensitivity of the detector to $100 \mathrm{keV}$ neutrons. The moderator dimensions are shown in Figure 6.8

The LND-252 was suspended $46.7 \mathrm{~cm}$ in front of the beam end with PICO-0.1 and its support table removed so as to minimize the number of rescattered neutrons. The calibration geometry is shown in Figure 6.9. All materials within $1 \mathrm{~m}$ of the neutron target, including detailed geometries of the beam ends shown in Figure 6.10, except those components highlighted in Figure 6.9, were simulated.

The composition and purity of materials in the simulation contributed to the calibration uncertainty, especially within the moderator of the calibration neutron detector. The acrylonitrile butadiene styrene (ABS) is approximated as equal parts of each monomer, resulting in a stoichiometric formula of $\mathrm{C}_{15} \mathrm{H}_{17} \mathrm{~N}$. The paraffin is modeled as $\mathrm{CH}_{2}$. The thickness of the moderator was designed to maximize the detector sensitivity to $50 \mathrm{keV}$ neutrons. A $3 \%$ uncertainty in the hydrogen content of the ABS and paraffin changes the detector count rate by less than $0.5 \%$ at all the calibration energies. A $30 \%$ uncertainty in the carbon content of ABS was used to model the uncertainty in its composition. Simulations with ABS composed of $\mathrm{C}_{15} \mathrm{H}_{17} \mathrm{~N}$ and $\mathrm{C}_{22} \mathrm{H}_{17} \mathrm{~N}$ differed by $1.5 \%$. In order to evaluate the cross-section uncertainty from steel in the vanadium target holder, simulations were performed at each energy with the density of the stainless steel decreased by $10 \%$ from the nominal density. The simulated 


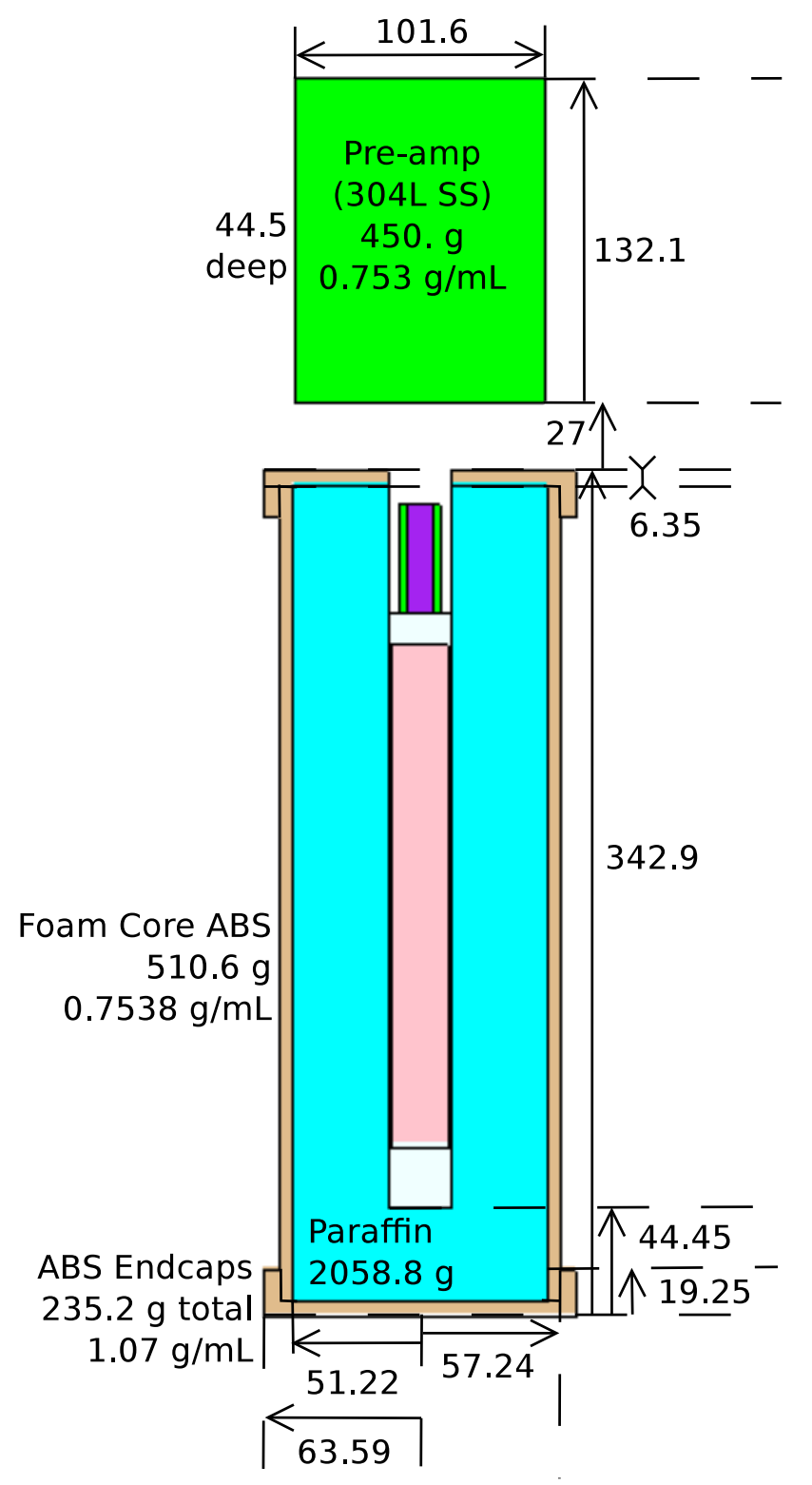

Figure 6.8: Geometry of the LND-252 ${ }^{3} \mathrm{He}$ detector moderator used to calibrate the University of Montreal neutron beam flux. The LND-252 inside the moderator uses the same geometry as that shown in Figure 4.4. The masses and dimensions of the moderator and preamplifier are shown; all dimensions are in $\mathrm{mm}$. 


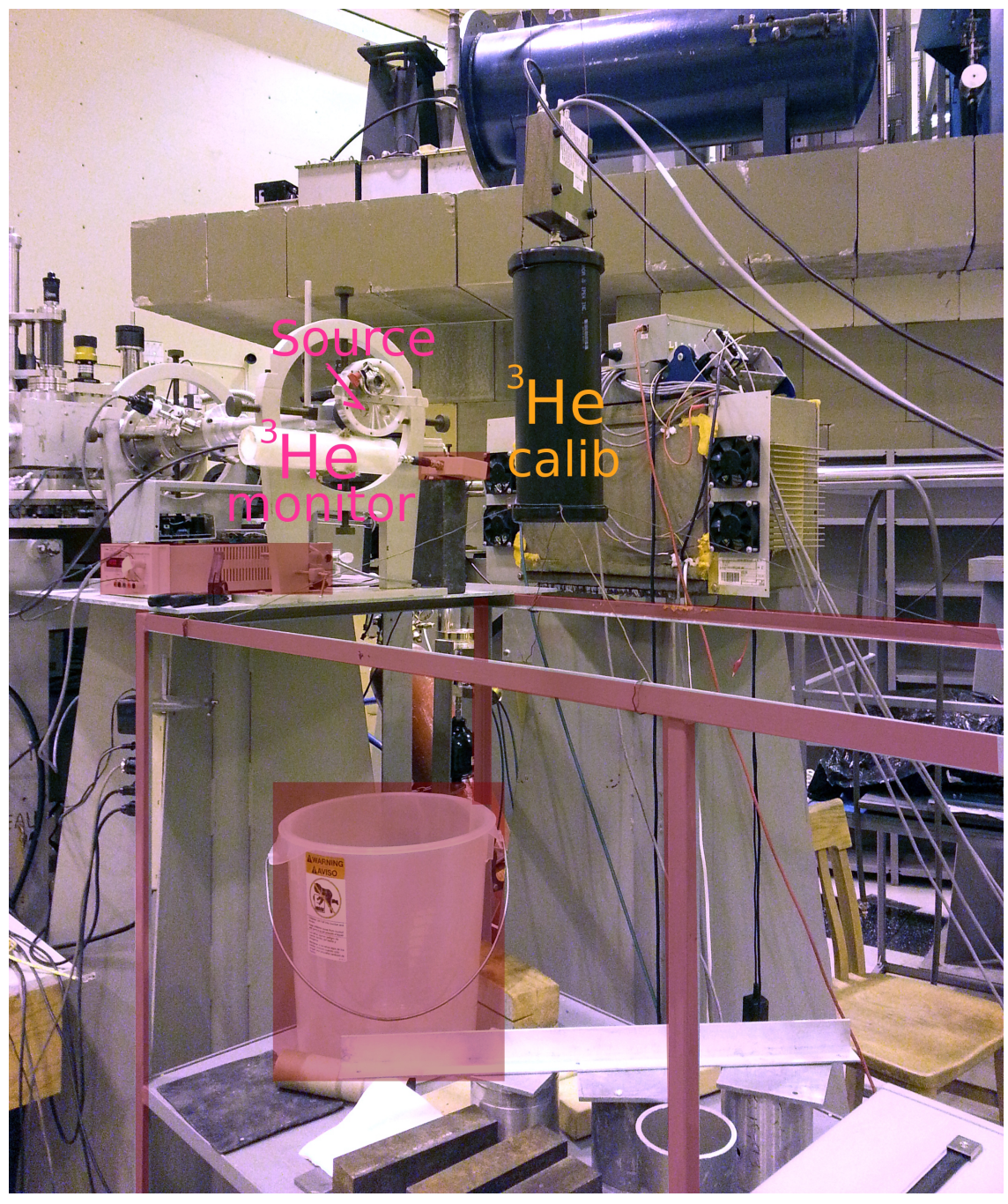

Figure 6.9: Layout of the University of Montreal neutron beam during neutron flux calibrations. The ${ }^{3}$ He detector labeled in magenta is used as a flux monitor and was calibrated against the ${ }^{3} \mathrm{He}$ detector labeled in yellow. All materials within $1 \mathrm{~m}$ of the source or the detectors was measured and simulated except for the red shaded bucket, table frame, power supply, and preamplifier. Neutrons scattering in these materials are expected to contribute negligibly to the count rate in the calibration ${ }^{3} \mathrm{He}$ detector. 


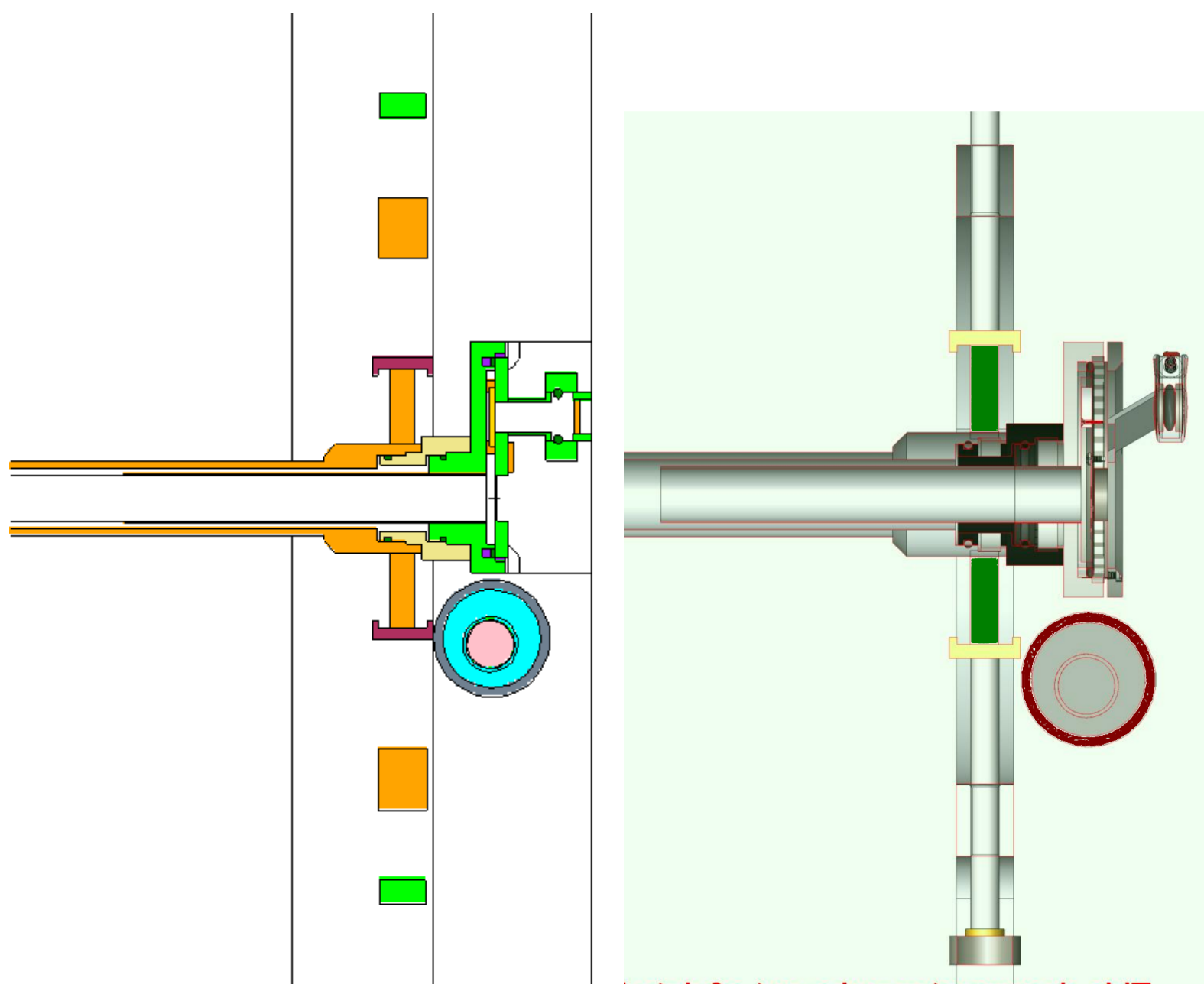

Figure 6.10: MCNPX-Polimi (left) and Solidworks (right) geometry cross-sections of the University of Montreal neutron beam-end. The Solidworks model was created by Mathieu Laurin at the Univerisity of Montreal using measurements of the disassembled beam-end. The MCNPX-Polimi geometry is based on this model. In the MCNP-geometry, steel components are in green, aluminum in orange, brass in fuchsia, and acetal components are in beige. Other quartz, PTFE, and fluorelastomer components are also visible. 


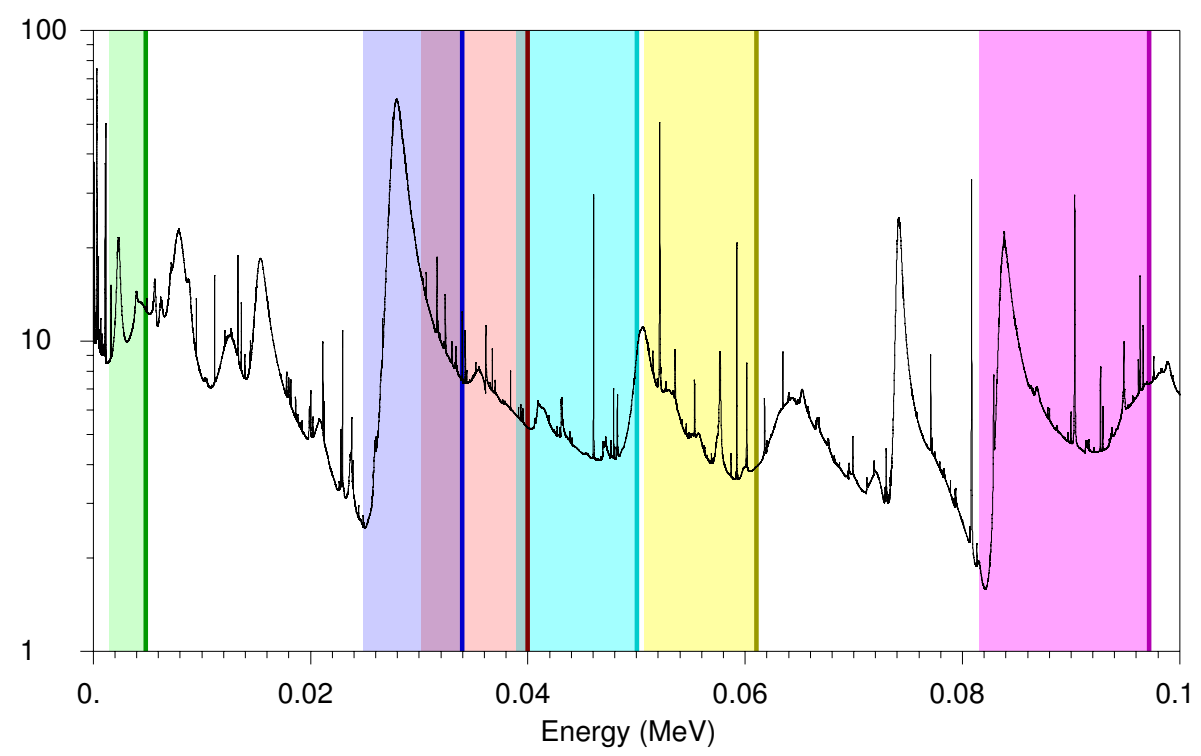

Figure 6.11: Neutron elastic scattering cross-section of 304L stainless steel. Lines show the calibration neutron energies in the forward direction while the shaded regions show the range of neutron energies emitted at each calibration point between $0^{\circ}$ and $90^{\circ}$. A strong resonance in the cross-section around $30 \mathrm{keV}$ increases the number of neutrons scattering in nearby materials, and thus increases the simulation uncertainty for the $34 \mathrm{keV}$ and $40 \mathrm{keV}$ run conditions.

flux changed by approximately $1.9 \%$ depending on the neutron energy. A strong elastic scattering resonance in steel, as shown in Figure 6.11, and in aluminum near $35 \mathrm{keV}$ may increase the simulation error due to scattering from nearby unaccounted materials.

The ratio of the count rates measured in the calibration and flux monitor ${ }^{3} \mathrm{He}$ detectors was measured. While the moderator around the LND-25291 for the beam flux monitor was unknown, the moderator geometry could be approximated based its outer dimensions. The simulated ratio of counts was compared to the measured ratio used in the calibration in order to validate the simulation geometry. Table 6.2 shows the measured and simulated ratio of count rates. The simulated and measured ratios are very similar at $50 \mathrm{keV}, 61 \mathrm{keV}$, and $97 \mathrm{keV}$, but they become discrepant at lower energies. Due to this discrepancy, the $40 \mathrm{keV}$ calibration data was not used in Amole et al. (2015) and will not be used here. 


\begin{tabular}{cccc}
\hline Neutron Energy & \multicolumn{2}{c}{ 3e counts ratio } & Simulated neutrons \\
& Simulated & Measured & per beam monitor count \\
\hline $4.8 \mathrm{keV}$ & 1.66 & $2.57 \pm 0.24$ & $192 \pm 18$ \\
$34 \mathrm{keV}$ & 2.84 & $3.18 \pm 0.30$ & $244 \pm 23$ \\
$40 \mathrm{keV}$ & 2.55 & $3.34 \pm 0.21$ & $266 \pm 17$ \\
$50 \mathrm{keV}$ & 2.26 & $2.28 \pm 0.07$ & $325 \pm 10$ \\
$61 \mathrm{keV}$ & 2.26 & $2.02 \pm 0.07$ & $383 \pm 13$ \\
$97 \mathrm{keV}$ & 2.21 & $2.07 \pm 0.10$ & $387 \pm 19$ \\
\hline
\end{tabular}

Table 6.2: The measured and simulated ratio of count rates between the beam flux monitor and the calibration ${ }^{3} \mathrm{He}$ detectors. Calibration values based on the measured ratio are also shown.

Using the calibrations from Table 6.2, simulations of the neutron beam with PICO0.1 in place were performed. Figure 6.12 shows the simulation geometry. In addition to the uncertainties due to the neutron flux calibration, additional uncertainties shown in Figure 6.2 are applied to the absolute count rates in PICO-0.1. Due to the poorly known angular distribution of neutrons from the ${ }^{51} \mathrm{~V}(p, n)$ reaction, a dipole moment uncertainty of $10 \%$ is applied to the neutron angular distribution, resulting in a $1 \%$ comparative rate error between the calibration ${ }^{3} \mathrm{He}$ detector and PICO-0.1 is applied. A $1 \mathrm{~mm}$ uncertainty in the thickness of the water bath surrounding PICO-0.1 adds a $6.4 \%$ uncertainty in the recoil rate.

With these simulations and calibrations, the data from Dahl and Jin (2014) as used in Amole et al. (2015) and shown in Table 6.4 are used to calculate the $\mathrm{C}_{3} \mathrm{~F}_{8}$ bubble nucleation efficiency function. As the background rate varied considerably between data and background runs in PICO-0.1, the background rate is measured using the highest threshold running condition from each data set. A $100 \%$ uncertainty is applied to the background rate. 


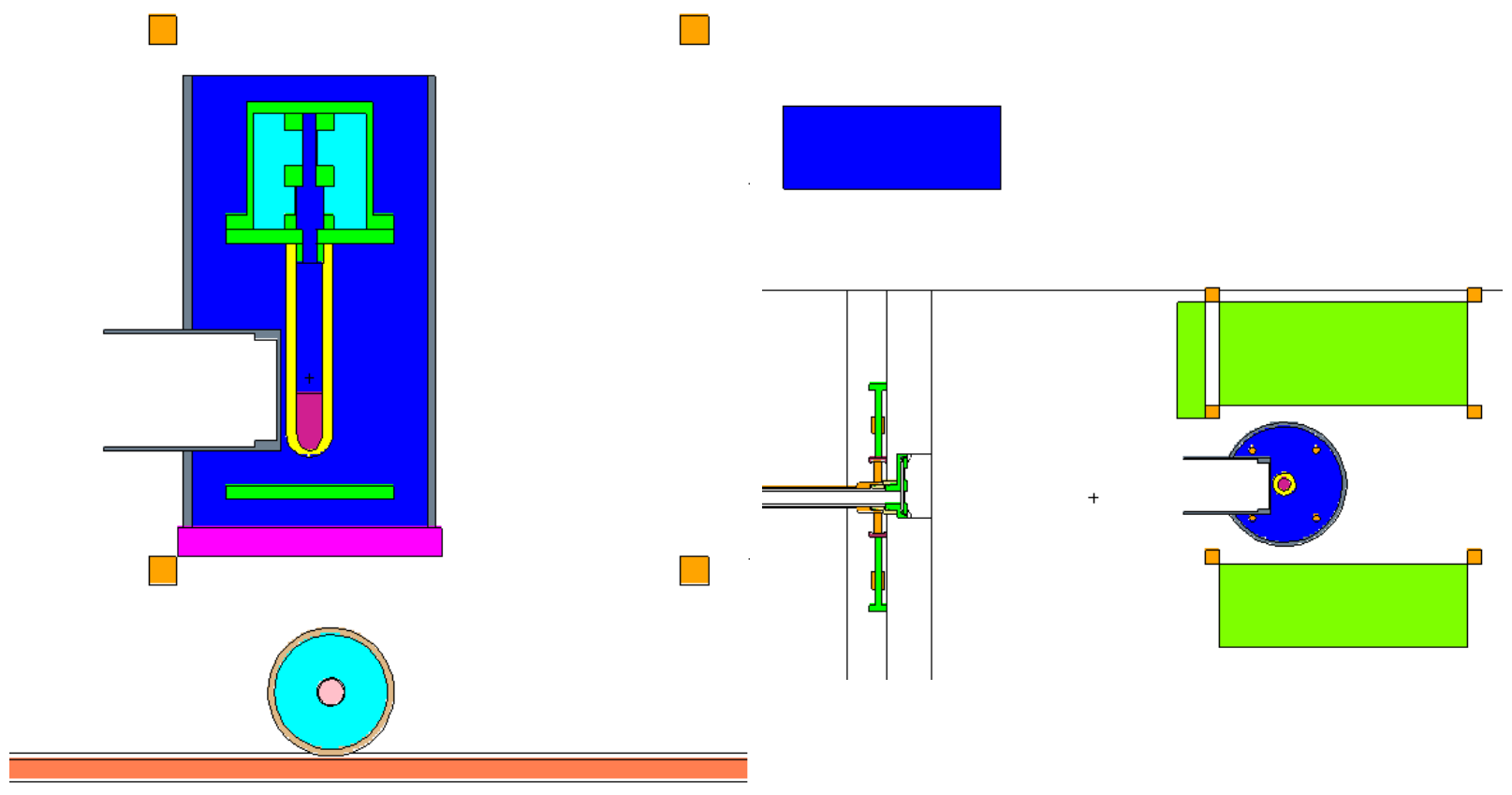

Figure 6.12: MCNPX-Polimi geometry of PICO-0.1.

\begin{tabular}{|c|c|c|}
\hline \multirow[t]{2}{*}{ Source of Uncertainty } & \multicolumn{2}{|c|}{ Uncertainty } \\
\hline & $\begin{array}{c}\text { at } 61 \mathrm{keV} \\
(\%)\end{array}$ & at $\begin{array}{c}97 \mathrm{keV} \\
(\%)\end{array}$ \\
\hline Neutron Flux & 5.0 & 5.9 \\
\hline Angular Distribution & \multicolumn{2}{|c|}{1.0} \\
\hline Fiducial Volume & \multicolumn{2}{|c|}{3.7} \\
\hline Water Bath Thickness & \multicolumn{2}{|c|}{6.5} \\
\hline Other materials & \multicolumn{2}{|c|}{2.0} \\
\hline$\sigma\left({ }^{19} \mathrm{~F}(n, \mathrm{el})\right)$ & 3.0 & 7.0 \\
\hline Total & 10 & 11 \\
\hline
\end{tabular}

Table 6.3: Count rate uncertainties or calibrations using PICO-0.1 at the University of Montreal. 


\begin{tabular}{|c|c|c|c|c|}
\hline $\begin{array}{l}\text { Seitz Threshold } \\
\qquad(\mathrm{keV})\end{array}$ & $\begin{array}{l}\text { Fiduci } \\
n=1\end{array}$ & $\begin{array}{l}\text { l event } \\
n=2\end{array}$ & $\begin{array}{l}n \text { bubbles } \\
n=3\end{array}$ & $\begin{array}{l}\text { Livetime } \\
\quad(\mathrm{sec})\end{array}$ \\
\hline \multicolumn{5}{|c|}{$97 \mathrm{keV}$ neutrons } \\
\hline $2.83 \pm 0.13$ & 22 & 1 & 0 & 765 \\
\hline $5.58 \pm 0.32$ & 24 & 3 & 0 & 1121 \\
\hline $7.4 \pm 0.5$ & 21 & 2 & 0 & 901 \\
\hline $10.1 \pm 0.7$ & 24 & 0 & 0 & 1723 \\
\hline $12.5 \pm 1.0$ & 20 & 0 & 0 & 2554 \\
\hline $13.9 \pm 1.1$ & 8 & 0 & 0 & 1604 \\
\hline $15.7 \pm 1.3$ & 7 & 0 & 0 & 3170 \\
\hline $17.0 \pm 1.5$ & 4 & 0 & 0 & 2481 \\
\hline $24.3 \pm 2.4$ & 5 & 0 & 0 & 2106 \\
\hline \multicolumn{5}{|c|}{$61 \mathrm{keV}$ neutrons } \\
\hline $2.83 \pm 0.13$ & 536 & 55 & 11 & 61030 \\
\hline $3.48 \pm 0.17$ & 467 & 23 & 2 & 66266 \\
\hline $4.35 \pm 0.23$ & 376 & 15 & 1 & 70117 \\
\hline $5.58 \pm 0.33$ & 203 & 3 & 1 & 83055 \\
\hline $7.4 \pm 0.5$ & 15 & 1 & 0 & 17321 \\
\hline $10.1 \pm 0.7$ & 6 & 0 & 0 & 19898 \\
\hline $22.1 \pm 2.1$ & 2 & 0 & 0 & 19054 \\
\hline
\end{tabular}

Table 6.4: Counts and livetimes from PICO-0.1.

\subsubsection{Simulations of PICO-2L ${ }^{241} \mathrm{Am} / \mathrm{Be}$}

PICO-2L ${ }^{241} \mathrm{Am} / \mathrm{Be}$ calibration data was combined with the Montreal neutron beam data to determine the bubble nucleation efficiency in Amole et al. (2015). The ${ }^{241} \mathrm{Am} / \mathrm{Be}$ neutrons in PICO-2L were simulated using MCNPX-Polimi using the geometry shown in Figure6.13 and using Geant4. Due to the large distance between the neutron source and the $\mathrm{C}_{3} \mathrm{~F}_{8}$, significant uncertainties exist in the simulated recoil rate. Except for a 3\% per recoil uncertainty discussed in Section 3.2 due to uncertainty in the ${ }^{19} \mathrm{~F}(n$, el $)$ cross-section, uncertainties were evaluated by resimulating the calibration neutrons in a geometry with modified parameters and comparing the simulated recoil rates above $3 \mathrm{keV}$. These uncertainties are summarized in Table 6.5, 
The rate of nuclear recoils in PICO-2L is especially sensitive to the hydrogen content of the mineral oil hydraulic fluid. Mineral oil is a mixture of linear $\left(\mathrm{C}_{n} \mathrm{H}_{2 n+2}\right)$, cyclic $\left(\mathrm{C}_{n} \mathrm{H}_{2 n}\right)$, and aromatic $\left(\mathrm{C}_{n} \mathrm{H}_{2 n-2}\right)$ saturated hydrocarbons with an average of 20-30 carbon atoms per molecule. Depending on the precise mixture, the hydrogen content may vary by up to $\sim 20 \%$ between different mineral oils. The mineral oil (Sonneborn Refined Products B. V. 2012) used in PICO-2L and the NO $\nu$ A neutrino experiment (Mufson et al. 2015), was analyzed for hydrogen, carbon, and nitrogen content by both Galbraith Laboratories Inc. of Knoxville, TN, USA and Midwest Microlab LLC of Indianapolis, IN, USA using combustion product analysis following the ASTM D5291 procedure (ASTM D5291-09 2009). Hydrogen contents of $13.37 \%$ and $14.07 \%$ by mass were measured by the respective laboratories, with a claimed relative uncertainty of $2.12 \%$ by Galbraith Laboratories. The average of these contents is adopted with a relative $3 \%$ uncertainty to account for the larger than expected spread between the measurements.

Eric Vasquèz-Jauregui independently modeled and simulated the PICO-2L ${ }^{241} \mathrm{Am} / \mathrm{Be}$ neutron calibration using Geant4.9.5 and ENDF-VII.1 libraries from Mendoza and CanoOtt (2011) with modifications from A. E. Robinson (2014, Appendix B). After reconciling our simulation geometries and source spectra, the Geant 4 simulation produced a $30 \%$ larger recoil rate for high-energy recoils than MCNP. The MCNP simulation output is adopted, with a $\pm 30 \%$ uncertainty applied to the recoil rate.

While the recoil rate has a large uncertainty, the ratio of multiple scattering events to the total number of events is unaffected by most of the uncertainties listed in Table 6.5. Only the target cross-section uncertainties and the target mass uncertainty remain, contributing an uncertainty of $\pm(3 \% \times(n-1))$ for multiple scattering events with $n$ recoiling nuclei.

Only single bubble events passing the recoil-like acoustic parameters cuts are used to calculate the allowed nucleation efficiency functions. These events are directly compared 


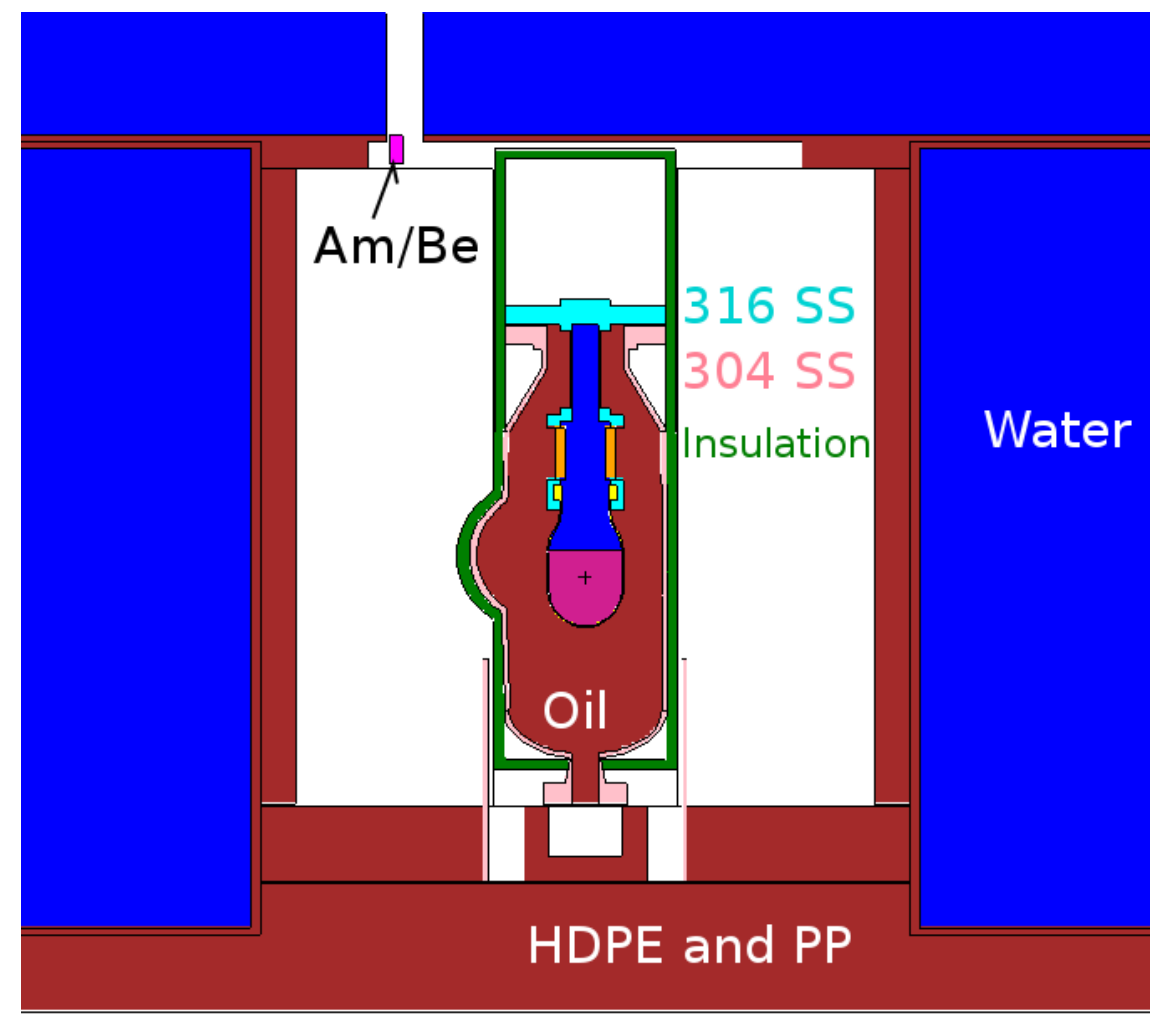

Figure 6.13: Position of the ${ }^{241} \mathrm{Am} /$ Be neutron source in the MCNPX-Polimi 4a PICO-2L geometry used for neutron calibrations.

to simulated events without reactions. In contrast to the COUPP-4kg calibration data, no additional cut is placed on the maximum simulated recoil energy. Any change in the simulated recoil rate due to the loss of this cut is hidden is sub-dominant to the applied $30 \%$ simulation uncertainty.

\subsubsection{Photoneutron Source Measurements}

Simulations of both the University of Chicago bubble chamber and the CYRTE bubble chamber used $10^{7}$ source photons and generated approximately $3 \times 10^{4}$ nuclear recoils each. Unlike the $\mathrm{CF}_{3} \mathrm{I}$ calibrations, the background rate varied considerably with Seitz threshold 


\begin{tabular}{lcc}
\hline Parameter & $\begin{array}{c}\text { Parameter } \\
\text { uncertainty }\end{array}$ & $\begin{array}{c}\text { Recoil rate } \\
\text { uncertainty }(\%)\end{array}$ \\
\hline Source $r$ position & $5 \mathrm{~mm}$ & 2.0 \\
Source $z$ position & $6 \mathrm{~mm}$ & 2.0 \\
Neutron spectrum & see Section 3.3 & 6.4 \\
${ }^{19} \mathrm{~F}(n, \mathrm{el})$ cross-section & $\sim 5 \%$ & 4.1 \\
${ }^{12} \mathrm{C}(n, \mathrm{el})$ cross-section & $0.5 \%$ & 0.3 \\
Mineral Oil hydrogen content & $3 \%$ & 4.2 \\
Water Shielding Tank Density & $5 \%$ & 0.1 \\
Pressure Vessel thickness & $15 \%$ & 3.6 \\
Insulation thickness & $100 \%$ & 0.5 \\
Silica cross-section & $2 \%$ & 0.1 \\
Source rate & $1.1 \%$ & 1.1 \\
Target mass & $0.15 \%$ & 0.15 \\
Total & & 9.9 \\
Geant4/MCNPX comparison & & 30 \\
\hline
\end{tabular}

Table 6.5: PICO-2L ${ }^{241} \mathrm{Am} / \mathrm{Be}$ calibration systematic uncertainties. While the evaluated uncertainties are $\pm 9.9 \%$, a $\pm 30 \%$ uncertainty is adopted due to disagreements with Geant 4 simulations.

in CYRTE when filled with $\mathrm{C}_{3} \mathrm{~F}_{8}$.

For the CYRTE ${ }^{124} \mathrm{Sb} /$ Be data, an exponential background model was fit

$$
R_{b g}=a e^{-E_{c} / b}+E_{c} / c+d
$$

where $a$ differed at $15^{\circ} \mathrm{C}\left(a=38.0 \mathrm{~s}^{-} 1\right)$ and $20^{\circ} \mathrm{C}\left(a=68.2 \mathrm{~s}^{-} 1\right)$ and the other parameters were fit to the combined data with $b=0.4572 \mathrm{keV}, c=25 \mathrm{MeV} \cdot \mathrm{s}$, and $d=0.0014 \mathrm{~s}^{-} 1$. This fit describes the background rate well. A $36 \%$ background rate uncertainty is applied based on the combination of the RMS of the fit residuals and half the difference in $a$ between the two temperatures. Data below a $3.0 \mathrm{keV}$ Seitz threshold was cut as the measured event rates in the presence of neutrons and photons was lower than the measured event rate in the presence of photons only (see Figure 4.25). 


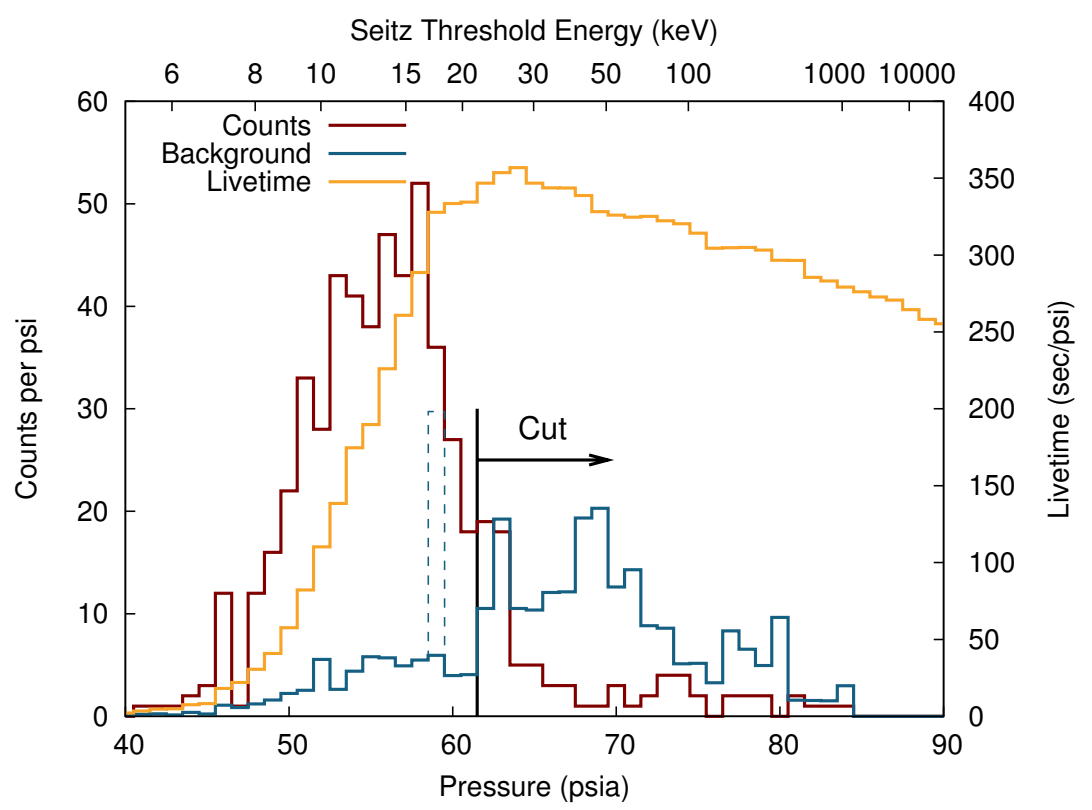

Figure 6.14: Counts, livetime, and expected background from the $\mathrm{C}_{3} \mathrm{~F}_{8}$ filled University of Chicago bubble chamber with a ${ }^{88} \mathrm{Y} /$ Be source in place at $14.0^{\circ} \mathrm{C}$. Data above 61.4 psia was not used in the analysis and the background rate at 59 psia (dashed blue) was adjusted to match the rate at nearby pressures.

While no abnormal background rate was observed in the presence of ${ }^{88} \mathrm{Y} / \mathrm{Be}$ source in CYRTE or the University of Chicago bubble chamber, a smooth background rate could not be expected. Instead of a background model, the background rate as measured at each operating point is used in the analysis. For the University of Chicago bubble chamber, a high rate of spontaneous nucleation occurred during the background run that affected data at Seitz thresholds above $20 \mathrm{keV}$. These thresholds are cut from the calibration data, as shown in Figure 6.14.

\subsection{4 $\quad \mathrm{C}_{3} \mathrm{~F}_{8}$ Efficiency Model}

Bubble nucleation efficiency limits with equal carbon and fluorine recoil efficiencies were calculated using the method described in Section 3.5. The allowed bubble nucleation efficiency functions are shown in Figure 6.15 at the thresholds used for the PICO-2L ${ }^{241} \mathrm{Am} / \mathrm{Be}$ 


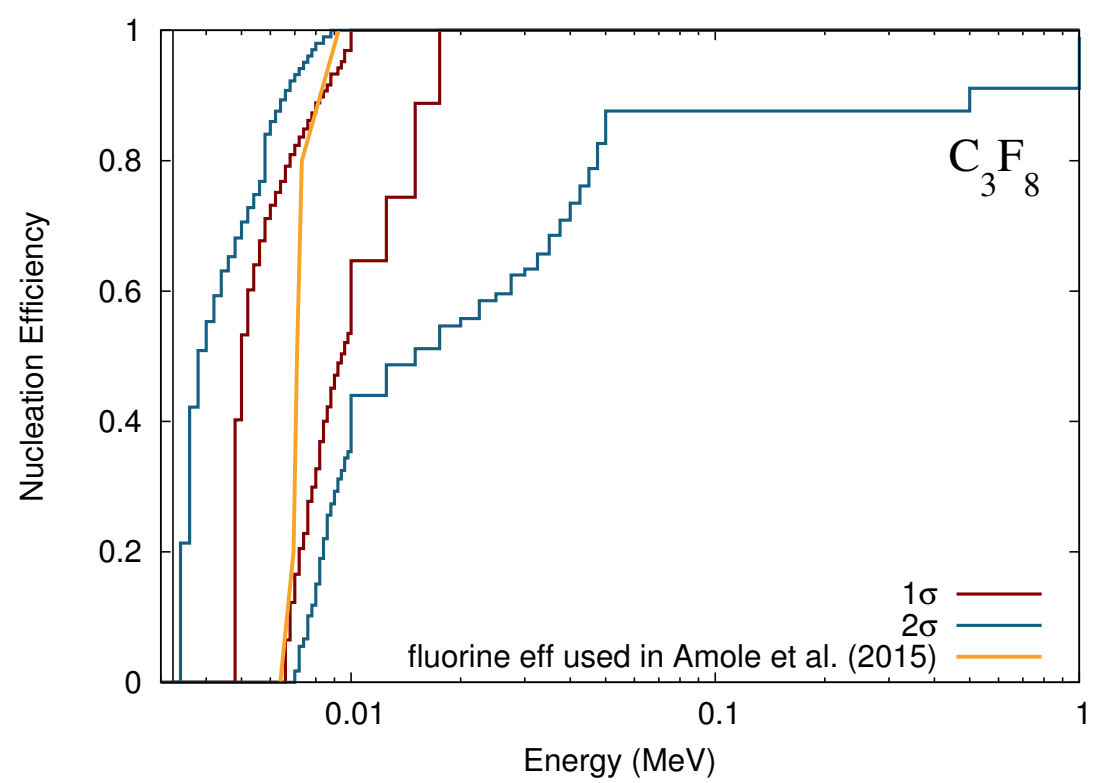

(a) $3.24 \mathrm{keV}$ : PICO-2L, CYRTE ${ }^{124} \mathrm{Sb} / \mathrm{Be}$, and University of Montreal $61 \& 97 \mathrm{keV}$

Figure 6.15: Allowed values of the bubble nucleation efficiency of carbon and fluorine recoils in $\mathrm{C}_{3} \mathrm{~F}_{8}$ at the specified Seitz threshold energies. Not every calibration experiment measured the bubble nucleation within $\pm 0.6 \mathrm{keV}$ of each energy shown. Fitted data sets are listed in the caption of each subfigure. Figure continues on the next two pages.

calculation. All data at Seitz thresholds within $\pm 0.6 \mathrm{keV}$ of the selected PICO-2L Seitz threshold are used to set limits.

The data from bubble multiplicities in ${ }^{241} \mathrm{Am} / \mathrm{Be}$ calibrations in PICO-2L and from CYRTE ${ }^{124} \mathrm{Sb} / \mathrm{Be}$ and the University of Montreal at $61 \mathrm{keV}$ are in severe tension at most of the listed energies, with $p$-values for obtaining the given data as little as $10^{-8}$. There is also severe disagreement with the fits used in Amole et al. (2015) that were based on a subset of the same calibration data. The fitted ${ }^{241} \mathrm{Am} /$ Be data prefers greater bubble nucleation efficiency function while the other two data sets prefer a lower efficiency near that used in Amole et al. (2015) (see Figure 6.17). Figure 6.15d shows efficiency function limits that are larger than at other energies where the latter two data sets are used. Confirmation of the neutron flux at the University of Montreal, and an ${ }^{124} \mathrm{Sb} / \mathrm{Be}$ measurement in a bubble 


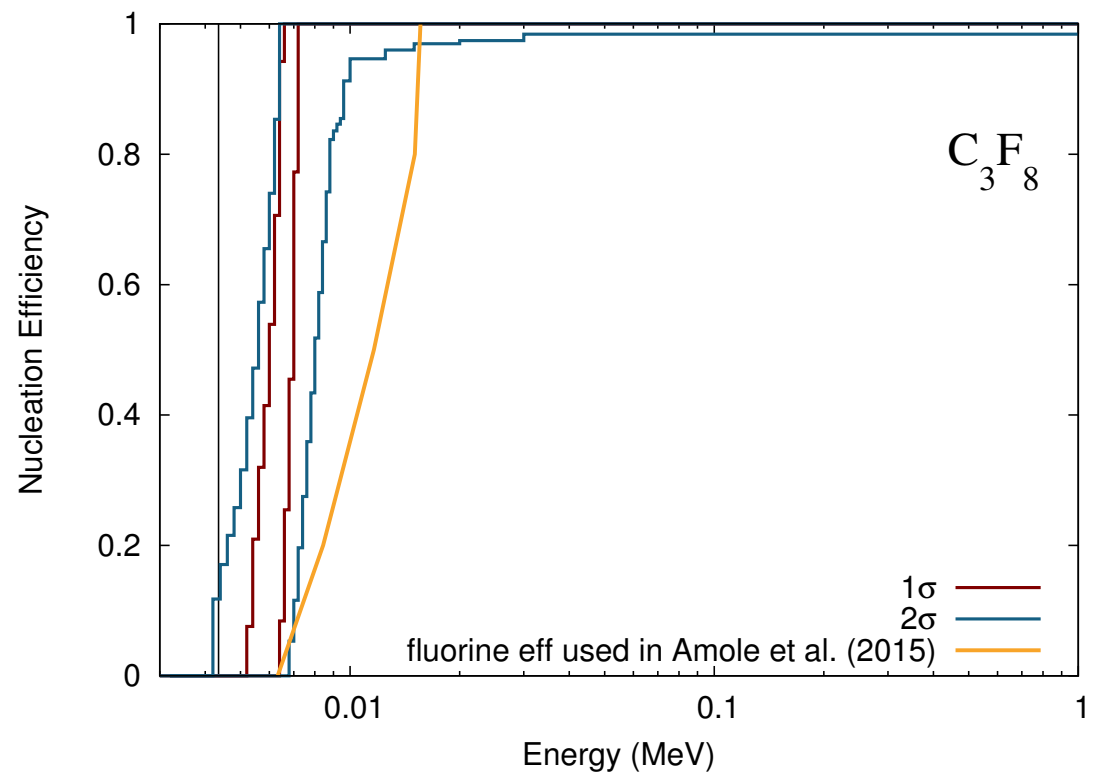

(b) Figure 6.15 continued. $4.35 \mathrm{keV}$ : PICO-2L, CYRTE ${ }^{124} \mathrm{Sb} / \mathrm{Be}$, and University of Montreal $61 \mathrm{keV}$.

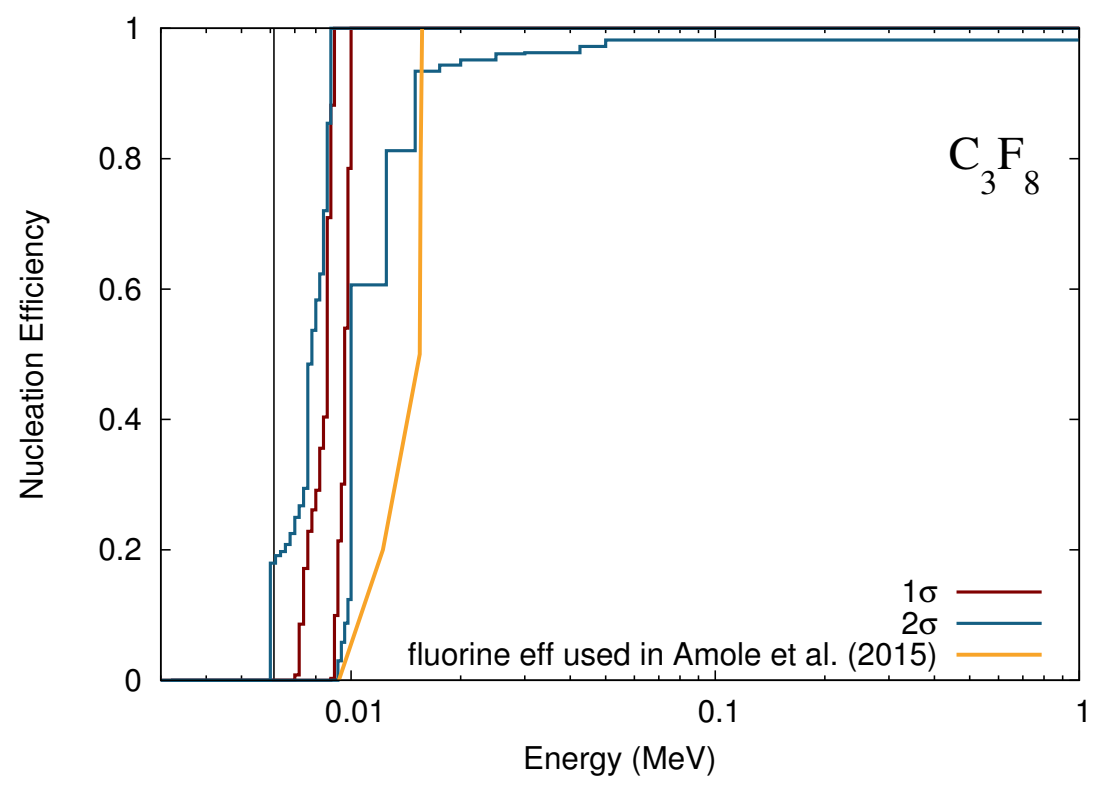

(c) Figure 6.15 continued. $6.14 \mathrm{keV}$ : PICO-2L, CYRTE ${ }^{88} \mathrm{Y} / \mathrm{Be}$, University of Montreal $61 \& 97 \mathrm{keV}$, and University of Chicago 


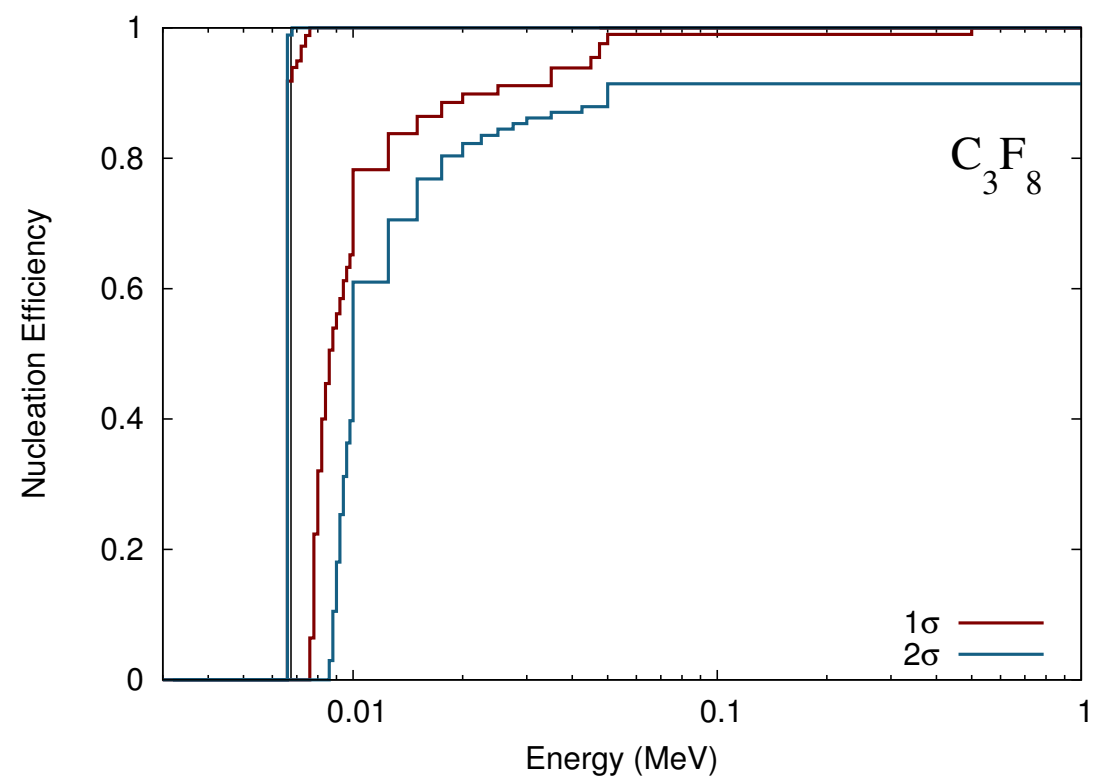

(d) Figure 6.15 continued. $6.75 \mathrm{keV}$ : PICO-2L, CYRTE ${ }^{88} \mathrm{Y} / \mathrm{Be}$, and University of Chicago

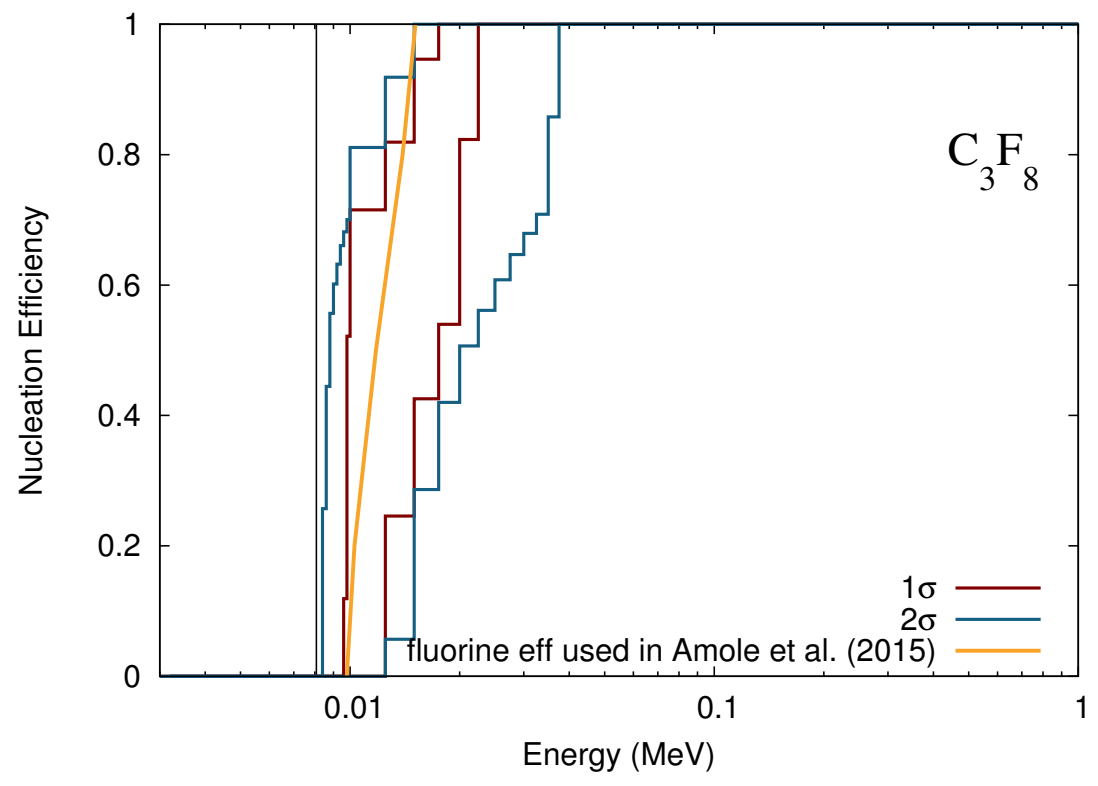

(e) Figure 6.15 continued. $8.08 \mathrm{keV}$ : PICO-2L, CYRTE ${ }^{88} \mathrm{Sb} / \mathrm{Be}$, and University of Chicago 


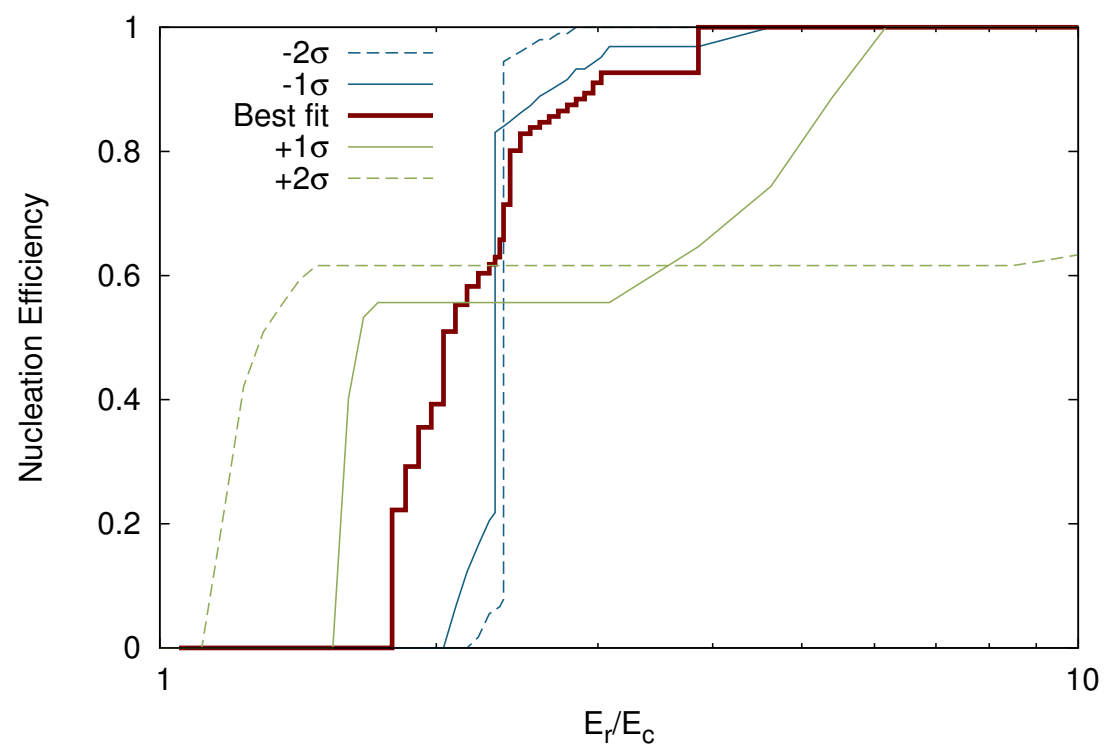

Figure 6.16: $\mathrm{C}_{3} \mathrm{~F}_{8}$ bubble nucleation efficiency functions compatible at $>1 \sigma$ and $>2 \sigma$ with calibration data near $3.24 \mathrm{keV}$. Functions that predict a low dark matter sensitivity $(-1 \sigma$ and $-2 \sigma)$ follow the lower limit bubble nucleation efficiency at low recoil energies and jump to the upper limit efficiency at high recoil energies. Functions that predict a high dark matter efficiency jump from the upper limit to the lower limit. The position of the jumps is set to maximize the consistency with calibration data. Effectively, these functions show how the shape of the efficiency function can varied while maintaining consistency with measured count rates during calibrations.

chamber without time-dependent electron recoil sensitivity are being pursued within the PICO collaboration in order to validate or correct the existing measurements and resolve these fit discrepancies.

The limits set at $3.24 \mathrm{keV}$ provide both the most consistent fit to data and the most conservative lower bound to the bubble nucleation efficiency when assuming that $\eta=\eta\left(E_{r} / E_{c}\right)$. Sample bubble nucleation efficiency curves based on the $3.24 \mathrm{keV}$ data shown in Figure 6.16 will be used to set the dark matter limits in Chapter 9 . 


\begin{tabular}{|c|c|c|c|c|}
\hline \multirow[t]{2}{*}{ Data } & & \multirow{2}{*}{$\begin{array}{c}E_{c} \\
(\mathrm{keV})\end{array}$} & \multicolumn{2}{|c|}{ Number of Counts } \\
\hline & & & Measured & Expected \\
\hline \multirow[t]{9}{*}{ PICO-2L ${ }^{241} \mathrm{Am} / \mathrm{Be}$} & $n=1$ & $3.24 \pm 0.15$ & 448 & $431 \pm 129$ \\
\hline & $n=2$ & & 245 & $278 \pm 16$ \\
\hline & $n=3$ & & 147 & $143 \pm 11$ \\
\hline & $n=4$ & & 73 & $80 \pm 8$ \\
\hline & $n=5$ & & 41 & $44 \pm 6$ \\
\hline & $n=6$ & & 24 & $25.4 \pm 4.0$ \\
\hline & $n=7$ & & 14 & $15.6 \pm 2.9$ \\
\hline & $n=8$ & & 4 & $9.1 \pm 2.0$ \\
\hline & $n \geq 9$ & & 4 & $13.0 \pm 3.6$ \\
\hline \multicolumn{2}{|c|}{ CYRTE ${ }^{124} \mathrm{Sb} / \mathrm{Be} 15^{\circ} \mathrm{C}$} & $3.52 \pm 0.14$ & 205 & $304 \pm 56$ \\
\hline \multicolumn{2}{|c|}{ CYRTE ${ }^{124} \mathrm{Sb} / \mathrm{Be} 20^{\circ} \mathrm{C}$} & $3.06 \pm 0.14$ & 238 & $225 \pm 64$ \\
\hline \multicolumn{2}{|c|}{ Montreal $61 \mathrm{keV} n=1$} & $2.83 \pm 0.13$ & 536 & $545 \pm 62$ \\
\hline \multicolumn{2}{|c|}{ Montreal $61 \mathrm{keV} n=1$} & $3.48 \pm 0.17$ & 467 & $457 \pm 56$ \\
\hline \multicolumn{2}{|l|}{ Montreal $97 \mathrm{keV}$} & $3.48 \pm 0.17$ & 22 & $16.0 \pm 1.8$ \\
\hline \multicolumn{2}{|l|}{ Montreal $n=2$} & & 79 & $111 \pm 15$ \\
\hline \multicolumn{2}{|l|}{ Montreal $n=3$} & & 13 & $8.2 \pm 1.2$ \\
\hline
\end{tabular}

Table 6.6: Measured and expected number of counts from calibrations of $\mathrm{C}_{3} \mathrm{~F}_{8}$ near $3.24 \mathrm{keV}$. The expected number of counts are the mean of the modeled Poisson distribution describing the measured number of counts. Only the Gaussian uncertainties in this mean are shown, not the additional uncertainties due to Poisson counting statistics. The University of Montreal $n=2$ and $n=3$ count rates are the summed rates from data using $61 \mathrm{keV}$ and $97 \mathrm{keV}$ neutrons at thresholds of $2.83 \mathrm{keV}$ and $3.48 \mathrm{keV}$. 


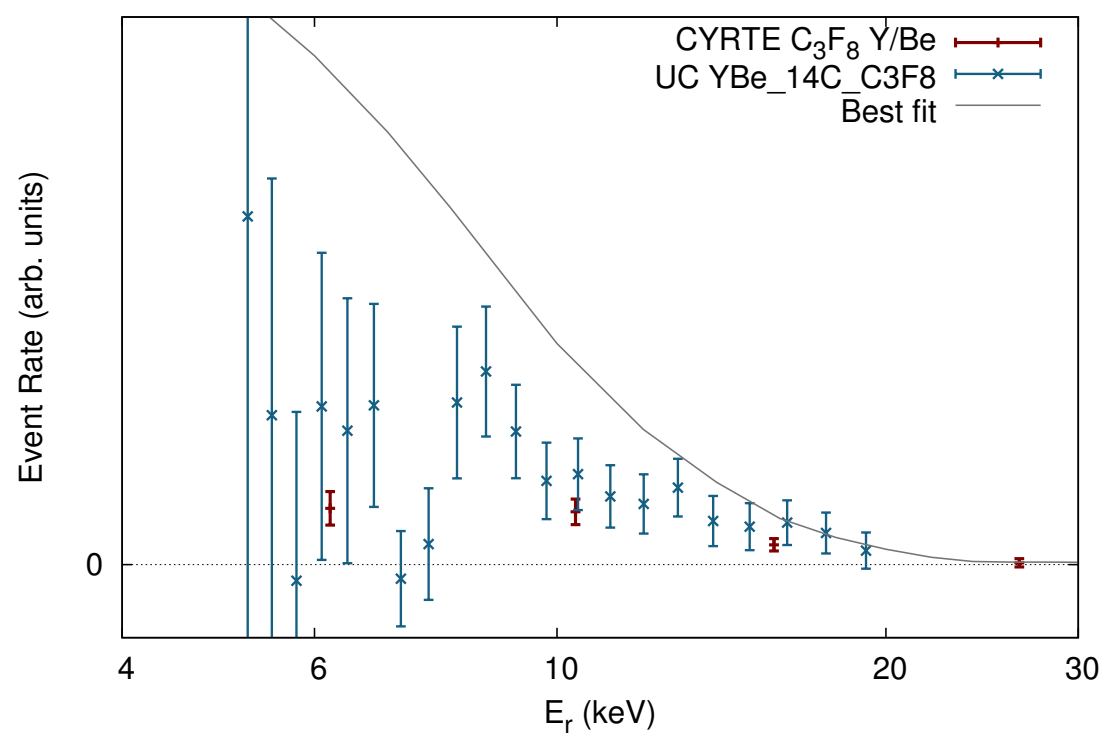

Figure 6.17: $\mathrm{C}_{3} \mathrm{~F}_{8}$ measured and predicted event rates in the presence of ${ }^{88} \mathrm{Y} /$ Be neutrons. The low measured event rates are in severe tension with ${ }^{241} \mathrm{Am} / \mathrm{Be}$ calibrations. 


\section{Chapter 7 \\ Background Studies in Bubble Chambers}

A nuclear recoil signal can be attributed to dark matter, neutron scattering, other radioactive backgrounds, or other detector backgrounds such as particulate caused bubble nucleation. In order to claim a dark matter signal, these other sources of events must be determined and discounted. This chapter will focus on the tools used to calculate neutron and other radioactive backgrounds while the next chapter will calculate the expected background rate for PICO-2L.

Neutron that may scatter in the dark matter detector can come from the spontaneous fission of actinide elements, notably ${ }^{238} \mathrm{U}$, or from energetic particles reacting with nuclei. Most neutrons that may interact in PICO-2L are produced by radioactive ${ }^{238} \mathrm{U},{ }^{232} \mathrm{Th}$, and their daughter isotopes by spontaneous fission and the $(\alpha, n)$ reaction.

\subsection{Radiopurity Assays}

The radioactive ${ }^{232} \mathrm{Th}$ and ${ }^{238} \mathrm{U}$ content of materials for use with the PICO and COUPP detectors is determined using either $\gamma$ emission spectroscopy (GES) at the University of Chicago or SNOLAB radioassay facilities, or by inductively coupled plasma mass spectroscopy (ICP-MS) at Pacific Northwest National Laboratory. The latter two facilities are well described by Lawson (2014) and Hoppe et al. (2014). The GES counting facility in the Collar Lab at the University of Chicago and the calculation of material activities from the counting spectrum are described in Fields (2014, Sec. 4.1).

GES is a non-destructive assay technique that measures the number and precise energy of photons emitted by a sample. A high-purity germanium crystal (HPGe) in a cryogenic environment is used to detect the photons. A bias volage is applied to the crystal, $3300 \mathrm{~V}$ in 
the case of the University of Chicago HPGe detector, and the charge ionization in the crystal from photon interactions is collected and recorded as a photon energy spectrum. In order to only measure photons emitted by a material sample, the detector and the sample are surrounded by a thick photon shield, generally composed of low radioactivity lead, copper, and/or steel.

The method for determining the activity of materials from the activity of individual photon energies differs from the method used by Fields. Rather than deriving a measurement from only the highest intensity peak for a given decay chain, activities were calculated from the weighed average of all measured peaks. To ensure that the selection of photon energies to average is not biased, all peaks with branching ratios above the smallest measured peak for a given decay chain are measured and included. A different random number seed is used to simulate the detector efficiency for each photon energy in order to ensure statistical independence of each component of the average. Table 7.1 shows the most commonly measured photon energies. Each measured spectrum was also inspected for additional uncommon photon energy peaks.

The consistency of peak strength at each measured photon energy with respect to the average is checked and inconsistent count rates are noted. A loss of equilibrium in the activity in the ${ }^{238} \mathrm{U}$ decay chain is particularly common, and its measurement is critical in calculating the neutron emission rate of materials. The long half-lives and different chemistries of ${ }^{226} \mathrm{Ra},{ }^{230} \mathrm{Th}$, and ${ }^{234} \mathrm{U}$ allow for their separation when materials are processed. Most of the photon activity that can be measured to determine the ${ }^{238} \mathrm{U}$ content of materials is generated by short-lived daughter isotopes of ${ }^{226}$ Ra. Low-energy and low branching ratio photon energies from the decay of ${ }^{234} \mathrm{Th},{ }^{234 m} \mathrm{Pr}$, and the ${ }^{235} \mathrm{U}$ decay chain are used to measure the amount of uranium present. When a break in equilibrium is found, the measured activity of ${ }^{214} \mathrm{~Pb}$ and ${ }^{214} \mathrm{Bi}$ is background subtracted from the combined $186.2 \mathrm{keV}$ 


\begin{tabular}{|c|c|c|c|c|c|c|}
\hline $\begin{array}{l}\text { Decay } \\
\text { Chain }\end{array}$ & $\begin{array}{c}E_{\gamma} \\
(\mathrm{keV})\end{array}$ & $\begin{array}{c}I_{\gamma} \\
(\%)\end{array}$ & $\begin{array}{l}\text { Parent } \\
\text { Nucleus }\end{array}$ & $\begin{array}{c}\text { Bckgnd } \\
\text { Rate } \\
\left(10^{-5} \mathrm{cps}\right)\end{array}$ & $\begin{array}{c}\text { Bckgnd for } \\
\text { a } 500 \mathrm{~mL} \\
\text { Marinelli } \\
(\mathrm{mBq})\end{array}$ & Notes \\
\hline \multirow[t]{19}{*}{${ }^{238} \mathrm{U}$} & 92.4 & 2.13 & ${ }^{234} \mathrm{Th}$ & $<7$ & & Near $\mathrm{Pb}$ x-ray lines \\
\hline & 92.8 & 2.10 & ${ }^{234} \mathrm{Th}$ & & & Fit combined with $92.8 \mathrm{keV}$ line \\
\hline & 186.2 & 3.64 & ${ }^{226} \mathrm{Ra}$ & $10.0 \pm 2.4$ & $112 \pm 27$ & Near $185.7 \mathrm{keV}{ }^{235} \mathrm{U}$ \\
\hline & 242.0 & 7.25 & ${ }^{214} \mathrm{~Pb}$ & $22.5 \pm 2.2$ & $151 \pm 15$ & Variable, near $241.0 \mathrm{keV}{ }^{224} \mathrm{Ra}$ \\
\hline & 295.2 & 18.42 & ${ }^{214} \mathrm{~Pb}$ & $57.2 \pm 2.5$ & $182 \pm 9$ & Variable, equivalent to $19 \mathrm{ppb}^{238} \mathrm{U}$ \\
\hline & 351.9 & 35.60 & ${ }^{214} \mathrm{~Pb}$ & $90.9 \pm 2.8$ & $189 \pm 8$ & Variable, near $351.1 \mathrm{keV}^{211} \mathrm{Bi}$ \\
\hline & 609.3 & 45.49 & ${ }^{214} \mathrm{Bi}$ & $82.6 \pm 2.4$ & $213 \pm 10$ & Variable \\
\hline & 768.4 & 4.89 & ${ }^{214} \mathrm{Bi}$ & $7.3 \pm 1.0$ & $240 \pm 30$ & Variable \\
\hline & 934.1 & 3.17 & ${ }^{214} \mathrm{Bi}$ & $3.3 \pm 0.8$ & $190 \pm 50$ & Variable \\
\hline & 1001.4 & 0.84 & ${ }^{234 m} \mathrm{~Pa}$ & $0.6 \pm 0.6$ & $130 \pm 130$ & Rarely observed. \\
\hline & 1120.3 & 14.92 & ${ }^{214} \mathrm{Bi}$ & $14.1 \pm 1.2$ & $197 \pm 19$ & Variable \\
\hline & 1239.0 & 5.83 & ${ }^{214} \mathrm{Bi}$ & $5.6 \pm 0.8$ & $220 \pm 30$ & Variable \\
\hline & 1377.7 & 3.99 & ${ }^{214} \mathrm{Bi}$ & $3.4 \pm 0.7$ & $210 \pm 40$ & Variable \\
\hline & 1408.0 & 2.39 & ${ }^{214} \mathrm{Bi}$ & $2.1 \pm 0.6$ & $220 \pm 60$ & Variable \\
\hline & 1509.2 & 2.13 & ${ }^{214} \mathrm{Bi}$ & $1.5 \pm 0.6$ & $200 \pm 80$ & Variable \\
\hline & 1729.6 & 2.88 & ${ }^{214} \mathrm{Bi}$ & $1.3 \pm 0.5$ & $120 \pm 50$ & Variable \\
\hline & 1764.5 & 15.30 & ${ }^{214} \mathrm{Bi}$ & $12.8 \pm 1.0$ & $266 \pm 26$ & Variable \\
\hline & 1847.4 & 2.03 & ${ }^{214} \mathrm{Bi}$ & $1.7 \pm 0.5$ & $300 \pm 90$ & Variable \\
\hline & 2204.1 & 4.92 & ${ }^{214} \mathrm{Bi}$ & $3.0 \pm 0.9$ & $270 \pm 80$ & Variable \\
\hline
\end{tabular}

Table 7.1: Commonly fitted photon energies. The sixth column calculates what activity would be required in a water filled $500 \mathrm{~mL}$ nominal $(775 \mathrm{~mL}$ actual) Marinelli beaker in order to produce the background count rate, providing a measure of the ultimate sensitivity of the detector. Continued on next page.

${ }^{226} \mathrm{Ra}$ and $185.7 \mathrm{keV}{ }^{235} \mathrm{U}$ peak to measure the ${ }^{235} \mathrm{U}$ activity.

One of the largest radioactive backgrounds during assays is due to gaseous ${ }^{222} \mathrm{Rn}$ entering the sample area during assays. The activity of the radon daughters ${ }^{214} \mathrm{Bi}$ and ${ }^{214} \mathrm{~Pb}$ varies over time as shown in Figure 7.1. In order to reduce the radon background, a $1 \mathrm{~cm}$ thick copper plate was often placed in front of the detector during both background and assay measurements when the size of the material to be assayed allowed. This plate reduced the volume of radon contaminated air within line-of-sight of the HPGe crystal. For each sample, a background spectrum is taken within 2 weeks either before or after (preferably both) the count spectrum. These background runs are used to verify that the count rate from radon daughters is stable. If these runs are consistent with older background runs using 


\begin{tabular}{|c|c|c|c|c|c|c|}
\hline $\begin{array}{l}\text { Decay } \\
\text { Chain }\end{array}$ & $\begin{array}{c}E_{\gamma} \\
(\mathrm{keV})\end{array}$ & $\begin{array}{l}I_{\gamma} \\
(\%)\end{array}$ & $\begin{array}{l}\text { Parent } \\
\text { Nucleus }\end{array}$ & $\begin{array}{l}\text { Bckgnd } \\
\text { Rate } \\
\left(10^{-5} \mathrm{cps}\right)\end{array}$ & $\begin{array}{l}\text { Bckgnd for } \\
\text { a } 500 \mathrm{~mL} \\
\text { Marinelli } \\
(\mathrm{mBq})\end{array}$ & Notes \\
\hline \multirow[t]{14}{*}{${ }^{232} \mathrm{Th}$} & 209.3 & 3.89 & ${ }^{228} \mathrm{Ac}$ & $2.6 \pm 2.1$ & $30 \pm 24$ & \multirow{14}{*}{$\begin{array}{l}\text { Equivalent to } 9.2 \mathrm{ppb}^{232} \mathrm{Th} \\
\text { Buried by } 242.0 \mathrm{keV}{ }^{214} \mathrm{~Pb} \\
\text { Near } 269.5 \text { and } 271.2 \mathrm{keV}{ }^{235} \mathrm{U} \text { chain }\end{array}$} \\
\hline & 238.6 & 43.60 & ${ }^{212} \mathrm{~Pb}$ & $25.9 \pm 2.3$ & $28.9 \pm 2.6$ & \\
\hline & 241.0 & 4.10 & ${ }^{224} \mathrm{Ra}$ & & & \\
\hline & 270.2 & 3.46 & ${ }^{228} \mathrm{Ac}$ & $2.5 \pm 1.7$ & $39 \pm 27$ & \\
\hline & 338.3 & 11.27 & ${ }^{228} \mathrm{Ac}$ & $4.4 \pm 1.4$ & $27 \pm 9$ & \\
\hline & 463.0 & 4.40 & ${ }^{228} \mathrm{Ac}$ & $2.1 \pm 1.0$ & $45 \pm 22$ & \\
\hline & 583.2 & 30.55 & ${ }^{208} \mathrm{Tl}$ & $15.6 \pm 1.3$ & $59 \pm 5$ & \\
\hline & 727.3 & 6.67 & ${ }^{212} \mathrm{Bi}$ & $2.5 \pm 0.8$ & $53 \pm 17$ & \\
\hline & 794.9 & 4.25 & ${ }^{228} \mathrm{Ac}$ & $2.1 \pm 0.8$ & $77 \pm 29$ & \\
\hline & 860.6 & 4.49 & ${ }^{208} \mathrm{Tl}$ & $0.4 \pm 0.6$ & $14 \pm 21$ & \\
\hline & 911.2 & 25.80 & ${ }^{228} \mathrm{Ac}$ & $6.8 \pm 1.0$ & $46 \pm 7$ & \\
\hline & 964.8 & 4.99 & ${ }^{228} \mathrm{Ac}$ & $0.6 \pm 0.6$ & $22 \pm 22$ & \\
\hline & 969.0 & 15.80 & ${ }^{228} \mathrm{Ac}$ & $4.1 \pm 0.8$ & $47 \pm 9$ & \\
\hline & 2614.5 & 35.85 & ${ }^{208} \mathrm{Tl}$ & $7.8 \pm 0.8$ & $95 \pm 12$ & \\
\hline \multirow[t]{8}{*}{${ }^{235} \mathrm{U}$} & 143.8 & 11.0 & ${ }^{235} \mathrm{U}$ & & & \multirow{8}{*}{$\begin{array}{l}\text { Near } 270.2 \mathrm{keV}{ }^{223} \mathrm{Ac} \\
\text { Near } 270.2 \mathrm{keV}{ }^{223} \mathrm{Ac} \\
\text { Buried by } 351.9 \mathrm{keV}{ }^{214} \mathrm{~Pb}\end{array}$} \\
\hline & 154.2 & 5.7 & ${ }^{223} \mathrm{Ra}$ & $5.2 \pm 2.4$ & $35 \pm 16$ & \\
\hline & 163.3 & 5.1 & ${ }^{235} \mathrm{U}$ & $4.2 \pm 2.3$ & $32 \pm 18$ & \\
\hline & 185.7 & 57.2 & ${ }^{235} \mathrm{U}$ & & & \\
\hline & 205.3 & 5.0 & ${ }^{235} \mathrm{U}$ & $6.4 \pm 2.2$ & $57 \pm 20$ & \\
\hline & 269.5 & 13.9 & ${ }^{223} \mathrm{Ra}$ & & & \\
\hline & 271.2 & 10.8 & ${ }^{219} \mathrm{Rn}$ & & & \\
\hline & 351.1 & 13.02 & ${ }^{211} \mathrm{Bi}$ & & & \\
\hline \multirow[t]{4}{*}{ other } & 661.7 & 85.1 & ${ }^{137} \mathrm{Cs}$ & $2.0 \pm 0.8$ & $2.9 \pm 1.2$ & \\
\hline & 1173.2 & 99.85 & ${ }^{60} \mathrm{Co}$ & $0.4 \pm 0.6$ & $0.9 \pm 1.3$ & \\
\hline & 1332.5 & 99.9826 & ${ }^{60} \mathrm{Co}$ & $-0.7 \pm 0.4$ & $-1.7 \pm 1.0$ & \\
\hline & 1460.8 & 10.66 & ${ }^{40} \mathrm{~K}$ & $11.2 \pm 1.0$ & $255 \pm 26$ & \\
\hline
\end{tabular}

Table 7.1: continued.

the same detector arrangement, the runs are combined and then fitted for the background subtraction peaks. If the radon count rate is not stable, the radon rate is interpolated between the background runs taken before and after the sample was counted. The identical fit procedure is performed to both the source count spectrum and a background count spectrum.

Assay samples placed near the detector can block the measurement of background activity. Simulations are used to evaluate this effect assuming that all of the background activity is generated isotropically by a spherical shell containing the detector and the sample. The 


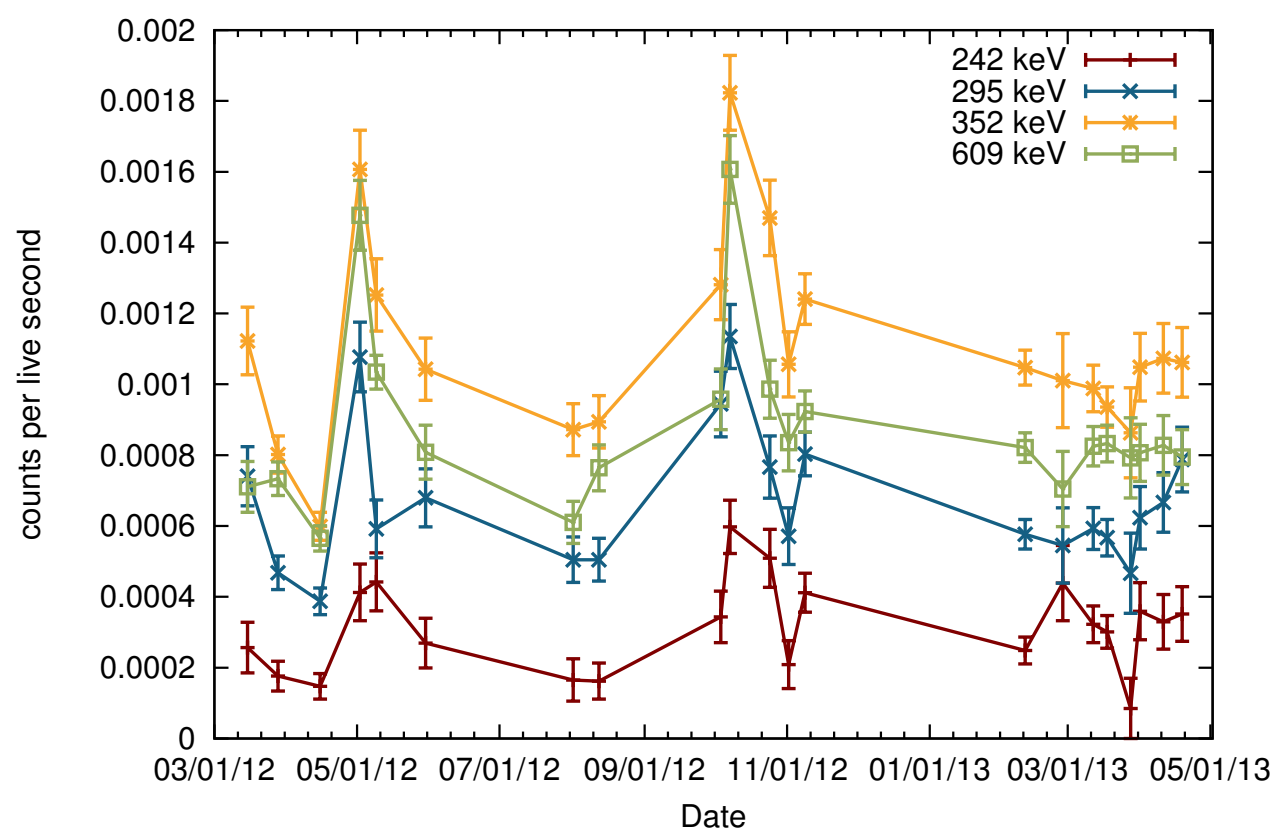

Figure 7.1: Variation of ${ }^{222} \mathrm{Rn}$ daughter activities in the detector volume. The $242 \mathrm{keV}$, $295 \mathrm{keV}$, and $352 \mathrm{keV}$ photons are from the decay of ${ }^{214} \mathrm{~Pb}$ while the $609 \mathrm{keV}$ photons are from the decay of ${ }^{214} \mathrm{Bi}$.

portion of the background activity that is not blocked by the sample is subtracted from the measured activity of the sample. $50 \%$ of the portion that is blocked is added to the systematic uncertainty of the assay.

Uncertainties for each assay are derived from counting and simulation statistics, a $10 \%$ simulation systematic uncertainty, and background blocking uncertainties. Uncertainties in the variation of the radon background, are not included.

The dimensions and mass of the GEM-10 HPGe crystal and vacuum can specified by ORTEC, the crystal manufacturer, were used in the simulation geometry, shown in Figure 7.2. The crystal is a p-type detector with a $1.35 \pm 0.04 \mathrm{~mm}$ thick inactive layer around the outside faces of the crystal that was included in the simulation. The inactive layer thickness was determined by adjusting the simulation to match the relative intensity of the $81 \mathrm{keV}$ and $276 \mathrm{keV}$ to $384 \mathrm{keV}$ photons from a $0.5 \mu \mathrm{Ci}{ }^{133} \mathrm{Ba}$ source. The inactive layer 


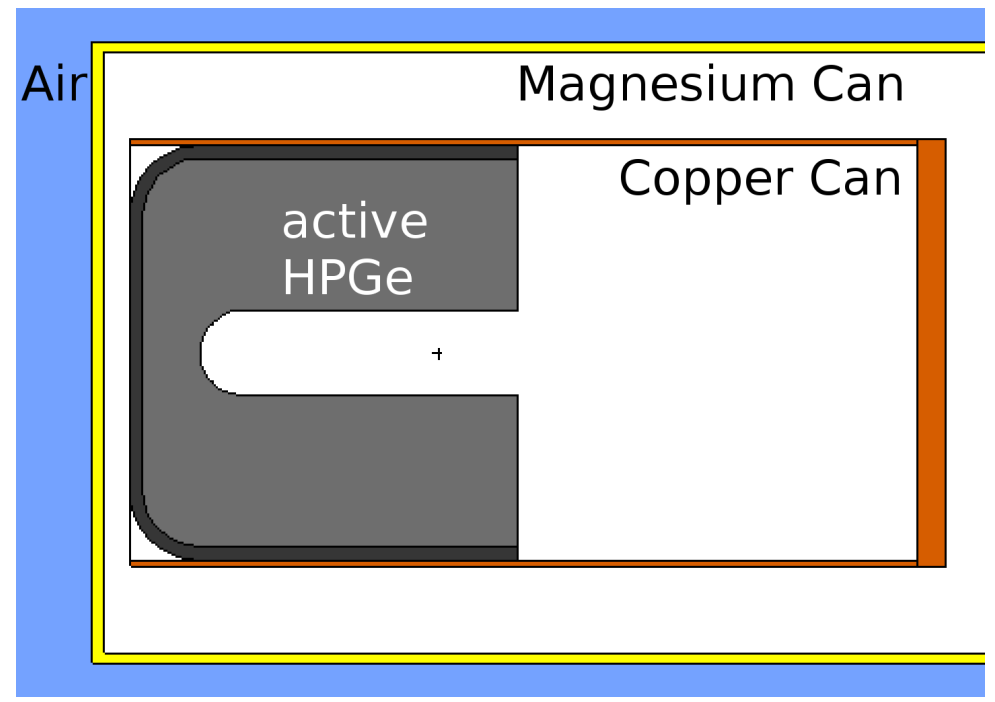

Figure 7.2: An x-y cross-section of the Collar Lab assay facility high-purity germanium (HPGe) detector simulation geometry.

thickness was tuned within the simulation to match the measured count rates.

The detector deadtime was not properly calculated by the modified XiA Polaris multichannel analyzer used with the HPGe detector. To verify the absolute detector sensitivity, the detector was exposed to calibrated sources, with activities of 7.7301 for ${ }^{133} \mathrm{Ba}$ and 6.923 $\mu \mathrm{Ci}$ for ${ }^{88} \mathrm{Y}$ on the day of calibration, placed at several distances from the front face of the detector in order to vary the detected count rate. The simulated and measured count rates are compared in Figure 7.3. A livetime subtraction of $142 \pm 4 \mu$ s per trigger in the presence of ${ }^{88} \mathrm{Y}$ reproduces the calibrated source strength. In the presence of ${ }^{133} \mathrm{Ba}$ photons a slightly longer deadtime of $220 \mu \mathrm{s}$ is preferred, possibly due to there being a higher proportion of hits below the $52 \mathrm{keV}$ trigger threshold. A $142 \mu$ s deadtime correction is applied to all assay results, although the correction is negligible for most samples.

The detector's energy scale and resolution was calibrated using a variety of photon energies, and is rechecked periodically using photons from ${ }^{60} \mathrm{Co}$. Fits to the energy calibration and resolution are shown in Figure 7.4. Linear functions fit both the energy scale and the 


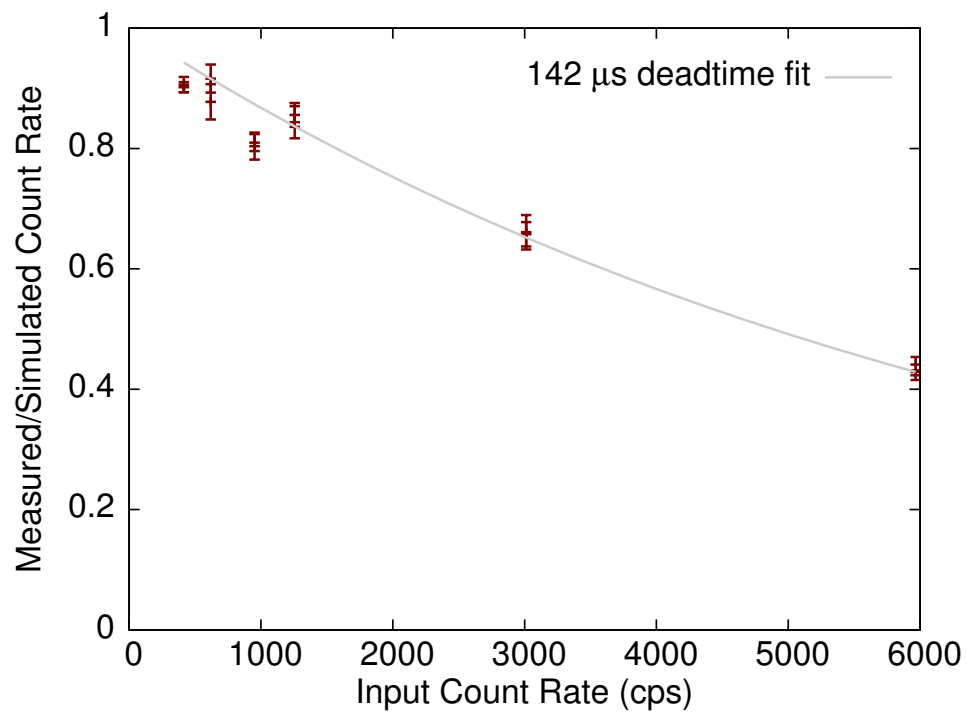

Figure 7.3: Simulated vs. measured absolute count rates from ${ }^{133} \mathrm{Ba}$ and ${ }^{88} \mathrm{Y}$ calibrations of the Collar Lab assay facility.
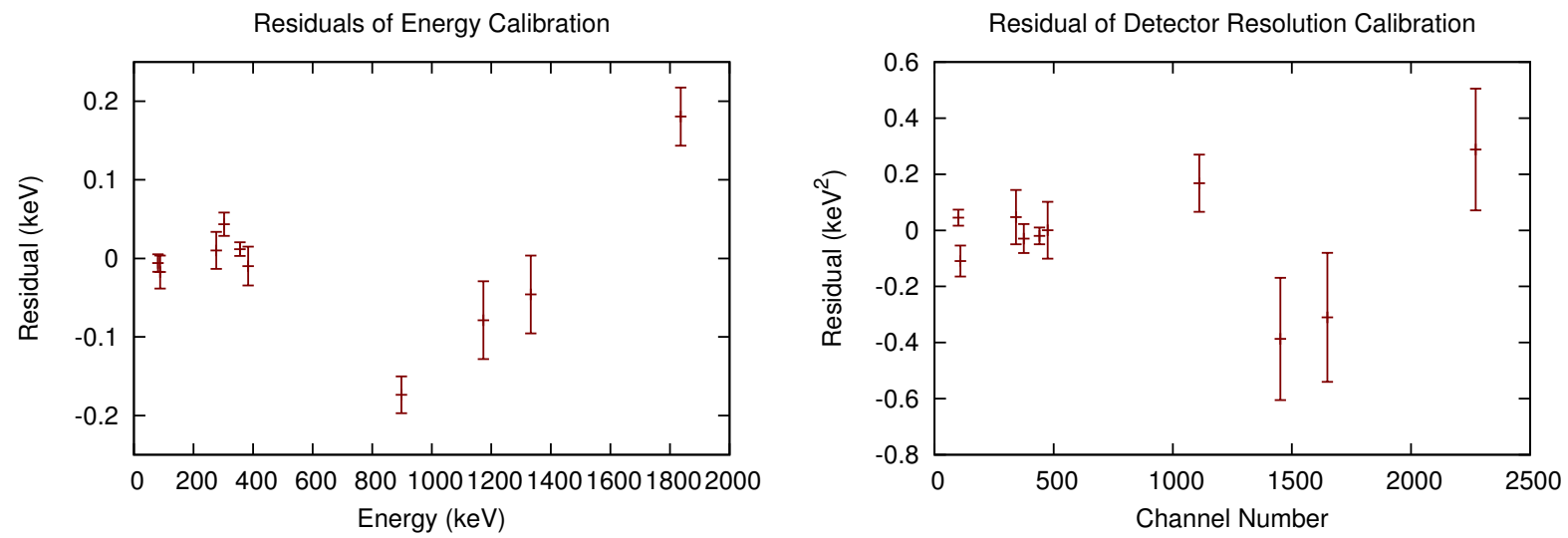

Figure 7.4: Fit residuals for the detector's energy and resolution calibrations, Apr 2013. 
variance of the energy measurement well. In Apr 2013, the functions were

$$
\begin{aligned}
& E=(0.80816(4) \times(\text { channel number })+0.129(21)) \mathrm{keV} \\
& \sigma_{E}^{2}=(1.53(3) \times(\text { channel number })+1.321(14)) \mathrm{keV}^{2}
\end{aligned}
$$

The energy scale has changed slightly whenever the high-voltage power supply for the detector has been readjusted. However, the energy resolution remains stable and is used as a fixed parameter when fitting photon energy spectra.

In comparison to the University of Chicago $\gamma$ assay system, the system at SNOLAB is significantly more sensitive. The Chicago crystal's active volume is 4 times smaller, and the background activity in ${ }^{222} \mathrm{Rn}$ daughters, ${ }^{232} \mathrm{Th}$, an ${ }^{40} \mathrm{~K}$ are approximately 140,20 , and 110 times higher respectively. The excess ${ }^{222} \mathrm{Rn}$ daughters are attributable to the lack of a radon purge system. However, the University of Chicago detector can accommodate much larger samples than at SNOLAB, precluding the need for destructive testing of such samples. The background rates of ${ }^{137} \mathrm{Cs}$ and ${ }^{60} \mathrm{Co}$ are also very similar between the two facilities.

\section{$7.2(\alpha, n)$ Reaction Rates}

Most neutrons emitted by PICO detector materials are produced via spontaneous fission or the $(\alpha, n)$ reaction. A modified version of SOURCES-4C (Wilson et al. 2002) is used to calculate the neutron flux and energy spectrum generated in the bulk of a material given the material composition and concentration of radioisotopes. As noted in Fustin (2012), SOURCES-4C's calculation is severely limited. The cross-section libraries provided with the program do not extend to energies above $6.5 \mathrm{MeV},(\alpha, n)$ production in heavy nuclei such as

iron is omitted, and cross-sections were provided based largely on nuclear models instead of experimental data. Tomasello and Kudryavtsev (2006) modified the SOURCES-4A source 
code and libraries to accept high energy $\alpha$ particles and with updated experimental results and nuclear model calculations extending to heavy nuclei. I have independently made similar modifications to the source code for SOURCES-4C.

SOURCES-4C calculates the neutron emission spectrum by comparing the ratio of the $\alpha$ stopping cross-section to the $(\alpha, n)$ reaction cross-section at an array of up to 4000 energies for every originating $\alpha$ energy. At each point, the energy of outgoing neutrons is calculated using the $Q$-value for each possible final state branching ratio of the two-body $(\alpha, n)$ reaction. The outgoing neutron energy was binned with bin sizes of $0.5 \mathrm{MeV}$ selected. The data used for these calculations is contained in 4 library files.

The first library, tape2, contains the coefficients for the $\alpha$ stopping cross-section given by ICRU (1993). tape2 was extended to include the coefficients for compounds included in the ICRU report including $\mathrm{CaF}_{2}$, cellulose nitrate, polyethylene, polycarbonate, PMMA, polystyrene, silica, NaI, and liquid water. Where coefficients are not given for compounds, the stopping power is approximated by the weighed average of the constituent elemental stopping powers.

tape3 contains $(\alpha, n)$ cross-sections. This file was completely replaced using the crosssections listed in Table 7.2. For magnesium and elements heavier than silicon, the crosssections calculated by Tomasello and Kudryavtsev are rescaled to match the experimental values to within $10 \%$.

The cross-section for deuterium breakup was rescaled from the ${ }^{2} \mathrm{H}(p, n)$ reaction crosssection in ENDF/B-VII (Chadwick et al. 2011) assuming that coulomb excitation is the only processes causing the breakup. The doubled charge of an $\alpha$ increased the cross-section by a factor of 4 when compared to a proton moving at the same non-relativistic speed. With four times the mass, an $\alpha$ has twice the energy of a proton with the same speed. Combining 


\begin{tabular}{|c|c|}
\hline Reference & $(\alpha, n)$ cross-section targets \\
\hline ENDF/B-VII.1 (Chadwick et al. 2011) & ${ }^{2} \mathrm{H}$ (see text) \\
\hline Murata, Matsunobu, and Shibata 2006 & $\begin{array}{l}{ }^{6,7} \mathrm{Li},{ }^{9} \mathrm{Be}(\text { see text }),{ }^{10,11} \mathrm{~B},{ }^{12} \mathrm{C},{ }^{14,15} \mathrm{~N}, \\
{ }^{17,18} \mathrm{O},{ }^{19} \mathrm{~F},{ }^{23} \mathrm{Na},{ }^{27} \mathrm{Al},{ }^{28,29,30} \mathrm{Si}\end{array}$ \\
\hline Harissopulos et al. 2005 & ${ }^{13} \mathrm{C}$ \\
\hline Cheng and King 1980 & ${ }^{24} \mathrm{Mg}$ \\
\hline See text & $25,26 \mathrm{Mg}$ \\
\hline Masumoto and Yagi 1983 & ${ }^{31} \mathrm{P}$ \\
\hline Woosley et al. 1975 & ${ }^{37} \mathrm{Cl}$ \\
\hline Howard et al. 1974 ; Abe et al. 1984 & ${ }^{46} \mathrm{Ti}$ \\
\hline Morton et al. 1992 & ${ }^{48} \mathrm{Ti}$ \\
\hline Morton et al. $\overline{1994}$ & ${ }^{50} \mathrm{Cr}$ \\
\hline Kocsonya et al. 2006 & ${ }^{52} \mathrm{Cr}$ (see text) \\
\hline Tims et al. 1993 & ${ }^{55} \mathrm{Mn}$ \\
\hline Tims et al. $\overline{\overline{1991}}$ & ${ }^{54} \mathrm{Fe}$ \\
\hline Jacobs and Liskien 1983 & $56,57,58 \mathrm{Fe}$ \\
\hline Tomasello and Kudryavtsev 2006 calculations & $\begin{array}{l}{ }^{39,40,41} \mathrm{~K},{ }^{42,43,44,46,48} \mathrm{Ca},{ }^{47,49,50} \mathrm{Ti}, \\
{ }^{53,54} \mathrm{Cr},{ }^{60,61,62,64} \mathrm{Ni}\end{array}$ \\
\hline
\end{tabular}

Table 7.2: Cross-sections and references used to construct the modified SOURCES-4C tape3 libraries.

the effects,

$$
\sigma_{2} \mathrm{H}(\alpha, n)(E)=4 \sigma_{2} \mathrm{H}(p, n)(E / 2)
$$

Kocsonya et al. (2006) measured the production of $411 \mathrm{keV}$ photons from the photon cascade of the ${ }^{52} \mathrm{Cr}(\alpha, n){ }^{55} \mathrm{Fe}$ reaction. The branching fraction of the $411 \mathrm{keV}$ photon from cascades of each of the reaction's final states was calculated using the final state branching fractions calculated by Tomasello and Kudryavtsev and photon branching ratios of the excited states of ${ }^{55} \mathrm{Fe}$ from Huo, Huo, and Ma (2007). The calculated production rate of $411 \mathrm{keV}$ photons matched the measured rate within $10 \%$.

tape4 contains $(\alpha, n)$ final state branching fractions and $Q$-values. These are taken from Tomasello and Kudryavtsev with updates from Murata, Matsunobu, and Shibata (2006) where available. Except for the ${ }^{9} \operatorname{Be}(\alpha, n+\alpha)$ reaction (see Section 3.3), only the branching 


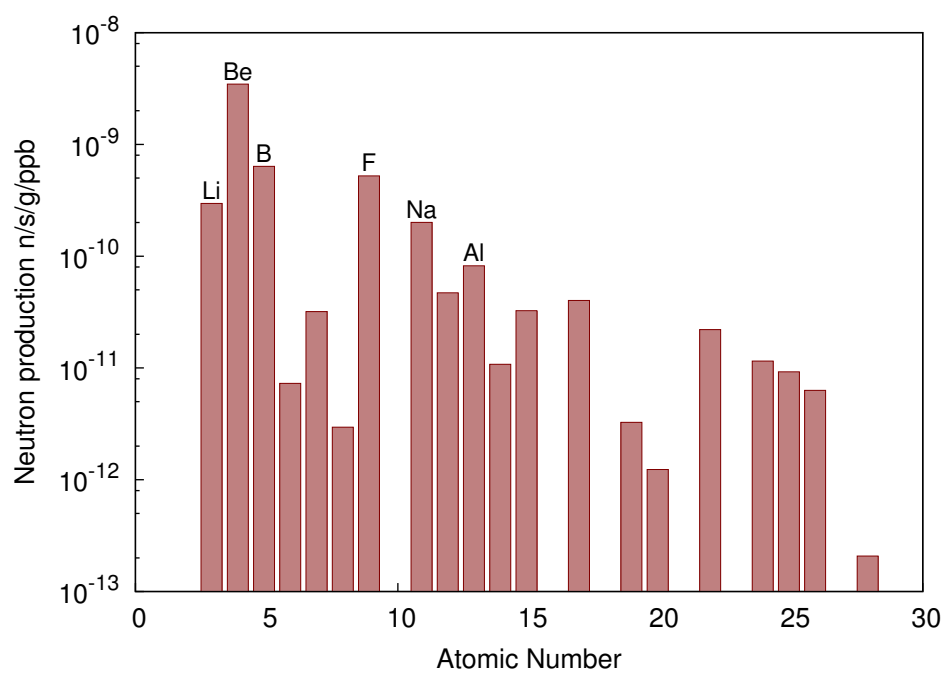

(a) ${ }^{232} \mathrm{Th}$

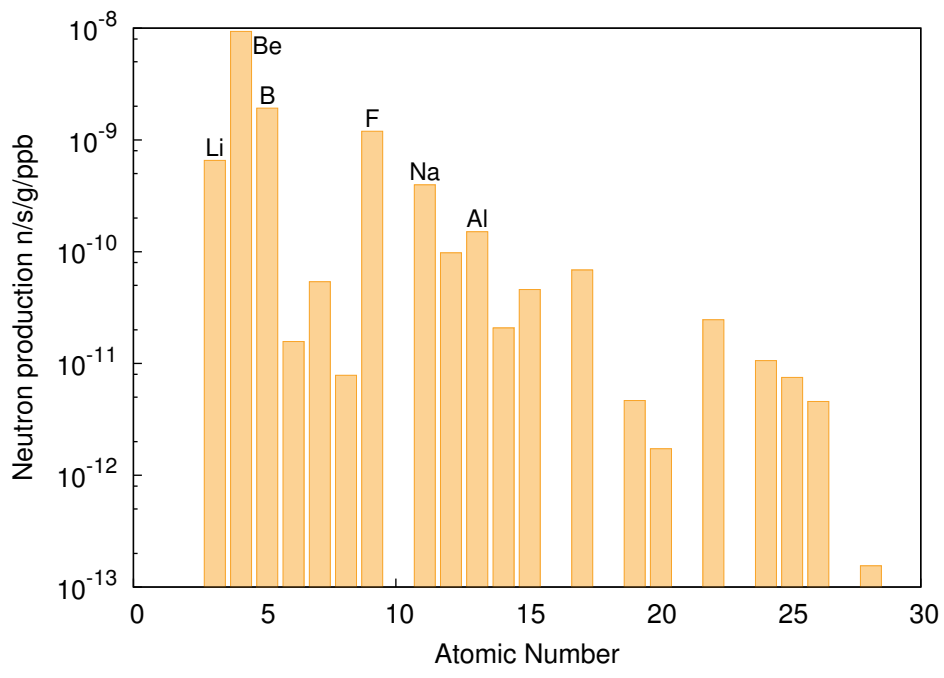

(b) ${ }^{238} \mathrm{U}$

Figure 7.5: Calculated neutron yields from ${ }^{232} \mathrm{Th}$ and ${ }^{238} \mathrm{U}$ decay chain activity in elemental targets of natural abundance. Calculations include all updates to the SOURCES-4C program from this section. Only the $(\alpha, n)$ neutron yield is shown for ${ }^{238} \mathrm{U} .{ }^{238} \mathrm{U}$ spontaneous fission adds an additional rate of $1.36 \times 10^{-11} \mathrm{n} / \mathrm{s} / \mathrm{g} / \mathrm{ppb}$ for all target materials with an average ${ }^{238} \mathrm{U}$ spontaneous fission neutron energy of $1.69 \mathrm{MeV}$. 


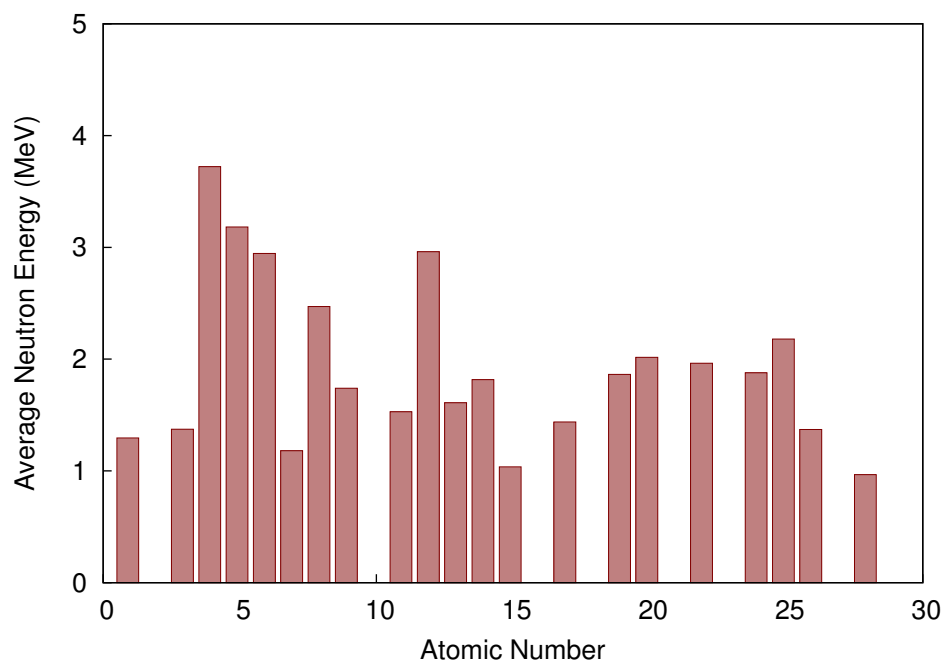

(a) ${ }^{232} \mathrm{Th}$

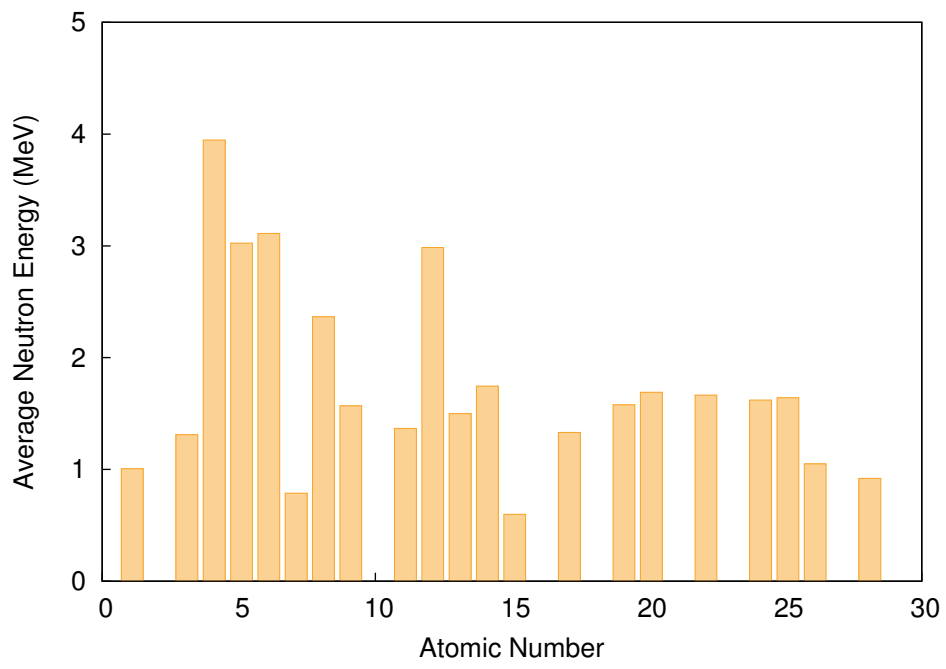

(b) ${ }^{238} \mathrm{U}$

Figure 7.6: Calculated average neutron energies from $(\alpha, n)$ reactions of ${ }^{232} \mathrm{Th}$ and ${ }^{238} \mathrm{U}$ decay chain $\alpha$ particles in elemental targets of natural abundance. Calculations include all updates to the SOURCES-4C program from this section. 


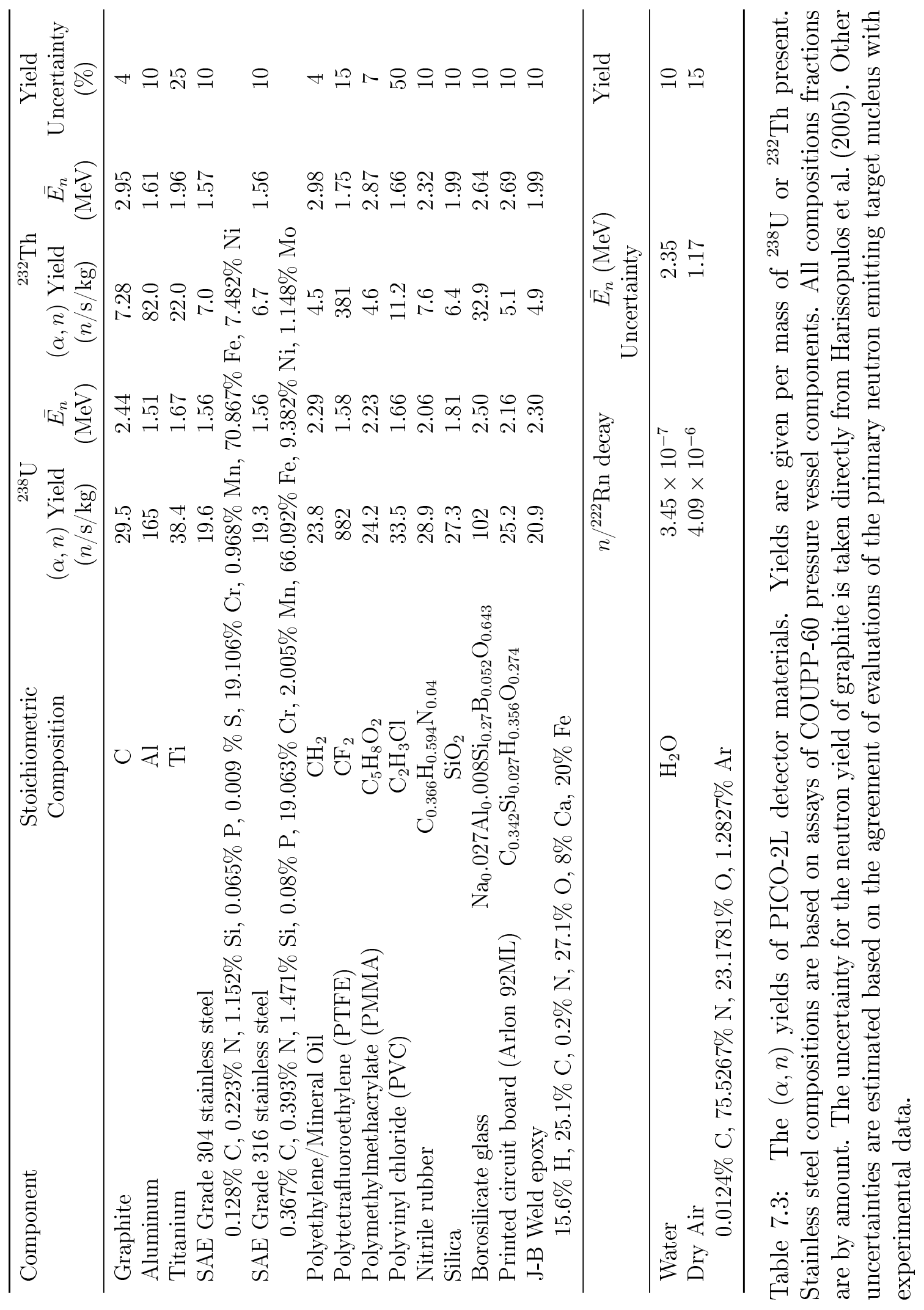


ratios of the $(\alpha, n)$ reaction are used. Calculations of the neutron energy spectrum from other isotopes with significant $(\alpha, n+\alpha)$ reaction cross-sections below $9 \mathrm{MeV},{ }^{10} \mathrm{~B},{ }^{14} \mathrm{~N}$, and

${ }^{17} \mathrm{O}$, produce neutrons with higher energies than if the three-body reaction were modeled.

tape5 contains the properties of radioisotopes including their $\alpha$ energies and branching ratios, spontaneous fission rates, Watt fission spectra parameters, and $\beta$-delayed neutron emission. The $\beta$-delayed neutron production from ${ }^{210} \mathrm{Tl}$ (Stetter 1961) was added to tape5 and the alpha energies and branchings for ${ }^{212} \mathrm{Bi}$ and ${ }^{214} \mathrm{Bi}$ (Bé et al. 2004) were updated.

Figures 7.5 and 7.6 show neutron yields and energies calculated using these updates to SOURCES-4C. When selecting material for use in dark matter detectors, elements with large $(\alpha, n)$ neutron yields should be avoided. The largest yields are produced by beryllium, boron, fluorine, lithium, magnesium, and aluminum. Table 7.3 lists $(\alpha, n)$ target materials and neutron yields used in PICO-2L. Borosilicate glass, PTFE, and aluminum have large neutron yield and their use in PICO-2L was minimized.

\subsection{COUPP-4kg Background Recalculation}

The COUPP-4kg neutron background estimates from Fustin (2012, Table 7.6) were recalculated given the updates in the calibration of the University of Chicago GES system and new $(\alpha, n)$ yield calculations. The new expected background rates are given in Table 7.4 . A $31 \%$ increase in the background rate is due to the updated SOURCES-4C calculation with a further $39 \%$ increase due to the GES calibration. The calculation assumes the flat $46 \%$ efficiency above the Seitz threshold for detecting neutrons used by Fustin. These recalculated background rates are now consistent with the multiple bubble event rate observed in COUPP-4kg (Behnke et al. 2012). 


\begin{tabular}{|c|c|c|c|c|c|}
\hline Material & Source & $\begin{array}{c}\text { Rate in } \\
\text { DM- } 34^{\circ} \mathrm{C}\end{array}$ & $\begin{array}{c}\text { Rate in } \\
\text { DM- } 37^{\circ} \mathrm{C}\end{array}$ & $\begin{array}{c}\text { Rate in } \\
\text { DM- } 40^{\circ} \mathrm{C}\end{array}$ & $\begin{array}{l}\text { New calc } \\
\div \text { old calc }\end{array}$ \\
\hline \multicolumn{6}{|c|}{$\left(10^{-3} \mathrm{cts} / \mathrm{kg}_{\mathrm{CF}_{3} \mathrm{I}} /\right.$ day $)$} \\
\hline Glass (viewports) & ${ }^{238} \mathrm{U}(\alpha, \mathrm{n}) 0.72 \mathrm{ppm}$ & 6.94 & 7.53 & 7.99 & 1.79 \\
\hline Glass (viewports) & ${ }^{232} \mathrm{Th}(\alpha, \mathrm{n}) 0.74 \mathrm{ppm}$ & 2.62 & 2.84 & 3.02 & 2.01 \\
\hline PZT (piezos) & ${ }^{238} \mathrm{U}$ s.f. $5.9 \mathrm{ppm}$ & 2.46 & 2.69 & 2.85 & 1.50 \\
\hline PZT (piezos) & ${ }^{238} \mathrm{U}(\alpha, \mathrm{n}) 5.9 \mathrm{ppm}$ & 1.46 & 1.60 & 1.67 & 2.40 \\
\hline \multicolumn{6}{|c|}{$\left(10^{-4} \mathrm{cts} / \mathrm{kg}_{\mathrm{CF}_{3} \mathrm{I}} /\right.$ day $)$} \\
\hline Glass (viewports) & ${ }^{238}$ U s.f. $0.72 \mathrm{ppm}$ & 6.82 & 7.41 & 7.50 & 1.40 \\
\hline Norite (rock walls) & ${ }^{238} \mathrm{U} /{ }^{232} \mathrm{Th} 1.2 / 3.3 \mathrm{ppt}$ & 3.0 & 3.0 & 3.0 & 2.4 \\
\hline PZT (piezos) & ${ }^{232} \mathrm{Th}(\alpha, \mathrm{n}) 1.77 \mathrm{ppm}$ & 1.92 & 2.13 & 2.28 & 3.05 \\
\hline PCB (preamps) & ${ }^{232} \mathrm{Th}(\alpha, \mathrm{n}) 2.81 \mathrm{ppm}$ & 1.10 & 1.19 & 1.26 & 2.24 \\
\hline Steel (pressure vessel) & ${ }^{238}$ U s.f. $\sim 1 \mathrm{ppb}$ & 0.92 & 1.00 & 1.07 & 1 \\
\hline \multicolumn{6}{|c|}{$\left(10^{-5} \mathrm{cts} / \mathrm{kg}_{\mathrm{CF}_{3} \mathrm{I}} /\right.$ day $)$} \\
\hline J-B Weld (epoxy) & ${ }^{238}$ U s.f. $0.242 \mathrm{ppm}$ & 8.16 & 8.88 & 9.44 & 1.40 \\
\hline PCB (preamps) & ${ }^{238} \mathrm{U}(\alpha, \mathrm{n}) 0.687 \mathrm{ppm}$ & 6.59 & 7.13 & 7.60 & 1.99 \\
\hline PCB (preamps) & ${ }^{238}$ U s.f. $0.687 \mathrm{ppm}$ & 6.17 & 6.72 & 7.15 & 1.40 \\
\hline J-B Weld (epoxy) & ${ }^{238} \mathrm{U}(\alpha, \mathrm{n}) \quad 0.242 \mathrm{ppm}$ & 5.57 & 5.98 & 6.29 & 2.65 \\
\hline Steel (pressure vessel) & ${ }^{232} \mathrm{Th}(\alpha, \mathrm{n}) \sim 1 \mathrm{ppb}$ & 3.97 & 4.36 & 4.65 & 102 \\
\hline Steel (pressure vessel) & ${ }^{238} \mathrm{U}(\alpha, \mathrm{n}) \sim 1 \mathrm{ppb}$ & 3.37 & 3.69 & 3.94 & 35.5 \\
\hline Quartz (flange) & ${ }^{238} \mathrm{U}(\alpha, \mathrm{n}) 73 \mathrm{ppb}$ & 3.56 & 3.88 & 4.17 & 2.54 \\
\hline Quartz (flange) & ${ }^{238} \mathrm{U}$ s.f. $73 \mathrm{ppb}$ & 3.34 & 3.63 & 3.84 & 1.74 \\
\hline J-B Weld (epoxy) & ${ }^{232} \mathrm{Th}(\alpha, \mathrm{n}) 0.14 \mathrm{ppm}$ & 2.00 & 2.13 & 2.24 & 4.94 \\
\hline Glycol (hydraulic fluid) & ${ }^{238} \mathrm{U}$ total $31 \mathrm{ppt}$ & 0.876 & 0.948 & 1.01 & 1.99 \\
\hline Quartz (flange) & ${ }^{232} \mathrm{Th}(\alpha, \mathrm{n}) 45 \mathrm{ppb}$ & 1.02 & 1.11 & 1.20 & 2.64 \\
\hline \multicolumn{6}{|c|}{$\left(10^{-6} \mathrm{cts} / \mathrm{kg}_{\mathrm{CF}_{3} \mathrm{I}} /\right.$ day $)$} \\
\hline Steel (pressure vessel) & $(\mu, \mathrm{n})$ & 4.55 & 4.87 & 5.12 & 1 \\
\hline Piezos and Quartz & ${ }^{210} \mathrm{Tl}$ from ${ }^{238} \mathrm{U}$ & 3.18 & 4.65 & 6.14 & $\mathrm{n} / \mathrm{a}$ \\
\hline $\mathrm{CF}_{3} \mathrm{I}$ (target) & $(\mu, \mathrm{n})$ & 3.74 & 3.99 & 4.21 & 1 \\
\hline \multicolumn{6}{|c|}{$\left(10^{-2} \mathrm{cts} / \mathrm{kg}_{\mathrm{CF}_{3} \mathrm{I}} /\right.$ day $)$} \\
\hline Total & & 1.53 & 1.68 & 1.76 & 1.82 \\
\hline
\end{tabular}

Table 7.4: Updated neutron background rates from each source, for the DM- $34^{\circ} \mathrm{C}, \mathrm{DM}-37^{\circ} \mathrm{C}$, and DM- $40^{\circ} \mathrm{C}$ data sets with comparisons to the rates from Fustin (2012, Table 7.6). 


\subsection{Neutron Calibration Backgrounds}

A population of events had been seen in neutron calibrations of both COUPP-4kg and PICO2L with an acoustic parameter between 2 and 5, and with a rate higher than that expected from the rate of $\alpha$ decays seen in background running. This excess is mostly explained by $\alpha$ particles generated by the $(n, \alpha)$ neutron capture reaction. These $\alpha$ particles have energies of $1-5 \mathrm{MeV}$, potentially explaining the lower acoustic parameter of the excess events. A total of $17.5 \pm 1.9 \alpha$ events are expected during PICO-2L AmBe calibrations at $3 \mathrm{keV}$ and $4 \mathrm{keV}$. Additional high-AP events observed in PICO-2L may be caused by energetic recoils, but further investigation into acoustic power generation is required to validate this hypothesis.

Table 7.5 shows all the possible neutron induced reactions during PICO-2L or COUPP$4 \mathrm{~kg}$ neutron calibrations. For both $\mathrm{C}_{3} \mathrm{~F}_{8}$ and $\mathrm{CF}_{3} \mathrm{I}$, the ${ }^{19} F(n, \alpha){ }^{16} N$ and ${ }^{19} F(n, n+\alpha){ }^{15} N$ reactions dominate the nuclear reaction rate. The cross-section of the former reaction has been measured several times since the 1950s. These measurements, including one taken by Bondarenko et al. 2013, disagree in absolute rate by factors of two. The cross-section for ${ }^{19} F(n, n+\alpha){ }^{15} N$ has not been experimentally verified but has been calculated with a theoretical uncertainty of $17 \%$ below $8 \mathrm{MeV}$ (Chadwick et al. 2011).

The ENDF/B-VII library (Chadwick et al. 2011) was used in MCNPX-Polimi to calculate the nuclear reaction rates during neutron calibrations. This library used a ${ }^{19} F(n, \alpha){ }^{16} N$ cross-section based on the measurements available in 1966. Figure 7.7 plots the various measured and evaluated reaction cross-sections. Smith, Meadows, and Whalen (1981) remeasured the cross-section in 1981 by comparing the short-lived ${ }^{16} \mathrm{~N}$ activation of a Teflon target against the ${ }^{238} \mathrm{U}(n, f)$ reaction rate in the same beam. This measurement had a well evaluated uncertainty of $15 \%$ mostly due to the detection efficiency of $\gamma$ 's from the ${ }^{16} \mathrm{~N}$ decays. The cross-section provided by ENDF/B-VII will be used with a separate rate printed rescaled to the cross-section from Smith, Meadows, and Whalen. 


\begin{tabular}{|c|c|c|c|c|}
\hline \multirow[t]{2}{*}{ Reaction } & \multirow[t]{2}{*}{$\mathrm{Q}(\mathrm{MeV})$} & \multicolumn{3}{|c|}{ Cross-section $(\mathrm{mb})$} \\
\hline & & at $4 \mathrm{MeV}$ & at $7 \mathrm{MeV}$ & at $10 \mathrm{MeV}$ \\
\hline${ }^{12} \mathrm{C}(n, \alpha){ }^{9} \mathrm{Be}$ & -5.702 & - & 1 & 162.4 \\
\hline${ }^{12} C(n, n+3 \alpha)$ & -7.275 & - & - & 15.5 \\
\hline${ }^{13} C(n, \alpha){ }^{10} B e$ & -3.836 & \multicolumn{3}{|c|}{ Not in ENDF } \\
\hline${ }^{13} C(n, 2 n){ }^{12} C$ & -4.946 & \multicolumn{3}{|c|}{ Not in ENDF } \\
\hline${ }^{19} F(n, \alpha)^{16} N$ & -1.525 & 45.8 & 150 & 63.3 \\
\hline$\left.{ }^{19} F(n, n+\alpha)\right)^{15} N$ & -4.014 & - & 122.7 & 413 \\
\hline${ }^{19} F(n, p){ }^{19} O$ & -4.038 & - & 37 & 35.9 \\
\hline${ }^{19} F(n, d){ }^{18} O$ & -5.769 & - & 0 & 9.89 \\
\hline${ }^{19} F(n, t){ }^{17} O$ & -7.557 & - & - & 1.5 \\
\hline${ }^{19} F(n, n+p){ }^{18} O$ & -7.994 & - & - & 2.88 \\
\hline${ }^{127} I(n, \alpha){ }^{124} S b$ & 4.284 & 0.001 & 0.028 & 0.192 \\
\hline${ }^{127} I(n, p){ }^{127} T e$ & 0.080 & 0.032 & 0.071 & 4.12 \\
\hline${ }^{127} I(n, 2 \alpha)^{120} I n$ & -0.034 & \multicolumn{3}{|c|}{ Not in ENDF } \\
\hline${ }^{127} I(n, n+\alpha){ }^{123} S b$ & -2.184 & 0 & 0 & 0.001 \\
\hline${ }^{127} I(n, p+\alpha)^{123} S n$ & -2.810 & 0 & 0 & 0.024 \\
\hline${ }^{127} I(n, d){ }^{126} T e$ & -3.983 & \multicolumn{3}{|c|}{ Not in ENDF } \\
\hline${ }^{127} I(n, 2 n)^{126} T e$ & -9.144 & - & - & 243 \\
\hline
\end{tabular}

Table 7.5: Q-values and cross-sections of relevant neutron induced charged particle reactions during AmBe calibrations. Rare reactions processes labelled "Not in ENDF" are not simulated. The effect of the missing libraries should be negligible.

A more recent measurement of the ${ }^{19} F(n, \alpha){ }^{16} N$ reaction by Bondarenko et al. (2013) published a cross-section significantly below those previously measured with very low uncertainties. They and Davis et al. (1961) measured the $\alpha$ particles from $\mathrm{CF}_{4}$ target gas in an ionization chamber. Bondarenko et al. do not obtain the same functional shape of cross-section vs. energy as the other measurements. Their paper compares their inclusive ${ }^{19} F(n, \alpha){ }^{16} N$ cross-section measurement with the exclusive measurements of other groups, and fail to recognize that they are measuring both the ${ }^{19} F(n, \alpha){ }^{16} N$ and ${ }^{19} F(n, n+\alpha){ }^{15} N$ reactions. Given the significant amount of event selection they require to produce their result (Bondarenko et al. 2013, Fig. 2), additional unpublished details of their experiment would be required to validate their cut efficiencies. Their data will not be considered. 


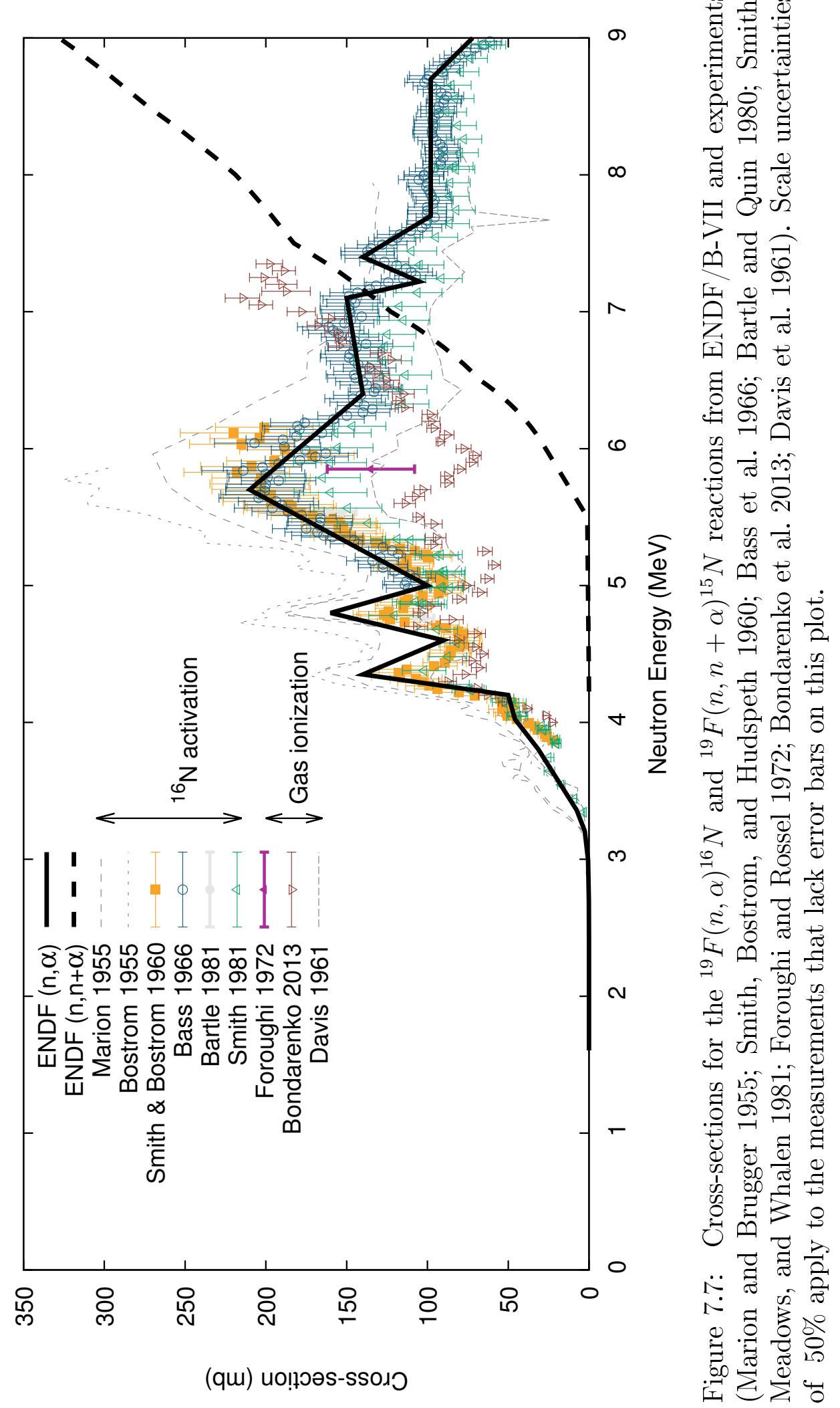




\begin{tabular}{|c|c|c|c|c|}
\hline \multirow[t]{2}{*}{ Reaction } & \multicolumn{2}{|c|}{ Single-bubble Events } & \multicolumn{2}{|c|}{ Multi-bubble Events } \\
\hline & \# Simulated & Rate (events/hour) & \# Simulated & Rate (events/hour) \\
\hline Elastic scatter & 63316 & $15.68_{ \pm 0.78 \text { syst. }}^{ \pm 0.06 \text { stat. }}$ & 71794 & $17.77_{ \pm 0.89 \text { syst. }}^{ \pm 0.07 \text { stat. }}$ \\
\hline${ }^{12} C(n, \alpha){ }^{9} B e$ & 105 & $0.0260_{ \pm 0.0026}^{ \pm 0.0025}$ stat. & 17 & $0.0042_{ \pm 0.0004}^{ \pm 0.0011 \text { stat. }}$ \\
\hline${ }^{12} C(n, n+3 \alpha)$ & 2 & $0.0005_{ \pm 0.0002}^{ \pm 0.0004 \text { stat. }}$ syst. & 4 & $0.0010_{ \pm 0.0005}^{ \pm 0.0006 \text { stat. }}$ \\
\hline${ }^{19} F(n, \alpha){ }^{16} N$ & 1770 & $0.438_{ \pm 0.044 \text { syst. }}^{ \pm 0.010 \text { stat. }}$ & 414 & $0.102_{ \pm 0.010}^{ \pm 0.005}$ stat. \\
\hline rescaled & & $0.357_{ \pm 0.054 \text { syst. }}^{ \pm 0.009 \text { stat. }}$ & & $0.083_{ \pm 0.013}^{ \pm 0.004 \text { stast. }}$ \\
\hline${ }^{19} F(n, n+\alpha){ }^{15} N$ & 473 & $0.117_{ \pm 0.016 \text { syst. }}^{ \pm 0.005 \text { stat. }}$ & 799 & $0.198_{ \pm 0.028}^{ \pm 0.007 \text { stat. }}$ \\
\hline${ }^{19} F(n, p){ }^{19} O$ & 242 & $0.060_{ \pm 0.010 ~ s y s t}^{ \pm 0.004 ~}$ & 44 & $0.0109_{ \pm 0.0017}^{ \pm 0.0017 \text { stast. }}$ \\
\hline${ }^{19} F(n, d){ }^{18} O$ & 8 & $0.0020_{ \pm 0.0006 \text { syst. }}^{ \pm 0.0007 \text { stat. }}$ & 0 & 0 \\
\hline Reactions total & 2600 & $0.562_{ \pm 0.057 \text { syst. }}^{ \pm 0.011 \text { stat. }}$ & 1278 & $0.297_{ \pm 0.030}^{ \pm 0.008 \text { stat. }}$ \\
\hline
\end{tabular}

Table 7.6: Simulated bubble events from $10^{9}$ AmBe neutrons in PICO-2L. Rates for the ${ }^{19} F(n, \alpha){ }^{16} N$ reaction are given for ENDF/B-VII cross-section and rescaled to the crosssection of Smith, Meadows, and Whalen (1981). Systematic uncertainties in the rates in this table are derived from uncertainties in the scaling of the reaction cross-sections only. Geometric uncertainties are ignored as they should not affect the ratio of reaction and multiple recoil events. The rate in events/hour does not include any analysis cuts or efficiencies. Step thresholds are assumed at $4 \mathrm{keV}$.

As the cross-section for charged particle reactions near threshold is negligible, all reactions in the bubble chamber produce nuclear recoils with sufficient energy to create a bubble. Figure 7.6 shows the expected number of events for each reaction type and run condition during PICO-2L ${ }^{241} \mathrm{Am} /$ Be source calibrations.

Systematic uncertainties in the simulated event rates are caused by uncertainties in scattering and reaction cross-sections, in the simulation geometry, and in the AmBe source neutron spectrum. The first uncertainty is considered in Table 7.6 and the second is canceled out by using the ratio of reaction to non-reaction events. Uncertainty due to AmBe source neutron energy spectrum remains. Most of the reactions shown in Table 7.6 are produced by neutrons with energies above $4 \mathrm{MeV}$. Applying the $35 \%$ variation in the rate of ${ }^{9} \operatorname{Be}\left(\alpha, n_{0}\right)$ neutrons produced by ${ }^{241} \mathrm{Am} / \mathrm{Be}$ source found in Section 3.3, a $14 \%$ systematic uncertainty is applied. 


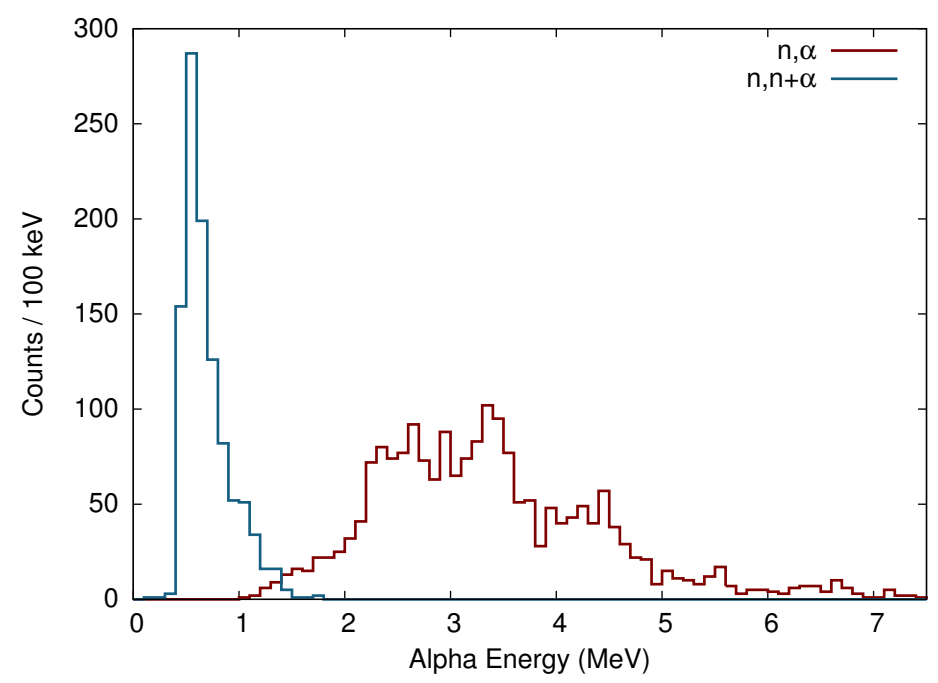

Figure 7.8: Energy spectrum of $\alpha$ particles generated by nuclear reactions during ${ }^{241} \mathrm{Am} / \mathrm{Be}$ source calibrations in PICO-2L. The $(n, n+\alpha)$ energy spectrum shown is narrower than it is in reality due an approximation of the 3-body kinematics (see the end of Section 3.2.

Figure 7.8 shows the energy distribution of $\alpha$ particles generated by the nuclear reactions. Most single-bubble nuclear reaction events are caused by the ${ }^{19} F(n, \alpha){ }^{16} N$ reaction with energies between $2 \mathrm{MeV}$ and $5 \mathrm{MeV}$, just below the energies of $\alpha$ particles from radon daughter decays. The ${ }^{19} F(n, n+\alpha){ }^{15} N$ and ${ }^{12} C(n, 3 \alpha)$ reactions produce low-energy $\alpha$ paricles with energies of $\sim 1 \mathrm{MeV}$.

AmBe at 36" and Cf-252 at 54" neutron runs in COUPP-4kg were simulated using Drew's geometry Fustin 2012, Results are shown in Table 7.7. Similar systematic uncertainties apply.

During ${ }^{241} \mathrm{Am} / \mathrm{Be}$ neutron calibrations in PICO-2L (see Table 5.6), 1382 single bubble recoil-like events, $137 \mathrm{AP}<\mathrm{AP}_{\alpha}$ high-AP events, and $60 \mathrm{AP}>\mathrm{AP}_{\alpha}$ events occurred. Applying the ratio of $\alpha$ producing reactions to single scatter events from simulations and using Smith, Meadows, and Whalen (1981), $44.1 \pm 1.5$ stat. \pm 5.0 syst. $\alpha$ reaction events are expected in addition to the background $\alpha$ rate. The $\alpha$ decay event rate during background running was fit assuming a constant component and a component decay with the half-life of ${ }^{222} \mathrm{Rn}$. The 


\begin{tabular}{|c|c|c|c|c|}
\hline \multirow[t]{2}{*}{ Reaction } & \multicolumn{2}{|c|}{ Single-bubble Events } & \multicolumn{2}{|c|}{ Multi-bubble Events } \\
\hline & \# Simulated & Rate (events/hour) & \# Simulated & Rate (events/hour) \\
\hline \multicolumn{5}{|l|}{ Cf-252 at $54^{\prime \prime}$} \\
\hline Elastic scatter & 61521 & $0.824_{ \pm 0.041 \text { syst. }}^{ \pm 0.003 \text { stat. }}$ & 21487 & $0.288_{ \pm 0.014}^{ \pm 0.002 \text { stat. }}$ syst. \\
\hline${ }^{12} C(n, \alpha){ }^{9} B e$ & 22 & $2.9_{ \pm 0.3 \text { syst. }}^{ \pm 0.6 \text { stat. }} \times 10^{-4}$ & 2 & $3_{ \pm 0.3 \text { syst. }}^{ \pm 2 \text { stat. }} \times 10^{-5}$ \\
\hline${ }^{19} F(n, \alpha){ }^{16} N$ & 562 & $0.0075_{ \pm 0.0008}^{ \pm 0.0003}$ stast. & 75 & $0.0010_{ \pm 0.0001}^{ \pm 0.0001 \text { stat. }}$ \\
\hline rescaled & & $0.0061_{ \pm 0.0009}^{ \pm 0.0003 \text { stat. }}$ & & $8.1_{ \pm 1.2 \text { syst. }}^{ \pm 0.9 \text { stat. }} \times 10^{-4}$ \\
\hline${ }^{19} F(n, n+\alpha){ }^{15} N$ & 156 & $0.0021_{ \pm 0.0003 \text { syst. }}^{ \pm 0.0002 \text { stat. }}$ & 86 & $0.0012_{ \pm 0.0002 \text { syst. }}^{ \pm 0.0001 \text { stat. }}$ \\
\hline${ }^{19} F(n, p){ }^{19} O$ & 49 & $6.6_{ \pm 1.0 \text { syst. }}^{ \pm 0.9 \text { stat. }} \times 10^{-4}$ & 8 & $1.1_{ \pm 0.2 \text { syst. }}^{ \pm 0.4 \text { stat. }} \times 10^{-4}$ \\
\hline${ }^{19} F(n, d){ }^{18} O$ & 4 & $5_{ \pm 2 \text { syst. }}^{ \pm 3 \text { stat. }} \times 10^{-5}$ & 0 & 0 \\
\hline${ }^{19} F(n, n+p){ }^{18} O$ & 1 & $1.3_{ \pm 0.2 \text { syst. }}^{ \pm 1.9 \text { stat. }} \times 10^{-5}$ & 1 & $1.3_{ \pm 0.2 \text { syst. }}^{ \pm 1.9 \text { stat. }} \times 10^{-5}$ \\
\hline${ }^{127} I(n, \alpha){ }^{124} S b$ & 0 & 0 & 1 & $1_{ \pm 4 \text { syst. }}^{ \pm 2 \text { stat. }} \times 10^{-5}$ \\
\hline \multicolumn{5}{|l|}{ AmBe at $36^{\prime \prime}$} \\
\hline Elastic scatter & 8040 & $19.7_{ \pm 0.4 \text { syst. }}^{ \pm 0.2 \text { stat. }}$ & 2717 & $6.67_{ \pm 0.33 \text { syst. }}^{ \pm 0.13 \text { stat. }}$ \\
\hline${ }^{12} C(n, \alpha){ }^{9} B e$ & 8 & $0.020_{ \pm 0.002}^{ \pm 0.007 \text { stat. }}$ syst. & 1 & $0.0025_{ \pm 0.0002}^{ \pm 0.0034 \text { sstat. }}$ \\
\hline${ }^{19} F(n, \alpha){ }^{16} N$ & 121 & $0.297_{ \pm 0.030 \text { syst. }}^{ \pm 0.027 \text { stat. }}$ & 15 & $0.037_{ \pm 0.004}^{ \pm 0.010 \text { stat. }}$ \\
\hline rescaled & & $0.241_{ \pm 0.036}^{ \pm 0.022 \text { stast. }}$ & & $0.030_{ \pm 0.004 \text { syst. }}^{ \pm 0.008 \text { stat. }}$ \\
\hline${ }^{19} F(n, n+\alpha){ }^{15} N$ & 55 & $0.135_{ \pm 0.019 \text { syst. }}^{ \pm 0.018 \text { stat. }}$ & 27 & $0.066_{ \pm 0.009}^{ \pm 0.013 \text { stast. }}$ \\
\hline${ }^{19} F(n, p){ }^{19} O$ & 19 & $0.046_{ \pm 0.007 \text { syst. }}^{ \pm 0.011 \text { stat. }}$ & 2 & $0.005_{ \pm 0.001}^{ \pm 0.004 \text { stat. }}$ syst. \\
\hline$\left.{ }^{19} F(n, d)\right)^{18} O$ & 1 & $0.0024_{ \pm 0.0007 \text { syst. }}^{ \pm 0.0035 \text { stat. }}$ & 0 & 0 \\
\hline${ }^{127} I(n, p){ }^{127} T e$ & 1 & $0.002_{ \pm 0.005}^{ \pm 0.003 \text { stat. }}$ & 0 & 0 \\
\hline
\end{tabular}

Table 7.7: Simulated bubble events from $10^{8}$ AmBe neutrons and $3.765 \times 10^{8}{ }^{252} \mathrm{Cf}$ neutrons in COUPP-4kg. Rates for the ${ }^{19} F(n, \alpha){ }^{16} N$ reaction are given for ENDF/B-VII cross-section and rescaled to the cross-section of Smith, Meadows, and Whalen (1981). A step threshold at $15.9 \mathrm{keV}$ for iodine, and $45 \mathrm{keV}$ for carbon and fluorine is assumed. 


\begin{tabular}{lccccc}
\hline Source & $\begin{array}{c}\text { Exposure } \\
\text { (kg-days) }\end{array}$ & $\mathrm{AP}>\mathrm{AP}_{\alpha}$ events & $\begin{array}{c}\text { Single bubble } \\
\text { events }\end{array}$ & $\begin{array}{c}\text { Expected \# } \\
\text { of } \alpha \text { decays }\end{array}$ & $\begin{array}{c}\text { Expected \# } \\
\text { of } \alpha \text { reactions }\end{array}$ \\
\hline Cf252-54" & 29.58 & 131 & 479 & 130 & $1.34 \pm 0.20$ \\
AmBe-36" & 12.56 & 84 & 1139 & 55.3 & $22.7 \pm 3.7$ \\
\hline
\end{tabular}

Table 7.8: Expected $\alpha$ event totals in COUPP-4kg from reactions and background decays during neutron calibrations. Reactions are required to explain the number of events observed during ${ }^{241} \mathrm{Am} /$ Be calibrations.

livetime and half-live weighted livetime corrected for the cut efficiency from each background data set was summed and the radon decay rate compared to the livetime before cuts was fitted.

$$
\begin{gathered}
R=R_{\text {const }}+0.5^{-\left(t-t_{o}\right) / t_{\frac{1}{2}}} R_{\text {decay }} \\
R_{\text {const }}=2.78 \pm 0.36 \mathrm{cnts} / \text { day } \quad R_{\text {decay }}=15.6 \pm 4.2 \mathrm{cnts} / \text { day } \\
\left.t_{o}=3465862188 \text { (the start of run } 20131028 \_5\right) \\
\chi^{2}=0.026 / 1 \text { dof }
\end{gathered}
$$

With this rate, $10.8 \pm 1.3 \alpha$ decay events are expected during neutron calibrations in PICO$2 \mathrm{~L}$, giving a total expectation of $54.9 \pm 5.4 \alpha$ events. An additional $5.5 \pm 1.0$ events from proton producing reactions are expected. This rate fully explains the $\mathrm{AP}>\mathrm{AP}_{\alpha}$ event rate, but does not explain the $\mathrm{AP}<\mathrm{AP}_{\alpha}$ high-AP tail to the recoil-like events.

It is possible that the $\mathrm{AP}<\mathrm{AP}_{\alpha}$ events are caused by very energetic nuclear recoils with track lengths on the order of the acoustic generation scale of $\sim 10 \mu \mathrm{m}$. To account for 137 observed high-AP events out of 1519 single bubble neutron scattering events, recoils with energies above $870 \pm 30 \mathrm{keV}$ must produce high-AP acoustic signals. A SRIM calculation (Ziegler, Biersack, and Ziegler 2008) of fluorine and carbon ions at this energy stopping in $\mathrm{C}_{3} \mathrm{~F}_{8}$ finds a projected range of $2.7 \mu \mathrm{m}$ and $3.1 \mu \mathrm{m}$ respectively. 
In COUPP-4kg, the large background $\alpha$ decay rate limited the ability to observe the effect of reactions on the $\alpha$ event rate during neutron calibrations. Both ${ }^{252} \mathrm{Cf}$ and ${ }^{241} \mathrm{Am} / \mathrm{Be}$ sources were used in neutron calibrations, and the expected reaction event rates are given in Table 7.7. Rescaling these rates to the observed single bubble event rate produces the expected event totals shown in Table 7.8. The combination of $\alpha$ decays and reaction events fully explain the observed AP distribution. Events with $1.3<\mathrm{AP}<2.0$ are not included in Table 7.8 but were discussed in Section 6.1.1 where it was found that if this high-AP population is attributed to high-energy recoils, events with energy greater than $560 \mathrm{keV}$ do not pass the AP acceptance cut. These recoils have a projected range greater than $1.8 \mu \mathrm{m}$ in $\mathrm{CF}_{3} \mathrm{I}$.

\subsection{High-energy Backgrounds in Bubble Chambers}

High-energy photons may produce $(\gamma, n)$ reactions on detector materials. PICO and COUPP bubble chambers are well shielded from both external neutrons and muons by a thick hydrogenated shield in a deep underground site. However, high energy photons from radioactive decay and neutron capture are only partially attenuated by the neutron shield, and may convert into neutrons near the active volume or lead directly to photonuclear reactions in the active volume itself.

\subsubsection{Photon Flux Measurements}

The high energy photon flux has been measured at the location of the COUPP-4kg and PICO-2L experiments in J-Drift at SNOLAB using a $1.78 \mathrm{~kg}$ low-background thallium doped sodium iodide (NaI[Tl]) crystal described in Fustin (2012, Section 8.2). A second $15.4 \mathrm{~kg} \mathrm{NaI}[\mathrm{Tl}]$ crystal was exposed to photons for 3031 hours in the same excavation, in Drift $\mathrm{C} 1$ near the junction with Drift A, at the location of the COUPP-60kg experiment. 
This larger crystal was used to measure the flux of photons with energies greater than $8 \mathrm{MeV}$ with greater efficiency than the $1.78 \mathrm{~kg}$ crystal. For the $\mathrm{SNO}$ experiment, a $15.6 \mathrm{~kg} \mathrm{NaI}$ crystal with an exposure of 1490 hours in the SNO cavern was used to measure the gamma flux (Perillo Isaac et al. 1997).

The simulations and spectral unfolding analysis of the $1.78 \mathrm{~kg}$ crystal from Fustin 2012 , Section 8.2.2) for low-energy photons was reperformed with slightly different intent and results. While Drew Fustin had simulated the NaI detector surrounded by the hydraulic fluid of COUPP-4kg, I simulated the detector crystal in its steel, PTFE, and quartz housings surrounded by vacuum. Drew Fustin's simulation measured the photon flux at an arbitrary sphere in COUPP-4kg while this revaluation is measuring the flux at the surface of the detector's outer casing. The response matrix bins the photon energy spectrum with bin edges at $0.1,0.66,1.32,1.66,2.47,2.91$, and $10 \mathrm{MeV}$. A response matrix was determined by simulating isotropic photon fluxes for each energy bin with photon energies evenly distributed in each bin. The simulated detector energy deposition was identically binned. The matrix

$$
\mathbb{R}=\left[\begin{array}{cccccc}
10.93 & 4.85 & 3.21 & 2.45 & 2.05 & 1.90 \\
0 & 5.56 & 3.20 & 1.98 & 1.20 & 0.540 \\
0 & 0 & 3.30 & 1.33 & 0.708 & 0.184 \\
0 & 0 & 0 & 3.57 & 2.94 & 0.511 \\
0 & 0 & 0 & 0 & 2.24 & 0.397 \\
0 & 0 & 0 & 0 & 0 & 5.95
\end{array}\right] \times 10^{-3} \mathrm{~m}^{2} 4 \pi \cdot \mathrm{sr}
$$

is related to the measured photon spectrum by

$$
R=\mathbb{R} \Phi
$$

where $\Phi$ is a vector of the physical photon flux, and $R$ is the measured flux. Using this new 


\begin{tabular}{cccc}
\hline $\begin{array}{c}E_{\gamma} \\
(\mathrm{MeV})\end{array}$ & $\begin{array}{c}\Phi_{\text {COUPP-4kg }} \\
\text { Fustin }(2012) \\
\left(\gamma / \mathrm{m}^{2} / \mathrm{s}\right)\end{array}$ & $\begin{array}{c}\text { Reanal. } \\
\left(\gamma / \mathrm{m}^{2} / \mathrm{s} / 4 \pi \mathrm{sr}\right)\end{array}$ & $\begin{array}{c}\Phi_{\text {Drift J }} \\
\left(\gamma / \mathrm{m}^{2} / \mathrm{s} / 4 \pi \mathrm{sr}\right)\end{array}$ \\
\hline $0.1-0.66$ & 68.44 & 714 & 25100 \\
$0.66-1.32$ & 147.2 & 257 & 8650 \\
$1.32-1.66$ & 72.54 & 112 & 5440 \\
$1.66-2.47$ & 37.90 & 66.4 & 1540 \\
$2.47-2.91$ & 24.46 & 41.7 & 1760 \\
$>2.91$ & 0 & 0 & 7.04 \\
\hline
\end{tabular}

Table 7.9: Measured photon flux at the position of the COUPP-4kg active volume

evaluation, $\Phi$ at the position of the COUPP-4kg inner vessel is given by Table 7.9 . Most of these values are consistent with those in Drew Fustin's thesis assuming he measured $\gamma / \mathrm{m}^{2} / \mathrm{s} / 2 \pi \mathrm{sr}$. The increased flux of $511 \mathrm{keV}$ and other low energy photons and the reduced flux of high-energy photons is due to having less material between the source and the detector in the reevaluation.

The $4.00 " \times 4.00 " \times 16.00 "(10.16 \mathrm{~cm} \times 10.16 \mathrm{~cm} \times 40.64 \mathrm{~cm})$ rectangular $15.4 \mathrm{~kg} \mathrm{NaI}[\mathrm{Tl}]$ detector was encapsulated in a thin steel casing coated with reflector. An Electron Tubes model 9306KB photomultiplier tube (PMT) with a model C636PFP positive high-voltage base was bonded to the 3.5" diameter window of the crystal enclosure using optical room temperature vulcanizing silicone rubber. An acetal plastic casing was machined, placed over the entire PMT and window, and sealed with a soft gasket to the crystal encapsulation to ensure stray light did not reach the PMT. The same data acquisition system using a Spectrum Techniques UCS-30 multi-channel analyzer was used for measurements with both the $1.78 \mathrm{~kg}$ and $15.4 \mathrm{~kg} \mathrm{NaI}$ detectors in the drift.

The energy spectrum measured by all three of these detectors is a combination of the internal background of the detector, and the response of the detector to external photon, $\mu$, and neutron radiation, the latter two of which are negligible at SNOLAB for this measurement. To measure the photon flux between $3 \mathrm{MeV}$ and $10 \mathrm{MeV}$, the high-energy 


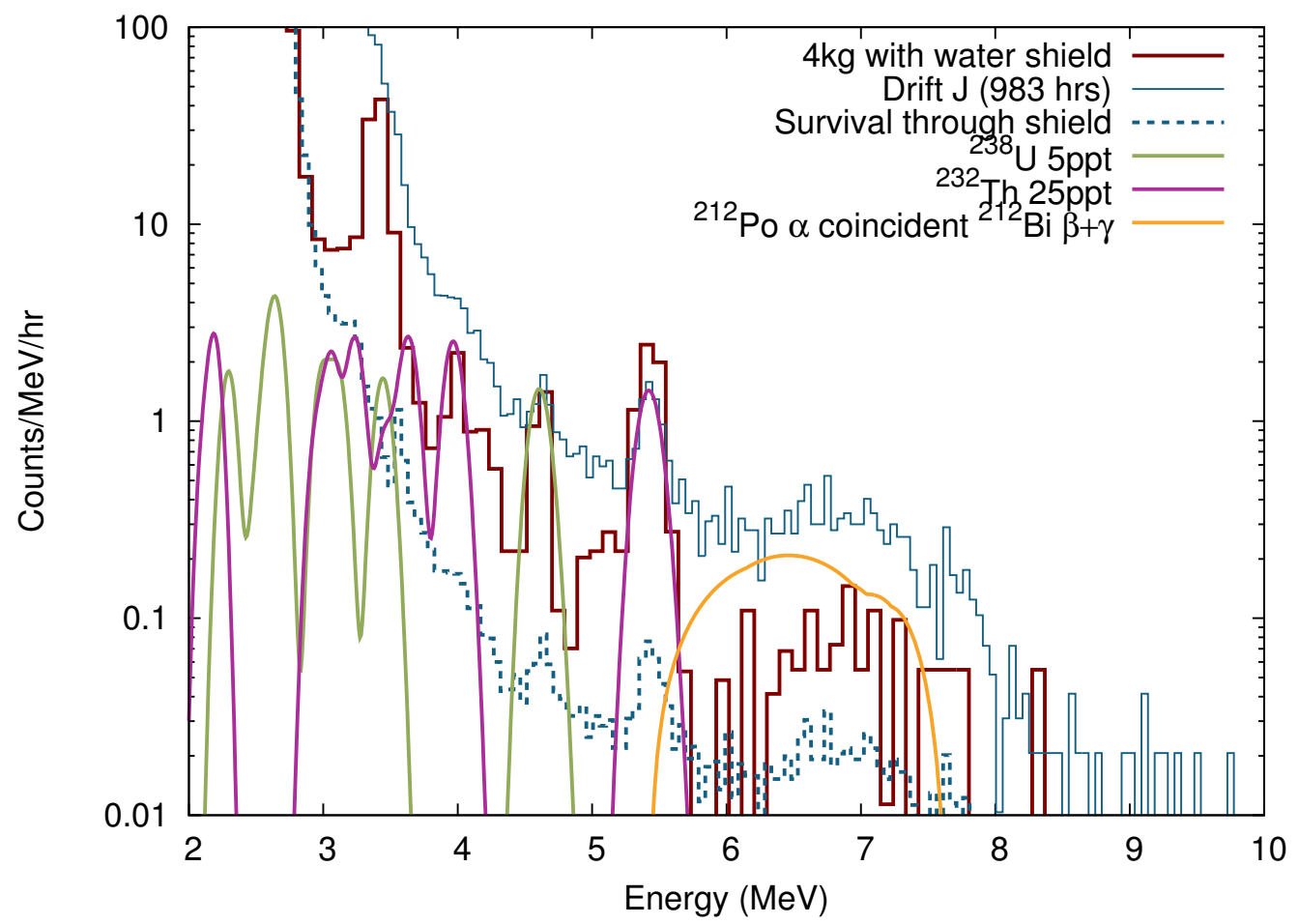

Figure 7.9: $\quad$ Spectra measured using the $1.78 \mathrm{~kg} \mathrm{NaI}[\mathrm{Tl}]$ crystal with various levels of shielding and potential internal alpha decaying contaminants.

internal background of the $1.78 \mathrm{~kg}$ was determined using the difference in the detected energy spectrum inside and outside of the COUPP-4kg neutron shield and pressure vessel. Figure 7.9 shows the two spectra, and the expected contribution from external neutrons passing through the COUPP-4kg shield and from internal contaminants. Most of the measured internal background above $3 \mathrm{MeV}$ are due to quenched $\alpha$ decays of ${ }^{232} \mathrm{Th}$ and ${ }^{238} \mathrm{U}$.

The measured activities and peak spectra are consistent with a ${ }^{238} \mathrm{U}$ contamination of 5 ppt and a ${ }^{232} \mathrm{Th}$ contamination of 25 ppt. The measured energies of $\alpha$ particles in NaI are quenched compared to the measured energies of photons or electrons at the same energy. The 6.778 MeV, $7.687 \mathrm{MeV}$, and 8.784 MeV $\alpha$ 's from the decays of ${ }^{216} \mathrm{Po},{ }^{214} \mathrm{Po}$, and ${ }^{212} \mathrm{~Pb}$ respectively are measured at $4008 \pm 16 \mathrm{keVee}$ (keV electron equivalent), $4642 \pm 22 \mathrm{keVee}$, and $5487 \pm 10 \mathrm{keVee}$. These correspond to a $59 \%$ to $62.5 \%$ quenching factor at these energies, 
slightly less than that measured by the DAMA experiment (Bernabei et al. 1996).

The peak intensity of the ${ }^{212} \mathrm{Po}$ decay is not consistent with the measured activities of other nuclei from the ${ }^{232} \mathrm{Th}$ decay chain: ${ }^{216} \mathrm{Po}, 220 \mathrm{Rn}$, and $212 \mathrm{Bi}$. The measured activity at $5.5 \mathrm{MeV}$ is also larger when the detector was exposed to less radiation inside the COUPP$4 \mathrm{~kg}$ shield than when outside the shield. The measurement using the $1.78 \mathrm{~kg} \mathrm{NaI}$ detector in the COUPP-4kg shield used a different data acquisition system, described by Fustin (2012), that was inefficient at low rates. As ${ }^{212}$ Po decays with a half-life of $0.299 \mu \mathrm{s}$, the fast coincidence between its decay and that of its parent, ${ }^{212} \mathrm{Bi}$, may have increased the efficiency for recording the ${ }^{212}$ Po decay.

The background subtracted spectrum is rebinned and unfolded using the same procedure as Drew Fustin in order to determine the gamma rate, shown in Figure 7.10. Above $8 \mathrm{MeV}$, the statistical uncertainties of the measurement using the $1.78 \mathrm{~kg}$ detector become large, and the spectrum from the $15.4 \mathrm{~kg}$ detector is used instead. Few internal background events are observed above $6 \mathrm{MeV}$, so the $15.4 \mathrm{~kg}$ detector spectrum is used without background subtraction. The measured photon flux is significantly higher in the SNOLAB area than in the SNO cavern.

The neutron shielding around COUPP chambers provides some attenuation to incoming photons. Table 7.11 shows the attenuation through the neutron shield and pressure vessel of photons in simulations of COUPP-4kg, and approximate geometries of COUPP-60 and the proposed PICO-250 detector with a 3 m radius water shield.

\subsubsection{Photonuclear Cross-sections in the Active Volume}

The Q-values for photonuclear processes in $\mathrm{CF}_{3} \mathrm{I}$ are shown in Table 7.12. Evaluated crosssection values exist in the ENDF/B-VII libraries (Chadwick et al. 2011) for processes on both ${ }^{12} \mathrm{C}$ and ${ }^{13} \mathrm{C}$ above threshold. The ${ }^{127} I(\gamma, x)$ cross-section has been evaluated by both 


\begin{tabular}{ccc}
\hline $\begin{array}{c}\text { Energy interval } \\
(\mathrm{MeV})\end{array}$ & $\begin{array}{c}\text { SNO cavern flux } \\
\left(\mathrm{y}^{-1} \mathrm{~m}^{-2} \mathrm{MeV}^{-1}(4 \pi \mathrm{sr})^{-1}\right)\end{array}$ & $\begin{array}{c}\text { SNOLAB drift flux } \\
\left(\mathrm{y}^{-1} \mathrm{~m}^{-2} \mathrm{MeV}^{-1}(4 \pi \mathrm{sr})^{-1}\right)\end{array}$ \\
\hline $3-4$ & $(1.7 \pm 0.2) \times 10^{4}$ & $(2.1 \pm 0.2) \times 10^{4}$ \\
$4-5$ & $91 \pm 11$ & $195 \pm 31$ \\
$5-6$ & $17.6 \pm 3.4$ & $46 \pm 17$ \\
$6-7$ & $11.2 \pm 2.1$ & $34 \pm 13$ \\
$7-8$ & $2.6 \pm 0.7$ & $52 \pm 10$ \\
$8-9$ & $<0.70$ & $13.2 \pm 1.4$ \\
$9-10$ & $<0.70$ & $0.64 \pm 0.11$ \\
$10-11$ & $\mathrm{n} / \mathrm{a}$ & $0.071 \pm 0.018$ \\
$11-13$ & $\mathrm{n} / \mathrm{a}$ & $0.012 \pm 0.004$ \\
$13-60$ & $\mathrm{n} / \mathrm{a}$ & $<2 \times 10^{-4}$ \\
\hline
\end{tabular}

Table 7.10: High-energy photon flux measured in the SNO Cavern (Perillo Isaac et al. 1997) and in the SNOLAB Drifts C1 and J. Combined statistical and 10\% systematic uncertainties are given. The SNOLAB drift flux is measured using the $1.78 \mathrm{~kg} \mathrm{NaI}$ crystal with background subtraction below $8 \mathrm{MeV}$ and the $15.4 \mathrm{~kg} \mathrm{NaI}$ crystal without background subtraction above $8 \mathrm{MeV}$.

\begin{tabular}{cccc}
\hline$E_{\gamma}(\mathrm{MeV})$ & COUPP-4 & COUPP-60 & PICO-250 \\
\hline 2.615 & $1.27 \%$ & $0.070 \%$ & $1.2 \times 10^{-7}$ \\
4 & $4.00 \%$ & $0.45 \%$ & $5.3 \times 10^{-6}$ \\
5 & $4.82 \%$ & $0.64 \%$ & $1.7 \times 10^{-5}$ \\
6 & $5.67 \%$ & $0.83 \%$ & $3.5 \times 10^{-5}$ \\
7 & $6.40 \%$ & $1.00 \%$ & $5.8 \times 10^{-5}$ \\
8 & $6.97 \%$ & $1.17 \%$ & $8.8 \times 10^{-5}$ \\
9 & $7.43 \%$ & $1.35 \%$ & $0.012 \%$ \\
10 & $7.76 \%$ & $1.49 \%$ & $0.016 \%$ \\
12 & $8.55 \%$ & $1.76 \%$ & $0.023 \%$ \\
\hline
\end{tabular}

Table 7.11: Simulated survival of full-energy photons through shielding for COUPP. Statistical uncertainties better than $12 \%$ were obtained for all the simulations. 


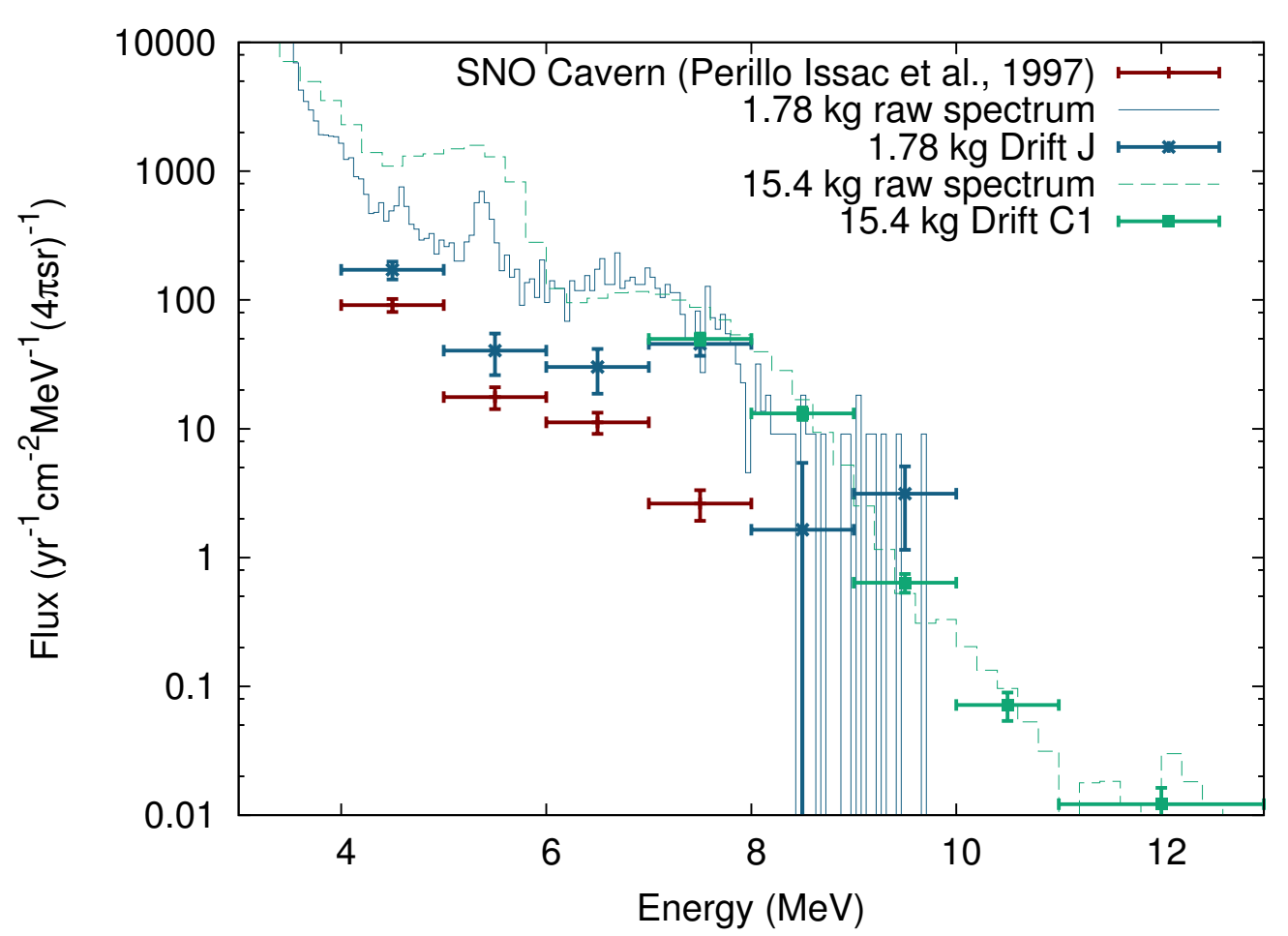

Figure 7.10: Comparison of NaI photon flux measurements at SNOLAB. Raw spectra are arbitrarily scaled. The $1.78 \mathrm{~kg}$ detector measurement in J-Drift subtracts internal backgrounds but does not discriminate gamma from neutron events as the SNO measurement does. No background subtraction is used with the $15.4 \mathrm{~kg}$ detector. The rebinning of the $\mathrm{UC}$ data and background exclude the ${ }^{212} \mathrm{Po} \alpha$ peak at $5.4 \mathrm{MeV}$ in order to avoid large uncertainties in the subtraction.

ENDF/B-VII and Varlamov et al. (2006). The latter evaluation is taken as it is derived from empirical data and has a cross-section about $60 \%$ higher than ENDF/B-VII. Alpha ejection from iodine is heavily suppressed by the coulomb barrier and is unimportant below $10 \mathrm{MeV}$. The photoneutron cross-section of fluorine has also been directly measured above $10 \mathrm{MeV}$ (Veyssiere et al. 1974).

No evaluation of the ${ }^{19} \mathrm{~F}(\gamma, x)$ cross-section could be found. Instead, the cross-section for the ejection of charged particles from fluorine below $10 \mathrm{MeV}$ is determined from measurements of resonances in the reverse kinematics: ${ }^{15} \mathrm{~N}(\alpha, \gamma){ }^{19} \mathrm{~F}$ and ${ }^{18} \mathrm{O}(p, \gamma){ }^{19} \mathrm{~F}$ (See A. Robinson 2012, for details). These resonances have been compiled by Tilley et al. (1995) 


\begin{tabular}{ll}
\hline Reaction & $\mathrm{Q}(\mathrm{MeV})$ \\
\hline${ }^{12} \mathrm{C}(\gamma, 3 \alpha)$ & -7.27 \\
${ }^{13} \mathrm{C}(\gamma, \alpha){ }^{9} \mathrm{Be}$ & -10.6 \\
${ }^{13} \mathrm{C}(\gamma, n){ }^{12} \mathrm{C}$ & -4.95 \\
${ }^{19} \mathrm{~F}(\gamma, \alpha){ }^{15} \mathrm{~N}$ & -4.01 \\
${ }^{19} \mathrm{~F}(\gamma, p){ }^{18} \mathrm{O}$ & -7.99 \\
${ }^{19} \mathrm{~F}(\gamma, n){ }^{18} \mathrm{~F}$ & -10.4 \\
${ }^{127} \mathrm{I}(\gamma, \alpha){ }^{123} \mathrm{Sb}$ & -2.18 \\
${ }^{127} \mathrm{I}(\gamma, p){ }^{126} \mathrm{Te}$ & -6.21 \\
${ }^{127} \mathrm{I}(\gamma, n){ }^{126} \mathrm{I}$ & -9.14 \\
\hline
\end{tabular}

Table 7.12: $\mathrm{Q}$ values for photonuclear processes in $\mathrm{CF}_{3} \mathrm{I}$ below $13 \mathrm{MeV}$.

\begin{tabular}{lcccc}
\hline Reaction & Unshielded Rate & \multicolumn{3}{c}{ Shielded Rate $\left((\mathrm{kg}-\mathrm{yr})^{-1}\right)$} \\
& $\left((\mathrm{kg}-\mathrm{yr})^{-1}\right)$ & COUPP-4 & COUPP-60 & COUPP-500 \\
\hline${ }^{12} \mathrm{C}(\gamma, 3 \alpha)$ & $4.9 \times 10^{-4}$ & $2.6 \times 10^{-5}$ & $3.0 \times 10^{-6}$ & $1.1 \times 10^{-8}$ \\
${ }^{13} \mathrm{C}(\gamma, n){ }^{12} \mathrm{C}$ & $1.8 \times 10^{-3}$ & $8.7 \times 10^{-5}$ & $9.5 \times 10^{-6}$ & $2.7 \times 10^{-8}$ \\
${ }^{19} \mathrm{~F}(\gamma, \alpha)^{15} \mathrm{~N}$ & $3.0 \times 10^{-4}$ & $1.4 \times 10^{-5}$ & $1.5 \times 10^{-6}$ & $3.2 \times 10^{-9}$ \\
${ }^{19} \mathrm{~F}(\gamma, p){ }^{18} \mathrm{O}$ & $1.6 \times 10^{-4}$ & $9.6 \times 10^{-6}$ & $1.3 \times 10^{-6}$ & $7.1 \times 10^{-9}$ \\
${ }^{127} \mathrm{I}(\gamma, n){ }^{126} \mathrm{I}$ & 0.046 & $2.5 \times 10^{-3}$ & $3.3 \times 10^{-4}$ & $1.7 \times 10^{-6}$ \\
\hline Total & 0.048 & $2.7 \times 10^{-3}$ & $3.5 \times 10^{-4}$ & $1.7 \times 10^{-6}$ \\
\hline Target Mass $(\mathrm{kg})$ & $\mathrm{n} / \mathrm{a}$ & 4.0 & 35.0 & 500 \\
Events/yr & $\mathrm{n} / \mathrm{a}$ & 0.011 & 0.089 & $8.5 \times 10^{-4}$ \\
\hline
\end{tabular}

Table 7.13: Photonuclear event rates in $\mathrm{CF}_{3} \mathrm{I}$ bubble chambers. As the ${ }^{127} \mathrm{I}(\gamma, n){ }^{126} \mathrm{I}$ dominates the event rate, the background in similarly sized $\mathrm{C}_{3} \mathrm{~F}_{8}$ filled bubble chambers would be much lower.

and the low-lying $(\alpha, \gamma)$ resonances have been measured by Wilmes et al. (2002). These cross-sections do not account for non-resonant scattering, which should be subdominant $\left(<10^{-9}\right.$ barns $)$.

The event rate, taken as the product of the cross-section and gamma flux, is shown in Table 7.13. With the full flux, a total event rate of up to $0.048 \mathrm{per} \mathrm{kg} \cdot \mathrm{yr}$ is found. With suitable neutron water shields in place, and after taking the self-shielding of the active volume into account, the event rate in actual and proposed detectors becomes negligible. 


\begin{tabular}{lccccc}
\hline Material & Isotope & $\begin{array}{c}\text { Mass Fraction } \\
\text { of Element } \\
\text { in Material }\end{array}$ & $\begin{array}{c}\text { Relative } \\
\text { Abundance }\end{array}$ & Q (MeV) & $\begin{array}{c}\text { Cross-section } \\
\text { at 10 MeV (mb) }\end{array}$ \\
\hline Glass & ${ }^{17} \mathrm{O}$ & $53 \%$ & $0.038 \%$ & -4.143 & 0.77 \\
& ${ }^{18} \mathrm{O}$ & & $0.205 \%$ & -8.05 & 4.8 \\
& ${ }^{29} \mathrm{Si}$ & $47 \%$ & $4.67 \%$ & -8.47 & 0.12 \\
$316 \mathrm{SS}$ & ${ }^{53} \mathrm{Cr}$ & $20 \%$ & $9.5 \%$ & -7.94 & 1.3 \\
& ${ }^{54} \mathrm{Cr}$ & & $2.4 \%$ & -9.72 & 1.6 \\
& ${ }^{61} \mathrm{Ni}$ & $10 \%$ & $1.14 \%$ & -7.82 & 1.7 \\
& ${ }^{64} \mathrm{Ni}$ & & $0.93 \%$ & -9.66 & 1.9 \\
& ${ }^{57} \mathrm{Fe}$ & $65 \%$ & $2.2 \%$ & -7.65 & 1.2 \\
& ${ }^{94} \mathrm{Mo}$ & $2.5 \%$ & $9.25 \%$ & -9.68 & 8.9 \\
& ${ }^{95} \mathrm{Mo}$ & & $15.9 \%$ & -7.37 & 10.7 \\
& ${ }^{96} \mathrm{Mo}$ & & $16.7 \%$ & -9.15 & 11.9 \\
& ${ }^{97} \mathrm{Mo}$ & & $9.6 \%$ & -6.82 & 18.7 \\
& ${ }^{98} \mathrm{Mo}$ & & $24.13 \%$ & -8.64 & 20.4 \\
& ${ }^{100} \mathrm{Mo}$ & & $9.63 \%$ & -8.29 & 20.7 \\
& & & $0.0115 \%$ & -2.225 & 2.6 at $5 \mathrm{MeV}$ \\
\hline
\end{tabular}

Table 7.14: Q-values less than $10 \mathrm{MeV}$ for $(\gamma, n)$ reactions within the water shield.

\subsubsection{Photoneutrons from Detector Materials}

The Q-values and abundances for $(\gamma, n)$ reactions on detector materials are given in Table 7.14. Of the isotopes within the shield, deuterium, molybdenum, ${ }^{17,18} \mathrm{O}$, and ${ }^{13} \mathrm{C}$ dominate the $(\gamma, n)$ rates. Backgrounds were evaluated for the COUPP-4kg experiment, with similar backgrounds expected in PICO-2L. Other dark matter detectors that do not effectively shield $2615 \mathrm{keV}$ photons from their innermost layer of neutron shielding may be susceptible to this background.

The ${ }^{2} H(\gamma, n)^{1} H$ reaction from $>3 \mathrm{MeV}$ photons was simulated using the COUPP-4kg geometry and isotropic neutron production. Using a neutron ejection rate of $3.9 \times 10^{-13}$ per $\mathrm{cm}^{3} \mathrm{~s}$ from deuterium in water or propylene glycol, an event rate of 0.0026 recoils $>10 \mathrm{keV}$ 


\begin{tabular}{lcc}
\hline Material & $\begin{array}{c}\text { Neutron Production } \\
\left(10^{-12} / \mathrm{s} / \mathrm{cm}^{3}\right)\end{array}$ & Events per year \\
\hline${ }^{2} \mathrm{H} 2615 \mathrm{keV} \gamma$ & 16 & 0.14 \\
Steel & 0.94 & 0.0010 \\
${ }^{2} \mathrm{H}>3 \mathrm{MeV} \gamma$ & 0.39 & 0.0026 \\
${ }^{17,18} \mathrm{O}$ in glycol & 0.035 & $2.3 \times 10^{-4}$ \\
Jar & 0.015 & $3 \times 10^{-5}$ \\
\hline
\end{tabular}

Table 7.15: $(\gamma, n)$ production rates and event rates in COUPP-4kg. Event rates assume a step threshold at $10 \mathrm{keV}$ for detecting nuclear recoils.

per year is expected. Neutrons from ${ }^{13} \mathrm{C}$ or ${ }^{17,18} \mathrm{O}$ increase the rate by approximately $9 \%$.

Deuterium has a sufficiently low threshold for neutron ejection that $2615 \mathrm{keV}$ photons from ${ }^{208} \mathrm{Tl}$ decay may produce neutrons from it with energies of $145-252 \mathrm{keV}$. While such low energy neutrons are easily shielded $\left(1.0 \mathrm{~cm}\right.$ mean free path in $\left.\mathrm{H}_{2} \mathrm{O}\right)$, these are produced within the shield itself. The cross-section for the ${ }^{2} \mathrm{H}(\gamma, n)^{1} \mathrm{H}$ reaction from ENDF/B-VII is $1.17 \mathrm{mb}$. With this, the neutron production rate in the propylene glycol surrounding the COUPP-4kg inner vessel is $5.1 \times 10^{-4} \mathrm{n} / \mathrm{yr} / \mathrm{cm}^{3}$. An MCNP-Polimi simulation of these neutrons in COUPP-4kg finds an event rate of 0.14 events per year.

The synthetic silica jar produces photoneutrons at a rate of $1.5 \times 10^{-14}$ per $\mathrm{cm}^{3} \cdot \mathrm{s}$. Assuming $50 \%$ of the neutrons on the $2 \mathrm{~mm} \times 655 \mathrm{~cm}^{2}$ glass surface of the 4 - $\mathrm{kg}$ jar enter the $\mathrm{CF}_{3} \mathrm{I}$, and all of those cause bubbles, an event rate of $3 \times 10^{-5}$ per year is expected, less than that expected from deuterium.

In stainless steel, molybdenum provides a combination of high $(\gamma, n)$ cross-section and low Q-value. Assuming that the steel is exposed to the photon flux in the drift attenuated

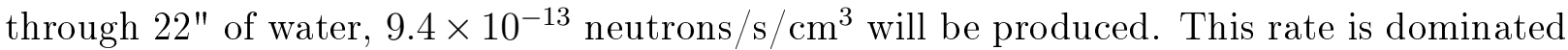
by a $2.5 \%$ concentration of molybdenum. For comparison, 1 ppb uranium and thorium in steel produce $2.1 \times 10^{-10}$ neutrons $/ \mathrm{s} / \mathrm{cm}^{3}$ with larger energies.

Lead in the piezos and the gold in the jar's seal both provide a high cross-section for 
the $(\gamma, n)$ reaction $(\sim 100 \mathrm{mb})$ and Q-values down to $-6.7 \mathrm{MeV}$. However, the small mass provided by these targets or the presence of internal neutron emitting contaminants makes the $(\gamma, n)$ reaction rate negligible. For other dark matter experiments with lead shields, these rates might not be negligible. 


\section{Chapter 8}

\section{Neutron Background Estimates for PICO-2L}

Neutrons from natural radioactivity dominate the expected radiation induced background rate in PICO-2L. This rate was determined by assaying detector materials, calculating their

neutron production yield, and simulating the neutron propagation into the detector. Assays and neutron yield calculations are described in the previous chapter and neutron simulations are described generally in Section 3.2 .

Neutron backgrounds were evaluated by assuming the detector operated at a $3 \mathrm{keV}$ step threshold. As shown in Figure 8.1, the efficiency for detecting single bubble events in PICO2L is nearly the same for any step threshold below $20 \mathrm{keV}$, however the multiple bubble event rate falls as threshold increases. As a consequence, this selection of step threshold will predict the correct background rate, but may overpredict the number of multiple bubble events that could be used to confirm the existence of a neutron background.

In order to track the background contribution of various components, a spreadsheet was created to track materials and the status of all aspects of the neutron background calculation. For each component, the spreadsheet contained their reference drawings (where available), composition, mass, location, assay results, simulation results, and background contribution.

\subsection{Inner Vessel Components}

Figure 8.2 and Table 8.1 show the components of the inner vessel assembly. For every component or group of components listed, a separate assay, simulation, and background evaluation was performed. Most assays used the same part or part of the same source material lot that was used for PICO-2L.

Due to the small size of the c-ring seals, their proximity to the active volume, and the 


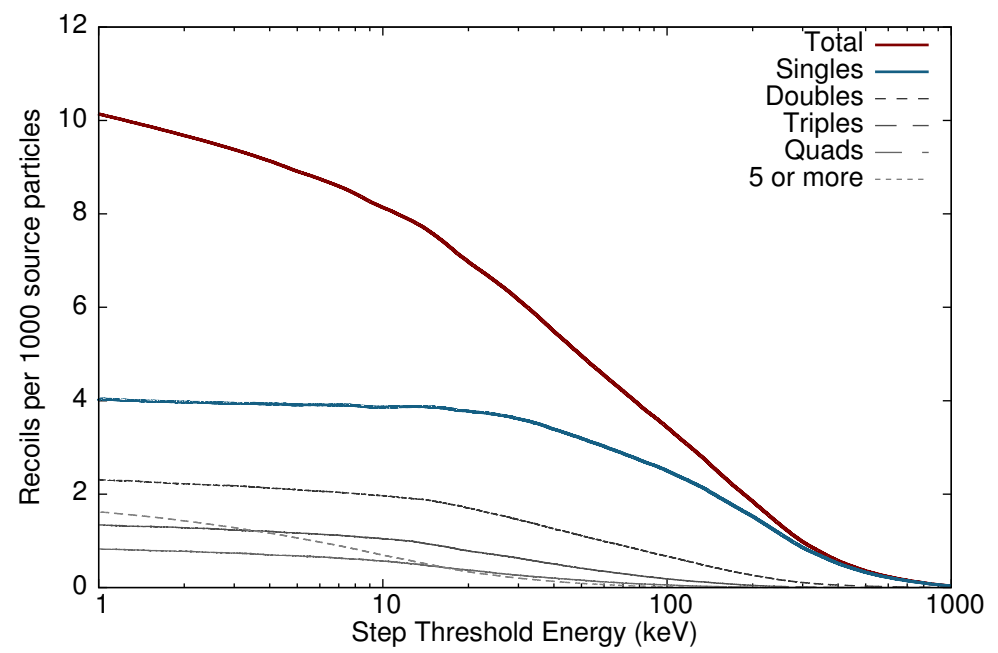

Figure 8.1: Neutron recoil event rates from ${ }^{238} \mathrm{U}$ generated neutrons in the retroreflector for different multiplicities as a function of the step threshold energy.

large neutron yield of polytetrafluoroethylene (PTFE), an assay of the c-rings was unable to achieve sufficient sensitivity to rule out a significant background contribution. A separate large plate of PTFE coated Inconel 718 was obtained and assayed. The Dupont Teflon High Build Topcoat Green (E. I. Dupont de Nemours \& Co. Fluoroproducts 2002) used to coat the c-rings contains PTFE and filler materials that often contain uranium and thorium, such as $\mathrm{TiO}_{2}$. Using the coated plate, both thorium and uranium activity was measured. While the majority of the measured radioactivity of the coated plate likely originated in the coating material, the radioactivity could have been in the Inconel, thus producing a negligible rate of neutrons. Asymmetrical uncertainties are applied to the measured activity, where the lower uncertainty bounds the conceivable lower limit of neutron production in the material.

In contrast to the PTFE coating, PTFE plastic parts generally have high purity and little or no measurable uranium and thorium (e.g. see Aprile et al. 2011). The assayed ${ }^{238} \mathrm{U}$ activity from the guiding rod sleeves was measured entirely using daughters of ${ }^{222} \mathrm{Rn}$ decay. These charged daughter particles are electrostatically attracted to the dielectric PTFE. Radon present during handling and assay of the sleeves would have been concentrated onto 


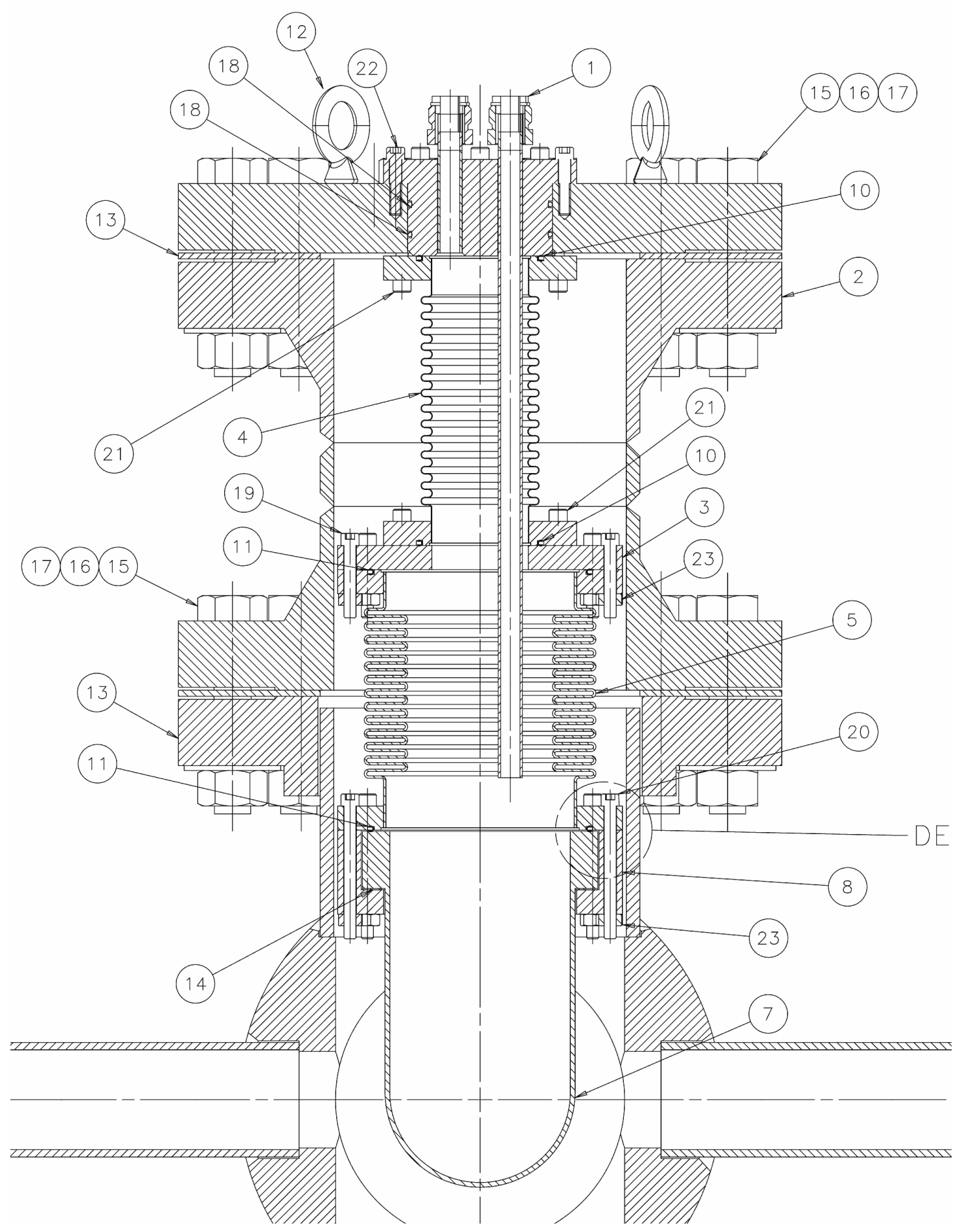

Figure 8.2: Cross-section drawing of the PICO-2L inner vessel. The illustrated jar and pressure vessel shown (items $2,7,9,15,16$, and 17) are not used in PICO-2L. 


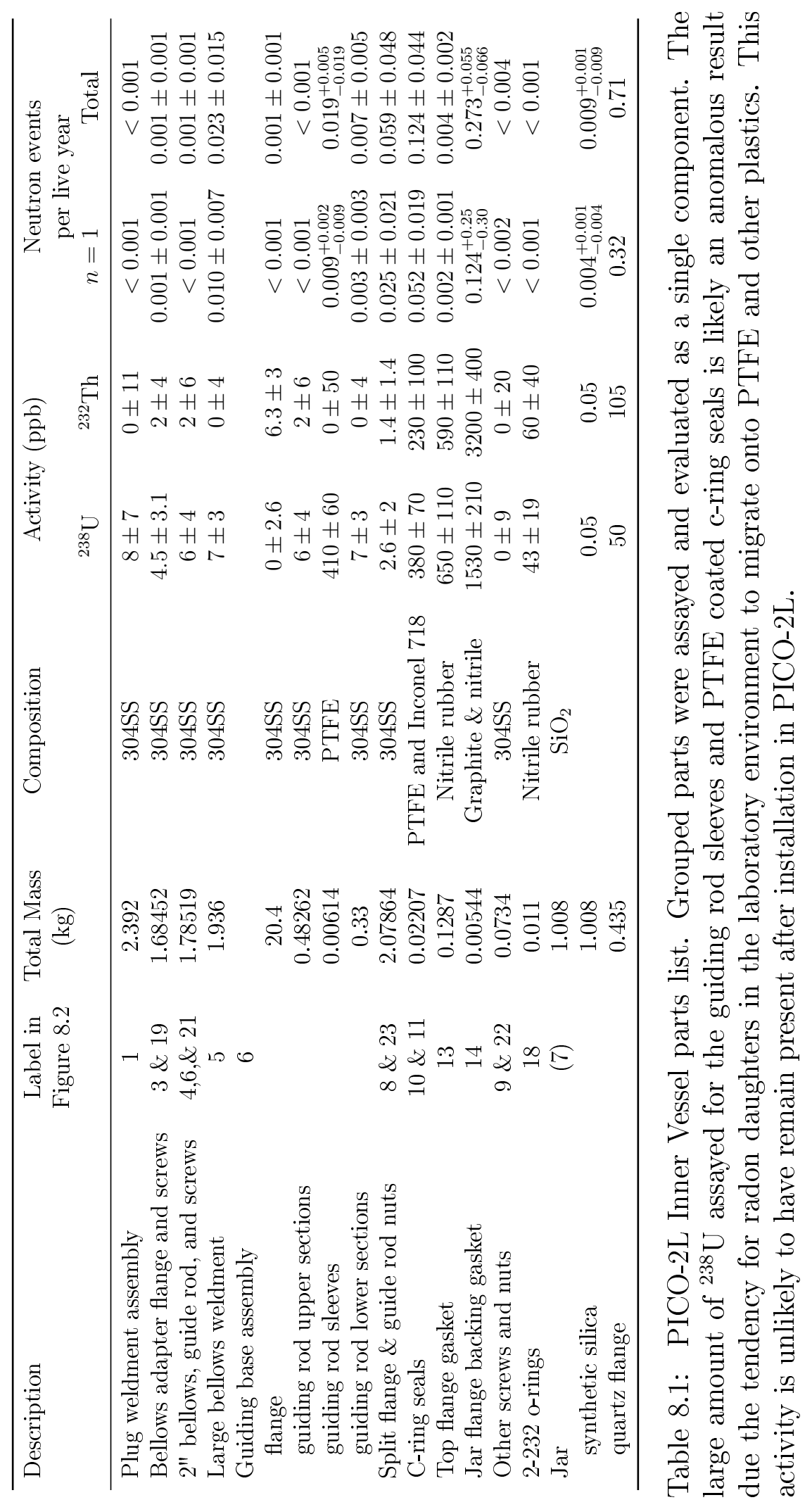




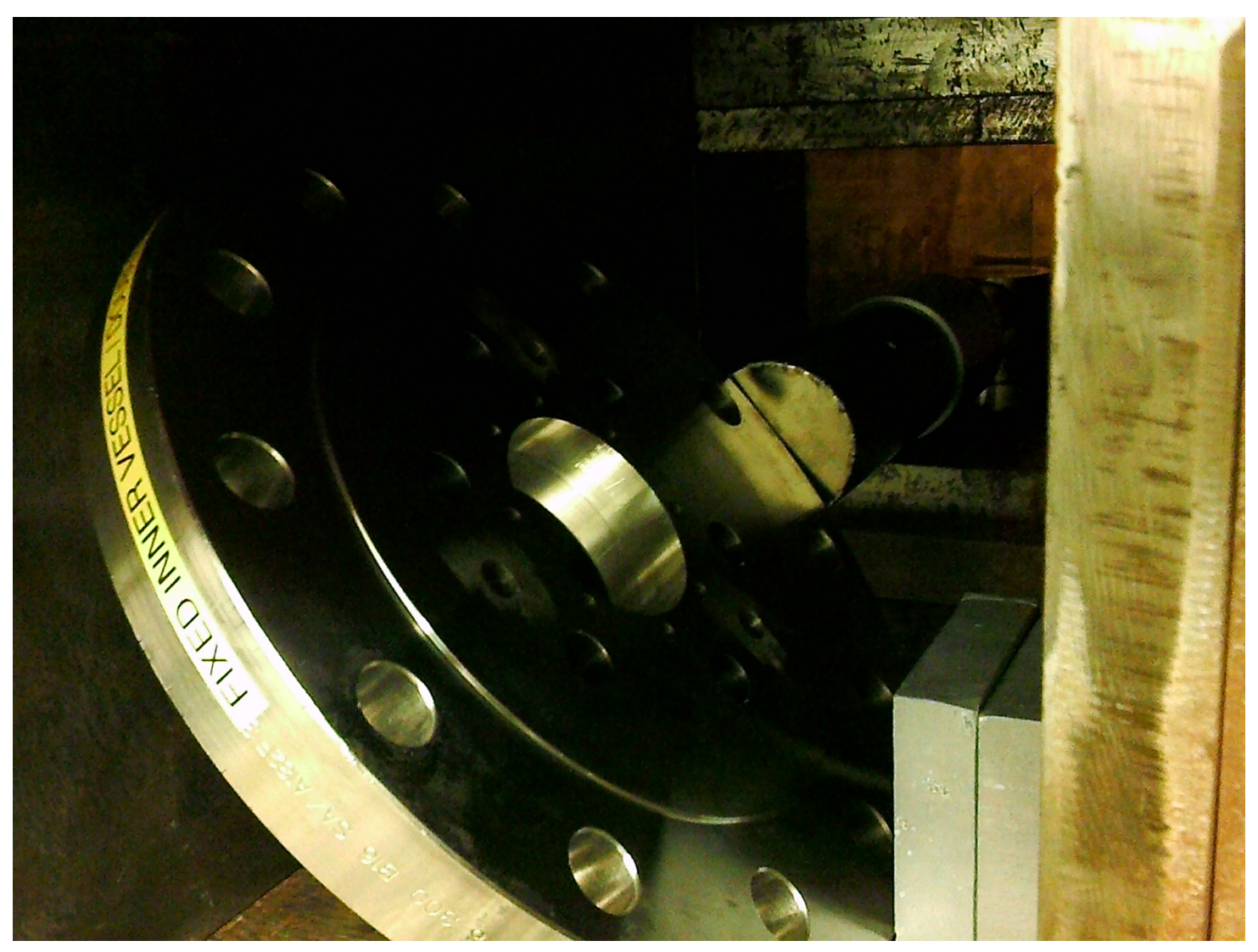

Figure 8.3: The inner vessel guiding base flange during $\gamma$ emission spectroscopy assaying. Most PICO-2L components were similarly assayed before assembly.

them. This activity would have rapidly decayed once the part was installed in PICO-2L, and would not contribute a neutron background. As this source for the assayed activity was not verified, the full assayed activity was used in the background evaluation. As with the PTFE coating, an asymmetric uncertainty is applied.

The top flange gasket, jar flange backing gasket, and o-rings are composed of nitrile rubber and filler materials including graphite and carbon black. The Garlock 9900 graphite fiber filled backing gasket consisted of domains of pure carbon and nitrile. The neutron background obtained by positing that the $\alpha$ activity was in only one of these sets of domains provided a worst-case neutron event background from the graphite domain, and a lowerbound background from the nitrile. The worst-case background rate is used with asymmetric 
uncertainties spanning the two cases. For the top flange gasket and o-rings, the carbon was well mixed with the nitrile, and the background rate assuming a pure nitrile gasket was calculated. Other unknown filler materials in these gaskets may increase or slightly decrease their neutron background contribution.

In addition to the major components of the inner vessel, hardware such as screws and nuts were also considered in the background estimate. Silver plated stainless steel screws were used. Most of the hardware was assayed with the components to which they connected. The additional background due to steel hardware components is negligible compared to other steel components. The uncoated stainless steel nuts and bolts used outside of the inner vessel were not assayed.

While the body of the inner vessel jar is composed of high-purity synthetic silica quartz, with a specified uranium and thorium content of less than $50 \mathrm{ppt}$, the $435 \mathrm{~g}$ jar flange was made of natural quartz. This portion of the jar was not assayed and its neutron background was unevaluated until the oversight was found while preparing this thesis. The activity of natural quartz varies from $\sim 10-500 \mathrm{ppb}$ of both ${ }^{238} \mathrm{U}$ and ${ }^{232} \mathrm{Th}$. Assuming an activity of $50 \mathrm{ppb}^{238} \mathrm{U}$ and $105 \mathrm{ppb}^{232} \mathrm{Th}$, the ratio of the neutron background to measured event rate of the jar backing gasket is used to estimate the neutron background. The neutron sources are in the same position, and the neutron yield of the jar backing gasket is similar to that of $\mathrm{SiO}_{2}$.

Neutron backgrounds from the inner volume fluids are negligible. $427 \alpha$-decay events were detected in the PICO-2L active volume during the dark matter search. Assuming that a similar amount of $\alpha$ activity was in the buffer fluid as is in the active fluid, $1.5 \times 10^{-4}$ neutrons and fewer neutron recoil events were expected during the run. An $\alpha$ activity capable of producing a neutron background from the inner volume fluids would prevent the bubble chamber from accumulating any useful livetime. Some inner vessel components are 
not included in Figure 8.2 and Table 8.1: 4 resistive thermometers (RTDs) embedded in the large bellows and bellow adapter flanges, the flange of the jar, and the piezoelectric transducer assemblies. The RTDs and their cables were assayed and found to contain $6 \pm 10 \mathrm{mBq}{ }^{238} \mathrm{U}$ and $10 \pm 4 \mathrm{mBq}{ }^{232} \mathrm{Th}$. The background from the RTDs and the clear epoxy resin used to attach them is considered negligible due to their high purity, low mass, and distant position.

The piezoelectric acoustic sensors were a major contribution to the neutron background of COUPP-4kg. For PICO-2L, low-background piezoelectric transducer elements, preamplifiers, and housings were developed. The piezo electric transducers were custom fabricated using selected low radioactivity salts described in Fustin (2012, Appendix C). These elements produce $12.0 \pm 3.5 \mathrm{n} / \mathrm{kg} / \mathrm{yr}$ mostly due to the $11 \pm 2.8 \mathrm{mBq} / \mathrm{kg}$ of ${ }^{210} \mathrm{~Pb}$ contained in the $\mathrm{Pb}_{2} \mathrm{O}_{3}$ salt used to construct the elements. $3.90 \mathrm{~g}$ of lead zirconate titanate is used in the experiment. The pre-amplifiers for the detectors were constructed on a CuFlon brand PTFE substrate using silver solder and silver containing epoxy to bond the components. Each piezo was encased in an Alloy 101 copper and MAS brand clear epoxy resin housing. Belden 8054 magnet wire was used to connect the sensors to the data acquisition system. All components except for the copper and the electronic circuit components were individually assayed. Activity was measured in the piezo salts, the solder $\left(7 \pm 4 \mathrm{ppb}{ }^{232} \mathrm{Th}\right)$, and from radon daughters attached to the PTFE circuilt board. Only upper limit activities were measured at the University of Chicago for all other components. The assembled piezo elements and preamplifier boards were assayed at SNOLAB, and an activity of $<0.75 \mathrm{mBq}{ }^{238} \mathrm{U}$ and $<0.08 \pm 0.68 \mathrm{mBq}^{232} \mathrm{Th}$ was measured for the three sensors. This activity was assumed to be within the PTFE circuit board in order to set an upper limit neutron background rate from the sensors of 0.005 single bubble events and 0.012 total events per live-year. The sensor's wires contribute $<0.001$ single bubble events and $<0.002$ total events per live-year. 
The acoustic sensors were attached to the inner vessel using J-B Weld brand epoxy resin. Unlike the other epoxy resins used in PICO-2L, J-B Weld contains a large fraction of filler materials and significant amounts of uranium and thorium. The face of the sensors was curved to match the shape of the jar at the attachment point and a piezo mounting procedure was developed in order to minimize the amount of J-B Weld required. Less than $1 \mathrm{~g}$ of J-B Weld was required to mount each sensor. A sample of the same resin used to mount the piezos was assayed and found to contain $88 \pm 43 \mathrm{ppb}^{238} \mathrm{U}$ and $240 \pm 80 \mathrm{ppb}^{232} \mathrm{Th}$. A background event rate of $0.004 \pm 0.002$ single bubble events and $0.009 \pm 0.004$ total neutron background events is expected from the J-B Weld.

\subsection{Pressure Vessel Components}

The pressure vessel components, listed in Table 8.1, were assayed and evaluated similarly to the inner vessel components. Instead of GES, ICP-MS was used for all of the steel pressure vessel component assays except for the bottom manifold. Most neutrons emitted from steel are expected to originate from ${ }^{238} \mathrm{U}$ spontaneous fission. ICP-MS provides vastly superior sensitivity to the ${ }^{238} \mathrm{U}$ content whereas GES is sensitive to its daughter isotopes, which are likely not in equilibrium with the ${ }^{238} \mathrm{U}$ parent.

For ICP-MS assayed component, at least three samples were analyzed. The variation of activity within the samples far exceeded the $\mathcal{O}(0.01 \mathrm{ppb})$ sensitivity of the measurements. Samples for analysis were obtained by cutting $\sim 1 \mathrm{~g}$ amounts of material from the installed pressure vessel. Samples were taken from the uncleaned surface of the steel, cleaned surfaces, and from the bulk of each component. The average of the bulk and clean surface samples is used to calculated the background expectation, with an uncertainty given by the standard deviation of the measurements. The uncleaned surface sample contained marginally more uranium and thorium than the cleaned samples. 


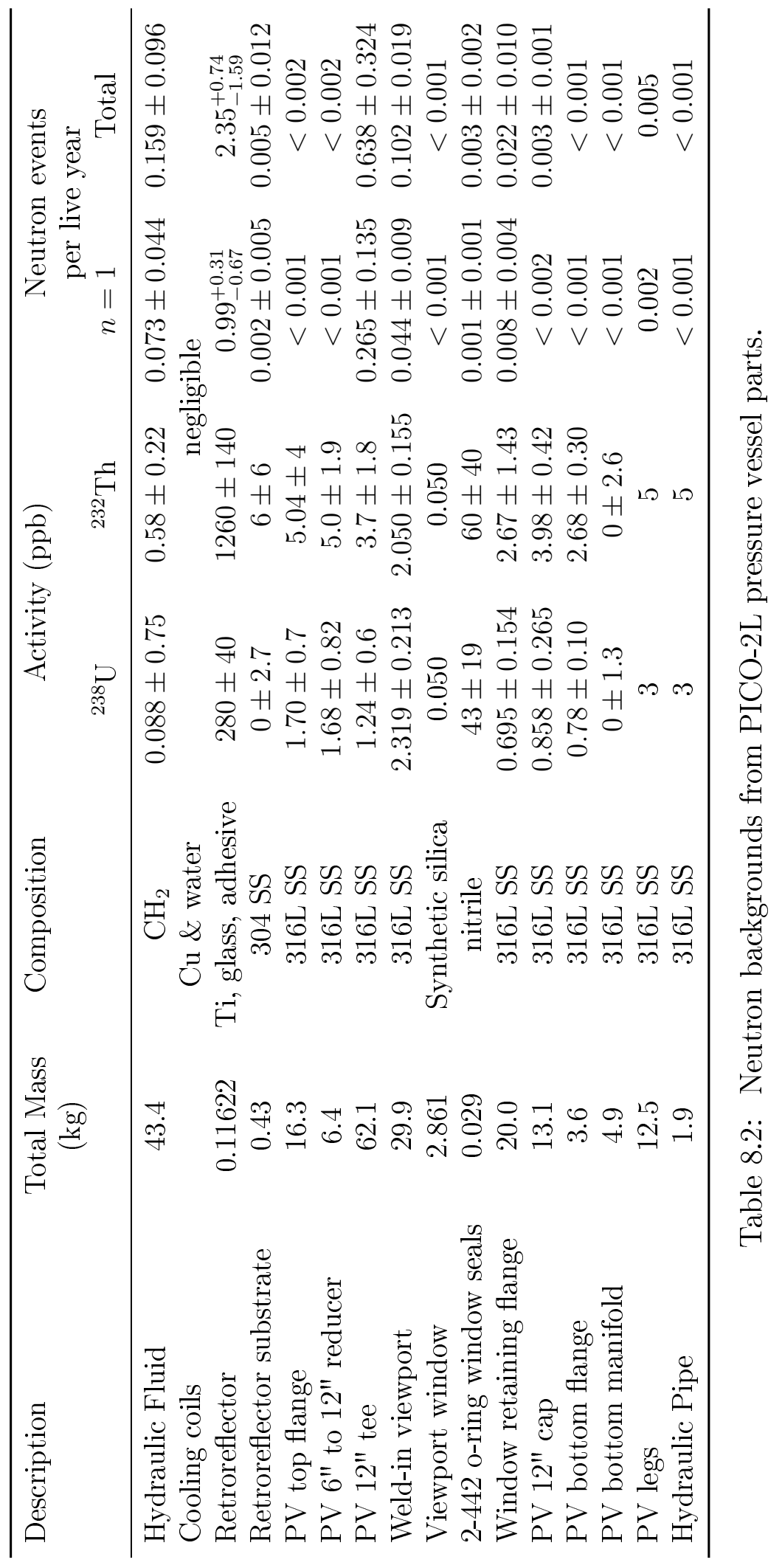




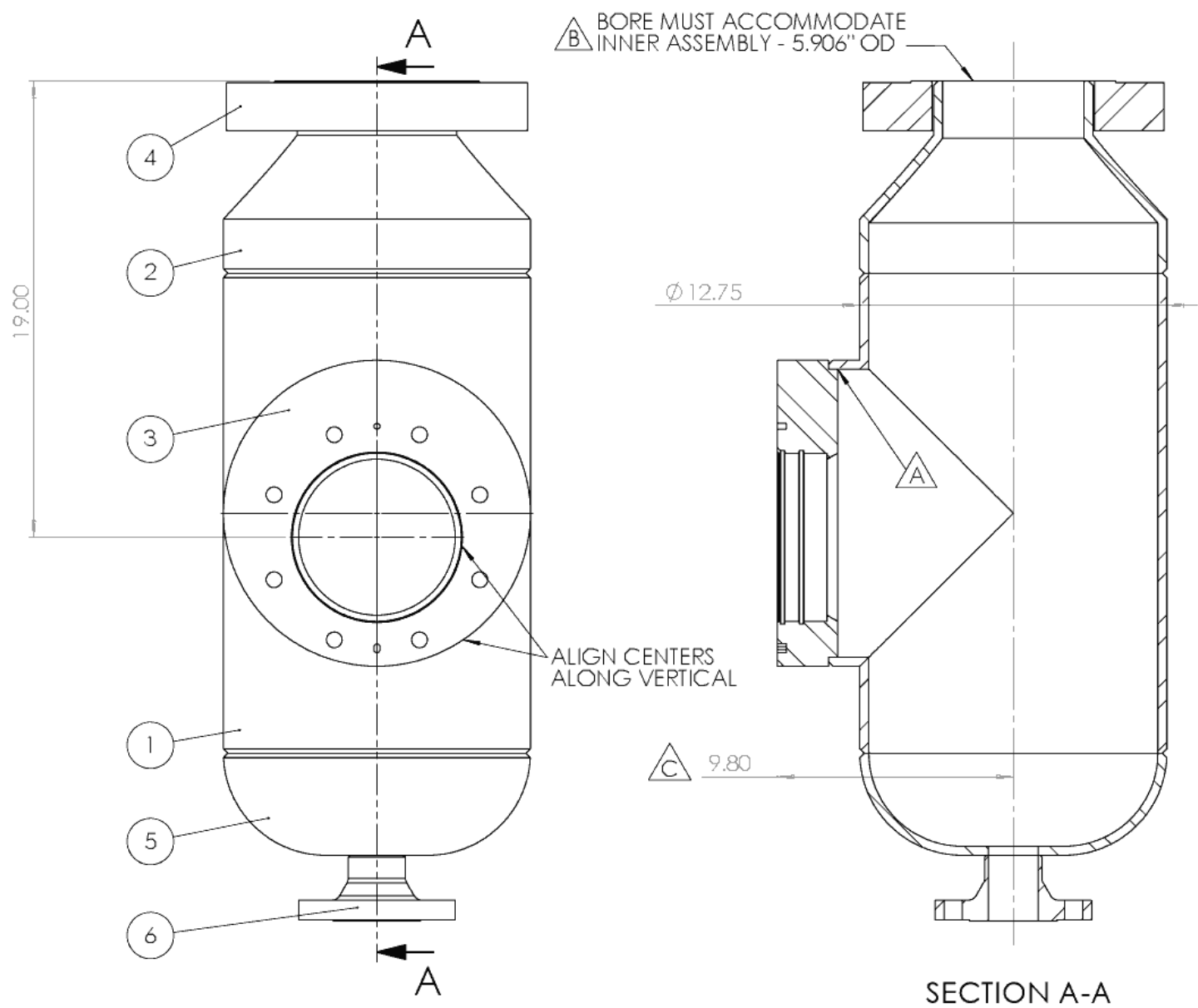

Figure 8.4: Extract from the PICO-2L pressure vessel (PV) weldment drawing. The PV 12" tee (1), PV 12" to 6" reducer (2), weld-in viewport (3), top flange (4), PV 12" cap (5), and PV bottom flange (6) are shown.

The PICO-2L pressure vessel was constructed from Schedule 40 pipe fittings as defined by the ASME $(1996 ; 2004 ; 2012)$. in order to obtain the correct distance between the pressure vessel window and top flange, both the $6 "$ to 12 " reducer and the 12 " tee were shortened by several inches. The nominal masses of these fittings provided by the manufacturer is used to evaluate the backgrounds, thus overcounting the background from the material that was removed. The pressure vessel legs, the pipe to the hydraulic cart, and the nuts and bolt used to secure the flanges were not assayed. For these components, 3 ppb of ${ }^{238} \mathrm{U}$ and $5 \mathrm{ppb}$ of ${ }^{232} \mathrm{Th}$ were assumed and evaluated. The pressure vessel legs contribute a small 
background while the pipe, the nuts, and the bolts contribute negligibly.

COUPP-4kg used PTFE coated threaded rods to secure the pressure vessel flanges. Due to the neutron background produced by these types of coatings, uncoated stainless steel bolts were used with PICO-2L. In order to prevent galling, grease was applied to the threads of these rods. The Locktite N-1000 grease used was assayed in its container and found to have at most $125 \pm 27 \mathrm{ppb}^{238} \mathrm{U}$ and $440 \pm 80 \mathrm{ppb}{ }^{232} \mathrm{Th}$. The background from the grease was neglected due to the small amount of grease used, its low radioactivity, its distant location, and the low neutron yield of hydrocarbons.

3M Scotchlite 3290 retroreflecting sheeting (3M 2008) was used to line the inside of the pressure vessel. The retroreflector is composed of glass beads embedded in metal. Microwave Plasma Atomic Emission Spectroscopy elemental analysis of the retroreflector found large amounts of titanium in the retroreflector, no aluminum or other high neutron yield targets were found (Borden, Levine, and Nania 2013). Over $1 \mathrm{ppm}$ of ${ }^{232} \mathrm{Th}$ was measured in the retroreflector during GES assays. In order to trigger effectively, PICO bubble chambers require a forming bubble to provide a high contrast to the background image. PICO uses an illumination source near the cameras and retroreflector behind the inner vessel to provide an evenly illuminated background. Light rays refracted at large angles by bubbles do not retroreflect toward the cameras, and bubbles appear dark in the images. 3M Scotchlite 3290 has been used in all COUPP and PICO bubble chambers with retroreflective optics to date. An alternative retroreflector, $3 \mathrm{M}$ Scotchlite 3430 retroreflector using plastic prismatic lenses was assayed and found to have $<2.6 \mathrm{ppb}^{238} \mathrm{U}$ and $2 \pm 5 \mathrm{ppb}^{232} \mathrm{Th}$. However, this design of retroreflector was quickly destroyed when exposed to high pressure hydraulic fluids.

The large activity measured in the retroreflector may be concentrated in either the titanium, glass, or adhesive. Titanium produces more neutrons by the $(\alpha, n)$ reaction than the other two materials and is therefore used to conservatively evaluate the neutron back- 
ground. Asymmetrical uncertainties are set so as to bound the possible neutron background rate from titanium and silica.

The inner vessel cooling coils are composed of Alloy 101 copper with ultra-high purity water flowing within them. As the specification for these materials guarantees sub-ppb uranium and thorium contents, the background for these materials is assumed to be negligible.

\subsection{DAQ Components}

Table 8.3 lists data aquisition (DAQ) components within the water shield. As with the retroreflector, other components of the bubble chamber optics contribute significant neutron backgrounds. Commercial machine vision cameras and lenses use aluminum bodies and custom glass formulations containing large amounts of uranium and thorium. The Computar H2Z0414C lenses used with PICO-2L contribute 0.59 single bubble events per live-year. Other machine vision camera lenses from Kowa have been assayed, and found to contain larger amounts of uranium, thorium, and lanthanum.

Red Luxeon STAR LEDs used to illuminate PICO-2L are mounted on custom circuit board constructed on an Arlon 92ML printed circuit board (PCB) substrate. Both the raw substrate and the LEDs were assayed. prior to installation. The LEDs are mounted on an Alloy 101 copper heat sink, and the entire LED and camera assembly are mounted to the pressure vessel using stainless steel brackets.

Signal cables run from the pressure vessel top flange and the cameras out through the bottom of the neutron shield towards the hydraulic cart and DAQ computers. The mass and length of cable inside of the neutron shield was estimated. The slow pressure transducers, acoustic transducers, and RTDs are fed through a breakout box at the top of the pressure vessel to 10 identical 2-pair cables. An additional single ethylene tetrafluoroethylene jacketed and PTFE insulated coaxial cable connected to the Dytran fast pressure transducer. This 


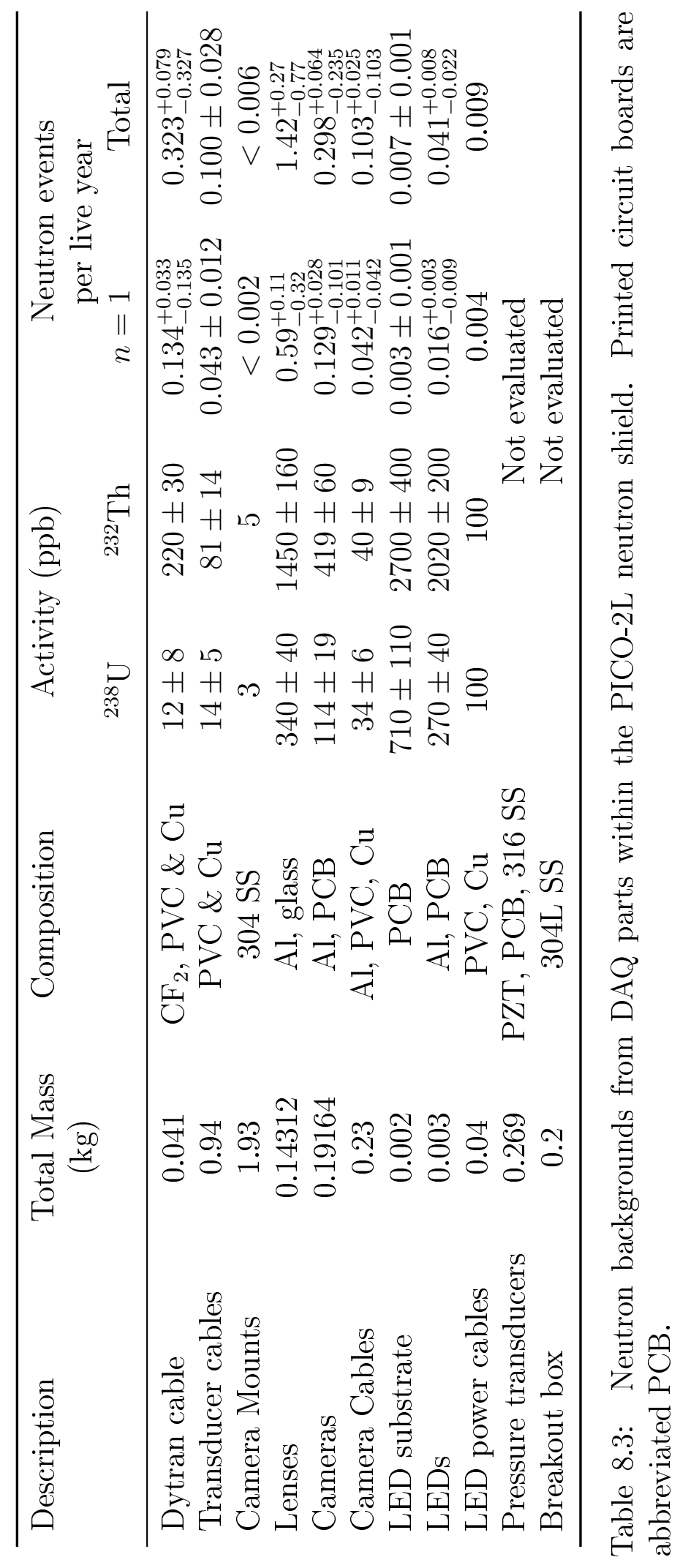


single cable contributes a larger neutron background than the other 10 cables combined. Two Firewire cables and two pairs of polyvinyl chloride (PVC) insulated wires supply the cameras and LEDs. All cables except the LED power cables were assayed.

Neutron backgrounds from the three pressure transducers, the breakout box, and the piezo and RTD feedthroughs on the inner vessel top flange were not evaluated.

\subsection{Environmental Neutrons}

Uranium and thorium in the concrete and norite rock surrounding PICO-2L produce approximately 4000 fast neutrons per $\mathrm{m}^{2}$ per day in Drift J (SNOLAB 2006). The energy spectrum of these neutrons is not well known. Using the same procedure as Fustin (2012, Sec. 7.2), the neutron spectrum for $1.2 \mathrm{ppm}$ of uranium and $3.3 \mathrm{ppm}$ of thorium in norite was recalculated, and the moderation of a uniformly distributed source of neutrons through a large $50 \mathrm{~cm}$ thick spherical shell was simulated. The inner diameter of the shell was set to $5 \mathrm{~m}$, much larger than that used by Drew Fustin. The new geometry increases the proportion of the simulated activity near the cavern wall, thus increasing the average tallied neutron energy.

Fustin (2012) calculated the expected neutron rate in COUPP-4kg assuming that the neutron rate in the drift was $4000 \mathrm{n} / \mathrm{m}^{2} /$ day $/ 2 \pi \mathrm{sr}$. The same assumption will be used here, although it may overpredict the expected event rate by a factor of 2 .

With this new neutron spectrum, a smaller pressure vessel with less moderator, and a higher nuclear recoil detection efficiency, the efficiency for external neutrons to scatter in PICO-2L is higher than that evaluated for COUPP-4kg: 1.28 single bubble events and 2.75 total events are expected per live-year. Due to the large uncertainties in the incoming neutron spectrum and rate, a $50 \%$ uncertainty is arbitrarily applied to these rates.

The air at SNOLAB contains $131.0 \pm 6.7 \mathrm{~Bq} / \mathrm{m}^{2}$ of ${ }^{222} \mathrm{Rn}$. While radon is not able 
to penetrate the sealed inner vessel and hydraulic systems, it can diffuse through into the neutron shield, the recirculating water bath, or into the air inside the neutron shield. In the air, ${ }^{222} \mathrm{Rn}$ and its daughter isotopes can produce $4.09 \times 10^{-6}$ neutrons per decay, or 1.93 neutrons $/ \mathrm{m}^{2} / \mathrm{hr}$ at SNOLAB. Within the $0.841 \mathrm{~m}^{2}$ volume within the neutron shield, this can produce up to $3.8 \pm 0.6$ single bubble events and $9.2 \pm 1.5$ total events per live year in PICO-2L, the largest background contribution. Most of these neutrons are produced by the $7.87 \mathrm{MeV} \alpha$ from the decay of ${ }^{214} \mathrm{Po}$ on ${ }^{14} \mathrm{~N}$. $\alpha$ 's from other radon daughters do not surpass the $6.09 \mathrm{MeV} \alpha, n$ threshold of ${ }^{14} \mathrm{~N} .{ }^{214} \mathrm{Po}$ decays on average $72 \mathrm{~min}$ after its ${ }^{222} \mathrm{Rn}$ parent, during which, the lead, polonium or bismuth ion is likely to implant onto a solid surface, thus likely reducing its neutron yield. An additional asymmetric uncertainty of $-50 \%$ is applied to the radon in air background rate.

The equilibrium concentration of radon in water is less than that in air. The volumetric partition coefficient in water is given by (Sander 2015),

$$
\frac{{ }^{222} \operatorname{Rn}(\mathrm{l})}{{ }^{222} \operatorname{Rn}(\mathrm{g})}=0.2084 \exp \left\{2600 \mathrm{~K}\left(T^{-1}-(298.15 \mathrm{~K})^{-1}\right)\right\}
$$

At the $9^{\circ} \mathrm{C}$ minimum operating temperature of the recirculating chiller, the partition coefficient is 0.342 . At $20^{\circ} \mathrm{C}$, the approximate temperature of the neutron shield, it is 0.242 . The copper cooling coils in the pressure vessel hold approximately $500 \mathrm{~mL}$ of water. In equilibrium with the air at SNOLAB, this would contain $22.4 \pm 1.1 \mathrm{mBq}$ of ${ }^{222} \mathrm{Rn}$ and produce 0.24 neutrons per year and $\sim 0.001$ single bubble event per live-year. The radon in the water tanks would produce $0.026 \pm 0.004$ single bubble events and $0.060 \pm 0.010$ total events per live-year. Both rates are likely overestimated and a $-100 \%$ asymmetric uncertainty is applied. 


\subsection{Other Background Sources}

The Reflectix aluminized bubble insulation wrapped around the pressure vessel was assayed and found to contain $24 \pm 11 \mathrm{ppb}{ }^{238} \mathrm{U}$ and $<23 \mathrm{ppb}{ }^{232} \mathrm{Th}$ (90\% conf.). The aluminum tape used to secure the insulation contained $20 \pm 6 \mathrm{ppb}{ }^{238} \mathrm{U}$ and $78 \pm 15 \mathrm{ppb}{ }^{232} \mathrm{Th}$. With approximately $850 \mathrm{~g}$ of insulation wrapping twice around the pressure vessel, a background of 0.09 single bubble events and 0.20 total events is expected. As the actual amount of insulation used was not measured, 100\% uncertainties are applied.

The polypropylene and polyethylene neutron shields around the detector have not been assayed. Assuming $1 \mathrm{ppb}$ of uranium and thorium in them, 0.024 single bubble events and 0.054 total events per year are expected.

The background rate from muon produced neutrons is negligible. Fustin 2012, Chapter 7) evaluated the $(\mu, n)$ rate at SNOLAB and found that less than 0.015 events per liveyear were produced in COUPP-4kg, mostly from muons generating neutrons in the pressure vessel steel. This rate is expected to be lower in PICO-2L due to the use of less steel mass in the detector design.

The rate of photon induced events is also negligible. Simulations of PICO-2L with the measured photon flux in Drift-J were compared to the electron recoil sensitivity limits set by exposing PICO-2L to photons from ${ }^{133} \mathrm{Ba}$. No photon induced events were detected, setting a photon sensitivity limit of $<3.5 \times 10^{-10}$ per electron recoil $>3.2 \mathrm{keV}$, and an upper limit of 0.2 events in the entire PICO-2L dataset was found.

\subsection{PICO-2L Neutron Simulations}

The design of PICO-2L was refined and changes as backgrounds were being evaluated for it. Figure 8.5 and Table 8.4 describe the 17 simulated versions of the PICO-2L geometry. 
The background contributions for most materials do not use the fully updated simulation geometry.

The jar flange backing gasket and the retroreflector were resimulated with the $4 \mathrm{~b}$ geometry and their original 41 , and $4 \mathrm{u}$ geometries respectively. The simulated neutron recoil rate changed by at most $10 \%$ between the simulations, subdominant to other background uncertainties.

\subsection{Neutron Background Uncertainties}

Each step of the calculation of neutron backgrounds, assays, neutron yield calculations, and simulations, contribute uncertainty to the final result. Total uncertainties from each part of are calculation are summed in quadrature to obtain the total uncertainty and the uncertainties for each component.

Both assays and simulations contribute statistical uncertainties that are all summed in

quadrature. Simulations were performed with a sufficient number of source particles, (up to $10^{9}$ ) to ensure that their statistical uncertainty was a factor of 10 less than the statistical uncertainty from the assays. The distribution of source particle energies in the simulation of neutrons external to the water shield was biased such that neutron with energies above $2 \mathrm{MeV}$ were more likely to be simulated and $94 \%$ fewer source neutrons with energies below 1.5 MeV were simulated. All other simulations were analog, with no biasing applied. A global systematic uncertainty of $10 \%$ was applied to all University of Chicago assay results, and a global 15\% uncertainty was applied to all simulations.

The uncertainties in the neutron yields of individual detector materials is given in Table 7.3. Uncertainties for each component were grouped by the material producing the neutrons. Uncertainties within each grouping were summed and the uncertainties between groups were summed in quadrature. Materials for which carbon is the primary $(\alpha, n)$ target 


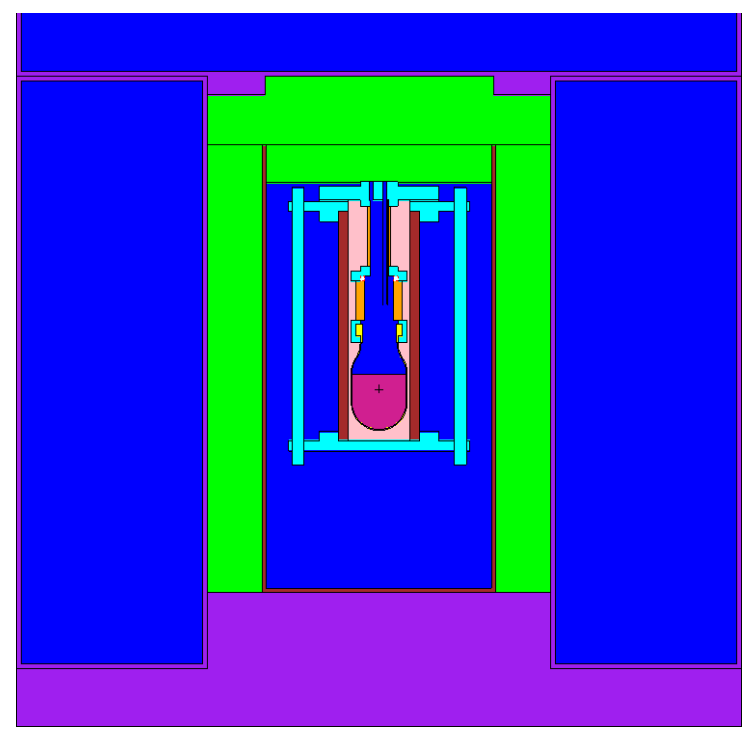

(a) Version 41

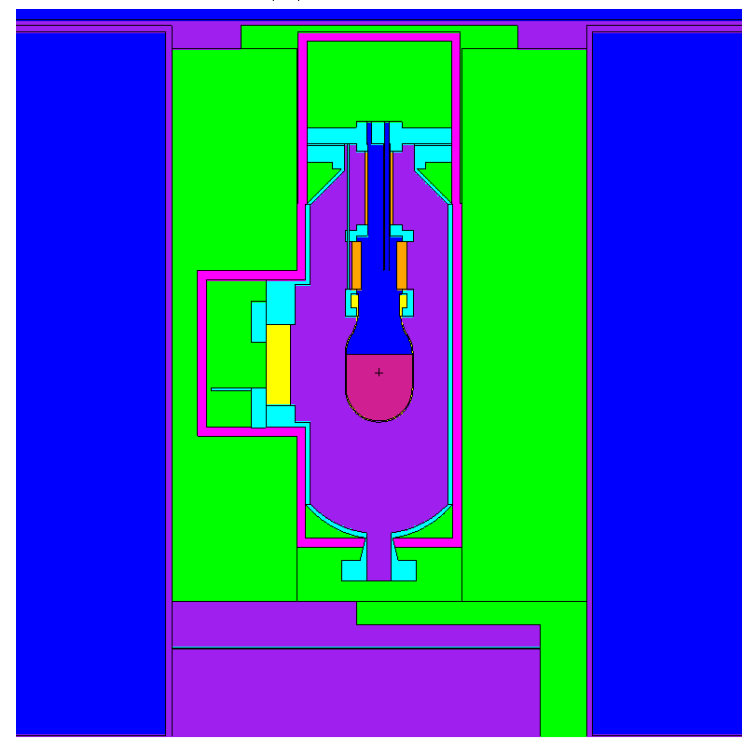

(c) Version $4 r$

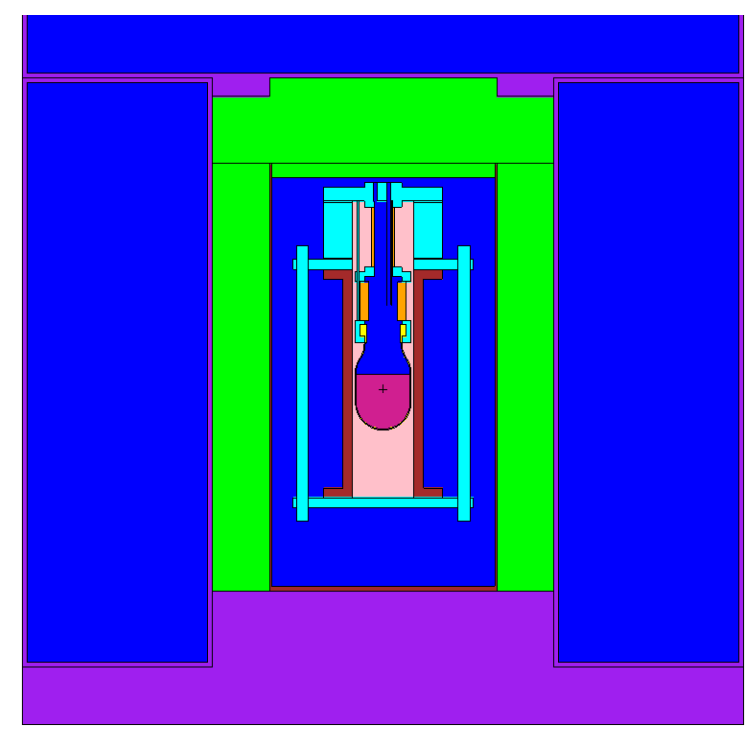

(b) Version $4 p$

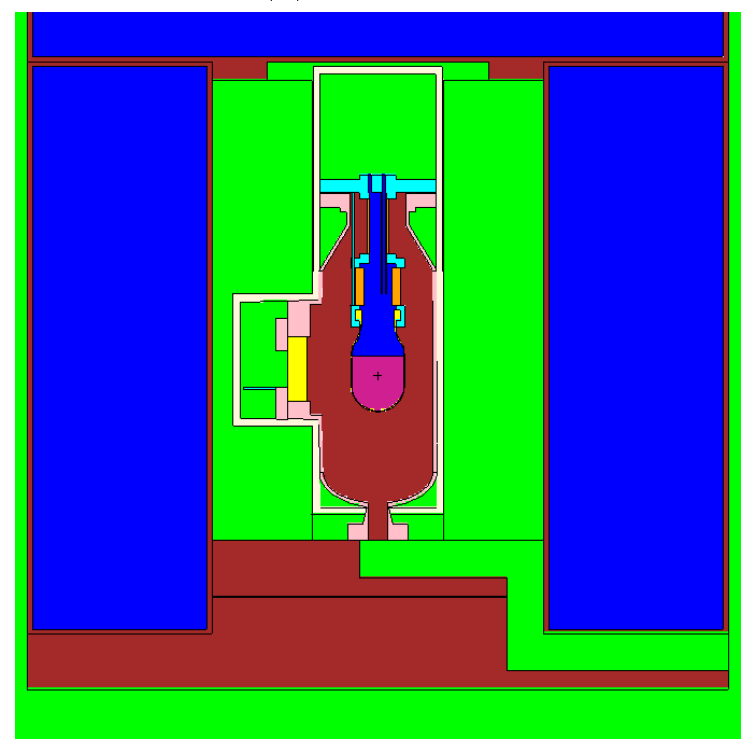

(d) Versions $4 \mathrm{a} \& 4 \mathrm{~b}$

Figure 8.5: Cross-sections of selected versions of the PICO-2L simulation geometry. 


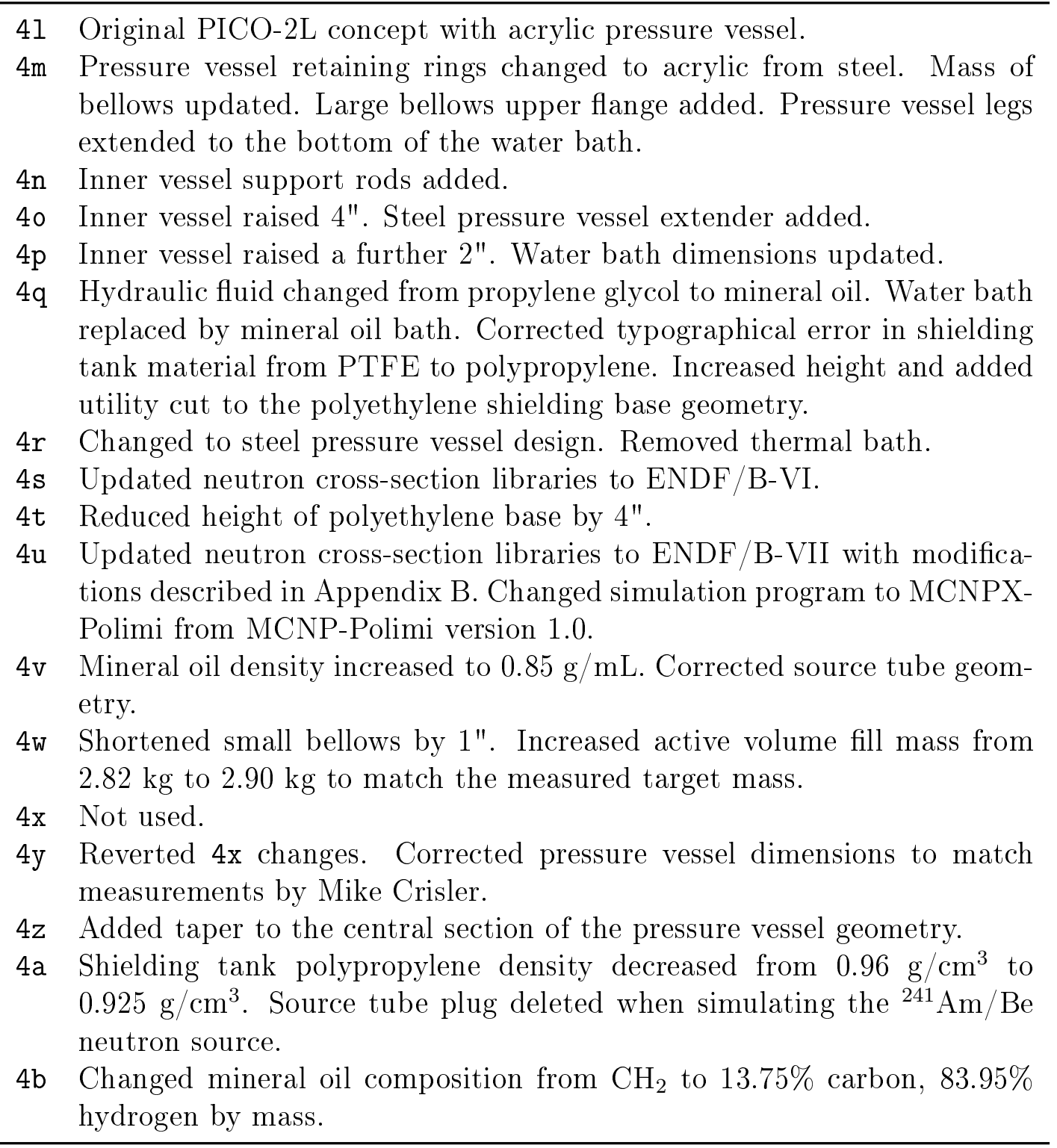

Table 8.4: Versions of the PICO-2L simulation geometry with notes and changes. The input file for the $4 \mathrm{~b}$ geometry is included in Appendix $\mathrm{D}$. 
(graphite, polyethylene, PMMA, nitrile, and $\mathrm{PCB}$ ) were grouped together. Materials for which the measurements of Bair and Campo (1979) and West and Sherwood (1982) were primarily used in the cross-section evaluation (aluminum, silica, borosilicate, water) were grouped together. Both SAE Grade 304 and SAE Grade 316 stainless steels were grouped. All other materials were considered individually.

Asymmetric uncertainties due to uncertainty in which $(\alpha, n)$ target material of a component contains the assayed activity was summed.

\subsection{Total Neutron Background Expectation}

The largest neutron background contribution in PICO-2L are listed in Table 8.5. With 0.200

live years of exposure, $1.6_{-0.9}^{+0.3}$ single bubble events and $2.2_{-1.3}^{+0.5}$ multiple bubble events would have been expected during the PICO-2L run. No multiple bubble events were observed. With this observed lack of multiple bubble events and using the predicted ratio of single to mutiple bubble events, at most 1.7 single bubble are expected in the data set at $90 \%$ confidence. Most of the background events observed in PICO-2L cannot have been caused by neutron scattering.

Many of the largest backgrounds in PICO-2L are smaller or eliminated in PICO-60 and the proposed PICO-250L due to the immersion of the detector in a water tank, and increased shielding depths. The neutron backgrounds due to components of the chamber optics will remain high as long as the present materials are used. 


\begin{tabular}{lccc}
\hline Component & $\begin{array}{c}\text { Simulation } \\
\text { Version }\end{array}$ & \multicolumn{2}{c}{$\begin{array}{c}\text { Neutron events } \\
\text { per live year }\end{array}$} \\
& & $n=1$ & Total \\
\hline Radon in Air & $4 \mathrm{~s}$ & $3.8_{-2.1}^{+0.6}$ & $9.2_{-5.0}^{+1.5}$ \\
External neutrons & $4 \mathrm{t}$ & $1.28 \pm 0.67$ & $2.75 \pm 1.44$ \\
Retroreflector & $4 \mathrm{~b}$ & $0.99_{-0.67}^{+0.31}$ & $2.35_{-1.59}^{+0.74}$ \\
Camera lenses & $4 \mathrm{~b}$ & $0.59_{-0.32}^{+0.11}$ & $1.42_{-0.77}^{+0.27}$ \\
Inner vessel jar flange & $4 \mathrm{~b}$ & 0.32 & 0.71 \\
Pressure Vessel Tee & $4 \mathrm{~s}$ & $0.265 \pm 0.135$ & $0.638 \pm 0.324$ \\
Dytran cable & $4 \mathrm{~s}$ & $0.134_{-0.135}^{+0.033}$ & $0.323_{-0.327}^{+0.079}$ \\
Cameras & $4 \mathrm{~s}$ & $0.129_{-0.101}^{+0.028}$ & $0.298_{-0.064}^{+0.0635}$ \\
Jar Flange Backing Gasket & $4 \mathrm{~b}$ & $0.124_{-0.30}^{+0.25}$ & $0.273_{-0.066}^{+0.055}$ \\
Other & $4 \mathrm{n}-4 \mathrm{~s}$ & $0.50_{-0.24}^{+0.17}$ & $1.17_{-0.47}^{+0.31}$ \\
\hline Total & & $8.1 \pm 0.7_{-4.5}^{+1.4}$ & $19.1 \pm 1.5_{-10.8}^{+3.3}$ \\
\hline
\end{tabular}

Table 8.5: Background contributions from components of the PICO-2L detector. Separate statistical and asymmetrical systematic uncertainties are given. 


\section{Chapter 9 PICO-2L Dark Matter Results}

\subsection{Time to Previous Non-timeout Cut}

In COUPP-4kg, both a neutron background an a time-clustered rate of single bubble events was observed (Behnke et al. 2012). Events of the latter type occurred within $1000 \mathrm{~s}$ of a bubble forming near the $\mathrm{CF}_{3} \mathrm{I}$ /water/silica triple interface. As described in Section 2.2.4. particulates concentrate at this location and can be liberated by the stirring caused by bubble formation. These liberated particulates can form bubbles. In both PICO-2L and COUPP-60, a similar population of events has been found. In both chambers, the inner volumes were assayed for particulates and particulates were found shown in Figure 9.1.

In addition to time clustering, several other anomalies were observed in the population of PICO-2L dark matter candidate events (Cooper et al. 2014). The average acoustic parameter of these events was approximately $5 \%$ larger than that of single bubble events from ${ }^{241} \mathrm{Am} / \mathrm{Be}$ calibrations. Several clusters of possible candidate events were observed during periods of low compression pressure that were cut from the data, including a cluster of 5 events within 90 minutes. The candidate events are also spatially clustered away from the central axis of the inner volume.

For PICO-2L, a time-clustering cut was developed to remove particulate caused events from the dark matter search. The cut eliminates dark matter candidate events within a set livetime of the previous formation of a bubble in PICO-2L. This time to previous nontimeout (TPNT) cut was set using the optimal interval method (Yellin 2002) modified for use in a threshold experiment. The optimum cut was set separately for each dark matter search threshold of PICO-2L and found to accept no candidate events. The livetime cuts 


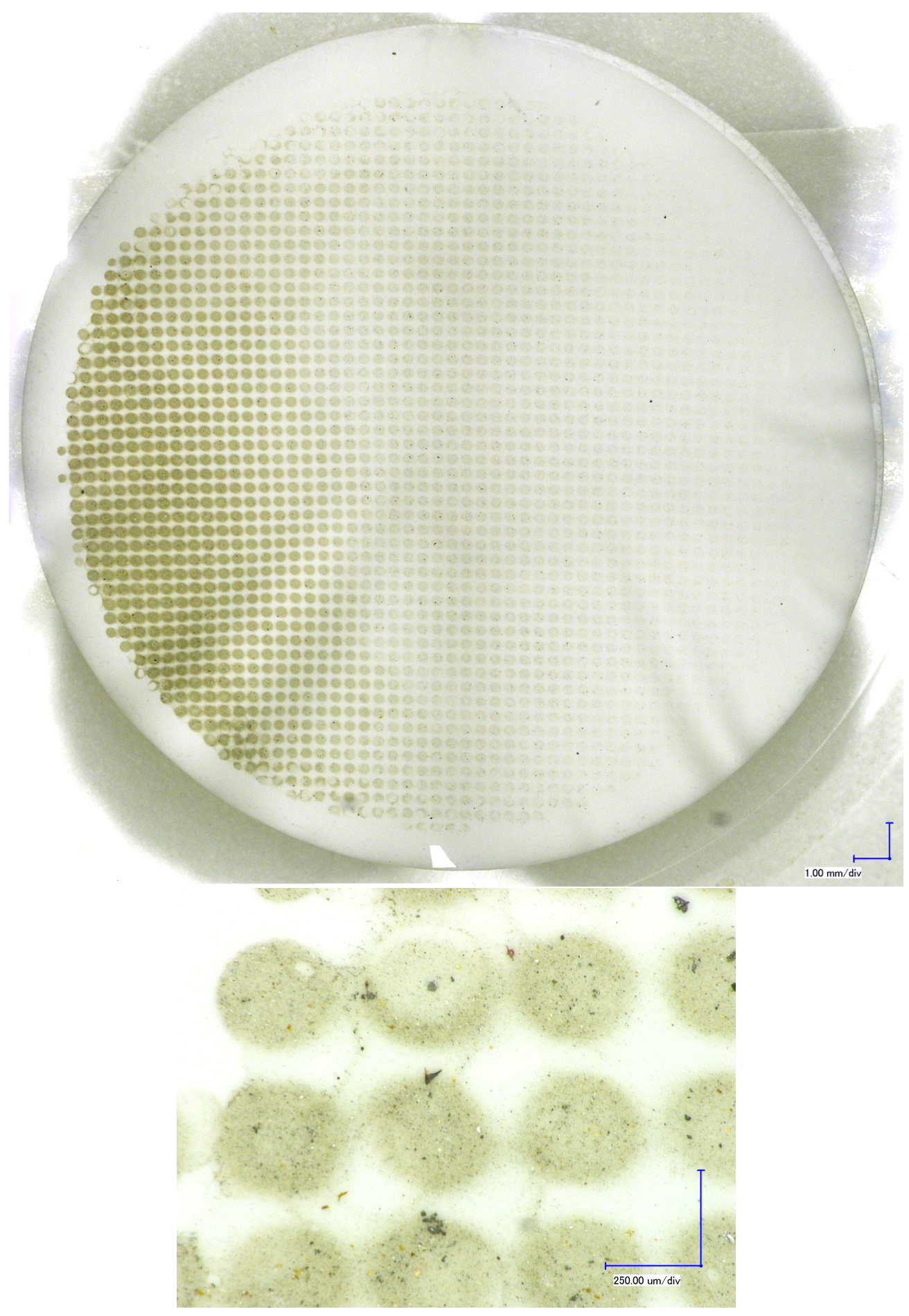

Figure 9.1: Particulates from PICO-2L on an assay filter. The PICO-2L inner volume was passed through the 1" diameter filter, collecting a significant number of silica and steel particulates. The whole filter (top) and an expanded image of the densest part or the filter (bottom) are shown. 


\begin{tabular}{ccc}
\hline Threshold & $\begin{array}{c}\text { TPNT cut } \\
(\mathrm{s})\end{array}$ & $\begin{array}{c}\text { Accepted Exposure } \\
(\mathrm{kg} \cdot \mathrm{days})\end{array}$ \\
\hline $3 \mathrm{keV}$ & 980 & 2.13 \\
$4 \mathrm{keV}$ & 0 & 1.38 \\
$5.5 \mathrm{keV}$ & 1700 & 1.69 \\
$7 \mathrm{keV}$ & 0 & 2.30 \\
\hline
\end{tabular}

Table 9.1: Time to previous non-timeout (TPNT) cuts used to calculate PICO-2L dark matter limits. The statistical penalty factors shown are the ratio of dark matter limits calculated using the modified optimal interval method (Yellin 2002) and those calculated using the accepted livetime with zero events.

and accepted livetime used in Amole et al. (2015) are shown in Table 9.1. As these cuts are made post priori, the optimum interval method calculates a dark matter event rate limit that compensates for the biased cut selection.

\subsection{Dark Matter Limits}

The spin-dependent WIMP-proton dark matter limits set by the COUPP-4kg and PICO2L experiments have been recalculated using the efficiency functions from Chapter 6 and are shown in Figure 9.2. Limits were calculated using the formalism and approximations of Lewin and Smith (1996) with spin-dependent form factors for fluorine and iodine from Engel, Pittel, and Vogel (1992).

The COUPP-4kg limits from Behnke et al. (2012, 2014) have been recalculated with the WIMP halo parameters used in Amole et al. (2015): $v_{o}=320 \mathrm{~km} / \mathrm{s}, v_{\mathrm{esc}}=544 \mathrm{~km} / \mathrm{s}$, $v_{e}=232 \mathrm{~km} / \mathrm{s}$, and $\rho_{\mathrm{DM}}=0.3 \mathrm{GeV} / \mathrm{cm}^{2}$. A limit band is shown between limits using two fluorine efficiency models used in Behnke et al. (2012), a flat 46\% efficiency above the Seitz threshold and a slowly rising efficiency function of the form

$$
\eta=1-\exp \left\{-0.15 \frac{E_{r}-E_{c}}{E_{c}}\right\}
$$


$100 \%$ iodine recoil efficiency above the Seitz threshold is assumed. In addition to the limit band, an updated limit based on the efficiency functions shown in Figure 6.6 was calculated. At each WIMP mass, the detector sensitivity using the five efficiency curves was calculated, and these sensitivities were converted into a probability distribution. This probability density function is flat between each calculated detector sensitivity with the area under each segment equal to either 0.34 or 0.135 for segments between sensitivity from the $\pm 1 \sigma$ and best fit fluorine efficiency functions, and between the $\pm 2 \sigma$ and $\pm 1 \sigma$ efficiency functions respectively. A delta function with an integral of 0.025 is added at the $\pm 2 \sigma$ points. This sensitivity probability distribution is convolved with the probability of the expected count rate given an observation of 20 events in COUPP-4kg to obtain a dark matter event rate distribution. The $90 \%$ confidence upper limit of this distribution is plotted.

The PICO-2L spin-dependent WIMP-proton dark matter limit from Amole et al. (2015) is also plotted in Figure 9.2. A new limit based on the efficiency function at a $3.24 \mathrm{keV}$ threshold from Figure 6.16 is calculated by rescaling the original limit. The dark matter sensitivity of PICO-2L was calculated for the exposures shown in Table 9.1 with both the efficiency function presented in Amole et al. (2015) and using the five efficiency functions shown in Figure 6.16. These five functions were combined in the same way as the $\mathrm{CF}_{3} \mathrm{I}$ efficiency functions were. The ratio of the PICO-2L sensitivities was used to scale the dark matter limit given in Amole et al.

The new efficiency functions shown in Figure 6.16 are significantly less sensitive than those shown previously. Calibrations for the previous PICO-2L and COUPP-4kg limits relied heavily on ${ }^{241} \mathrm{Am} / \mathrm{Be}$ neutrons. At masses above $200 \mathrm{GeV},{ }^{241} \mathrm{Am} / \mathrm{Be}$ neutron calibrations provide a good proxy for WIMP sensitivity. The reduced sensitivity at these masses is due to the inconsistency between ${ }^{241} \mathrm{Am} / \mathrm{Be}$ data and the fits found in Chapter 6 . Should this discrepancy, or an error in the fitting program or method used in Chapter 6 be found, 


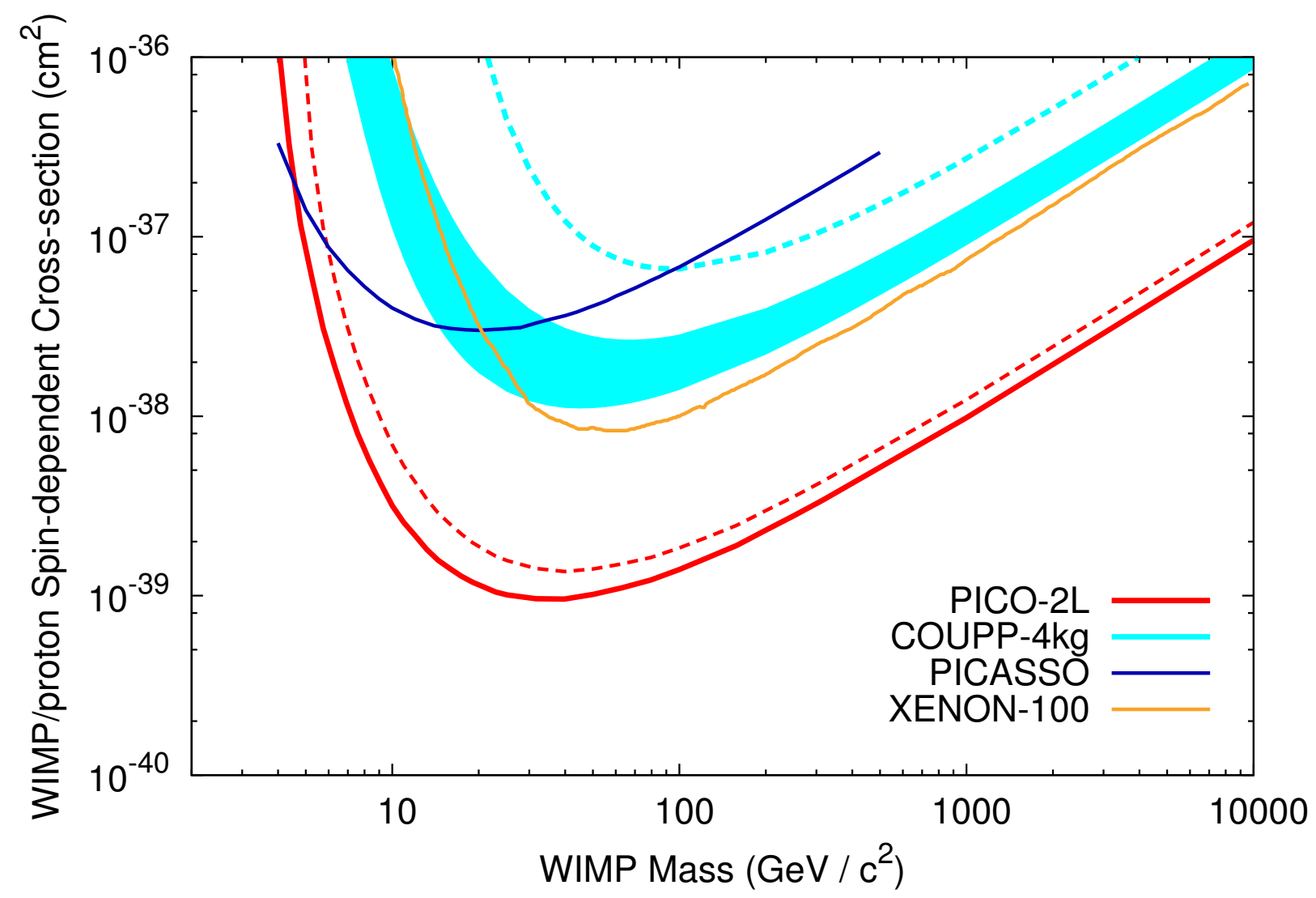

Figure 9.2: Limits on the spin-dependent WIMP-proton scattering cross-section. Published limits from PICO-2L (Amole et al. 2015) in solid red, and other prior dark matter direct search experiments PICASSO (Archambault et al. 2009) in blue and XENON-100 (Aprile et al. 2013) in yellow are shown. The dashed red and cyan lines are the recalculated PICO-2L and COUPP-4kg limit using the efficiency functions shown in Figure 6.16 (see text). 


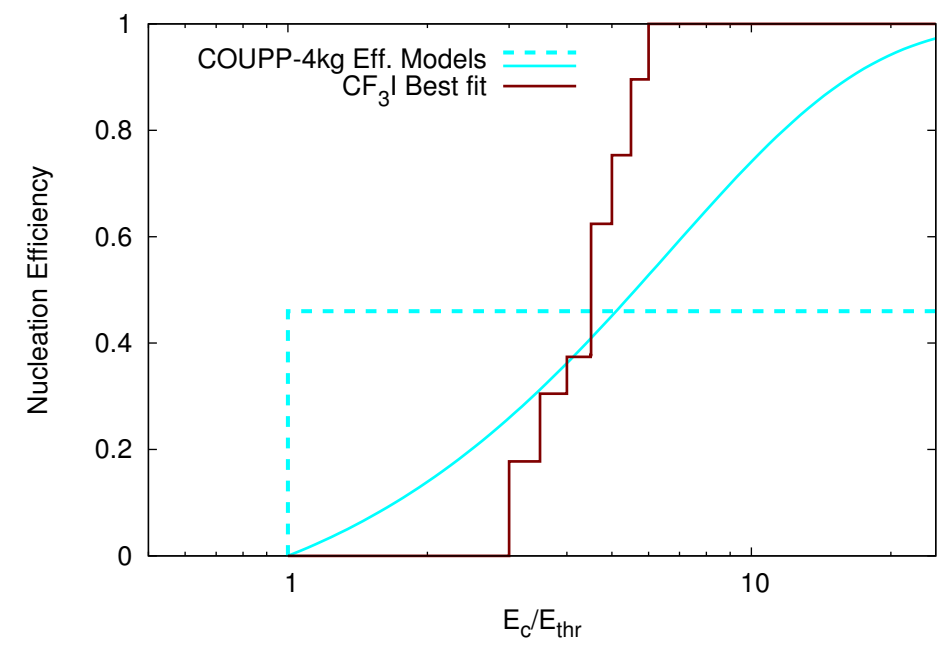

Figure 9.3: Efficiency models used for COUPP-4kg (Behnke et al. 2012; Fustin 2012) compared to the best fit efficiency model from Figure 6.6. The ${ }^{241} \mathrm{Am} / \mathrm{Be}$ neutron calibrations from COUPP-4kg measured the bubble nucleation efficiency averaged over a broad recoil energy $E_{r}$ range. Sample efficiency models were chosen that provided bubble nucleation efficiency approching the Seitz threshold $E_{c}$. With low-energy neutron calibrations, no nucleation efficiency was found for recoil energies within a factor of three of the Seitz threshold.

limits at these masses would return to their previously published values.

At low WIMP masses, the sensitivity of the COUPP-4kg experiment is significantly less than that previously proposed based on efficiency models with sensitivity approaching the Seitz threshold, as shown in Figure 9.3. Previous calibrations of nuclear recoils in other superheated fluid detectors (Archambault et al. 2011) had found efficiency at the Seitz threshold. A low stopping power threshold that could cause the observed inefficiency was also disfavored by fits to the ${ }^{241} \mathrm{Am} /$ Be calibration data at threshold energies (Fustin 2012 , Sec. 6.4).

With either the efficiency models presented here or used in Amole et al. (2015), PICO-2L sets the world's best spin-dependent WIMP-proton limits for a direct search experiment. Results from other competitive experiments, XENON-100 (Aprile et al.2013) and PICASSO (Archambault et al. 2009) are shown on Figure 9.2 for comparison. Results from the SIM- 
PLE experiment (Felizardo et al. 2014) are not shown. They used superheated $\mathrm{CF}_{3} \mathrm{I}$ and assume a high fluorine recoil detection efficiency that has been disproven in this thesis. Their data analysis also uses an unwarranted background subtraction (Dahl, Hall, and Lippincott 2012). Anticipated results from the $250 \mathrm{~kg}$ xenon-filled LUX experiment (Akerib et al. 2014) will likely be a factor of 3-5 stronger than the XENON-100 limit, still less sensitive than the $2.90 \mathrm{~kg}$ PICO-2L detector.

With lower background event rates and the elimination of particulates from the active volume, future $\mathrm{C}_{3} \mathrm{~F}_{8}$ filled bubble chamber will continue to provide the best spin-dependent WIMP-proton dark matter cross-section limits. 


\section{Appendix A Results of $\mathrm{CF}_{3} \mathrm{I}$ Darkening Tests}

The plots on the following pages presents the history of three test stands used to measure the darkening rate of $\mathrm{CF}_{3} \mathrm{I}$ in a large variety of conditions and in the presence of a variety of illuminator. Section 2.1 describes the apparatus and illuminators used for the tests. Test stand operations between October 2010 to May 2011 are presented. One thin pressure vessel and three thick pressure vessels were used in the tests.

The upper portion of each plot shows the history of each test stand and the conditions to which it was subjected. Unique outline colors identify the test stands while fill colors identify the illuminator used. Discontinuities in the lines indicate that the stands were emptied and refilled.

The lower portion of each plot shows the measurement of darkening in each stand. The color of the lines again identifies the test stand while the color and shape of the points identifies the measurement method. In addition to the presence of various illuminators and materials, other variables were changed as annotated on the plots and in their companion tables. In addition to the parameters of each fill, the tables calculate the darkening rates achieved in the precisely measured units of \%/day, or converted to \%-cm/W-day and mb with larger uncertainties.

On occasion, the chambers were inverted to allow the $\mathrm{CF}_{3} \mathrm{I}$ to contact the steel in the plumbing. This allowed for the addition and removal of steel without having to empty and refill the chamber. Whenever steel was in contact with the active fluid, molecular iodine was eliminated. To test whether the mixing action or the contact with steel from inverting

the chamber reduced the iodine concentration, a darkened stand was inverted and righted 10 times within 5 minutes. No change in darkening was observed. 
To test whether dissociation reactions were being caused by interactions at the water $/ \mathrm{CF}_{3} \mathrm{I}$ interface, on December 9, masks were used to reduce to either hide or illuminate the interface region. When the interface was exposed, the bottom of the chamber was masked so as to mask $90 \%$ of the illumination in each case.

From January 7 to January 21st, the '1003' stand was exposed to red light through an optical filter that cut-off light with wavelengths shorter than $630 \mathrm{~nm}$ and reduced the illumination intensity to $45 \%$ of its unfiltered value.

The inhibiting effects of $\mathrm{Na}_{2} \mathrm{SO}_{3}$ were tested in two ways: the salt was added to the water buffer or the $\mathrm{CF}_{3} \mathrm{I}$ was bubble through the $\mathrm{Na}_{2} \mathrm{SO}_{3}$ solution before filling the test stand. In the latter case, no $\mathrm{Na}_{2} \mathrm{SO}_{3}$ was added directly to the test stand.

Some test stands with nominally identical conditions did not always darken at the same rate. Darkening occurred with delayed onset, suddenly slowed, or reversed itself. Other chemicals beyond $\mathrm{CF}_{3} \mathrm{I}$, water, and molecular iodine must be present, either as catalysts, buffers, or inhibitors, to cause this variation. 


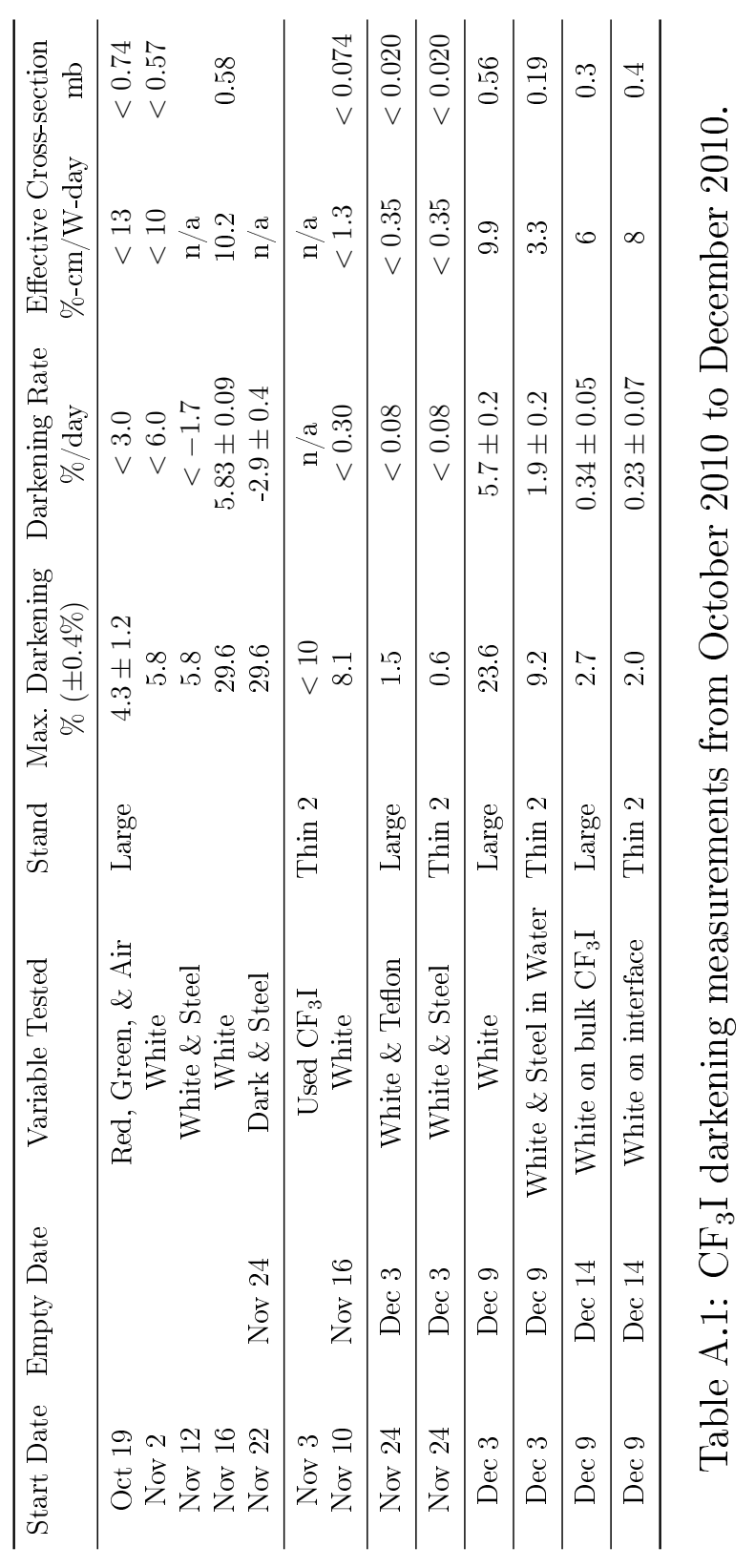



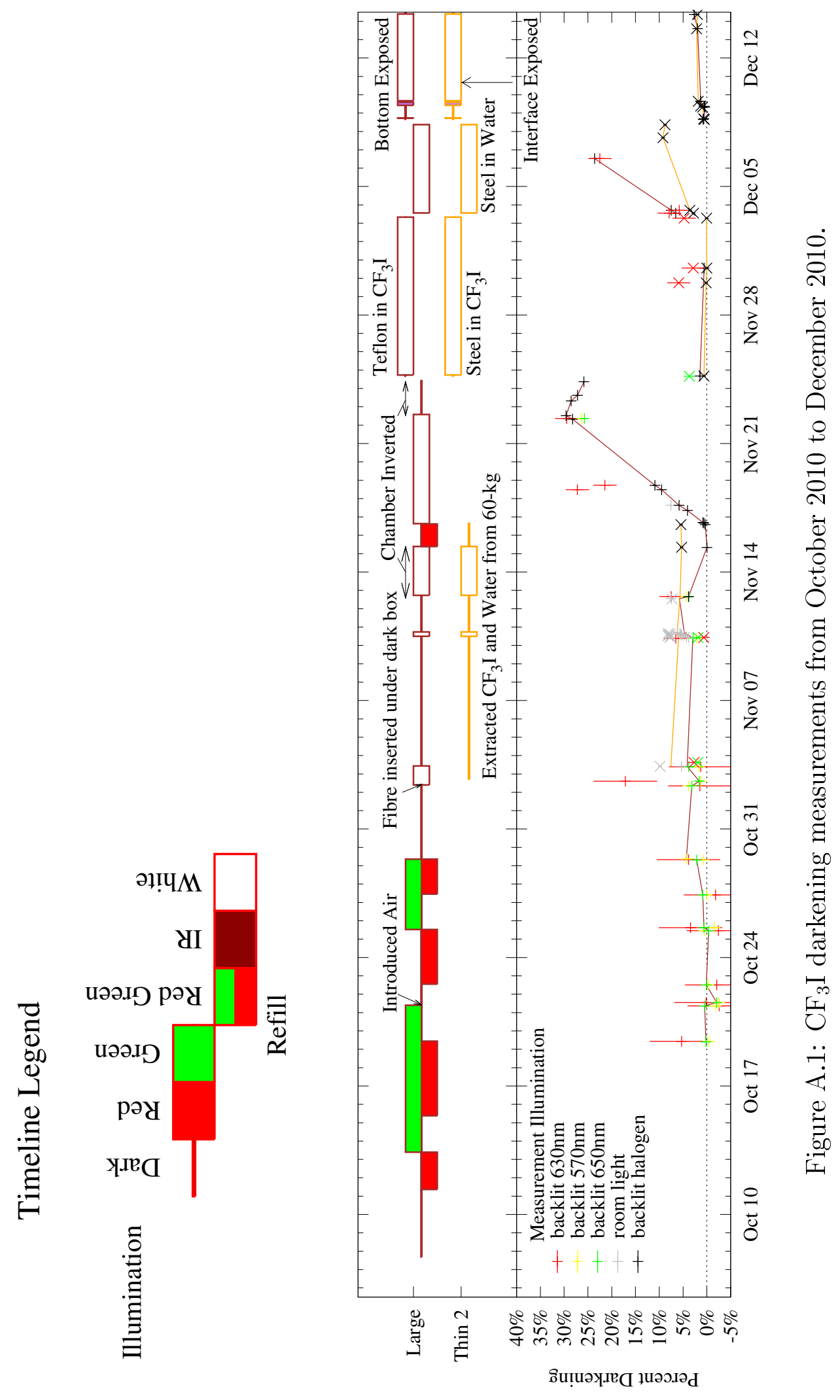


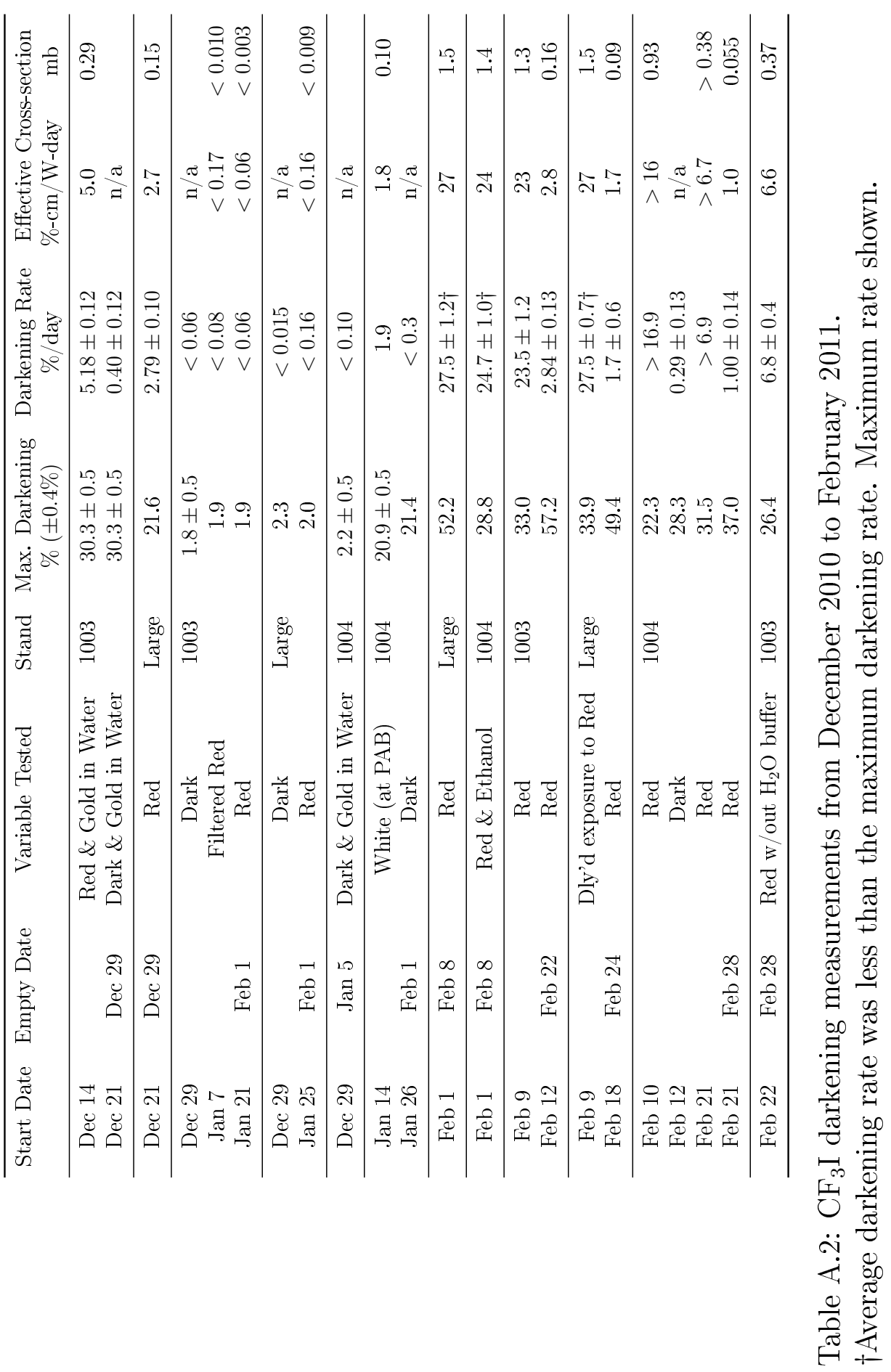




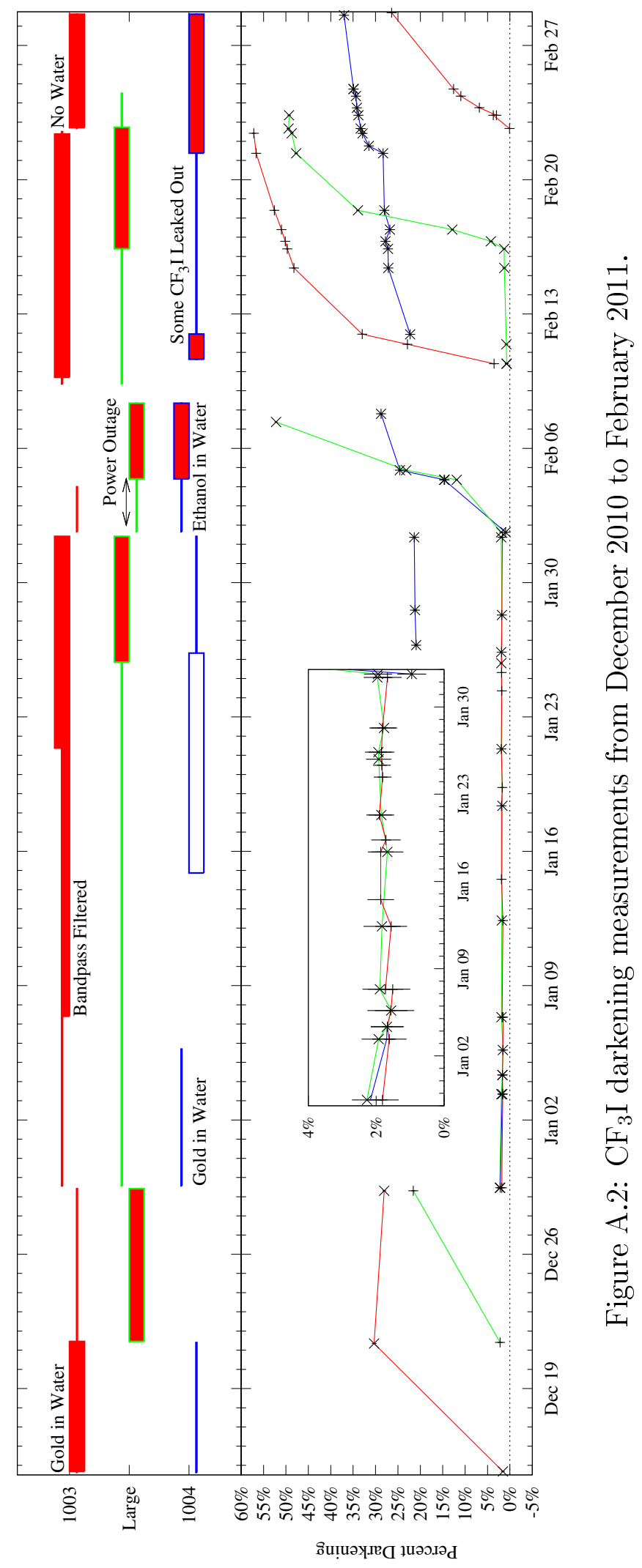




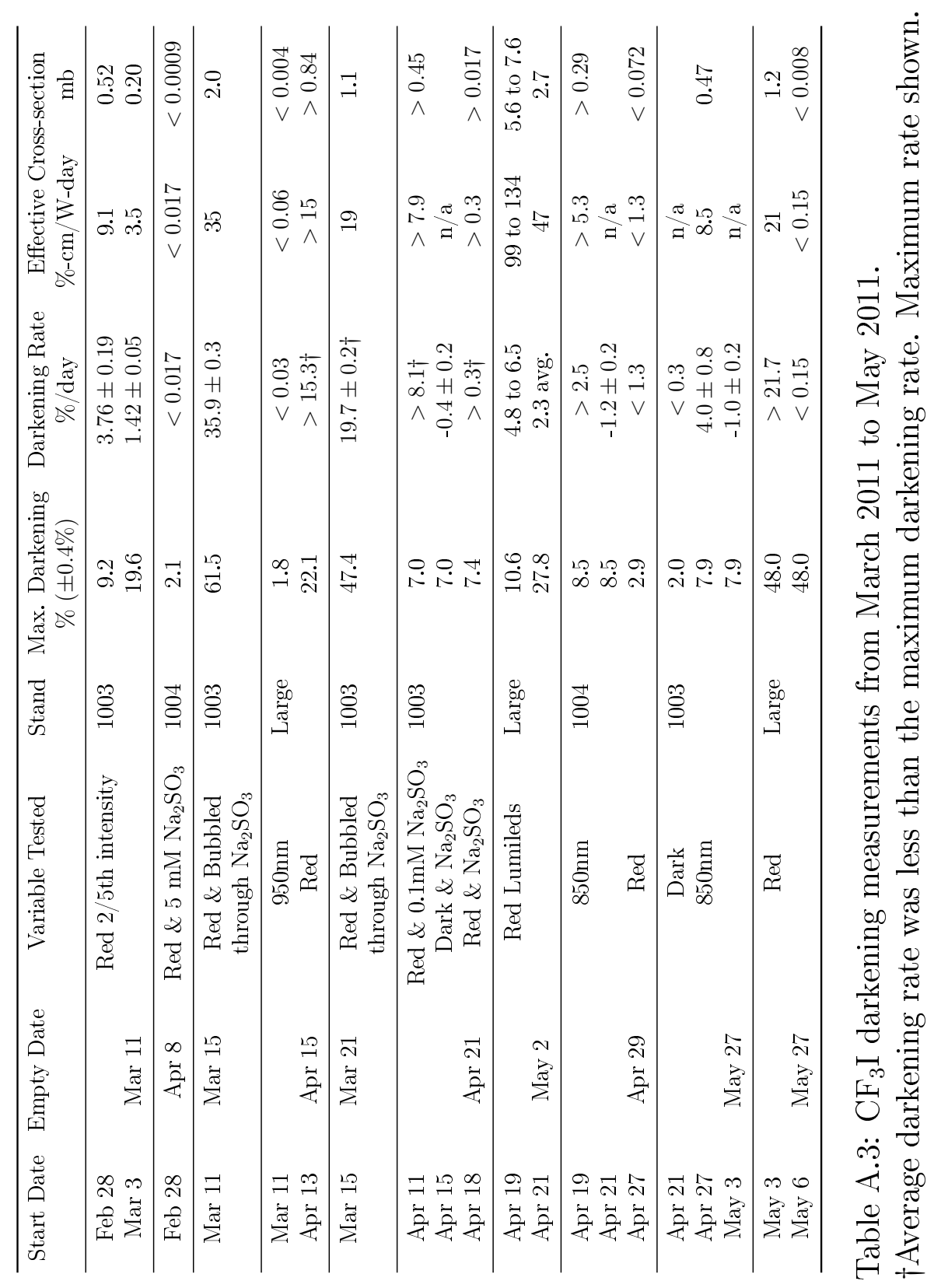




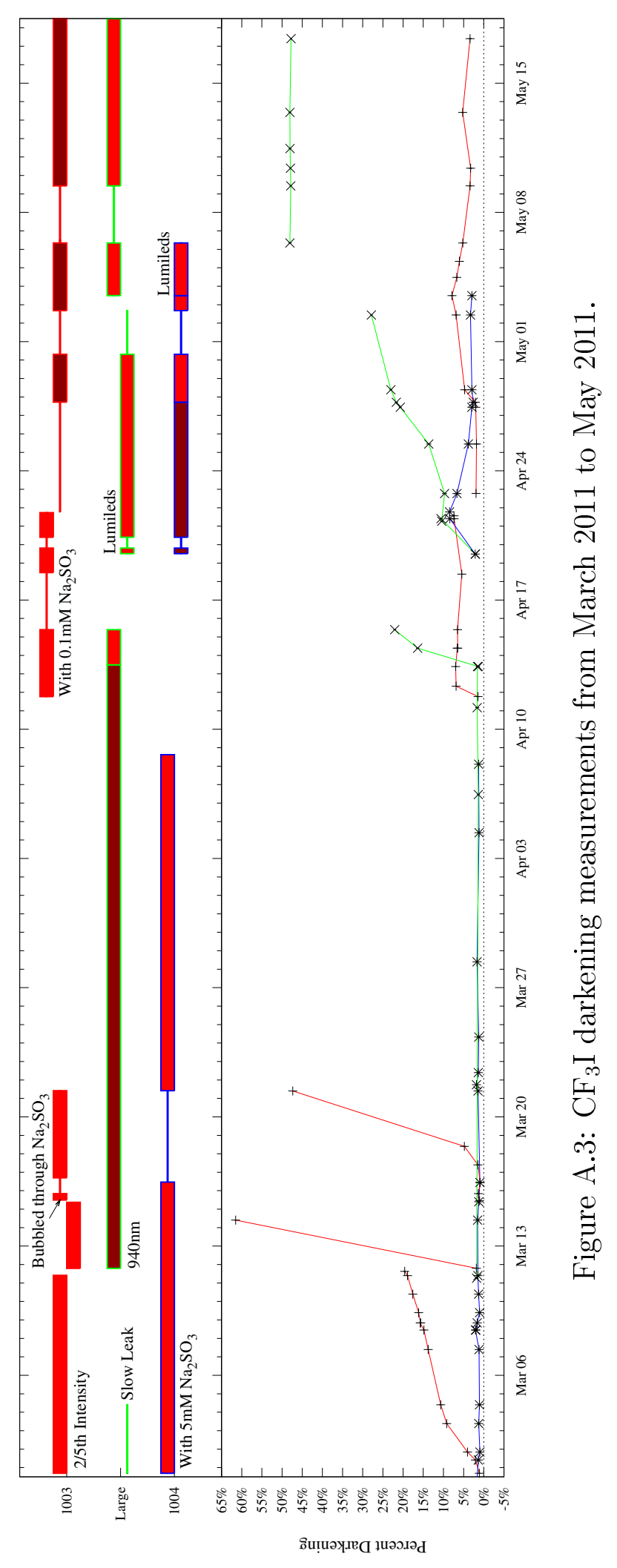




\section{Appendix B}

\section{New libraries for simulating neutron scattering in dark matter detector calibrations}

This appendix has been published as an article in Physical Review C (A. E. Robinson 2014) and is reproduced here.

At neutron energies up to a few $\mathrm{MeV}$, neutron elastic scattering is well described by optical model scattering off a nuclear potential plus scattering off resonances of excited and compound nuclear states (Mughabghab 2006). Elastic scattering cross-sections and angular distributions can be calculated using R-matrix formalism from a list of nuclear potential shape and resonance parameters. Many modern nuclear data evaluations using the Evaluated Nuclear Data Format (ENDF-6) (Herman, Tkrov, and Brown 2011) provide these parameters instead of pointwise elastic scattering cross-sections ฤ. For an introduction to nuclear data evaluations in ENDF-6 format, see McFarlane (McFarlane 1998). The MCNP and Geant4 Monte Carlo radiation transport programs require pointwise cross-section libraries that are generated by either the NJOY (MacFarlane and Kahler 2010) or PREPRO (Cullen 2012) codes from the ENDF evaluations.

Both PREPRO and NJOY calculate neutron elastic scattering cross-sections from the resonance parameters using R-matrix formalism (Blatt and Biedenharn 1952), but not the differential cross-section 2 . Instead, these codes translate the angular distribution found in File 4 of the ENDF evaluations verbatim. For all stable nuclei lighter than ${ }^{16} \mathrm{O}$, the most modern ENDF/B-VII (Chadwick et al. 2011) and JENDL-4 (Shibata et al. 2011) evaluations contain accurate angular distributions either from R-matrix calculations or from

1. Modern ENDF evaluations can be found though Nuclear Data Services at http://www-nds.iaea.org

2. There is a hidden option under development in NJOY2012 for calculating the differential cross-section from resonance parameters. Robert MacFarlane, private communication. 
high resolution experimental data. However, the ENDF File 4 evaluations of almost all heavier nuclei either assume isotropy, ignore the resolved resonance contributions to the angular distributions, or are based on incomplete experimental data.

The nuclear recoil response of dark matter detectors are most often calibrated using the nuclear recoils produced by neutron elastic scattering. The simulated nuclear recoil energy distribution against which detectors are calibrated can be affected in at least three ways by incorrect elastic scattering angle distributions.

- Any change in the recoil energy distribution at a given neutron energy is a change in the scattering angle distribution as $E_{r} \propto \cos \theta$.

- The probability for low energy neutrons to propagate into the active volume of the detector can change.

- The energy loss and diversion of neutrons in the active volume of the detector can change, affecting multiple scattering distributions.

Calibrations that rely on simulating the absolute nuclear recoil distribution (Barnabé-Heider et al. 2005: Horn et al. 2011; Collar 2013a; Bernabei et al. 1996, Ref 20. in Agnese et al. 2013) are vulnerable to all three effects while calibrations that determine the recoil energy and rate by tagging the outgoing neutron (Barbeau 2009; Alexander et al. 2013, Collar 2013b; Manzur et al. 2010) are only affected by changes in the multiple scattering distributions. Some heavy nuclei used in detector construction have resolved resonances for neutron energies only below $20 \mathrm{keV}$ (producing nuclear recoils at $<1 \mathrm{keV}$ ), including ${ }^{127} \mathrm{I}$, ${ }^{133} \mathrm{Cs}, \mathrm{W}$, and most isotopes of Xe (Mughabghab 2006). These neutron recoils are below the threshold of most dark matter detectors and the use of existing neutron cross-section libraries can be used in confidence. However, most elements heavier than oxygen have resolved resonances above $100 \mathrm{keV}$ that are important in simulating the response of dark matter detectors to neutron scattering. 


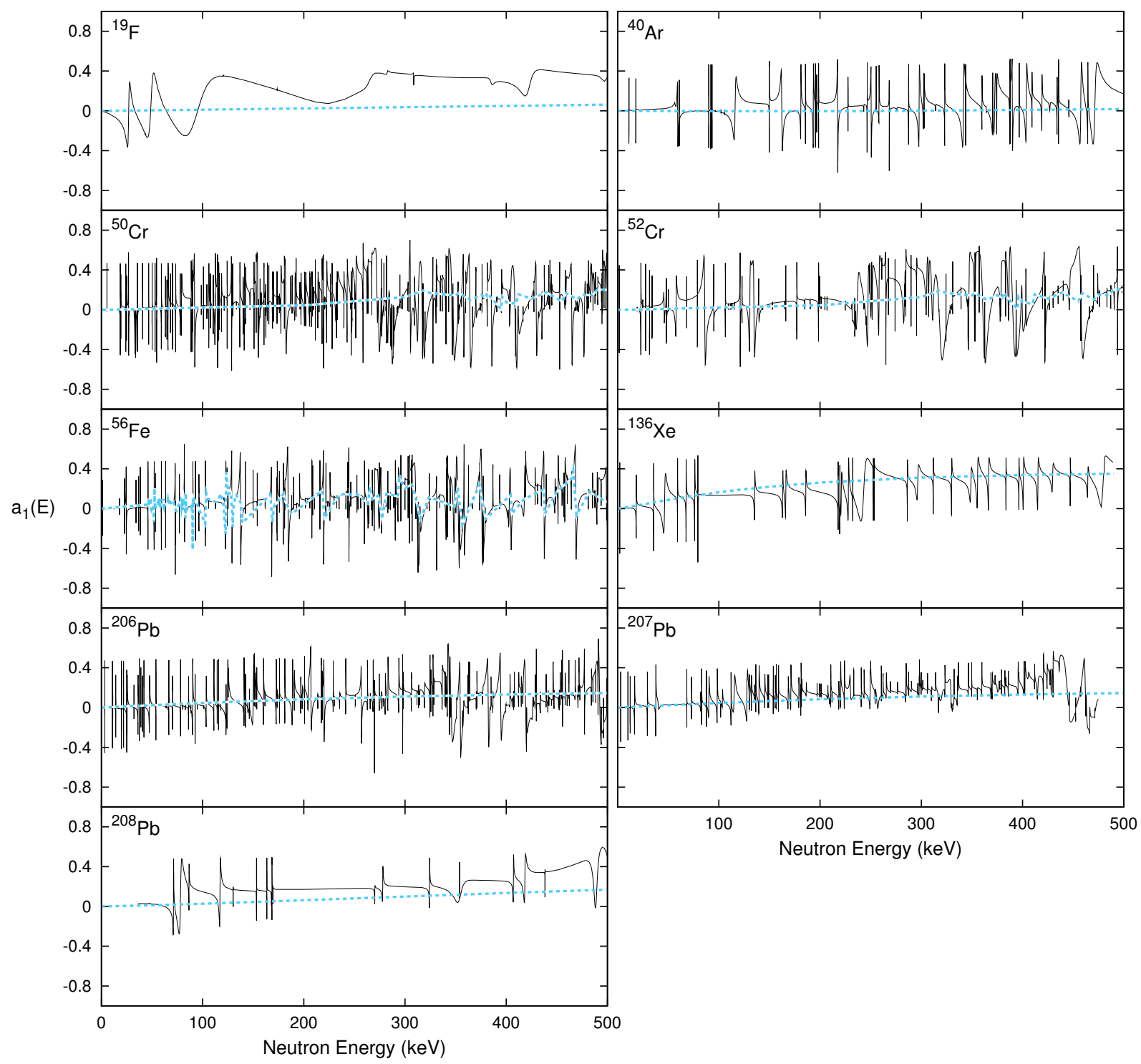

Figure B.1: Dipole anisotropy terms of neutron elastic scattering for ENDF/B-VII (dashed) and for R-matrix calculations (solid). The R-matrix calculations are used in a set of new libraries for MCNP and Geant4. The ENDF/B-VII iron and chromium evaluations are based on experimental data and follow the R-matrix calculated angular distribution. The lead, xenon, and argon evaluations have angular distributions calculated using optical model calculations without resonance contributions. The ${ }^{19} \mathrm{~F}$ evaluation has no angular distribution data below $1 \mathrm{MeV}$. 
Using SAMMY (Larson 2008) or other R-matrix codes, the neutron scattering angular distributions can be calculated. The SAMMY auxiliary program SAMRML can calculate the cross-section at specific angles directly from an ENDF-6 formated file. For use in simulations, I have edited ENDF/B-VII based MCNP and Geant4 (Mendoza et al. 2012) libraries for ${ }^{19} \mathrm{~F}$, ${ }^{50,52} \mathrm{Cr},{ }^{56} \mathrm{Fe},{ }^{136} \mathrm{Xe}$, and ${ }^{206,207,208} \mathrm{~Pb}$ with high-resolution angular distributions generated by SAMMY using R-matrix formalism. Libraries for $\mathrm{Si}, \mathrm{Al}$, and $\mathrm{Ge}$ are planned. The dipole term of the angular distributions of the ENDF/B-VII and new libraries are shown in Figure B.1. The grids in energy and angle used by these libraries were selected to reproduce the calculated differential cross-section to better than $1 \%$ except for ${ }^{50} \mathrm{Cr}$ for which a $5 \%$ tolerance was adopted. The total memory usage of the MCNP libraries was increased by $76 \%$ as compared to the same libraries for ENDF-VII. To investigate the effect of the new libraries, simulation of the response of dark matter detectors to low energy neutrons with ENDF-VII.0 and these new libraries were compared.

These new libraries are being used by the now merged PICASSO (Archambault et al. 2009) and COUPP (Behnke et al. 2012) (PICO) collaboration to study the response of fluorinated superheated fluid detectors. There is an ongoing calibration of $\mathrm{C}_{3} \mathrm{~F}_{8}$ in the $20 \mathrm{~mL}$ PICO-0.1 bubble chamber using $4.8 \mathrm{keV}$ to $97 \mathrm{keV}$ mono-energetic neutrons at the Université de Montreal's EN tandem accelerator via the ${ }^{50} \mathrm{~V}(p, n){ }^{50} \mathrm{Cr}$ reaction. The calibration compares the rate of bubble formation to the expected rate of nuclear recoils above the detector's threshold energy to obtain the bubble nucleation efficiency as a function of recoil energy, temperature, and pressure. As the bubble formation rate is a convolution of the nuclear recoil energy spectrum and the bubble nucleation efficiency, the efficiency function is measured by setting the threshold energy right below the endpoint of the nuclear recoil spectrum and producing bubbles from a single known recoil energy. The neutron energy is then changed while keeping temperature and pressure constant, and the efficiency 


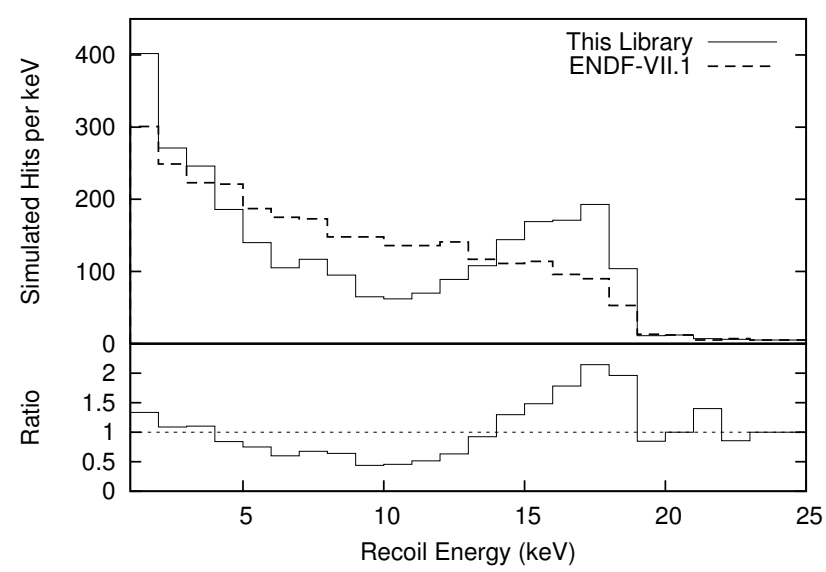

Figure B.2: Simulated nuclear recoil distributions from $97 \mathrm{keV}$ neutrons on $\mathrm{C}_{3} \mathrm{~F}_{8}$ in the PICO-0.1 bubble chamber calibration experiment. The calibration of the detector's bubble nucleation efficiency depends critically on the number of recoils at the endpoint of the simulated nuclear recoil distribution. A factor of 2 discrepancy is found between the Rmatrix calculation used in this library release and ENDF/B-VII.

function is deconvolved from the nuclear recoil energy spectrum. This deconvolution is very sensitive to the measured efficiency at the recoil spectrum endpoint. Figure B.2 shows the simulated nuclear recoil energy spectrum for a $97 \mathrm{keV}$ neutron beam using the ENDF/B-VII evaluation and R-matrix calculations. At a $15 \mathrm{keV}$ threshold, the ENDF/B-VII evaluation over-predicts the bubble nucleation efficiency at the endpoint by a factor of 2 .

These new libraries affect multiple scattering distributions by several mechanisms. With an increase in the number of forward scatters, the neutron loses less energy at each interaction and travels further in both total track length and distance from the origin. A simulation of $900 \mathrm{keV}$ to $1 \mathrm{MeV}$ neutrons propagating an infinite volume of $\mathrm{C}_{3} \mathrm{~F}_{8}$ have $8 \%$ greater track length and travel $16 \%$ further from the origin with the new F-19 library than with the ENDF/B-VII libraries. The effect of the new libraries on multiple scattering distributions will depend on a detector's particular geometry and energy threshold. The simulated probability of detecting a multiple scatter may either increase (due to more collisions) or decrease (due to particles passing through or recoils falling below threshold). 


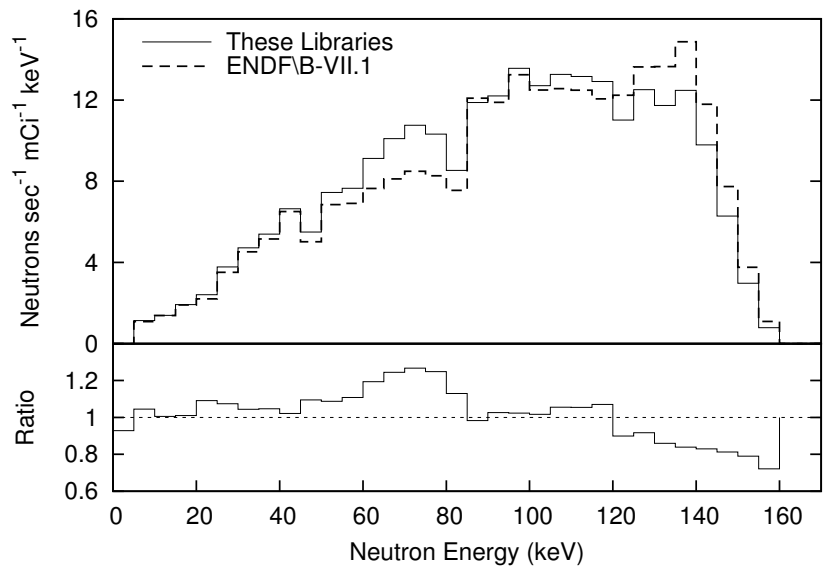

Figure B.3: Simulated neutron energy spectra from an ${ }^{88} \mathrm{Y} / \mathrm{Be}$ surrounded by $20 \mathrm{~cm}$ of lead as used in the ongoing XCD experiment at Fermilab (see text).

$\mathrm{XCD}$ is a new experiment ongoing at Fermilab to calibrate a liquid xenon TPC with low energy neutrons using neutrons from an ${ }^{88} \mathrm{Y} /$ Be neutron source, as described by Collar (Collar 2013a). The $152 \mathrm{keV}$ neutrons from the ${ }^{9} \mathrm{Be}(\gamma, n){ }^{8} \mathrm{Be}$ reaction propagate through a large amount of lead, steel, and PTFE before interacting in the liquid xenon detector. The hit rate in the detector will be compared against the expected number of nuclear recoils from a simulation of the neutron propagation, similar to the PICO-0.1 calibration. As with PICO-0.1, this calibration is more sensitive to high energy neutrons that are able to produce higher energy recoils in the active volume. A simulation of the neutron energy from the lead surrounded ${ }^{88} \mathrm{Y} /$ Be source, Figure B.3. indicates a $17 \%$ reduction in the number of neutrons above $130 \mathrm{keV}$ exiting the lead using the new libraries as compared to using the ENDF/B-VII based libraries.

One additional new MCNPX library of the ${ }^{9} \mathrm{Be}(\gamma, n)^{8} \mathrm{Be}$ reaction is provided in this package to allow simulations of $(\gamma, n)$ neutron sources for XCD, PICO, and similar experiments. The library implements the measured resonance parameters and branching ratios for the reaction from Arnold et al. (Arnold et al. 2012) up to a maximum energy of 5.2 $\mathrm{MeV}$. A ${ }^{88} \mathrm{Y} /$ Be source has a $5 \%$ dipole anisotropy in both the lab-frame neutron energy 
and angle when converting from isotropic neutron production in the center-of-mass frame. This anisotropy cannot be correctly coded into a spatially extended MCNP neutron source. This library is required by MCNPX in order to obtain the correct energy-angle relationship of the neutrons.

In conclusion, a package of libraries for the simulation of low energy neutron propagation in dark matter detectors with MCNP and Geant4 is presented. These libraries can dramatically change, by factors of 2 in some instances, the results of simulations of detector calibrations as compared to the use of presently available libraries. The difference is especially apparent for ${ }^{19} \mathrm{~F}$ and is present at neutron energies above $20 \mathrm{keV}$ for all stable isotopes with $16<A<67$ and some heavier isotopes. 


\section{Appendix C}

\section{Efficiency Limit Calculator}

\#/usr/bin/python

\# A script to calculate limits on efficiency functions from neutron calibrations.

import sys, math

import $n p$. as $n p$

import os path

from collections import defaultdict

from scipy.stats import norm, poisson

from scipy.optimize import brenth

threshold $=1.0 \#$ Dynamic

thrBins $=$ np. concatenate $((n p$. arange $(1.0,7.99,0.5)$, np. arange

$(8.0,20.0,2.0)$, np.arange $(20.0,2000.1,1980.0)))$

thr_mult_range $=(-1.0,1.1,1.0)$

ambe_eff $=0.8507$

def loadSim (filename, thr_mult $=1.0, z_{-}$cut $\left.=[-999.0,999.0]\right)$ :

nucl_dict $=$ defaultdict (lambda: 3, \{6000: 0, 9019: 1, 53127:2\})

polimi_load $=$ np.loadtxt (filename, usecols $=(0,3,4,6,10,11)$, dtype='f, f, f, f, f, f')

\# Assume nuclear reactions remaining in the polimi file are fully efficient

polimi_load ['f3'] $*=$ thr_mult

polimi_load [np.greater (polimi_load ['f1'],0.5) ]['f3'] $=100$.

polimi_load [np.logical_and (np.less (polimi_load ['f3'], thrBins [1])

, np. less (polimi_load ['f2'],100000.)) ] [' $\left.\mathrm{f}^{\prime}{ }^{\prime}\right]=0.0$

single_scat $=$ np.zeros $((\operatorname{thrBins}$. shape $[0]-1,4))$

total_scat $=$ np.zeros $(($ thrBins.shape $[0]-1,4))$

\# Sort Polimidata by energy within an event in order to easily

calculate the pointers later

polimi_cut $=$ polimi_load [np.greater( polimi_load ['f3'], threshold )]

polimi_data $=$ np.sort (polimi_cut, order $=[$ 'f0', , f3 ', , f2 ', , f4 ', , f5

, ]). view (np.float 32 ). reshape (polimi_cut.shape [0],6)

$[:,(0,2,3,5,4)]$

multiple_mask $=$ np.zeros $(($ polimi_data.shape $[0])$, dtype $=\mathbf{b o o l})$ 
multiple_mask $[1:]=$ np.equal (polimi_data $[:-1,0]$, polimi_data $[1:, \overline{0}])$

multiple_mask [:-1] = np. logical_or (multiple_mask [:-1], multiple_mask [1:])

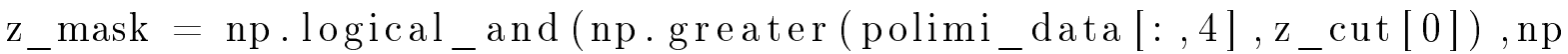
. less (polimi_data [:,4], z_cut[1]))

\# Construct singles array binned in energy and recoiling nucleus

for nucl in nucl_dict.keys ():

polimi_data $[\mathrm{np}$. equal (polimi_data $[:, 1], \mathrm{nucl}), 1]=$ nucl_dict [ nucl ]

polimi_data $[n p . g r e a t e r($ polimi_data $[:, 1], 3), 1]=3$

for $i$ in range (4):

mask $=$ np. logical_and (np.logical_and (np.logical_not ( multiple_mask), np.equal (polimi_data $[:, 1]$, i $)), z_{-}$mask) single_scat $[:, \mathrm{i}]=$ np.histogram (polimi_data $[$ mask, 2$]$, bins $=$ thrBins, weights=polimi_data [mask, 3$]$, density=False ) [0] mask $=n p . \operatorname{logical}$ and $\left(n p\right.$. equal $($ polimi_data $[:, 1]$, i $), z_{-}$mask $)$ total_scat $[:, \mathrm{i}]=\mathrm{np}$. histogram (polimi_data[mask, 2$],$ bins $=$ thrBins, weights=polimi_data [mask, $\overline{3}], \quad$ density=False $)[0]$

\# Return all multiples in array sorted by energy, per row ( energy, nucl, weight, fiducial_passed, pointer prev, pointer next)

mult_arg $=$ np.argsort (polimi_data [multiple_mask, 2])

mult_data $=$ polimi_data [multiple_mask][mult_arg]

multiple_scat $=$ np.zeros $(($ mult_data.shape $[0], 6))$

multiple_scat $[:, 0: 3]=$ mult_data $[:,[2,1,3]]$

multiple_scat [z_mask[multiple_mask], 3$]=1.0$

\# Find the pointer to the previous hit in an event, sorted by energy

event_mask $=$ np.equal (polimi_data [multiple_mask,0][: - 1], polimi_data[multiple_mask, 0$][1:])$

mult_pointer $=$ np.ones $(($ mult_data.shape $[0], 2)) *-1 \#$ Null

pointer is -1

mult_inv $=$ np.argsort (mult_arg)

mult_pointer [: $-1,1][$ event_mask] = mult_inv [1:][event_mask] mult_pointer $[1:, 0][$ event_mask $]=$ mult_inv $[:-1][$ event_mask] 


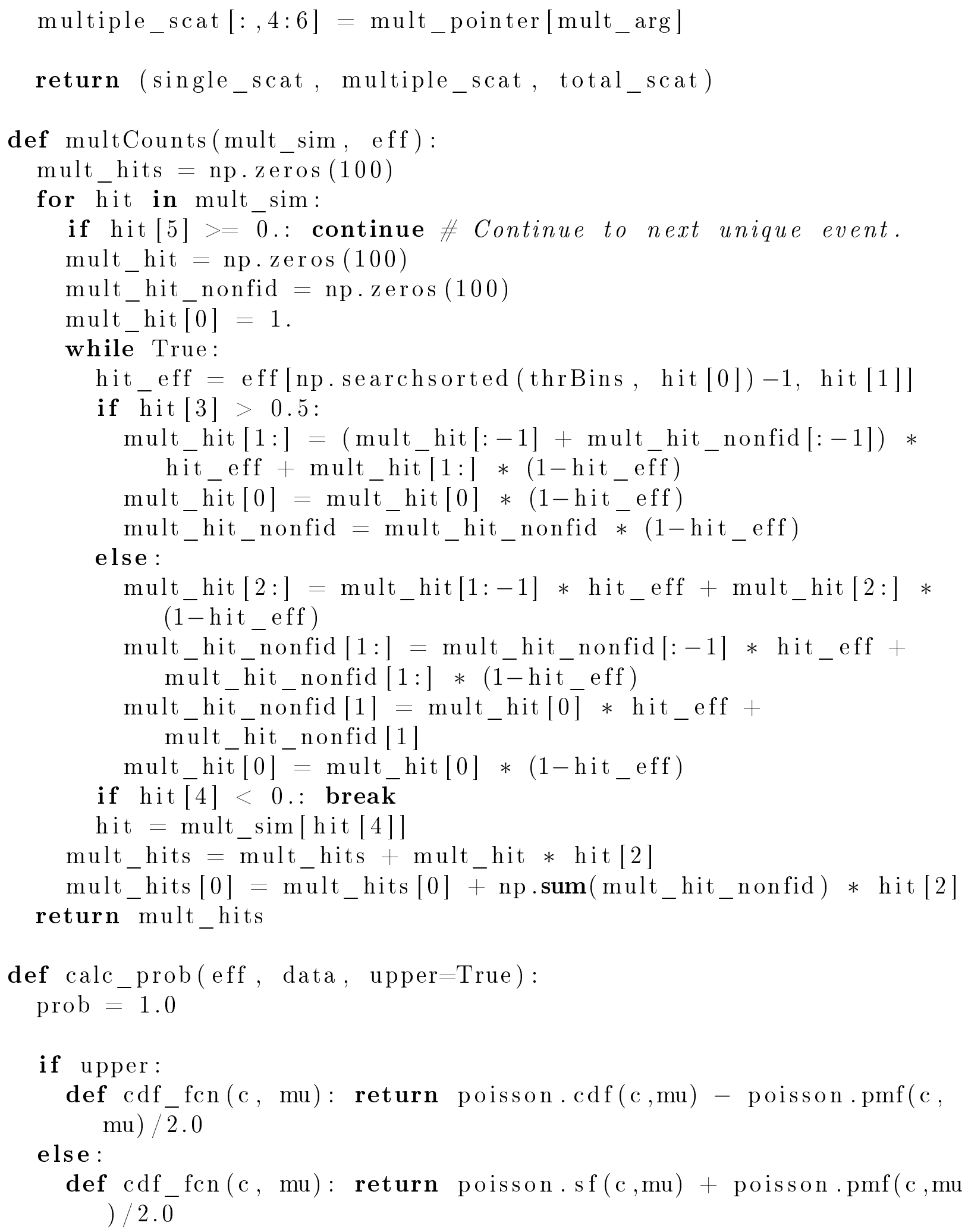


exp_rate $=$ np.zeros $((4,3))$ \# array of measured \#, expected \#,
gaussian expected uncertainty.

for datum in data[0]:

counts $=$ multCounts (datum[2][1], eff $)$

counts[1] $*=(1$-datum[0][9]) \# Cut high AP (reaction) singles.

high AP recoil singles are already cut from the main loop \# Add in recoil events and reaction singles.

counts $+=$ multCounts (datum [1][1], eff)

counts [1] $+=$ np.sum(eff $*$ datum[1][0]) + np.sum(eff $*$ datum

$[2][0]) *(1-\operatorname{datum}[0][9])$

counts $*=$ datum[0][6] / datum[3][0] \# * livetime /

simulated_time

exp_rate $[0]+=$ np.array $(($ datum $[0][2]$, counts $[1] *$ ambe_eff + datum [0][7] * datum[0][6], np.sqrt ( (counts [1] * ambe_eff $*$ datum [3][1]) $* * 2+(\operatorname{datum}[0][8] * \operatorname{datum}[0][6]) * * 2)))$

counts $*=$ datum[0][2] / (counts[1] * ambe_eff + datum[0][7]* datum[0][6]) \# Take multiplicity ratio

exp_rate $[0]+=$ np. array $(($ datum $[0][3]$, counts [2], counts [2] * math.sqrt $(0.03 * * 2+1 /(\operatorname{datum}[0][2]+1))))$ \# Doubles

exp_rate $[1]+=$ np. array $(($ datum $[0][4]$, counts $[3]$, counts $[3] *$ math. $\operatorname{sqrt}(0.06 * * 2+1 /(\operatorname{datum}[0][2]+1))))$ \# Triples

exp_rate [2] += np.array ((datum[0][5], np.sum(counts [4:]), np. $\operatorname{sum}(\operatorname{counts}[4:]) *$ math.sqrt $(0.09 * * 2+1 /(\operatorname{datum}[0][2]+1))))$ $\#>=4$

\# Update prob with multiples. Combine norm and poisson statistics.

$\mathrm{x}=$ norm.ppf(np.arange $(0.005,1.0,0.01))$

$\mathrm{c}=\mathrm{np}$.floor $(\exp$ _rate $[:, 0]+0.5)[:, \mathrm{np}$.newaxis] \# Guard against float precision error

$\mathrm{mu}=\mathrm{np} \cdot \operatorname{maximum}\left(\exp \_\right.$rate $[:, 1, \mathrm{np}$. newaxis $]+$ exp_rate $[:, 2, \mathrm{np}$. newaxis $] * \mathrm{x}[\mathrm{np}$. newaxis,$:], 0.01)$

prob $*=n p \cdot \operatorname{prod}\left(n p \cdot \operatorname{maximum}\left(n p \cdot \operatorname{sum}\left(\operatorname{cdf} \_f c n(c, m u)\right.\right.\right.$, axis $\left.=1\right) / x \cdot$ shape $[0], 1 \mathrm{e}-100))$

$\exp _{\text {rate }}=\mathrm{np} . \mathrm{zeros}((2, \mathrm{x}$. shape $[0]))$

for data_set in data [1]:

for datum in data_set[0]:

counts $=$ np. $\operatorname{sum}(\operatorname{mult}$ Counts $($ datum [1][0][1], eff $)[1:])+n p$.

$\operatorname{sum}(\operatorname{datum}[1][0][0] *$ eff $)$

counts $*=$ datum[0][4] / data_set [1][0]

dcounts $=$ np.sum $(\operatorname{multCounts}(\bar{d} \operatorname{atum}[1][1][1]$, eff $)[1:])+n p$. 


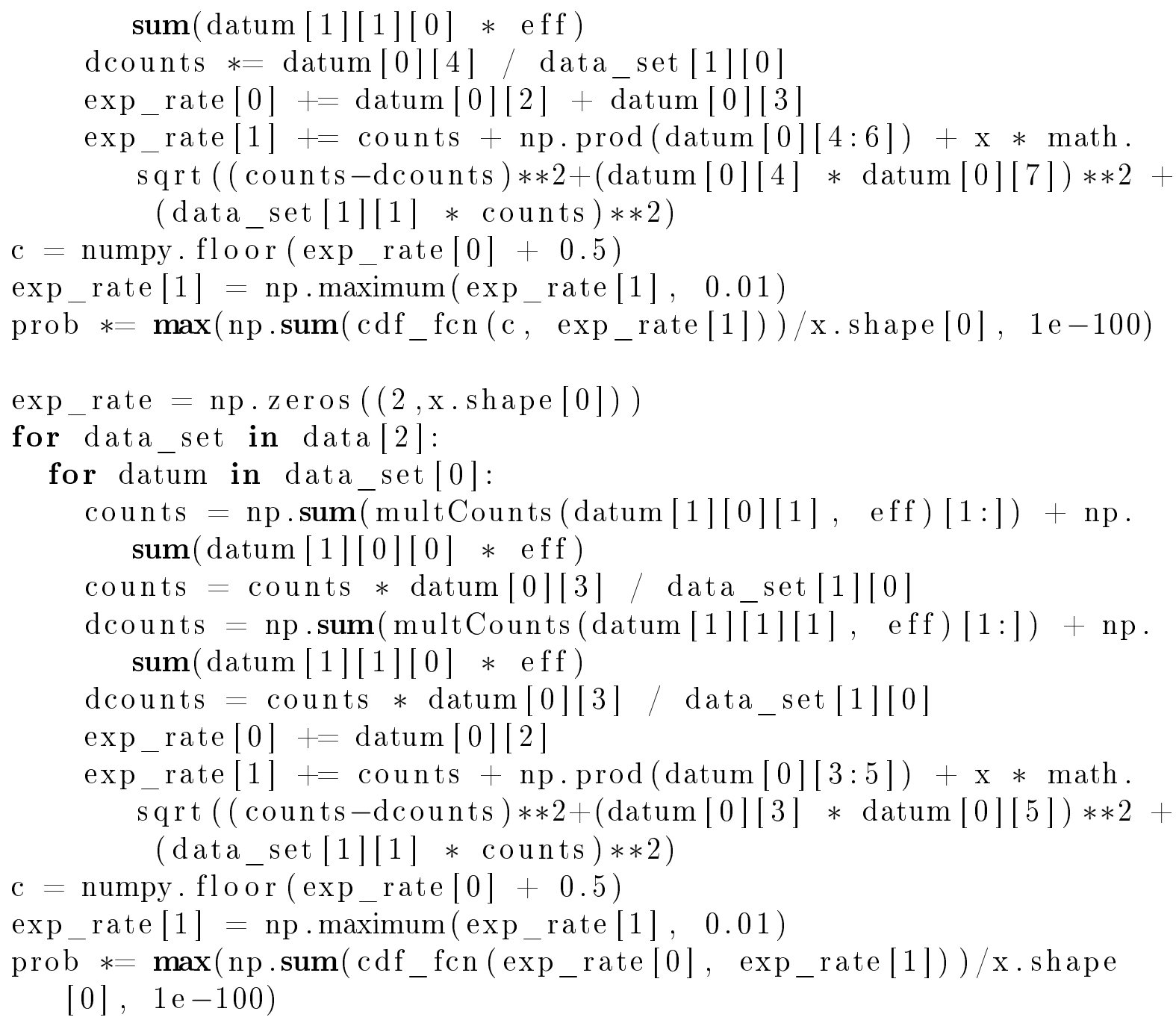


return 0.0

return prob - fit_prob

def prob_min_lower(cur_eff, data, max_eff, min_eff, Er, fit_prob): eff $=n p . \operatorname{copy}($ max_eff $)$

eff $[: \operatorname{Er}+1,: 2]=n p . \operatorname{minimum}\left(\operatorname{cur}_{-}\right.$eff, $\left.\operatorname{eff}[: \operatorname{Er}+1,: 2]\right)$

$\min \_$bins $=n p .1$ ess $\left(\operatorname{eff}[:, 0], \max \left(\min \_\right.\right.$eff $\left.\left.[\operatorname{Er}, 0], \min _{-} \operatorname{eff}[\operatorname{Er}-1,0]\right)\right)$

if $\mathrm{np}$.any $\left(\min \_\right.$bins $):$min_bins $\left[\min \_\right.$bins $][-1]=$ False

eff $[$ min_bins,$: 2]=\min \_$eff $\left[\min \_\right.$bins,$\left.: 2\right]$

prob $=$ math. $\log ($ calc_prob $($ eff, data, upper $=$ False $))$

if $c u r \_$eff $<\max \left(\min \_\right.$eff $[\mathrm{Er}, 0], \min \_$eff $\left.[\mathrm{Er}-1,0]\right)+0.00001$ and

prob > fit_prob:

return 0.0

if $c u r \_$eff $>$max_eff $[\mathrm{Er}, 0]-0.00001$ and prob $<$ fit_prob:

return 0.0

return prob - fit_prob

if __name__ $=$ ' main__, :

\# . dat files formated with columns

\# (Threshold sig Singles Doubles Livetime Singles_BG Doubles_BG Singles_BG_sig Doubles_BG_sig)

\# last 3 elements of each tuple below are the

Simulation_filename, simulation_time(sec), z_position_cut

data_ANL $=(($ np.loadtxt ('Data/ANL_37C.dat', skiprows $=2), \quad$ 'Sim/ anl_octfull.d', (0.1539, 0.087), (0.55,3.8)),

( $\overline{n p}$. loadtxt ('Data/ANL.dat', skiprows=2), 'Sim/anl_eoctfull. $\left.\left.\mathrm{d}^{\prime},(0.1497,0.087),(0.55,3.8)\right)\right)$

data_AmBe $=$ np. loadtxt ('Data/COUPP4_AmBe.dat', skiprows=2)

data_Cf $=$ np.loadtxt ('Data/COUPP4_Cf.dat', skiprows=2, ndmin $=2$ )

data_UC $=\left(\left(\right.\right.$ np.loadtxt $\left(' D a t a / U C \_37 C . d a t '\right.$, skiprows $\left.=2\right), '$ Sim $/$

ybe_115.d', (35.6, 0.111), (-999.9,999.9) ), \

(np.loadtxt ('Data/UC_39C.dat', skiprows=2), 'Sim/ybe_115.d $,(37.7,0.111),(-999.9,999.9)), \backslash$

(np.loadtxt ('Data/UC_39C_s.dat', skiprows=2), 'Sim/ybe_116 $\left.. \mathrm{d}^{\prime},(31.3,0.111),(-999.9,999.9)\right)$,

(np.loadtxt ('Data/CYRTE_cfi_37C.dat', skiprows=2), 'Sim/ cyrte_cfi.d', $(5.01, \overline{0} .12),(-999.9,-10.0)))$

singles_cutoff $=0.56$ 
\# Set data structure, load simulations.

data $=[[],[],[]]$

for datum in data_AmBe:

sim_recoil $=$ loadSim('Sim $/ 4 \mathrm{k} 402$ _recoil.d', thr_mult $=1000.0 /$ datum $[0])$

if datum [9]:

sim_recoil [0][np.searchsorted (thrBins, singles_cutoff * $1000.0 /$ datum $[0]):]=0$.

data $[0]$. append ( (datum, sim_recoil, loadSim ('Sim $/ 4$

$\mathrm{k} 402$ reaction_c.d', thr_mult $=1000.0 /$ datum $[0]),(68.73$, $0.3))$ )

for datum in data_Cf:

sim_recoil $=$ loadSim('Sim $/ 4 \mathrm{k} 400 \_$recoil.d', thr_mult $=1000.0 /$ datum [0])

if datum [9]:

sim_recoil [0][np.searchsorted (thrBins, singles_cutoff * $1000.0 /$ datum $[0]):]=0$.

data $[0]$. append ((datum, sim_recoil, loadSim ('Sim $/ 4$ k400_reaction_c.d', thr_mult $=1000.0 /$ datum $[0]),(2590$, $0.2))$ )

for datum in data_ANL:

datum_b $=[]$

for point in datum [0]:

datum_b.append ( (point, [loadSim (datum [1], thr_mult $=1000.0$ $/($ point $[0]+$ point $[1] * \mathrm{x}), \mathrm{z}_{-}$cut $\left.=\operatorname{datum}[3]\right)$ for $\mathrm{x}$ in $(0.0,-1.0)]))$

\#print point [0], datum[1]

if datum b:

data $[1]$. append ( (datum_b, datum [2]))

for datum in data_UC:

datum_c $=[]$

for point in datum [0]:

datum_c.append ( (point, [loadSim(datum [1], thr_mult $=1000.0$

$/($ point $[0]+$ point $\left.[1] * \mathrm{x}), \mathrm{z}_{\mathbf{c}} \mathrm{cut}=\operatorname{datum}[3]\right)$ for $\mathrm{x}$ in $(0.0,-1.0)]))$

if datum_c:

$\operatorname{data}[2]$. append ((datum_c, datum [2]))

test_eff $=$ np.zeros $(($ thrBins.shape $[0]-1,4))$

test_eff $[:, 2:]=1.0$

test_eff $[11:]=1.0$

test_eff $[4: 11,: 2]=$ np.arange $(0,1,0.15625)[:$, np.newaxis $]$ 
prob1 $=$ math. $\log ($ calc_prob $($ test_eff, data, upper $=$ False $))$

prob2 $=$ math. $\log ($ calc_prob $($ test_eff, data $))$

fit_probs $=[\min (\operatorname{prob} 1, \operatorname{prob} 2), \overline{ }$

fit_prob $=\max ($ prob1, prob2)

outfile $=\operatorname{open}($ 'fit_all.dat', 'a')

fail_probs $=[0$,

\# Execute a binary search for the maximum likelihood

while True:

max_eff $=$ np.ones $(($ thrBins.shape $[0]-1,4))$

min_eff $=$ np.zeros $((\operatorname{thrBins}$. shape $[0]-1,4))$

min_eff $[:, 2:]=1.0 \# 100 \%$ iodine and reaction efficiency

\# Iterate fit a fixed number of times

for main_fit_iter in range (3):

print time.clock () - start, main_fit_iter, fit_prob,

fit_probs, fail_probs

\# Fit upper limit efficiency

for $\operatorname{Er}$ in range(thrBins. shape $[0]-3,-1,-1)$ :

max_eff $[\operatorname{Er},: 2]=$ brenth $\left(\right.$ prob_min_upper, min $\left(\max \_\right.$eff $[\operatorname{Er}, 0]$, max_eff $[\operatorname{Er}+1,0])$, min_eff $[\operatorname{Er}, 0], \quad$ args $=($ data, max_eff, min_eff, Er, fit_prob), xtol $=0.00001$, rtol $=0.01$, maxiter $=20)$

if $\mathrm{np} \cdot \operatorname{amax}\left(\max _{-} \operatorname{eff}[:, 0]-\right.$ min_eff $\left.[:, 0]\right)<0.01$ :

fail_probs.append (fit_prob)

break

for $\operatorname{Er}$ in range(1, thrBins. shape[0]-1):

$\min \_$eff $[\mathrm{Er},: 2]=$ brenth $\left(\right.$ prob_min_lower, $\max \left(\min \_\right.$eff $[\operatorname{Er}, 0]$, min_eff $[\operatorname{Er}-1,0]), \max _{\text {eff }}[\mathrm{Er}, 0], \operatorname{args}=($ data, max_eff, min_eff, Er, fit_prob), xtol $=0.00001$, rtol $=0.01$, maxiter $=20$ )

if $\mathrm{np} \cdot \operatorname{amax}\left(\max _{-}\right.$eff $[:, 0]-$ min_eff $\left.[:, 0]\right)<0.01$ :

fail_probs.append (fit_prob)

\section{break}

if main_fit_iter $=2$ :

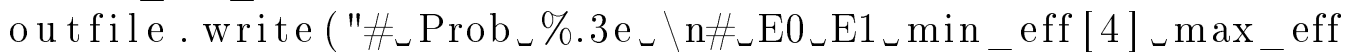

$[4] \backslash \mathrm{n} " \%$ fit_prob)

np. savetxt (outfile, np. concatenate ( ( thrBins [: -1 , np. newaxis

], thrBins [1:,np.newaxis ], min_eff, max_eff ), axis =1))

if fit_prob fail_probs $[-1]$ :

fit_prob $=($ fit_prob $+\max ($ fit_probs $)) / 2.0$ 
else :

fit_probs . append (fit_prob)

fit_prob $=(\max ($ fit_probs $)+\min ($ fail_probs $)) / 2.0$

if (fit_prob $-\max ($ fit_probs $))<\operatorname{math} . \overline{l o g}(1.03)$ :

break

base_prob $=$ fit_prob

\# Find 1 and 2 sigma bounds

for fit_iter in range(2):

fit_prob $=$ base_prob $-0.5-1.5 *$ fit_iter

max_eff $=$ np.ones $(($ thrBins.shape $[0]-1,4))$

min_eff $=$ np.zeros $(($ thrBins.shape [0]-1, 4) $)$

min_eff $[:, 2:]=1.0 \# 100 \%$ iodine and reaction efficiency

\# Iterate fit a fixed number of times

for main_fit_iter in range(3):

print time.clock () - start, main_fit_iter, fit_prob

\# Fit upper limit efficiency

for $\operatorname{Er}$ in range(thrBins. shape $[0]-3,-1,-1)$ :

max_eff $[\operatorname{Er},: 2]=$ brenth (prob_min_upper, min $\left(\max \_\right.$eff $[\operatorname{Er}, 0]$, max_eff $[\mathrm{Er}+1,0])$, min_eff $[\mathrm{Er}, 0], \operatorname{args}=($ data, max_eff, min_eff, Er, fit_prob), xtol $=0.00001$, rtol $=0.01$, maxiter $=20$ )

for $\operatorname{Er}$ in range(1, thrBins.shape[0]-1):

min_eff $[$ Er,$: 2]=$ brenth $\left(\right.$ prob_min_lower, $\max \left(\min \_\right.$eff $[\operatorname{Er}, 0]$,

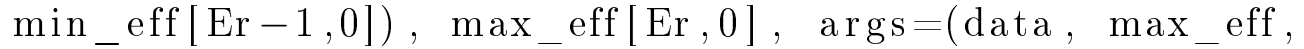
min_eff, Er, fit_prob), xtol=0.00001, rtol $=0.01$, maxiter $=20$ )

if main_fit_iter $=2$ :

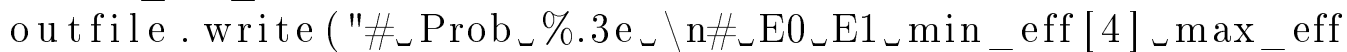
$[4] \backslash \mathrm{n} " \%$ fit_prob)

np. savetxt (outfile, np. concatenate ( (thrBins [: -1, np. newaxis ], thrBins [1:,np.newaxis ], min_eff, max_eff $)$, axis $=1$ ))

outfile.close () 


\section{Appendix D MCNPX-Polimi Input Files}

\section{D.1 PICO-2L 4b Geometry}

4b27a.i - U-238 neutrons in the titanium of the retroreflector

c $: \because$ cell cards

c cel\# mat\# density definition w.r.t. surfaces

$c$ spaces define the intersection of volumes, colons define unions,

$c$ \# defines other cells to exclude

c C3F8 (2040 cc, 2.90 kg)

$11-1.3781(-12-5):(-2-4):(5-6-3)$

c water

$22-1.00(3-6-7):(7 \quad 8-9-10):(10-11-19): \&$

$(((-2119-22):(22-222-27):(222-23-21):(23-31-26)) \&$

$(-37: 36:-35)):((-35:-38) 31-33)$

c quartz jar

$33-2.203\left(\begin{array}{llll}1 & -12 & -5\end{array}\right):(-24-13):(5-7 \quad 6-14):(7-10-815-9)$

$43-2.203(10-17 \quad 11-16):\left(\begin{array}{lllll}17 & -19 & 11 & -18\end{array}\right)$

c steel flanges below bellows

55 -7.00 (10 -17 16 -20):(17 -19 18 -20):(21 -20 19 -22)

c steel-water-glycol mix for large bellows

$66-3.15(22-222 \quad 27-28)$

c Mid-bellows flanges

75 -8.00 ((222 -23 $21-20):(23-2426-20):(24-2526-21)) 616263$

c steel-water-glycol mix for small bellows

$86-1.27(26-2925-30)$

$c$ steel flanges above bellows

$95-8.00(26-2130-31):(31-34-333538(-32:-21))$

c Fireplug top

107 -8.00 (-34:-44) $40-41$ (42:-43 44:-943:-45) (46:849) 47

c Fireplug mid-section

117 -8.00 -47 (849:949) (-848 -948:-850 -60) (851:60:-52) 67 (53:52) 55

c Fireplug bottom

$127-8.0070(-6768-69:(-71-72:-73-74) 75)-948$

c Window

$133-2.203-53-5455$

c Window Retaining Flange

147 -8.00 -55 -956 5657 (58:-59:-64:65) 66

c $P V$ legs 
$157-8.0076-8182-87131-89(-77: 80:-83: 86)(-78: 79)(-84: 85) 948$

c Fill tube

$167-8.00(35-3637-31)$

c IV guide rods

175 -8.00 22 -31 (-61:-62:-63)

c Camera Mounts

$185-8.0090-9192-93-6694: 95-96(97-98: 99-100) 91-101$

c Cameras

$1911-1.3395-96-101102(98-103: 104-99)$

c Lenses

$2014-0.930-105(-106:-107) 96$

c mineral oil

$2191-0.856$ (75 -70:-68) -67:(-849 -949-46:-851 $52-60: 54-52-53:-40) \&$

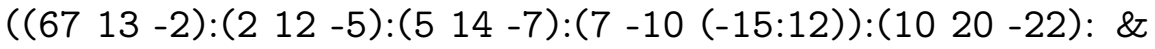

$(616263((22-222$ 28):(222 -24 20):(24 -25 21):(25 -30 29): \&

$(30-3121))))$

c Insulation

$2215-0.0269-110-111(112: 34) 45$ 47:117 -116 -115: \&

(-47 (848:948) (-114:-115 -60 116)(850:60) 118 (956:-66:55): \&

$119-118-11472) \# 15$

c PTFE Gasket

$2310-1.441-34-3140$

c polyethylene above plate

$249-0.925131-132135-136137-138(-210:-213-214: 211:-215) \&$

$(-76: 78-79: 81:-82: 84-85: 87)$

$c$ steel plate

255 -8.00 $130-131135-136137-138$ (-210:-213:211)

c polyethylene base below plate

$319-0.925(-130140135-136137-138(-210:-213: 211)): \&$

(-140 $141142-143144-145$ (-210:-213:211:-212))

c polyethylene water shields

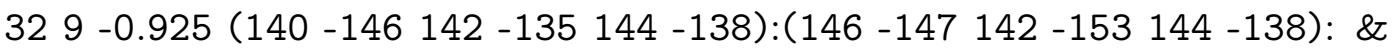
$\left(\begin{array}{lllllllllll}146 & -147 & 154 & -135 & 144 & -138):(146 & -147 & 153 & -154 & 144 & -159): \&\end{array}\right.$

(146 -147 $153-154162-138):\left(\begin{array}{lllllll}147 & -148 & 142 & -135 & 144 & -138): \&\end{array}\right.$

(139 -152 $135-169137-167)$

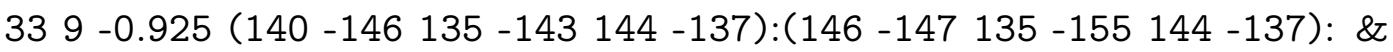
(146 -147 $155-158144-159):\left(\begin{array}{lllllll}146 & -147 & 155 & -158 & 160 & -137): \&\end{array}\right.$

(146 -147 $158-143144-137):\left(\begin{array}{lllllll}147 & -148 & 135 & -143 & 144 & -137): \&\end{array}\right.$

(139 -152 $166-136137-171)$

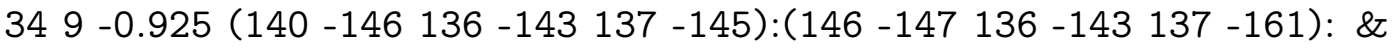
(146 -147 $136-157161-164):\left(\begin{array}{lllllll}146 & -147 & 158 & -143 & 161 & -164): \&\end{array}\right.$

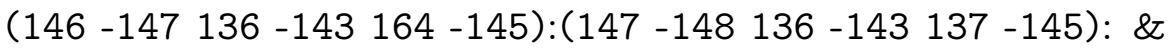




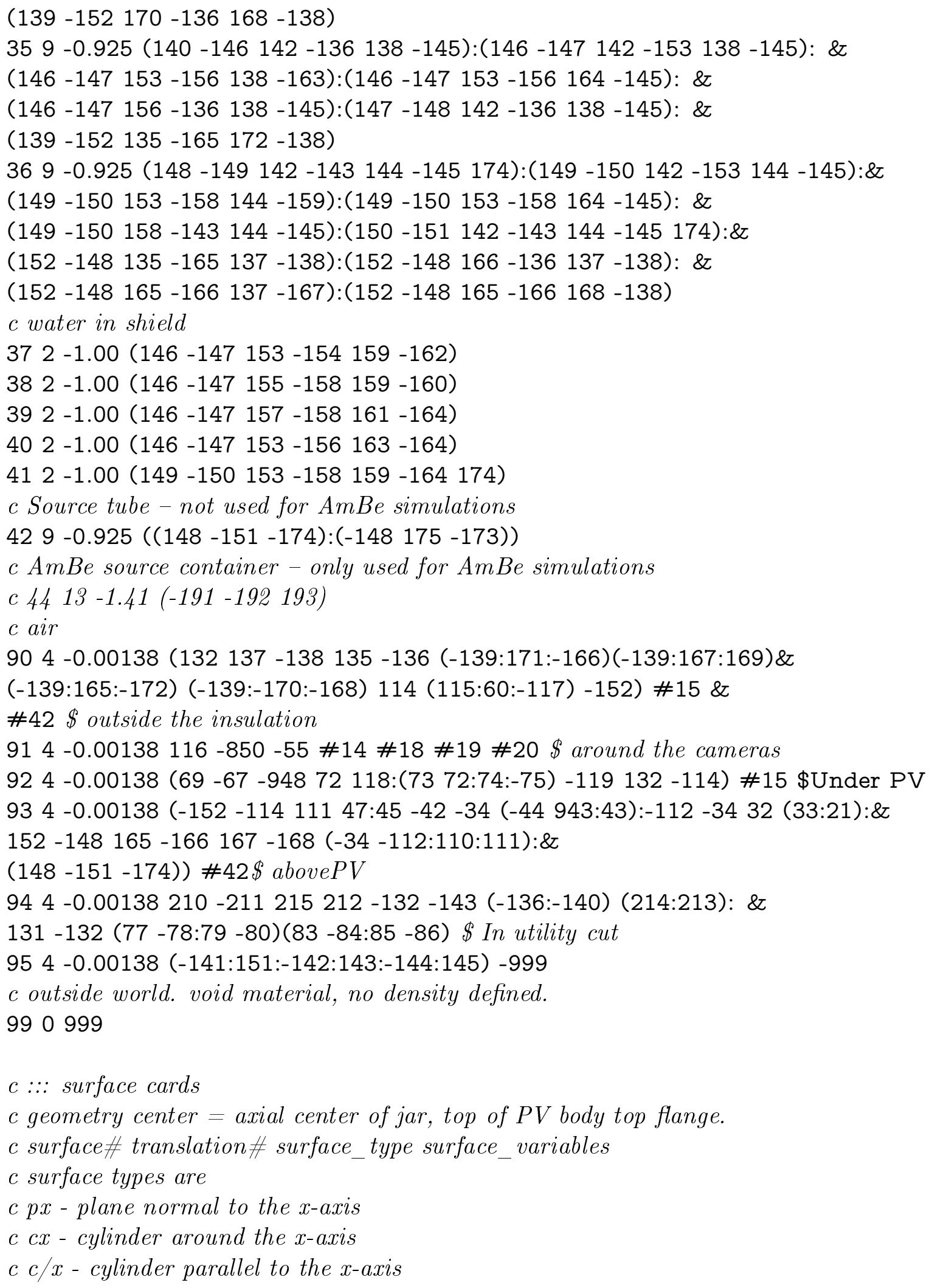


c sx-sphere centered on the $x$-axis

$c$ tx - toroid rotated around the $x$-axis

$c k x$ - cone rotated around the $x$-axis

$c k / x$ - cone parallel to the $x$-axis

c $g q$ - an arbetrary conic section

c sq-an arbetrary ellipsoid

c $C 3 F 8$

$1 \mathrm{cz} 7.25$

$23 \mathrm{pz}-50.84$

$33 \mathrm{pz}-42.933$

$43 \mathrm{sz}-50.847 .25$

c water

$53 \mathrm{pz}-43.22$

$63 \mathrm{sz}-43.227 .25$

$73 \mathrm{pz}-39.02$

83 tz $000-35.0312 .507 .757 .75$

$9 \mathrm{cz} 12.50$

$103 \mathrm{pz}-35.03$

$11 \mathrm{cz} 4.50$

$c$ quartz

$12 \mathrm{cz} 7.50$

$133 \mathrm{sz}-50.847 .50$

$143 \mathrm{sz}-43.227 .50$

$153 \mathrm{tz} 00-35.0312 .507 .507 .50$

$16 \mathrm{cz} 5.00$

$173 \mathrm{pz}-33.13$

$18 \mathrm{cz} 6.20$

$193 \mathrm{pz}-30.13$

$c$ steel bellows and flanges

$20 \mathrm{cz} 7.50$

$21 \mathrm{cz} 5.00$

$223 \mathrm{pz}-29.06$

$2223 \mathrm{pz}-18.65$

$233 \mathrm{pz}-17.38$

$243 \mathrm{pz}-16.11$

$253 \mathrm{pz}-14.84$

$26 \mathrm{cz} 2.50$

c steel bellows convolutions

$27 \mathrm{cz} 3.89$

$28 \mathrm{cz} 6.10$

$29 \mathrm{cz} 3.11$ \$ small bellows OD

c top flange 
$30 \mathrm{pz}-1.27$

$31 \mathrm{pz} 0.30$

$32 \mathrm{pz} 3.96$ \$ ordered w.r.t rods (surf 56) in cell 22

$33 \mathrm{pz} 5.23$

$34 \mathrm{Cz} 15.88$

c fill tube

$35 \mathrm{c} / \mathrm{z} 1.600 .51$

$36 \mathrm{c} / \mathrm{z} 1.600 .635$

$37 \mathrm{pz}-27.46$

$38 \mathrm{c} / \mathrm{z}-2.200 .51$

c $P V$ top flange

$40 \mathrm{cz} 7.70$

$412 \mathrm{pz} 0$

$422 \mathrm{pz}-3.66$

$43 \mathrm{cz} 10.31$

$943 \mathrm{cz} 8.5$

$442 \mathrm{pz}-4.92$

$452 \mathrm{kz} 5.78340 .36-1$

$462 \mathrm{kz} 4.06670 .36-1$

$472 \mathrm{pz}-21.2$

$48 \mathrm{cz} 16.19$

$49 \mathrm{cz} 15.16$

$c$ Cones defining the pressure vessel tee.

9482 gq $110000.032 .13360-0.50856-298.2268228$

8482 gq $11000-0.03-0.609600 .50856-250.6789372$

9492 gq $110000.032 .133600 .0 .47766-263.7387148$

8492 gq $11000-0.03-0.609600 .47766-217.6035772$

c $P V$ window area

$502 \mathrm{c} / \mathrm{x} 0-45.7216 .19$

$512 \mathrm{c} / \mathrm{x} 0-45.7215 .16$

$8502 \mathrm{k} / \mathrm{x}-1120.410-45.720 .0002184$

$8512 \mathrm{k} / \mathrm{x}-1050.710-45.720 .0002184$

$522 \mathrm{px}-18.54$

$532 \mathrm{c} / \mathrm{x} 0-48.268 .89$

$542 \mathrm{px}-19.66$

$552 \mathrm{px}-24.89$

$9562 \mathrm{c} / \mathrm{x} 0-48.2613 .91$

$562 \mathrm{c} / \mathrm{x}-3.175-48.265 .08$

$572 \mathrm{c} / \mathrm{x} 3.175-48.265 .08$

$582 \mathrm{pz}-43.18$

$592 \mathrm{pz}-53.34$

$602 \mathrm{px} 0$ 
642 py -3.175

652 py 3.175

$662 \mathrm{px}-28.22$

$c$ Rods

$61 \mathrm{c} / \mathrm{z} 2.8936 .2050 .635$

$62 c / z \quad 3.927-5.6080 .635$

$63 \mathrm{c} / \mathrm{z}-6.8210 .5970 .635$

c PV bottom cap and flange

$672 \mathrm{pz}-76.72 \$-71.12$

682 sq $113.208000-229.8256000-76.72$

$692 \mathrm{sq} 112.908000-262.116100-76.72$

$70 \mathrm{cz} 2.63$

$71 \mathrm{pz}-85.19$

$722 \mathrm{kz}-74.350 .06171-1$

$732 \mathrm{pz}-90.86$

$74 \mathrm{cz} 8.25$

$752 \mathrm{pz}-95.34$ \$ Includes both flanges.

c PV legs

$762 \mathrm{px}-14.35$

$772 \mathrm{px}-13.71$

$782 \mathrm{px}-8.63$

$792 \mathrm{px} 8.63$

802 px 13.71

$812 \mathrm{px} 14.35$

822 py -14.35

832 py -13.71

842 py -8.63

852 py 8.63

862 py 13.71

872 py 14.35

$882 \mathrm{pz}-110.50$ \$ Not used. should match 131 using tr1

$892 \mathrm{pz}-66.34$

c Cameras, mounts, and LEDs

$902 \mathrm{pz}-53.98$

$912 \mathrm{pz}-53.34$

922 py -8.26

932 py 8.26

$942 \mathrm{px}-37.11$

$952 \mathrm{px}-36.50$

$962 \mathrm{px}-30.60$

972 py -5.53

982 py -4.9 


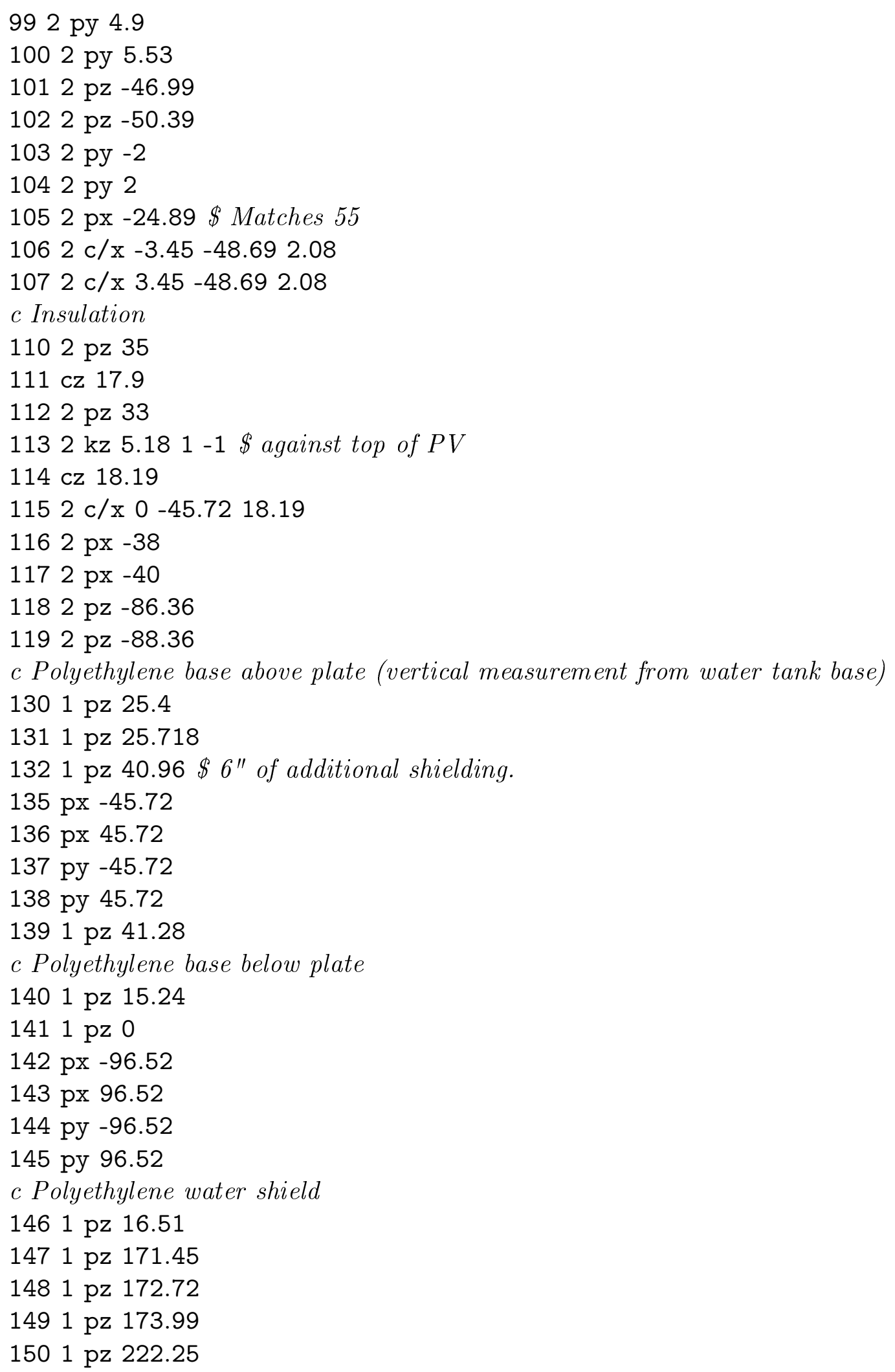




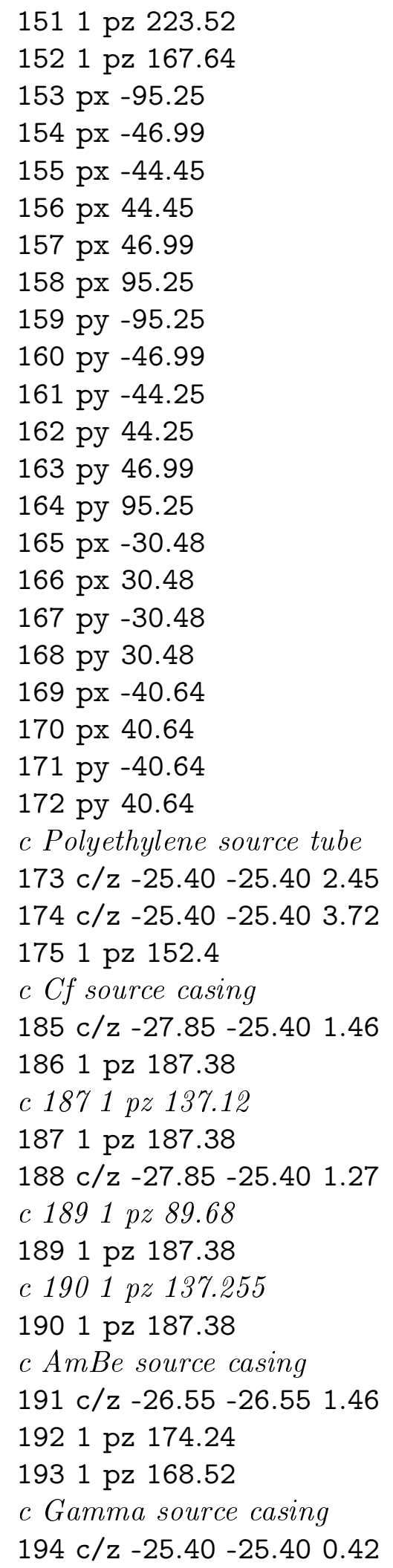


$1951 \mathrm{pz} 83.12$

$1961 \mathrm{pz} 84.02$

$1971 \mathrm{pz} 86.77$

$198 \mathrm{c} / \mathrm{z}-27.85-25.400 .95$

c utility 4 " $x 4$ " cut

210 py -5.08

211 py 5.08

$2121 \mathrm{pz} 5.08$

213 px 35.56

$2141 \mathrm{pz} 30.8$

$215 \mathrm{px}-5.08$

c Norite rock wall

999 sz -24.84 180

c :.: Problem definition

$c * * *$ translation card

tr1 $00-136.378$ \& Height of top flange off ground.

tr2 $000 \$$ Height of $P V$ extender

tr3 $00-1.67 \$$ Adjust height of $I V$.

$c$ *** modes and importances

mode $\mathrm{n}$

imp:n,p 1 42r 0

$c^{* * *}$ material cards

$\mathrm{m} 1$ 6000.70c 3 9019.19c 8 \& $C 3 F 8$

$\mathrm{m} 2$ 1001.70c 2 8016.70c 1 \$ H2O

m3 14028.70c 0.9222314029 .70 c 0.04685 14030.70c $0.030928016 .70 c 2$ \$ SiO2

c Air - Approximately a moist atmosphere

$\mathrm{m} 47014.70 \mathrm{c}-0.77227015 .70 \mathrm{c}-0.0028$ 8016.70c $-0.2105318040 .70 c-0.0128$ \&

1001.70c -0.00137 6000.70c -0.0003

c Steel $-304 \mathrm{~L}$ grade stainless steel

m5 6000.70c - $0.00027014 .70 c-0.0005$ 14028.70c $-0.004515031 .70 c-0.0003 \&$ $22048.70 c-0.00324050 .19 c-0.008124052 .19 c-0.157024053 .70 c-0.0178$ \& $24054.70 c-0.004425055 .70 c-0.01326054 .70 c-0.041026056 .19 c-0.6434 \&$ $26057.70 c-0.014826058 .70 c-0.002027059 .70 c-0.003928058 .70 c-0.0553 \&$ $28060.70 c-0.021328061 .70 c-0.000928062 .70 c-0.003028064 .70 c-0.0008 \&$ $29063.70 c-0.003329065 .70 c-0.0015$

c 73\%/27\% water-steel mix

m6 1001.70c 0.4867 8016.70c 0.2433 24050.19c 0.002 24052.19c 0.042 \&

24053.70c $0.00525055 .70 c 0.00426054 .70 c 0.012$ 26056.19c 0.178 \&

26057.70c 0.00427059 .70 c 0.001 28058.70c 0.015 28060.70c 0.007

c Steel - 316L grade stainless steel

$\mathrm{m} 7$ 6000.70c $-0.00027014 .70 \mathrm{c}-0.0005$ 14028.70c $-0.004515031 .70 c-0.0003$ \& 
$22048.70 c-0.00324050 .19 c-0.007324052 .19 c-0.140324053 .70 c-0.0159 \&$ $24054.70 c-0.004025055 .70 c-0.01326054 .70 c-0.039126056 .19 c-0.6152 \&$ $26057.70 c-0.014226058 .70 c-0.001927059 .70 c-0.003928058 .70 c-0.0688 \&$ $28060.70 c-0.026528061 .70 c-0.011528062 .70 c-0.003728064 .70 c-0.0009 \&$ $29063.70 c-0.003329065 .70 c-0.001542092 .70 c-0.00342094 .70 c-0.0019 \&$ $42095.70 c-0.003242096 .70 c-0.003442097 .70 c-0.00242098 .70 c-0.005 \&$ $42100.70 \mathrm{c}-0.002$

m8 1001.70c 0.62 6000.70c 0.23 8016.70c 0.15 \$ propylene glycol (C3H8O2) m9 1001.70c 2 6000.70c 1 \$ HDPE/Mineral Oil m91 1001.70c-0.1375 6000.70c-0.8395 \$ Mineral oil - degassed m10 6000.70c 1 9019.19c 2 \& PTFE c 70\% Al, 15\% SiO2 15\% plastic for cameras $\mathrm{m} 11$ 1001.70c $0.16000 .70 \mathrm{c} 0.05$ 8016.70c $0.1013027 .70 \mathrm{c} 0.7$ \& 14028.70c 0.0458 14029.70c $0.002514030 .70 \mathrm{c} 0.0017$ m12 1001.70c 8 6000.70c 5 8016.70c 2 \$ PMMA acrylic (C5H8O2) m13 1001.70c 2 6000.70c 1 8016.70c 1 \$ Delrin polyoxymethylene (CH20) c Lenses, 40\% Al 60\% acrylic $\mathrm{m} 14$ 1001.70c 0.32 6000.70c 0.2 8016.70c 0.08 13027.70c 0.4 m15 1001.70c-0.091 6000.70c -0.542 7014.70c -0.040 8016.70c -0.011 \& $13027.70 \mathrm{c}-0.316$ \$ Reflectix insulation $c^{* * *}$ source cards

c Surface source at retroreflector sdef erg $=\mathrm{d} 1 \mathrm{axs}=001 \mathrm{rad}=15.14 \mathrm{ext}=\mathrm{d} 3$ c U-238 in $\mathrm{Ti}$ si1 H $0.0010 .51 .01 .52 .02 .53 .03 .54 .04 .55 .05 .56 .06 .57 .0 \&$ 7.58 .08 .59 .09 .510 .010 .511 .011 .512 .0 $\begin{array}{llllllllllllll}\text { sp1 } & 0 & 0.133 & 0.1932 & 0.205 & 0.1342 & 0.1171 & 0.0825 & 0.0543 & 0.0336 & 0.0192 & 9.4 \mathrm{E}-3 & \&\end{array}$ 5.1E-3 2.81E-3 1.59E-3 8.86E-4 4.64E-4 2.82E-4 1.66E-4 8.17E-5 4.39E-5 \& $2.51 \mathrm{E}-5$ 1.43E-5 8.07E-6 4.55E-6 2.55E-6 si2 5.216 .19 si3 H $-78.39-25.05$ sp3 D 01 $c^{* * *}$ tally specifications

c Reaction flux tally, over the C3F8 volume.

f4:n 1

fm4 -1 1 (22) (28) (107)

$c$ *** run specifications

phys:n j 20

cut:n j 0.001

nps $1 \mathrm{e} 7$

$c^{* * *}$ PoliMi specifications

ipol $000002 \mathrm{j} 11$ 
rpol 0 100. j 1

files 21 dumn 1

$c$ random number seed

dbcn 18405

\section{D.2 STAR runs 20111010_1-20111011_2}

c Model of the STAR bubble chamber at Argonne

c by original by Alan Robinson, Sept 20, 2010

c Updated geometry as-built Sept 12, 2011

c Geometry for oct13_2 source position

c New Libraries, Apr 9, 2014

c 8 May 2015, redefined source.

$c$ Volumes

$11-1.967-14:(-813-10)$ \& $13 F 3 I$

c Hydraulic Fluid (water) and pipes (steel)

$22-1.010-8-7$ \$ near interface

$32-1.07-1-2 \$$ above chamber

$42-1.0-5355-56 \$$ Horizontal tube

$52-1.0-57-5830 \$$ Vertical tube

$64-7.874-32-1$ \$above chamber

$74-7.8753-5455-56$ \$ horizontal tube

$84-7.87-5758-5930$ \$ vertical tube

$c^{*}$

$93-2.2(-1314-15):(138-9-6):(7 \quad 3-5-4)$ \$ Glass vessel

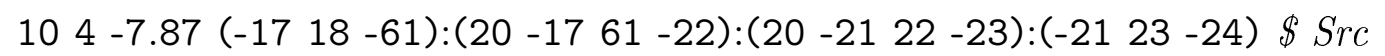

$1110-2.85(-6061-62):(63-6462-66) \mathrm{fcl}: \mathrm{p}=1 \$ B e O$

$128-1.37$ (21 $26-25-28)$ \& PVC Source Holder

$134-7.87-2931-33$ (28:-30:32) \$ Containment Vessel

$145-2.22-37-4 \$$ Teflon connector

$159-1.5-501551-52$ \$ Epoxy (and piezo)

$167-2.7040-41-4330((45-47):(44-46))$ \$ Diffuser Hld

$173-2.241-42-4330$ (-46:-47) \$ Glass Diffuser

$1811-2.471-70-72$ \$ Cement Wall

$199-1.5-6362-66$ \$ Epoxy in source.

c Air

$906-0.0012-2830-32-4342 \$$ Back of chamber

$916-0.0012-2830-32439$ \#10 \#11 \#12 \#4 \#5 \#7 \#8 \#19 \$ Front of Chamber

$926-0.001230-32-4340-42$ \#16 \#17 \$ Around diffusers

$936-0.001230-32-43-409$ \#4 \#5 \#7 \#8 \$ Behind vessel

$946-0.001230-32-9$ \#1 \#2 \#3 \#4 \#6 \#7 \#9 \#14 \#15 \$ Centre 
$956-0.0012-7270-73$ (29:-31:33) \$ outside of chamber $990-71: 72: 73 \$$ Graveyard

c Inner Vessel. All PZ positions are in decending order.

$1 \mathrm{pz} 30.8$

2 cz 0.79

$3 \mathrm{cz} 1.07$

$4 \mathrm{pz} 10.48$

$5 \mathrm{cz} 1.495$

$6 \mathrm{pz} 5.72$

$7 \mathrm{pz} 5.72 \$$ Must be $<=$ to surf 6

$8 \mathrm{cz} 1.495 \$$ Must be $<=$ to surf 5

$9 \mathrm{cz} 1.883$

$10 \mathrm{pz} 4.217$

$13 \mathrm{pz} 0.5$

$14 \mathrm{sz} 0.51 .495 \$$ Match 13 and 8

15 sz $0.51 .883 \$$ Match 13 and 9

$c \mathrm{BeO}$

$601 \mathrm{CX} 1.335$

611 PX 0.17 \$ Bottom of Steel cup

621 PX 0.82 \$ Top of BeO wafers.

$631 \mathrm{CX} 0.685$

$641 \mathrm{CX} 1.36$

661 PX 3.99

$c$ Steel

$171 \mathrm{CX} 1.53$

$181 \mathrm{PX} 0 \$$ Add 1.16 to SDEF

$201 \mathrm{CX} 1.365$

$211 \mathrm{CX} 3.49$

221 PX 3.9

$231 \mathrm{PX} 5.17$

241 PX 6.44

c Source Holder

$251 \mathrm{cx} 4.45$

$261 \mathrm{px} 3.32$

c Diffusers

$40 \mathrm{cz} 13.6$

$41 \mathrm{cz} 14.3$

$42 \mathrm{cz} 15$

$43 \mathrm{px} 0$

44 gq $0.50 .51-100000-53.3 \$$ Cylinder on $x=-y$ axis

45 gq $0.50 .51100000-53.3 \$$ Cylinder on $x=y$ axis 
46 gq $0.50 .51-10000000-90.7$

47 gq $0.50 .5111000000-90.7$

$c$ Containment Vessel

$28 \mathrm{cz} 25$

$29 \mathrm{cz} 27$

$30 \mathrm{pz}-8.9$

$31 \mathrm{pz}-10.8$

$32 \mathrm{pz} 36.8$

$33 \mathrm{pz} 37.7$

c Plumbing

$53 \mathrm{C} / \mathrm{Y} 015.20 .856$

$54 \mathrm{C} / \mathrm{Y} 015.21 .067$

55 PY 1.4

56 PY 11.9

$57 \mathrm{PZ} 14.1$

$58 \mathrm{C} / Z 012.40 .856$

$59 \mathrm{C} / \mathrm{Z} 012.41 .067$

c Piezos

$50 \mathrm{KZ}-0.90 .27-1$

$51 \mathrm{PZ}-5.21$

$52 \mathrm{CZ} 0.95$

c Concrete

$\begin{array}{lllllllllllll}70 & \mathrm{P} & 1 & 0 & -75\end{array}$

$\begin{array}{lllllll}71 & \mathrm{P} & 1 & 1 & 0 & -154\end{array}$

72 GQ $0.50 .51-1000000-22500$

73 P 11040

mode $\mathrm{n} \mathrm{p}$

$\operatorname{tr} 14.785-0.0320 .716$

$\mathrm{m} 160000.290190 .6531270 .2 \$ C F 3 I$

$\mathrm{mx} 1: \mathrm{p} 000$

m2 10010.666780160 .3333 \& Water

$\mathrm{mx} 2: \mathrm{p} 00$

m3 $5010-0.0085011-0.0328016-0.54111023-0.0314028-0.348$ \&

14029 -0.016 $14030-0.01313027-0.012$ \$ Type I Class A Borosilicate Glass

$\mathrm{mx} 3: \mathrm{p} 07 \mathrm{r}$

c Steel

c 316L grade stainless steel

m4 6000.70c $-0.00027014 .70 c-0.000514028 .19 c-0.004515031 .70 c-0.0003$ \& $22048.70 c-0.00324050 .19 c-0.007324052 .19 c-0.140324053 .70 c-0.0159 \&$ $24054.70 c-0.004025055 .70 c-0.01326054 .70 c-0.039126056 .19 c-0.6152 \&$ $26057.70 c-0.014226058 .70 c-0.001927059 .70 c-0.003928058 .70 c-0.0688 \&$ 
$28060.70 c-0.026528061 .70 c-0.011528062 .70 c-0.003728064 .70 c-0.0009 \&$ $29063.70 c-0.003329065 .70 c-0.001542092 .70 c-0.00342094 .70 c-0.0019 \&$ $42095.70 c-0.003242096 .70 c-0.003442097 .70 c-0.00242098 .70 c-0.005 \&$ $42100.70 c-0.002$

$\mathrm{mx4}$ :p $028 \mathrm{r}$

m5 60000.33390190 .667 \& PTFE

$\mathrm{mx5}: \mathrm{p} 00$

$c$ Air

c Approximately a moist atmosphere

m6 $7014-0.7758016-0.2105318040-0.01281001-0.001376000-0.0003$

$\mathrm{mx6}$ :p $04 \mathrm{r}$

c Aluminium Alloy 6061

m7 $13027-0.97514028-0.00626056-0.00329063-0.00229065-0.001 \&$ $12024-0.00812025-0.00112026-0.00124052-0.00230000-0.001$

$\mathrm{mx} 7: \mathrm{p} 0$ 9r

m8 $1001460002170350.7576170370 .2424 \$ P V C$

$\mathrm{mx8}: \mathrm{p} 03 \mathrm{r}$

m9 10010.55160000 .34870140 .05880160 .043 \$ Epoxy, approx.

$\mathrm{mx} 9: \mathrm{p} 03 \mathrm{r}$

m10 $40090.580160 .5 \$ B e O$

$\mathrm{mx10:p} 40090$

m11 $1001-0.0038016-0.55313027-0.00314028-0.36214029-0.017 \&$

$14030-0.01316032-0.00120040-0.048$ \$ Concrete

$\mathrm{mx} 11: \mathrm{p} 07 \mathrm{r}$

imp:n $124 \mathrm{r} 0$

imp:p 0 7r 1 2r 06 r $10104 \mathrm{r}$

$c$ Biased photonuclear production.

phys:n 4. 1. 2 j 21.

phys:p j 101

cut:p $2 \mathrm{j} 0$

$c * * * * * * * * * * * * * * * * * * * * * * * * * * * * * * * * * * * * * * * * * * * * * * * * * * * * * * * * * * * * * * * * * * * * * * * * *$

c Monocromatic neutron source at cell 11, weighted by rî gamma flux

$\mathrm{SDEF} E R G=\mathrm{d} 4 \mathrm{PAR}=2 \mathrm{AXS}=100 \mathrm{RAD}=\mathrm{d} 1 \mathrm{POS}=5.945-0.0320 .716$

SI1 00.3

SI4 L 1.8360632 .7343 .2197

SP4 0.9920 .00710 .00007

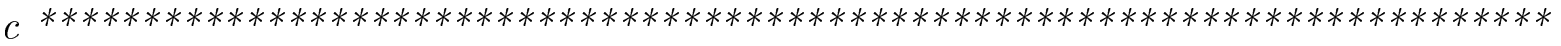

c Neutron flux in CF3I.

f4:n 1

e4 0.0176 13I 0.15760 .9370 .9631 .3631 .401

cut:n j $0.017600 \$$ Kill neutrons with carbon recoils $<5 \mathrm{keV}$

nps $1 \mathrm{e} 7$ 
c Polimi options

ipol 0000 2J 12129101112131415161719

rpol 1e-3 1e10

files 21 DUMN1

\section{D.3 University of Chicago Bubble Chamber, YBe_14C_C3F8}

c Model of the U chicago bubble chamber

c by Alan Robinson, Feb 10, 2012

c updated Mar 20, 2012 - added insulation

c updated May 9, 2012 - fill level for new runs, $15.5 \mathrm{~mL}$

c updated Nov 22, 2013 - New fluorine libraries

c Inner Vessel

$11-1.375(-14-13):(-11-1213) \$ C 3 F 8$

$22-112-11-10 \$$ H2O above interface

$32-1(10-3-7):(7-2-1) \$$ H2O above chamber

$43-2.2(14-15-13):(1311-9-10):(-8103-9): \$$ Glass

$(3-5-48)$

$54-7.872-3-17 \$$ Bushing

c Temperature Bath

$102-1-155-59-646573$ (-72:-56 )\#1 \#2 \#3 \#4 \#5 \$ Water

$117-2.7-50-59((5152-6168):(5868-6176):(5375-6267$

$(-57:-71)))(-54: 63:-66:-74:(5672))$ \$ Aluminum shell

$128-1-15052-6168-59(-55: 56: 64:-65)$ \& Rubber Gasket

$135-2.2-5054-636674-59$ (-55:64:-65:-73) (-72:-56) \$ Teflon

$143-2.259-60-6168-6976$ \$ Glass

$159-1$-77 78 -51 76 -61 68 (62:-75:-67) \$ Temperature control loop

$168-0.2479-53-5175-626780$ \$ Insulation on back of chamber

c Flange and Bellows

204 -7.87 (1 -20 -48 422 )(-45:-46) \$ Center Flange

$214-7.8720-23-2221$ \$ Bellows Flange

$224-7.872321-24-25$ \& Bellows

$234-7.8725-26-22 \$$ Bellows Top Flange

$242-1((-42:-2) 1-20):(20-21-25)$ \$ Water

$254-7.8727-28-48$ \$ Piston Flange

c $Y$-Be source

$3010-2.8523-5381-80 \mathrm{fcl}: \mathrm{p}=1 \$ B e O$

$31 \quad 12-1.18-8183-82 \$ Y-88$

c Support stuct and other nearby mass

$407-1.43-9694$ (-90:-91:-92:-93) \$ Vertical struts

$417-1.35-148-9596 \$$ Top horizontal struts (approx.) 
c Air

906 -0.0012 45 (46:20 )-27 -48 \#21 \#22 \#23 \#24 \$ Above middle flange

$916-0.0012-152-60-616876$ (-79:62:-67:-75: 57 71 ) )\#11 \#12 \#14

\#30 \#31 \#15 \$ surrounding inner volume

926 -0.0012 -1 (-52:60:61:-68:-76 )-99 94 \#30 \#31 \#40 \#41 \$ Below CF

$936-0.0012148-99-28$ \$ outside center flange

$99099:-94: 28$ \& Graveyard

$c$ Origin is at the center bottom of the top flange.

c Inner Vessel. All PZ positions are in decending order.

$1 \mathrm{pz} 0$

$2 \mathrm{cz} 0.635 \$$ Assumed 0.5" dia.; measured $>0.45^{\prime \prime}$

$3 \mathrm{cz} 1.27$ \&st.

$4 \mathrm{pz}-0.8$ \$Est. \$ Top of glass = bottom of steel at the seal.

$5 \mathrm{cz} 1.47$ \$ Est.

$6 \mathrm{cz} 7.5$

$7 \mathrm{pz}-3 \$$ Est.

c Approximation of pressure vessel neck using a stepped cylinder.

$8 \mathrm{pz}-5$

$9 \mathrm{cz} 1.89 \$+/-0.025$

$10 \mathrm{pz}-5.4$

$11 \mathrm{cz} 1.49 \$+/-0.025$

$12 \mathrm{pz}-9.07 \$$ Fill level $15.5 \mathrm{~mL}$

$13 \mathrm{pz}-10.3 \$=\operatorname{surf} 4-9.5 \mathrm{~cm}$

$14 \mathrm{sz}-10.31 .49$ \$ Match 13 and 9

15 sz $-10.31 .89 \$$ Match 13 and 11

c Containment vessel.

c All measurements from spec unless noted, surfaces 20-49.

c Bellows.

c All PZ positions are in ascending order.

$20 \mathrm{pz} 2.38$

$21 \mathrm{cz} 2.46$

$22 \mathrm{cz} 7.62$

$23 \mathrm{pz} 4.29$

$24 \mathrm{cz} 3.09$

25 pz 13.09

$26 \mathrm{pz} 15 \$$ measured

$27 \mathrm{pz} 18 \$$ est

28 pz 20.38

c Steel flange.

c All PZ positions are in decending order.

$42 \mathrm{kz} 1.271 .4141$ 
$45 \mathrm{pz} 2.22$

$46 \mathrm{cz} 8.1$

$48 \mathrm{cz} 11.43$

c Aluminum + Teflon container

c All positions measured.

$50 \mathrm{pz}-0.3$ \$ Est. rubber seal thickness

$51 \mathrm{pz}-0.59$

$52 \mathrm{px}-4$

$53 \mathrm{px}-2.66$ \$ check against 81 to 84 .

$54 \mathrm{px}-2.37$

$55 \mathrm{px}-2.25 \$+/-0.10$

$56 \mathrm{px} 2.12$

$57 \mathrm{px} 2.41$

$58 \mathrm{px} 2.90$

$59 \mathrm{px} 3.19$

$60 \mathrm{px} 4.27$

61 py $3.8 \$$ To outside of glass and flanges. (assumed equal)

62 py 2.55

63 py 2.26

64 py 2.14

65 py -2.14

66 py -2.26

67 py -2.55

68 py -3.8

$69 \mathrm{pz}-0.7 \$$ Glass top est.

$70 \mathrm{pz}-0.1 \$$ flange top est.

$71 \mathrm{pz}-1.5 \$$ curve top est.

$72 \mathrm{pz}-1.89 \$$ ditto

$73 \mathrm{pz}-12.48$

$74 \mathrm{pz}-12.6$

c from bottom of Al. flange. Dist to bottom of $P V$ (3mm) agrees.

$75 \mathrm{pz}-12.89$

$76 \mathrm{pz}-14.16$

77 px 0.5

$78 \mathrm{px}-1$

$79 \mathrm{px}-3.26$ \$ insulation

c Source recheck SDEF after changing.

c $\mathrm{BeO}$

80 c/x $0-10.351 .335$

$81 \mathrm{px}-3.68 \$$ Check w/ 53

c $Y-88$

$82 \mathrm{c} / \mathrm{x} \quad 0-10.351 .29$ 
$83 \mathrm{px}-4.35 \$$ including backing insulation

c Aluminum 80/20

$90 \mathrm{c} / \mathrm{z} 10.210 .21 .3$

$91 \mathrm{c} / \mathrm{z} 10.2-10.21 .3$

$92 \mathrm{c} / \mathrm{z}-10.2-10.21 .3$

$93 \mathrm{c} / \mathrm{z}-10.210 .21 .3$

$94 \mathrm{pz}-30.48$

$95 \mathrm{cz} 13.97$

$96 \mathrm{pz}-2.54$

$99 \mathrm{cz} 17$

mode $\mathrm{n} \mathrm{p}$

$\mathrm{m} 1$ 6000.70c 3 9019.19c 8 \& C3F8

$\mathrm{mx} 1: \mathrm{p} 00$

m2 10010.666780160 .3333 \& Water

$\mathrm{mx} 2: \mathrm{p} 00$

m3 $5010-0.0085011-0.0328016-0.54111023-0.0314028-0.348$ \& 14029 -0.016 14030 -0.013 13027 -0.012 \$ Type I Class A Borosilicate Glass $\mathrm{mx3}: \mathrm{p} 07 \mathrm{r}$

c Steel

c 316L grade stainless steel

$\mathrm{m} 4$ 6000.70c $-0.00027014 .70 \mathrm{c}-0.0005$ 14028.19c $-0.004515031 .70 \mathrm{c}-0.0003$ \& $22048.70 c-0.00324050 .19 c-0.007324052 .19 c-0.140324053 .70 c-0.0159 \&$ $24054.70 c-0.004025055 .70 c-0.01326054 .70 c-0.039126056 .19 c-0.6152 \&$ $26057.70 c-0.014226058 .70 c-0.001927059 .70 c-0.003928058 .70 c-0.0688 \&$ $28060.70 c-0.026528061 .70 c-0.011528062 .70 c-0.003728064 .70 c-0.0009 \&$ $29063.70 c-0.003329065 .70 c-0.001542092 .70 c-0.00342094 .70 c-0.0019 \&$ 42095.70c - $0.003242096 .70 c-0.003442097 .70 c-0.00242098 .70 c-0.005$ \& $42100.70 c-0.002$

$\mathrm{mx} 4: \mathrm{p} 028 \mathrm{r}$

m5 60000.33390190 .667 \& PTFE

$\mathrm{mx} 5: \mathrm{p} 00$

$c$ Air

c Approximately a moist atmosphere

m6 $7014-0.7758016-0.2105318040-0.01281001-0.001376000-0.0003$

$\mathrm{mx} 6: \mathrm{p} 04 \mathrm{r}$

c Aluminium Alloy 6061

m7 $13027-0.97514028-0.00626056-0.00329063-0.00229065-0.001 \&$ $12024-0.00812025-0.00112026-0.00124052-0.00230000-0.001$

$\mathrm{mx} 7: \mathrm{p} 0$ 9r

m8 1001260001 \$ Butyl rubber / polypropylene / insulation mx8:p 00 
c Epoxy, aluminum, $\mathrm{FeO}$, $\mathrm{H} 2 \mathrm{O}$

m9 10010.360000 .170140 .0180160 .2260540 .02260560 .12130270 .25

$\mathrm{mx9}$ : 0 $0 \mathrm{r}$

m10 $40090.580160 .5 \$ B e O$

$\mathrm{mx10:p} 40090$

m11 $1001-0.0038016-0.55313027-0.00314028-0.36214029-0.017 \&$

$14030-0.01316032-0.00120040-0.048$ \$ Concrete

$\mathrm{mx} 11: \mathrm{p} 07 \mathrm{r}$

m12 $100186000580162 \$$ PMMA acrylic (C5H8O2)

$\mathrm{mx} 12 \mathrm{p} 02 \mathrm{r}$

imp:n 1 25r $0 \$ 1,99$

imp:p 0 17r 110 2r 11110

c Three Required Cards for Polimi, to ensure an analogue simulation.

phys:n j 20.

phys:p 0101

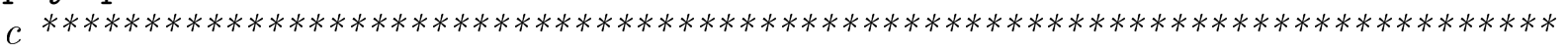

c Monocromatic neutron source at cell 30, weighted by r̂े gamma flux

sdef $A X S=100 \mathrm{PAR}=2 \mathrm{RAD}=\mathrm{d} 1 \mathrm{POS}=-3.70-10.35 \mathrm{ERG}=\mathrm{d} 4$ ext=d2

si1 00.25

si2 $-1.8745-1.557$

SI4 L 1.8360632 .7343 .2197

SP4 0.9920 .00710 .00007

c $* * * * * * * * * * * * * * * * * * * * * * * * * * * * * * * * * * * * * * * * * * * * * * * * * * * * * * * * * * * * * * * * * * * * * * * * * *$

$\mathrm{f} 4: \mathrm{n} 1$

e4 0.0176 13I 0.15760 .9370 .9631 .3631 .401

cut:p j 1.664

cut:n $2 \mathrm{j} 00$

nps $1 \mathrm{e} 7$

ipol $000002 \mathrm{~J} 11$

rpol 1e-3 1e-3

files 21 DUMN1

\section{D.4 CYRTE Geometry, June $2013 \mathrm{C}_{3} \mathrm{~F}_{8}$ fill}

c Model of the CYRTE bubble chamber

$c$ by Alan Robinson, Jun 20, 2013

c 210 updated BeOxs

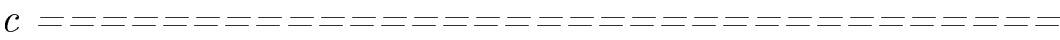

$c$ Volumes

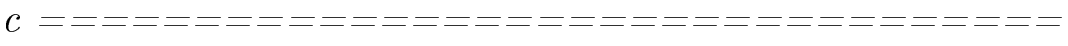

c Inner Vessel 
$11-1.375-29-8 \$ C 3 F 8$

$22-1.0-2-1$ (8:-9) 10:-12 \$ H2O above and below interface

$33-2.232(-1-3$ 5:-5 $10-6):-1012-11$ \$ Glass

$45-1.41-167-4$ (3:-5) \$ Bushing and clamp

c Bath, Tophat, and structure

$102-1.026-27-23$ (-34 22 35:-35 36 39) (6:-10) 11 (41 42 43:-44) \$ Water

$1111-1.18(-2021-2425-28 \quad 29:-2930-3132-33(37: 38) 40) \&$

(-22:23:-26:27:(-36 -35):-39) ((41 42 43):-44) \$ Acrylic

$1212-6.3(-41:-42:-43) 44-45 \$$ Water bath ports

$137-1.2((50-51: 52-53) 1-99: 51-5220-1)(54-55: 56-57): \&$

$20-158-5960-54 \$$ Aluminum frame

147 -2.70 -20 (-61:-62:-63:-64)65 -55 \$ Aluminum posts

$152-0.970-75-74 \$$ tophat fluids

$164-8.021-2454-571-70: 70-71-72$ 74:72 -75 74 -73:75 -76 -73 \$topsteel

$177-1.079-78-77 \$$ Camera

c Source

$2010-2.85-85-8687 \mathrm{fcl}: \mathrm{p}=1 \$ B e O$

$2111-1.18-87-8889 \$ Y$-88 source

$228-0.94-83-8485 \$ P E$ spacer

$237-2.7-82(83: 84)(-80-81:-92-93) 94 \$$ Al cup

$2412-8.5-90-9195$ (81:-94) (-94:93:92) \$ brass holder

$254-7.87-95-96-99 \$$ Piston

$c$ Air

$906-0.001220-99(1:-7: 4)(7: 6) \# 13 \# 15 \# 16 \$$ Above $z=-2.54$

916 -0.0012 -99 -20 \#1 \#2 \#3 \#10 \#11 \#12 \#14 \#17 \#20 \#21 \#22 \#23 \#24 \#25

99099 \& Graveyard

c Inner Vessel. All PZ positions are in decending order.

$c$ Origin is at the center bottom of the top flange.

$1 \mathrm{pz} 0$

$2 \mathrm{cz} 0.5$

$3 \mathrm{cz} 1.25$

$4 \mathrm{cz} 2.25$

$5 \mathrm{pz}-0.5$

$6 \mathrm{cz} 0.593$

$7 \mathrm{pz}-1.7$

$8 \mathrm{pz}-7.4$

$9 \mathrm{pz}-15.5$

$10 \mathrm{pz}-18.1$

$11 \mathrm{sz}-18.10 .6$

$12 \mathrm{sz}-18.10 .5$

c Bath 
$20 \mathrm{pz}-2.54$

$21 \mathrm{px}-7.62$

$221 \mathrm{px}-3.33$

$231 \mathrm{px} 3.33$

$24 \mathrm{px} 7.62$

25 py -4.3

26 py -3.33

27 py 3.33

28 py 4.3

$29 \mathrm{pz}-3.81$

$301 \mathrm{px}-3.81$

$311 \mathrm{px} 3.81$

32 py -3.81

33 py 3.81

$34 \mathrm{pz}-5.0$

$35 \mathrm{pz}-9.68$

$361 \mathrm{px}-0.635$

$37 \mathrm{pz}-10.16$

$381 \mathrm{px}-1.111$

$39 \mathrm{pz}-22.38$

$40 \mathrm{pz}-22.86$

$41 \mathrm{c} / \mathrm{x} 0-5.71 .5$

$42 \mathrm{c} / \mathrm{x} \quad 0-12.7 \quad 1.5$

$43 \mathrm{c} / \mathrm{x} 0-20.51 .5$

$441 \mathrm{px} 2.8$

$451 \mathrm{px} 4.7$

c Aluminum Frame

$50 \mathrm{px}-12.54$

$51 \mathrm{px}-10$

$52 \mathrm{px} 10$

53 px 12.54

54 py -7.1

55 py -4.56

56 py 4.56

57 py 7.1

$58 \mathrm{px}-4.3$

$59 \mathrm{px}-1.76$

60 py -28

$61 c / z-5.8-5.80 .635$

$62 \mathrm{c} / \mathrm{z}-5.85 .80 .635$

$63 \mathrm{c} / \mathrm{z} 5.8-5.80 .635$

$64 \mathrm{c} / \mathrm{z} 5.85 .80 .635$ 
$65 \mathrm{pz}-24.5$

$c$ Tophat

$70 \mathrm{pz} 1.27$

$71 \mathrm{cz} 7.62$

$72 \mathrm{pz} 2.54$

$73 \mathrm{cz} 5.715$

$74 \mathrm{cz} 5.08$

$75 \mathrm{pz} 11.4$

$76 \mathrm{pz} 12.7$

c Camera

77 c/y $0-102.0$

78 py -13.5

79 py -24.5

$c$ Source marked from center of front face.

$802 \mathrm{px} 0$

$812 \mathrm{cx} 1.58$

822 py 1.17

$832 \mathrm{px}-0.13$

$842 \mathrm{cx} 1.349$

$852 \mathrm{px}-0.26$

$862 \mathrm{cx} 1.33$

$872 \mathrm{px}-1.26$

$882 \mathrm{cx} 1.27$

$892 \mathrm{px}-1.895$

$902 \mathrm{px}-1.905$

$912 \mathrm{cx} 2.54$

$922 \mathrm{px}-2.286$

932 cx 1.899

$942 \mathrm{px}-2.54$

$952 \mathrm{px}-3.81$

$962 \mathrm{cx} 0.32$

c Simulation boundary

99 sz -530

mode $\mathrm{n} \mathrm{p}$

$\operatorname{tr} 10.02300 \$$ no larger than $0.043 x$ or less than -0.5

$\operatorname{tr} 2-1.2380 .2-15.70 .9955-0.09500 .0950 .99550001$

c Materials

$\mathrm{m} 1$ 6000.70c 3 9019.19c 8 \$C3F8

$\mathrm{mx1:p} 00$

m2 10010.666780160 .3333 \& Water

$\mathrm{mx} 2: \mathrm{p} 00$ 
m3 80162140280.92223140290 .04685140300 .03092 \& Quartz Glass $\mathrm{mx3}: \mathrm{p} 03 \mathrm{r}$

$c$ Steel

c 316L grade stainless steel

$\mathrm{m} 4$ 6000.70c $-0.00027014 .70 \mathrm{c}-0.0005$ 14028.19c-0.0045 15031.70c -0.0003 \& $22048.70 c-0.00324050 .19 c-0.007324052 .19 c-0.140324053 .70 c-0.0159 \&$ $24054.70 c-0.004025055 .70 c-0.01326054 .70 c-0.039126056 .19 c-0.6152 \&$ $26057.70 c-0.014226058 .70 c-0.001927059 .70 c-0.003928058 .70 c-0.0688 \&$ $28060.70 c-0.026528061 .70 c-0.011528062 .70 c-0.003728064 .70 c-0.0009 \&$ $29063.70 c-0.003329065 .70 c-0.001542092 .70 c-0.00342094 .70 c-0.0019 \&$ $42095.70 c-0.003242096 .70 c-0.003442097 .70 c-0.00242098 .70 c-0.005 \&$ $42100.70 c-0.002$

$\mathrm{mx} 4: \mathrm{p} 028 \mathrm{r}$

m5 $100126000180161 \$$ Delrin/Acetal $\mathrm{mx} 5: \mathrm{p} 02 \mathrm{r}$

$c$ Air

c Approximately a moist atmosphere

m6 $7014-0.7758016-0.2105318040-0.01281001-0.001376000-0.0003$

$\mathrm{mx} 6: \mathrm{p} 04 \mathrm{r}$

m7 $13027-0.982514000-0.00826000-0.00312000-0.0065$ \$Aluminium 6105-T5 $\mathrm{mx} 7: \mathrm{p} 03 \mathrm{r}$

m8 1001260001 \$ Butyl rubber / polypropylene / insulation

$\mathrm{mx8}: \mathrm{p} 00$

c Epoxy, aluminum, $\mathrm{FeO}$, $\mathrm{H} 2 \mathrm{O}$

m9 10010.360000 .170140 .0180160 .2260540 .02260560 .12130270 .25

$\mathrm{mx} 9: \mathrm{p} 06 \mathrm{r}$

m10 $40090.580160 .5 \$ B e O$

$\mathrm{mx10:p} 40090$

m11 $100186000580162 \$$ PMMA acrylic (C5H8O2)

$\mathrm{mx} 11: \mathrm{p} 02 \mathrm{r}$

m12 $29063-.42529065-.19030000-0.35582204-0.0004282206-0.00723 \&$

$82207-0.0066282208-0.01572$ \$ Brass alloy 360

$\mathrm{mx} 12: \mathrm{p} 0 \mathrm{6r}$

c $70 \% \mathrm{Al}, 15 \%$ SiO2 $15 \%$ plastic for cameras

m13 1001. 0.16000 . 0.05 8016. 0.10 13027. 0.7 \&

14028. 0.0458 14029. 0.0025 14030. 0.0017

$\mathrm{mx13: \textrm {p } 0} 6 \mathrm{r}$

c Lenses, $40 \%$ Al $60 \%$ SiO2

m14 8016. 0.4 13027. 0.4 14028. 0.1844 14029. 0.0936 14030. 0.062

$\mathrm{mx} 14: \mathrm{p} 04 \mathrm{r}$

$c * * * * * * * * * * * * * * * * * * * * * * * * * * * * * * * * * * * * * * * * * * * * * * * * * * * * * * * * * * * * * * * * * * * * * * * * * *$

imp:n 1 19r 0 
imp:p 0 11r 1 3r 0 2r 10

$c$ Three Required Cards for Polimi, to ensure an analogue simulation.

phys:n 4. 1. 2 j 21.

phys:p j $101 \$$ Force photoneutron production

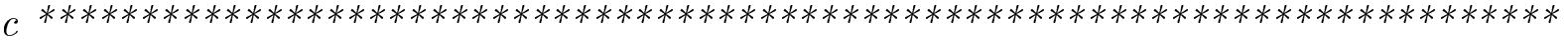

c Gamma source disk for $Y-88$

sdef $\mathrm{AXS}=0.99550 .0950 \mathrm{PAR}=2 \mathrm{RAD}=\mathrm{d} 1 \mathrm{POS}=-1.2380 .2-15.7 \mathrm{ERG}=\mathrm{d} 4$ ext $=\mathrm{d} 2$

si1 00.25

si2 $-1.8745-1.557$

SI4 L 1.8360632 .7343 .2197

SP4 0.9920 .00710 .00007

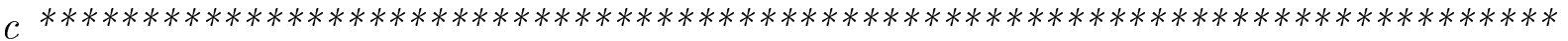

f4:n 1

e4 $024 \mathrm{I} .325$

cut:p j 1.664

cut:n $2 \mathrm{j} 00$

nps 1 e7

ipol $000002 \mathrm{~J} 11$

rpol 1e-3 1e-3

files 21 DUMN 1

\section{D.5 Montreal He-3 Calibration}

c Model of COUPP-0.1 at Universite de Montreal

c by Alan Robinson, Feb 13, 2013

$c========================$

$c$ Volumes

$c======================$

14 -8.00 -1 2 -8 (4 5 7:-3) 6 (208 209210211212 213:214) \& Target holder

1014 -8.00 (-202:-203:-204:-205:-206:-207) 201 -2 \$ screw heads

$24-8.0068-323-326(-11: 13)$ \& KF flange

$10217-1.41-10323-324 \$$ KF clamp handle

$10319-1.8-320 \$ 2-314 K F$ oring

$1204-4.00326-1213-14320$ \$ KF Clamp steel

$1214-8.00324-15-326325 \$$ KF outside flange

$1226-3.97321-322-325$ \$ KF flange window

$10418-2.203-217215-2 \$$ Quartz

$1056-2.7218-219216-2$ (217:-215) \& Quartz Holder

$317-1.498-1817-16220$ \$ Beam Tube End Retainer (Delrin)

c Handle

$1066-2.78222-221-227229-228(-223:-225:-336)(224$ 335:226) 233234 
1074 -7.5 8 -232 (-230:-231) (223:-233:-234) \$ Screws

$10810-2.212-235-236$ \$ Target Holder Sleve

$10919-1.6-2237239-238 \$$ oring 243

$11010-2.2-237239-238240$ \$ beam tube end ring

$11119-1.6-245246-250251$ \$ oring 223

c Beam Tube End

1124 -8.00 -16 -235 241242 (236:-2) (243:-216) (19:-249) (245:-250) \&

\#109 \#110 \#111

$1136-2.7-241-216253252$ \& Inner tube Al?

$11419-1.6260-259-250251$ \$ oring 223

c Beam Tube Isolator

$11520-1.05-19261264-262(-245: 249)(-254: 250)(255:-249)(256:-250)$ \#114

$46-2.7-25523-21-999(-256: 249)(-265: 250)(-266$ 20:-20 -22) \$ Beam tube

59 -2e-15 -2 -243 216 (219:-218 -215) \#101 \$ approx e-10 torr Vacuum

$1169-2 \mathrm{e}-15-999-216-252$ \$ approx e-10 torr Vacuum

$1179-2 \mathrm{e}-15$-999 -242 252 -250 (-264:254:-261) (-23:265) (241:-253) \$ Vacuum

$1186-2.721-267268269$-270 $271-272$ (273 -274:275 -276) \$ Alignment Spacers

$11921-8.07((277-281: 282-278) 291-292:(279-283: 284-280) 289-290) \&$

-285 288 (-269:270:-271:272:286:-287) \$ alignment screw foot

64 -8.00 (-25:-24:-294:295:-298:299) (-301:-302) $293-296297-300 \&$

(-277:278:-279:280) \$ Alignment screws

c Centering Mounts

$76-2.7242526(-27:-318-316$ 317) -28 29 (303 -304:-305) \&

(306:-307:308:319 (309-420:310 421)) 315 : $313-314311-312135-315$

c Table

$84-8.00-30$ (31 $34-3536-37:-3238-39: 39-45(-4142:-4344)$ (-32:40) 151

9 like 8 but $\operatorname{trcl}=5 \$$ Table 2

$102-0.85110-111112-113114-115$ \$ PICASSO Crate

$113-11.3446-13047-311135-49$ \$ Pb Brick at UM He-3

$123-6.0156-157152154-155-158$ \$3 Pb Bricks/steel stands/DAQ box $p=-4.5$

$136-2.7-130131-132133134-135 \$ 3 / 8^{\prime \prime}$ Al table

c He-3 Chicago

$317-0.000500$ (-61 -62 63) \$ He-3 chamber

$3211-2.70$ (-64 $61-62$ 63):(-64 62 -65):(-64 -63 66) \$ Aluminium canister

$334-4.5674-7565-76$ \$ Steel HN connector

$3410-2.2-7465-76 \$$ PTFE dielectric

$355-0.898$ (64:-66) $78-79-80 \$$ Paraffin

$3612-1.07$ (-78:79:81) (64:-66) $84-86-82$ (-83:85) \$ ABS Solid

$3712-0.75387880-79-81 \$ A B S$ Foam Core

c He-3 Near Beam

$417-0.000625(-123124-125) \$ H e-3$ chamber

$424-8.0-12697-96(127:-128: 125)$ \$ steel canister 
434 -4.56 -97-98 (99:-120 121) 122 \$ steel MHV connector

$4410-2.2-122-97121 \$$ PTFE dielectric

$455-0.92-126-929596 \$ H D P E$

$465-0.898-9091-93$ (92:126) \$ Paraffin

$478-1.18-909193-94 \$$ Acrylic

$489-0.001291-97-9698 \$$ Air

$4913-2.5$ (-127 123:-124 128) -125 \$ Ceramic Insulator

c Other

$504-0.753106-101102-103104-105$ \$ Preamp

$5715-0.64-152153154-155150-999$ \& Table

$5816-2.3-999-151 \$$ Floor

c Air

$c$ near front of target

909 -0.0012 2 -15 -16 (235:-17) \#1 \#2 \#102 \#103 \#120 \#121 \#3 \#106 \#107 \#108 $\&$

$\# 122$

$c$ behind target

$919-0.0012285-15262$ (-19:16) 135 (314:-311:312:315) (90:94:-121) $154-155 \&$ -999 \#11 \#106

$929-0.0012121-94-91$ \#43 \#44 \#45 \& Air near He-3 end

939 -0.0012 -285 $313154-155135$-999 22 (-20:266:21) (262:-255) \&

\#118 \#119 \#6 \#7 \#11 \$Air around alignment mount

949 -0.0012 -313 135 -999 $22154-155$ \#11 \$ Air behind alignment mount

959 -0.0012 -135 -999 $154-155151$ \#8 \#11 \#12 \#13 \#57 \& Air below tables

96 -0.0012 -999 $15135154-155$ \#31 \#32 \#33 \#34 \#35 \#36 \#37 \#50 \$ Air in front

$979-0.0012-999155151 \$$ Air $+y$

$989-0.0012-999-154151$ \#9 \#10 \#13 \& Air -y

990999 \& Graveyard

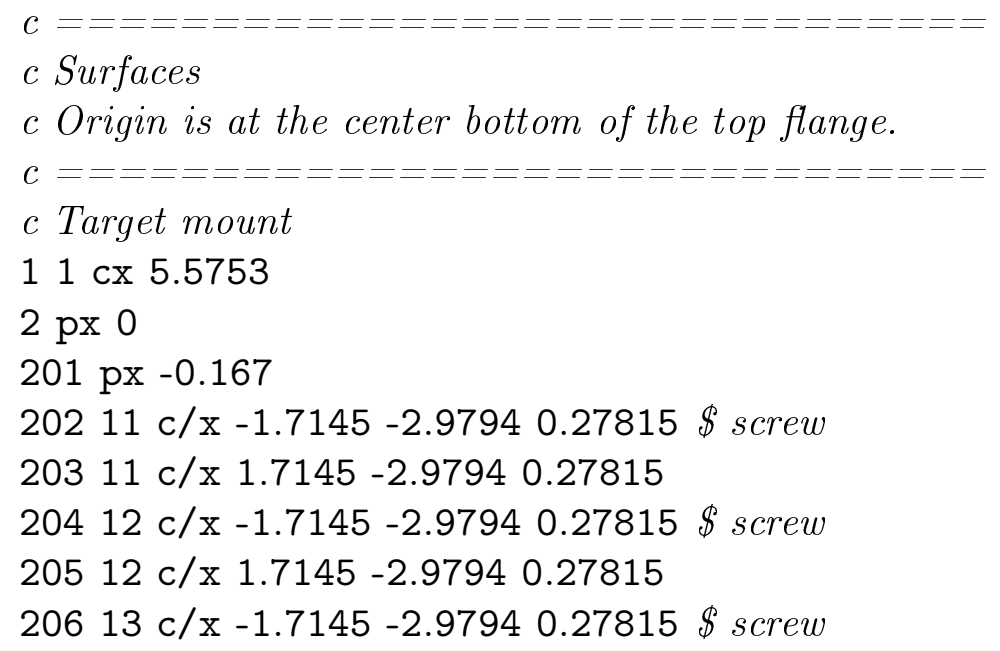


$20713 c / x 1.7145-2.97940 .27815$

$2081 \mathrm{c} / \mathrm{x} .985-.9850 .11305$ \$ screw holes

$2091 \mathrm{c} / \mathrm{x}-.985 .9850 .11305$ \$ screw holes

$2101 \mathrm{c} / \mathrm{x}-.985-.9850 .11305 \$$ screw holes

$2111 \mathrm{c} / \mathrm{x} 3.32-3.320 .11305$ \$ screw holes

$2121 \mathrm{c} / \mathrm{x}-3.323 .320 .11305 \$$ screw holes

$2131 \mathrm{c} / \mathrm{x}-3.32-3.320 .11305 \$$ screw holes

$214 \mathrm{px} 0.5842$

$215 \mathrm{px}-0.3175$

$216 \mathrm{px}-0.4826$

$3 \mathrm{px} 0.0808 \$ .03$ thicker due to foils approx.

$41 \mathrm{c} / \mathrm{x}-2.112 .111 .1811$

$51 \mathrm{c} / \mathrm{x} 2.11-2.111 .1811$

$61 \mathrm{c} / \mathrm{x} 2.112 .110 .79325$

$71 \mathrm{c} / \mathrm{x}-2.11-2.111 .1811$

$8 \mathrm{px} 0.635$

c KF Flange

$101 \mathrm{c} / \mathrm{x} 5.12 .110 .635$

$111 \mathrm{c} / \mathrm{x} 2.112 .110 .9522$

$121 \mathrm{c} / \mathrm{x} 2.112 .112 .286 \$ O D$ of clamp, not including hinge or nut.

$13 \mathrm{px} 2.617$

$14 \mathrm{px} 4.2322$

15 px 4.9942

$2171 \mathrm{c} / \mathrm{x} 2.112 .111 .5081$

$2181 \mathrm{c} / \mathrm{x} 2.112 .111 .27$

$2191 \mathrm{c} / \mathrm{x} 2.112 .111 .905$

32015 tx 3.252001 .190 .2380 .238

$321 \mathrm{px} 4.1052$

$322 \mathrm{px} 4.3592$

$323 \mathrm{px} 2.933$

$324 \mathrm{px} 3.9874$

$3251 \mathrm{c} / \mathrm{x} 2.112 .110 .889$

$3261 \mathrm{c} / \mathrm{x} 2.112 .111 .27$

$c$ Retainer

$161 \mathrm{cx} 6.35$

$171 \mathrm{cx} 5.023875$

$18 \mathrm{px} 1.2954$

$2201 \mathrm{kx}-4.260851$

c Handle

$2211 \mathrm{pz} 0.794$

$2221 \mathrm{pz}-0.794$

223 px 0.9525 
2241 p $1-0.66666670-1.905$

2251 p $1-0.66666670-1.6408$

3351 p $10.66666670-1.905$

3361 p $10.66666670-1.6408$

$226 \mathrm{px} 3.175$

227 px 3.4925

2281 py 9.525

2291 py -9.525

$2301 \mathrm{c} / \mathrm{x} 1.1430 .27815$

$2311 \mathrm{c} / \mathrm{x}-1.1430 .27815$

$232 \mathrm{px} 1.1322$

$2331 \mathrm{c} / \mathrm{x} 1.14300 .14225$

$2341 \mathrm{c} / \mathrm{x}-1.14300 .14225$

c Sleeve

$235 \mathrm{px} 0.5334$

2361 cx 5.7785

c Beam Tube End

$19 \mathrm{px}-1.2446$

$237 \mathrm{px}-0.254$ \$ oring

2381 cx 5.5753

$2391 \mathrm{cx} 5.1943$

$240 \mathrm{px}-0.635$ \& teflon

$241 \mathrm{cx} 1.27$

$242 \mathrm{px}-3.4036$

$2431 \mathrm{cx} 4.9911$

$245 \mathrm{px}-2.54$

246 px -2.794

$247 \mathrm{px}-3.175$

249 cx 2.413

250 cx 2.2479

251 cx 2.032

c Inner tube

252 cx 1.1938

$253 \mathrm{px}-19.2151$

c Beam Tube Isolator

$254 \mathrm{px}-3.556$

$255 \mathrm{px}-3.81$

$256 \mathrm{px}-4.64$

$258 \mathrm{px}-5.08$

$259 \mathrm{px}-5.334$

260 px -5.715

261 px -5.969 
262 cx 3.175

264 cx 1.7145

c Vacuum Pipe

$20 \mathrm{px}-8.89$

21 cx 2.794

22 cx 1.905

23 cx 1.5875

$265 \mathrm{px}-6.096$

$266 \mathrm{kx}-11.0491$

c Alignment Spacer

$267 \mathrm{px}-4.1529$

268 px -5.4229

269 py -6.6797

270 py 6.6797

$271 \mathrm{pz}-6.6797$

272 pz 6.6797

273 py -1.27

274 py 1.27

$275 \mathrm{pz}-1.27$

$276 \mathrm{pz} 1.27$

c Alignment Screws

277 py -7.3147

278 py 7.3147

$279 \mathrm{pz}-7.3147$

280 pz 7.3147

281 py -6.3622

282 py 6.3622

$283 \mathrm{pz}-6.3622$

$284 \mathrm{pz} 6.3622$

$285 \mathrm{px}-3.175$

$286 \mathrm{px}-3.5179$

$287 \mathrm{px}-6.0071$

$288 \mathrm{px}-6.35$

2897 py -1.016

2907 py 1.016

$2917 \mathrm{pz}-1.016$

2927 pz 1.016

$247 \mathrm{cz} 0.635$

257 cy 0.635

293 py -20.9672

294 py -19.6972

295 py 19.6972 


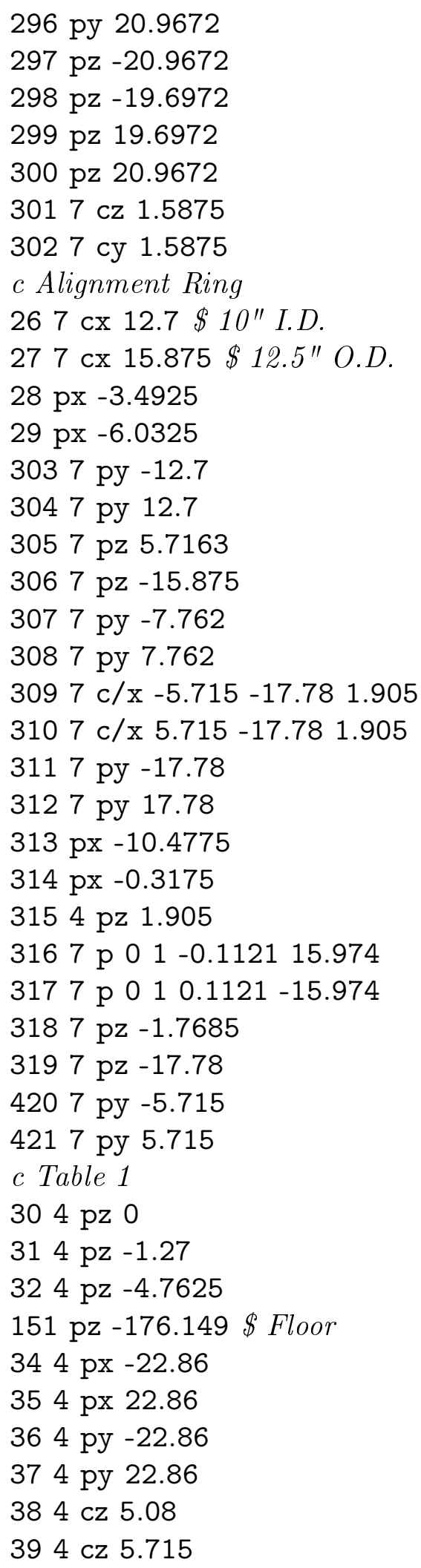


$404 \mathrm{cz} 10.16$

$414 \mathrm{p} 1100.45$

$424 \mathrm{p} 110-0.45$

$434 \mathrm{p} 1-100.45$

$444 \mathrm{p} 1-10-0.45$

$454 \mathrm{kz} 2000.016188$

c $\mathrm{Pb}$ Brick

$46 \mathrm{px}-11.1125$

477 py -22.86

$494 \mathrm{pz} 20.955$

c PICASSO

$1106 \mathrm{px}-20$

1116 px 20

1126 py -15.24

1136 py 0

$1146 \mathrm{pz} 0$

$1156 \mathrm{pz} 31.75$

c He3 chamber

$612 \mathrm{cz} 1.181$ \$ Nominal.

$622 \mathrm{pz} 11.223 \$$ Nominal. Gives slightly higher effective volume.

$632 \mathrm{pz}-9.1$ \$ Measured using center of mass

c Aluminium canister of He3 chamber

$642 \mathrm{cz} 1.27$ \$ Nominal

$652 \mathrm{pz} 12.50 \$$ Nominal. Matches measured $+/-0.05 \mathrm{~cm}$

$662 \mathrm{pz}-11.55 \$$ Measured

c Steel HN connector top

$742 \mathrm{cz} 0.545$

$752 \mathrm{cz} 0.84$

762 pz 16.91 \$subtract 1.31 for PTFE mass comparison

c Paraffin

$782 \mathrm{pz}-15.36$

$792 \mathrm{pz} 17.66$

802 cz 5.122

812 cz 5.724

822 cz 6.359

$832 \mathrm{pz}-14.07$

$842 \mathrm{pz}-15.995$

$852 \mathrm{pz} 16.39$

862 pz 18.295

c Near He-3

903 py 14.5415

913 py -11.811 


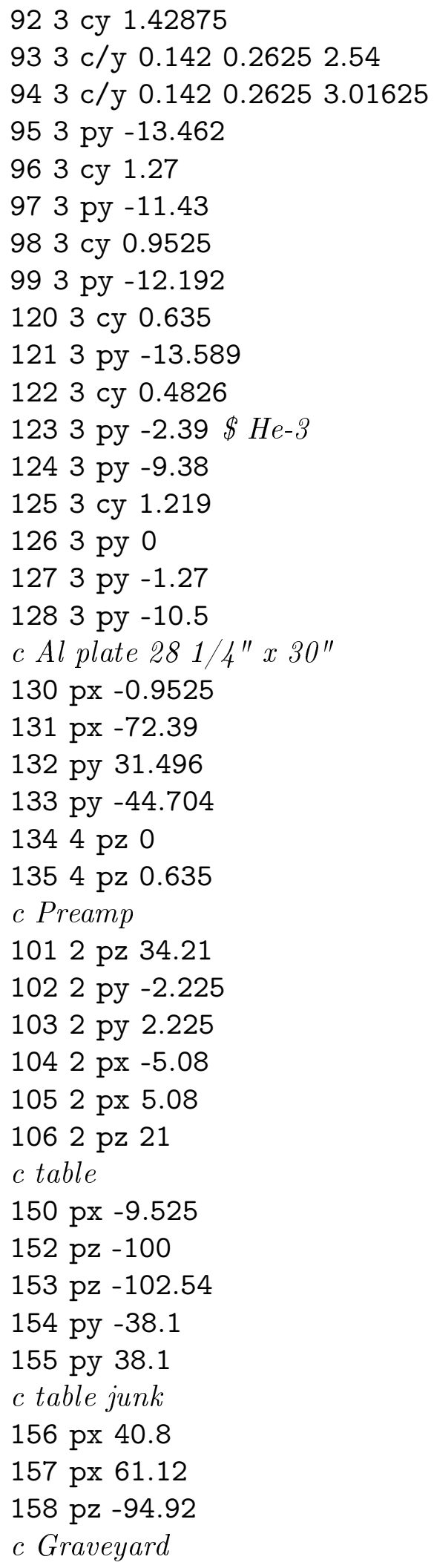


999 so 300

mode $\mathrm{n}$

imp:n 1 62r 0

tr1 $0-2.112 .11$ \$ target $23 \mathrm{deg}$

tr2 $46.6725-1.12917-2.7406$ \& Uchicago counter

tr3 -0.168 5.7785-7.522 \$ Montreal counter

tr4 $-50.1651 .016-31.369$ \& table 1

tr5 $49.8983-80.70850 \$$ table 1 to table 2

tr6 -2.2733-56.8325-31.369 \$ picasso

tr7 $-4.76251 .016-0.254$ \$ centering mount

$c \operatorname{tr} 1$ with 45 deg rotation

$\operatorname{tr} 110-2.112 .1110000 .7071 \quad-0.707100 .70710 .7071$

$\operatorname{tr} 120 \begin{array}{lllllllllll}2.11 & 2.11 & 1 & 0 & 0 & 0 & -0.7071 & -0.7071 & 0 & -0.7071 & 0.7071\end{array}$

$\operatorname{tr} 130-2.112 .111000-0.7071-0.707100 .0 .70710 .7071$

tr15 004.22 \$ center of surface 320. Related to tr1.

$c====================$

$c$ Materials

$c====================$

c HDPE

m1 1001. 2 \$MAT

6000. 1

c Hydraulics/Electronics Mixture of steel and mineral oil

$\mathrm{m} 2$ 1001. 2 6000. 1 26056. 1240520.15

c Lead

m3 822040.014822060 .241822070 .221822080 .524

c Steel

c $304 \mathrm{~L}$ grade stainless steel

m4 6000. -0.0002 7014. -0.0005 14028. -0.0045 15031. $-0.0003 \&$ 22048. -0.003 24050. -0.0081 24052. -0.1570 24053. $-0.0178 \&$

24054. -0.0044 25055. -0.013 26054. -0.0410 26056. $-0.6434 \&$ 26057. -0.0148 26058. -0.0020 27059. -0.0039 28058. $-0.0553 \&$ 28060. -0.0213 28061. -0.0009 28062. -0.0030 28064. $-0.0008 \&$ 29063. -0.0033 29065. -0.0015

c Mineral Oil / Parrafin

m5 6000. 1 \$MAT

1001. 2

c Aluminium 6061 - 80/20 is 6105 alloy, similar.

m6 13027. -0.975 \$MAT

14028. -0.006 26056. -0.003 29063. -0.002

29065. -0.001 12024. -0.008 12025. -0.001

12026. -0.001 24052. -0.002 30000. -0.001 
c $\mathrm{He}-3$

M7 20031

c PTFE

m10 6000190192

$c$ Air

c Approximately a moist atmosphere

m9 7014. -0.775 \$MAT

8016. -0.21053 18040. -0.0128 1001. -0.00137

6000. -0.0003

c Acrylic

m8 1001. 8 \$MAT

6000. 5 8016. 2

c Pure Aluminum

m11 130271

c $A B S+40 \%$ carbon black by weight

m12 1001. 17. 6000. 22 7014. 1

c Ceramic - kaolinite

m13 $50101 e-45011$ 4e-4 80160.636130270 .18140280 .163140290 .0083 \& 140300.0055220464 e-4 22047 3e-4 220480.003222049 2e-4 22050 2e-4 \& 26054 le-4 260560.0023

c Polystyrene

m14 1001. 1 6000. 1

c Wood

m15 1001. 0.462423 6000. 0.323389 7014. 0.002773 8016. 0.208779

12000. 0.000639 16000. 0.001211 19000. 0.000397 20000. 0.000388

c NIST Concrete

m16 10010.30533060000 .00288080160 .500407110230 .009212 \& 120240.000573120250 .000073120260 .00008130270 .010298 \& $140280.139295140290 .007076140300 .00467190390 .003337 \&$ 190410.000241200400 .014467200420 .000097200430 .00002 \& $200440.000312200480 .000028260540 .000094260560 .001473 \&$ 260570.000034260580 .000005

c Acetal, update with fillers

m17 100126000180161

c Quartz

m18 80162 140280.92223140290 .04685140300 .03092

c Viton

c 15 part viton, 4.5 parts filler (BaSO4, $\mathrm{CaCO}, \mathrm{CaSiO3,} \mathrm{carbon} \mathrm{black)}$

c 0.06 parts wax/release agent, 0.3-0.5 parts acrylate or Zn acrylate

c 0.5 - 1.5 parts metal oxide ( $\left.\mathrm{ZnO}, \mathrm{MgO}, \mathrm{CaOH}, \mathrm{Mg} 6 \mathrm{Al2} \mathrm{Co} 3(\mathrm{OH}) 16^{*} 4 \mathrm{H} 2 \mathrm{O}\right)$

c important resonances, $24 \mathrm{Mg}, \mathrm{Zn}$ *

m19100126000 9.59019880160 .75120240 .6120250 .075120260 .08 
$c$ Noryl

c PPO $(4,6,1)$ Polystyrene $(8,8)$

$c$ other possibilities: Nylon CPVC Acetal ABS

m20100166000880161

c Brass (typical)

$\mathrm{m} 21260560.001002$ 29063. 0.46671290650 .20821300000 .320956 \&

501160.00021501170 .00012501180 .00035501190 .00012 \&

501200.00047501220 .00007501240 .00009 \&

822060.0004822070 .00004822080 .00009

c Sapphire

$\mathrm{m} 2280163130272$

$c$ Three Required Cards for Polimi, to ensure an analogue simulation.

phys:n $\mathrm{j} 20$.

phys:p 010

cut:n $2 \mathrm{j} 0.00352$

c $* * * * * * * * * * * * * * * * * * * * * * * * * * * * * * * * * * * * * * * * * * * * * * * * * * * * * * * * * * * * * * * * * * * * * * * * * *$

c Neutrons at $97 \mathrm{keV}$

sdef pos=-0.03 00 ERG fdir $\mathrm{d} 2 \mathrm{vec}=100 \mathrm{dir}=\mathrm{d} 1$

si1 $-1-0.7079-0.4304-0.16600 .08670 .32870 .56110 .78461$

sp1 $00.1257 \mathrm{r}$

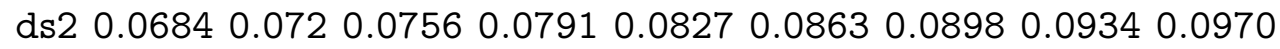

$c$ Neutrons at $61 \mathrm{keV}$

c si1 -1 - $0.6948-0.4097-0.1421-0.11030 .34940 .57650 .7931$

c sp $100.125 \mathrm{rr}$

$\begin{array}{lllllllllll}c & 0.039 & 0.0418 & 0.0445 & 0.0473 & 0.05 & 0.0527 & 0.0555 & 0.0582 & 0.061\end{array}$

$c * * * * * * * * * * * * * * * * * * * * * * * * * * * * * * * * * * * * * * * * * * * * * * * * * * * * * * * * * * * * * * * * * * * * * * * * * *$

c Neutrons in both He-3 counters

f14:n 31

fm14 -1 7103

f24:n 41

fm24 -1 7103

nps 1 e 7

dbcn 18405 


\section{Bibliography}

3M. 2008. Engineer Grade and Utility Grade Reflective Sheeting: Series 3200, 3260 and 5200. St. Paul, MN, USA 55144-1000: 3M Traffic Safety Systems Division.

Abe, Katunori, Atsushi Iizuka, Akira Hasegawa, and Shotaro Morozumi. 1984. "Induced Radioactivity of Component Materials by 16-MeV Protons and 30-MeV Alpha Particles." Journal of Nuclear Materials 123 (1-3): 972-976. doi:10.1016/0022-3115(84)902034.

Agnese, R., Z. Ahmed, A. J. Anderson, S. Arrenberg, D. Balakishiyeva, R. Basu Thakur, D. A. Bauer, et al. 2013. "Silicon Detector Results from the First Five-Tower Run of CDMS II." Physical Review D 88 (3): 031104. doi:10.1103/PhysRevD.88.031104.

Agostinelli, S., J. Allison, K. Amako, J. Apostolakis, H. Araujo, P. Arce, M. Asai, et al. 2003. "Geant4-A Simulation Toolkit." Nuclear Instruments and Methods in Physics Research Section A: Accelerators, Spectrometers, Detectors and Associated Equipment 506 (3): 250-303. doi:10.1016/S0168-9002(03)01368-8.

Akerib, D. S., H. M. Araújo, X. Bai, A. J. Bailey, J. Balajthy, S. Bedikian, E. Bernard, et al. 2014. "First Results from the LUX Dark Matter Experiment at the Sanford Underground Research Facility." Physical Review Letters 112 (9): 091303. doi:10.1103/ PhysRevLett.112.091303.

Alburger, D. E., R. E. Chrien, R. J. Sutter, and J. F. Wishart. 2004. "Search for the ThreeBody Photodisintegration of Be." Physical Review $C 70$ (6): 064611. doi:10.1103/ PhysRevC.70.064611.

Alexander, T., H. O. Back, H. Cao, A. G. Cocco, F. DeJongh, G. Fiorillo, C. Galbiati, et al. 2013. "Observation of the Dependence on Drift Field of Scintillation from Nuclear Recoils in Liquid Argon." Physical Review D 88 (9): 092006. doi:10.1103/PhysRevD. 88.092006.

Allen, W. D. 1955. Flux Measurements with the BF 3 'Long Counter'. Technical report A. E.R. E. NP/R 1667. Harwell, UK: Atomic Energy Research Establishment, June.

Amole, C. 2014. "Dytran Tech Note." COUPP internal document, no. 1356.

Amole, C., M. Ardid, D. M. Asner, D. Baxter, E. Behnke, P. Bhattacharjee, H. Borsodi, et al. 2015. "Dark Matter Search Results from the PICO-2L $\mathrm{C}_{3} \mathrm{~F}_{8}$ Bubble Chamber." Physical Review Letters 114 (23): 231302. doi:10.1103/PhysRevLett.114.231302.

Angulo, C., M. Arnould, M. Rayet, P. Descouvemont, D. Baye, C. Leclercq-Willain, A. Coc, et al. 1999. "A Compilation of Charged-Particle Induced Thermonuclear Reaction Rates." Nuclear Physics A 656 (1): 3-183. doi:10.1016/S0375-9474(99)00030-5. 
Aprile, E., M. Alfonsi, K. Arisaka, F. Arneodo, C. Balan, L. Baudis, B. Bauermeister, et al. 2013. "Limits on Spin-Dependent WIMP-Nucleon Cross Sections from 225 Live Days of XENON100 Data." Physical Review Letters 111 (2): 021301. doi:10.1103/ PhysRevLett.111.021301.

Aprile, E., K. Arisaka, F. Arneodo, A. Askin, L. Baudis, A. Behrens, K. Bokeloh, et al. 2011. "Material Screening and Selection for XENON100." Astroparticle Physics 35 (2): 43-49. doi:10.1016/j.astropartphys.2011.06.001.

Archambault, S., F. Aubin, M. Auger, E. Behnke, B. Beltran, K. Clark, X. Dai, et al. 2009. "Dark Matter Spin-Dependent Limits for WIMP Interactions on ${ }^{19} \mathrm{~F}$ by PICASSO." Physics Letters B 682 (2): 185-192. doi:10.1016/j.physletb.2009.11.019.

Archambault, S., F. Aubin, M. Auger, M. Beleshi, E. Behnke, J. Behnke, B. Beltran, et al. 2011. "New Insights into Particle Detection with Superheated Liquids." New Journal of Physics 13 (4): 043006.

Arkles, Barry. 2006. "Hydrophobicity, Hydrophilicity and Silanes." Paint and Coatings Industry 22 (10): 114-135.

Arnold, C. W., T. B. Clegg, C. Iliadis, H. J. Karwowski, G. C. Rich, J. R. Tompkins, and C. R. Howell. 2012. "Cross-Section Measurement of ${ }^{9} \mathrm{Be}(\gamma, n){ }^{8} \mathrm{Be}$ and Implications for $\alpha+\alpha+n \rightarrow{ }^{9}$ Be in the $r$ Process." Physical Review C 85 (4): 044605. doi:10.1103/ PhysRevC.85.044605.

Artna-Cohen, Agda. 1997. "Nuclear Data Sheets for A = 224." Nuclear Data Sheets 80 (1): 227-262. doi:10.1006/ndsh.1997.0003.

ASME. 1996. ASME B16.5-1996: Pipe Flanges and Flanged Fittings: NPS 1/2 Through NPS 24 Metric/Inch Standard. New York: ASME (American Society of Mechanical Engineers).

—. 2004. ASME B36.10M: Welded and Seamless Wrought Steel Pipe. New York: ASME.

—. 2012. ASME B16.9: Factory Made Wrought Buttweld Fittings. New York: ASME.

ASTM D5291-09. 2009. Standard Test Methods for Instrumental Determination of Carbon, Hydrogen, and Nitrogen in Petroleum Products and Lubricants. West Conshohocken, PA, USA: ASTM International. http://www.astm.org.

Bair, J. K., and J. Gomez del Campo. 1979. "Neutron Yields from Alpha-Particle Bombardment." Nuclear Science and Engineering 71:18.

Barbeau, Phillip S. 2009. "Neutrino and astroparticle physics with p-type point contact high purity germanium detectors." PhD diss., University of Chicago. http://search . proquest.com/docview/305051617?accountid=14657. 
Barker, F. C. 1983. "The First Excited State of ${ }^{9}$ Be." Canadian Journal of Physics 61 (10): 1371-1373. doi:10.1139/p83-175.

. 2000. "The Low-Energy ${ }^{9} \operatorname{Be}(\gamma, n){ }^{8}$ Be Cross Section." Australian Journal of Physics 53:247-257. doi:10.1071/PH99065.

Barnabé-Heider, M., M. Di Marco, P. Doane, M.-H. Genest, R. Gornea, R. Guénette, C. Leroy, L. Lessard, et al. 2005. "Response of Superheated Droplet Detectors of the PICASSO Dark Matter Search Experiment." Nuclear Instruments and Methods in Physics Research Section A: Accelerators, Spectrometers, Detectors and Associated Equipment 555 (1-2): 184-204. doi:10.1016/j.nima.2005.09.015.

Bartle, C. M., and P. A. Quin. 1980. "Total Cross Sections for the ${ }^{40} \mathrm{Ca}(\mathrm{n}, \mathrm{p})$ and $(\mathrm{n}, \alpha)$ Reactions between 2.7 and 5.5 MeV." Annals of Nuclear Energy 8:43.

Bass, R., P. Haug, K. Kruger, and B. Staginnus. 1966. Fast Neutron Excitation Functions by Activation Techniques. Technical report EANDC(E)-66. European-American Nuclear Data Committee.

Basunia, M. Shamsuzzoha. 2013. "Nuclear Data Sheets for A = 28." Nuclear Data Sheets 114 (10): 1189-1291. doi:10.1016/j.nds.2013.10.001.

Baxter, D., and C. E. Dahl. 2015. "CYRTE Conclusions." COUPP internal document, no. 1584 .

Bé, M.-M., E. Browne, V. Chechev, V. Chisté, R. Dersch, C. Dulieu, R. G. Helmer, D. McMahon, et al. 2004. Monographie BIPM-5 - Table of Radionuclides, Vol. 1832. Data available at http://www.nucleide.org/DDEP_WG/DDEPdata.htm. Bureau International des Pois et Measures.

Becher, Paul. 2001. Emulsions: Theory and Practice. 3rd. New York: Oxford University Press.

Behnke, E., J. Behnke, S. J. Brice, D. Broemmelsiek, J. I. Collar, A. Conner, P. S. Cooper, et al. 2012. "First Dark Matter Search Results from a 4-kg $\mathrm{CF}_{3} \mathrm{I}$ Bubble Chamber Operated in a Deep Underground Site." Physical Review D 86 (5): 052001. doi:10 . 1103/PhysRevD . 86.052001.

2014. "Erratum: First Dark Matter Search Results from a 4-kg $\mathrm{CF}_{3} \mathrm{I}$ Bubble Chamber Operated in a Deep Underground Site [Physical Review D 86 , 052001 (2012)]." Physical Review D 90 (7): 079902. doi:10.1103/PhysRevD.90.079902.

Behnke, E., T. Benjamin, S. J. Brice, D. Broemmelsiek, J. I. Collar, P. S. Cooper, M. Crisler, et al. 2013. "Direct Measurement of the Bubble-Nucleation Energy Threshold in a $\mathrm{CF}_{3}$ I Bubble Chamber." Physical Review D 88 (2): 021101. doi:10.1103/PhysRevD. 88.021101 . 
Behnke, E., J. I. Collar, P. S. Cooper, K. Crum, M. Crisler, M. Hu, I. Levine, et al. 2008. "Spin-Dependent WIMP Limits from a Bubble Chamber." Science 319 (5865): 933-936. doi:10.1126/science.1149999.

Berman, B. L., R. L. Van Hemert, and C. D. Bowman. 1967. "Threshold Photoneutron Cross Section for ${ }^{9}$ Be." Physical Review 163 (4): 958-963. doi:10.1103/PhysRev.163.958.

Bernabei, R., P. Belli, V. Landoni, F. Montecchia, Nicolantonio W. Di, A. Incicchitti, D. Prosperi, C. Bacci, Dai C. J., et al. 1996. "New Limits on WIMP Search with LargeMass Low-Radioactivity NaI(Tl) Set-Up at Gran Sasso." Physics Letters B 389 (4): 757-766. doi:10.1016/S0370-2693(96)80020-7.

Bernett, Marianne K., and W. A. Zisman. 1969. "Effect of Adsorbed water on Wetting Properties of Borosilicate Glass, Quartz, and Sapphire." Journal of Colloid and Interface Science 29 (3): 413-423. doi:10.1016/0021-9797(69)90120-9.

Biodex. 2015. Nuclear Medicine and Molecular Imaging Devices and Supplies. 20 Ramsey Road, Shirley New York, USA 11967.

Blatt, John M., and L. C. Biedenharn. 1952. "The Angular Distribution of Scattering and Reaction Cross Sections." Review of Modern Physics 24 (4): 258-272. doi:10.1103/ RevModPhys.24.258.

Blumenthal, George R., S. M. Faber, Joel R. Primack, and Martin J. Rees. 1984. "Formation of Galaxies and Large-Scale Structure with Cold Dark Matter." Nature 311:517-525.

Bondarenko, I. P., V. A. Khryachkov, T. A. Ivanova, B. D. Kuzminov, N. N. Semenova, and A. I. Sergachev. 2013. "Investigation of the ${ }^{19} \mathrm{~F}(\mathrm{n}, \alpha){ }^{16} \mathrm{~N}$ Reaction Excitation Function in Neutron Energy Range of 4-7.35 MeV." Bulletin of the Russian Academy of Sciences: Physics 77 (4): 459-461. doi:10.3103/S1062873813040060.

Borden, Nickolas, Ilan Levine, and Thomas Nania. 2013. "Second Look at Retroreflector." COUPP internal document, no. 924.

Browne, E. 2005. "Nuclear Data Sheets for A = 212." Nuclear Data Sheets 104 (2): 427-496. doi:10.1016/j.nds.2005.01.002.

Browne, E., and J. K. Tuli. 2007. "Nuclear Data Sheets for A = 234." Nuclear Data Sheets 108 (3): 681-772. doi:10.1016/j.nds.2007.02.003.

- 2010. "Nuclear Data Sheets for A =65." Nuclear Data Sheets 111 (9): 2425-2553. doi:10.1016/j.nds.2010.09.002.

. 2013. "Nuclear Data Sheets for A =60." Nuclear Data Sheets 114 (12): 1849-2022. doi:10.1016/j.nds.2013.11.002. 
Burda, O., P. von Neumann-Cosel, A. Richter, C. Forssén, and B. A. Brown. 2010. "Resonance Parameters of the First $1 / 2^{+}$State in ${ }^{9} \mathrm{Be}$ and Astrophysical Implications." Physical Review C 82 (1): 015808. doi:10.1103/PhysRevC.82.015808.

Cameron, John A., and Balraj Singh. 2008. "Nuclear Data Sheets for A = 38." Nuclear Data Sheets 109 (1): 1-170. doi:10.1016/j.nds.2007.12.001.

Chadwick, J., and M. Goldhaber. 1935. "The Nuclear Photoelectric Effect." Proceedings of the Royal Society of London. Series A, Mathematical and Physical Sciences 151 (873): 479-493. http://www.jstor.org/stable/96561.

Chadwick, M. B., M. Herman, P. Obložinský, M. E. Dunn, Y. Danon, A. C. Kahler, D. L. Smith, et al. 2011. "ENDF/B-VII.1 Nuclear Data for Science and Technology: Cross Sections, Covariances, Fission Product Yields and Decay Data." Nuclear Data Sheets 112 (12): 2887-2996. doi:10.1016/j.nds.2011.11.002.

Chartier, J.-L. 2009. "Revision of the ISO 8529 Standards Calculations of the (AmBe) Neutron Spectrum." Slides from presentation at Laboratori Nazionali di Frascati, Rome, June 18. https://agenda.infn.it/conferenceDisplay.py?confId=1503.

Cheng, C. W., and J. D. King. 1980. "Cross Sections and Thermonuclear Reaction Rates for the ${ }^{24} \mathrm{Mg}(\alpha, \mathrm{n}){ }^{27} \mathrm{Si},{ }^{25} \mathrm{Mg}(\mathrm{p}, \mathrm{n}){ }^{25} \mathrm{Al},{ }^{27} \mathrm{Al}(\mathrm{p}, \mathrm{n}){ }^{27} \mathrm{Si}$, and ${ }^{28} \mathrm{Si}(\alpha, \mathrm{n}){ }^{31} \mathrm{~S}$ Reactions." Canadian Journal of Physics 58 (5): 697-702. doi:10.1139/p80-095.

Clerc, H.-G., K. J. Wetzel, and E. Spamer. 1968. "Inelastic Electron Scattering from ${ }^{9}$ Be: Levels Below 10 MeV." Nuclear Physics A 120 (2): 441-459. doi:10 . 1016/03759474(68)90777-X.

Cole, Robert. 1974. "Boiling Nucleation." In Advances in Heat Transfer, edited by James P. Hartnett and Thomas F. Irvine, 10:85-166. Elsevier. doi:10.1016/S0065-2717(08) 70110-2.

Collar, J. I. 2013a. "Applications of an ${ }^{88} \mathrm{Y} /$ Be Photoneutron Calibration Source to Dark Matter and Neutrino Experiments." Physical Review Letters 110 (21): 211101. doi:10. 1103/PhysRevLett.110.211101.

2013b. "Quenching and Channeling of Nuclear Recoils in NaI(Tl): Implications for Dark-Matter Searches." Physical Review C 88 (3): 035806. doi:10.1103/PhysRevC.88. 035806 .

Cooper, P., O. Harris, H. Lippincott, and R. Neilson. 2014. "PICO-2L Run I Anomalies in Recoil Candidate Events." COUPP internal document, no. 1229.

Cullen, Dermott E. 2012. PREPRO 2012: 2012 ENDF/B Pre-Processing Codes. Technical report IAEA-NDS-39. IAEA: Nuclear Data Section, October. https : //www-nds .iaea. org/public/endf/prepro/.

Dahl, C. E. 2011. "Seitz Model Tech Note." COUPP internal document, no. 460. 
Dahl, C. E. 2013. "Seitz Model Revisited." COUPP internal document, no. 791.

Dahl, C. E., J. Hall, and W. H. Lippincott. 2012. "Comment on "First Results of the Phase II SIMPLE Dark Matter Search"." Physical Review Letters 108 (25): 259001. doi:10. 1103/PhysRevLett.108.259001.

Dahl, C. E., and M. Jin. 2014. "PICO 0.1 Neutron Calibrations, Tech Note." COUPP internal document, no. 1320.

Davis, E. A., F. Gabbard, T. W. Bonner, and R. Bass. 1961. "The Disintegration of ${ }^{10} \mathrm{~B}$ and ${ }^{19}$ F by Fast Neutrons." Nuclear Physics 27:448.

De Juren, J. W., and J. Chin. 1955. "Absolute Calibration of the National Bureau of Standards Photoneutron Standard .2. Absorption in Manganese Sulfate." Journal of Research of the National Bureau of Standards (Washington, DC 20402-9325 USA) 55 (6): 311-316. doi:10.6028/jres.055.037.

De Juren, J. W., D. W. Padgett, and L. F. Cutis. 1955. "Absolute Calibration of the National Bureau of Standards Photoneutron Standard: I." Journal of Research of the National Bureau of Standards (Washington, DC 20402-9325 USA) 55 (2): 63-69.

Devices, Analog. 2013. High Performance, 145 MHz FastFET Op Amps. Technical report AD8065/AD8066. August.

DiGiovine, B., D. Henderson, R. J. Holt, R. Raut, K. E. Rehm, A. Robinson, A. Sonnenschein, G. Rusev, A. P. Tonchev, and C. Ugalde. 2015. "Bubble Chambers for Experiments in Nuclear Astrophysics." Nuclear Instruments and Methods in Physics Research Section A: Accelerators, Spectrometers, Detectors and Associated Equipment 781:96104. doi:10.1016/j.nima.2015.01.060.

Dixit, S., W. Bertozzi, T. N. Buti, J. M. Finn, F. W. Hersman, C. E. Hyde-Wright, M. V. Hynes, et al. 1991. "Structure of ${ }^{9}$ Be from Proton Scattering at $180 \mathrm{MeV}$." Physical Review C 43 (4): 1758-1776. doi:10.1103/PhysRevC.43.1758.

Duncan, F., A. J. Noble, and D. Sinclair. 2010. "The Construction and Anticipated Science of SNOLAB." Annual Review of Nuclear and Particle Science 60 (1): 163-180. doi:10. 1146/annurev.nucl.012809.104513.

E. I. Dupont de Nemours \& Co. Fluoroproducts. 2002. Materials Safety Data Sheet - High Build Topcoat Green, 8 October. Willmington, DE, 19898 USA.

Dussan V., E. B., and Robert Tao-Ping Chow. 1983. "On the Ability of Drops or Bubbles to Stick to Non-Horizontal Surfaces of Solids." Journal of Fluid Mechanics 137 (December): 1-29. doi:10.1017/S002211208300227X.

Eckert \& Ziegler. 2007. Reference \& Calibration Sources: Product Information. Valencia, CA 91355 USA.: Eckert \& Ziegler Isotope Products Inc. 
Engel, J., S. Pittel, and P. Vogel. 1992. "Nuclear Physics of Dark Matter Detection." International Journal of Modern Physics E 1:1-37. doi:10.1142/S0218301392000023.

Felizardo, M., T. A. Girard, T. Morlat, A. C. Fernandes, A. R. Ramos, J. G. Marques, A. Kling, et al. 2014. "The SIMPLE Phase II Dark Matter Search." Physical Review D 89 (7): 072013. doi:10.1103/PhysRevD.89.072013.

Fields, Nicole E. 2014. "C ${ }^{o}$ sI: Development of a Low Threshold Detector for the Observation of Coherent Elastic Neutrino-Nucleus Scattering." PhD diss., University of Chicago.

Fordham, S. 1948. "On the Calculation of Surface Tension from Measurements of Pendant Drops." Proceedings of the Royal Society of London. Series A, Mathematical and Physical Sciences 194 (1036): 1-16.

Foroughi, F., and J. Rossel. 1972. "Étude des Sections Efficaces Differentielles des Reactions (n, p) et (n, $\alpha)$ sur ${ }^{19} \mathrm{~F},{ }^{29} \mathrm{Si},{ }^{32} \mathrm{~S}$ et ${ }^{40} \mathrm{Ca}$ a $5.85 \mathrm{MeV} . "$ Helvetica Physica Acta 45:439.

Fox, H. W., and W. A. Zisman. 1950. "The Spreading of Liquids on Low Energy Surfaces. I. Polytetrafluoroethylene." Journal of Colloid Science 5 (6): 514-531. doi:10.1016/00958522(50)90044-4.

Frenne, D. De, and E. Jacobs. 2005. "Nuclear Data Sheets for A = 105." Nuclear Data Sheets 105 (4): 775-958. doi:10.1016/j.nds.2005.10.001.

Fuchs, Udi, and Niels Kristian Bech Jensen. 2014. "UFRaw." http://ufraw.sourceforge. net/.

Fujishiro, M., T. Tabata, K. Okamoto, and T. Tsujimoto. 1982. "Cross Section of the Reaction ${ }^{9} \operatorname{Be}(\gamma, N)$ near Threshold." Canadian Journal of Physics 60 (11): 1672-1677. doi:10.1139/p82-224.

Fustin, Drew A. 2012. "First Dark Matter Limits from the COUPP $4 \mathrm{Kg}$ Bubble Chamber at a Deep Underground Site." PhD diss., University of Chicago.

Geiger, K. W., and L. van der Zwan. 1975. "Radioactive Neutron Source Spectra from ${ }^{9} \operatorname{Be}(\alpha, n)$ Cross Section Data." Nuclear Instruments and Methods 131:315-321.

Gibbons, J. H., R. L. Macklin, J. B. Marion, and H. W. Schmitt. 1959. "Precision Measurement of the $\operatorname{Be}^{9}(\gamma, n)$ Cross Section." Physical Review 114 (5): 1319-1323. doi:10. 1103/PhysRev.114.1319.

Gibbons, J. H., R. L. Macklin, and H. W. Schmitt. 1958. "V ${ }^{51}(p, n) \mathrm{Cr}^{51}$ Reaction as a 5- to 120-keV Neutron Source." Physical Review 100, no. 1 (October): 167-168.

Girifalco, L. A., and R. J. Good. 1957. "A Theory for the Estimation of Surface and Interfacial Energies. I. Derivation and Application to Interfacial Tension." The Journal of Physical Chemistry 61 (7): 904-909. doi:10.1021/j150553a013. 
Halban, H. von. 1938. "Sur l'Énergie et l'Intensité des Photoneutrons, du Beryllium et du Deuton." Comptes Rendus hebdomadaires des séances de l'Académie des sciences 1938/01:1170-1172.

Halper, L. A., C. O. Timmons, and W. A. Zisman. 1972. "Spreading of Liquid Perfluorinated Hydrocarbons on Water." Journal of Colloid and Interface Science 38 (2): 511-516. doi:10.1016/0021-9797(72)90268-8.

Hamermesh, Bernard, and Clyde Kimball. 1953. "The Photodisintegration Cross Section of Beryllium at 2.185 Mev." Physical Review 90 (6): 1063-1065. doi:10.1103/PhysRev . 90.1063.

Harissopulos, S., H. W. Becker, J. W. Hammer, A. Lagoyannis, C. Rolfs, and F. Strieder. 2005. "Cross Section of the ${ }^{13} \mathrm{C}(\alpha, n){ }^{16}$ O Reaction: A Background for the Measurement of Geo-Neutrinos." Physical Review C 72 (6): 062801. doi:10 . 1103/ PhysRevC . 72 . 062801 .

Harris, O., H. Lippincott, and R. Neilson. 2014. "PICO-2L 2013-2014 Analysis Tech Note." COUPP internal document, no. 1364.

Herman, M., A. Tkrov, and D. A. Brown. 2011. ENDF-6 Formats Nanual: Data Formats and Procedures for the Evaluated Nuclear Data File, ENDF/B-VI and ENDF/B-VII. Technical report BNL-90365-2009. BNL: Cross Sections Evaluation Working Group, November. http://www.nndc.bnl.gov/endf/b7.1/endf-manual-vii.1.pdf.

Herrera, María S., Gustavo A. Moreno, and Andrés J. Kreiner. 2014. "Revisiting the ${ }^{7} \mathrm{Li}(\mathrm{p}, \mathrm{n}){ }^{7}$ Be Reaction near Threshold." 15th International Congress on Neutron Capture Therapy Impact of a new radiotherapy against cancer, Applied Radiation and Isotopes 88:243-246. doi:10.1016/j.apradiso.2013.11.042.

Hoppe, E. W., B. D. LaFerriere, T. C. Maiti, and A Soin. 2014. Determination of Method Detection Limits for Trace 232-Thorium and 238-Uranium in Copper using Ion Exchange and ICPMS. Technical report PNNL-23293. Richland, WA: Pacific Northwest National Laboratory.

Horn, M., V. A. Belov, D. Yu. Akimov, H. M. Araújo, E. J. Barnes, A. A. Burnkov, V. Chepel, et al. 2011. "Nuclear Recoil Scintillation and Ionisation Yields in Liquid Xenon from ZEPLIN-III Data." Physics Letters B 705 (5): 471-476. doi:10.1016/j ·physletb. 2011.10.038.

Howard, A. J., H. B. Jensen, M. Rios, W. A. Fowler, and B. A. Zimmerman. 1974. "Measurement and Theoretical Analysis of some Reaction Rates of Interest in Silicon Burning." The Astrophysical Journal 188 (February): 131-140. doi:10.1086/152694.

Huo, Junde, Su Huo, and Chunhui Ma. 2007. "Nuclear Data Sheets for A = 52." Nuclear Data Sheets 108 (4): 773-882. doi:10.1016/j.nds.2007.03.001. 
Hwang, Hyun Jin, and Mostafa A. El-Sayed. 1992. "Photodissociation of Trifluoroiodomethane at $304 \mathrm{~nm}$ : Effects of Photon Energy and Curve Crossing on the Internal Excitation of Trifluoromethyl." The Journal of Physical Chemistry 96 (22): 8728-8735. doi:10.1021/j100201a014.

ICRU. 1993. Stopping Powers and Ranges for Protons and Alpha Particles. Technical report ICRU Report 49. Bethesda, MD, USA: International Commission on Radiation Units and Measurements.

ISO. 2001. ISO 8529-1: Reference Neutron Radiations - Part 1: Characteristics and Methods of Production. Geneva, Switzerland: ISO (International Organization for Standardization).

Jacobs, G. J.H., and H. Liskien. 1983. "Energy Spectra of Neutrons Produced by $\alpha$-Particles in Thick Targets of Light Elements." Annals of Nuclear Energy 10 (10): 541-552. doi:10. 1016/0306-4549(83)90003-8.

Jakobson, Mark J. 1961. "Photodisintegration of Be ${ }^{9}$ from Threshold to 5 Mev." Physical Review 123 (1): 229-230. doi:10.1103/PhysRev.123.229.

Janczuk, B., and A. Zdziennicka. 1994. "A Study on the Components of Surface Rree Energy of Quartz from Contact Angle Measurements." Journal of Materials Science 29 (13): 3559-3564. doi:10.1007/BF00352063.

Jawerth, Nicole. 2015. "IAEA Commemorates 50th Anniversary of IAEA Nuclear Data Section," February 14. https ://www.iaea.org/newscenter/news/iaea-commemorates50th-anniversary-iaea-nuclear-data-section.

John, Walter, and John M. Prosser. 1962. "Photodisintegration Cross Section of Beryllium near Threshold." Physical Review 127 (1): 231-235. doi:10.1103/PhysRev.127.231.

Jungman, Gerard, Marc Kamionkowski, and Kim Griest. 1996. "Supersymmetric Dark Matter." Physics Reports 267:195-373.

Katakura, J., and Z. D. Wu. 2008. "Nuclear Data Sheets for A = 124." Nuclear Data Sheets 109 (7): 1655-1877. doi:10.1016/j.nds.2008.06.001.

Kocsonya, A., Z. Szőkefalvi-Nagy, A. Torri, E. Rauhala, and J. Räisänen. 2006. "Absolute Alpha Particle Induced Gamma-Ray Yields for Ti-Zn." Nuclear Instruments and Methods in Physics Research Section B: Beam Interactions with Materials and Atoms 251 (2): 367-370. doi:10.1016/j.nimb.2006.07.020.

Kondev, F. G., and S. Lalkovski. 2011. "Nuclear Data Sheets for A = 207." Nuclear Data Sheets 112 (3): 707-853. doi:10.1016/j.nds.2011.02.002.

Kuechler, G., A. Richter, and W. von Witsch. 1987. "Line Shape and Excitation Strength of the First Excited State in ${ }^{9}$ Be." Zeitschrift für Physik A Atomic Nuclei 326 (4): 447-454. doi:10.1007/BF01289549. 
Larson, Nancy M. 2008. Updated User's Guide for SAMMY: Multilevel R-Matrix Fits to Neutron Data Using Bayes' Equations. Technical report ORNL/TM-9179/R8. ORNL, October. http : / / web . ornl .gov/sci/nuclear_science_technology/nuclear_ data/sammy/.

Lawson, Ian. 2014. Acquistion and Processing of Ge Detector Data at SNOLAB. Technical report SNOLAB-STR-2014-004. Lively, ON, Canada: SNOLAB.

Lemal, David M. 2004. "Perspective on Fluorocarbon Chemistry." The Journal of Organic Chemistry 69 (1): 1-11. doi:10.1021/j00302556.

Lemmon, E. W. 2006. Preliminary Evaluation of $\mathrm{CF}_{3} \mathrm{I}$ Equation of State. Provided in the NIST REFPROP database.

Lemmon, E. W., M. L. Huber, and M. O. McLinden. 2013. NIST Standard Reference Database 23: Reference Fluid Thermodynamic and Transport Properties-REFPROP, Version 9.1. Technical report. Gaithersburg, MD, USA: National Institute of Standards and Technology, Standard Reference Data Program.

Lemmon, E. W., and R. Span. 2006. "Short Fundamental Equations of State for 20 Industrial Fluids." Journal of Chemical and Engineering Data 51.

Levine, I. 2014. "Acoustic Sensor Progress: Overview of Program \& Resources." COUPP internal document, no. 1406.

Levine, S., B. D. Bowen, and S. J. Partridge. 1989a. "Stabilization of Emulsions by Fine Particles .1. Partitioning of Particles between Continuous Phase and Oil-Water Interface." Colloids and Surfaces 38 (4): 325-343. doi:10.1016/0166-6622(89)80271-9.

. 1989b. "Stabilization of Emulsions by Fine Particles .2. Capillary and Van der Waals Forces between Particles." Colloids and Surfaces (Amsterdam) 38, no. 4 (August): 345364. doi:10.1016/0166-6622(89)80272-0.

Lewin, J. D., and P. F. Smith. 1996. "Review of Mathematics, Numerical Factors, and Corrections for Dark Matter Experiments Based on Elastic Nuclear Recoil." Astroparticle Physics 6 (1): 87-112. doi:10.1016/S0927-6505(96)00047-3.

Lippincott, H. 2010. " $\mathrm{I}_{2}$ Concentration in the $60 \mathrm{~kg}$ vs. Time." COUPP internal document, no. 368 .

LND Inc. 2015. 252 Cylindrical He3 Neutron Detector. Oceanside, New York, USA: LND Inc. Accessed May 14. http://www. Indinc.com/products/pdf/538/.

MacFarlane, R. E., and A. C. Kahler. 2010. "Methods for Processing ENDF/B-VII with NJOY." Nuclear Data Sheets 111 (12): 2739-2890. doi:10.1016/j.nds.2010.11.001. 
Manzur, A., A. Curioni, L. Kastens, D. N. McKinsey, K. Ni, and T. Wongjirad. 2010. "Scintillation Efficiency and Ionization Yield of Liquid Xenon for Nonoenergetic Nuclear Recoils Down to 4 keV." Physical Review C 81 (2): 025808. doi:10.1103/PhysRevC. 81.025808 .

Marion, Jerry B., and Robert M. Brugger. 1955. "Neutron-Induced Reactions in Fluorine." Physical Review 100 (1): 69-74. doi:10.1103/PhysRev.100.69.

Martin, M. J. 2007. "Nuclear Data Sheets for A = 208." Nuclear Data Sheets 108 (8): 15831806. doi:10.1016/j.nds.2007.07.001.

Masumoto, K., and M. Yagi. 1983. "Charged Particle Activation Analysis of Phosphorus in Biological Materials." Journal of Radioanalytical Chemistry 78 (2): 233-239. doi:10 . 1007/BF02530433.

McCutchan, E. A., and A. A. Sonzogni. 2014. "Nuclear Data Sheets for A $=88$." Nuclear Data Sheets 115:135-304. doi:10.1016/j.nds.2013.12.002.

McFarlane, Robert. 1998. An Introduction to ENDF Formats. Technical report LA-UR-981779. LANL: T-2 Nuclear Information Service, January. \%5Curl\%7Bhttp://t2.lanl. gov/nis/endf\%7D.

McGarry, E. Dale, and Edward W. Boswell. 1988. NBS Measurement Services: Neutron Source Strength Calibrations. Technical report, National Bureau of Standards Special Publication 250-18. National Bureau of Standards, March.

Mendoza, E., and D. Cano-Ott. 2011. "New Evaluated Neutron Cross-Section Libraries for the Geant4 Simulation Package." IAEA Nuclear Data Services. https : //wwwnds.iaea.org/geant4/.

Mendoza, E., D. Cano-Ott, C. Guerrero, and R. Capote. 2012. New Evaluated Neutron Cross Section Libraries for the Geant4 Code. Technical report INDC(NDS)-0612. Data available online at http://www-nds.iaea.org/geant4. IAEA.

Morton, A. J., A. F. Scott, S. G. Tims, V. Y. Hansper, and D. G. Sargood. 1994. "The ${ }^{50} \mathrm{Cr}(\alpha, \mathrm{n}){ }^{53} \mathrm{Fe}$ and ${ }^{50} \mathrm{Cr}(\alpha, \mathrm{P})^{53} \mathrm{Mn}$ Cross Sections." Nuclear Physics A 573 (2): 276290. doi:10.1016/0375-9474(94)90171-6.

Morton, A. J., S. G. Tims, A. F. Scott, V. Y. Hansper, C. I.W. Tingwell, and D. G. Sargood. 1992. "The ${ }^{48} \mathrm{Ti}(\alpha, \mathrm{n})^{51} \mathrm{Cr}$ and ${ }^{48} \mathrm{Ti}(\alpha, \mathrm{P}){ }^{51} \mathrm{~V}$ Cross Sections." Nuclear Physics A 537 (1-2): 167-182. doi:10.1016/0375-9474(92)90163-E.

Mufson, S., B. Baugh, C. Bower, T. E. Coan, J. Cooper, L. Corwin, J. A. Karty, et al. 2015. "Liquid Scintillator Production for the NOvA Experiment." ArXiv e-prints (April). arXiv: 1504.04035 [physics.ins-det].

Mughabghab, S. F. 2006. Atlas of Neutron Resonances. 5th ed. Amsterdam: Elsevier. 
Murata, Toru, Hiroyuki Matsunobu, and Keiichi Shibata. 2006. Evaluation of the ( $\alpha$, Xn) Reaction Data for JENDL/AN-2005. Technical report 2006-052. JAEA-Research, July.

Neilson, R. 2014. "PICO-2L Run I Temperature, Pressure and Thresholds." COUPP internal document, no. 1349.

Nesaraja, Caroline D., Scott D. Geraedts, and Balraj Singh. 2010. "Nuclear Data Sheets for $\mathrm{A}=58 . "$ Nuclear Data Sheets 111 (4):897-1092. doi:10.1016/j.nds.2010.03.003.

Neumann, A. W. 1974. "Contact Angles and their Temperature Dependence: Thermodynamic Status, Measurement, Interpretation and Application." Advances in Colloid and Interface Science 4 (2-3): 105-191. doi:10.1016/0001-8686(74)85001-3.

Ngoc, H. Nguyen, M. Hors, and J. Perez Y. Jorba. 1963. "Study of Some Excited Levels of ${ }^{9}$ Be by Inelastic Electron Scattering." Nuclear Physics 42:62-74. http : / / www . sciencedirect.com/science/article/pii/0029558263907144

Padovani, Enrico. 2014. Private communication.

Padovani, Enrico, Sara A. Pozzi, Shaun D. Clarke, and Eric C. Miller. 2012. MCNPXPolimi Users Manual. 2.0. P.O. Box 2008, Oak Ridge, TN, USA 37831: Radiation Safety Informational Computer Center (RSICC).

Perillo Isaac, M. C., Y. D. Chan, D. L. Hurley, K. T. Lesko, M. E. Moorhead, E. B. Norman, A. R. Smith, et al. 1997. High Energy Gamma-Ray Measurements in the SNO Cavity. Technical report SNO-97-009. Sudbury Neutrino Observatory. http://www. sno.phy . queensu.ca/sno/str/rad.html.

Petke, F. David, and B. Roger Ray. 1969. "Temperature Dependence of Contact Angles of Liquids on Polymeric Solids." Papers Presented at the 43rd National Colloid Symposium, Journal of Colloid and Interface Science 31 (2): 216-227. doi:10.1016/00219797(69)90329-4.

Peyrou, Ch. 1967. "Bubble Chamber Principles." In Bubble and Spark Chambers, edited by R. P. Shutt. New York: Academic Press.

Phillips, M. C., and Riddifor.A. C. 1965. "Temperature Dependence of Contact Angles." Nature 205 (4975): 1005. doi:10.1038/2051005b0.

Planck Collaboration. 2014. "Planck 2013 Results. XVI. Cosmological Parameters." Astronomy and Astrophysics 571, A16 (November): A16. doi:10.1051/0004-6361/201321591.

Riepe, G., and B. Hahn. 1961. "Untersuchungen zum Mechanismus der Blasenbildung in Freon-12 und Propan durch Rückstosskerne einiger $\alpha$-Strahler." Helvetica Physica Acta 38 (VIII): 865-892.

Robinson, A. 2012. "High Energy Gamma-Ray Flux and Photonuclear Rates in COUPP-4kg at SNOLAB." COUPP internal document, no. 630. 
Robinson, Alan E. 2014. "New Libraries for Simulating Neutron Scattering in Dark Matter Detector Calibrations." Physical Review C 89 (3): 032801. doi:10.1103/PhysRevC.89. 032801 .

Rubin, V. C., and W. K. Ford Jr. 1970. "Rotation of the Andromeda Nebula from a Spectroscopic Survey of Emission Regions." The Astrophysical Journal 159 (February): 379. doi:10.1086/150317.

Russell, B., D. Sachs, A. Wattenberg, and R. Fields. 1948. "Yields of Neutrons from PhotoNeutron Sources." Physical Review 73 (6): 545-549. doi:10.1103/PhysRev.73.545.

Saiz-Lopez, A., R. W. Saunders, D. M. Joseph, S. H. Ashworth, and J. M. C. Plane. 2004. "Absolute Absorption Cross-Section and Photolysis Rate of $\mathrm{I}_{2}$." Atmospheric Chemistry and Physics 4 (5): 1443-1450. doi:10.5194/acp-4-1443-2004.

Sander, R. 2015. "Henry's Law Constants." In NIST Standard Reference Database Number 69: NIST Chemistry WebBook, edited by P. J. Linstrom and W. G. Mallard. Gaithersburg, MD, USA 20899: National Institute of Standards / Technology. Accessed August 16. http: //webbook.nist.gov.

Seitz, Frederic. 1958. "On the Theory of the Bubble Chamber." The Physics of Fluids 1:213.

Shibata, Keiichi, Osamu Iwamoto, Tsuneo Nakagawa, Nobuyuki Iwamoto, Akira Ichihara, Satoshi Kunieda, Satoshi Chiba, et al. 2011. "JENDL-4.0: A New Library for Nuclear Science and Engineering." Journal of Nuclear Science and Technology 48 (1): 1-30. doi:10.1080/18811248.2011.9711675.

Shores, E. F., G. E. Mueller, and G. A. Schlapper. 2003. "A New ${ }^{9} \operatorname{Be}(\alpha, n)$ Cross-Section Evaluation for use in the SOURCES Computer Code." Applied Radiation and Isotopes 59 (2-3): 151-158. doi:10.1016/S0969-8043(03)00157-X.

Singh, Sukhjeet, A. K. Jain, and Jagdish K. Tuli. 2011. "Nuclear Data Sheets for A = 222." Nuclear Data Sheets 112 (11): 2851-2886. doi:10.1016/j.nds.2011.10.002.

Smith, D. L., J. W. Meadows, and J. F. Whalen. 1981. "Cross Sections for ${ }^{19} \mathrm{~F}(\mathrm{n}, \mathrm{p}){ }^{19} \mathrm{O}$ and ${ }^{19} \mathrm{~F}(\mathrm{n}, \alpha){ }^{16} \mathrm{~N}$ from Threshold to $9 \mathrm{MeV}$." Nuclear Science and Engineering 77:256.

Smith, D. M., N. A. Bostrom, and E. L. Hudspeth. 1960. "Excitation Study of ${ }^{19} \mathrm{~F}(\mathrm{n}, \alpha){ }^{16} \mathrm{~N}$ with a $\mathrm{BaF}_{2}$ Crystal." Physical Review 117:514.

Snell, Arthur H., E. C. Barker, and R. L. Sternberg. 1950. "Photo-Disintegration Cross Sections of Deuterium and Beryllium for the Gamma-Rays of Sodium 24 and Gallium 72." Physical Review 80 (4): 637-642. doi:10.1103/PhysRev.80.637.

SNOLAB. 2006. SNOLAB User Handbook. Version rev. 2. Lively, ON, Canada. 
Sonneborn Refined Products B. V. 2012. PAROL White Mineral Oil, 1 March. Mainhavenweg 6, 1043 AL Amsterdam, The Netherlands.

Span, R., and W. Wagner. 1996. "A New Equation of State for Carbon Dioxide Covering the Fluid Region from the Triple-Point Temperature to $1100 \mathrm{~K}$ at Pressures up to 800 MPa." Journal of Physical and Chemical Reference Data 25 (6).

Spencer, R. R., G. C. Phillips, and T. E. Young. 1960. "Energy Levels of ${ }^{9}$ Be and ${ }^{9}$ B." Nuclear Physics 21:310-326. http://www. sciencedirect. com/science/article/ pii/0029558260900560.

Stetter, Georg. 1961. Investiation of the Decay of Tl-210 ( $\left.R a C^{\prime \prime}\right)$. Technical report TID14880. OSTI Identifier: 4809427. Vienna. Universitat. Physikalisches Institut.

Tilley, D. R., H. R. Weller, C. M. Cheves, and R. M. Chasteler. 1995. "Energy Levels of Light Nuclei A = 18-19." Nuclear Physics A 595 (1): 1-170. doi:10.1016/03759474(95) 00338-1.

Tims, S. G., A. J. Morton, C. I.W. Tingwell, A. F. Scott, V. Y. Hansper, and D. G. Sargood. 1991. "The ${ }^{54} \mathrm{Fe}(\alpha, n){ }^{57} \mathrm{Ni}$ and ${ }^{54} \mathrm{Fe}(\alpha, p){ }^{57} \mathrm{Co}$ Cross Sections." Nuclear Physics A 524 (3): 479-494. doi:10.1016/0375-9474(91)90281-A.

Tims, S. G., A. F. Scott, A. J. Morton, V. Y. Hansper, and D. G. Sargood. 1993. "Cross Sections of the Reactions ${ }^{58} \mathrm{Fe}(p, \gamma){ }^{59} \mathrm{Co},{ }^{58} \mathrm{Fe}(p, n){ }^{58} \mathrm{Co},{ }^{55} \mathrm{Mn}(\alpha, n){ }^{58} \mathrm{Co},{ }^{55} \mathrm{Mn}(\alpha, p){ }^{58} \mathrm{Fe}$ and ${ }^{57} \mathrm{Fe}(p, n){ }^{57}$ Co." Nuclear Physics A 563 (3): 473-493. doi:10.1016/0375-9474(93) 90124-G.

Tolman, Richard C. 1949. "The Superficial Density of Matter at a Liquid-Vapor Boundary." The Journal of Chemical Physics 17:118-127.

Tomasello, V., and V. A. Kudryavtsev. 2006. "Calculation of Neutron Yield from Radioactivity in Materials Relevant to Dark Matter Searches." In The Identification of Dark Matter, edited by M. Axenides, G. Fanourakis, and J. Vergados, 537. World Scientific.

Tsujimura, N., T. Yoshida, and T. Momose. 2007. "Calculations of Anisotropy Factors for Radionuclide Neutron Sources due to Scattering from Source Encapsulation and Support Structures." Radiation Protection Dosimetry 126 (1-4): 168-173. doi:10.1093/ $\mathrm{rpd} / \mathrm{ncm} 036$.

Tucker SN, Komarov VV, Treacy PB. 1970. "The 1.67 MeV Level in ${ }^{9}$ Be." Australian Journal of Physics 23 (1): 651-654. doi:10.1071/PH700651.

Utsunomiya, H., Y. Yonezawa, H. Akimune, T. Yamagata, M. Ohta, M. Fujishiro, H. Toyokawa, and H. Ohgaki. 2000. "Photodisintegration of ${ }^{9} \mathrm{Be}$ with Laser-Induced Compton Backscattered $\gamma$ Rays." Physical Review C 63 (1): 018801. doi:10.1103/PhysRevC. 63.018801 . 
Varlamov, V. V., B. S. Ishkhanov, I. V. Makarenko, V. N. Orlin, and N. N. Peskov. 2006. "Evaluation of ${ }^{127}$ I Photoneutron Reaction Cross Sections." Retrieved from NNDC EXFOR, Moscow State Univiversity Institute of Nuclear Physics Reports 2006 (9): 808. http: / / www . nndc . bnl.gov/exf or / servlet / X4sGetSubent? reqx=135408\&subID = 220693002 .

Veyssiere, A., H. Beil, R. Bergere, P. Carlos, A. Lepretre, and A. de Miniac. 1974. "A Study of the Photoneutron Contribution to the Giant Dipole Resonance of s-d Shell Nuclei." Nuclear Physics A 227:513.

Walker, R. L. 1946. Absolute Calibration of a Ra-Be Neutron Source. Technical report MDDC-414. Argonne National Laboratory: Atomic Energy Commission, October.

Wen, Wen-Yang, and JohnA. Muccitelli. 1979. "Thermodynamics of some perfluorocarbon gases in water." Journal of Solution Chemistry 8 (3): 225-246. doi:10.1007/ BF00648882.

West, D., and A. C. Sherwood. 1982. "Measurements of Thick-Target $(\alpha, n)$ Yields from Light Elements." Annals of Nuclear Engineering 9:551-577.

Wilmes, S., V. Wilmes, G. Staudt, P. Mohr, and J. W. Hammer. 2002. "The ${ }^{15} \mathrm{~N}(\alpha, \gamma){ }^{19} \mathrm{~F}$ Reaction and Nucleosynthesis of ${ }^{19}$ F." Physical Review $C 66$ (6): 065802. doi:10.1103/ PhysRevC.66.065802.

Wilson, W. B., R. T. Perry, E. F. Shores, W. S. Charlton, T. A. Parish, G. P. Estes, T. H. Brown, et al. 2002. SOURCES 4C: A Code for Calculating (alpha,n), Spontaneous Fission, and Delayed Neutron Sources and Spectra. Technical report LA-UR-02-1839. LANL, April.

Woosley, S. E., W. A. Fowler, J. A. Holmes, and B. A. Zimmerman. 1975. Tables of Thermonuclear Reaction Rate Data for Intermediate Mass Nuclei. Technical report OAP422. Pasedena, CA, USA: Kellogg Radiation Laboratory, California Institute of Technology.

Wu, S.-C. 2009. "Nuclear Data Sheets for A = 214." Nuclear Data Sheets 110 (3): 681-748. doi:10.1016/j.nds.2009.02.002.

Yellin, S. 2002. "Finding An Upper Limit in the Presence of an Unknown Background." Physical Review D 66 (3): 032005. doi:10.1103/PhysRevD.66.032005.

Ziegler, James F., J. P. Biersack, and M. D. Ziegler. 2008. SRIM - the Stopping and Range of Ions in Matter. Raleigh, NC, USA 27607: Lulu Press. 